\title{
Discriminating between spatial and temporal variations in seismic anisotropy at active volcanoes
}

\author{
Jessica Helen Johnson
}

September 22, 2011

A thesis submitted to Victoria University of Wellington as fulfilment for the degree of

Doctor of Philosophy in Geophysics

School of Geography, Environment and Earth Science

Victoria University of Wellington

Te Whare Wānanga o te Upoko o te Ika a Māui

Wellington, New Zealand 



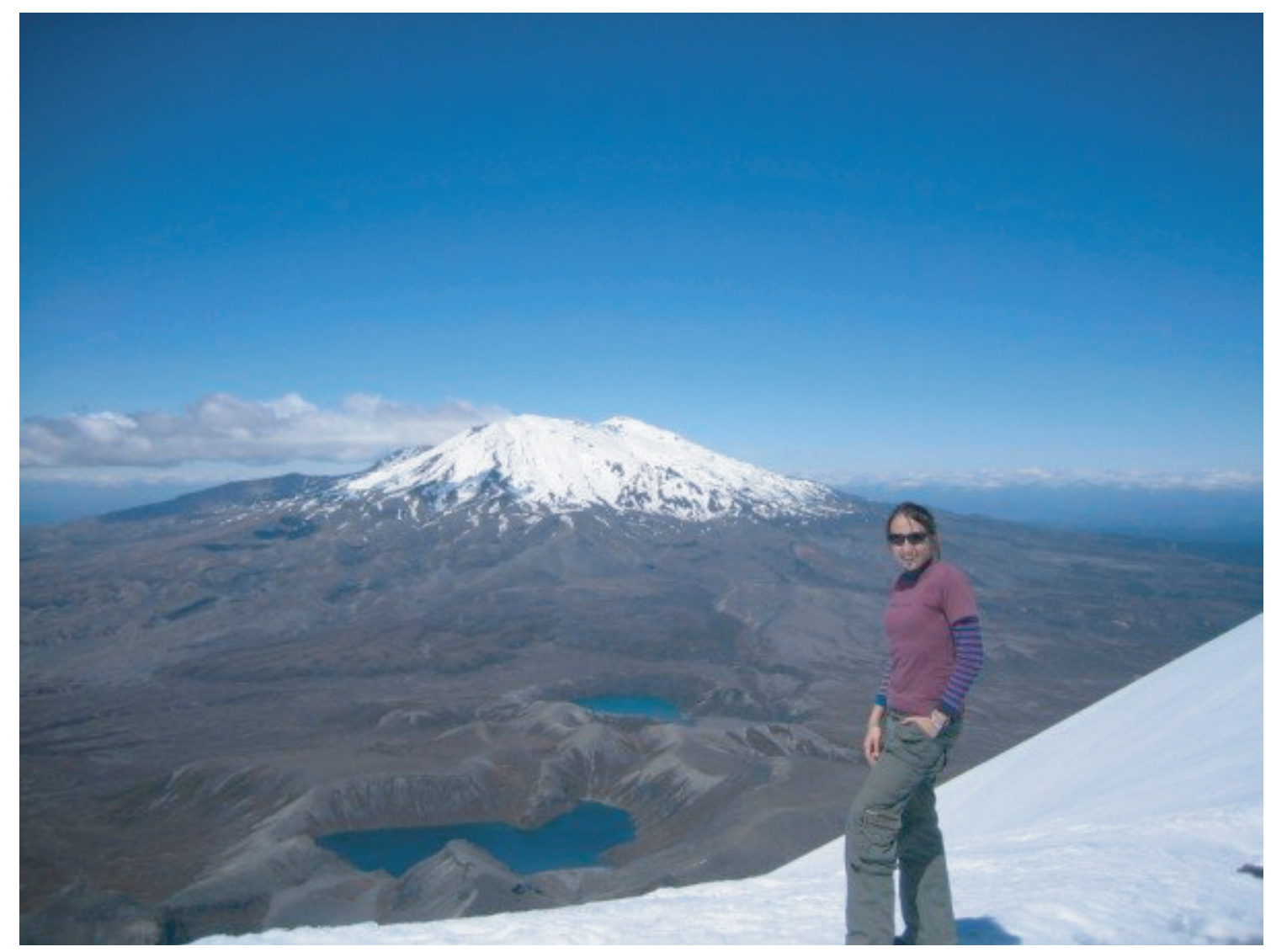

View of Mt. Ruapehu from the summit of Mt. Ngauruhoe

"Great things are done

when men and mountains meet."

William Blake

c. $1807-1809$ 



\section{Abstract}

This thesis addresses the measurement and interpretation of seismic anisotropy around active volcanoes via shear wave splitting analysis.

An overpressured magma reservoir will exert a stress on the surrounding country rock that may or may not be manifest as observable strain. Shear wave splitting analysis can be a useful indicator of stress in the crust and hence, the pressure induced by magma movement. Changes in shear wave splitting have already been observed at Mt. Ruapehu following eruptions in 1995/1996 and are inferred to be caused by changes in local stress in response to magma pressure. One of the main problems with the interpretation of temporal changes in shear wave splitting is the possibility of spatial variations being sampled along differing raypaths and being interpreted as temporal changes. Using a dense observational network and an automated shear wave splitting analysis, we examine local earthquakes occurring in 2008 within $100 \mathrm{~km}$ of Mt. Ruapehu. We note a strong azimuthal dependence of the fast direction of anisotropy $(\phi)$ and so introduce a spatial averaging technique and a two-dimensional tomography of recorded delay times $(\delta \mathrm{t})$, to observe the spatial variation in more detail. Using this new method of mapping shear wave splitting parameters, we have created a benchmark of spatial variations in shear wave anisotropy around Mt. Ruapehu, against which future temporal changes may be measured. The observed anisotropy is used to define regions in which $\phi$ agrees with stress estimations from focal mechanism inversions, suggesting stress-induced anisotropy, and those in which $\phi$ aligns with structural features such as fault strikes, suggesting structural anisotropy. Data from past deployments of three-component seismometers have been analysed in the same way as those recorded during the 2008 experiment and the results compared. We identify a stable region of strong anisotropy, interpreted to be caused by schistose mineral alignment, and a transient region of strong anisotropy centred on the volcano during the major magmatic eruption of 1995 .

We also introduce a method of analysing temporal variations in seismic anisotropy 
at active volcanoes by using tight clusters of earthquakes and highly correlated multiplets. At Mt. Ruapehu, changes in shear wave splitting parameters associated with the 2006 and 2007 phreatic eruptions are detected using a cluster of earthquakes to the west of the volcano. Similar analyses using another cluster and multiplets from the stable region of strong anisotropy do not reveal temporal changes, although examination of the waveform codas of the repeating earthquakes reveals systematic changes that we interpret as being caused by seismic scatterers associated with the 2006 and 2007 eruptions. These scatterers appear to contaminate the shear wave coda and so inhibit the detection of any subtle changes in shear wave splitting parameters.

Finally, we apply some of these methods to data from the 2008 eruption of Okmok volcano, Alaska. Shear wave splitting analysis at Okmok reveals a change in anisotropy associated with the 2008 eruption. This change however, is attributed to a change in dominant hypocentre location. Multiplet analysis at Okmok volcano reveals a similar scatterer contamination of the shear wave arrival. This spurious phase is interpreted to be an $\mathrm{S}$ to $\mathrm{P}$ conversion from interaction with the magma reservoir. 


\section{Acknowledgements}

It is a pleasure to thank the many people who travelled on this roller-coaster ride with me and made this thesis possible.

It is difficult to overstate my gratitude to my Ph.D. supervisors, Prof. Martha Savage and Dr. John Townend. Martha has been an inspiration to me, not only through her passion for all things seismically anisotropic, but also her enthusiasm for life and her ability to cope when all the deadlines pile up. Despite all of the demands on her time, Martha was always happy to discuss my latest results, and I thank her for treating me more as a colleague than a student. John's ability to think outside the box and his attention to detail have undoubtedly strengthened this thesis. His guidance has cultivated my approach to science for years to come.

This thesis would not have been possible without the data, and so I thank my field team, particularly Mark Henderson, who is a priceless component of the Geophysics department. A large proportion of my time has been dedicated to picking phases so I would like to thank Denise Fernandez and Rob Holt for helping with that mundane task and saving my eyesight. I am also indebted to Andrew Mellanby, who has shown the patience of a saint through all of the computer problems the department has faced. I gratefully acknowledge GeoNet for providing other earthquake data used in this study, and am particularly grateful to Kevin Fenaughty and Mark Chadwick for help with extracting waveform data from the GeoNet catalogue.

Funding for this project, including field work and travel further afield, was provided by Victoria University, The Earthquake Commission, The New Zealand Marsden Fund, and The Foundation for Research, Science and Technology. Their help was very much appreciated.

I and my work have indescribably benefitted from contact with a long list of friends and colleagues. Euan Smith, Tim Stern, Art Jolly, Tony Hurst and Steve Sherburn have greatly improved this work through interesting and insightful discussions. Welcome distractions and intense scientific debates have come from my office mates 
Sapi Karallyadda, Katrina Jacobs, Adrian Shelley and Sonja Greve, and the Friday morning/Monday afternoon crew: Carolin Boese, Kathi Unglert, Yannik Behr and Luke Jackson. I have to thank my original office mates, Hannu Seebeck and Dan Bassett, who set me up and started me going, and would like to mention those students who inspired me as much as I helped them: Sofia Kufner and Brook Keats. A great big thank you goes to the divers, Tony Howell, Andy Lett, Steve Journee, and others, who helped me escape.

Finally, a special and heartfelt thanks goes to Janie, Brian, Max and Ramsey, who, despite the time difference, were always there (and to skype for making that possible). And to Ben McLeod, Anya Seward and Rosie Cody, who shared my triumphs and kept my chin up when things were looking bleak, this thesis is for you. 


\section{Contents}

$\begin{array}{ll}\text { Abstract } & \text { iii }\end{array}$

Acknowledgements $\quad$ v

Contents vii

List of Figures . . . . . . . . . . . . . . . . . . . . . . . . . . . . . . . . . xi

List of Tables . . . . . . . . . . . . . . . . . . . . . . . . . . . . $\mathrm{xV}$

1. Overview and motivation $\quad 1$

2. Background 5

2.1. Introduction . . . . . . . . . . . . . . . . . 5

2.2. Mount Ruapehu . . . . . . . . . . . . . . . . . . . . 5

2.2.1. Introduction . . . . . . . . . . . . . . . . 5

2.2.2. Tectonic setting . . . . . . . . . . . . . 8

2.2.3. Crater Lake . . . . . . . . . . . . . . . . . . . . . . 9 9

2.2.4. History of hazards at Mount Ruapehu . . . . . . . . . . . . 11

2.2.5. Monitoring . . . . . . . . . . . . . . . 16

2.2.6. Seismology . . . . . . . . . . . . . . . . 19

2.2.7. Other geophysical observations . . . . . . . . . . . . 30

2.2.8. Some geological observations . . . . . . . . . . . . . . . . 32

2.3. Seismic anisotropy . . . . . . . . . . . . . . . . . . . . . 37

2.3.1. Introduction . . . . . . . . . . . . . . . 37

2.3.2. Theoretical models of anisotropy . . . . . . . . . . . . 37

2.3.3. Types of anisotropy . . . . . . . . . . . . . . . . . . . . . 40

2.3.4. Shear wave splitting . . . . . . . . . . . . . . . 45

2.3.5. Determination of shear wave splitting parameters . . . . . . . 48

2.3.6. Ambiguities and limitations . . . . . . . . . . . . 51

2.3.7. Temporal variation of shear wave splitting in the crust . . . . 60

2.3.8. Shear wave splitting around volcanoes . . . . . . . . . . 61

2.3.9. Spatial variation of shear wave splitting . . . . . . . . . 68 
2.4. Earthquake multiplets . . . . . . . . . . . . . . . . 71

2.4.1. Introduction . . . . . . . . . . . . . . . . 71

2.4.2. Identification of repeating earthquakes . . . . . . . . . . 71

2.4.3. Uses of repeating earthquakes . . . . . . . . . . . . . 75

2.5. Summary . . . . . . . . . . . . . . . . . . . . . . 81

3. Methodology 83

3.0.1. Introduction . . . . . . . . . . . . . . . . . 83

3.1. Data acquisition . . . . . . . . . . . . . . . . 83

3.1.1. Spatial Anisotropy Deployment At Ruapehu . . . . . . . . . . 83

3.1.2. GeoNet . . . . . . . . . . . . . . . . . . . . 84

3.1.3. Past deployments . . . . . . . . . . . . . . . 86

3.1.4. Alaska Volcano Observatory . . . . . . . . . . . . . . . . . . . 89

3.2. Data processing . . . . . . . . . . . . . . . . . 9 90

3.2.1. Waveform cross-correlation . . . . . . . . . . . . . 90

3.2.2. Double-difference relocation . . . . . . . . . . . . . . . 93

3.2.3. Shear wave splitting . . . . . . . . . . . . . . . . . 95

3.2.4. Circular statistics . . . . . . . . . . . . . . . . 103

3.2.5. Anisotropy analysis . . . . . . . . . . . . . . . . 106

3.2.6. Stress analysis . . . . . . . . . . . . . . . 109

4. Distinguishing between stress-induced and structural anisotropy at Mount Ruapehu volcano, New Zealand 111

4.1. Introduction . . . . . . . . . . . . . . . . . . . . . . . . . . 112

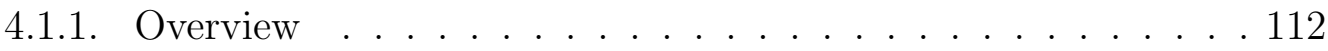

4.1.2. Previous studies of seismic anisotropy . . . . . . . . . . . . . . 114

4.1.3. Regional Geology . . . . . . . . . . . . . . . . . . . . 115

4.1.4. Stress Estimates . . . . . . . . . . . . . . . . 117

4.2. Data . . . . . . . . . . . . . . . . . . 122

4.3. Method . . . . . . . . . . . . . . . . . . . . . 124

4.3.1. Shear wave splitting . . . . . . . . . . . . . . . . . . 124

4.3.2. Delay Time Tomography . . . . . . . . . . . . . . . . . . . . . 127

4.3.3. Spatial Averaging . . . . . . . . . . . . . . . . . 135

4.4. Results . . . . . . . . . . . . . . . . . . . . 137

4.4.1. Shear wave splitting . . . . . . . . . . . . . . 137

4.4.2. Spatial Averaging . . . . . . . . . . . . . . . . . 142

4.4.3. Delay Time Tomography . . . . . . . . . . . . . . . . . . . . . 143 
4.5. Interpretation . . . . . . . . . . . . . . . . . . 146

4.5.1. Stress and Structure . . . . . . . . . . . . . . . . . 148

4.5.2. Anisotropy, Stress and Structure . . . . . . . . . . . . . 148

4.5.3. Delay Time Tomography . . . . . . . . . . . . . . . . . . 150

4.5.4. Modelling . . . . . . . . . . . . . . . . . . 151

4.6. Conclusions . . . . . . . . . . . . . . . . . . . . . 155

4.7. Obtaining and Using the Programs . . . . . . . . . . . 156

5. Multiplets and multiple projects at Mount Ruapehu: tools for the de$\begin{array}{ll}\text { tection of temporal variations in seismic anisotropy } & 157\end{array}$

5.1. Introduction . . . . . . . . . . . . . . . . . . . . . . . 158

5.2. Data . . . . . . . . . . . . . . . . . . . . 161

5.3. Method . . . . . . . . . . . . . . . . . . 162

5.3.1. Delay time tomography and spatial averaging of $\phi \ldots . . .162$

5.3.2. Shear wave splitting using clusters . . . . . . . . . . 163

5.3.3. Identification of multiplets . . . . . . . . . . . . . . . 165

5.4. Results and discussion . . . . . . . . . . . . . . . . . . . . 169

5.4.1. Delay time tomography . . . . . . . . . . . . . . 169

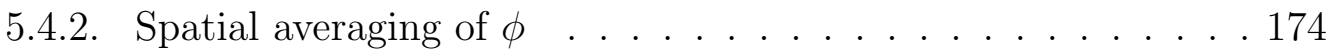

5.4.3. Shear wave splitting using clusters . . . . . . . . . . . 174

5.4.4. Shear wave splitting using multiplets . . . . . . . . . . . 178

5.4.5. Scatterers . . . . . . . . . . . . . . . 181

5.5. Conclusions . . . . . . . . . . . . . . . . . . . . . . . . 184

6. Anisotropy, repeating earthquakes and seismicity associated with the 2008 eruption of Okmok volcano, Alaska 187

6.1. Introduction . . . . . . . . . . . . . . . . . . . . . . . 188

6.1.1. Background . . . . . . . . . . . . . . . . 189

6.1.2. Seismic anisotropy ... . . . . . . . . . . . 193

6.2. Cross-correlation . . . . . . . . . . . . . . . . . . . 195

6.2.1. Method .................... 195

6.2.2. Results . . . . . . . . . . . . . . . 196

6.3. Relocation . . . . . . . . . . . . . . . . . . . . 198

6.3.1. Method . . . . . . . . . . . . . . . . 198

6.3.2. Results . . . . . . . . . . . . . . . 200

6.4. Shear wave splitting . . . . . . . . . . . . . . . 201

6.4.1. Method ..................... 201

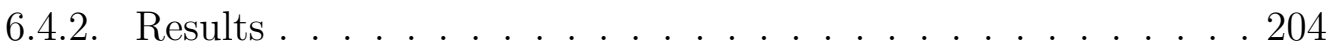


6.5. Discussion and interpretation . . . . . . . . . . . . . . 210

6.5.1. The multiplet . . . . . . . . . . . . . . . 210

6.5.2. Relocation . . . . . . . . . . . . . . . 211

6.5.3. Shear wave splitting . . . . . . . . . . . . . . 212

6.6. Conclusions . . . . . . . . . . . . . . . . . . . . 217

$\begin{array}{lr}\text { 7. Synthesis and conclusions } & 219\end{array}$

A. Appendix: Eruptive activity at Ruapehu 253

$\begin{array}{ll}\text { B. Appendix: Station details } & 265\end{array}$

C. Appendix: Data acquisition 277

C.1. Deployment logistics . . . . . . . . . . . . . . . . . . . . . . 277

C.2. Data processing manual . . . . . . . . . . . . . . . . . . 278

D. Appendix: Scripts $\quad 285$

D.1. CWB data retrieval . . . . . . . . . . . . . . . . . . 285

D.2. Phase picking . . . . . . . . . . . . . . . . . 287

E. Appendix: Parameters used in multiplet identification, double-difference relocation, anisotropy analysis, and Coulomb modelling 289

$\begin{array}{ll}\text { F. Appendix: Earthquake multiplets } & 301\end{array}$

G. The Erua earthquake cluster and seismic anisotropy in the Ruapehu region, New Zealand 315

G.1. Introduction . . . . . . . . . . . . . . . . . . 316

G.2. Data . . . . . . . . . . . . . . . . . . 317

G.3. Method . . . . . . . . . . . . . . . . 317

G.4. Results . . . . . . . . . . . . . . . . . . . . . 319

G.5. Discussion and conclusions . . . . . . . . . . . . . . . . . 323 


\section{List of Figures}

2.1. Photograph of Mount Ruapehu . . . . . . . . . . . . . . . . 6

2.2. Maps of the Ruapehu Region . . . . . . . . . . . . . . . . . . 7

2.3. Map of the TVZ . . . . . . . . . . . . . . . . . . . . 9 9

2.4. Ruapehu Crater Lake temperature since 2000 . . . . . . . . . . . . . 10

2.5. Sketches of Crater Lake dynamics . . . . . . . . . . . . . . . . . 12

2.6. Webcam images of Ruapehu . . . . . . . . . . . . . . . . . . 17

2.7. Tongariro Volcanic Centre seismograph network . . . . . . . . . . 18

2.8. Types of earthquakes recorded at Mt. Ruapehu . . . . . . . . . . . 20

2.9. Earthquakes in the Ruapehu region . . . . . . . . . . . . . . . . 22

2.10. Clusters of earthquakes in the Ruapehu region . . . . . . . . . . . . 25

2.11. Velocity models for the Tongariro National Park . . . . . . . . . . . . 27

2.12. New Zealand terranes . . . . . . . . . . . . . . . . . . . . 33

2.13. Ruapehu region faults . . . . . . . . . . . . . . . . . . 34

2.14. Geological models of Mount Ruapehu . . . . . . . . . . . . . . . . . . 35

2.15. Seismic wave propagation through anisotropic media . . . . . . . . . 42

2.16. Shear wave splitting in an anisotropic medium . . . . . . . . . . . . 46

2.17. Example of cycle skipping . . . . . . . . . . . . . . . . . 53

2.18. Example of window selection . . . . . . . . . . . . . . . . 54

2.19. Example of a null measurement . . . . . . . . . . . . . . . 55

2.20. $\phi$ as a function of incoming polarisation . . . . . . . . . . . . 56

2.21. Schematic of shear wave splitting in the case of two anisotropic layers 58

2.22. Volcano shear wave splitting investigations . . . . . . . . . . . . 62

2.23. Schematic stress and anisotropy model at Mt. Ruapehu . . . . . . . . 67

2.24. Multiplet waveforms in California . . . . . . . . . . . . . . . . . 72

2.25. Cross-correlation of similar earthquakes . . . . . . . . . . . . 73

2.26. Cross-correlation coefficients between earthquake pairs . . . . . . . . 74

2.27. Multiplet waveforms at Okmok . . . . . . . . . . . . . 76

3.1. SADAR network and earthquakes . . . . . . . . . . . . . 84

3.2. Flow diagram and directory structure for data processing . . . . . . . 85 
3.3. Map of past deployments . . . . . . . . . . . . . . . . . . . 87

3.4. Seismic network of Okmok volcano . . . . . . . . . . . . . . . . 90

3.5. Example of time window used for cross-correlation analysis . . . . . . 93

3.6. Schematic of the double-difference residual . . . . . . . . . . . . . . . 94

3.7. Examples of split shear wave particle motions . . . . . . . . . . . . . 96

3.8. Schematic of the steps to determine shear wave splitting parameters . 97

3.9. Example of cluster analysis . . . . . . . . . . . . . . . . . 98

3.10. Cluster types . . . . . . . . . . . . . . . . . . . . . 99

3.11. Flowchart of data processing steps for MFAST . . . . . . . . . . . . 100

3.12. Schematic of time windows around S arrival for cluster analysis . . . 101

3.13. Eigenvalue contour plot from shear wave spitting . . . . . . . . . . 102

3.14. Schematic of the vector resultant . . . . . . . . . . . . . . . . . . . . 104

3.15. Examples of output from 2-D delay time tomography steps . . . . . . 108

4.1. Map of the Ruapehu region showing network and 2008 seismicity . . 113

4.2. Zones of faults and clusters of earthquakes at Ruapehu . . . . . . . . 116

4.3. Steps to calculate $S_{\text {Hmax }}$ from focal mechanism inversions . . . . . . . 119

4.4. Relocated hypocentres of the 2008 catalogue . . . . . . . . . . . . . . 123

4.5. Representative raypaths . . . . . . . . . . . . . . . . . . . . . 124

4.6. Rose diagrams of $\phi$ at Ruapehu in 2008 . . . . . . . . . . . . . . . . . 128

4.7. Ruapehu region divided into grid blocks using quadtree gridding . . . 131

4.8. Delay time tomography . . . . . . . . . . . . . . . . . . . . . 132

4.9. Checkerboard test for tomographic inversion of delay time . . . . . . 133

4.10. Contours of statistical significance . . . . . . . . . . . . . . . 135

4.11. Spatial averages of $\phi$ in 2008 using various weighting functions . . . . 136

4.12. Spatial averages of $\phi$ in 2008 using $1 / d^{2}$ weighting function . . . . . . 138

4.13. Rose diagrams of $\phi$ from shallow and deep earthquakes . . . . . . . . 139

4.14. Analysis of earthquake depth with $\delta \mathrm{t} \ldots$. . . . . . . . . . . . 139

4.15. Temporal analysis of shear wave splitting data in 2008 . . . . . . . . 140

4.16. Azimuthal analysis of $\phi$ for stations TWVZ and WPVZ . . . . . . . . 141

4.17. Azimuthal analysis of $\phi$ and $\delta$ t for station TWVZ . . . . . . . . . . 143

4.18. Azimuthal analysis of fast polarisation data for all stations (a) . . . . 144

4.19. Azimuthal analysis of fast polarisation data for all stations (b) . . . . 145

4.20. Comparison of data and inferred anisotropy mechanisms around Mount Ruapehu . . . . . . . . . . . . . . . . . . . . . 147

4.21. Results from Coulomb modelling of a dike . . . . . . . . . . . . 152

5.1. Map of the Tongariro Volcanic Centre . . . . . . . . . . . . . . . . 160 
5.2. Grids, rays and stations of past deployments . . . . . . . . . . . . . 164

5.3. Flowchart of multiplet construction . . . . . . . . . . . . . . . 165

5.4. Temporal evolution of multiplets and instrument operation times . . . 168

5.5. Delay time tomography for past deployments . . . . . . . . . . . . . . 170

5.6. Jackknife tests . . . . . . . . . . . . . . . . . . . . . . . . 171

5.7. Checkerboard tests . . . . . . . . . . . . . . . . 173

5.8. Spatial averaging of fast direction for past deployments . . . . . . . . 175

5.9. Spatial averaging of fast direction compared to the 2008 deployment . 176

5.10. Waiouru shear wave splitting . . . . . . . . . . . . . . . 177

5.11. Geothermal system at Tongariro . . . . . . . . . . . . . . . . . 178

5.12. Multiplet shear wave splitting . . . . . . . . . . . . . . . . 179

5.13. Multiplet incoming polarisations . . . . . . . . . . . . . . . . . . 180

5.14. Shear wave splitting over time using multiplets . . . . . . . . . . . . 181

5.15. Waveforms of multiplet 14 . . . . . . . . . . . . . . . . 182

5.16. Cross-correlation matrix for multiplet $14 \ldots \ldots$. . . . . . . 183

6.1. Map of Okmok volcano . . . . . . . . . . . . . . . . . 190

6.2. Seismicity leading up to eruption at Okmok . . . . . . . . . . . . 191

6.3. Multiplet earthquakes . . . . . . . . . . . . . . . . . . 197

6.4. Relocations of Okmok earthquakes . . . . . . . . . . . . . . . . 201

6.5. Relocation of multiplet earthquakes . . . . . . . . . . . . . . . . 202

6.6. Shear wave splitting results from Okmok earthquakes . . . . . . . . . 203

6.7. Example of shear wave splitting cluster analysis . . . . . . . . . 205

6.8. Shear wave splitting analysis results from station $\mathrm{OKSO}$. . . . . . 206

6.9. Shear wave splitting results for the multiplet over time . . . . . . . 208

6.10. Coulomb modelling at Okmok . . . . . . . . . . . . . . . . . 213

C.1. Guralp (CMG3-ESP) . . . . . . . . . . . . . . . . . . 278

C.2. Reftek seismograph, batteries and cables . . . . . . . . . . . . 279

C.3. Complete set up of seismic station . . . . . . . . . . . . . . . . . 280

F.1. Multiplet 1-5 waveforms and $C C C$ matrices . . . . . . . . . . . . . . 309

F.2. Multiplet 6-10 waveforms and $C C C$ matrices . . . . . . . . . . . . 310

F.3. Multiplet 11-15 waveforms and $C C C$ matrices . . . . . . . . . . . . . 311

F.4. Multiplet 16-20 waveforms and $C C C$ matrices . . . . . . . . . . . . . 312

F.5. Multiplet 21-25 waveforms and $C C C$ matrices . . . . . . . . . . . . . 313

F.6. Multiplet 26-29 waveforms and $C C C$ matrices . . . . . . . . . . . . 314

G.1. Earthquake relocations ar Erua . . . . . . . . . . . . . . . . 318 
G.2. Moving average results at FWVZ . . . . . . . . . . . . . . . 321

G.3. Moving average results at stations FWVZ, MOVZ, TWVZ and WNVZ322

G.4. Maps of earthquake location coloured by $\phi$. . . . . . . . . . . . . . . 324 


\section{List of Tables}

2.1. Anisotropy of Earth's materials . . . . . . . . . . . . . . . . . . . 41

4.1. Previous stress estimates in the Ruapehu region . . . . . . . . . . 118

4.2. Comparison of fault strike, metamorphic fabric, $S_{H \max }$ and $\phi$. . . . 121

4.3. Shear wave splitting measurements in 2008 . . . . . . . . . . . . . . . 125

4.4. Parameters used in $\delta$ t 2-D tomography and spatial averaging . . . . . 130

4.5. Coulomb models tested . . . . . . . . . . . . . . . . . . . . . 154

5.1. Parameters used in tomography and spatial averaging. . . . . . . . 163

5.2. Multiplet earthquakes . . . . . . . . . . . . . 166

6.1. Catalogue details of the events belonging to the multiplet . . . . . . 199

6.2. Number of events used in shear wave splitting at Okmok . . . . . . . 204

6.3. Shear wave splitting analysis parameters applied to the multiplet . . . 205

6.4. Statistical analysis of shear wave splitting results using the multiplet 209

A.1. Historical activity of Mount Ruapehu . . . . . . . . . . . . . . . . . 254

A.2. Phases of the 1995/1996 Ruapehu Eruption . . . . . . . . . . . . . 262

B.1. Station details with number of earthquakes recorded . . . . . . . . 266

B.2. Stations used in past deployments . . . . . . . . . . . . . 268

E.1. Repeating earthquake parameters . . . . . . . . . . . . . . . 290

E.2. Parameters used in quake searches . . . . . . . . . . . . . . . . . . 294

E.3. Parameters used during double-difference relocation . . . . . . . . . . 295

E.4. Weighting of iteration sets in hypoDD analysis . . . . . . . . . . 297

E.5. Filters tested during MFAST . . . . . . . . . . . . . . 298

E.6. Quality criteria for grading during MFAST . . . . . . . . . . . . . . 298

E.7. Parameters used in Coulomb modelling . . . . . . . . . . . . . . 300

F.1. Shear wave splitting results using the multiplets . . . . . . . . . . . 302

G.1. Velocity model used during earthquake relocation . . . . . . . . . . . 319 
G.2. Statistical analysis at FWVZ . . . . . . . . . . . . . . . . . 320 


\section{Overview and motivation}

Modern geophysical techniques enable changes to be observed at some volcanoes before magmatic eruptions: detection of seismicity from magma pushing through cold country rock is one of the most common and successful monitoring techniques and can lead to short-term predictions. The famous example of the $1980 \mathrm{Mt}$. St. Helens eruption is one such case, in which elevated rates of seismicity $3-7 \mathrm{~km}$ beneath the point of eruption for 56 days prior to the eruption suggested to scientists that an eruption was imminent, enabling certain actions to try to prevent loss of life [Geophysics Program, University of Washington, 1980; McNutt, 1996]. Another example of geophysical precursers to eruptions is surface deformation from inflation or deflation of a volcano due to magma movement, which has been detected with Interferometric Synthetic Aperture Radar (InSAR) at Okmok Volcano in the Aleutians [Lu et al., 2005] and in the Afar region of Ethiopia [Barisin et al., 2009] among others. However, some volcanoes do not display these clues, and there remains a need for techniques that are sensitive to other physical attributes that might change in conjunction with the eruption process.

Lu et al. [2005] were able to observe the surface deformation of magma pooling in a shallow reservoir at Okmok. Any overpressured magma storage reservoir, be it a system of dikes, sills, conduits, spherical chamber or a combination of these, will exert a stress on the surrounding country rock that may or may not be manifest as observable strain. Detecting and understanding this stress may be a key to predicting if and when a volcano will erupt.

The underlying motivation of this project is to determine whether anisotropy measured from shear wave splitting changes around a volcano prior to and during magmatic eruptions. The temporal variation of shear wave splitting and its interpretation is highly controversial [e.g. Aster et al., 1990; Bokelmann and Harjes, 2000; Seher and Main, 2004; Liu et al., 2004; do Nascimento et al., 2004; Peng and BenZion, 2005; Liu et al., 2008]. One of the main problems with the interpretation of temporal changes in shear wave splitting is the possibility that spatial variations 
are sampled at different times when measurements are made along different propagation paths, and that these spatial variations masquerade as temporal variations [e.g. Kaneshima, 1990; Liu et al., 2004; Boness and Zoback, 2006a]. Changes in anisotropy have already been reported at Mt. Ruapehu following the 1995/1996 eruptions (see Section 2.2.6 and Miller and Savage [2001] for more details) and interpreted to be caused by changes in local stresses in response to magma pressure. In this study we aim to develop and extend the investigation of these changes. This will lead to new understanding of both seismic anisotropy and the magmatic plumbing system so that methods of monitoring can be advanced and improved, ideally leading to more reliable eruption prediction.

In order to achieve these goals, three investigations were conducted. First, a dense network of seismometers was deployed between January and December 2008 to supplement the permanent network [GeoNet, Last accessed 17 April 2011, http: //www . geonet.org.nz] and resolve the spatial extent of the anisotropy around Mt. Ruapehu, which differs from the regional trend. Using an automated shear wave splitting analysis, we examined local earthquakes that occurred in 2008 within 100 $\mathrm{km}$ of the volcano. We observe a strong azimuthal dependence of fast direction of anisotropy $(\phi)$ and so introduce a spatial averaging technique and a two-dimensional tomography of recorded delay times $(\delta \mathrm{t})$, to observe the spatial variation in more detail. This yields a reference map of anisotropy against which to measure future changes. The anisotropy can be divided into regions in which $\phi$ agrees with stress estimations from focal mechanism inversions, suggesting stress-induced anisotropy, and those in which $\phi$ is aligned with structural features such as fault strikes, suggesting structural anisotropy. This investigation is described in Chapter 4.

Second, as shear wave splitting can be highly dependent on the raypath, methods have been sought to mitigate the problem of spatial variations being erroneously interpreted as temporal changes. We have repicked and re-analysed temporary deployments of three-component seismometers around Mt. Ruapehu in 1994, 1995, 1998, 2001, 2002 and 2008. The shear wave splitting results are inverted for twodimensional delay time tomography and a spatial averaging of fast directions undertaken. This analysis takes into account the differing earthquake and sensor locations during each of the deployments and enables data from each time period to be compared. We also use clusters of earthquakes, families of similar earthquakes and permanent seismic stations to analyse temporal variations of shear wave splitting along similar paths. This investigation is described in Chapter 5.

Finally, the method of identifying repeating earthquakes and using them to mon- 
itor anisotropy before, during and after an eruption has been tested at Okmok volcano in the Aleutian Arc, Alaska. Okmok has recently experienced a large magmatic eruption, recorded by a network of seismometers and by GPS as part of the Alaska Volcano Observatory's (AVO) [Alaska Volcano Observatory, Last accessed 22 Feb 2011, http://www.avo.alaska.edu] monitoring system. As mentioned above, Okmok has displayed other evidence of magma pooling and charging, so this location was chosen to investigate how magma charging is linked to stress changes causing shear wave anisotropy. This investigation is described in Chapter 6 .

In summary, this project develops the interpretation of shear wave splitting at volcanoes and provide a method of monitoring stress caused by filling of a magma reservoir before an eruption. This method uses repeating sources so that the changes in seismic anisotropy observed occur along a specific path, in order to eliminate the uncertainty caused by different raypaths.

Chapter 2 of this thesis contains a review of the relevant literature about Mt. Ruapehu, including geophysical and geological observations. This is included because a good interpretation of the data needs to be consistent with all available evidence.

Chapter 3 outlines in detail most of the methods and software used in this project. In some cases important or useful scripts are included in the appendices. Chapters 4, 5 and 6 have been written as stand-alone papers. Chapter 4 is in review and has been resubmitted after minor revisions to the Journal of Geophysical Research at the time of writing. Chapter 5 is in preparation for submission. Chapter 6 is published in a Journal of Geophysical Research special edition for the 2008 eruptions of Okmok and Kasatochi volcanoes. The structure of this thesis means that there is some repetition of methodology and background as some explanation was needed in the individual papers, however, the explanations in the background and methodology chapters are more thorough.

Appendices A-G provide supplementary material. Appendix A summarises the eruptive activity of Mt. Ruapehu. Appendix B provides information about the temporary and permanent seismic stations around Mt. Ruapehu. Appendix C contains instructions for the acquisition of seismic data. Appendix D provides some useful scripts for processing seismic data. Appendix E lists useful parameters that are used in multiplet identification, double-difference relocation, anisotropy analysis, and Coulomb modelling. Appendix F contains results from Chapter 5, using the multiplets. Appendix G also includes a paper that has been published in Geophysical Research Letters. I provided guidance to the author during the research for 
the Honor's thesis [Keats, 2010], prepared the paper for publication and was corresponding author for the submission. This paper ties closely with Chapter 5 and a lot of the work was done in conjunction with this thesis. 


\section{Background}

\subsection{Introduction}

The subject of temporally varying anisotropy is a hotly debated topic [e.g. Aster et al., 1990; Bokelmann and Harjes, 2000; Seher and Main, 2004; Liu et al., 2004; do Nascimento et al., 2004; Peng and Ben-Zion, 2005; Liu et al., 2008; Crampin and Peacock, 2008]. Spatial variations of anisotropy masquerading as temporal variations is one of the main caveats associated with the interpretation [Liu et al., 2004]. The temporal variations in anisotropy associated with major magmatic eruption at Mount Ruapehu [Miller and Savage, 2001; Gerst and Savage, 2004] have been subject to these doubts and so in this thesis we aim to test whether changes in anisotropy with time can be reliably used as an eruption forecasting tool. To do this we must first have a full understanding of the problem at hand. We must gain an understanding of the spatial variations in anisotropy at Mt. Ruapehu before temporal changes can be examined. This chapter contains an introduction to shear wave splitting and a review of the geophysical and geological literature that is relevant to the interpretation in Chapters 4 and 5. This chapter also includes a review of the use of multiplets, which have been widely used to detect changing path properties, while mitigating the problem of heterogeneity [e.g. Baisch et al., 2008, and references therein]. Understanding the occurrence, detection and use of multiplets underpins the analyses employed in Chapters 5 and 6.

\subsection{Mount Ruapehu}

\subsubsection{Introduction}

Mount Ruapehu is an active, predominantly andesitic, composite volcano (Figure 2.1). It is the highest mountain $(2797 \mathrm{~m})$ on the North Island of New Zealand and is 


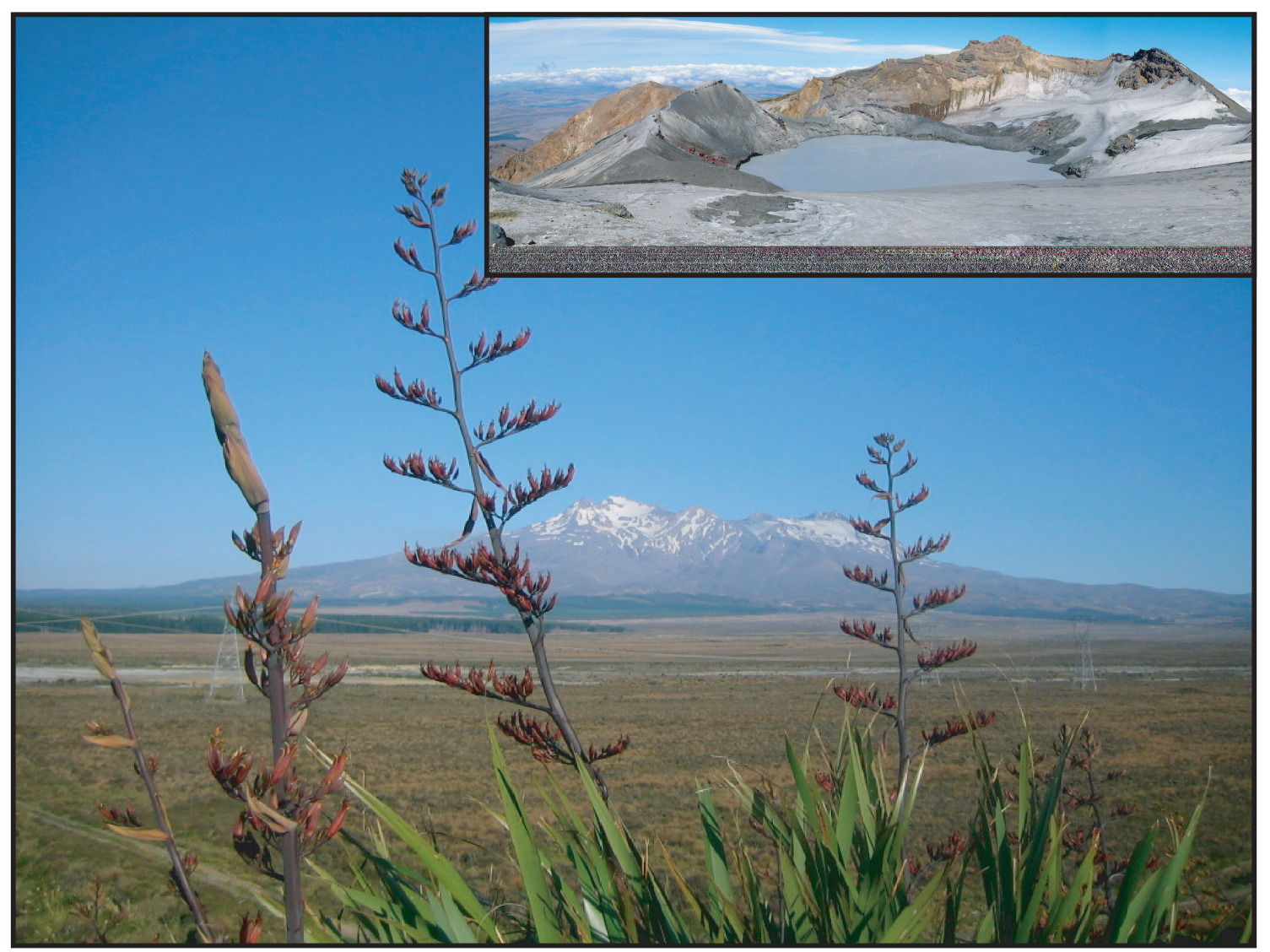

Figure 2.1. Photograph of Mount Ruapehu from the east in spring. Inset: photograph of Crater Lake in summer.

one of a small number of active volcanoes that are topped by a crater lake that fills the active vent (Figure 2.1 inset). The summit is composed of overlapping craters that have been active since the Holocene [Hackett and Houghton, 1989], the currently active one of which is South Crater [Nairn et al., 1979], filled by Crater Lake (Figure 2.2 top). Major magmatic eruptions occurred in 1945 and 1995/1996; the latter was the largest historical eruption of Mt. Ruapehu, producing a $12 \mathrm{~km}$-high volcanic ash plume and lahars on the flanks of the volcano [Bryan and Sherburn, 1999].

Phreatic and phreatomagmatic eruptions frequently occur at Mt. Ruapehu [Hurst et al., 2004], which also threaten lives and property [Johnston et al., 2000]. There are three ski fields on the mountain, Whakapapa, Turoa and Tukino (Figure 2.2 top), all three of which lie in high risk zones [Houghton et al., 1987]. There is also a hydrolectric power scheme around Mt. Ruapehu with a major dam at Lake Moawhango (Figure 2.2 bottom). The principal road and rail routes for the North Island cross the ring plain east and west of the volcano (Figure 2.2 bottom). 


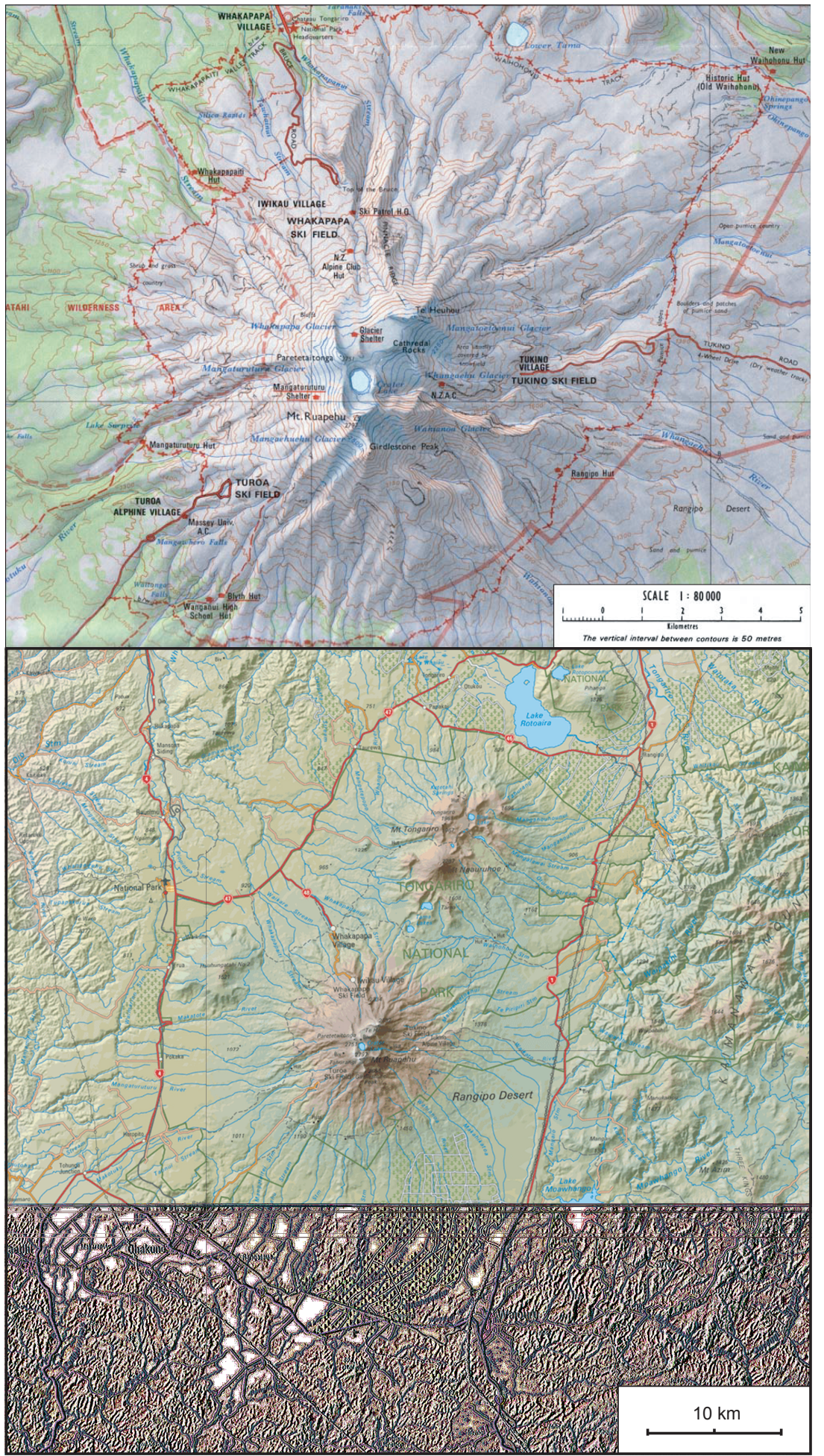

Figure 2.2. Top: Map of Ruapehu Mountain showing trails and ski fields [New Zealand Map Series (NZMS) 273 Tongariro National Park, from http://www.doc.govt.nz Last accessed 22 February 2011]. Bottom: Road map of the Ruapehu region [from http://www.wises.co.nz Last accessed 22 February 2011]. 


\subsubsection{Tectonic setting}

New Zealand forms part of the boundary between the Australian and Pacific tectonic plates (Figure 2.3 left). To the south of the South Island this plate interface dips steeply eastward as the Australian plate subducts beneath the Pacific plate at the Puysegur subduction zone [e.g. Lamarche and Lebrun, 2000]. Northeast of the North Island the Pacific plate subducts beneath the Australian plate along the obliquelywestward dipping Hikurangi subduction zone [e.g. Walcott, 1987]. Along the western side of the South Island the transpressive Alpine Fault accommodates the change in subduction sense [e.g. Wellman, 1971].

Mount Ruapehu is the southernmost of the large active volcanoes on the North Island (Figure 2.3). It is located at the southern end of the Taupo Volcanic Zone (TVZ), an extending continental back-arc system resulting from the subduction of the Pacific Plate beneath the Australian Plate (Figure 2.3 left) [Stern et al., 2010]. The TVZ is regarded as the southern onshore continuation of the Havre trough, an extending basin NNE of New Zealand defined by active normal faulting and volcanism [e.g. Walcott, 1987; Wright, 1993]. Within the TVZ, extension occurs within the volcanic arc, as opposed to most of the Havre Trough, which has a well defined back-arc basin to the west of the subduction zone and the active volcanic arc to the east of the tectonic basin [Villamor and Berryman, 2006]. Most models depict extension of the TVZ/Havre Trough gradually decreasing from up to 30-40 mm/yr in the north [Davey et al., 1995] to a point of zero extension at the southern termination [Beanland and Haines, 1998; Rowland and Sibson, 2001; Wallace et al., 2004]. The TVZ is characterised by high crustal heat flow (between $600 \mathrm{~mW} / \mathrm{m}^{2}$ in the south and $2000 \mathrm{~mW} / \mathrm{m}^{2}$ in areas of rhyolitic melts [Hochstein, 1995]), numerous shallow earthquakes ( $<8 \mathrm{~km}$ [Bryan and Sherburn, 1999]), and active extensional faulting [Bibby et al., 1995]. Volcanism within the TVZ is predominantly rhyolitic in the centre with andesite stratovolcanoes at the northern and southern extremities [Wilson et al., 1995]. Rhyolite volcanism is generally manifest in catastrophic caldera-forming eruptions, the last of which was the AD 180 Hatepe eruption of Taupo caldera [Wilson et al., 1995]. Other activity takes the form of geysers and hotsprings in the ca. 20 geothermal areas [Bibby et al., 1995] (Figure 2.3). 


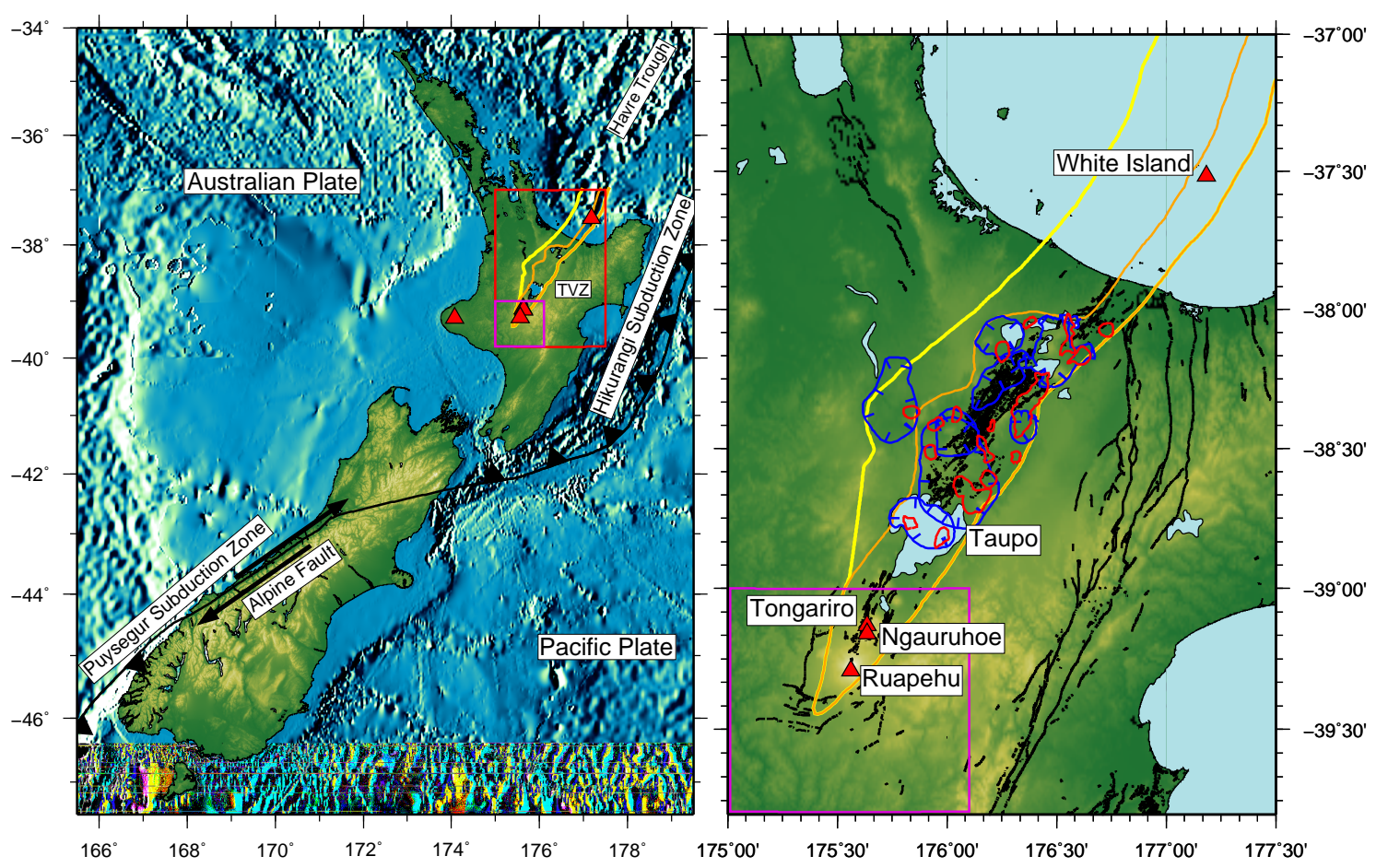

Figure 2.3. Left: Map of New Zealand showing the Hikurangi margin, Alpine Fault and Puyseger margin in black. Red box is area of TVZ map. Right: Map of the TVZ showing mapped faults in black, calderas in blue and active geothermal areas in red. Red triangles show locations of active stratovolcanoes. Orange and yellow curves are the young and old TVZ outlines respectively, after Wilson et al. [1995]. Magenta boxes show main study area.

\subsubsection{Crater Lake}

Crater Lake is the source of the most frequent hazards at Mt. Ruapehu. The incorporation of large amounts of water into magmatic eruptions often results in more explosive and violent phreatomagmatic events [Starostin et al., 2005]. Hydrothermal or phreatic eruptions frequently occur at Ruapehu Crater Lake when the surface lake temperature is above $40^{\circ} \mathrm{C}$ [Hurst and Dibble, 1981]. These conditions are probably only possible when the Crater Lake has a depth of $175 \mathrm{~m}$ or greater: this is the greatest depth for which Hurst and Dibble [1981] found that a small scale convection model fitted the data, implying that at depth the lake is on the margin of a boiling instability.

Lahars from the Crater Lake pose one of the greatest threats to people on the ski fields and also in the low lying areas of the ring plain [Hurst et al., 2004]. A breakout lahar caused one of the worst natural disasters in the history of New Zealand, the Tangiwai disaster, killing 151 people as their train was caught in the flow [Manville et al., 2007].

Prior to the 1995 eruption, Crater Lake was about 150 m deep lying over a breccia- 


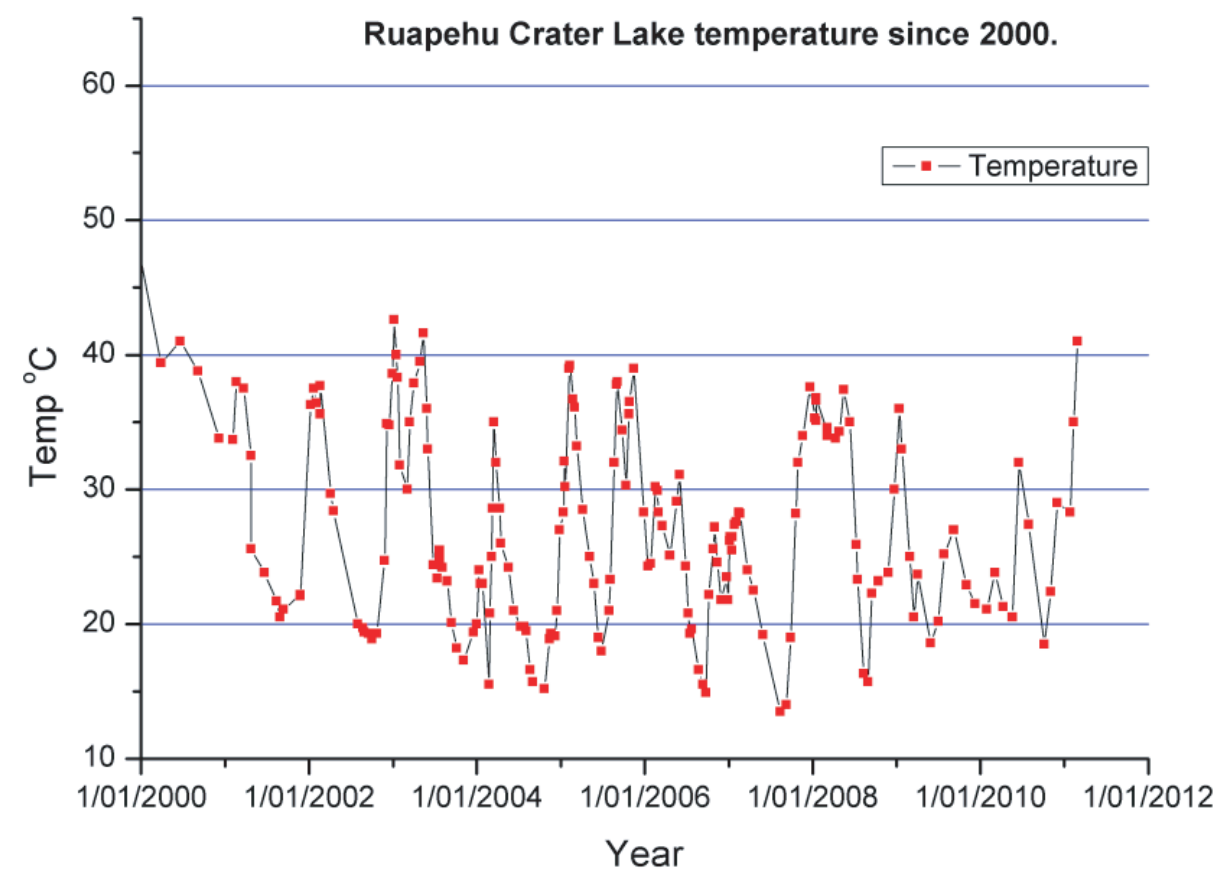

Figure 2.4. Ruapehu Crater Lake temperature since 2000 [courtesy of GeoNet, Last accessed 17 April 2011, http://www.geonet.org.nz/volcano/alert-bulletins/archives/2011/ alert-bulletin-feb-21-2011-4-00-pm-ruapehu-volcano.html].

filled vent in which a pool of molten sulphur controlled heat flow into the lake [Hurst et al., 1991; Christenson, 1994]. Crater Lake's volume varies as lava emplacement or explosive volcanism modifies the bathymetry, and has a catchment for precipitation of about $0.8 \mathrm{~km}^{2}$ [Hurst et al., 1991]. It is usually maintained at the overflow level by meltwater from ice and precipitation on the summit catchment and by condensation of magmatic steam, but the explosions can expell water and lower the lake level to far below overflow. During the 1980s the temperature of Crater Lake was found to cycle between $\sim 15$ and $40^{\circ} \mathrm{C}$ with a 6-12 month periodicity [Hurst et al., 1991]. This phenomenon is, as of 18 April 2011, currently observed with a similar periodicity (Figure 2.4). Hurst et al. [1991] analysed heat and mass input and output of Crater Lake in order to derive a model of the dynamics of the lake (Figure 2.5) and the phreatic eruptions described by Latter [1981b]. They found that only $50 \%$ of heat going into the lake is from magmatic steam and so to account for the other heat input, they concluded that at lower levels $(>100 \mathrm{~m})$ the lake will consist of a series of immiscible liquids or suspensions separated by definite boundaries. Within the throat of the volcano, Hurst et al. [1991] suggested that the mechanism 
for transferring heat from depth is in the form of a heat pipe with steam rising and liquid falling through a porous or fractured medium. This model proposed by Hurst et al. [1991] was stable and provided enough heat without additional net mass flux; however, it did not explain the cyclic heating and cooling that was observed in the 1980s [Hurst et al., 1991] and 2000s (Figure 2.4), with a periodicity of 6 months to a year. The cooling can be achieved by a thin impermeable layer at the upper boundary of the heat pipe as conduction will not provide sufficiently rapid heat transfer. Hurst et al. [1991] suggested that a layer of sulphur could play an important role. Elemental sulphur has been observed to exist within the waters of Crater Lake [Giggenbach, 1974], and the density of sulphur relative to water favours accumulation on the bottom of the lake. Sulphur pools have also been observed at volcanoes in Costa Rica [Oppenheimer and Stevenson, 1989] and submarine volcanoes in the Kermadec Arc [C. de Ronde pers. comm. 2009]. The viscosity of liquid sulphur is highly temperature dependant (the viscosity changes by four orders of magnitude in the temperature range of $159^{\circ} \mathrm{C}$ to $200^{\circ} \mathrm{C}$ ) and Hurst et al. [1991] demonstrated that this property provides a mechanism for the cycles of low and high heat input into the lake. Recent integrated finite-difference heat-mass transport simulations [Christenson et al., 2010] agree with this model (Figure 2.5).

\subsubsection{History of hazards at Mount Ruapehu}

Phreatic and phreatomagmatic explosive eruptions have occurred on average every 5 years historically (see Appendix A.1 for details). All historic eruptions have originated from the southern summit crater occupied by the $0.16 \mathrm{~km}^{2}$ Crater Lake with $10^{7} \mathrm{~m}^{3}$ of hot $\left(20-50^{\circ} \mathrm{C}\right)$, acidic $(\mathrm{pH}<1)$ water [Kilgour et al., 2010]. Phreatic eruptions, when a small amount of basic andesite interacts with Crater Lake water, have dominated historic eruptions [Houghton et al., 1987; Kilgour et al., 2010], although six major magmatic eruptions have happened in historic times; in 1861, 1945, 1969, 1971, 1975 and 1995-1996.

\section{The 1945 eruption}

In 1945, magma extrusion took place with dome-building. The dome completely filled the crater and was later destroyed by explosions [Houghton et al., 1987]. Activity was first recorded on 8 March 1945, when a steam plume was observed coming 
Hurst et al. (1991)
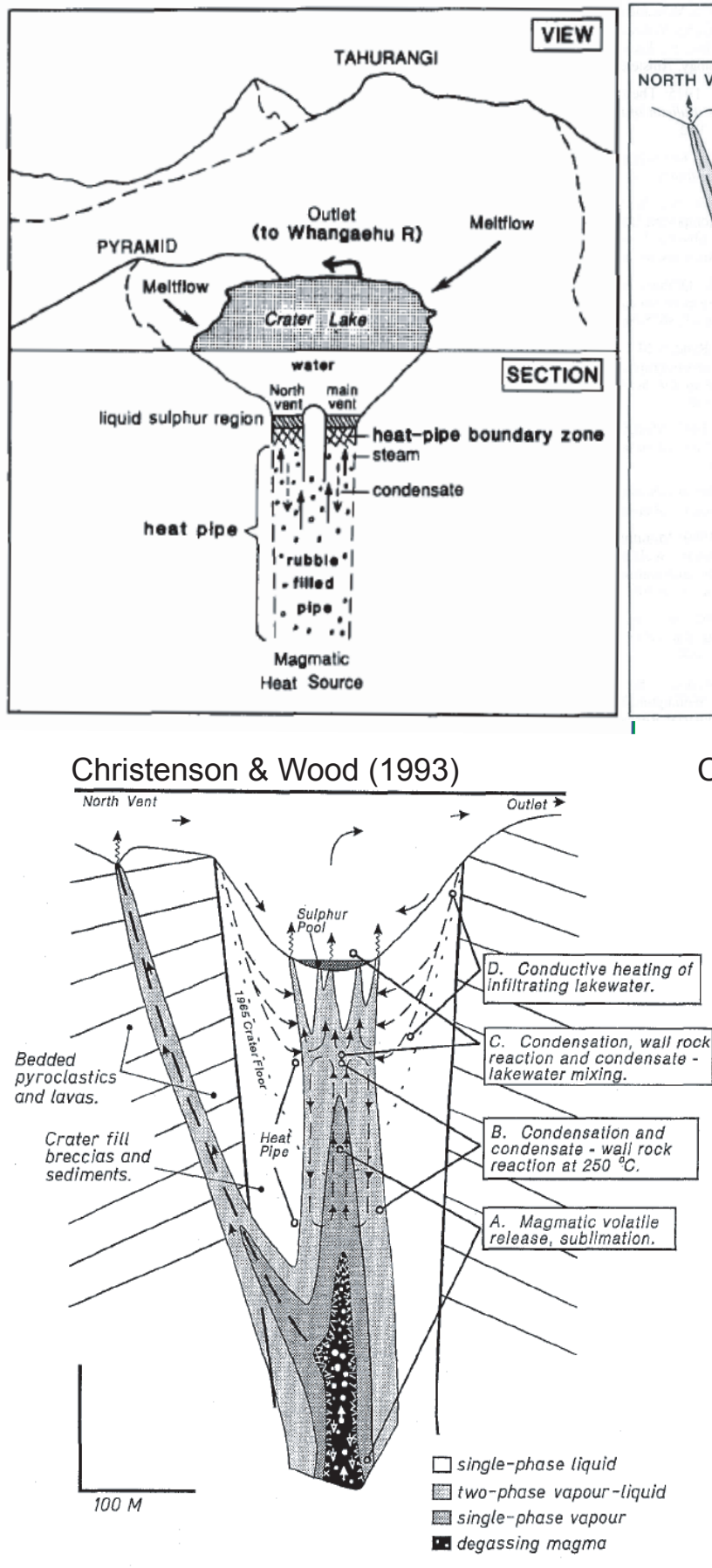

Hurst \& Sherburn (1993)

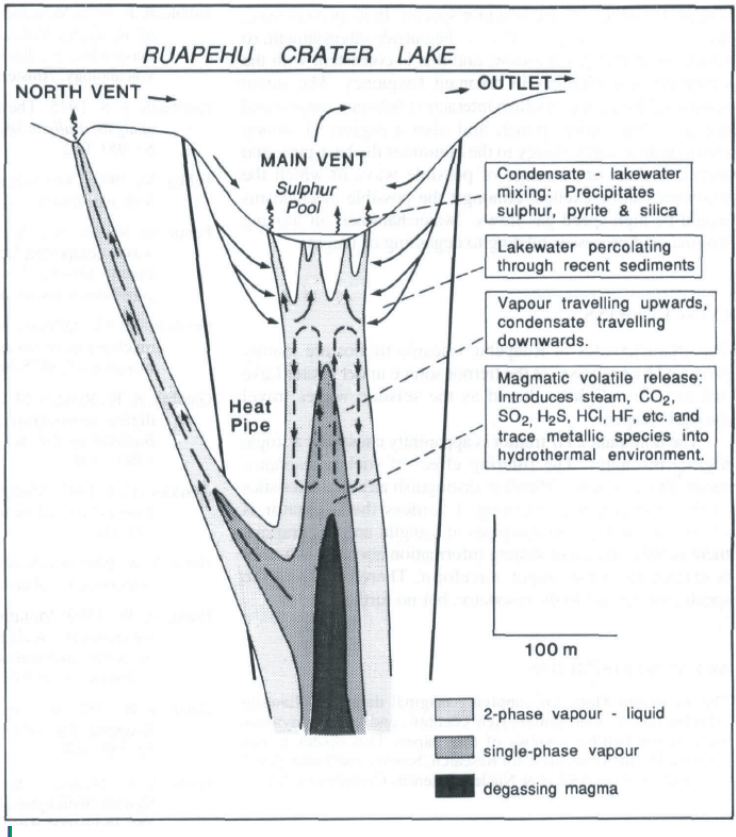

Christenson et al. (2010) September, 2007

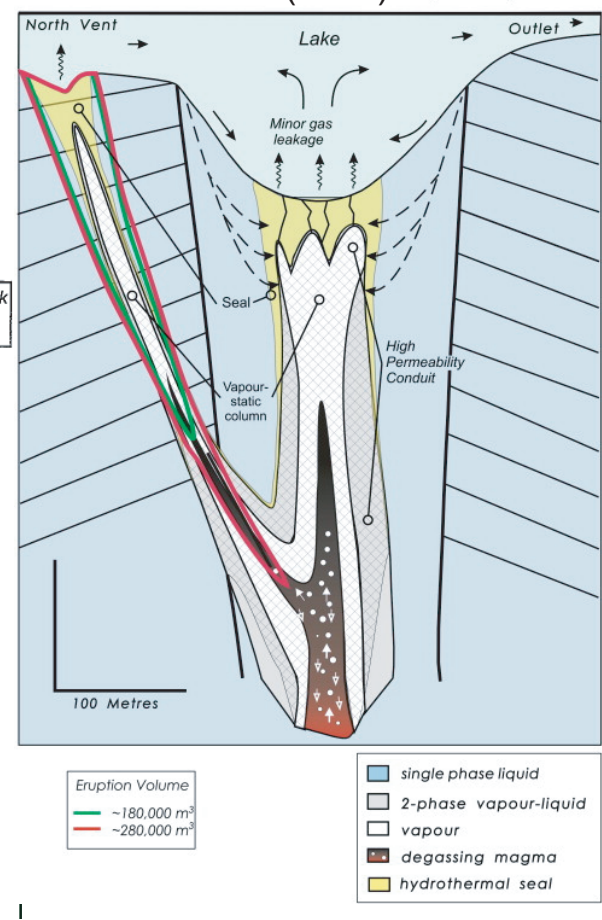

Figure 2.5. Sketches of Crater Lake dynamics showing multiple phase flows and sulphur hydrothermal seal. After Hurst et al. [1991], Hurst and Sherburn [1993], Christenson and Wood [1993] and Christenson et al. [2010].

from Crater Lake. Steam was the only observation until 19 March, when a lava dome was seen emerging from Crater Lake. Spectacular eruptions commenced on 26 March, sending a plume of ash and steam to heights of $2.5 \mathrm{~km}$ above the summit [Johnston and Neall, 1995]. Activity increased and culminated in a series of explo- 
sive eruptions from late July to December. Ash was distributed up to $250 \mathrm{~km}$ away from the vent, polluting water supplies and causing loss of crops [Johnston et al., 2000]. Two hikers were injured by hot ballistics close to the summit [Johnston et al., 2000].

\section{The 1966 to 1975 eruptions}

Phreatomagmatic eruptions due to failure of the "roof-rock" are the most violent type of eruptions at Ruapehu and give very little warning through seismicity; about 25 minutes of isolated earthquakes and 105 seconds of tremor in 1969 and nine minutes of earthquakes and 75 seconds of tremor in 1975 [Latter, 1981b]. The volcanic earthquakes increased in size before both the 1969 and 1975 eruptions of Ruapehu [Hurst et al., 2004]. Both of these eruptions consisted of a single large explosion that ejected lake water, mud and rocks onto the summit, causing damage to Whakapapa ski field [Ruapehu Surveillance Group, 1996]. These eruptions both occurred at night so no-one was harmed [Scott and Travers, 2009] but there are also no known eyewitness accounts. A different type of phreatomagmatic eruption, such as that in 1971, was preceded by several months of warning in the form of tremor, which was interpreted by Latter [1981b] as being from rising magma.

The 1966, 1968 and 1971 eruptions of Ruapehu were preceded by fumarolic heating of the Crater Lake. In the case of the 1966 eruption, the surface lake temperature was above $40^{\circ} \mathrm{C}$ [Hurst and Dibble, 1981].

\section{The 1995/1996 eruptions}

In the five or six weeks preceding the 1995 eruption, there was an $18 \%$ increase in the concentration of sulphate ions in the Crater Lake [Hurst et al., 2004] and geodetic measurements showed a small $(2-3 \mathrm{~cm})$ inflation of the crater area [Ruapehu Surveillance Group, 1996]. There was a gradual increase of the lakewater's Mg/Cl ratio (see Section 2.2.5 for explanation) in the six months leading up to the 1995 eruption [Hurst et al., 2004] and temperature fluctuations were accompanied by minor steam eruptions [Ruapehu Surveillance Group, 1996]. A telemetered hydrophone and temperature sensor were suspended at $20 \mathrm{~m}$ depth in Crater Lake at the time of the eruption [Hurst and Vandemeulebrouck, 1996]. This equipment recorded a strong acoustic pulse and a rapid increase in temperature on 26 April 1995, about the same time that the $\mathrm{Mg} / \mathrm{Cl}$ increase started. A small eruption on 29th June 1995 destroyed 
the equipment and so temperature measurements could only be obtained by manual sampling in conjunction with $\mathrm{Mg} / \mathrm{Cl}$ measurements [Hurst and Vandemeulebrouck, 1995]. Readings at the outlet showed a temperature decrease between May and September and so further activity was unexpected. With hindsight, the decrease in temperature may have been due to small eruptions causing large amounts of snow and ice to fall into the lake [Hurst and Vandemeulebrouck, 1995].

The 1995 eruption sequence began when an $\mathrm{M}_{L} 3.6$ volcanic earthquake occurred on the 18th September 1995 and was followed by a large lahar [Ruapehu Surveillance Group, 1996]. Ruapehu Surveillance Group [1996] provide a detailed account of observations during the 1995 eruption.

Bryan and Sherburn [1999] divided the 1995 and 1996 eruptions into 10 phases based on changes in seismicity, the details of which are presented in Appendix A.2. Lowfrequency $(1 \mathrm{~Hz})$ volcanic tremor was first recorded on 18 September, about $1 \mathrm{hr}$ before the eruption, and continued for several days [Ruapehu Surveillance Group, 1996; Bryan and Sherburn, 1999]. In contrast, 2 and $7 \mathrm{~Hz}$ tremor was regularly recorded throughout 1994 and 1995. Enhanced $7 \mathrm{~Hz}$ tremor occurred about $10 \mathrm{hrs}$ prior to the eruption and $2 \mathrm{~Hz}$ tremor continued throughout the eruption period. Long-period earthquakes with a dominant frequency of $2 \mathrm{~Hz}$ dominated the earthquake activity and there was a marked lack of high-frequency earthquakes throughout the eruption period. Bryan and Sherburn [1999] demonstrated that the source of the $1 \mathrm{~Hz}$ tremor was significantly deeper (a few $\mathrm{km}$ ) than that of the $2 \mathrm{~Hz}$ tremor $(<1000 \mathrm{~m}$ ) and that it was associated with magma intrusion. In early October 1995, a new pattern of seismicity was established with a change from predominantly $2 \mathrm{~Hz}$ tremor and LP earthquakes to a wider-band $(2-10 \mathrm{~Hz})$ tremor, coinciding with the ejection of the last of the water from Crater Lake and the change from a phreatomagmatic to a magmatic eruption style. In late October and early November 1995, small volcanic earthquakes accompanied eruptions of ash to several hundred metres above the crater, the last of which was observed on 9 November 1995.

The 1996 eruption was preceded by several days of strong tremor [Hurst et al., 2004] and phreatomagmatic eruptions resumed on 17 June 1996. The small amount of water that had accumulated in Crater Lake between eruptions was quickly expelled and the eruption style and seismicity was very similar to that of the later 1995 eruptions [Bryan and Sherburn, 1999]. Sub-Plinian ash fell over a large section to the north and west of Ruapehu on 17 and 18 June and smaller eruptions continued through July and the first week of August, spreading ash over much of the North Island [Johnston et al., 2000]. 
In 1995 the northwestern lahars passed by a T-bar drive station on Whakapapa ski field, which had fortunately been closed an hour previously and so was deserted. The repeated ashfall and acid rain caused considerable damage to facilities on Ruapehu's three ski fields [Miller et al., 1999]. Flights over the North Island were severely disrupted by the ash plumes of the 1995 and 1996 eruptions [Hurst et al., 2004]. In 2000, Johnston et al. [2000] estimated the total cost of economic loss, damage of equipment and response activities from the 1995 and 1996 eruptions to be in excess of NZ\$130 million.

\section{The 2006 and 2007 phreatic eruptions}

On 4 October 2006 a small eruption occurred in Ruapehu Crater Lake. At the time of the eruption, the lake temperature was at a local low of $\sim 15^{\circ} \mathrm{C}$ and there was no anomalous gas flux (A. Jolly pers. comm. 2010). The 2006 eruption was associated with a $\mathrm{M}_{L} 2.9$ volcanic earthquake but no airwave, or ash or steam projection into the atmosphere. It created a $4-5 \mathrm{~m}$-high wave deposit on the summit snow [Mordret et al., 2010]. There was also a $1.3 \mathrm{~m}$ rise in lake level in the following fortnight [Kilgour et al., 2010].

The Crater Lake level was again at overflow on 25 September 2007, when another phreatic eruption occurred. Jolly et al. [2010] analysed seismic data from the 2007 eruption. The eruption was preceded by two small volcano-tectonic earthquakes and minor tremor. It began with a $\mathrm{M}_{L} 3.2$ volcanic earthquake and produced a strong VLP (Very-Long Period) earthquake, which lasted for about $20 \mathrm{~s}$. The main eruptive phase lasted for about 4 min and included an explosive phase, lasting less than $60 \mathrm{~s}$ that was detected on microbarographs 3.1 and $9.4 \mathrm{~km}$ away. The eruption also created $\mathrm{a} \sim 4.5 \mathrm{~km}$-high steam column and a $\sim 2 \mathrm{~km}$-high Surtseyan jet, which ejected ballistics, ash and water, producing lahars in the Whangaehu and Whakapapaiti catchments. Two climbers staying in the Dome Shelter $(\sim 600 \mathrm{~m}$ from Crater Lake $)$ were caught in the eruption: one was seriously injured by the ejecta from Crater Lake, but was rescued later in the evening [Kilgour et al., 2010]. The ballistics, some up to $2 \mathrm{~m}$ diameter, showed evidence of intense hydrothermal alteration and pores were often filled with elemental sulphur. Juvenile glass, making up $5 \%$ of the ash, confirmed the presence of magma [Christenson et al., 2010]. The eruption has been interpreted to have been triggered by the development of pressurised, vapour-static gas columns beneath the elemental sulphur seal (see Section 2.2.3 and Christenson et al. [2010]). 


\section{Lahars}

As well as explosive eruptions, break-out lahars occur at Mt. Ruaephu when the dam of ash and ice, usually created by previous eruptions, forming the southeastern rim of Crater Lake fails. A break-out lahar occurred on Christmas Eve 1953, sending a $1.6 \times 10^{6} \mathrm{~m}^{3}$ lahar down the Whangaehu River, causing the destruction of the Tangiwai rail bridge and the loss of 151 lives [Healy, 1954]. Since then, Crater Lake has been continuously monitored and measures have been put in place, such as lahar warnings and the raising of the bridge, to reduce the risk from break-out lahars. In March 2007, a similar break-out lahar occurred but this one resulted in very little damage due to planning and monitoring [Manville et al., 2007].

\subsubsection{Monitoring}

The Whakapapa ski field, on the northwest flank of Mt. Ruapehu, is exposed to a high hazard with a very short response time, should an eruption occur beneath the summit Crater Lake. For this reason an automatic Eruption Detection System (EDS) is in place [Sherburn and Bryan, 1999]. It is operated by the Department of Conservation (DoC) and works on parameters developed by GNS Science [Hurst et al., 2004]. The EDS has recently been updated to include air pressure sensors to help detect eruptions, a purpose-built computer program to quickly evaluate seismic and air pressure data for tell-tale signs of an eruption, and a monitoring system that notifies emergency responders and activates speakers to send out warnings [GNS Science, 2011].

GeoNet is a government-funded project to build and operate a modern geological hazard monitoring system in New Zealand. The following description of monitoring techniques is based on information from the GeoNet website [GeoNet, Last accessed 17 April 2011, http://www.geonet.org.nz/volcano/monitoring-methods/], unless otherwise stated. The chemistry of the Crater Lake is monitored closely by GeoNet to obtain information about the relative abundances of gases and interaction of the water with juvenile magma. An increase in magnesium to chloride ratio suggests water interaction with juvenile magma [Hurst and Vandemeulebrouck, 1995]. Neither chloride nor magnesium ions enter the lake through precipitation. The main source of chloride is fumarolic gases containing hydrogen chloride entering the lake. Magnesium ions however, enter the lake through direct contact between water and high-temperature rock. Magnesium and chloride ions are only lost though seepage 


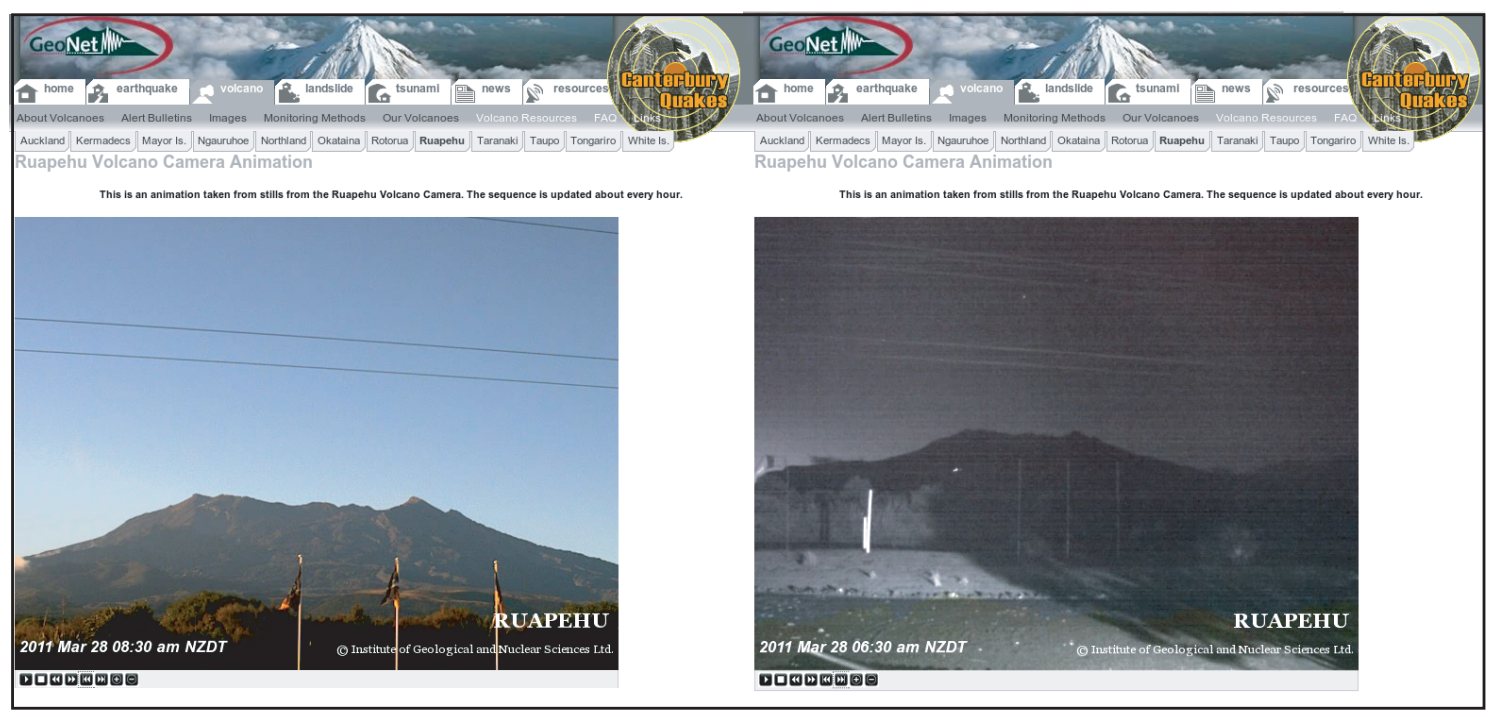

Figure 2.6. Day and night images of Mt. Ruapehu from the GeoNet Webcam [GeoNet, Last accessed 17 April 2011, http://www.geonet.org.nz].

and outflow [Hurst et al., 1991], thus an increase in magnesium to chloride ratio is indicative of contact between high temperature rock and water. Sampling is carried out at the surface of the lake, which is assumed to be representative of the whole lake because of mixing to considerable depths, as discussed in Section 2.2.3.

The bathymetry of Crater Lake was determined in the second half of the last century and a summary of depth measurements was published by Hurst and Dibble [1981]. In 1965, depth soundings were made with a Kelvin sounding winch and a metered pulley. Prior to this, depth estimations had been carried out by the New Zealand Canoeing Association. In 1970, the lake was resurveyed with an echo sounder. Other surveys included spot depth measurements in 1982 and depth soundings. After the 1995 and 1996 eruptions, all of the water in the crater lake was expelled and the form of the lake bottom could again be recorded [Harry Keys, pers. comm. 2008].

Temperature, heatflow, density and mineral concentrations suggest that Crater Lake is convecting vigorously, although there may be a layer $\sim 0.2 \mathrm{~m}$ deep on the surface in which the cold, fresh, less dense water from melt and precipitation does not mix thoroughly with the rest of the lake [Hurst and Dibble, 1981].

GeoNet uses a Correlation Spectrometer (COSPEC) or a FLYSPEC (a miniaturised, lightweight correlation spectrometer) to measure the absorption of ultraviolet light by sulphur dioxide $\left(\mathrm{SO}_{2}\right)$. Measurements since 2003 have shown a correlation between carbon dioxide $\left(\mathrm{CO}_{2}\right)$ and $\mathrm{SO}_{2}$ production and Crater Lake temperature [Christenson et al., 2010]. 


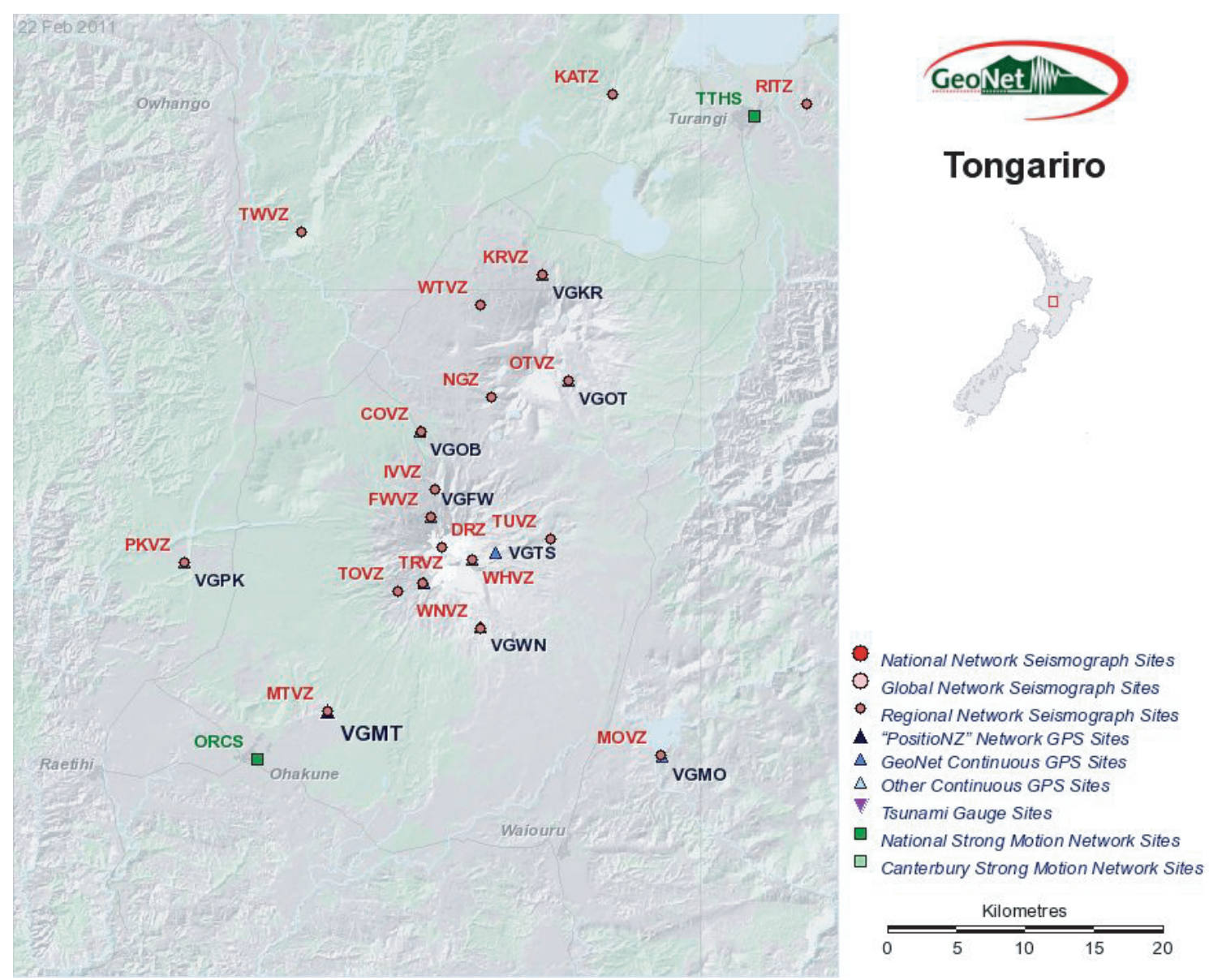

Figure 2.7. Tongariro Volcanic Centre seismograph network as of 22 February 2011 [GeoNet, Last accessed 17 April 2011, http://www.geonet.org.nz/resources/network/static-maps.html].

Two real-time GeoNet Webcams are a valuable source for the remote monitoring of Mt. Ruapehu (Figure 2.6). Visual observations such as rockfalls may indicate changes in the state of the volcano, and when analysed with seismic observations, can aid interpretation of signals. New cameras are also infrared-capable, allowing pictures to be taken at night when there is enough ambient light such as from a full moon (Figure 2.6 right).

Seismicity around Mt. Ruapehu has been monitored since the installation of the Tongariro seismometer in 1952 [Smith, 1981]. Since then, the network has been expanded and upgraded, and complemented with many temporary deployments. As of 28 March 2011, the network consists of seventeen three-component seismometers and two borehole seismometers distributed throughout the Tongariro Volcanic Centre (Figure 2.7). Triangulation and levelling measurements [e.g. Otway, 1979] have been replaced by twelve continuous GPS monuments to monitor ground deformation. 


\subsubsection{Seismology}

\section{Types of seismicity}

Four types of earthquakes are recorded at Mt. Ruapehu; these are volcanic or longperiod (LP), volcano-tectonic (VT), regional and teleseismic [Latter, 1981a; Sherburn et al., 1999] (Figure 2.8). These earthquakes are described in detail below and examples can be seen in Figure 2.8. Figure 2.9 displays the local seismicity since 1990 in the top panel and the deeper, regional seismicity since 1990, which mostly occurs on the subducted slab in the bottom panel.

Latter [1981a] described detailed observations of seismicity in the 1960s and 1970s around Mt. Ruapehu: The majority of observed earthquakes at Mt. Ruapehu were VTs, with high-frequency ( $\geq 3 \mathrm{~Hz}$ ), usually sharp, well-defined phases. The LPs observed at Mt. Ruapehu were often multiple rather than discrete events, with poorly defined phases, emergent onsets and frequencies $\leq 3 \mathrm{~Hz}$, with $2 \mathrm{~Hz}$ being the most common. Latter [1981a] noted that it was sometimes difficult to identify an LP earthquake purely on the basis of its frequency content because of marked attenuation of high frequencies along the path. Dominant low frequencies were ascribed to the earthquake's occurence in heat-weakened material and high frequencies to instantaneous source mechanisms operating in competant rock. Latter [1981a] found no difference in phase structure, waveform, or frequency content between LPs that were accompanied by eruptions and those that were not. The majority of LP earthquakes occurred directly beneath Crater Lake, either immediately beneath the lake or 0.5 to $1.3 \mathrm{~km}$ below [Latter, 1981a; Hurst and McGinty, 1996]. Occasionally, LP earthquakes took place at locations in the Tongariro National Park where no previous vent was in existence, suggesting a magmatic intrusion. When a sequence of LPs was of long duration, and the spacing between events was very small, Latter [1981a] observed that the sequence would grade into volcanic tremor. Mt. Ruapehu was anomalous in having long periods of volcanic tremor that did not precede eruption [Hurst et al., 2004].

Hurst [1992] analysed the most common volcanic tremor produced by Mt. Ruapehu, which was a continuous signal with a dominant frequency of about $2 \mathrm{~Hz}$, and found that it had a sharply peaked spectrum and an autocorrelation function with a high degree of coherence. Comparisons of this tremor with various stochastic simulations, with either the characteristics of the oscillator, or the forcing function containing a random element, revealed that the cause of the tremor was most likely to be 

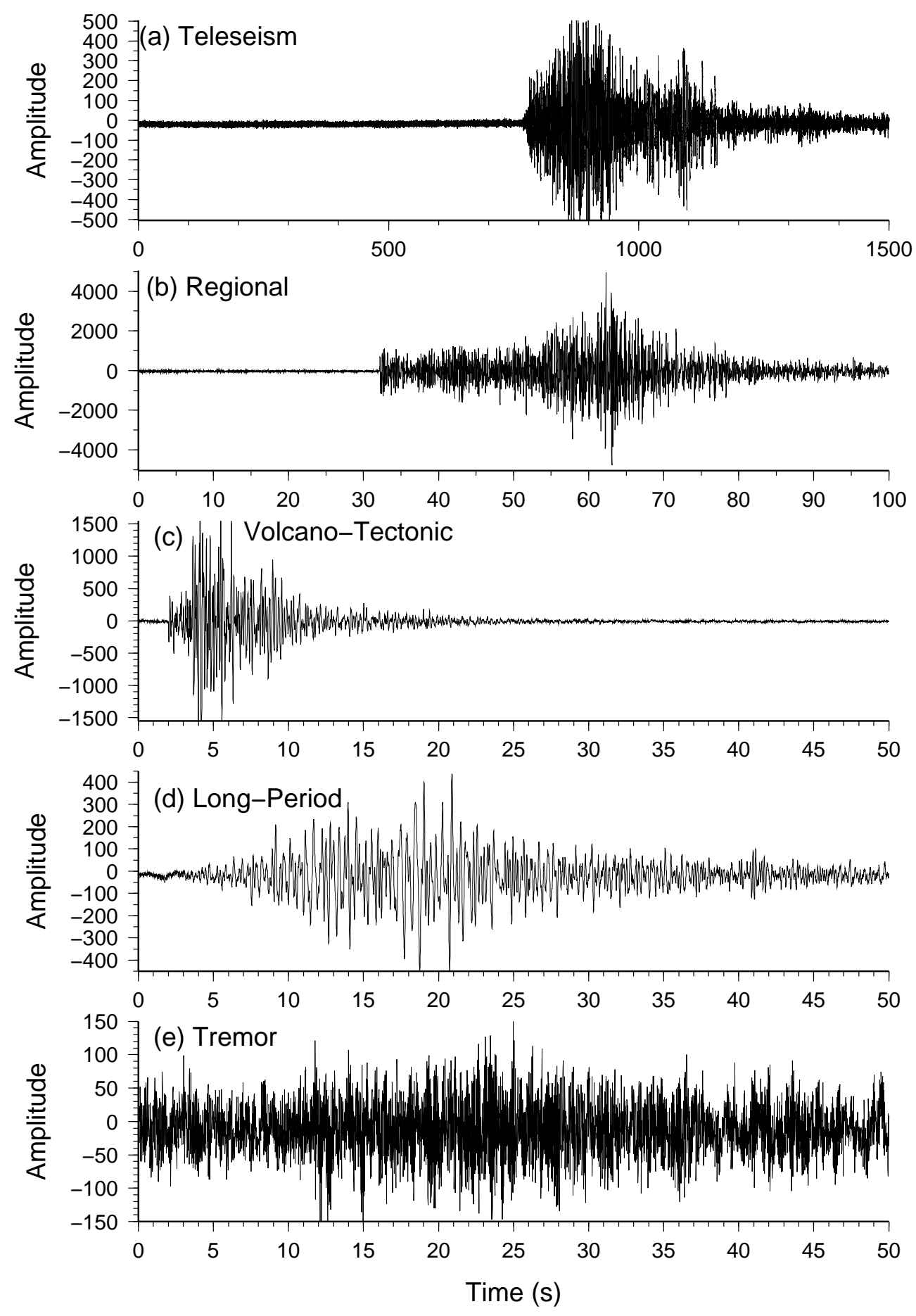

Figure 2.8. Example waveforms of typical earthquakes recorded at station TUVZ at Mt. Ruapehu. (a) Recording of the M 9.0 Japan earthquake on Friday, March 11, 2011, recorded at 05:59 UTC. (b) Recording of a $\mathrm{M}_{L} 5.0$ earthquake on the subducted slab beneath the North Island on Thursday, January 6, 2011 (cusp ID 3443657). (c) Recording of a $\mathrm{M}_{L} 2.0$ volcano-tectonic earthquake at Mt. Ruapehu on March 25, 2011 (cusp ID 3485488). (d) Recording of a $\mathrm{M}_{L} 1.7$ long-period earthquake at Mt. Ruapehu on Jauary 27, 2011 (cusp ID 3455916). (e) Recording of volcanic tremor at Mt. Ruapehu on October 24, 2007, recorded at 14:54 UTC. 
resonance in a cavity, such as an "organ-pipe", filled by a compressible fluid. The "organ pipe" resonator was interpreted to be a quarter of a wavelength long (65 $\mathrm{m}$ if it were filled with superheated steam and $200-400 \mathrm{~m}$ in the case of magma) and of low attenuation (high $Q$ ), which meant that it had little interaction with the surrounding material. The tremor was best modelled by a random damping factor on the oscillator, representing energy input to the resonant system, although a "white noise" forcing function, representing high pressure steam, also fit the data well. Hurst [1992] also noted that, while the $2 \mathrm{~Hz}$ tremor and LP earthquakes probably originated from the same source, the tremor was not the result of the repeated ocurrence of small LPs, contrary to previous belief [Latter, 1981a], but the product of a resonator with positive feedback sustaining oscillation. The dominant $2 \mathrm{~Hz}$ tremor was further analysed by Hurst and Sherburn [1993] in an attempt to characterise the source. They concluded that the $2 \mathrm{~Hz}$ tremor was caused by a single, asymmetric, low-attenuation resonator and suggested that the resonator was in the single-phase vapour region beneath the main vent (Figure 2.5).

\section{Earthquake locations}

In 1981, Latter [1981b] found that earthquake epicentres were strongly concentrated beneath the east and northeast slopes of Mt. Ruapehu and there was a tendency for deeper earthquakes in the south and west compared to the north and east.

A two month temporary deployment of 14 broadband seismometers around $\mathrm{Mt}$. Ruapehu in early 1994 was carried out by Leeds University, the University of Memphis and IGNS (Institute of Geophysical and Nuclear Science, now GNS Science), and analysed by Hurst [1998]. Seismicity associated with the active vent of Mt. Ruapehu as well as tectonic earthquakes were recorded. Hurst [1998] found that VT earthquakes and bursts of volcanic tremor both had sources under Crater Lake. The general crustal seismicity was found to show a transition from shallow $(<10 \mathrm{~km})$ TVZ-type events to the north, to deeper crustal activity to the south. Three main clusters around Mt. Ruapehu were identified by Hurst [1998] (Figure 2.10): a wellknown long-lived centre near Waiouru (referred to hereafter as the Waiouru swarm), an area on the north flank of Tongariro volcano, and a cluster about $15 \mathrm{~km} \mathrm{WNW}$ of Mt. Ruapehu (henceforth referred to as the Erua swarm). Both high-frequency (VT) and low-frequency (LP) earthquakes were located by Hurst [1998] to be in the immediate vicinity of the Crater Lake with similar depths; less than $1000 \mathrm{~m}$ below the surface of Crater Lake. However, the epicentral position of the low-frequency 

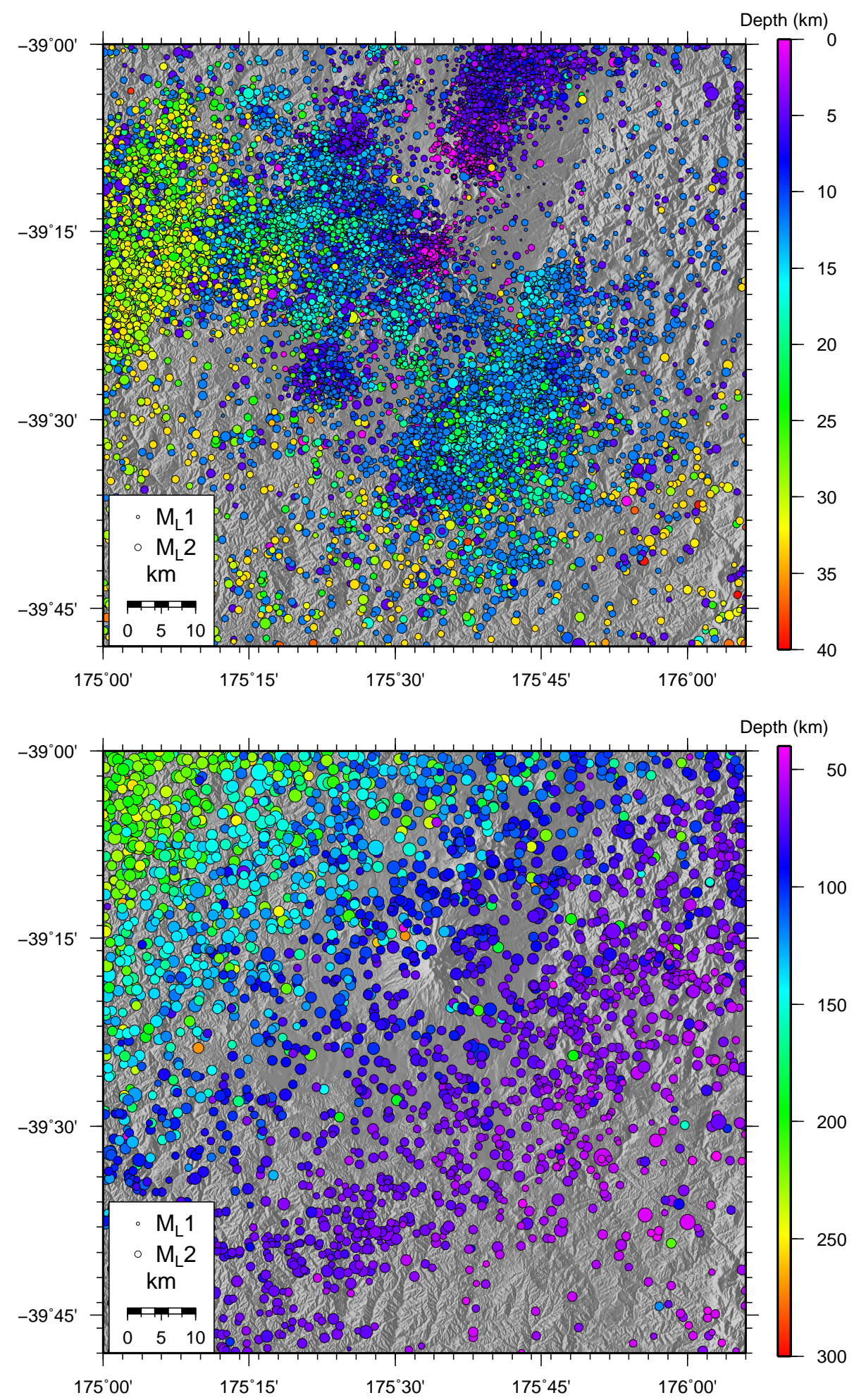

Figure 2.9. All earthquakes in the Ruapehu region from 1 January 1990 to 1 January 2011 from the GeoNet catalogue [GeoNet, Last accessed 17 April 2011, http://www.geonet.org.nz]. Top: Shallow $(<40 \mathrm{~km})$ seismicity, colour coded by depth and scaled by magnitude. Bottom: Seismicity deeper than $40 \mathrm{~km}$, colour coded by depth and scaled by magnitude. 
earthquakes was in a gap in the high-frequency events, lending support to the idea that the VTs occurred in the wall-rock next to the source of the LPs; a fluid filled conduit (Figure 2.5).

\section{Seismicity in relation to eruptions}

Bryan and Sherburn [1999] observed that the background seismicity at Mt. Ruapehu altered after the 1995 and 1996 eruptions, likely reflecting a change in the volcanic plumbing system. Prior to 1995, seismicity at Mt. Ruapehu was characterised by volcanic tremor with spectral peaks at 2 and $7 \mathrm{~Hz}$, and by shallow VT earthquakes occurring at rates of about 5 per day. The $7 \mathrm{~Hz}$ tremor was continuous and only detected at station DRZ (Figure 2.7), the closest station to the Crater Lake, whereas the $2 \mathrm{~Hz}$ tremor tended to occur in bursts. On and off throughout the late 1980s, 3 $\mathrm{Hz}$ tremor was also observed at DRZ [Sherburn et al., 1999], which mainly occurred when the temperature of Crater Lake was above $30^{\circ} \mathrm{C}$. Sherburn et al. [1999] suggested an origin related to the source of additional heat input into the lake. There were very few VT earthquakes prior to and during the 1995 eruption but many between the 1995 and 1996 eruptions. During the latter part of the 1995 eruption and the 1996 eruption volcanic tremor at Mt. Ruapehu was dominated by wideband $(2-10 \mathrm{~Hz})$ tremor. Two factors are suggested by Bryan and Sherburn [1999] to have affected the tremor frequency; structural modifications within the conduit and the loss of Crater Lake. In addition, only after the loss of the Crater Lake were ground-coupled airwaves observed. The airwaves were used to assess whether the LP earthquakes were associated with explosions when visual observations were not possible [Sherburn et al., 1999].

The style of seismicity and its relation to eruption at Mt. Ruapehu was analysed by Sherburn et al. [1999]. They observed that, although large eruptions are always accompanied by LP earthquakes and/or strong volcanic tremor, the converse was not true; periods of enhanced volcanic tremor and large or more numerous LPs did not always coincide with eruptions. No consistency could be found between correlations of smaller eruptive events and seismicity or lake temperature.

A later temporary deployment of broadband seismometers in 1998 was used to characterise the changes in seismicity from before and after the 1995/1996 eruptions. Bryan and Sherburn [2003] also observed the change in dominant frequency of strong tremor from before to after the 1995-1996 eruptions. The post eruption tremor was found to be dominated by $0.8-1.4 \mathrm{~Hz}$ energy although the $2 \mathrm{~Hz}$ energy 
was still present. The change in dominant frequency was attributed to changes in the volcanic plumbing system but it was suggested that the two types of tremor had discrete sources and the change in the system was the creation of the new source rather than an alteration of the former. It was also noted that no very-long-period (VLP) seismicity, with periods of 3-20 s, had been observed at Ruapehu [Bryan and Sherburn, 2003].

\section{Distal swarms and their relationship with eruptions}

Hurst and McGinty [1999] observed that, although there were very few VT earthquakes at Mt. Ruapehu imediately prior to and during the 1995/1996 eruptions, within the year preceeding the eruptions there were a series of swarms of earthquakes $15-22 \mathrm{~km}$ to the west of the volcano (close the the Erua swarm, Figure 2.10). All of the several hundred located earthquakes showed sharp onsets and appeared to be typical tectonic events, but the swarms were larger than typical swarms in the area. Comparison of this seismicity with other observations at Mt. Ruapehu showed that the two main bursts of seismicity in the swarms could be related to the two periods of heating of Crater Lake, and the latter of the heating phases was immediately followed by increases in the $\mathrm{Mg}^{2+}$ ion concentration. Relocation of the swarm seismicity revealed three distinct clusters close to the Raurimu fault (Section 2.2.8) with most of the earthquake depths between 10 and $15 \mathrm{~km}$ below the surface. Hurst and McGinty [1999] found no apparent migration and calculated the $b$-value to be 0.74 , which is on the low end of those typical of tectonic earthquake swarms. Tectonic environments typically have $b$-values of $0.8-1.2$ while $b$-values in volcanic swarms tend to be higher $(\geq 1.3)$ [Wiemer and Wyss, 2002]. Hurst and McGinty [1999] also calculated composite and individual focal mechanisms and found that the two southern clusters displayed oblique-normal mechanisms, which is consistent with the Raurimu fault. The northern cluster displayed an oblique-reverse composite mechanism, which is in agreement with a branch of the Raurimu fault $2 \mathrm{~km}$ to the north. The inference was that the seismicity was not caused directly by magma movement but by the changes in stress caused by magma movement [Hurst and McGinty, 1999].

We analysed seismicity and anisotropy of the persistent section of those earthquakes analysed by Hurst and McGinty [1999]; the Erua swarm (Keats et al. [2011], Appendix G and Figure 2.10). We found that the $b$-value of the Erua swarm increased from 1.2 to 1.8 around the time of the 2006 and 2007 phreatic eruptions of Mt. 


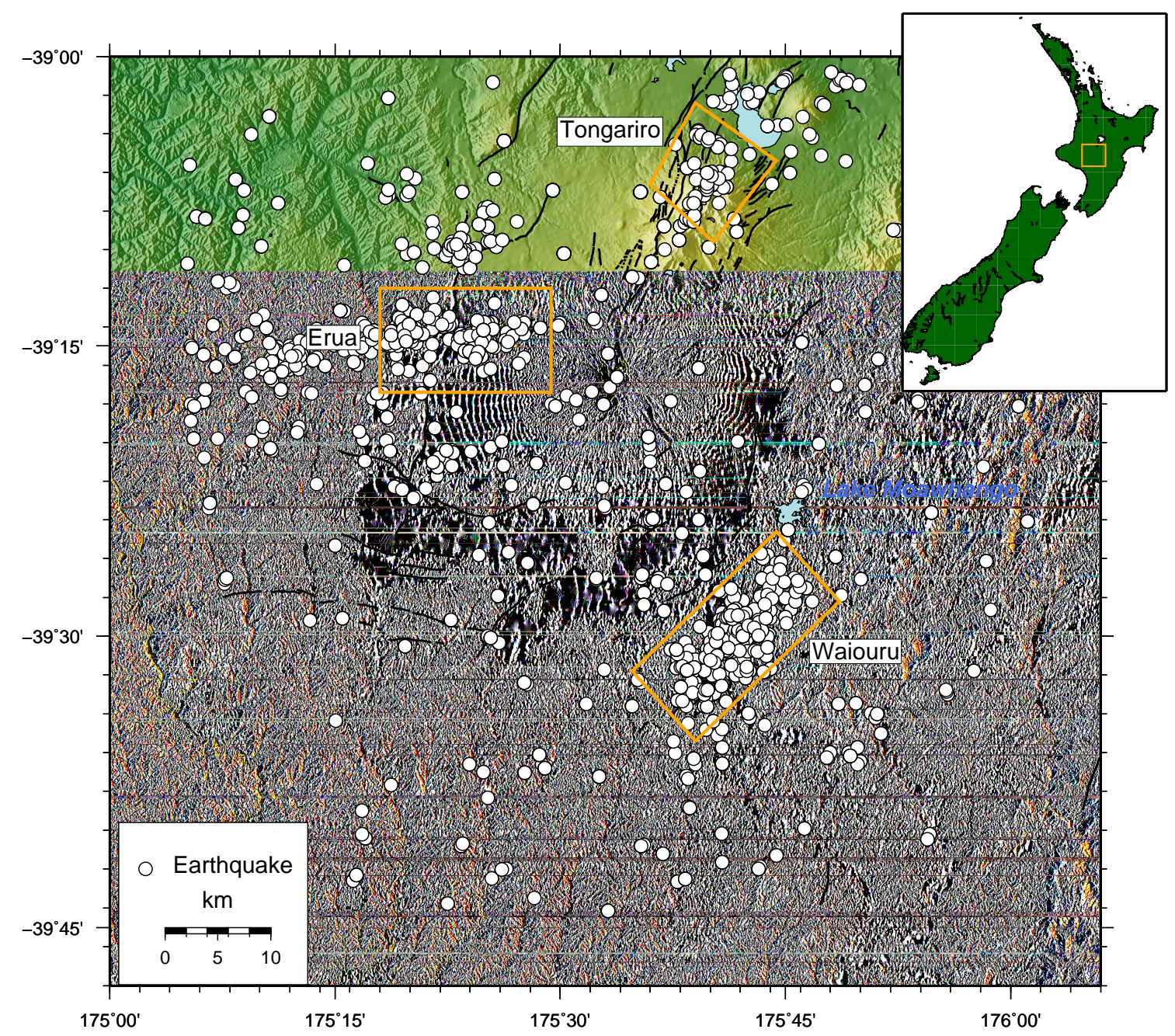

Figure 2.10. Earthquakes recorded during the 2008 temporary deployment, clustered in the Waiouru swarm, the Erua swarm and the Tongariro swarm.

Ruapehu. We also found that the fast direction of anisotropy changed and delay time decreased locally around the same time period. These results were interpreted as an increase in fluid in the region associated with the trigger of the eruptions.

The cluster of earthquakes to the southeast of the summit of Mt. Ruapehu, known as the Waiouru swarm (Figure 2.10), was first analysed by Reyners [1980] and later by Hayes et al. [2004]. During a six month temporary seismic deployment in 2001, 319 earthquakes in the cluster were detected and relocated. Hayes et al. [2004] found that the seismicity defined a vertical sheet striking NE-SW with the majority of the events occurring at 11-21 km depth. Focal mechanisms and stress inversions were found to indicate strike-slip motion on a near-vertical fault parallel to the strike of the swarm, which is in contrast to the nearby, sub-parallel, normal Snowgrass fault (Section 2.2.8). Analysis gave a $b$-value of 1.02, which is more consistent 
with tectonic, rather than volcanic swarms, although analysis of $b$-value with time showed large fluctuations after the 1995 eruption of Mt. Ruapehu. The conclusion was that the swarm was due to fluid movement from the dehydrating subducted slab, tectonic in origin and reflected the regional stress rather than local stress caused by the volcano. Hayes et al. [2004] found that the activity of the swarm did correlate with volcanic activity but to a lesser extent than Hurst and McGinty [1999] found around the Raurimu fault to the west.

\section{Velocity models}

Several velocity models have been proposed for the Ruapehu Region using various methods including refraction surveys [e.g. Sissons and Dibble, 1981; Dibble et al., 1985], joint earthquake location and velocity estimation [e.g. Latter, 1981b; Hurst and McGinty, 1996], and velocity tomography [e.g. Rowlands et al., 2005]. A summary of 1-D velocity models in the Ruapehu region is shown in Figure 2.11. 

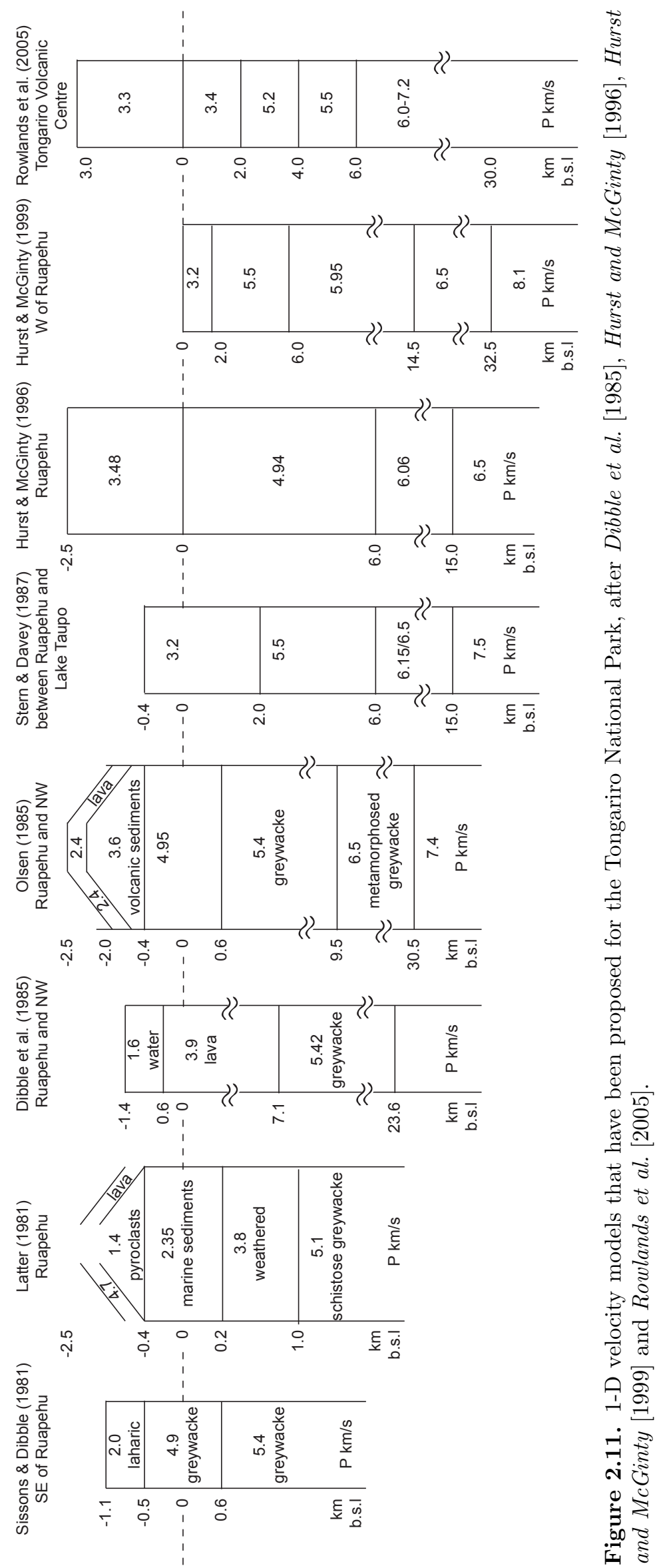


\section{Anisotropy, attenuation and ambient noise}

Miller and Savage [2001] measured shear wave splitting from shallow $(<30 \mathrm{~km})$ and deep (> $50 \mathrm{~km}$ ) earthquakes in 1994 and 1998 and observed a change in the dominant fast polarisation $(\phi)$ spanning the time of the last major magmatic eruption in 1995/1996. This study was extended by Gerst and Savage [2004], who used the same techniques and an additional deployment of three-component seismometers in 2002 to observe further changes in $\phi$. The results of both studies were interpreted as being caused by a dike-shaped magma reservoir, or system of dikes, trending NE-SW. According to this model, the magma reservoir was pressurised before the eruption, producing a local stress field different from the regional stress field. Following the eruption the reservoir was less full and correspondingly less pressurised so the local stress returned to that of the surrounding region. The Gerst and Savage [2004] study suggested that the later changes in $\phi$ were due to repressurising of the reservoir in response to an increase of magma in the system. The stress in the surrounding crust caused by the pressurised magma reservoir is thought to preferentially align randomly oriented fluid-filled microcracks and cause seismic anisotropy that is detected through shear wave splitting [Crampin, 1994] (see Section 2.3.4).

Shear wave splitting results in the North Island from teleseisms consistently yield trench parallel fast directions [Hofmann, 2002; Audoine et al., 2004; Greve et al., 2008], with larger delay times within the Central Volcanic Region (CVR) [Greve et al., 2008]. Using local events generally results in more scatter within the CVR [Hofmann, 2002; Audoine et al., 2004], although Morley et al. [2006] found that, using earthquakes from the subducted slab, fast directions rotated from trench parallel east of the CVR to trench perpendicular within the CVR. Seward et al. [2009] examined anisotropy in the North Island using Pn velocity modelling and also found trench parallel fast directions to the east and within the CVR. Audoine et al. [2004] examined anisotropy in the North Island from shear wave splitting and spatial averaging and found a complex pattern. The general fast direction from shallow and deep earthquakes was trench-parallel, which was attributed to fluid-filled cracks in the crust and asthenospheric flow in the mantle. They found that in the extension region of the TVZ the results were more varied with a dominant trench-normal fast direction, which was attributed to corner flow.

Eberhart-Phillips and Reyners [2009] used earthquake arrival time data to invert for a 3-D $v_{p}$ azimuthal anisotropy model for the central North Island. They found that at most depths there was strong anisotropy to the east of the TVZ, with the 
fast axis striking NE-SW (trench parallel). This was associated with the Haast schist terrane mineral orientation in the crust and bending or fossil anisotropy in the subducted slab at depth. Beneath the TVZ and to the west Eberhart-Phillips and Reyners [2009] found less anisotropy, generally striking N-S-NW-SE, which is trench-normal. This was attributed to corner flow in the mantle wedge. In the vicinity of Mt. Ruapehu, anisotropy in the crust was found to be generally parallel to extension (WNW-ESE) and was attributed to microcracks aligning with local stress.

Seismic attenuation around Mt. Ruapehu was investigated by Syuhada [2010]. He found that there was higher attenuation within the TVZ than outside. This attenuation was frequency dependant, suggesting intrinsic attenuation rather than scattering. Syuhada [2010] also found that the attenuation was anisotropic, with higher attenuation in the N-S direction in the shallow crust, and NW-SE direction in the lower crust, agreeing with the hypothesis of Eberhart-Phillips and Reyners [2009], that fluid-filled cracks are oriented to local stress in these directions. Titzschkau et al. [2010] used a repeating source within the Waiouru swarm to investigate changes in attenuation related to the 1995/1996 eruptions of Mt. Ruapehu. They found a high attenuation anomaly in waveforms that travelled through the volcano, which increased over the time of the eruption and a decrease in attenuation of waves that travelled close to but not through the volcano. These changes in attenuation were attributed to the same source mechanism as the shear wave anisotropy studies [Miller and Savage, 2001; Gerst and Savage, 2004].

Using Interferometry on retrieved cross-correlation functions from ambient seismic noise (IRCCSN) is a newly emerging technique used to monitor changes in stress at volcanoes [e.g. Brenguier et al., 2008]. Mordret et al. [2010] employed this method at five stations around Mt. Ruapehu to investigate changes associated with the 2006 and 2007 phreatic eruptions of Mt. Ruapehu. By comparing surface wave Green Functions constructed from ambient seismic noise relative to a reference Green Function, they found a $0.8 \%$ decrease of relative seismic velocity starting two days before the 2006 eruption, which was modelled as a point source at $5 \mathrm{~km}$ depth, either centred on the volcano or offset to the NE, with volume change of $\sim 0.0017$ $\mathrm{km}^{3}$. This volume change was found to be the threshold at which no deformation would be detected by the GPS network. There was no significant velocity change associated with the 2007 eruption and this was attributed to a more open system than the 2006 eruption even though other observations suggest the same eruption mechanisms. 


\subsubsection{Other geophysical observations}

\section{Gravity and magnetics}

The regional gravity map (not presented) shows a NNE-trending regional gradient [Cassidy et al., 2009, and references therein] and shows little effect of the volcanoes in the Tongariro Volcanic Centre. However, when integrated with 38 newer gravity stations, Cassidy et al. [2009] were able to determine a gravity residual along the saddle between Mts. Ruapehu and Ngauruhoe. They found an asymmetric negative anomaly, which was modelled along with two aeromagnetic data sets that follow the same profile. Regional magnetic data showed positive anomalies associated with the volcanic centres [Cassidy et al., 2009]. The aeromagnetic data of Cassidy et al. [2009] were from elevations of 1500 and $2500 \mathrm{~m}$ a.s.l, and both profiles also displayed a positive anomaly over the volcanoes. The optimum model from the joint inversion was found to be an extensive upper layer with density $2200 \mathrm{~kg} / \mathrm{m}^{3}$ and a maximum thickness of $1000 \mathrm{~m}$ over a $2670 \mathrm{~kg} / \mathrm{m}^{3}$ layer with three abrupt depth changes, and a denser magnetic material in the centre. This was interpreted as mixed volcanic deposits over greywacke with a minimum basement subsidence of 650 $\mathrm{m}$ and steep-sided bodies of lavas and dikes at the centre. The depth changes were correlated with mapped faults. These interpretations agree with those of Horspool [2003] from gravity profiles across Mt. Ruapehu, restrained by seismic refraction surveys. Horspool [2003] found a broad anomaly and a short-wavelength anomaly. The broad anomaly was interpreted as the displacement of basement rocks by low density material. The subsidence was found to be accommodated mainly by faulting (1000 m), but $400 \mathrm{~m}$ of subsidence was attributed to flexure due to the load of the volcano. The short-wavelength anomaly was interpreted to be a dense andesite dike in a low density cone.

\section{Magnetotellurics}

A 3-D inversion of data from 40 broadband magnetotelluric (MT) soundings was conducted by Ingham et al. [2009]. Phase tensor analysis suggested that a shallow conductive layer corresponding to a zone of acid alteration lies between a high resistivity layer of dry volcanic rock and ash and a higher resistivity layer corresponding to the basement greywacke. The 3-D inversion indicated a dike-like structure of low resistivity extending from the summit of Mt. Ruapehu to the NE and persisting to at least $10 \mathrm{~km}$ depth. This structure was interpreted to be part of the volcanic feeder 
system supplying fluids, hot gases and magma to the volcanoes. A more localised high frequency MT survey was carried out by Jones et al. [2008] to investigate the hydrothermal vent system of Mt. Ruapehu. They found that the entire summit plateau was underlain with hydrothermally alterred low resistivity material. However, Jones et al. [2008] did identify two regions of higher resistivity that coincided with past eruption centres and are believed to be the location of heat pipes that have been altered at a higher temperature.

\section{Deformation}

Deformation surveys conducted by Otway [1979] were initiated in 1970 and consisted of accurate horizontal angle observations supplemented with electromagnetic distance measurements. Local extension across Crater Lake was observed in the six weeks prior to the May 1971 phreatomagmatic eruptions. Over the following three years, a reversal of the pre-eruption deformation was observed as volcanic activity declined. During this time there were two phreatic eruptions, which correlated with sharp rises in lake temperature and seismicity, but not with significant extension. Two weeks before the 1975 eruption, significant extension was observed, which extended further from the lake than previously measured extension phases. The 1975 eruption destroyed the survey markers and so continuity with further surveys was lost. Otway [1979] interpreted the observed deformation as magmatic intrusions or increases in shallow gas pressure.

A pilot GPS survey was carried out by Miller et al. [2003] to identify potential permanent site locations and detection thresholds. Unfortunately the atmospheric conditions around Mt. Ruapehu in the troposphere mean that at times the noise had a higher amplitude than the changes expected (centimetres) [Hurst and Fournier, 2009].

Synthetic aperture radar interferometry (InSAR) has recently been carried out on Mt. Ruapehu using ALOS PALSAR. The results indicated that there has been no major deformation around Ruapehu since 2007, although resolution on the summit was limited due to snow cover [Joyce et al., 2009].

\section{Stress estimations}

Villamor and Berryman [2006] note that all of the Quaternary faults in the area are normal, suggesting that the maximum compressive stress, $S_{1}$, is close to vertical. In 
a normal faulting regime the minimum compressive stress, $S_{3}$, is the minimum horizontal compressive stress, $S_{H \text { min }}$, and is orthogonal to the fault strike. Therefore the maximum horizontal compressive stress, $S_{H \max }$, is parallel to fault strike. However, the faults may be at a high angle to the stress [e.g. Townend and Zoback, 2001], may have been formed at a time when the local stresses were different, or may have been deformed since their creation, in which case the current $S_{H \max }$ may be different to that inferred from the faults. The crosscutting of contemporary fault sets with three different strikes suggest that the $S_{3}$ and $S_{2}$ axes of the stress tensor have to be similar in magnitude, suggesting that small changes in horizontal stress could rotate $S_{\text {Hmax }}$ [Villamor and Berryman, 2006]. Further evidence that $\left|S_{2}\right| \approx\left|S_{3}\right|$ in the Ruapehu region comes from Wallace et al. [2004], who modelled GPS observations with block rotation that allows opening of the Taupo rift and implies a change in stress orientation over a short distance due to observed contraction at the southern termination of the TVZ. The temporal changes in shear wave splitting in the region observed by Miller and Savage [2001] and Gerst and Savage [2004] are also consistent with this hypothesis.

Estimates of local stress parameters have been calculated by inverting first motions and focal mechanisms of local earthquakes. A summary of these stress estimates can be found in Section 4.1, and Table 4.1.

\subsubsection{Some geological observations}

\section{Structural geology}

The basement greywacke in the Ruapehu region consists of the Torlesse and Waipapa terranes to the east and west respectively [Adams et al., 1998; Mortimer, 2004] (Figure 2.12). The geological textures of the basement rocks around Mt. Ruapehu were investigated by Beetham and Watters [1985] during the Tongariro Power development project. They found no discernible internal structure in the basement greywacke with the exception of a couple of small areas that display textural zone $2 \mathrm{~B}$, semi-schistose rocks (on the metamorphic sequence described by Turnbull et al. [2001]); these were to the north of the Kaimanawa Range and around lake Moawhango (Figure 2.10).

Figure 2.13 displays four zones identified by Villamor and Berryman [2006] as having different fault strikes. The area of most dense faulting is to the NE of Mt. Ruapehu, around Mt. Ngauruhoe and Mt. Tongariro, called the Tongariro graben [Rowland 


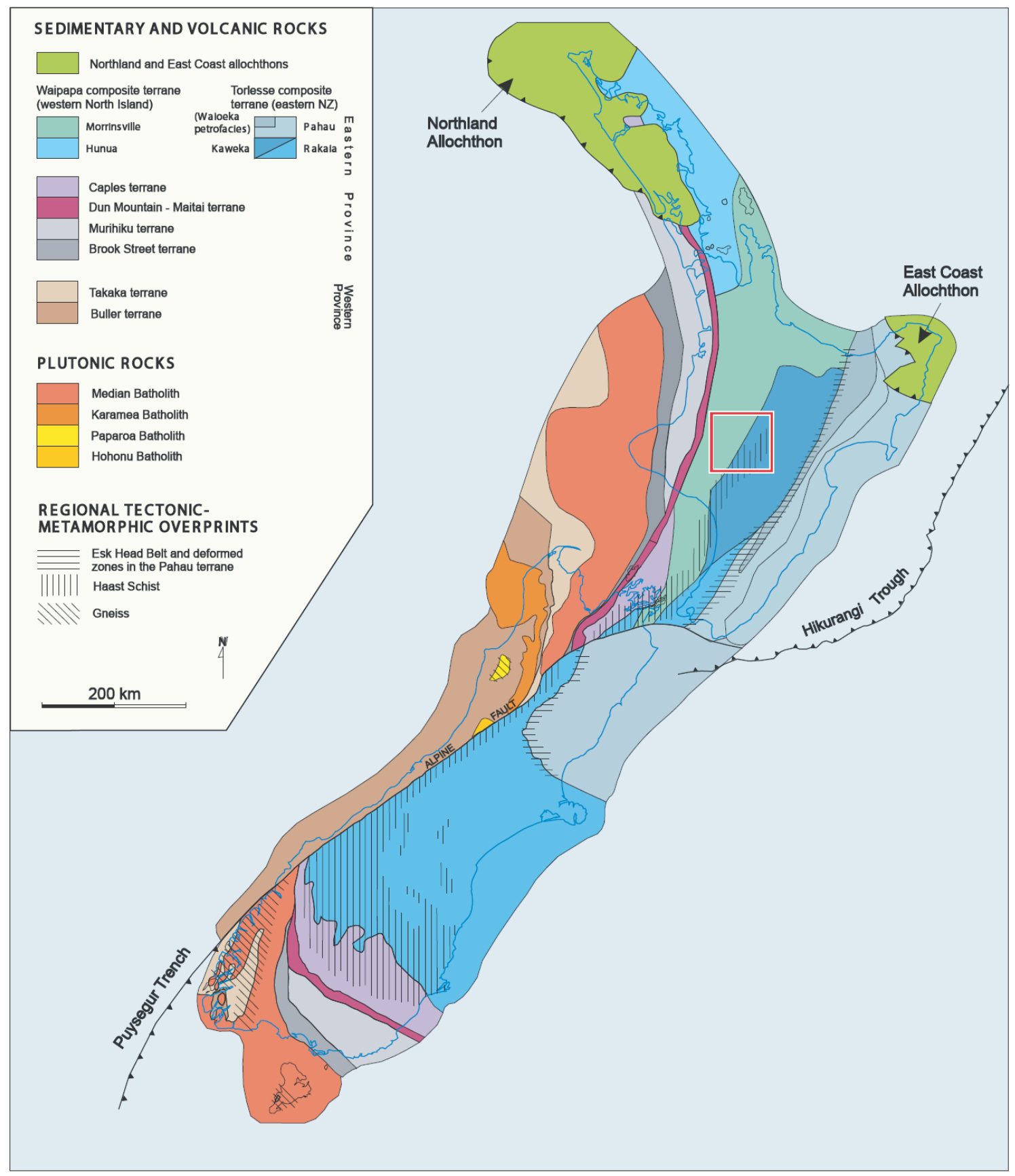

Figure 2.12. Terranes of New Zealand [after Mortimer, 2004]. Red box indicates Ruapehu region. 


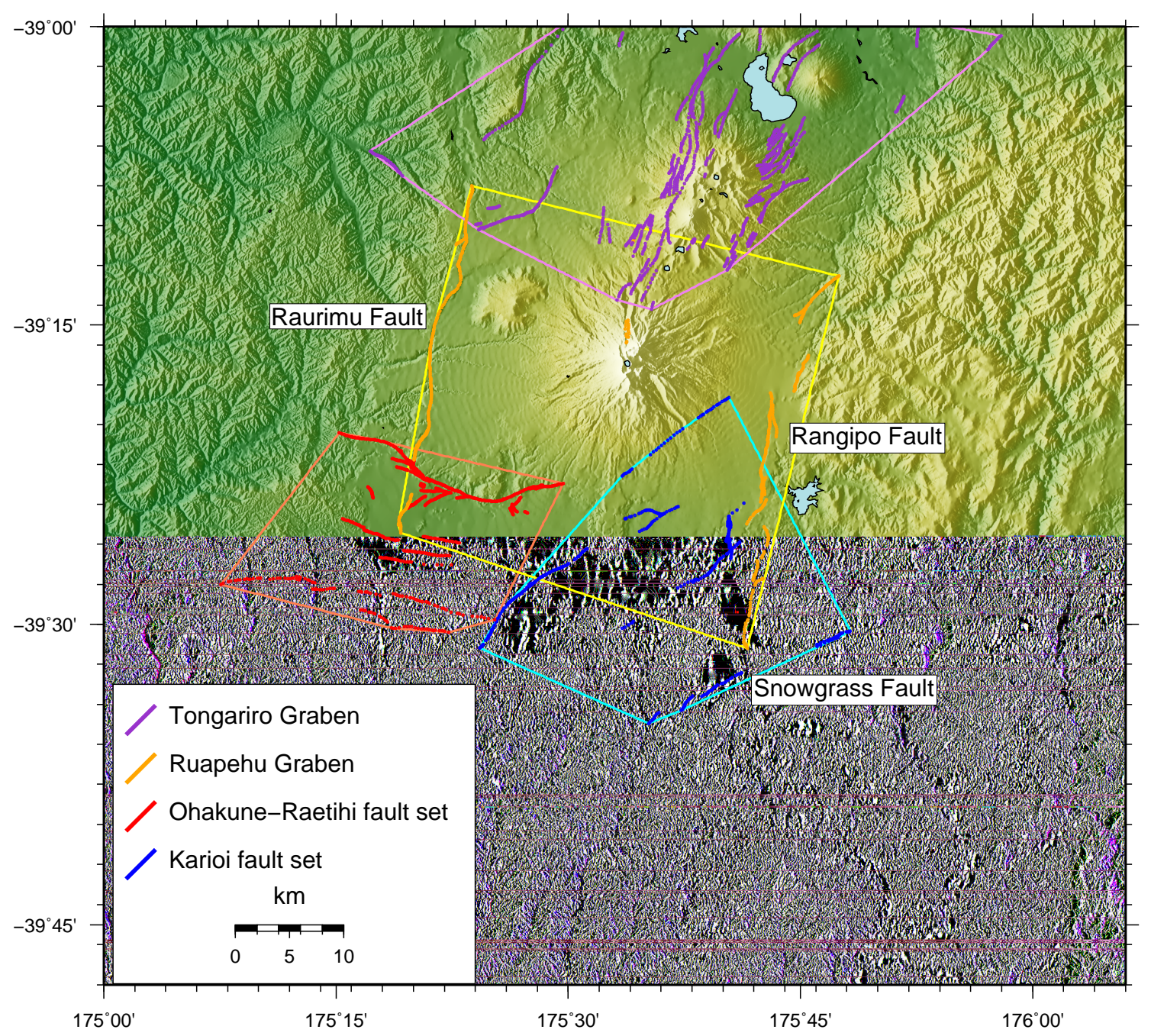

Figure 2.13. Map of the Ruapehu region showing mapped faults (from the NZ Active Faults Database of GNS, after Villamor and Berryman [2006]). Colours show different fault sets and pale polygons show limits of the overlapping zones of similar strike.

and Sibson, 2001]. The average strike of these faults is $030^{\circ}$ [Rowland and Sibson, 2001], similar to metamorphic fabric in the basement rock. The strike of faults around Mt. Ruapehu describe the axis of the Ruapehu graben, which at $010^{\circ}$, is closer to N-S than the Tongariro graben to the north. The Rangipo fault forms the east boundary of the Ruapehu graben and crosscuts the faults of the Karioi fault set to the southeast. Faults to the southwest (the Ohakune-Raetihi fault set) strike WNW-ESE in an area of Tertiary marine deposits and Quaternary volcanics, however there is no information about deeper basement fabric for this zone. The Ohakune-Raetihi fault set are cross cut to the east by the Karioi fault set and to the north by the Raurimu fault, which bounds the west side of the Ruapehu graben. 


\section{Petrology and geochemistry}

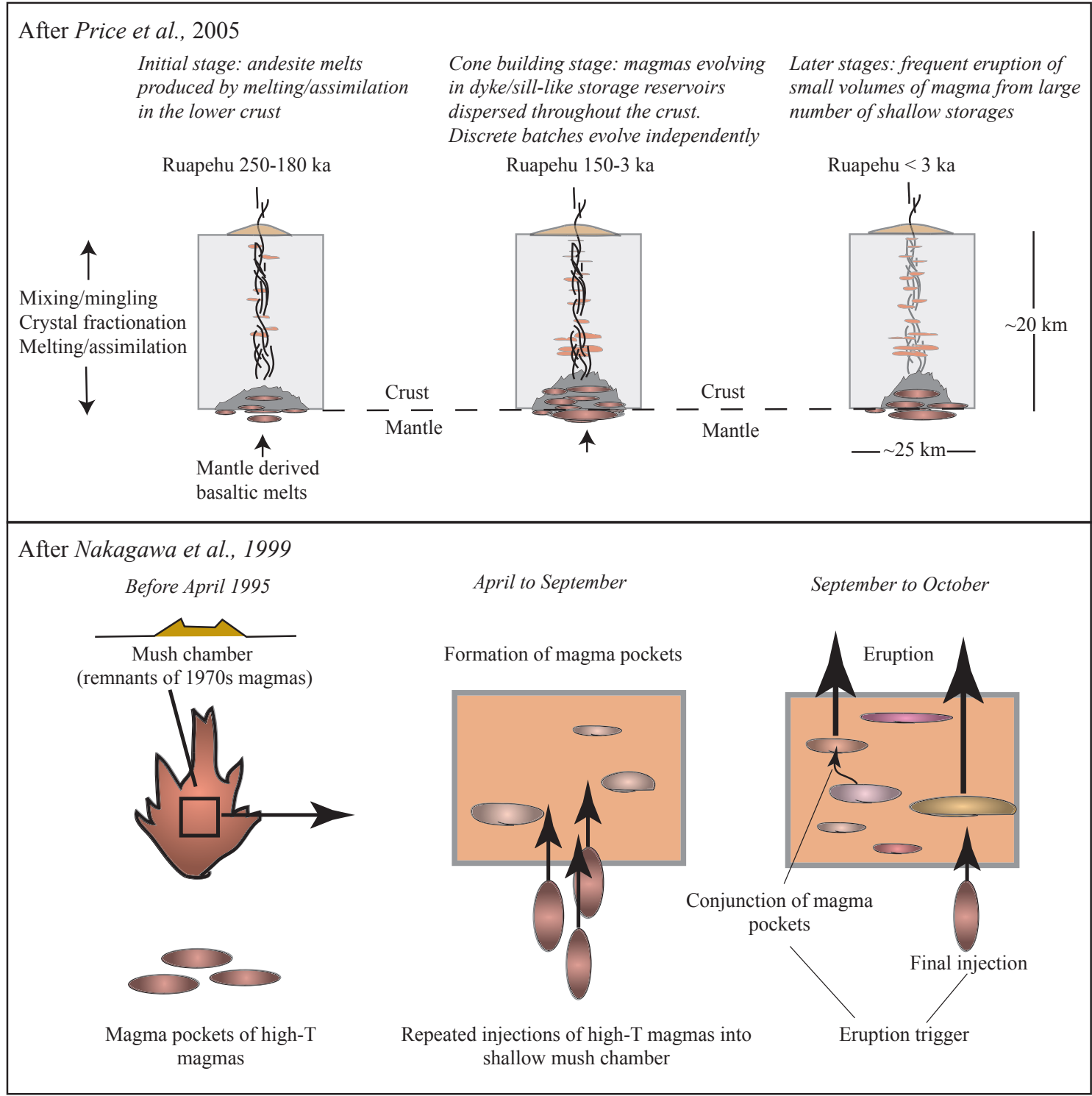

Figure 2.14. Petrological and geochemical models of Mt. Ruapehu [after Nakagawa et al., 1999; Price et al., 2005].

Petrologic and geochemical evidence suggests that rather than having one main magma reservoir, magma is stored at Mt. Ruapehu in evolving dikes and sills dispersed throughout the crust [Price et al., 2005] (Figure 2.14). These magma pockets are suggested to be discreet, enabling different levels of crust assimilation [Graham et al., 1995], until just before or during an eruption, when mixing occurs [Donoghue et al., 1995]. Magma composistions fluctuate through wide ranges over relatively short time intervals, although geochemical evidence [Gamble et al., 1999] from collective data suggest that the general trend is increasing $\mathrm{SiO}_{2}$ abundance. Gamble et al. 
[1999] showed that the temporal geochemical and petrographic variations among erupted magmas are modulated by processes of mixing and mingling between fresh magma from below and stagnant melt and entrained crystals from earlier events remaining in the volcanic edifice. Evidence of mixing comes from complexly zoned phenocryst assemblages, banded pumices and variable melt inclusion compositions in phenocrysts, although zoned feldspar phenocrysts suggest long existences of some magma before extrusion [Gamble et al., 1999]. The conjunction of magma pockets has been suggested as a possible eruption trigger for the 1995/1996 eruptions by Nakagawa et al. [1999] (Figure 2.14), although the main trigger was thought to be injection of high temperature magmas into the shallow plumbing system. Nakagawa et al. [1999] propose that, prior to 1995 there was a shallow low-temperature magma storage system comprising crystal-rich mush and remnant magma from preceding eruptive episodes. Geochemical evidence from tephras show that at least two discrete high-temperature magmas were then repeatedly injected into the mush zone, forming discrete and mixed magma pockets within the shallow system. The intermittent 1995 and 1996 eruptions sequentially tapped these magma pockets. Geochemical evidence suggests that the high-temperature magmas that are stored in the lower crust $(\sim 20 \mathrm{~km})$ are recharged on a time scale of the order of thousands of years [Price et al., 2007; Stewart, 2010] and it is the migration of batches of this more mafic magma up through the crust (on time scales of the order of 10-100 years), interacting with the more evolved magma at shallow levels, that triggers eruptions. Magma flux has been found to be consistent $\left(0.8 \mathrm{~km}^{3} \mathrm{ka}^{-1}\right)$, through ${ }^{40} \mathrm{Ar} /{ }^{39} \mathrm{Ar}$ dating, over time scales of thousands of years although production may be in short bursts of activity, creating fluxes up to 20 times greater [Gamble et al., 2003]. 


\subsection{Seismic anisotropy}

\subsubsection{Introduction}

Seismic anisotropy is the variation of seismic wavespeed with direction. It is an indicator of geometric ordering in a material, where features smaller than the seismic wavelength (e.g. crystals, cracks, pores, layers or inclusions) have a dominant alignment. This alignment leads to a directional variation of elastic wavespeed. Measuring the effects of anisotropy in seismic data can provide important information about processes and mineralogy in the Earth. Measurements of seismic anisotropy have been used to detect fabric and stress in the Earth's crust [e.g. Christensen and Mooney, 1995], flow in the upper mantle [e.g. Ribe, 1989], topography of the core-mantle boundary [e.g. Kendall and Silver, 1996] and differential rotation of the inner core [e.g. Song and Richards, 1996].

Hess [1964] first made a significant observation of large-scale anisotropy when seismic refraction measurements in oceans showed that the $\mathrm{P}$ wave velocity of the upper mantle (Pn) was consistently higher for profiles recorded perpendicular to an oceanic spreading centre (i.e. parallel to the direction of spreading or plate movement) than for profiles recorded parallel to the spreading centre. These measurements were attributed to the alignment of olivine crystals in the mantle lithosphere because of flow during the formation of the oceanic plate at the ridge. Since the 1970s improvements in computing power and memory and in seismic field observation have led to a greater understanding of the seismic anisotropy of the Earth at all levels and scales.

\subsubsection{Theoretical models of anisotropy}

The derivation of the equations governing seismic body wave propagation in anisotropic media presented here follows the approaches of Crampin [1984], Babuška and Cara [1991] and Stein and Wysession [2003].

In a homogeneous anisotropic medium the elastodynamic equations of motion can be written as

$$
\rho \frac{\partial^{2} u_{j}}{\partial t^{2}}=\frac{\partial \sigma_{j k}}{\partial x_{k}}
$$

for $j, k=1,2,3$. Here $u_{j}$ is the component of displacement in the direction $j$ of the displacement vector $\vec{u}, x_{k}$ is position, $t$ is time and $\rho$ is density. $\sigma_{j k}$ are the 
components of the second-order stress tensor, which is related to infinitesimal strain by Hooke's Law:

$$
\sigma_{j k}=C_{j k m n} \epsilon_{m n}
$$

for $j, k, m, n=1,2,3$, where $C_{j k m n}$ is the fourth-order tensor of elastic moduli that defines the properties of the medium and $\epsilon_{m n}$ is the second-order strain tensor:

$$
\epsilon_{m n}=\frac{1}{2}\left(\frac{\partial u_{m}}{\partial x_{n}}+\frac{\partial u_{n}}{\partial x_{m}}\right) .
$$

Due to the symmetry of $\epsilon_{m n}$, Equation 2.2 can be written as

$$
\sigma_{j k}=C_{j k m n} \frac{\partial u_{m}}{\partial x_{n}}
$$

meaning that Equation 2.1 becomes

$$
\rho \frac{\partial^{2} u_{j}}{\partial t^{2}}=C_{j k m n} \frac{\partial^{2} u_{n}}{\partial x_{k} \partial x_{m}}
$$

The fourth-order tensor of elastic moduli $C_{j k m n}$ has the following symmetries:

$$
C_{j k m n}=C_{k j m n}
$$

due to the symmetry of $\sigma_{j k}$,

$$
C_{j k m n}=C_{j k n m}
$$

due to the symmetry of $\epsilon_{m n}$, and from thermodynamic arguments

$$
C_{j k m n}=C_{m n j k}
$$

These symmetry arguments reduce the number of independent elastic parameters from $3^{4}=81$ to 21 , and enables the tensor of elastic moduli to be written as a symmetric $6 \times 6$ matrix [e.g. Babuška and Cara, 1991]:

$$
C_{j k m n}=\left[\begin{array}{llllll}
C_{1111} & C_{1122} & C_{1133} & C_{1123} & C_{1131} & C_{1112} \\
C_{2211} & C_{2222} & C_{2233} & C_{2223} & C_{2231} & C_{2212} \\
C_{3311} & C_{3322} & C_{3333} & C_{3323} & C_{3331} & C_{3312} \\
C_{2311} & C_{2322} & C_{2333} & C_{2323} & C_{2331} & C_{2312} \\
C_{3111} & C_{3122} & C_{3133} & C_{3123} & C_{3131} & C_{3112} \\
C_{1211} & C_{1222} & C_{1233} & C_{1223} & C_{1231} & C_{1212}
\end{array}\right]
$$


The displacement vector $\vec{u}$ of a plane wave can be expressed as

$$
u_{j}=a_{j} e^{i \omega\left(t-\frac{n_{l} x_{l}}{c}\right)}
$$

where $a_{j}$ is the amplitude vector defining the polarisation, $\omega$ is the angular frequency, $c$ is the phase velocity and $n_{l}$ are the components of a normal vector $\vec{n}$ in the direction of propagation.

The derivatives of $\vec{u}$ in space and time can be written as

$$
\begin{gathered}
\frac{\partial^{2} u_{j}}{\partial t^{2}}=a_{j}(i \omega)^{2} e^{i \omega\left(t-\frac{n_{l} x_{l}}{c}\right)} \\
\frac{\partial^{2} u_{n}}{\partial x_{k} \partial x_{m}}=\frac{a_{n} n_{k} n_{m}}{c^{2}}(i \omega)^{2} e^{i \omega\left(t-\frac{n_{l} x_{l}}{c}\right)} .
\end{gathered}
$$

Inserting these derivatives into Equation 2.5, we find that

$$
\rho a_{j}=\frac{C_{j k m n} a_{n} n_{k} n_{m}}{c^{2}}
$$

where we have omitted the common multiplier $(i \omega)^{2} e^{i \omega\left(t-\frac{n_{l} x_{l}}{c}\right)}$.

In order to derive a simple relationship for the propagation of seismic waves in an anisotropic medium, we will restrict our attention to propagation in the direction $x_{1}$. This is not a severe restriction because we can rotate the tensor of elastic moduli to consider propagation in other directions. Equation 2.13 becomes

$$
\begin{aligned}
& \rho c^{2} a_{1}=C_{1111} a_{1}+C_{1112} a_{2}+C_{1113} a_{3} \\
& \rho c^{2} a_{2}=C_{2111} a_{1}+C_{2112} a_{2}+C_{2113} a_{3} \\
& \rho c^{2} a_{3}=C_{3111} a_{1}+C_{3112} a_{2}+C_{3113} a_{3}
\end{aligned}
$$

These equations can be written as the linear eigenvalue problem

$$
\left(\mathbf{M}-\rho c^{2} \mathbf{I}\right) \mathbf{a}=0
$$

where the $3 \times 3$ matrix $\mathbf{M}$ is given by

$$
\mathbf{M}=\left[\begin{array}{lll}
C_{1111} & C_{1112} & C_{1113} \\
C_{2111} & C_{2112} & C_{2113} \\
C_{3111} & C_{3112} & C_{3113}
\end{array}\right]
$$


$\mathbf{I}$ is the identity matrix and $\mathbf{a}$ is the amplitude vector.

In the general case this becomes

$$
\left(m_{j n}-c^{2} \delta_{j n}\right) a_{n}=0
$$

where $\delta_{j n}$ is the Kronecker delta function ( 1 if $i=j, 0$ if $i \neq j$ ), and $m_{j n}$ is the Christoffel Tensor $\underline{M}$

$$
m_{j n}=\frac{C_{j k m n} n_{k} n_{m}}{\rho}
$$

the components of which are dependent on the propagation direction $\vec{n}$.

To solve the classic eigenvalue problem in Equation 2.17, we form the determinant:

$$
\operatorname{det}\left(\underline{M}-c^{2} \mathbf{I}\right)=0
$$

This gives us three orthogonal, linearly independent eigenvectors, $\vec{a}$, which are the polarisation vectors.

The eigenvalue for the $i^{\text {th }}$ eigenvector is $c_{i}^{2}$, which represents the squared phase velocity for a polarisation direction parallel to $\vec{a}_{i}$. The result is mutually orthogonal polarisations for the quasi-P and two quasi-S waves, which also all have different velocities. The terminology "quasi" is adopted because the polarisations of the waves in an anisotropic medium are no longer strictly parallel or perpendicular to the direction of propagation. A key implication is that a body wave that is polarised in the direction of one of these three eigenvectors does not experience a polarisation change. A body wave entering the medium that is not polarised in one of these directions, however, will be split into a $\mathrm{P}$ wave and two quasi-S waves, which are often called the fast and slow $S$ waves. This phenomenon is called shear wave splitting or acoustic birefringence.

\subsubsection{Types of anisotropy}

The equations in Section 2.3.2 describe the most general system of anisotropy. Specific systems contain additional symmetries, which reduce the number of independent elements of the elastic tensor. For instance, an orthorhombic crystal system has nine independent elements and a hexagonal system has five. An isotropic solid has only two independent elements, commonly referred to as the Lamé coefficients 
$\lambda$ and $\mu$, and a fluid has only one (the bulk modulus, $k$ ). Table 2.1 displays the orders of anisotropy found in the Earth and typical causes.

Table 2.1. Number of independent elastic coefficients for selected symmetry systems and typical minerals or Earth's materials. After Babuška and Cara [1991].

\begin{tabular}{|l|l|l|}
\hline Type of symmetry & $\begin{array}{l}\text { Number of } \\
\text { independent elastic } \\
\text { coefficients }\end{array}$ & Typical material \\
\hline \hline triclinic & 21 & plagioclase \\
\hline monoclinic & 13 & horneblende \\
\hline orthorhombic & 9 & olivine \\
\hline tetragonal & 6 & stishovite \\
\hline trigonal I & 7 & ilmenite \\
\hline trigonal II & 6 & quartz \\
\hline hexagonal & 5 & ice \\
\hline cubic & 3 & garnet \\
\hline isotropic & 2 & volcanic glass \\
\hline
\end{tabular}

\section{Hexagonal symmetry}

Here we give a detailed explanation of anisotropy with hexagonal symmetry because this is the most relevant type in the crust [Babuška and Cara, 1991].

The elastic tensor for hexagonal materials has the form

$$
C_{j k m n}=\left[\begin{array}{cccccc}
C_{1111} & C_{1122} & C_{1133} & 0 & 0 & 0 \\
C_{2211} & C_{2222} & C_{2233} & 0 & 0 & 0 \\
C_{3311} & C_{3322} & C_{3333} & 0 & 0 & 0 \\
0 & 0 & 0 & C_{2323} & 0 & 0 \\
0 & 0 & 0 & 0 & C_{3131} & 0 \\
0 & 0 & 0 & 0 & 0 & C_{1212}
\end{array}\right]
$$

for an axis of symmetry parallel to axis $x_{3}$ in Cartesian coordinates (e.g. Figure 2.15). This is because $C_{j k m n}=0$ when either one or three of $j k m n$ are equal. This can be expressed as five independent coefficients $A, C, F, L$ and $N$, Love's 

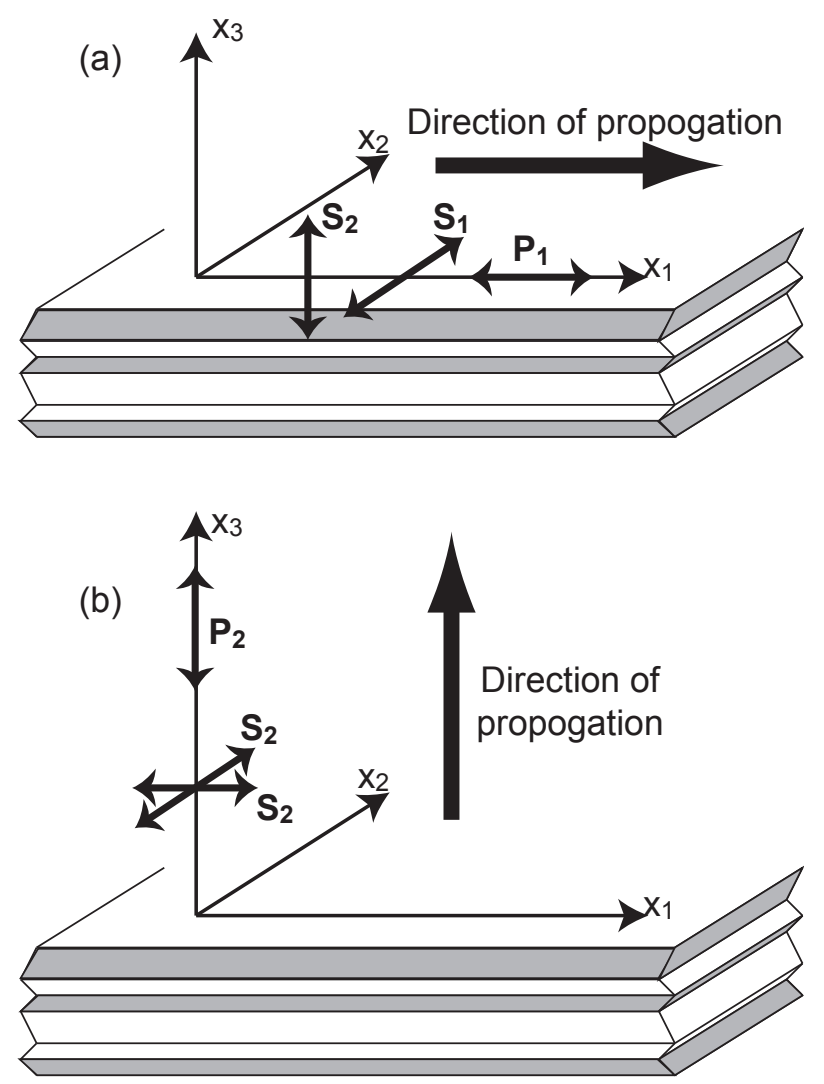

Figure 2.15. Three possible seismic plane-waves propagating through material with alternating fast and slow velocity layers. (a) propagation direction $\left(x_{1}\right)$ is orthogonal to axis of hexagonal symmetry, (b) propagation direction $\left(x_{3}\right)$ is parallel to axis of hexagonal symmetry. After Babuška and Cara [1991].

coefficients, arranged as follows:

$$
C_{j k m n}=\left[\begin{array}{cccccc}
A & A-2 N & \mathrm{~F} & 0 & 0 & 0 \\
A-2 N & A & \mathrm{~F} & 0 & 0 & 0 \\
F & F & \mathrm{C} & 0 & 0 & 0 \\
0 & 0 & 0 & \mathrm{~L} & 0 & 0 \\
0 & 0 & 0 & 0 & \mathrm{~L} & 0 \\
0 & 0 & 0 & 0 & 0 & \mathrm{~N}
\end{array}\right] .
$$

One example of hexagonal anisotropy is a stack of alternating layers of materials with fast and slow seismic velocities (Figure 2.15). The system will have a vertical axis of symmetry and so a vertically propagating $\mathrm{S}$ wave will have a speed independent of its polarisation and will not be split. A wave travelling perpendicular to the axis of symmetry (Figure 2.15 a) however, will have a velocity that is dependent on its polarisation. An $\mathrm{S}$ wave with a polarisation vector perpendicular to the plane of 
the layers will have a velocity that is a function of both seismic velocities. An $\mathrm{S}$ wave with a polarisation vector parallel to the plane can travel in the layers with faster seismic velocity without being severely influenced by the layers with a slower seismic velocity. To examine this, consider a wave propagating in the $x_{1}$ direction. The Christoffel tensor becomes

$$
m_{j k}=\frac{1}{\rho}\left[\begin{array}{ccc}
A & 0 & 0 \\
0 & N & 0 \\
0 & 0 & L
\end{array}\right] .
$$

As long as $L \neq N$, there are three distinct eigenvalues, which are equal to the diagonal terms. The fastest wave is polarised parallel to the direction of propagation and has the velocity

$$
\alpha_{1}=\sqrt{\frac{A}{\rho}} .
$$

If $N>L$, as is the case in Figure 2.15, the second wave has a polarisation in the $x_{1}-x_{2}$ plane, parallel to the layers, and a velocity

$$
\beta_{1}=\sqrt{\frac{N}{\rho}} .
$$

The slowest wave propagates with phase velocity

$$
\beta_{2}=\sqrt{\frac{L}{\rho}}
$$

and is polarised parallel to the axis of symmetry.

In the case of propagation parallel to the axis of symmetry, $x_{3}$ (Figure $2.15 \mathrm{~b}$ ), the Christoffel tensor becomes

$$
m_{j k}=\frac{1}{\rho}\left[\begin{array}{ccc}
L & 0 & 0 \\
0 & L & 0 \\
0 & 0 & C
\end{array}\right],
$$

and two of the three eigenvalues are degenerate. There will be one $\mathrm{P}$ wave polarised in the propagation direction with velocity

$$
\alpha_{2}=\sqrt{\frac{C}{\rho}},
$$


and one $\mathrm{S}$ wave with arbitrary polarisation in the $x_{1}-x_{2}$ plane, and velocity

$$
\beta_{2}=\sqrt{\frac{L}{\rho}} .
$$

Comparison of these two cases shows that the $\mathrm{P}$ wave in the second case (propagation parallel to $x_{3}$ ) has a slower velocity than that in the first case (propagation parallel to $x_{1}$ ) and the $\mathrm{S}$ wave in the second case has the same velocity as the slow wave in the first case.

\section{Systems with lower orders of symmetry}

The majority of anisotropic rocks in the Earth have, or can be approximated to have, hexagonal symmetry. This is because the most common symmetries have patterns that do not differ significantly for horizontal fast axis alignment and near-vertical incidence angles [Savage, 1999]. The simplest models used to explain variations in two orthogonal directions are hexagonally symmetric models. Therefore shear wave splitting is usually interpreted in terms of transverse anisotropy with a horizontal symmetry axis [Savage, 1999]. However, in Section 2.3.2 and Table 2.1 we saw that a lot of common minerals have lower orders of symmetry. These other types of anisotropy usually make up a small percentage of the total and so, if the contributions of different symmetry systems are known, averages can be calculated. For example, for a layered medium that also contains aligned cracks, the combined result is usually orthorhombic symmetry [Babuška and Cara, 1991]. Several averaging processes can be used to define the overall elastic properties of a material on a macroscopic scale. Voight's average is calculated by averaging the stiffnesses of the material (i.e. the rigidities, $\mu_{n}$ ). Reuss's average is computed by averaging the compliances $\left(1 / \mu_{n}\right)$. These are upper and lower bounds and so the Hill's average [Hill, $1952]$ is commonly applied, which is an average of the Voight and Ruess averages [see Mavko et al., 1998, for a detailed comparison].

\section{Intrinsic and extrinsic anisotropy}

The overall anisotropy of a rock depends not only on the minerals of which it is made, but also on the physical conditions that the rock has experienced, and on the length scale of investigation. Most microscopic constituents of the Earth are intrinsically anisotropic (see Table 2.1), meaning that the individual crystals are themselves 
anisotropic. However, if the crystals are randomly orientated, then on the length scales of seismic waves (of order $10^{-1} \mathrm{~m}$ in seismic exploration techniques and $10^{2}$ $\mathrm{km}$ in long-period seismology) the rock will appear isotropic. If this rock then undergoes shearing (plastic and viscous flow), the anisotropic minerals will preferentially align and the rock will be extrinsically anisotropic. This situation is called latticepreferred orientation (LPO) anisotropy [Mainprice and Nicolas, 1989]. Conversely, two isotropic rocks with different physical properties in alternating layers, such as in sedimentary rocks, will commonly be anisotropic on the scale of seismic investigation. In this case, the gravity field has created the rock to be anisotropic. Isotropic rocks that have undergone tectonic stress can also become anisotropic due to aligned fractures or cracks. Practically all rocks contain small cracks and the spatial distribution of these cracks exerts a strong influence on the bulk elastic anisotropy. These situations are called shape-preferred orientation (SPO) anisotropy [Mainprice and Nicolas, 1989] and result from a certain geometric order. In the upper crust, anisotropy is most likely to be caused by stress closing microcracks perpendicular to the maximum compressive stress (SPO) [Crampin, 1994]. At a high confining pressure (when the effect of cracks on seismic velocity is eliminated), anisotropy is controlled by the preferred orientation of anisotropic minerals (LPO) [Babuška and Cara, 1991, and references therein].

\subsubsection{Shear wave splitting}

As discussed in Section 2.3.2, a shear body wave entering an anisotropic medium will typically be split into a quasi-P wave, which is usually small in amplitude and can be neglected [Crampin, 1984], and two quasi-S waves, which are the fast and slow $\mathrm{S}$ waves. The polarisation of the fast $\mathrm{S}$ wave is usually referred to as $\phi$ and the delay time between the fast and slow waves is $\delta$ t (Figure 2.16). Both of these parameters give information about the anisotropic medium: $\phi$ indicates the fast direction of the anisotropic medium and $\delta$ t contains information about the strength of anisotropy and the length of the raypath in the anisotropic medium. Savage [1999] provided a list of shear wave anisotropy studies that give insights into processes at various depths throughout the Earth. Anisotropy in the upper mantle is mainly caused by the preferred orientation of olivine crystals [Mainprice et al., 2005]. This thesis is concerned primarily with crustal anisotropy and so a more detailed explanation of anisotropy in the crust is presented below. 


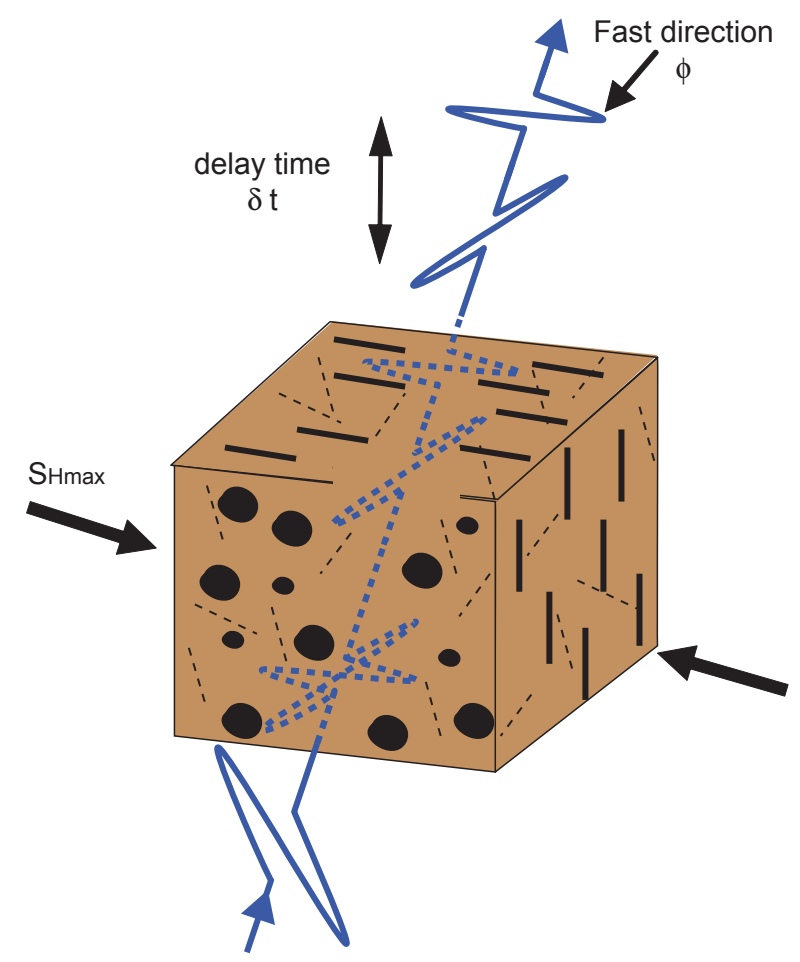

Figure 2.16. Shear wave splitting in an anisotropic medium. Anisotropy is caused by preferentially aligned cracks due to a maximum horizontal compressive stress $\left(S_{H \max }\right)$. A vertically propagating shear wave that is arbitrarily polarised gets split into a fast wave with polarisation $(\phi)$ parallel to crack alignment, and a slow wave, which is polarised at $90^{\circ}$ to $\phi$. The waves are separated with delay time $\delta$ t.

\section{In the crust}

Most of the rocks of which the crust is composed are anisotropic due to preferred mineral orientations (LPO) or small scale compositional layering (SPO) [Mainprice and Nicolas, 1989; Babuška and Cara, 1991]. Compositional layering, as mentioned in Section 2.3.3, has hexagonal symmetry and will usually have its axis of symmetry aligned vertically, yielding transverse anisotropy, if the rock hasn't undergone too much deformation. A vertically propagating wave will therefore not experience any shear wave splitting and will have the velocity of the "slow" direction.

As mentioned earlier, in the upper crust, anisotropy is most likely to be caused by stress closing microcracks perpendicular to the maximum compressive stress (SPO) [Crampin, 1994] (Figure 2.16). For vertical propagation, the shear wave with the displacement in the plane of the open cracks will travel faster than one crossing the plane of the cracks, and so a fast shear wave with orientation $\phi$, and a slow shear wave orthogonal to $\phi$, with a delay time, $\delta t$, will be observed [Babuška and Cara, 1991]. In the simple case of fluid-filled cracks with infinite aspect ratios (flat ellipsoids), 
the system will also have hexagonal symmetry. Often the axis of symmetry will be aligned horizontally, creating azimuthal anisotropy, perpendicular to the direction of maximum horizontal compressive stress. A vertically propagating wave will therefore experience shear wave splitting. When the anisotropy is crack-induced, it can be considered a direct indicator of present stress [e.g. Nur, 1971; Boness and Zoback, $2006 \mathrm{a}]$, with $\phi$ giving information about the orientation of maximum stress and $\delta \mathrm{t}$ giving information about the strength of anisotropy and the amount of time that the wave spent in the anisotropic medium. If the normals to the plane of the cracks are aligned parallel to the $x_{1}$ axis, the stiffness matrix may be written as:

$$
C_{j k m n}=\left[\begin{array}{cccccc}
\lambda+2 \mu & \lambda & \lambda & 0 & 0 & 0 \\
\lambda & \lambda+2 \mu & \lambda & 0 & 0 & 0 \\
\lambda & \lambda & \lambda+2 \mu & 0 & 0 & 0 \\
0 & 0 & 0 & \mu & 0 & 0 \\
0 & 0 & 0 & 0 & \mu(1-\epsilon) & 0 \\
0 & 0 & 0 & 0 & 0 & \mu(1-\epsilon)
\end{array}\right]
$$

where $\lambda$ and $\mu$ are the Lamé coefficients and $\epsilon=N a^{3} / V$ is the crack density, $N$ being the number of cracks in the volume $V$, and $a$ being the radius of the cracks [Crampin, 1985].

Shear wave velocity depends strongly on the porosity of a rock and the degree of saturation [Babuška and Cara, 1991]. In a dry rock, the $\mathrm{S}$ wave velocity is barely influenced by the presence of porosity. If the pore pressure equals the confining pressure, microcracks and grain boundaries are kept open and both $\mathrm{P}$ and $\mathrm{S}$ velocities are reduced, and continue to decrease with increasing pressure. For this reason shear wave splitting analysis has also been used in industry to examine the small changes associated with injection and removal of fluids from reservoirs [Hatchell and Bourne, 2005; Sayers, 2007].

SPO anisotropy from cracks is not always on a microscopic scale. Macroscopic fractures are often aligned because they are caused by faulting in a regional stress field [Boness and Zoback, 2006b]. These macroscopic structures also cause anisotropy and shear wave splitting that often aligns with the maximum compressive stress. Exceptions arise when strike-slip faults initially align at $45^{\circ}$ to the direction of maximum compressive stress, faults are a product of a palaeo-stress, or the rock has been deformed since the faulting.

Velocity anisotropy is also strongly dependent on rock fabrics and metamorphic 
rocks with distinct foliations can have anisotropies of up to $20 \%$ even in the absence of cracks [Babuška and Cara, 1991, and references therein]. This is mainly due to the preferred alignment of intrinsically anisotropic minerals (LPO) such as biotite and hornblende [Babuška and Cara, 1991]. The order of symmetry in this case is dependent on the composition of the rock and the relative abundances of minerals. Shear wave splitting will also depend on the orientation of the rock with respect to the direction of propagation. A metamorphic rock will often have an axis of symmetry that is neither vertical nor horizontal, making interpretation of seismic analyses more challenging (see Section 2.3.6 for a discussion).

\subsubsection{Determination of shear wave splitting parameters}

The following section derives the equations for shear wave splitting parameters based on the method of Silver and Chan [1991].

The displacement of a shear wave arriving at the surface at time $T_{o}$ in an isotropic, homogeneous medium can be expressed (after Equation 2.10) as

$$
\mathbf{u}(\omega)=w(\omega) e^{-i \omega T_{o}} \hat{\mathbf{p}}
$$

where $w(\omega)$ is the wavelet function, incorporating the source time function, the attenuation operator, the instrument response and the vector amplitude, and $\hat{\mathbf{p}}$ is the unit vector pointing in the direction of displacement contained in the plane orthogonal to the direction of propagation $\hat{\mathbf{b}}$.

Splitting due to anisotropy is modelled by projecting $\hat{\mathbf{p}}$ onto the fast and slow polarisation directions $\hat{\mathbf{f}}$ and $\hat{\mathbf{s}}$ and then time shifting them by $\delta t / 2$ and $-\delta t / 2$. The polarisation matrix $\mathbf{V}$ is defined by

$$
\rho V_{i l}=C_{i j k l} \hat{b}_{j} \hat{b}_{k}
$$

For small amounts of anisotropy, $\delta$ t can be expressed in terms of the relative perturbation of shear velocity $\beta$ :

$$
\delta \hat{\beta}=\frac{\delta \beta_{1}-\delta \beta_{2}}{\beta_{0}}
$$

where $\delta \beta_{1,2}=\beta_{1,2}-\beta_{0}$, and $\beta_{0}$ is the isotropic shear velocity, so that

$$
\delta t=\beta_{0}^{-1} L \delta \hat{\beta}
$$


The split waveform can be written as

$$
\mathbf{u}(\omega)=w(\omega) e^{-i \omega T_{0}} \boldsymbol{\Gamma}(\phi, \delta t) \hat{\mathbf{p}}
$$

where $\phi$ is the angle between $\hat{\mathbf{f}}$ and $\hat{\mathbf{p}}$, and the splitting operator is

$$
\boldsymbol{\Gamma}=e^{i \omega \delta t / 2} \hat{\mathbf{f}} \hat{\mathbf{f}}+e^{-i \omega \delta t / 2} \hat{\mathbf{s}} \hat{\mathbf{S}}
$$

or more simply

$$
\boldsymbol{\Gamma}=e^{i \omega \delta \mathbf{T}(\phi, \delta t)}
$$

where

$$
\delta \mathbf{T}=\delta t / 2(\hat{\mathbf{f f}}-\hat{\mathbf{s}} \hat{\mathbf{s}})
$$

A straightforward way to determine the parameters $\phi$ and $\delta t$ is to correct for the anisotropy by returning Equation 2.36 to the form of Equation 2.32 by searching for the inverse operator $\boldsymbol{\Gamma}^{-1}$. Equation 2.37 shows that the operator is unitary and so the inverse is simply the complex conjugate $\Gamma^{*}$.

A measure of linearity can be found by computing the eigenvalues of the twodimensional time-domain covariance matrix $c_{i j}$ of the particle motion:

$$
c_{i j}(\phi, \delta t)=\int_{-\infty}^{\infty} u_{i}(t) u_{j}(t-\delta t) d t
$$

There will be one non-zero eigenvalue in the absence of anisotropy:

$$
\lambda_{1}=\int_{-\infty}^{\infty} w(t)_{2} d t
$$

In the presence of anisotropy $\mathbf{c}$ will have two non-zero eigenvalues $\lambda_{1}$ and $\lambda_{2}$, and thus a search for $\Gamma^{-1}$ such that the corrected seismogram

$$
\tilde{\mathbf{u}}_{s}(\omega)=\Gamma^{-1} \mathbf{u}_{s}(\omega)
$$

possesses a singular covariance matrix will produce the splitting parameters $\phi$ and $\delta t$.

For any pair of values of $\phi$ and $\delta t$, the covariance matrix $\tilde{\mathbf{c}}(\phi, \delta t)$ for the rotated and shifted seismograms may be expressed in terms of the covariance $\mathbf{c}(\delta t)$ of a reference 
coordinate system. The components of $\tilde{\mathbf{c}}(\phi, \delta t)$ are therefore

$$
\begin{aligned}
\tilde{\mathbf{c}}_{11}(\phi, \delta t) & =\int_{-\infty}^{\infty} \tilde{\mathbf{u}}_{1}^{2}(t+\delta t / 2) d t \\
& =\tilde{\mathbf{c}}_{11}(\phi, 0) \\
& =R_{1 i}(\phi) c_{i j}(0) R_{1 j}(\phi) \\
\tilde{\mathbf{c}}_{22}(\phi, \delta t) & =\int_{-\infty}^{\infty} \tilde{\mathbf{u}}_{2}^{2}(t+\delta t / 2) d t \\
& =\tilde{\mathbf{c}}_{22}(\phi, 0) \\
& =R_{2 i}(\phi) c_{i j}(0) R_{2 j}(\phi) \\
\tilde{\mathbf{c}}_{12}(\phi, \delta t) & =\int_{-\infty}^{\infty} \tilde{\mathbf{u}}_{1}(t+\delta t / 2) \tilde{\mathbf{u}}_{2}(t+\delta t / 2) d t \\
& =R_{1 i}(\phi) c_{i j}(\delta t) R_{2 j}(\phi) \\
& =\tilde{\mathbf{c}}_{21}(\phi, \delta t)
\end{aligned}
$$

where the rotation tensor $\mathbf{R}$ defines the change of coordinate system from the initial reference frame of north-south, east-west. In the presence of noise, $\tilde{\mathbf{c}}(\phi, \delta t)$ will not be singular but the grid search over $\phi$ and $\delta t$ will be for the matrix that is most nearly singular. This is equivalent to finding the minimum of $\lambda_{2}\left(\lambda_{2}^{\min }\right)$, which gives a measure of variance of the noise processes. For a noise process that is approximately Gaussian, the squares of the noise components will be $\chi^{2}$-distributed, and $\lambda_{2}^{\text {min }}$ is the sum-of-squares of the noise. This enables the calculation of the confidence region from

$$
\frac{\lambda_{2}}{\lambda_{s}^{\min }} \leq 1+\frac{k}{n-k} f_{k, n-k}(1-\alpha)
$$

where $k$ is the number of parameters $(\phi, \delta t=2), \alpha$ is the confidence level $(0.05$ for $95 \%$ confidence) and $f$ is the inverse of the $F$ probability distribution with $n$ degrees of freedom.

The magnitude of elastic anisotropy is sometimes represented by the coefficient of anisotropy [Babuška and Cara, 1991]

$$
\begin{aligned}
\mathrm{k} & =\frac{\left(\beta_{\max }-\beta_{\min }\right)}{\bar{\beta}} \times 100 \% \\
& =\delta t\left(\frac{1}{t t_{\min }}-\frac{1}{t t_{\max }}\right) \times 100 \%
\end{aligned}
$$

which is also referred to as the percentage anisotropy [Savage, 1999], and sometimes 
by the differential shear wave anisotropy [Crampin, 1994]

$$
\mathrm{SWA}=\frac{\left(\beta_{\max }-\beta_{\min }\right)}{\beta_{\max }} \times 100 \%
$$

Another parameter used is the anisotropy parameter $\xi=N / L[$ Montagner and Kennett, 1996], where $N$ and $L$ are the Love parameters from Section 2.3.3. $\xi$ can be related to $\mathrm{k}$ as follows:

$$
\mathrm{k}=\frac{200(\sqrt{\xi}-1)}{\sqrt{\xi}+1}
$$

Having calculated the strength of anisotropy in a cracked medium, the crack density and crack radius can be estimated using $\epsilon=N a^{3} / V$ from Section 2.3.4, which can be normalised to $\epsilon=a^{3}$ for a unit cube where $\epsilon$ is the crack density and $a$ is the crack radius. Obviously one of these parameters needs to be estimated before the other can be computed.

Another parameter that can be used to characterise shear wave splitting is the splitting intensity, which is measured by the amplitude of the transverse component, and depends on the angle between the backazimuth of the earthquake and the direction of the symmetry axis, and on the delay time between the two quasi-shear waves [Chevrot, 2000]. It can therefore be used to determine $\phi$ and $\delta \mathrm{t}$, or conversely, can be calculated from the splitting parameters and used to compare measurements.

\subsubsection{Ambiguities and limitations}

\section{Subjectivity}

Visual inspection methods of determining shear wave splitting parameters can suffer problems associated with observer bias or subjectivity [Teanby et al., 2004a]. Automated methods can be used to analyse large data sets, although visual inspection is useful for checking the results [Liu et al., 2008]. There are currently several automated methods used in shear wave splitting analysis; the cross-correlation method [e.g. Fukao, 1984], the covariance matrix method [Silver and Chan, 1991] and the aspect ratio method [Shih et al., 1989]. 


\section{Time window and cycle skipping}

All of the methods mentioned above use a 'window' in time around the $\mathrm{S}$ wave arrival. An ideal time window for the covariance matrix method should begin right before the fast arrival and end after the slow arrival [Liu et al., 2008]. However, the analysis window may include a significant portion of $\mathrm{P}$ wave coda before the $\mathrm{S}$ wave arrival or other phases after the slow arrival so the choice of window can significantly affect the results. If the $\mathrm{S}$ waveform is sinusoidal (contains a narrow band of frequencies, e.g. Figure 2.17 a) and the onset is not sharp, or if the time window doesn't allow for any low-energy time before the arrival, then cycle skipping can occur. This is when the match of the fast and slow waveforms has a factor of $T / 2$ ambiguity where $T$ is the dominant period, i.e. a shift of the slow direction by $n \times T / 2$ for integer $n$ will give the same degree of fit and waveform linearity.

Cycle skipping can often be identified by examination of the error contours from a shear wave splitting measurement: if there are multiple minima, separated by $T / 2$ on the delay time axis (Figure $2.17 \mathrm{~d}$ ). Usually the correct solution is chosen but sometimes the wrong one is selected, which leads to a false delay time. If one or more cycles are skipped so that the slow component lags behind, the fast direction is not affected, however, if the slow component is shifted in front of the fast component then the slow direction will be recorded as the fast direction. This leads to an error of $90^{\circ}$ in the recorded fast direction. Several attempts have been made to find the optimal window [e.g. Peng and Ben-Zion, 2004; Liu et al., 2008] but Teanby et al. [2004a] devised an automated method using cluster analysis to provide an objective choice of window that gives the most stable result for shear wave splitting (Figure 2.18, see Section 3.2.3 for more details).

\section{The shear wave window}

The particle motion recorded at the Earth's surface does not always represent the particle motion of an incoming $S$ wave along its path. Due to interaction of the incoming wave with the free surface, an $\mathrm{S}$ to $\mathrm{P}$ conversion can occur and the measured particle motion can adopt an elliptical waveform even if travelling in an isotropic medium [Nuttli, 1961]. This leads to apparent shear wave splitting with the apparent fast direction pointing to the direction of the incoming wave. A characteristic of measurements that were obtained from rays with a large deviation from vertical is a $180^{\circ}$ periodicity of the fast directions with backazimuth [Crampin, 1985]. 
(a)

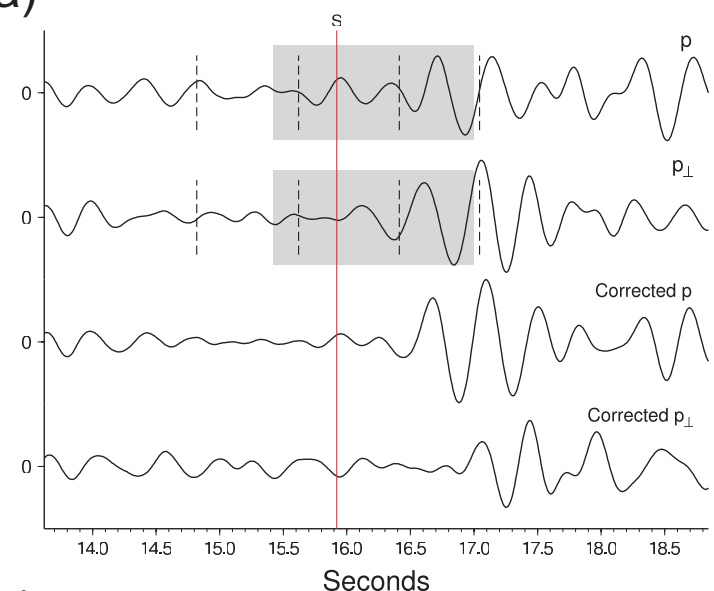

(c)

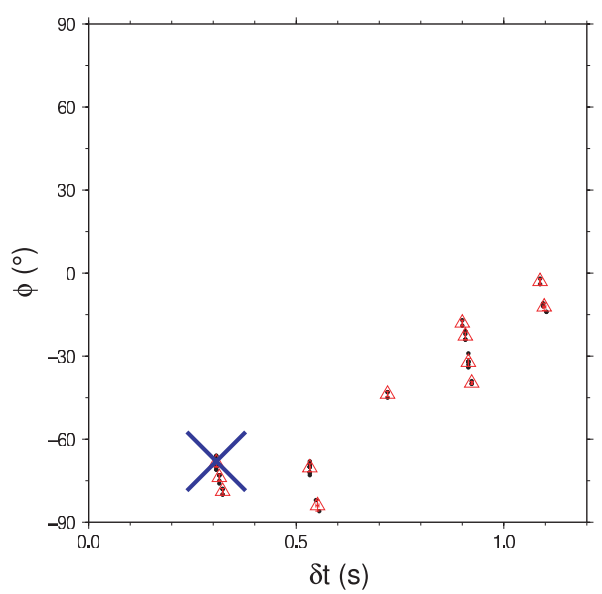

(b)
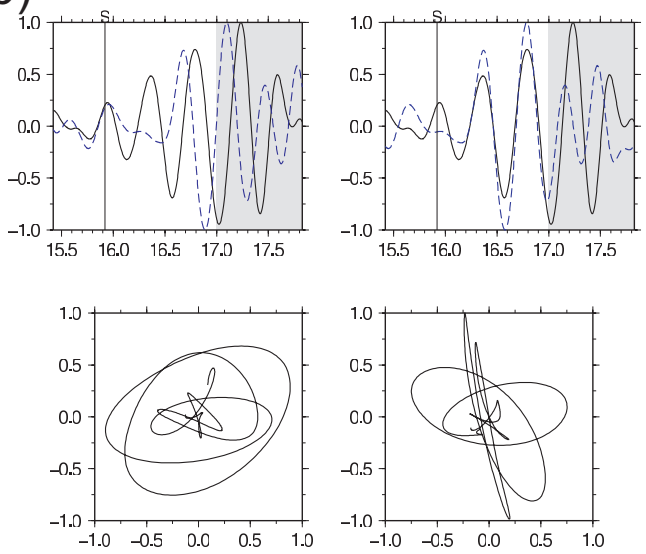

(d)

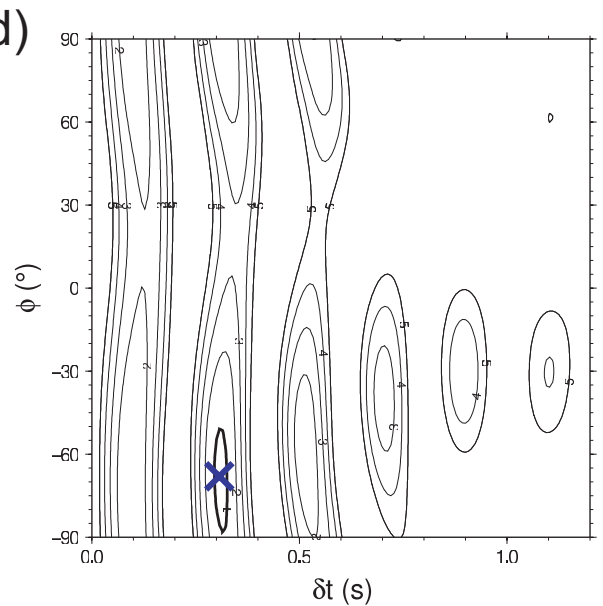

Figure 2.17. Example of measurement with potential cycle skipping from earthquake 3014562 at station OTVZ filtered with $2-3 \mathrm{~Hz}$ band pass. (a) Normalised waveforms of radial and transverse waves and radial and transverse waves corrected for splitting (b) Overlaid waveforms rotated to fast (black) and slow (blue dashed) directions and particle motion showing split (left) and corrected (right) waves. (c) $\phi$ and $\delta$ t results for different time windows. (d) Error surface showing repeating $\phi$.

Nuttli [1961] showed that the distortion is only significant if the incidence angle of the $\mathrm{S}$ wave is larger than the critical $\mathrm{S}_{V}$ to $\mathrm{P}$ conversion angle at the free surface:

$$
i_{c}=\sin ^{-1}\left(\frac{v_{s}}{v_{p}}\right)
$$

where $v_{p}$ and $v_{s}$ are the near-surface $\mathrm{P}$ and $\mathrm{S}$ wave velocities respectively.

Assuming a normal $v_{s} / v_{p}$ ratio of $\sim 1 / \sqrt{3}$ (Poisson's ratio of 0.25 ), the critical angle, $i_{c}$, is found to be close to $35^{\circ}$, i.e. near-vertical incidence [Babuška and Cara, 1991]. Due to this phenomenon, measurements of shear wave splitting with incidence angles larger than $35^{\circ}$ at the surface should not be included in the results as the complex particle motion can result in erroneous splitting parameters. However, 

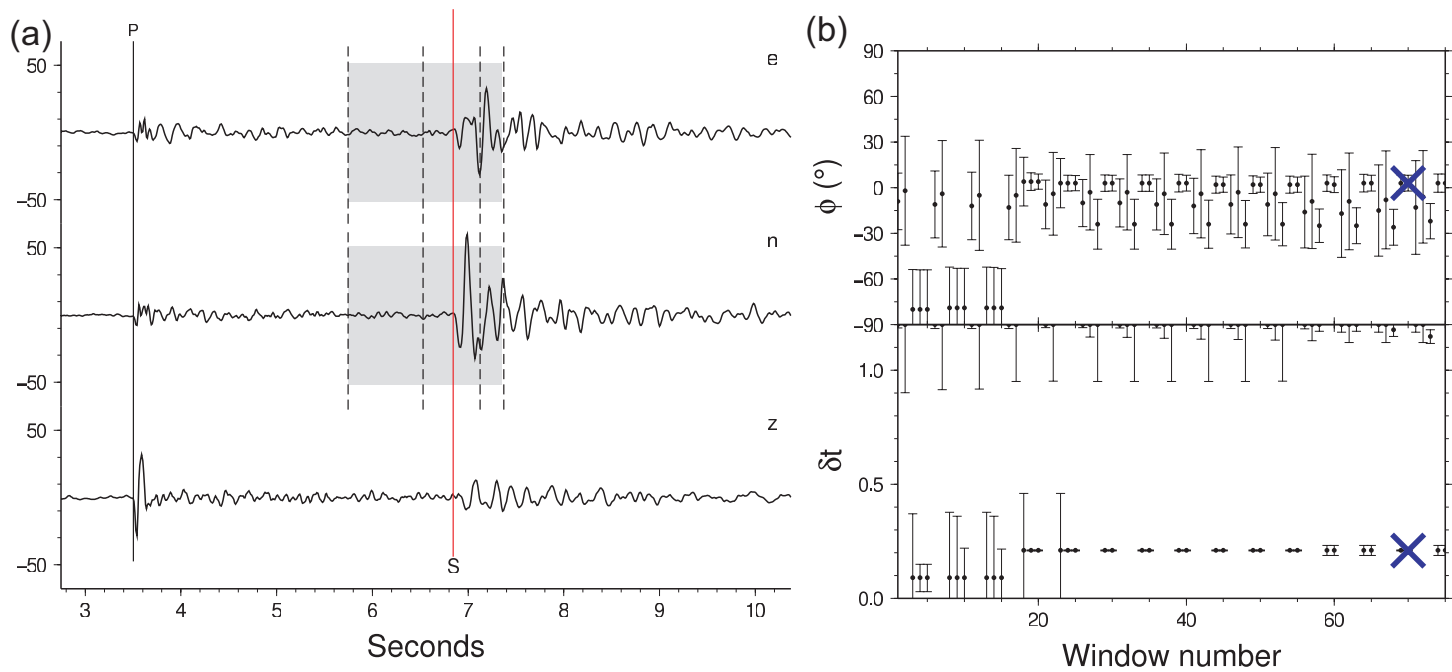

Figure 2.18. Example of window selection from earthquake 2845512 at station MOVZ. (a) Waveforms of east, north and vertical components showing $\mathrm{P}$ and $\mathrm{S}$ (red) arrival picks and maximum and minimum windows. Grey box indicated final chosen window. (b) $\phi$ and $\delta$ t results for different time windows.

shear wave splitting studies [Crampin, 1986; Currie et al., 2001; Boness and Zoback, 2006a] often use an incidence angle threshold of up to $i \leq 50^{\circ}$. This is because the calculation of $i_{c}$ is a simplification and assumes straight line rays. In reality lower velocity layers at the surface will curve the wavefront and effectively extend the shear wave window by several degrees [Gledhill, 1991].

\section{Null measurements}

As mentioned in Section 2.3.2, a shear wave will only be split if the incoming polarisation differs from the fast or slow directions of the medium it travels through. Sometimes an unsplit wave, when analysed for shear wave splitting, can yield splitting parameters due to a matching of the first wavelet with a scattered wave arriving later in the coda. The corresponding value of $\delta \mathrm{t}$ is meaningless and $\phi$ has a $90^{\circ}$ ambiguity as it could be either the fast or slow direction (Figure 2.19). These measurements are known as "nulls" and in practice, result from the shear wave splitting analysis that give $\phi$ to within $20^{\circ}$ of the polarisation of the incoming wave are often considered null results [Peng and Ben-Zion, 2004] and are not included in the interpretation. Nulls signify that no splitting was reliably detected [Silver and Chan, 1991], although sometimes their direction can assist with interpretation of shear wave splitting results as the measured direction will either be the fast or slow direction of the medium. When looking for some of the characteristics described 
(a)

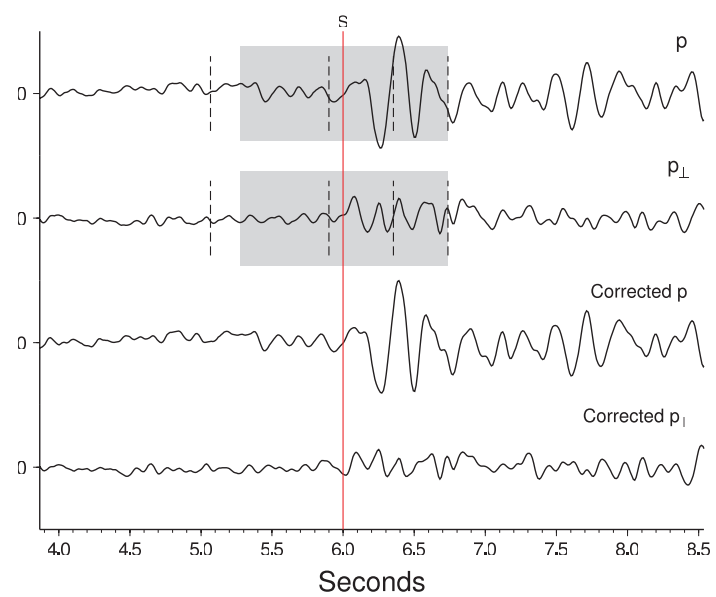

(c)

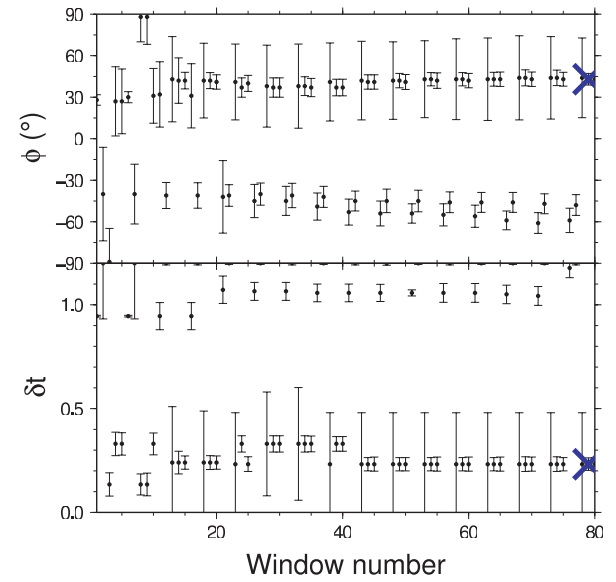

(b)
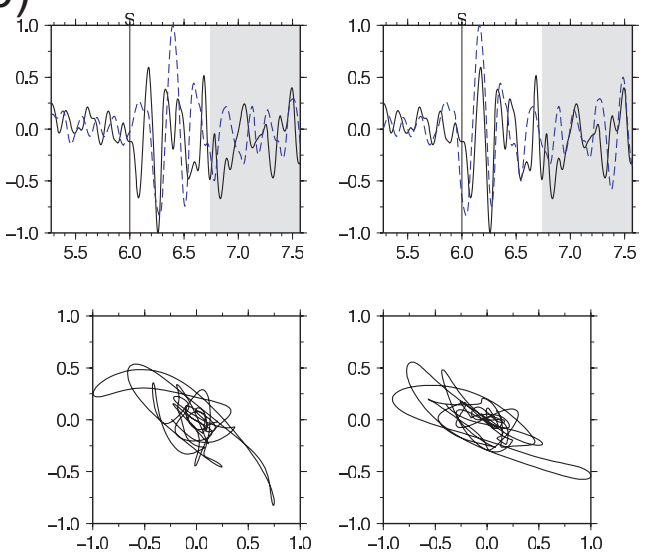

(d)

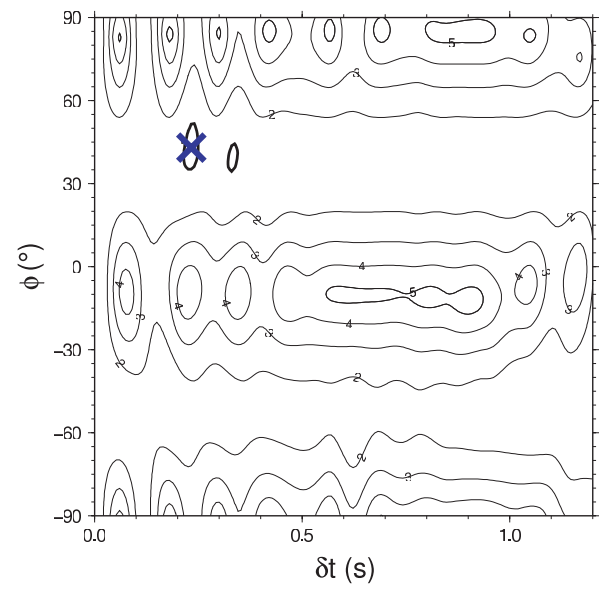

Figure 2.19. Example of null measurement from earthquake 2999693 at station FWVZ. (a) Normalised waveforms of radial and transverse waves and corrected radial and transverse waves. (b) Overlaid waveforms rotated to fast (black) and slow (blue dashed) directions and particle motion showing split (left) and corrected (right) waves. (c) $\phi$ and $\delta$ t results for different time windows. (d) Error surface showing two distinct bars of $\phi$.

subsequently in this section, a diagnostic plot of $\phi$ against incoming polarisation is sometimes useful. In this case the null measurements must be included, otherwise bars of gradient 1 and thickness $40^{\circ}$ will appear with no measurements, as can be seen in Figure 2.20.

\section{Frequency dependence}

Another hurdle that must be overcome in shear wave splitting analysis is that the results (both $\phi$ and $\delta$ t) can be heavily frequency-dependent [e.g. Marson-Pidgeon and Savage, 1997]. Boness and Zoback [2006a] showed that the polarisation of the split shear waves and the amount of anisotropy recorded are strongly dependent 


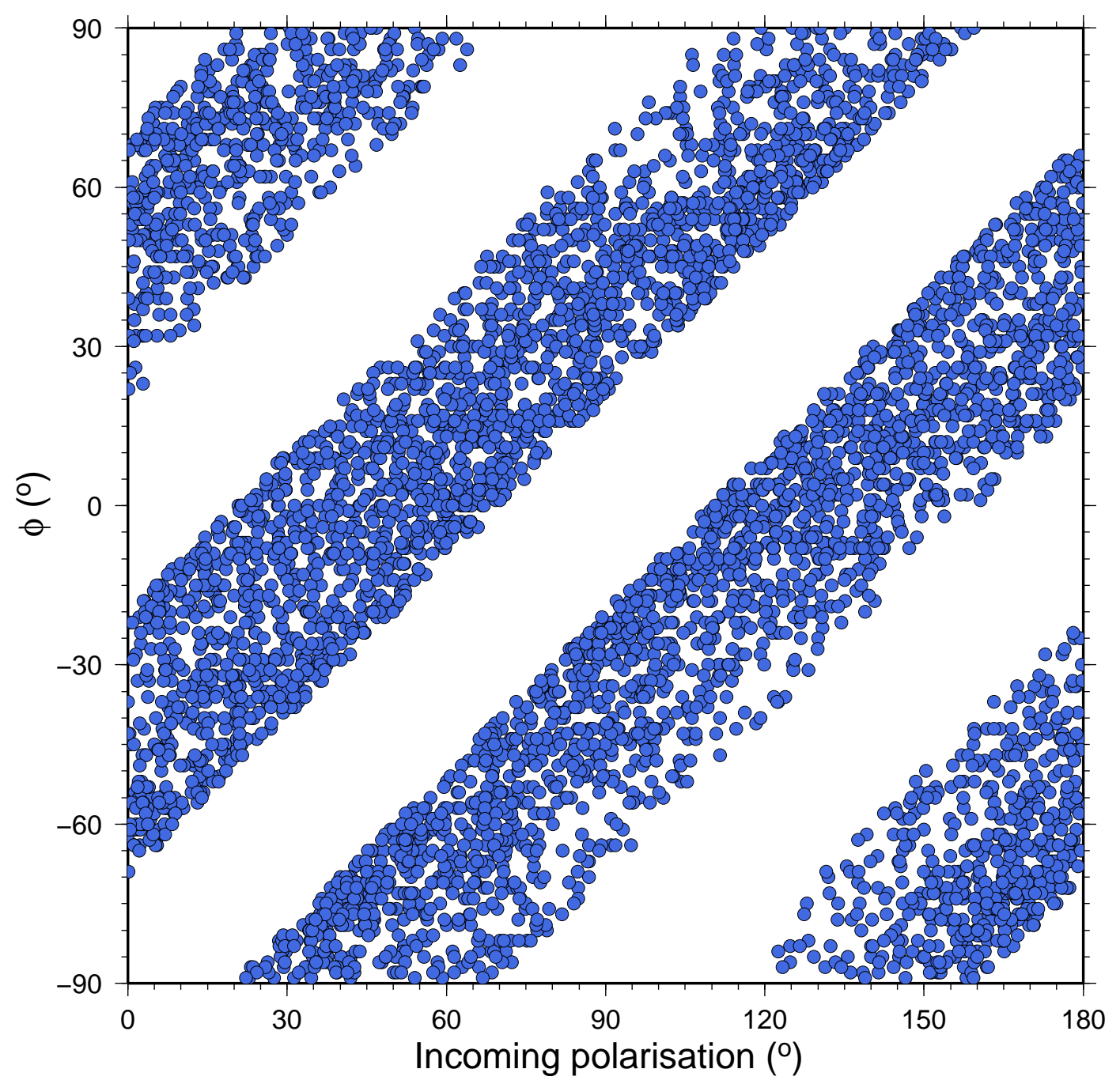

Figure 2.20. Fast polarisation measurements as a function of incoming polarisation displaying the bands, with gradient 1 , that have no data because the measurements that fall into these bands are null measurements.

on the frequency and length scale of investigation. Nistala and McMechan [2005] explained the frequency-dependence of anisotropy by showing that the polarisation at the receiver depends only on the orientation of the anisotropy within two wavelengths of the receiver. Liu et al. [2003] analysed this phenomenon and discussed two mechanisms giving rise to it: scattering of seismic waves by preferentially aligned inhomogeneities, such as fractures, oriented inclusions or fine layers; and fluid flow in porous rocks containing micro-cracks and macro-fractures [Tod and Liu, 2002]. Maultzsch et al. [2003] and Chapman [2003] attributed the frequency-dependence to the length scale of fractures in the rock and suggested that this dependence could be used to calculate fracture size. 


\section{Interpreting shear wave splitting: Some assumptions}

Most shear wave splitting observations are interpreted under some assumptions:

1. That the medium possesses hexagonal symmetry,

2. That the medium has a horizontal axis of symmetry,

3. That the anisotropic medium is in a single, homogeneous layer.

4. That the anisotropic medium is localised beneath the receiver.

These assumptions are rarely completely true in the Earth. The following paragraphs explore these common caveats, although this project will mainly focus on the last of these issues.

\section{Symmetry of anisotropy}

The existence of lower orders of symmetry than hexagonal may produce large variations in shear wave splitting such as frequency-dependence of splitting parameters if the symmetry systems are on different length-scales, as was mentioned above. A single layer of anisotropy that has a dipping axis of symmetry will produce splitting parameters with a certain symmetry with backazimuth even when the incidence angles are near-vertical [Silver and Savage, 1994]. An examination of splitting parameters over a range of backazimuths and angles of incidence can help to identify the symmetry in the medium [Liu et al., 1993]. However, laterally heterogeneous anisotropy may also display a dependence on backazimuth [Zinke and Zoback, 2000] and so care must be taken.

\section{Vertically-layered anisotropy}

When a shear wave passes through two anisotropic layers, the observed splitting parameters depend strongly on the thickness and strength of anisotropy of the layers, the relative fast directions, and on the wavelength of the wave [Silver and Savage, 1994]. If the $\mathrm{S}$ wave has been sufficiently split in the first layer that the fast and slow waves are separated, then when it enters the second layer, which has a fast direction $20-70^{\circ}$ different, both of the quasi-shear waves will be split again [Silver and Savage, 1994] (Figure 2.21). In this case both waves will now have the fast and slow directions of the second layer. When the shear wave splitting parameters are 


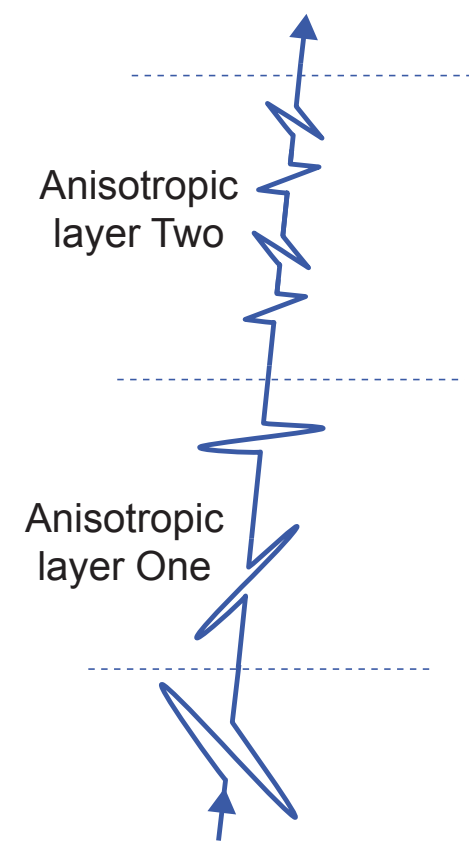

Figure 2.21. Schematic of shear wave splitting in the case of two anisotropic layers. The incoming shear wave is split twice, leading to four individual waves at the receiver. In most cases the individual arrivals are unresolved. After Silver and Savage [1994].

measured, it is only the first arriving wavelets that are taken into account and so the measurement will only show the second layer. One way to determine whether this has happened is to look at the incoming polarisation of the split shear waves. If all of the measurements indicate the same incoming polarisation then it is likely that there is more than one layer of anisotropy. However, when the splitting from the first layer is weak so that the two quasi-shear waves aren't more than one wavelength apart, both waves are still resplit, but the result is a complex waveform that is difficult to interpret, but is still meaningful. Silver and Savage [1994] showed that if apparent splitting measurements are obtained for this case then a characteristic $90^{\circ}$ periodicity in the measured fast direction and delay time as a function of incoming polarisation occurs. This pattern can also help to identify more than one layer of anisotropy.

When there are multiple layers of anisotropy that have a gradual transition, rather than an interface such as in Figure 2.21, the shear waves will not resplit. Ray theory predicts that the two orthogonal waves can rotate slowly around the ray in a smoothly varying anisotropic medium [Babuška and Cara, 1991]. This means that the fast direction recorded at the surface will only reflect that of the uppermost anisotropic medium but the delay time could be a sum of all of the splitting. This could lead to anomalously high delay times. In Chapter 5, we use a simple test 
involving multiplets to determine whether multiple layers are present in the Ruapehu region and find that the data suggest that there is one layer.

\section{Varying incoming polarisation}

As we have seen, several factors may affect the splitting parameters as a function of incoming polarisation. Having a wide range of incoming polarisations may help to identify one or more of these factors. However, if individual or few measurements are used to diagnose changes in anisotropy, be they spatial or temporal, then care must be taken that the variation is not a function of the earthquake source mechanism rather than the raypath. We overcome this in Chapters 5 and 6 by using repeating sources in the form of multiplet earthquakes (see Section 2.4 for more details on multiplets).

\section{Spatially varying anisotropy}

Most shear wave splitting results are plotted as rose diagrams (circular histograms) of fast direction at the station at which the measurements were made (e.g. Section 4.4.1). This implicitly assumes that the anisotropy is localised beneath the station. In many regions this may be appropriate, however when there is lateral heterogeneity, the rose diagrams will become scattered or multi-modal. In Chapter 4 we detect distinct splitting parameters for measurements using earthquakes from several different clusters in the region of Mt. Ruapehu. This backazimuthal dependence suggests that the $\phi$ value obtained from shear wave splitting analysis is highly dependent upon the path that the ray takes, which has also been found in other regions by Zinke and Zoback [2000] in California and Liu et al. [2004] in Taiwan, for example. Furthermore, this suggests that the anisotropy changes over shorter distances than is often observed for mantle anisotropy [Greve et al., 2008] and that averaging $\phi$ over the whole region [Gerst and Savage, 2004] may not be appropriate. If the causes of the different regions of anisotropy are known, it is easier to identify and map the differences using shear wave splitting analysis. Zinke and Zoback [2000] detected both stress-induced and structure related anisotropy at the same station in California, and distinguished between the two mechanisms by observing that different clusters of earthquakes gave different fast polarisations, thereby showing that the shallow crust beneath the station does not always influence the shear wave splitting. If the crustal stresses or fabrics are more complex or unknown, then it is more difficult to map and interpret heterogeneous anisotropy, although a denser array of seismic 
stations and a broad range of backazimuths increases the likelihood of identifying the source of heterogeneity.

\subsubsection{Temporal variation of shear wave splitting in the crust}

The temporal variation of shear wave splitting and its interpretation is highly controversial [e.g. Aster et al., 1990; Bokelmann and Harjes, 2000; Seher and Main, 2004; Liu et al., 2004; do Nascimento et al., 2004; Peng and Ben-Zion, 2005; Liu et al., 2008]. The main point of dispute is whether the accumulation of stress before earthquakes allows the time and magnitude of impending large earthquakes to be stress-forecast through shear wave splitting monitoring. The effects have been reported with hindsight before 15 earthquakes ranging in magnitude from an M1.7 seismic swarm event in Iceland [Gao and Crampin, 2004] to the M7.7 Chi-Chi Earthquake in Taiwan [Crampin and Peacock, 2005], including an apparently successfully stress-forecast M5.0 earthquake in SW Iceland [Crampin et al., 1999]. Crampin and Peacock [2008] present a biased summary of observations of temporal variations in shear wave splitting attributed to stress-aligned fluid-saturated microcracks and contrary interpretations. Among the discussions about the identification of temporal variations there are accusations of observer bias in data selection [Aster et al., 1990], unsound statistical analyses [Seher and Main, 2004], misinterpretation of spatial variation [ Liu et al., 2004] and lack of correlation with other stress determining factors/correlation with structural evidence [do Nascimento et al., 2004].

Clear evidence has been obtained that crustal shear wave splitting can vary over short distances and can be caused by structural features in the crust, which would not change with changing stress [Zinke and Zoback, 2000; Boness and Zoback, 2006a]. However, it is also clear that anisotropy due to stress-aligned fluid-saturated microcracks can change with time, as has been demonstrated in industry when small changes associated with injection and removal of fluids from reservoirs were examined [Hatchell and Bourne, 2005; Sayers, 2007; De Meersman et al., 2009].

The use of similar earthquakes (i.e. those that have the same source mechanism and location) for shear wave splitting analysis helps to eliminate some of the discrepancies with interpretation. Peng and Ben-Zion [2005] used similar earthquakes to analyse spatiotemporal variations of crustal anisotropy in the aftershock regions of the 1999 M7.4 Izmit and M7.1 Düzce earthquakes. They found pronounced changes in the spatiotemporal anisotropy patterns but explained them by the spatial variations of raypaths due to the changing seismicity, rather than changes in the properties of the 
anisotropic medium. Results using the similar earthquakes did not show systematic precursory changes in anisotropy before the Düzce main-shocks.

A spatial and temporal analysis of shear wave anisotropy around the San Andreas Fault was conducted by Liu et al. [2008] using similar events so that the raypaths were almost identical. They found strong spatial variations in both the measured $\phi$ and $\delta$ t, which revealed the extreme complexity of shear wave anisotropy in the area. However, the results also showed that there were no appreciable precursory, coseismic, or post-seismic temporal changes in a region near the rupture of an M6.0 earthquake, about $15 \mathrm{~km}$ away from its epicentre.

Temporal variation of shear wave splitting parameters as a stress indicator has also been employed at several volcanoes. These observations are summarised in Section 2.3.8

\subsubsection{Shear wave splitting around volcanoes}

Even though we have seen that shear wave splitting analysis can be used as an indicator of stress and of fluid saturation in the crust, surprisingly few studies have been conducted on shear wave splitting around volcanoes (Figure 2.22). This is due, in part, to the generally noisy waveforms and complicated interpretation of such observations when taking into account heterogeneity and complex stress regimes. This section contains an overview of some shear wave splitting studies around active volcanoes. These studies are often coupled with other stress or strain indicators in order to minimise the ambiguity in the interpretation of shear wave splitting parameters. It should be noted that there is significant literature about shear wave splitting in the mantle beneath active volcanoes because these regions are invariably of interest tectonically, but this section focuses on crustal studies only.

\section{Hawaii}

Munson et al. [1993] and Munson et al. [1995] found that $\phi$ was consistent with the direction of maximum horizontal compressive stress in Southern Hawaii. Savage et al. [1989] also found that around the East Rift Zone of Hawaii, fast polarisations tended to be parallel to the strike of the East Rift Zone and the regional maximum horizontal compressive stress from earthquake focal mechanisms. They found that the average anisotropy was about $5 \%$ but also found evidence of near-station effects. 


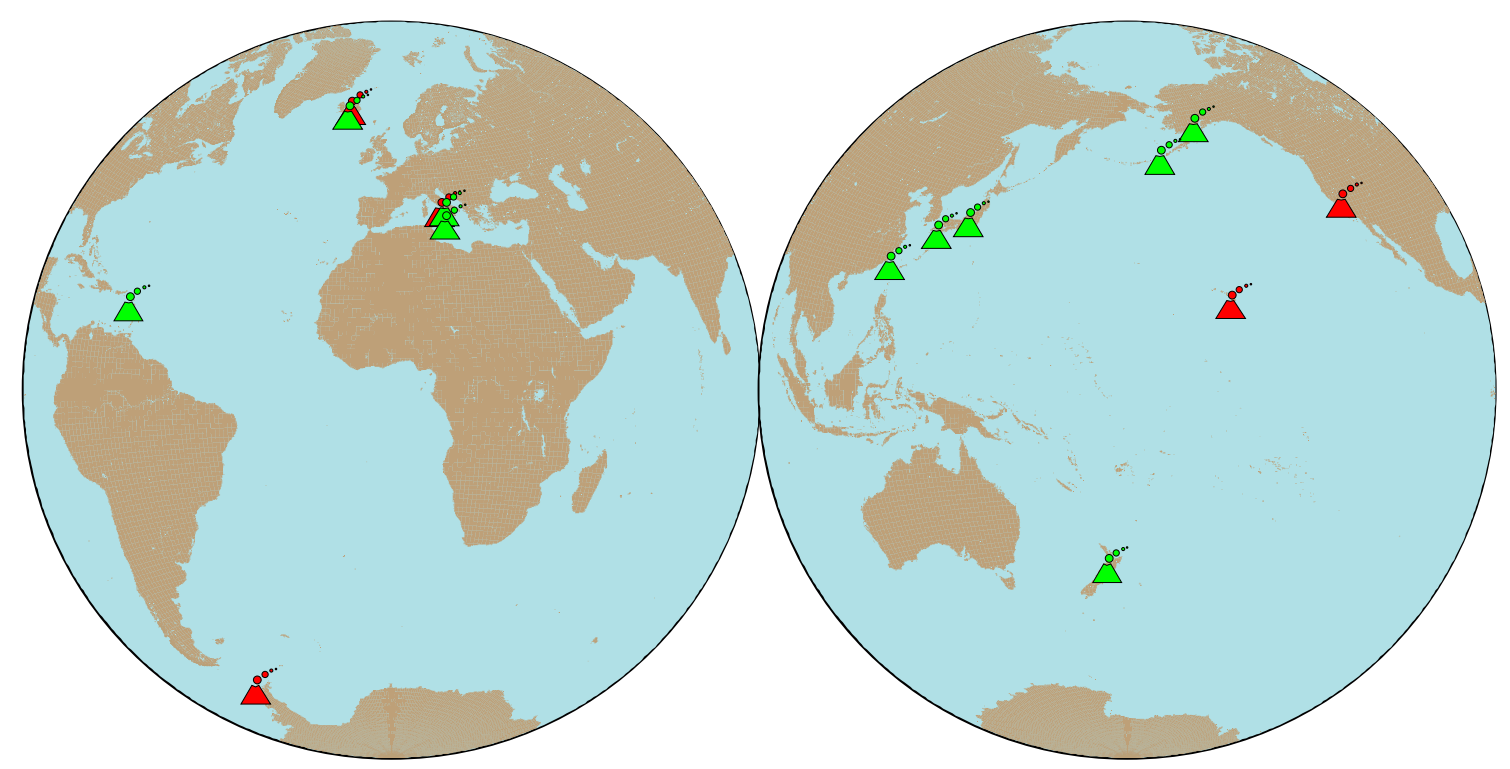

Figure 2.22. Map showing volcanoes at which shear wave splitting investigations have been carried out. Green volcano symbol indicates that temporal variations were observed, red indicates that no temporal variations were observed. Details of the studies are given in the text.

Local to the Kaoiki Fault Zone, Booth et al. [1992] found that $\phi$ was consistent with anisotropy being caused by cracks aligned approximately perpendicular to the direction of least principal stress. However, they did not observe time-dependent variation of $\phi$ associated with the $1983 \mathrm{M}_{L} 6.6$ earthquake, either because there was no change or the change was smaller than the scatter of the data. Munson et al. [1993] found that, in the Kaoiki region, delay time measurements showed evidence for both dominant shallow and weaker pervasive anisotropy and a crack density of 6-12\% was obtained. However, Munson et al. [1995] later found large station-tostation variations in $\phi$ and a relationship between delay time measurements and event depth that provided evidence of predominantly shallow anisotropy. A search for temporal changes in anisotropy associated with the 1983 Kaoiki main shock was also unsuccessful. Deviation from the general trend was detected at a different array in Hilea, where Munson et al. [1993] found that $\phi$ was rotated by $30^{\circ}$ in relation to the general direction of maximum horizontal compressive stress in Southern Hawaii.

\section{Long Valley}

Savage et al. [1990] examined shear wave splitting from local earthquakes around Long Valley Caldera and found that the fast directions had a strong lateral variation but in most cases lined up with fault strikes and with the $\mathrm{P}$ axes of focal mechanism 
groupings. The lateral variation suggested that the anisotropy was primarily nearstation and shallow in this region. Further shear wave splitting analysis by Shih and Meyer [1990], who also found lateral variation in $\phi$, indicated interaction between the inflating caldera and the Sierra Nevada range-front faults.

\section{Alaska}

Gardine and Roman [2010] investigated shear wave splitting and earthquake faultplane solutions at Redoubt volcano around the time of the March 2009 eruption. They found that after the eruption, $\phi$ had a direction more consistent with the regional stress than before the eruption.

In Chapter 6 we use shear wave splitting analysis, a multiplet of 25 similar earthquakes and double-difference relocation to examine temporal variations in seismic properties prior to and accompanying magmatic activity associated with the 2008 eruption of Okmok volcano. We find a general change in $\phi$ but cannot rule out dependence on backazimuth, and no significant change using the multiplet. Using earthquakes originating from the subducted slab in order to reduce the effect from changing paths, Kufner [2010] examined shear wave splitting beneath Okmok volcano. Several modes of $\phi$ were identified, relating to the anisotropy of the mantle wedge, regional stress direction, and local stress induced by the pressurisation and depressurisation of the magma reservoir. These modes were found to have different prominence at different times throughout the eruptive cycle.

\section{Montserrat}

Roman et al. [2011] investigated local stress field reorientations preceding changes in volcanic activity at the Soufrière Hills volcano using local earthquake fault-plane solutions and measurements of shear wave splitting in regional earthquakes. They observed that local stress orientation from fault-plane solutions during a six-month period preceding the onset of eruptive activity in 1999 was highly localised and spatiotemporally variable, although the variation in fault-plane solutions and shear wave splitting measurements were correlated. The spatial pattern of precursory local stress orientations was modelled by Roman et al. [2011] in terms of the pressurisation of a vertical dike. 


\section{Etna}

Bianco et al. [1996] investigated shear wave splitting on the east slope of Mt. Etna Volcano, Sicily, and found consistent $\phi$ aligned with the maximum compressive stress regime calculated from fault-plane solutions. Musumeci et al. [2005] conducted a similar analysis in southeastern Sicily, where focal mechanism inversions and shear wave splitting confirmed the regional alignment. Temporal variations of shear wave splitting parameters related to eruption were identified by Bianco et al. [2006] and Zaccarelli et al. [2009]. Bianco et al. [2006] found that time delays of shear wave splitting before the 17 July-9 August 2001 flank eruption of Mt. Etna showed a systematic increase starting several days before and a sudden decrease hours before the start of the eruption. On several occasions before the eruption, Bianco et al. [2006] also observed a $90^{\circ}$ flip of $\phi$, whereby the faster and slower split shear waves exchanged polarisations. The observed changes of the splitting parameters were interpreted in terms of changes in the stress field acting in the area, in turn producing changes in the aspect ratio of the cracks. Coda wave interferometry and shear wave splitting analysis using earthquake doublets were employed by Zaccarelli et al. [2009] to track stress-related wave propagation effects during the waning phase of the 2002 NE fissure eruption at Mt. Etna. They detected temporal changes in both wave velocities and anisotropy, consistent with observed eruptive activity, and inferred a depressurisation of the system correlating with the termination of the eruption.

\section{Vesuvius}

Bianco et al. [1998] carried out a detailed structural and geophysical study of the Somma-Vesuvius volcanic complex by integrating mesostructural measurements, focal mechanisms and shear wave splitting analysis. They found that the movements of faults were mainly related to the regional stress field and that a local stress field was also present but that there was no evidence of magma- or gravity-induced stresses. Seismic swarms were found to correlate with variations in shear wave splitting parameters at Vesuvius by Del Pezzo et al. [2004] and Bianco and Zaccarelli [2009]. Del Pezzo et al. [2004] compared S coda decay rate $\left(\mathrm{Q}_{C}^{-1}\right), b$-values and shear wave splitting associated and found correlations between $\mathrm{Q}_{C}^{-1}, \phi$ and $\delta \mathrm{t}$ with earthquake activity but no variations in $b$-value. When compared to strain measurements from a nearby dilatometer, sensitivities of the order of $1.4 \times 10^{9}\left(\mathrm{Q}_{C}^{-1} /\right.$ strain units $)$ for $\mathrm{Q}_{C}^{-1}$ and $2 \times 10^{10}$ (msec/strain units) for $\delta$ t were calculated. Bianco and Zaccarelli [2009] calculated a background seismicity anisotropy of $4 \%$ but at times of seismic 
crisis, the average anisotropy was $8 \%$ and the fast directions tended to flip by $90^{\circ}$. The mechanism of this flip is described by Crampin et al. [2002] to be due to an increase in pore-fluid pressure to the extent that it is greater than the maximum horizontal stress and the cracks are therefore re-orientated at $90^{\circ}$.

\section{Phlegraean Fields}

At the Phlegraean Fields, Savage et al. [1989] observed the average shear wave splitting delay time to be $0.2 \mathrm{~s}$, corresponding to a minimum anisotropy of $7 \%$. The strike of the inferred maximum compressive stress was consistent with values obtained from fault-plane solutions. In their reprocessed data, Bianco and Zaccarelli [2009] obtained 7\% anisotropy and irregular fast directions with a general trend in agreement with results of Savage et al. [1989].

\section{Iceland}

Analysis of shear wave splitting within the Krafla-Leirhnùkur geothermal field, Iceland, was carried out by Tang et al. [2008]. They found evidence for at least two major crack systems of microfractures, which agreed with microearthquake locations and a simultaneous MT (magnetotelluric) survey.

Volti and Crampin [2003] observed temporal increases in time delays for 5 months at 240-km distance before the 1996 Gjálp Eruption on the Vatnajökull Icefield. The increasing time delays were interpreted as indicating the accumulation of stress as the ascending magma fractured the upper crustal surface layers.

\section{Japan}

At Mount Asama, Savage et al. [2010a] correlated Global Positioning System (GPS) baseline length measurements with shear wave splitting measurements to analyse stress changes accompanying the eruption in 2004. They found that the best model from the GPS analysis of a vertical dike and conduit also fit the shear wave splitting measurements, as did the temporal variations. From this a crack aspect-ratio of $2.6 \times 10^{-5}$ was calculated and a differential horizontal stress of $6 \mathrm{MPa}$ at $3 \mathrm{~km}$ depth was inferred. 
Unglert et al. [2010] performed shear wave splitting analyses on local earthquakes around Aso Volcano between 2001 and 2008 and compared the results to strain from GPS measurements in the area. They observed, using clusters with relatively stable epicentres, that two stations showed a significant change in $\phi$ in $2004-2005$. Models from seismic tomography and receiver functions were found to fit both the anisotropy and strain measurements.

\section{Taiwan}

Konstantinou et al. [2009] compared focal mechanism inversions with shear wave splitting analysis at the Tatun Volcano Group in Taiwan. They observed that an area of vigorous hydrothermal activity exhibited a localised stress field that is different from the regional stress field. The suggestion was that such a stress configuration likely causes opening of microcracks, favouring the ascent and circulation of fluids in the upper crust. Shear wave splitting measurements seemed to confirm these results.

\section{Antarctica}

Martinez-Arevalo et al. [2003] used seismic attenuation and shear wave splitting around Deception Island volcano to demonstrate the complex and heterogeneous structure there. They did not investigate the possibility of temporal changes and found that the results were too varied to compare to regional trends.

\section{Ruapehu}

Temporal variation of shear wave splitting at Mt. Ruapehu was investigated by Miller and Savage [2001] and Gerst and Savage [2004]. Miller and Savage [2001] measured shear wave splitting from earthquakes in 1994 and 1998 and observed a change in the dominant $\phi$ spanning the time of the last major magmatic eruption in 1995/1996. That study was extended by Gerst and Savage [2004], who used the same techniques and an additional deployment of three-component seismometers in 2002 to observe further changes in $\phi$ (Figure 2.23). The results of both studies were interpreted as being caused by a dike-shaped magma reservoir, or system of dikes, trending NE-SW. According to this model, the magma reservoir was pressurised before the eruption, producing a local stress field different from the regional stress field. 


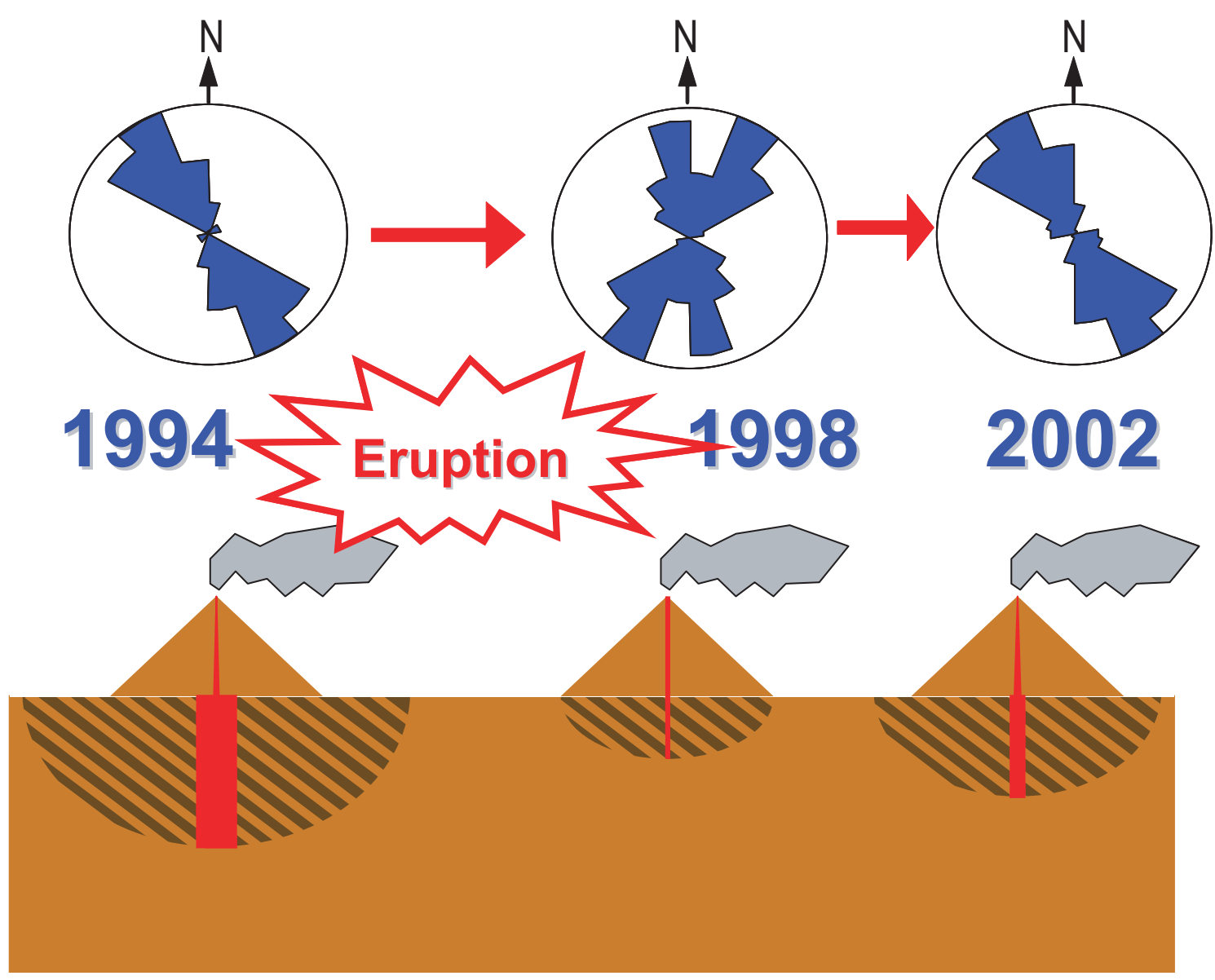

Figure 2.23. Schematic stress and anisotropy model after Gerst and Savage [2004]. In 1994, a pressurised dike system created a local stress field. In 1998, after the eruption, when the dike system was depressurised, stress directions partially returned to the regional trend. In 2002, the dike system refilled, and the stress field in the anomalous region was dominated by the dike again. The alignment of cracks was not as strong as in 1994, so the anisotropy in the anomalous region was not strong enough to affect fast directions from deep events.

This interpretation is favoured above one in which dikes are intruded and solidify, causing a new structural anisotropy, because of the lack of detectable deformation and seismicity associated with dike intrusion. The model suggests that following the eruption the reservoir was less full and correspondingly less pressurised, meaning that the local stress returned to that of the surrounding region. The Gerst and Savage [2004] study suggested that the later changes in $\phi$ were due to repressurising of the reservoir in response to an increase of magma in the system because $\phi$ from deep earthquakes displayed the regional trend, while $\phi$ from shallow earthquakes was orientated to the pre-eruption direction. The return of the anisotropy to the pre-eruptive state also supports the interpretation of stress-controlled anisotropy as the intrusion of dikes would be a permanent change. 
In Chapter 4 we investigate the spatial variations in anisotropy in more detail in order to compare future changes in anisotropy and to identify the regions and causes of past changes in anisotropy with more confidence. These changes are examined in more detail in Chapter 5, where we test new methods of detecting temporal changes in anisotropy.

\section{Geothermal fields}

Shear wave splitting has been used as a tool to characterise fractured reservoirs (geometry, distribution and density of subsurface cracks) and monitor fluid pressure in many geothermal areas. Shear wave splitting analysis is particularly useful in these environments because the assumption that the observed shear wave splitting is due solely to the mechanical anisotropy induced by aligned cracks and microcracks in an otherwise isotropic matrix has been found to be reliable [Rial et al., 2005]. Vlahovic et al. [2003] characterised crack orientations at Coso Geothermal field, California and also inferred a temporal increase in crack density from a $3 \%$ increase in percentage anisotropy over 5 years. Tang et al. [2005] found that time delays increased at times of fluid injection at Krafla Geothermal field, Iceland and also at Coso. High quality observations of shear wave splitting at The Geysers Geothermal fields allowed Elkibbi et al. [2005] to characterise the crack distribution there. Crack orientations obtained from observed polarisation orientations were in good agreement with independent field evidence at The Geysers.

\subsubsection{Spatial variation of shear wave splitting}

As outlined in Section 2.3.7, it is important to mitigate the chance of spatial variations of shear wave splitting being erroneously interpreted as temporal variations. One way to do this would be to map spatial variations in detail at a time when there are no hypothesised temporal changes. This becomes difficult, but essential, when the anisotropy is very heterogeneous and the seismograph array is less dense than the spatial changes. This section outlines some examples of shear wave splitting tomography studies, which attempt to solve the problem of spatial variation. Shear wave anisotropy tomography is difficult because of the nonlinear effect of multiple layers of anisotropy on waveforms.

Audoine et al. [2004] presented a simple method of 2D spatial averaging to examine heterogeneous anisotropy in the crust. The method treated $\phi$ separately from $\delta$ t. 
A grid was constructed with nodes regularly spaced between each earthquake and station. This grid was then treated as a new data set and $\phi$ for each node within a polygon, or within a box of a regular lattice, was averaged. This created average $\phi$ values at regular intervals that could be more dense than the station spacing.

Abt and Fischer [2008] carried out 3-D shear wave splitting tomography for the mantle. The method parametrised the mantle as a 3-D block model of crystallographic orientations with the elastic properties of olivine and orthopyroxene. Splitting was calculated using the Christoffel equation (Equation 2.20) to progressively split the horizontal components of a synthetic wavelet in each block of the model, and shear wave splitting parameters were predicted with an eigenvalue minimisation technique. A linearised, damped least-squares inversion was used to calculate partial derivatives and to solve for a best-fitting model of crystallographic orientations. Non-linear properties of shear wave splitting were accounted for by applying the inversion iteratively and recalculating partial derivatives after each iteration. Using this method, Abt and Fischer [2008] modelled an idealised subduction zone with uniform stations and sources. They found that both the azimuth and dip of crystallographic axes were resolvable to a depth of $100-150 \mathrm{~km}$, as were lateral heterogeneities in anisotropy on a scale of $50 \mathrm{~km}$. When applied to real data, Abt and Fischer [2008] found that the geometry of stations and observed seismicity in the Nicaragua-Costa Rica subduction zone yielded partial to good resolution.

Long et al. [2008] described a theoretical framework for the computation of finitefrequency sensitivity kernels for wave equation shear wave splitting intensity tomography aimed at the upper mantle. They found that the partial differential equations that govern wave equation shear wave splitting tomography are, upon linearisation with the Born approximation, similar to the equations that describe transmission and reflection tomography. Solving for the dip of the symmetry axis with respect to the horizontal plane and the anellipticity parameter, which represents the strength of the anisotropy, they derived sensitivity kernels in the same way.

Zhang et al. [2007] developed a 3-D shear wave splitting tomography method by back projecting shear wave splitting delay times along raypaths derived from a $3-\mathrm{D}$ shear velocity model, assuming the delay times are accumulated along the ray paths. This tomography used only $\delta$ t from local earthquakes to investigate anisotropy strength in the crust. In this way, regions of high anisotropy were identified but information regarding fast directions was not accounted for.

In Chapter 4, we develop a method to quantify spatial variations of shear wave 
splitting that has similarities with the method of Audoine et al. [2004], and that of Zhang et al. [2007]. 


\subsection{Earthquake multiplets}

\subsubsection{Introduction}

Repeatable sources of seismic energy have been used to study changes in seismic velocity due to changes of stress in the Earth's crust for over a century (Reasenberg and Aki [1974] and references therein). The sources first considered were usually explosions in quarries and were used to detect stress variations due to the Earth's tides. Poupinet et al. [1984] used microearthquake doublets to monitor stress around the Calaveras Fault in California. The choice of using doublets was influenced by the accuracy of timing measurements, the provision of more direct sampling of the seismogenic zone and the fact that earthquake sources are richer in shear wave energy than explosions. It is this latter value that makes repeating earthquakes attractive in this study.

For two earthquakes to have seismograms that are indistinguishable to within uncertainties, they require near-identical source parameters (hypocentre and moment tensor) and invariant seismic properties along the propagation path [Poupinet et al., 1984]. Figure 2.24 displays similar waveforms recorded near the Calveras Fault in California from Schaff and Beroza [2004]. The first pulse of each phase is mainly determined by the radiation pattern. Waveform similarity therefore places a limit on differences of the underlying source processes [Got and Frechet, 1993]. First motions could be reproduced by events located in different positions [Cattaneo et al., 1999] and so similarities in the coda are also sought. The seismograms of earthquakes that have closely spaced hypocentres tend to be similar due to the similarity of the Green's functions characterising the source-receiver paths [Baisch et al., 2008]. Therefore the coda of the seismogram, which is only affected by the propagation, is unlikely to be replicated by a different raypath. Geller and Mueller [1980] concluded that the hypocentres of similar earthquakes cannot be separated from each other by more than a quarter of the dominant wavelength $(\lambda / 4)$.

\subsubsection{Identification of repeating earthquakes}

There are several methods of multiplet identification; cross-spectral techniques [Got et al., 1994], pattern recognition [Joswig, 1995], cross-correlation analysis on $\mathrm{P}$ and S waves checked independently [Maurer and Deichmann, 1995] and a fractal approach [Smalley et al., 1987]. One of the methods, BCSEIS [Du et al., 2004a], 


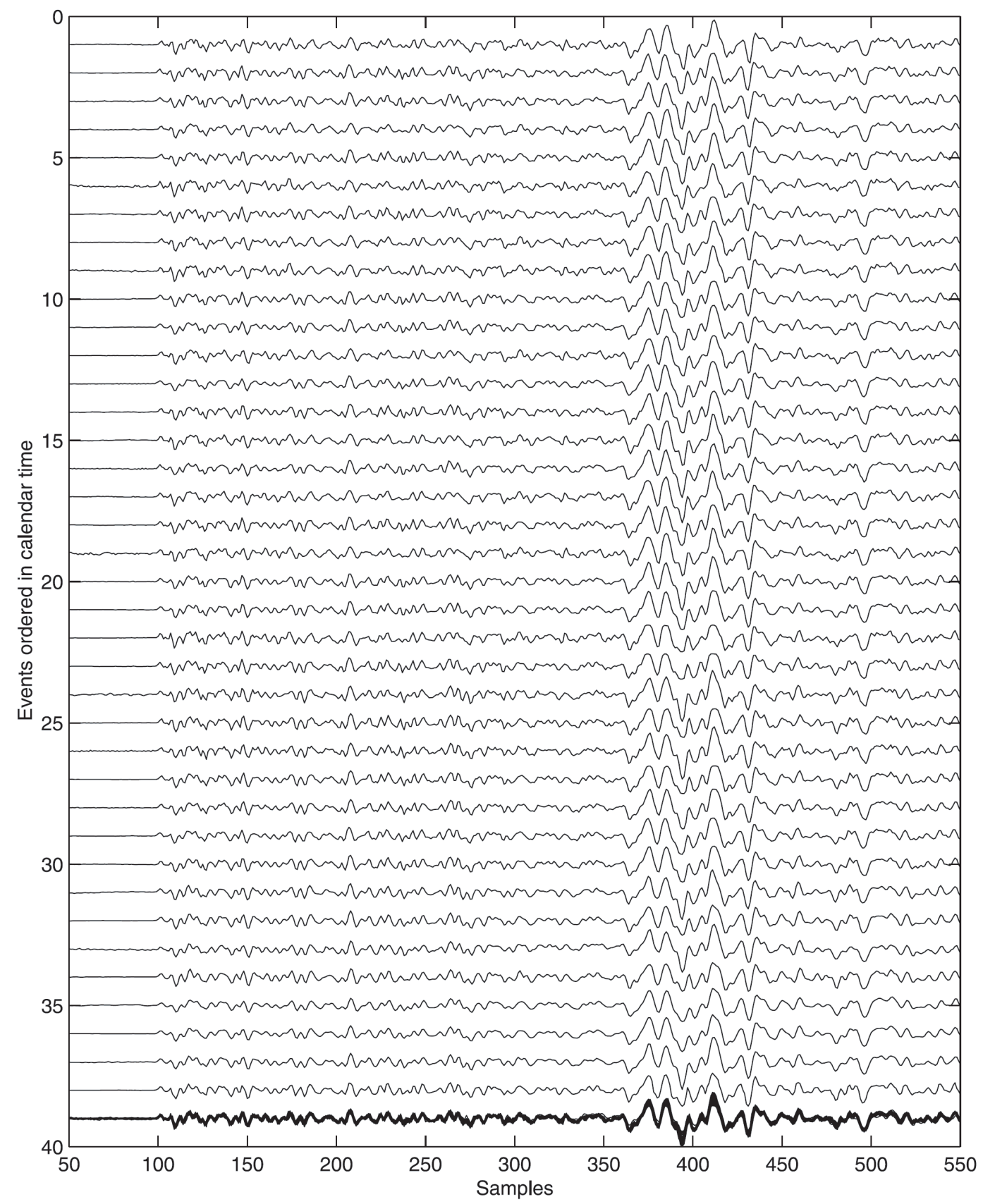

Figure 2.24. Unfiltered waveforms of 38 multiplet earthquakes from Schaff and Beroza [2004], recorded near the Calveras Fault in California. Bottom trace shows superposed individual traces. 

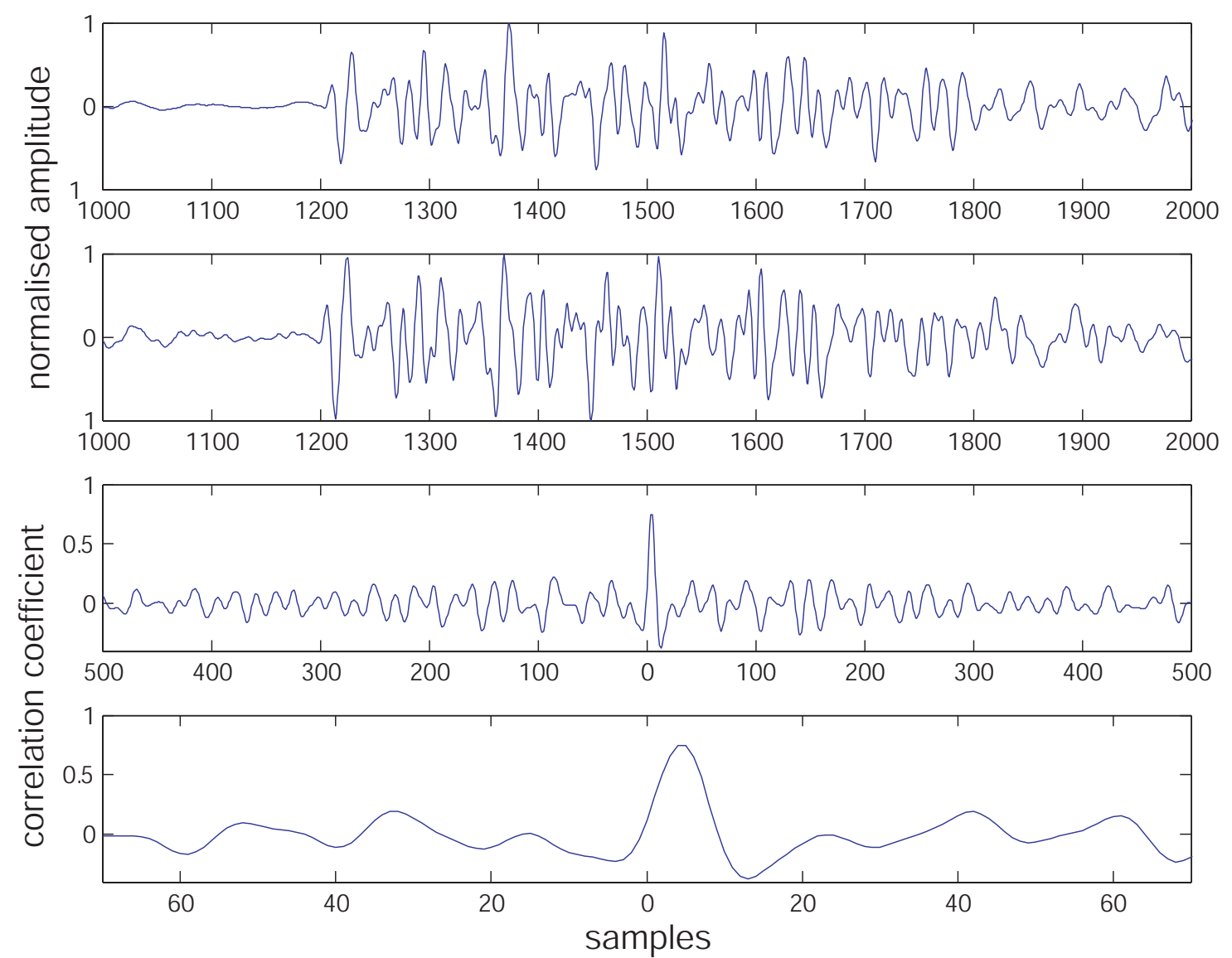

Figure 2.25. Two earthquakes from the multiplet from Chapter 6. Earthquakes 10196 and 10230 , recorded at station OKWE. Correlation coefficients for the cross-correlation show a peak near zero. Bottom is zoom of correlations around zero time shift.

cross-correlates band-pass filtered waveforms and then verifies (selects or rejects) the estimated time delay by comparing the result to that from cross-correlation of both raw and band-pass filtered data in the third-order spectral domain. This method is described in more detail in Section 3.2.1.

The cross-correlation results can depend on several parameters. Filtered waveforms are often used and the type and band of filter has an effect on the cross-correlations [Du et al., 2004a]. For example, the narrower the band, the less complex the waveform and therefore the two waveforms will usually correlate better. The use of lower frequencies will also tend to produce more similar waveforms because more distant earthquakes will be within $\lambda / 4$ separation. Other parameters include the time window for comparison, the sampling frequency and the size of the time steps that the waveforms are shifted during the cross-correlation. A larger time window will result in more of the coda being compared and therefore waveforms with a certain cross-correlation coefficient $(C C C)$ will be more similar. However, using large 


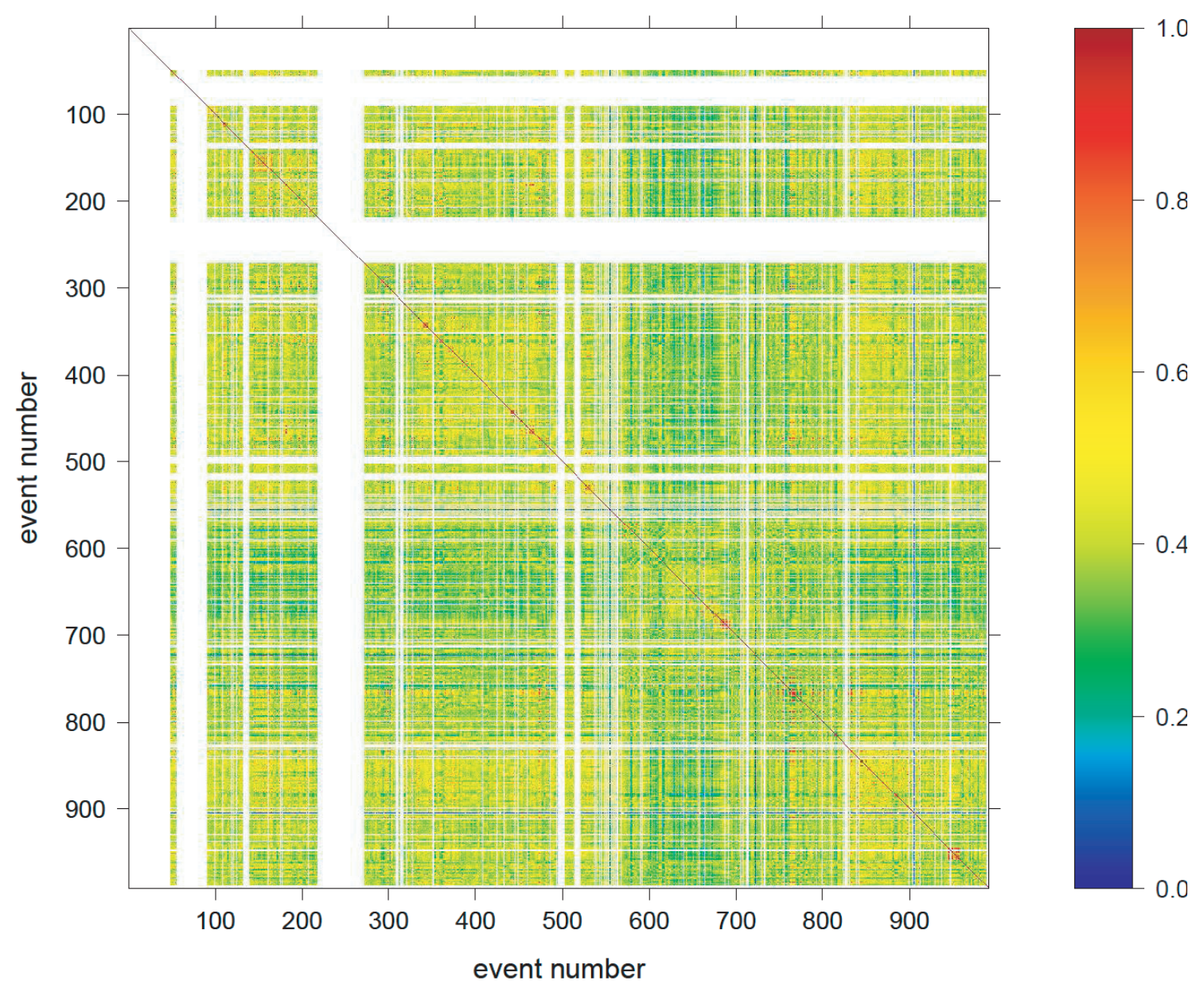

Figure 2.26. Plot showing cross-correlation coefficients between all earthquake pairs recorded at station OKSO on Okmok Volcano between January 2003 and February 2009 from Chapter 6. Warm colours indicate a higher cross-correlation coefficient.

time windows will be more computationally expensive [Du et al., 2004a] and give generally lower $C C C$ [Schaff and Beroza, 2004], yielding fewer pairs meeting imposed $C C C$ criteria. Smaller time steps will give a more precise time shift for the matching arrivals but will likewise take more time to run. Once the cross-correlation has been conducted, a search for $C C C$ over a certain threshold $\left(C C^{l i m}\right)$ is carried out. The $C C C$ is a measure of similarity of the waveforms (for example see Figure 2.25). Many studies use different parameters and thresholds as they have varying data quality and the aim of the studies are different. However, Baisch et al. [2008] quantify the $C C C$ threshold at which the $\lambda / 4$ criterion can be applied to the data as 0.95 with a correlation window length $2.8 \times$ the travel-time difference between the $\mathrm{P}$ and $\mathrm{S}$ arrivals. Appendix E.1 lists some of these different studies.

The next step is to identify clusters, families or multiplets of earthquakes. Some studies only need two earthquakes (a doublet) for analysis [e.g. Poupinet et al., 
1984], but some require many earthquakes over certain time periods [e.g. Rubinstein and Ellsworth, 2010]. There are also several ways to identify multiplets. Figure 2.26 displays the cross-correlation coefficients between all earthquake pairs recorded at station OKSO on Okmok Volcano between January 2003 and February 2009, colour coded by $C C C$. The simplest method is to identify events that have $C C C \geq C C^{\text {lim }}$ using a cross-correlation matrix such as that in Figure 2.26. In this way if earthquake $\mathrm{A}$ and $\mathrm{B}$ are similar, and $\mathrm{B}$ and $\mathrm{C}$ are similar, then $\mathrm{A}, \mathrm{B}$ and $\mathrm{C}$ will all be in the same multiplet [Rubinstein and Ellsworth, 2010]. This is the Equivalence Class algorithm [Press et al., 1986]. The drawback with this method is that A and C might not be as similar as the study requires. An additional constraint can be applied so that A and $\mathrm{C}$ also have to have a $C C C \geq C C^{\text {lim }}$. In this way all earthquakes within a multiplet will have $C C C \geq C C^{\text {lim }}$ with all other earthquakes within the multiplet. This is the method used to identify the multiplet (Figure 2.27) in Chapter 6. Additional requirements are usually put on the multiplet having to have passed the $C C^{\text {lim }}$ criteria at a certain number of stations. For example, in Chapter 6 the earthquakes had to have $C C C \geq C C^{l i m}$ at three or more stations to be included in the multiplet. This is because the source could look similar from one azimuth but still be quite different at different azimuths and would therefore have a different location or source mechanism [Baisch et al., 2008]. However, conditions are sometimes set so that if the $C C C$ at one station is greater than an upper limit $C C^{\lim (u)}$, then the $C C C$ only has to pass a lower threshold $C C^{\text {lim(l) }}$ at other stations for the earthquake to be included [Du et al., 2004a].

Most studies that use multiplets do so because the source locations are indistinguishable. For this reason some authors put additional constraints on the distance between the hypocentres [e.g. Rubinstein and Ellsworth, 2010]. This can be done with relocated earthquakes or with catalogue locations, although some catalogues have automatic depths and locations might not be accurate if the network is sparse.

\subsubsection{Uses of repeating earthquakes}

Multiplets are a valuable tool for investigating processes within the Earth. The waveform similarity of these repeating earthquakes enables changes in seismic properties manifest as subtle changes in the waveforms to be monitored. The fact that repeating earthquakes rupture a single location also enables monitoring of slip and fault strength as a function of space and time. Other authors exploit the fact that 


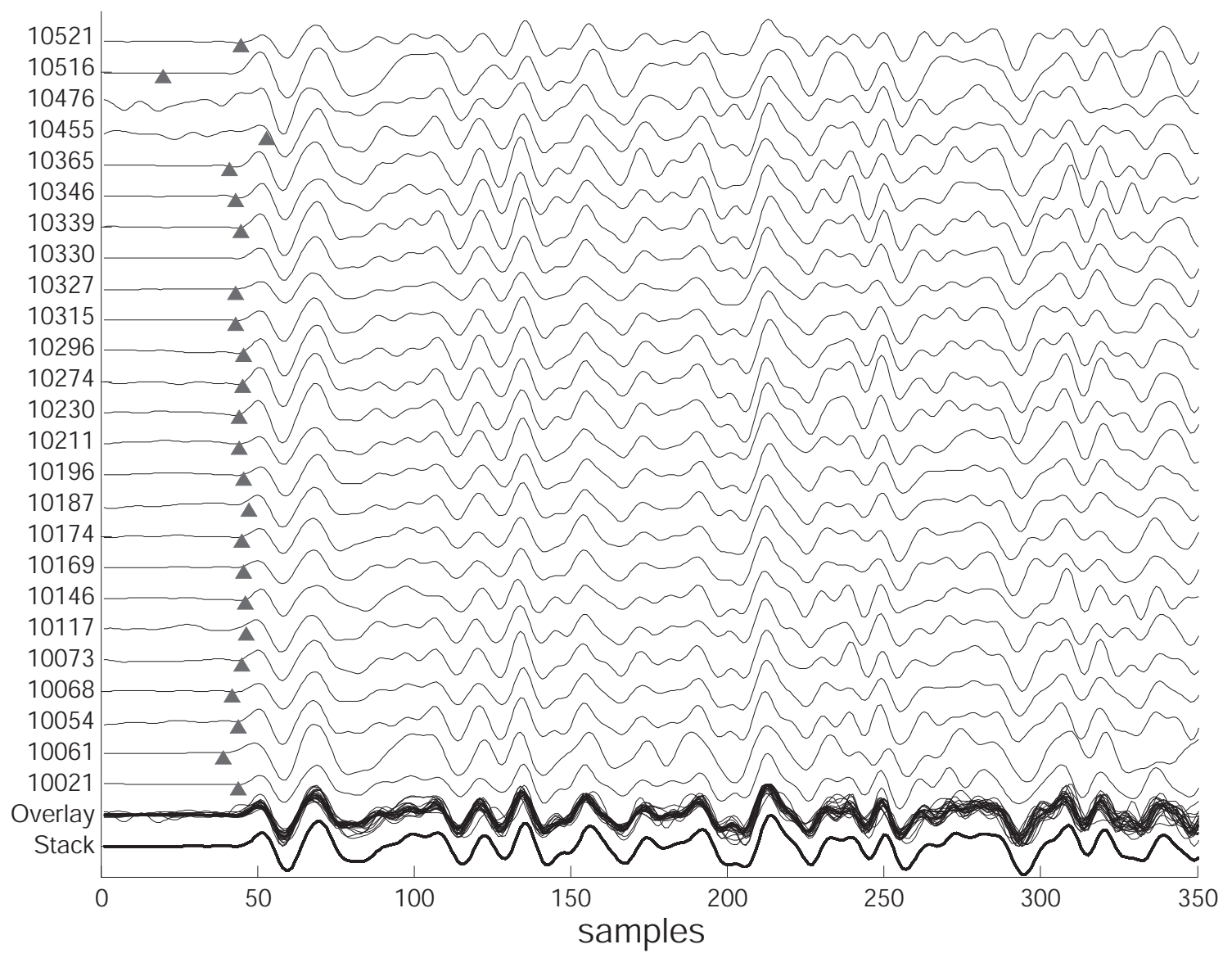

Figure 2.27. Multiplet from Chapter 6, recorded is station OKWE. Bottom traces show overlayed individual traces and stack of all traces.

small changes in phase arrival times can indicate a slight shift in earthquake location, and therefore relocate similar events to understand fracture geometry. Studies using multiplets generally fall into one of these three categories. Some important studies are summarised below and in Appendix E.1.

\section{Understanding earthquake rupture}

Several conceptual models have been suggested to explain the phenomenon of repeating earthquakes: repeated stress release at an asperity or stress concentration along the fault [e.g. Nadeau and Johnson, 1998; Igarashi et al., 2003], and highpressure fluid injection into the crust [e.g. Evans et al., 2005; Baisch et al., 2006]. Observations that suggest that similar earthquakes are caused by repeating slips of small asperities can be used to estimate the cumulative amount of aseismic slip in the region around the repeating earthquakes [Igarashi et al., 2003], provided the aseismic slip in these asperities is negligable. 
Seismicity due to fluid injection is usually explained by the reduction of effective normal stress acting on pre-existing fractures [Baisch et al., 2008]. Shear slip occurs when the ratio of shear to effective normal stress exceeds the frictional strength [King et al., 1994]. This type of earthquake can have associated stress drops that are several orders of magnitude smaller than that resolved on the fracture planes [e.g. Jost et al., 1998]. Therefore coseismic changes in the stress field would be relatively minor and, as long as the fluid pressure inside the fracture keeps increasing, the same fracture plane may slip repeatedly [Baisch and Harjes, 2003]. If the stress conditions are constant, subsequent slip events on the same fracture may exhibit approximately the same source function, thus forming a series of repeating earthquakes. Subtle changes in the size, recurrence interval or location of these repeating earthquakes can be used to monitor the spatial and temporal evolution of fluid injection [Lees, 1998], slip [Igarashi et al., 2003; Rau et al., 2007] and fault strength [Marone et al., 1995; Dreger et al., 2007; Allmann and Shearer, 2007].

Lack of repeating earthquakes can also provide insights into seismogenic processes. Du et al. [2004b] examined earthquakes in the Wellington region and failed to identify repeating earthquakes. This was suggested to be consistent with the hypothesised strong coupling between the Pacific and Australian plates under the Wellington region.

\section{Understanding seismogenetic structures}

Small changes in phase arrival times can indicate a slight shift in earthquake location. Relocation of similar earthquakes using methods similar to double-difference relocation (see Section 3.2.2 for more details) can be used to identify subsurface fractures and fracture networks. Relative relocation of similar events has been widely used to understand earthquake geometry in geothermal areas [Lees, 1998; Moriya et al., 2003], volcanic areas [Got et al., 1994, 2002; Brancato and Gresta, 2003; Battaglia et al., 2003; Alparone and Gambino, 2003; Yamawaki et al., 2004], subduction interfaces [Igarashi et al., 2003; Kimura et al., 2009], large fault zones [Cattaneo et al., 1997; Stich et al., 2001; Waldhauser et al., 2004; Rau et al., 2007; Li et al., 2007; Dreger et al., 2007] and in mines [Spottiswoode and Milev, 1998]. 


\section{Monitoring changes in path properties}

\section{Velocity changes}

Reasenberg and Aki [1974] first used repeated air gun shots to measure seismic velocities in a granite quarry. They recognised that, with a repeating source, the only changes observed should occur in the physical properties along the path of propagation, and so detected a change in seismic velocity due to the Earth's tides. Poupinet et al. [1984] used microearthquake doublets to monitor seismic velocity, and by inference stress, around the Calaveras Fault in California. They found a delay in coda arrivals after the Coyote Lake earthquake (M5.9) and interpretted the reduction in S wave velocity as a decrease in stress. Schaff and Beroza [2004] used repeating earthquakes in the aftershock zones of the 1989 Loma Prieta and 1984 Morgan Hill, California, earthquakes. They measured a coseismic velocity decrease of about $1.5 \%$ for $\mathrm{P}$ waves and $3.5 \%$ for $\mathrm{S}$ waves, and demonstrated that the amplitude of the velocity decrease decayed logarithmically with time following the main shock. Schaff and Beroza [2004] suggested that, because the fractional change in $\mathrm{S}$ wave velocity was greater than the fractional change in $\mathrm{P}$ wave velocity, the opening or connection of fluid-filled fractures was the underlying cause, while the magnitude of the velocity change implied that low effective pressures were present in the source region of the velocity change. Zhao and Peng [2008] systematically investigated the velocity contrast along the Calaveras fault that ruptured during the 1984 Morgan Hill earthquake using fault zone head waves that refract along the fault interface. They stacked waveforms in 353 sets of repeating clusters, and aligned the direct $\mathrm{P}$ waves to obtain velocity contrasts of $2-3 \%$ and $12-14 \% \mathrm{NW}$ and SE.

Earthquake multiplets were used to monitor the shear wave velocity in Merapi Volcano, Indonesia, by Ratdomopurbo and Poupinet [1995]. They found a gradual increase in velocity before the 1992 eruption and attributed this to an increase of pressure in the volcanic plumbing system closing cracks. Pandolfi et al. [2006] also used doublets to detect pressurisation in the volcanic plumbing system at Mt. Vesuvius. They found a systematic velocity increase followed by a rapid drop just before a sustained seismic swarm including a M3.6 earthquake.

\section{Attenuation changes}

Got et al. [1990] analysed the spectral ratio of the coda of doublets of microearthquakes with the purpose of finding evidence for temporal changes in the 
attenuation in the crust before a large magnitude earthquake. They found that there were numerous kinds of variations but they could not directly relate them to the forecasting of earthquakes. Beroza et al. [1995] analysed 21 earthquake doublets that spanned the preseismic, coseismic and postseismic intervals of the The Loma Prieta earthquake and measured the change in coda Q. They also found that the changes were neither spatially coherent nor correlated between adjacent frequency bands. Aster et al. [1996] and Antolik et al. [1996] used pairs and clusters of earthquakes to search for temporal variations in coda Q along the San Jacinto fault zone (Parkfield, California). They found that when using earthquakes with $C C C \geq 0.7$, coda $\mathrm{Q}$ was stable with time but with a large standard deviation, and attributed this variability to random fluctuations in the coda driven by source variability and not by any systematic temporal variability in coda $\mathrm{Q}$. Using earthquakes with $C C C \geq 0.95$, the mean value of coda $\mathrm{Q}$ did not vary by more than approximately $5 \%$ during the study period, indicating that coda $\mathrm{Q}$ shows no sign of sensitivity to processes before large earthquakes. However, using a multiplet, Chun et al. [2010] found a sharp rise in the $\mathrm{P}$ wave attenuation operator about 18 months before the 2004 Parkfield Earthquake, accompanied by a rapid fall in $\mathrm{P}$ to $\mathrm{S}$ amplitude ratio in both the frequency and time domains. These changes were attributed to a decline in pore-fluid saturation due to microcracking that culminated in catastrophic rock failure during the fault rupture.

Titzschkau et al. [2010] used a repeating source within the Waiouru swarm to investigate changes in attenuation related to the 1995/1996 eruptions of Mt. Ruapehu. They did not find any direct dependence of coda $Q$ on volcanic activity although they did find a high attenuation anomaly in waveforms that travelled through the volcano when they examined relative changes in integrated direct wave attenuation. The anomaly increased over the time of the eruption and decreased in waves that travelled close to but not through the volcano and was attributed to changing stress caused by the magma plumbing system.

\section{Scattering changes}

Got and Coutant [1997] used the S wave coda of multiplets to investigate scatterers along the propagation path at Kilauea, Hawaii. They found that scattered waves were emitted anisotropically along some preferred directions and that travel time delay variation in the coda could arise purely due to geometrical propagation effects. Baisch and Bokelmann [2001] investigated the character of multiplet wave- 
forms before and after the Loma Prieta earthquake. They found that the coherence between the waveforms reduced after the earthquake due to coseismically opened cracks causing increased scattering, and gradually recovered over 5 years due to the cracks closing through crack healing, fluid diffusion or after deformation. Niu et al. [2003] observed a change of the order of $10 \mathrm{~m}$ in the location of scatterers in the Parkfield region by monitoring them using repeating earthquakes. The motion of the scatterers was coincident with the onset of an aseismic deformation event in 1993 and was thought to be the result of a stress-induced redistribution of fluids in fluid-filled fractures caused by the transient event.

\section{Shear wave splitting changes}

Peng and Ben-Zion [2005] used similar earthquakes to analyse spatiotemporal variations of crustal anisotropy in the aftershock regions of the 1999 M7.4 Izmit and M7.1 Düzce earthquakes, and Liu et al. [2008] carried out a similar investigation around the San Andreas Fault. The results of these studies are discussed in Section 2.3.7.

Bokelmann and Harjes [2000] used induced seismicity multiplets in the German Continental Deep Drilling Program to investigate time varying shear wave splitting. They observed a decrease in $\mathrm{S}$ wave anisotropy of $\sim 2 \%$ associated with fluid injection into aligned cracks, and then stable velocity after a larger (M1.2) earthquake, and suggested that the temporal variation was due to tectonic stress release. De Meersman et al. [2009] found a correlation between multiplet groups and temporal variations in seismic anisotropy from sensors in a single borehole in the North Sea Valhall oil field in 1998. They explained these findings in the context of a cyclic recharge and dissipation of cap-rock stresses in response to production-driven compaction of the underlying oil reservoir. They suggested that the cyclic nature of this mechanism drove short-term variations in seismic anisotropy and the reactivation of microseismic source mechanisms over time.

\section{Detection of earthquakes}

Recently the occurrence of highly similar waveforms in geothermal areas has been taken advantage of to detect low magnitude earthquakes [Rawlinson et al., 2010]. Waveform templates of master events are cross-correlated with continuous seismic data and earthquakes as small as $\mathrm{M}_{L} 0$ have been systematically identified in low 
SNR data. Waveform similarity analysis has also been adapted and implemented in a declustering procedure to identify foreshocks and aftershocks to obtain instrumental catalogues that are cleaned of dependent events [Barani et al., 2007].

\subsection{Summary}

In this chapter we have reviewed the previous work that is relevant when trying to discriminate between temporally amd spatially varying anisotropy at active volcanoes. An overview of the detection and quantification of anisotropy has been given that will assist in the understanding and interpretation of shear wave splitting results. This chapter has reviewed the geophysical and geological literature that is relevant to the interpretation of anisotropy at Mt. Ruapehu in Chapters 4 and 5. We have also reviewed the detection and use of multiplets, which are used in Chapters 5 and 6 as a tool to measure temporally changing anisotropy. 



\section{Methodology}

\subsubsection{Introduction}

This chapter explains the steps that were taken and the methods used in this project. Not all analysis tools were used on all datasets and individual parameters used for each dataset can be found in the associated chapter. New processing programs written by this author are described in the associated chapter. All data were processed using Seismic Analysis Code (SAC) [Goldstein et al., 2003; Goldstein and Snoke, 2005]; important scripts can be found in the appendicies.

\subsection{Data acquisition}

Data for this project were obtained from several sources including a temporary deployment of seismometers around Mt. Ruapehu.

\subsubsection{Spatial Anisotropy Deployment At Ruapehu}

The Spatial Anisotropy Deployment At Ruapehu (SADAR) was part of a project funded by the Earthquake Commission (EQC) and Victoria University of Welligton (VUW) to investigate seismic anisotropy at Mt. Ruapehu. The deployment consisted of sixteen temporary three-component seismometers deployed around Mt. Ruapehu to complement the permanent (GeoNet) network of fifteen three-component seismometers (Figure 3.1) active at the time. The SADAR stations operated throughout 2008 and during that time recorded 929 local earthquakes, as small as magnitude 1.0, that were also recorded at the permanent sites and routinely located for the catalogue. More details about the seismic stations used in this analysis can be found in Appendix B.1. Information about deployment equipment and logistics can be found in Appendix C.1. 


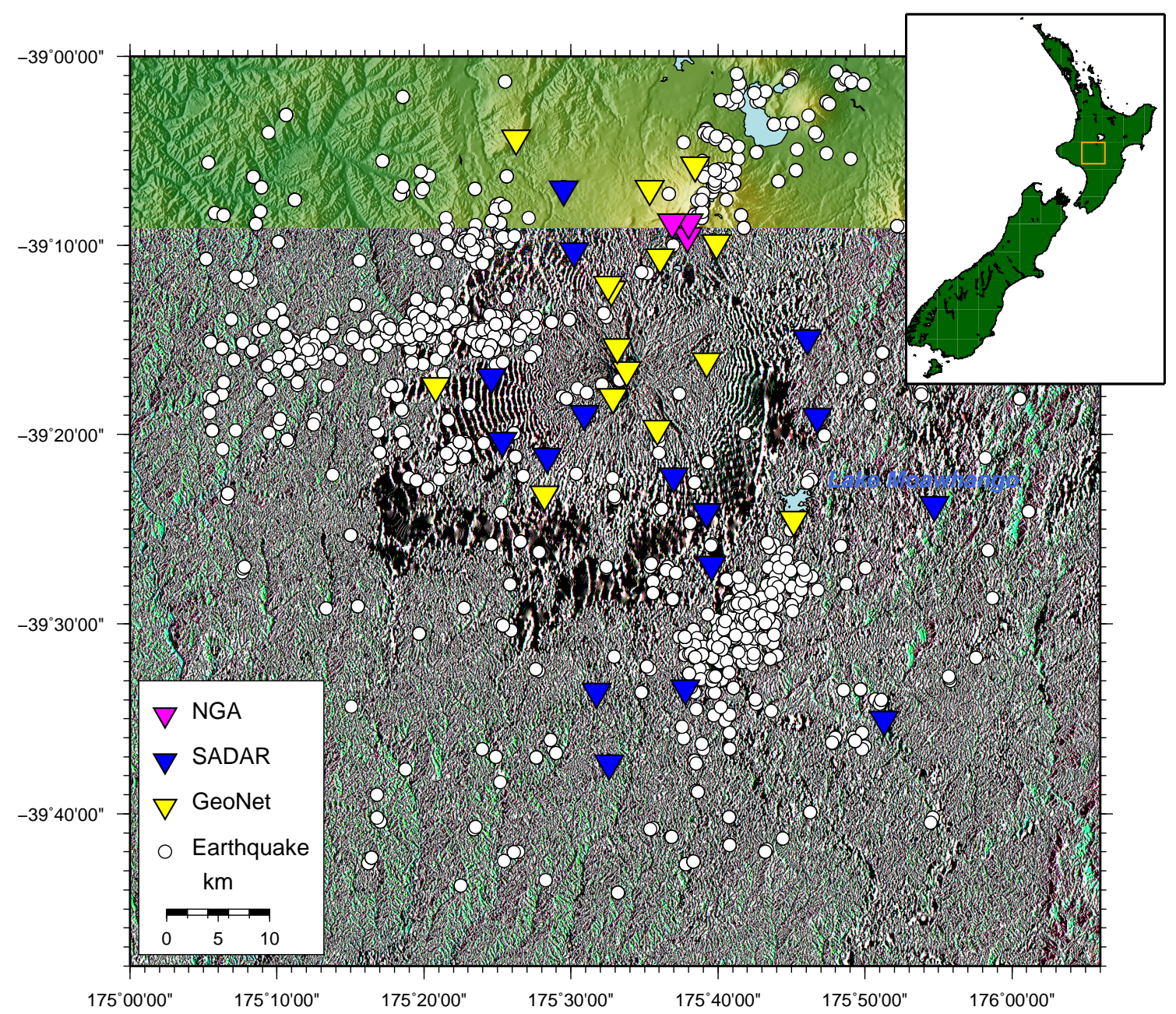

Figure 3.1. Map of the area around Mount Ruapehu (central peak). The white circles indicate the catalogue locations of the earthquakes in 2008 , used in this study. The inverted triangles represent 3-component seismometers; the yellow are permanent GeoNet stations, the blue are temporary SADAR stations and the pink are temporary Ngauruhoe stations (NGA).

The PASSCAL program suite was used to convert the data into Reftek format and then continuous mini-seed format. The mini-seed files were cut around earthquakes of interest and finally converted into SAC format for picking. Figure 3.2 displays a flow diagram of the steps taken to get the RT130 data into cut SAC files and Appendix C.2 contains a more detailed explanation of how the conversions were performed.

\subsubsection{GeoNet}

GeoNet [GeoNet, Last accessed 17 April 2011, http://www.geonet.org.nz/] is a project to build and operate a modern geological hazard monitoring system in New 


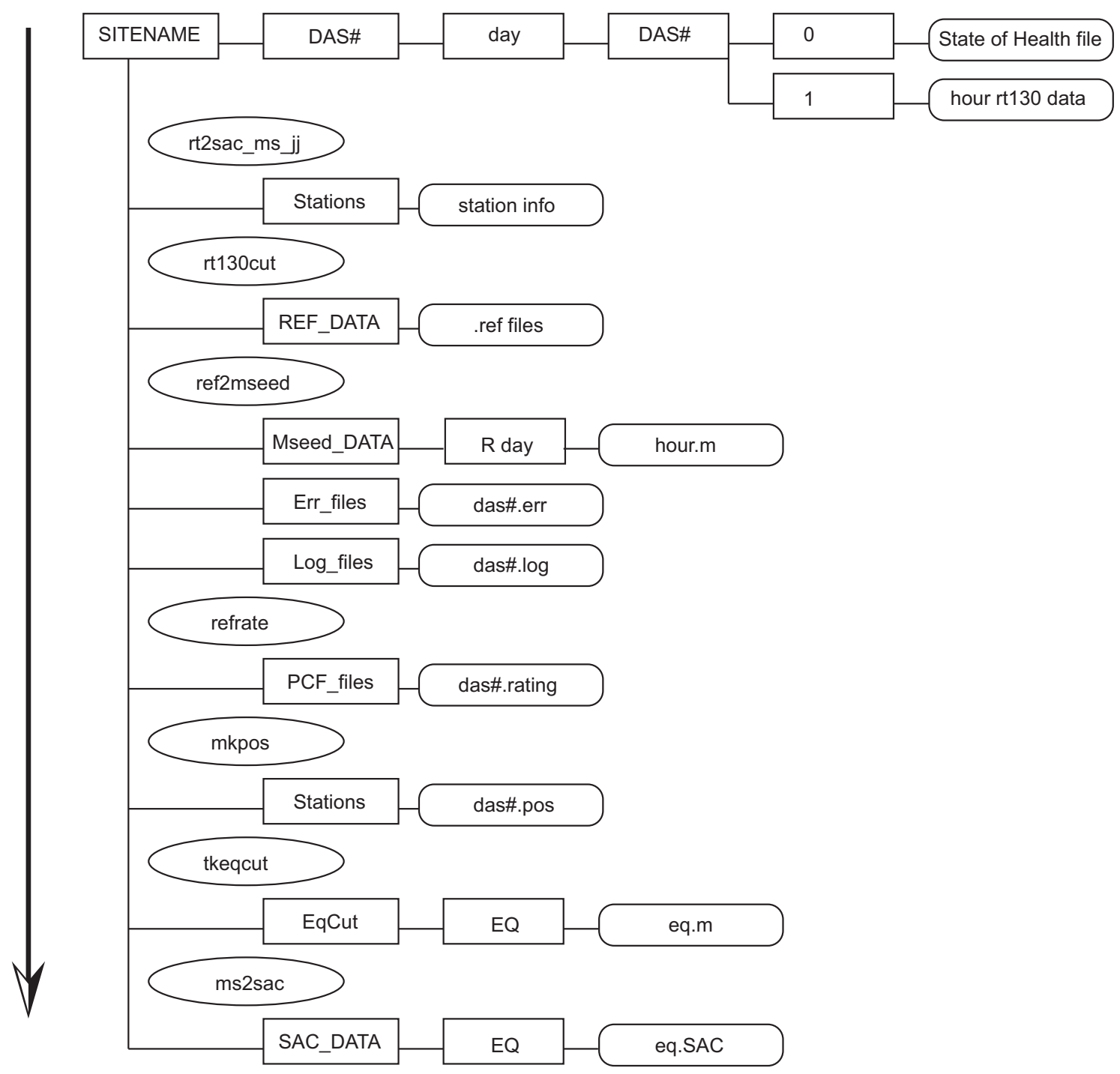

Figure 3.2. Flow diagram and directory structure outlining the conversion of RT130 data to cut SAC files. Rectangles represent directories, ellipses represent program steps and rounded rectangles represent data files. Script $r t 2 s_{a}$ ms $_{-} j \mathrm{j}$ prepares the data and directory structure, other scripts are part of the PASSCAL package. More details can be found in Appendix C.2. 
Zealand. It is funded by the Earthquake Commission (EQC) and designed, built and operated by GNS Science. The New Zealand National Seismograph Network is made up of 46 backbone stations as of 22 February 2011, located throughout the country to provide a homogeneous location and data collection capability. Regional seismograph networks provide enhanced volcano monitoring and improved depth control for subduction-related earthquakes along the Hikurangi margin. They use predominantly short-period seismometers, remote digitisers and spread-spectrum radio links to backbone stations or directly to the data centres. One of these regional networks surrounds the Tongariro Volcanic Centre (TVC) (Figure 2.7).

The TVC network consists of 19 seismograph sites, including two borehole seismometers. For the purpose of this study seismic data were obtained by first selecting the earthquakes of interest using GeoNet's "quakesearch" [GeoNet, Last accessed 17 April 2011, http://magma.geonet.org.nz/resources/quakesearch/]. The parameters used in this project are listed in Appendix E.2. Once the CUSP (CaltechUSGS Seismic Processing) IDs of the earthquakes had been listed, the waveform data were downloaded using the Common Waveform Buffer (CWB) client, which is also available from the GeoNet website [GeoNet, Last accessed 17 April 2011, http: //www.geonet.org.nz/resources/basic-data/waveform-data/]. Appendix D.1 contains the shell script used to download data.

Once the waveform data had been obtained, the $\mathrm{P}$ and $\mathrm{S}$ arrivals were manually picked in SAC (see Appendix D.2 for scripts) for further analysis.

\subsubsection{Past deployments}

\section{4}

A deployment of 14 three-component seismometers was carried out around Mt. Ruapehu between 28 January and 13th March 1994 by Leeds University, the University of Memphis and IGNS (Institute of Geophysical and Nuclear Science, now GNS Science) to characterise the seismicity beneath Crater Lake [Hurst, 1998]. The station details are listed in Appendix B.2 and displayed in Figure 3.3. Data from that deployment were used by Miller and Savage [2001] and Gerst and Savage [2004] in their analyses of shear wave splitting. However, the processed data were not archived and so had to be retrieved from DAT tapes stored at GNS Science, Wairakei. The data were recorded by Lennarz MARS-88 data-loggers and so had to be read by a DAT reader, converted with mars2mseed (PASSCAL package) then merged, converted 


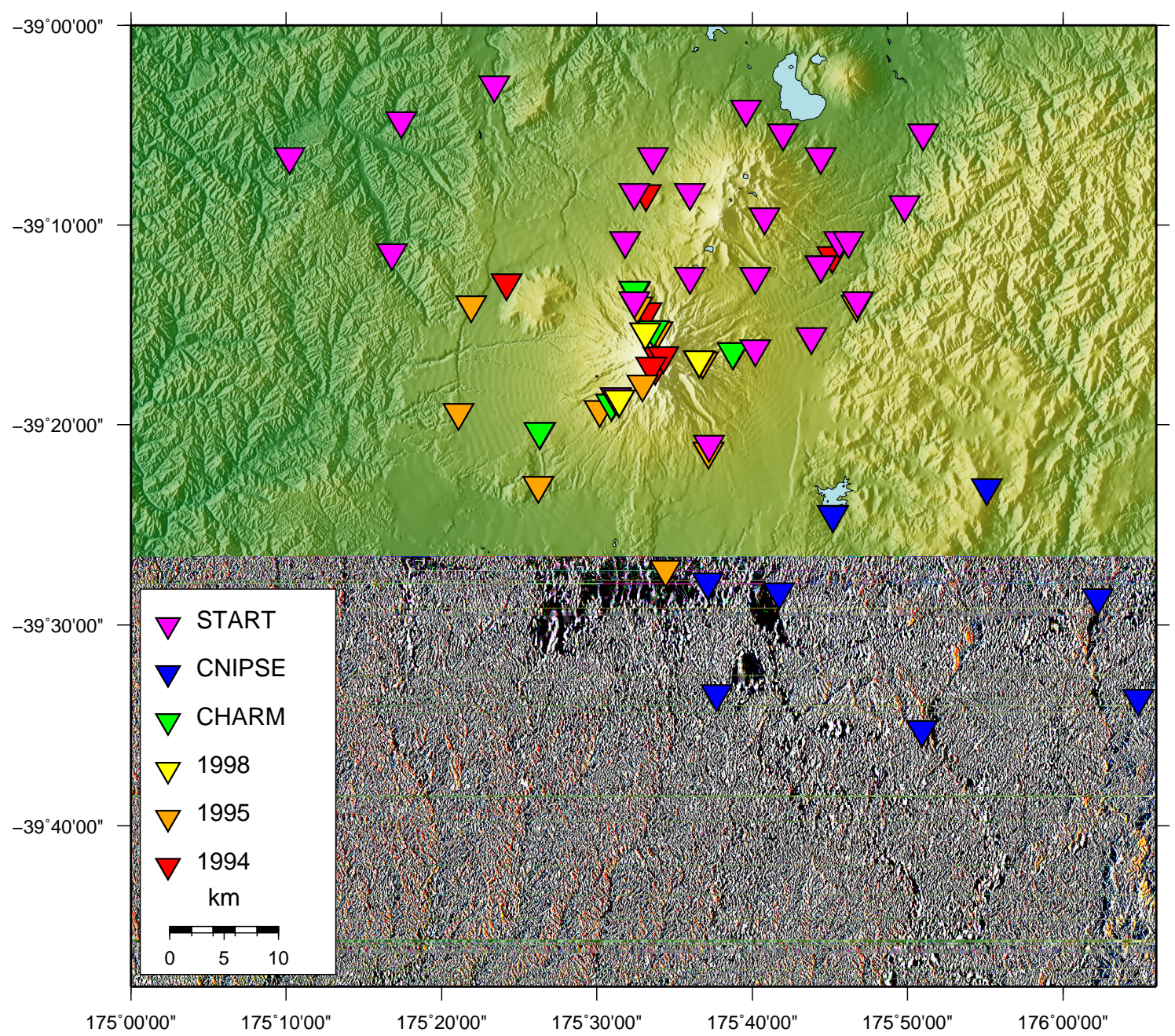

Figure 3.3. Map of past deployments.

and cut to obtain SAC earthquake files. It was also noted that the previous analyses only used five out of nine available stations (the other five stations had timing errors and so the data was not used or archived). Data from the nine stations were picked and analysed for shear wave splitting using MFAST (Section 3.2.3) in this investigation.

\section{5}

Twelve three-component seismometers were deployed around Mt. Ruapehu between September and December 1995 by IGNS to observe the 1995 eruption sequence and to act as a backup in case the permanent stations were destroyed. The station details are listed in Appendix B.2 and displayed in Figure 3.3. The data are archived at GeoNet and can be accessed in the same way as the permanent network using the 
CWB request tool. Once obtained, the data were picked for $\mathrm{P}$ and $\mathrm{S}$ arrivals and analysed for shear wave splitting using MFAST.

\section{8}

A deployment of three three-component seismometers was carried out around Mt. Ruapehu between February and July 1998 by Leeds University and IGNS. The purpose of this deployment was to characterise the post-eruption background seismicity [Sherburn et al., 1999]. The station details are listed in Appendix B.2 and displayed in Figure 3.3. Those data were also used by Miller and Savage [2001] and Gerst and Savage [2004] in their studies of shear wave splitting, but the processed data were not archived at the time and so had to be retrieved from tapes stored at GNS Science, Wairakei. The data were processed in the same way as for the 1994 deployment although the waveform data had not been stored with details about the deployment and so comparison of waveforms with those previously published was used to identify stations.

\section{START}

The START experiment was carried out between January and June 2001 by University of Cambridge student Daniel Rowlands. It was designed to create uniform coverage over the central and northern Tongariro volcanic centre for use in seismic tomography [Rowlands et al., 2005]. The station details are listed in Appendix B.2 and displayed in Figure 3.3. Those data are archived on the IRIS DMC (Incorporated Research Institutions for Seismology Data Management Center) and were accessed using the BREQ-FAST (Batch REQuests, FAST) system. The P and S arrivals were retrieved, picked and analysed for shear wave splitting by Rob Holt using MFAST.

\section{CNIPSE}

The deployment around Waiouru in 2001 was conducted in order to characterise earthquakes in the Waiouru swarm [Hayes et al., 2004]. This deployment was a smaller part of the CNIPSE (Central North Island Passive Seismic Experiment) deployment [Henrys et al., 2003]. The station details are listed in Appendix B.2 and displayed in Figure 3.3. Data from this deployment are 
archived at GeoNet and so can be accessed in the same way as for the permanent network using the CWB request tool. Phase picks are also archived at GeoNet but separately from the waveform data. Phase picks were downloaded from ftp://ftp.geonet.org.nz/quake/bulletin/ and added to the SAC headers. Shear wave splitting analysis was carried out using MFAST.

\section{CHARM}

The CHanging Anisotropy at Ruapehu Mountain (CHARM) experiment was carried out between January and July 2002 by Victoria University of Wellington (VUW) Masters student Alex Gerst. It was designed to reoccupy the stations from the 1994 and 1998 deployments to further investigate changes in shear wave splitting around Mt. Ruapehu [Gerst and Savage, 2004]. The station details are listed in Appendix B.2 and displayed in Figure 3.3. As this deployment was carried out by VUW, the data were still on file. They were repicked and reprocessed by Prof. Martha Savage using MFAST [see Savage et al., 2010b] and the results of that reprocessing are used in this project.

\subsubsection{Alaska Volcano Observatory}

The Alaska Volcano Observatory (AVO) [Alaska Volcano Observatory, Last accessed 22 Feb 2011, https://www.avo.alaska.edu/] is a joint program of the United States Geological Survey (USGS), the Geophysical Institute of the University of Alaska Fairbanks (UAFGI), and the State of Alaska Division of Geological and Geophysical Surveys (ADGGS). The backbone of AVO's volcano monitoring program consists of networks of continuously recording seismometers installed at selected volcanoes in Alaska. Okmok volcano is one of the best instrumented, with four broadband three-component seismometers and eight short-period, single-component seismic stations (Figure 3.4). Seismic data are relayed to AVO facilities in Fairbanks and Anchorage where they are analysed both automatically and manually. AVO operates a network of telemetered GPS receivers at Okmok volcano that provides a continuous record of ground deformation. AVO also conducts periodic field-based GPS surveys as well as measuring deformation with satellite radar interferometry (InSAR) techniques. Seismic data were personally obtained from the AVO archive in Anchorage and $\mathrm{P}$ and $\mathrm{S}$ picks from the catalogue were added to the SAC headers. 


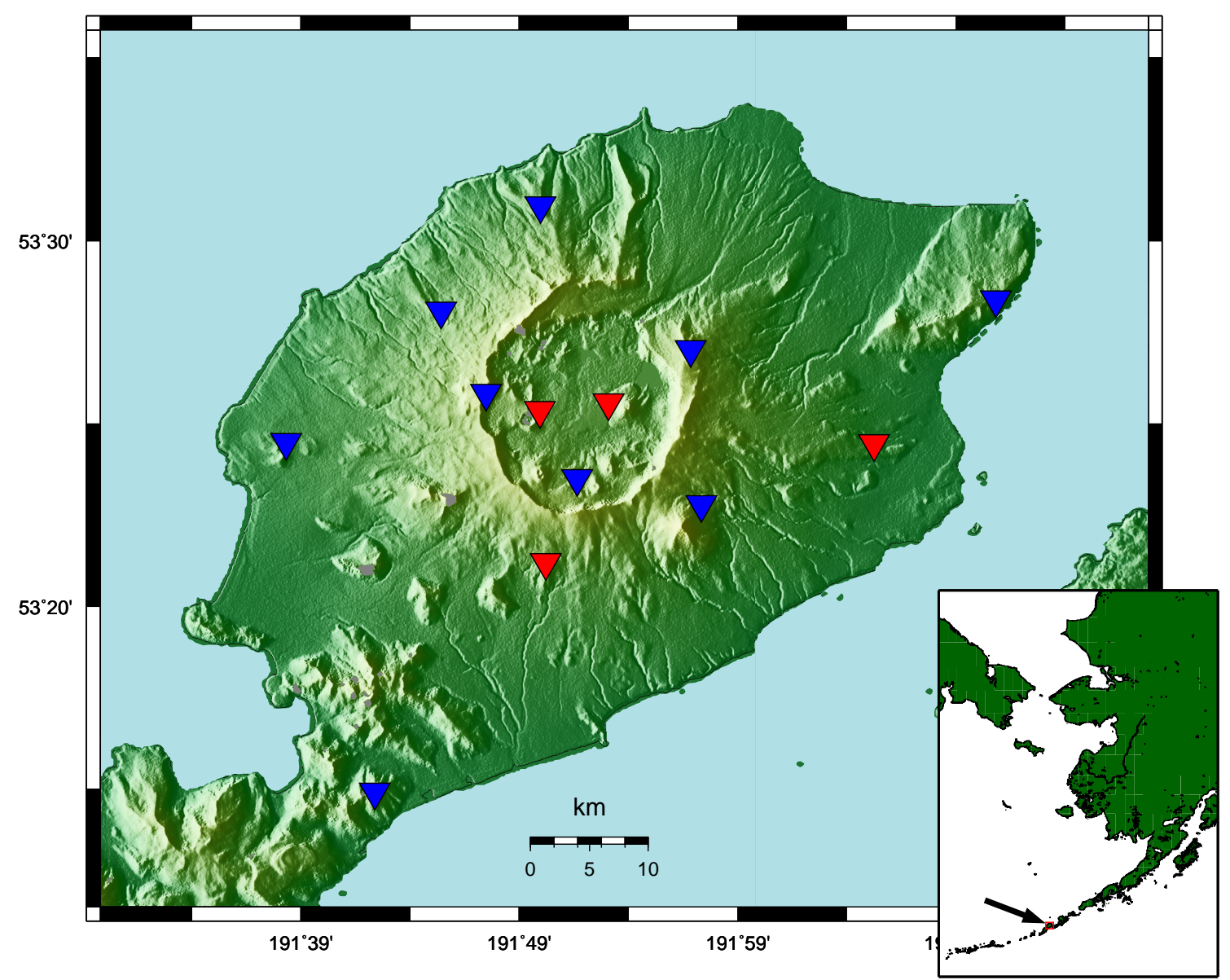

Figure 3.4. Map of Okmok Volcano. Red triangles are three-component broadband seismic stations with continuous GPS; blue triangles are single component short-period seismic stations.

\subsection{Data processing}

\subsubsection{Waveform cross-correlation}

We use the Bispectrum Cross-correlation package for SEISmic events (BCSEIS) of Du et al. [2004a] to obtain cross-correlation coefficients and time delay estimates. Cross-correlation using BCSEIS has been performed in this study to identify multiplets and to assist in the relocation of hypocentres (see Section 3.2.2). BCSEIS cross-correlates band-pass filtered waveforms and then verifies (selects or rejects) the estimated time delay by comparing the result to that from cross-correlation of both raw and band-pass filtered data in the third-order spectral domain. This makes the method less sensitive than cross-correlation delay time estimates alone to Gaussian noise [Du et al., 2004a].

The cross-correlation is carried out on specified windows around the $\mathrm{P}$ and $\mathrm{S}$ wave 
arrival picks (e.g. Figure 3.5) on pairs of seismograms recorded at a common station. The cross-correlation function is:

$$
C C(\tau)=n \int_{-\infty}^{\infty} x(t) y(t+\tau) d t
$$

where $x$ and $y$ are the two seismograms, $\tau$ is the time delay between the two seismograms and $n$ is a normalisation factor that ensures the function takes values between -1 and 1:

$$
n=\left(\int x^{2} d t \int y^{2} d t\right)^{-1 / 2}
$$

The maximum value of $C C(\tau)\left(C C(\tau)_{\max }\right)$ represents the similarity between the two time series and $\tau$ is the time delay corresponding to $C C(\tau)_{\max }$. The BCSEIS algorithm returns the maximum cross-correlation coefficient and the corresponding time delays of event pairs at each station (absolute cross-correlation coefficients are given between 0 and 1, 1 being perfect correlation).

The cross-correlation approach works in the second-order spectral domain. This is because a cross-correlation is a convolution with a time-reversed sequence, or a multiplication with a complex-conjugate in the frequency domain. Given that $X(f)$ and $Y(f)$ are the Fourier transforms of $x(t)$ and $y(t)$ respectively, and $X^{*}$ is the complex conjugate of $X$, the cross-correlation, $C$, is

$$
C=X^{*} \cdot Y
$$

Non-linear multiplications, for example the bispectrum (Equation 3.4), can be used in this case because Gaussian processes in spectra of order higher than two are identically zero [Du et al., 2004a]. Even if the noise is non-Gaussian, if it has zero skewness then it will still be suppressed by the bispectrum method. The bispectrum cross-correlation, $B$ is given by

$$
B\left(f_{1}, f_{2}\right)=E\left[Y\left(f_{1}\right) \cdot X\left(f_{2}\right) \cdot X^{*}\left(f_{1}+f_{2}\right)\right]
$$

where $E[\cdot]$ is the expectation operator. When the time series are divided into $k$ overlapping data segments [Wong et al., 2009], this becomes

$$
B\left(f_{1}, f_{2}\right)=\frac{1}{k} \sum_{i=1}^{k} Y_{i}\left(f_{1}\right) \cdot X_{i}\left(f_{2}\right) \cdot X_{i}^{*}\left(f_{1}+f_{2}\right) \text {. }
$$


The bispectrum method is used to calculate a time delay on both raw and bandpassed waveforms by locating the lag of the peak of the bispectrum cross-correlation series, which is then compared to $\tau$. If they are sufficiently close then both $C C(\tau)$ and $\tau$ from the cross-correlation are accepted.

A Hanning tapered band-pass filter between 1 and $10 \mathrm{~Hz}$ was used for all cases. Our results agree with observations made by $D u$ et al. [2004a]; choosing a different filter in the range $0.5-5 \mathrm{~Hz}$ to $5-20 \mathrm{~Hz}$ was not found to change the final results appreciably.

In Chapter 6 multiplets were identified by having a $\mathrm{P}$ wave cross-correlation coefficient $\left(C C^{\text {lim }}\right)$ of more than 0.95 at three or more stations; if one or more of the stations showed a cross-correlation coefficient of more than $0.98\left(C C^{\lim (u)}\right)$ then the threshold at the other stations was lowered to $0.85\left(C C^{\lim (l)}\right)$. These criteria have been adopted based on studies by Shearer [1997] and Du et al. [2004a], although the thresholds are higher in this study, and a slightly different method was used and is described in Chapter 5. Choosing only those events with high cross-correlation coefficients ensures extremely similar waveforms [Hemmann et al., 2003; Igarashi et al., 2003; Du et al., 2004b; Pandolfi et al., 2006]. Examples of the resulting multiplets are discussed in Chapters 5 and 6. A higher correlation in the $\mathrm{P}$ waveforms than in the $\mathrm{S}$ waveforms was sought because the nature of this study required the inclusion of S waveforms that change over time. The cross-correlation analysis used the vertical-component because the temporal changes observed in shear wave splitting were expected to be more pronounced in the horizontal components of the $\mathrm{S}$ waveform. For input into the double-difference relocation algorithm the cross-correlation thresholds were lowered to $C C^{\lim (l)}$ of $0.5, C C^{\lim }$ of 0.7 and $C C^{\lim (u)}$ of 0.8 . This is because it is the accurate differential times that are important for this step rather than highly similar waveforms.

Schaff et al. [2004] tested a variety of window lengths and found that although smaller windows generally produced higher-similarity measurements than longer ones they also produced larger residuals after double-difference relocation. We used a window of $2.54 \mathrm{~s}$ around the $\mathrm{P}$ wave, making a total of 254 sample points with 30 of them before the $\mathrm{P}$ arrival (Figure 3.5). This window was large enough to contain several cycles of the seismic wavetrain, producing precise delay time measurements, and yielding events with highly similar waveforms. A longer time window of 3.82 $\mathrm{s}$ was used for the $\mathrm{S}$ wave calculation to accommodate the uncertainty associated with the catalogue $\mathrm{S}$ arrival picks and the longer periods of the $\mathrm{S}$ waves. This corresponded to 382 sample points, 50 of them before the picked S arrival. The windows 


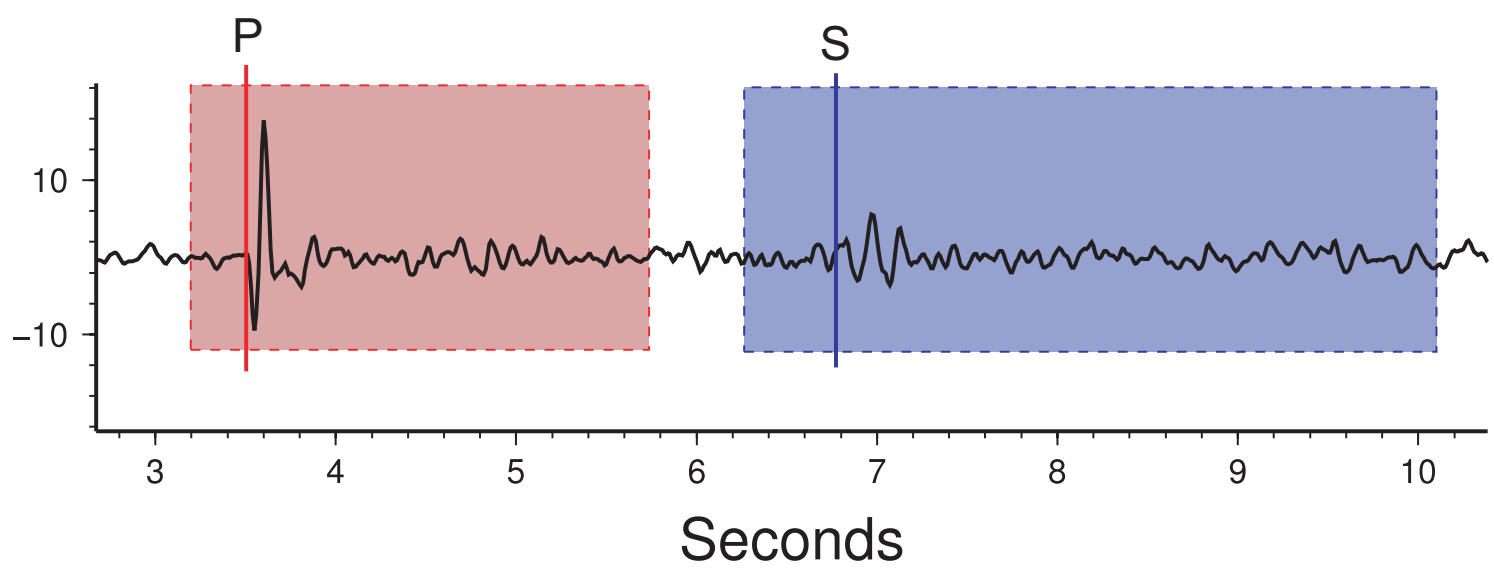

Figure 3.5. Example of time window used for cross-correlation analysis. Vertical component of earthquake 2246324 recorded at station MOVZ.

were allowed to overlap.

\subsubsection{Double-difference relocation}

HypoDD is a package for relocating earthquakes based on the double-difference (DD) algorithm of Waldhauser and Ellsworth [2000]. The DD method can be used when the inter-event distance between two earthquakes is small compared to the eventstation distance and the scale of velocity heterogeneity. In this case the ray paths between the event and station can be considered to be highly similar and the only difference, accounting for the differential arrival time, is the distance between the events (Figure 3.6). This results in accurate relative locations between the events but does not constrain absolute locations. HypoDD employs an iterative method to adjust the relative positions of the events and origin times to minimise the residual between the observed and calculated travel time differences.

$$
d r_{k}^{i j}=\left(t_{t}^{i}-t_{k}^{j}\right)^{o b s}-\left(t_{k}^{i}-t_{k}^{j}\right)^{c a l}
$$

Here $d r_{k}^{i j}$ is the DD for station $k,\left(t_{t}^{i}-t_{k}^{j}\right)^{o b s}$ is the difference in observed arrival times for events $i$ and $j$, and $\left(t_{k}^{i}-t_{k}^{j}\right)^{c a l}$ is the difference in calculated arrival time for events $i$ and $j$. The DD travel time residual can be linearly related to perturbations in the hypocentres using a truncated Taylor series expansion and using the appropriate slowness vector for each event [Waldhauser and Ellsworth, 2000].

$$
d r_{k}^{i j}=\frac{\partial t_{k}^{i}}{\partial \mathbf{m}} \Delta \mathbf{m}^{i}-\frac{\partial t_{k}^{i}}{\partial \mathbf{m}} \Delta \mathbf{m}^{j}
$$




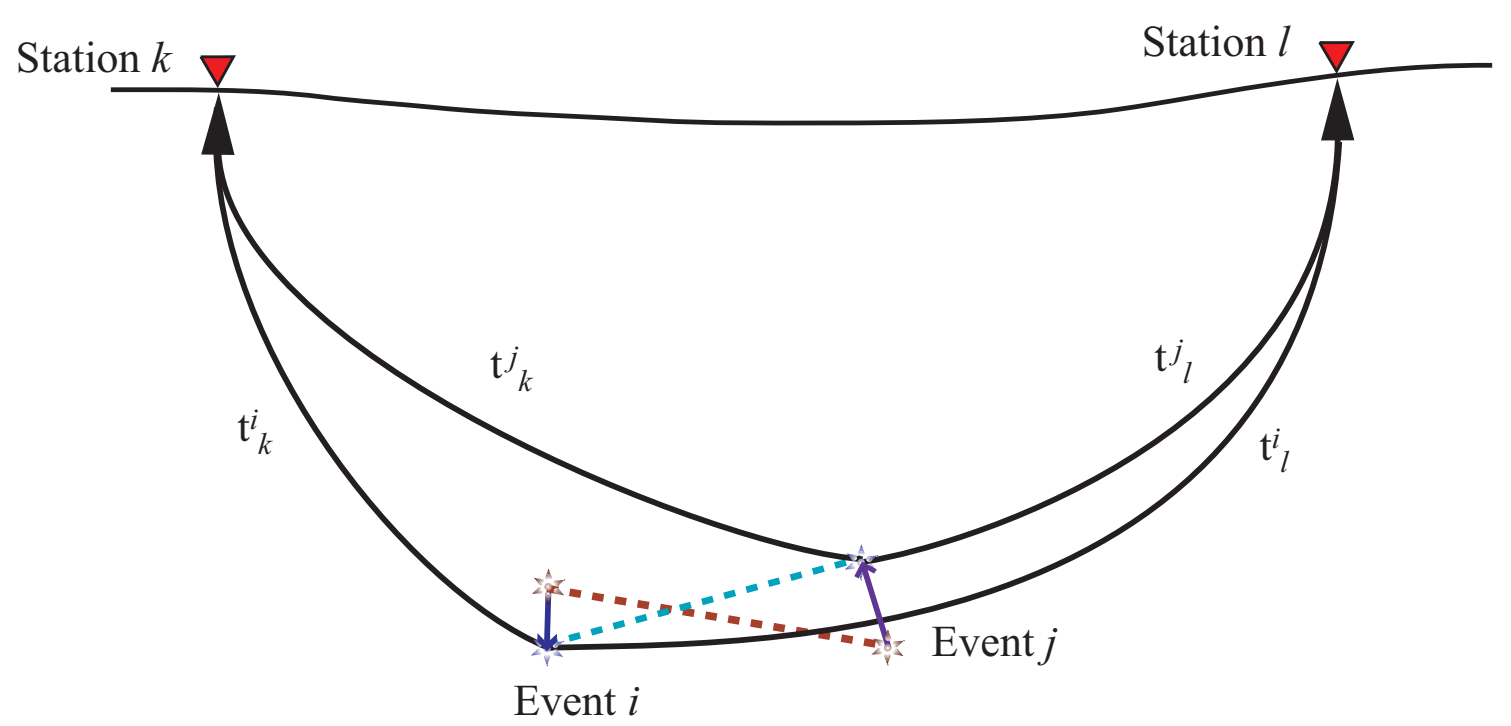

Figure 3.6. Schematic diagram illustrating the calculation of the double difference residual.

Here $\mathbf{m}^{i}$ and $\mathbf{m}^{j}$ are the estimated hypocentres ( $x, y, z$ and origin time), and $\Delta \mathbf{m}^{i}$ and $\Delta \mathbf{m}^{j}$ are the adjustments to the estimated hypocentres required for a better fit to the observed phase arrival times. Equation 3.6 can be used for both absolute travel times or cross-correlation relative travel time differences, a fact that helps to mitigate the problem of unconstrained absolute locations if only the cross-correlation differential times are used.

Collation of the DD residuals (Equation 3.7) for all earthquake pairs and all stations yields a system of linear equations that can be solved iteratively:

$$
\mathbf{W G m}=\mathbf{W d}
$$

Here $\mathbf{G}$ contains the partial derivatives, $\mathbf{d}$ contains the double-differences, $\mathbf{m}$ contains the changes in hypocentral parameters of interest, and $\mathbf{W}$ is a diagonal weighting matrix. The mean shift of the centroid of the cluster (of $N$ events) can be restricted to zero by including

$$
\sum_{i=1}^{N} \Delta \mathbf{m}_{i}=0
$$

The number of earthquake pairs grows as the square of the number of earthquakes to be relocated and therefore the system of linear equations (Equation 3.8) can become very large and the inversion can be computationally expensive and time consuming. For this reason the inversion can be performed using either the conjugate gradient method [LSQR see Paige and Saunders, 1982] or the method of singular value de- 
composition (SVD). SVD is more robust and produces more reliable estimates of uncertainties but is far less efficient and so cannot be used for more than about 100 earthquakes. In this case Waldhauser and Ellsworth [2000] suggest that the LSQR method be used, however smaller selections of earthquakes should still be relocated using SVD to estimate uncertainties.

There are several parameters to customise to the dataset in order to optimise the HypoDD inversion. The first set of parameters defines the linkage between the events and selects events for the relocation. Appendix E.3 displays the parameters, a description, and a typical value. The second set of parameters are involved in the inversion itself and are mainly related to the weightings and errors. These parameters can change after each iteration and Appendix E.4 displays the chosen parameters for the relocation carried out in Section 6.3.1. Here we employed five sets of iterations with ten iterations per set. The weightings were chosen so that in the initial iterations the catalogue $\mathrm{P}$ and $\mathrm{S}$ picks were used almost exclusively, then the cross-correlations were phased in such that in later iterations the cross-correlation relative times were used almost exclusively. This weighting scheme was employed to ensure that the catalogue data mainly constrained the absolute positions of events without sacrificing the highly accurate relative cross-correlation data.

\subsubsection{Shear wave splitting}

We use the automatic shear wave splitting package (MFAST) of Savage et al. [2010b] in order to compute shear wave splitting parameters. MFAST incorporates Silver and Chan's [1991] algorithm for calculating shear wave splitting using a grid-search inversion over the azimuth of the fast polarisation direction $\phi$ and delay time $\delta$ t, for a given time window, and Teanby et al.'s [2004b] SPLIT code, which conducts cluster analysis over a range of time windows to find the most stable result. These methods are explained in more detail in this section.

\section{Silver and Chan algorithm}

The mathematics behind the Silver and Chan [1991] automatic shear wave splitting algorithm are outlined in detail in Section 2.3.5. In this section we focus on the practicalities of running the algorithm. 
(a)
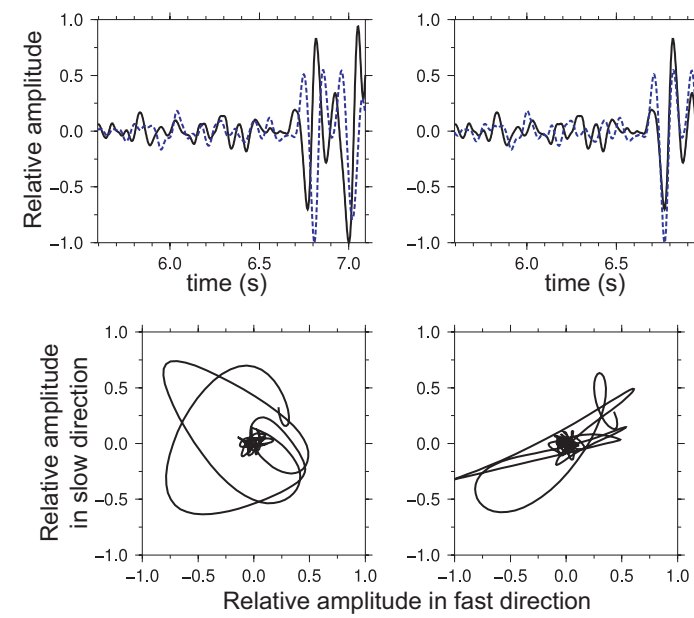

(b)
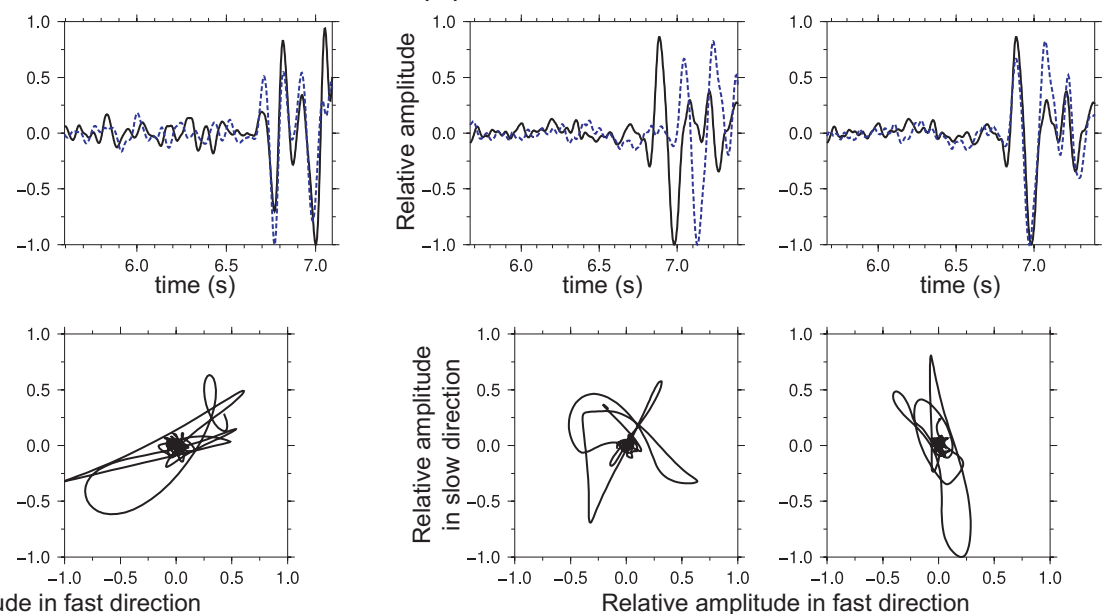

Figure 3.7. Examples of split shear wave particle motions. Top row shows time series of fast (black) and slow (blue) shear waves. Bottom row shows hodograms (display of particle motion). Left column of each panel shows time series and hodogram of the unprocessed signal, right column of each panel shows the result after shear wave splitting analysis. (a) Event 2257819 at station MOVZ. Unprocessed hodogram displays elliptical particle motion. (b) Event 2246324 at station MOVZ. Unprocessed hodogram displays cruciform particle motion.

The first step is to identify the shear wave arrival and define a shear wave analysis window around it, which was originally picked manually. If anisotropy is present, the particle motion within this window will be elliptical or cruciform, examples of which can be seen in Figure 3.7. A grid search over $\phi$ and $\delta$ t is then performed (see Section 2.3.5), during which both horizontal components of the seismogram are rotated by $\phi$ and one component is lagged by $\delta$ t. The grid search here uses increments of $1^{\circ}$ over $180^{\circ}$ and increments of 0.01 seconds between 0.0 and 1.0 seconds. The result that has the lowest second eigenvalue $\lambda_{2}$ of the corrected particle-motion covariance matrix indicates linear particle motion after correction and is the solution that best corrects for the splitting. Figure 3.8 conceptualises these steps. An F test is used to calculate the $95 \%$ confidence interval for the optimum values of $\phi$ and $\delta$. The $\lambda_{2}$ of the particle-motion covariance matrix provides a measure of the match of the corrected waveforms in the analysis window. The smaller the value of $\lambda_{2}$, the better the match. A threshold value for $\lambda_{2}$ and the confidence interval are applied in order to discard events that do not give good shear wave splitting parameters.

\section{SPLIT}

Results are often sensitive to the exact choice of shear wave analysis window. Teanby et al. [2004b] incorporated the Silver and Chan [1991] algorithm into codes (SPLIT) 
(a)

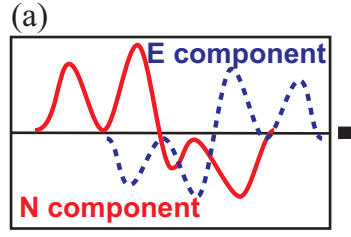

(b)

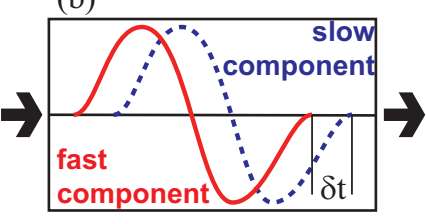

(c)

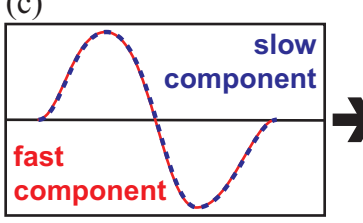

(d)

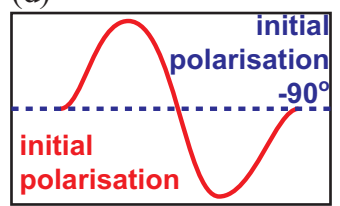

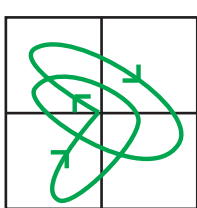
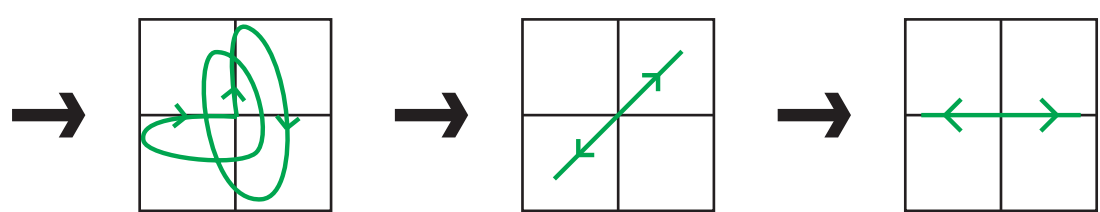

Figure 3.8. Schematic illustration of the steps to determine shear wave splitting parameters [after Gerst, 2003]. (a) North and east components of a seismogram and a hodogram (display of particle motion) oriented to north of a split $\mathrm{S}$ wave. Seismogram is complex and particle motion is elliptical. (b) Seismogram and hodogram oriented to fast and slow directions. The waveforms are similar but are separated by $\delta$ t. (c) The fast component is shifted back by $\delta$ t so that the waveforms line up. The particle motion becomes linear. (d) Seismogram and hodogram rotated into the direction of the initial polarisation. All of the energy is within this plane.

that calculate $\phi$ and $\delta \mathrm{t}$ for a range of windows and perform a cluster analysis on the results to find the most stable result.

The choice of shear wave analysis window should be representative of the shear wave and ideally be long enough to include several periods of the dominant frequency to prevent cycle skipping (see Section 2.3.6) and decrease the influence of noise. However, if spurious secondary phases in later parts of the waveform are included, they can degrade splitting estimates [Teanby et al., 2004b]. Teanby et al. [2004b] found that results from windows shorter than one period tend to become unstable and have unrealistically low errors because only small fragments of a wavelength need to be matched.

The grid of windows to be tested is set up by the SPLIT algorithm as follows. The beginning of the analysis window $t_{b e g}$ is allowed to vary between $t_{b e g 0}$ and $t_{b e g 1}$, with $n_{\text {beg }}$ steps of $\Delta t_{\text {beg }}$. Similarly, the end of the analysis window $t_{\text {end }}$ is allowed to vary between $t_{\text {end } 0}$ and $t_{\text {end1 } 1}$, with $n_{\text {end }}$ steps of $\Delta t_{\text {end }}$. The total number of analysis windows $n$ is given by $n=n_{\text {beg }} \times n_{\text {end }}$, and the shear wave analysis window is defined by

$$
\begin{array}{cc}
t_{\text {beg }}=t_{\text {beg } 1}-(i-1) \Delta t_{\text {beg }} & \text { for } i=1,2,3, \ldots, n_{\text {beg }}, \\
t_{\text {end }}=t_{\text {end } 0}-(i-1) \Delta t_{\text {end }} & \text { for } i=1,2,3, \ldots, n_{\text {end }} .
\end{array}
$$

$t_{\text {beg } 0}, t_{\text {beg } 1}, t_{\text {end } 0}$ and $t_{\text {end } 1}$ are chosen, initially manually, to fulfill the above criteria.

Stability over a wide range of different analysis windows ensures that the measurement is robust and SPLIT reduces the time taken to test using manual window 
(a)

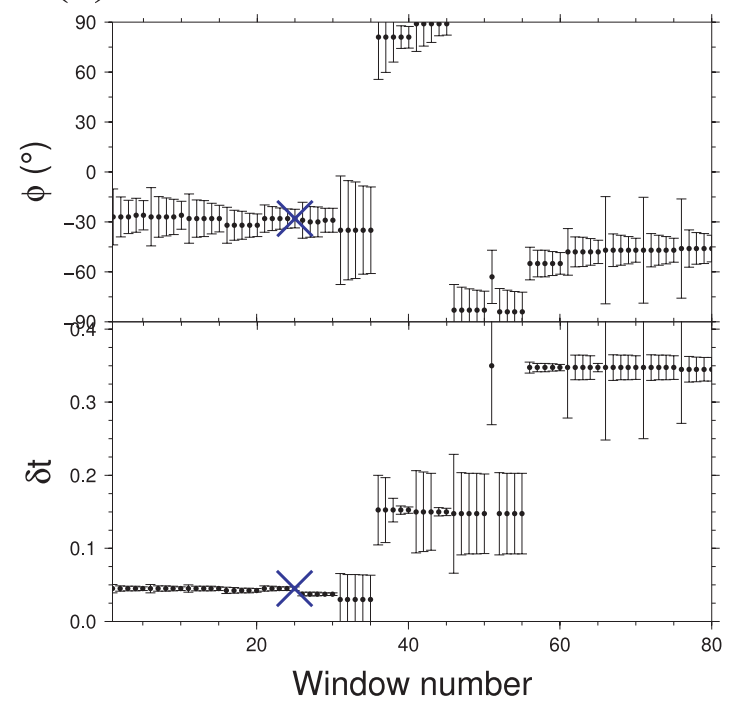

(b)

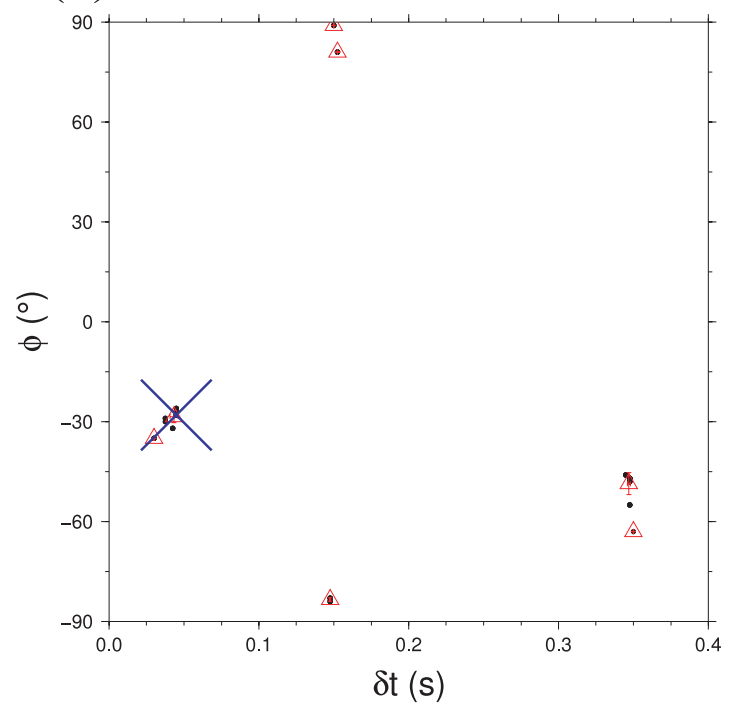

Figure 3.9. Example of cluster analysis from event 2241298 at station MOVZ. (a) $\phi$ and $\delta \mathrm{t}$ plotted against window number, plateaus indicate similar results. (b) $\phi$ plotted against $\delta$ t tight clusters indicate similar results. Blue cross is chosen "best" result

selection. Simply searching for the window that gives the lowest error bars in the measurements is not a good criterion as an unstable result, which is sensitive to small window changes, may be selected. The Teanby et al. [2004b] method searches over the range of window start/end times defined above to find measurements that are stable over many different analysis windows. Plateaus in $\phi$ and $\delta$ t when plotted against window number (Figure 3.9 a), or tight clusters when $\phi$ is plotted against $\delta$ t (Figure $3.9 \mathrm{~b}$ ), indicate stable splitting measurements, so that once the proper plateau has been identified the window that gives the smallest error can be selected. The unsupervised hierarchical cluster analysis technique of Everitt et al. [2001] is used to analyse clusters. To analyse the clusters, the scales (of $\phi$ and $\delta$ t) are standardised in order to compare distances between points. To start there are the same number of clusters as there are data points and all the inter-cluster distances are calculated, taking into account the cyclic nature of $\phi$. The two nearest clusters are then combined so that the number of clusters decreases by one. SPLIT continues combining clusters until there is only one cluster comprising the whole dataset. The result is a hierarchy of clusters. The final clusters are selected by maximising the between-cluster variance with respect to the within-cluster variance, indicating tight clusters that are widely spaced. The final clusters also have to satisfy the criterion that the ratio of within-cluster variances to that when two clusters are combined is lower than a critical value. This is the null hypothesis that the two clusters should be combined as a single cluster [Milligan and Cooper, 1985]. 
(a)

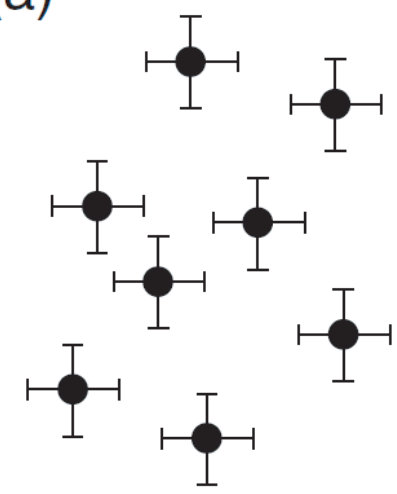

(b)

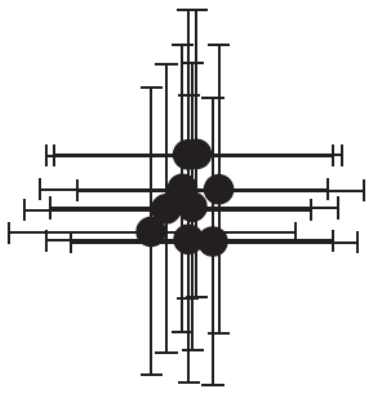

(c)

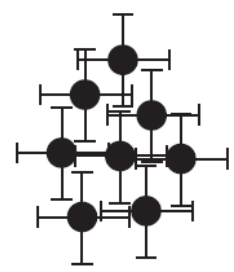

Figure 3.10. Three different cluster types [after Teanby et al., 2004b]: (a) diffuse cluster with low individual measurement errors, (b) tight cluster with high individual measurement errors, and (c) tight cluster with low individual measurement errors. SPLIT chooses cluster (c).

The criteria employed to select the optimum cluster are based on the number of points and the variance of the cluster. The number of points in the optimum cluster must not be less than a cycle's worth of points. The optimum cluster is selected by minimising both the within-cluster variance, and the mean data variance. This avoids the selection of diffuse clusters with low measurement errors and tight clusters with high measurement errors, as illustrated in Figure 3.10. The best measurement is the one with the lowest variance from within the best cluster.

\section{MFAST}

The final step to fully automate the shear wave splitting analysis process is to automatically pick the SPLIT window start and end times and to automatically choose the filter that is applied to the data before the shear wave splitting analysis. MFAST, the method of Savage et al. [2010b], incorporates the Silver and Chan [1991] algorithm and the SPLIT codes to create a program that can automatically analyse large data sets for shear wave splitting. Figure 3.11 illustrates the processing steps involved in MFAST.

As we have seen in Section 2.3.6, shear wave splitting can be highly frequencydependent and so the choice of filter can bias the shear wave splitting results. Different types of earthquakes recorded at different stations require different filters to maximise the signal-to-noise ratio (SNR). However, it has also been observed that using a narrow band filter can encourage cycle skipping (Section 2.3.6). MFAST uses a predefined set of 14 band-pass filters (Appendix E.5) to identify the best filter. 


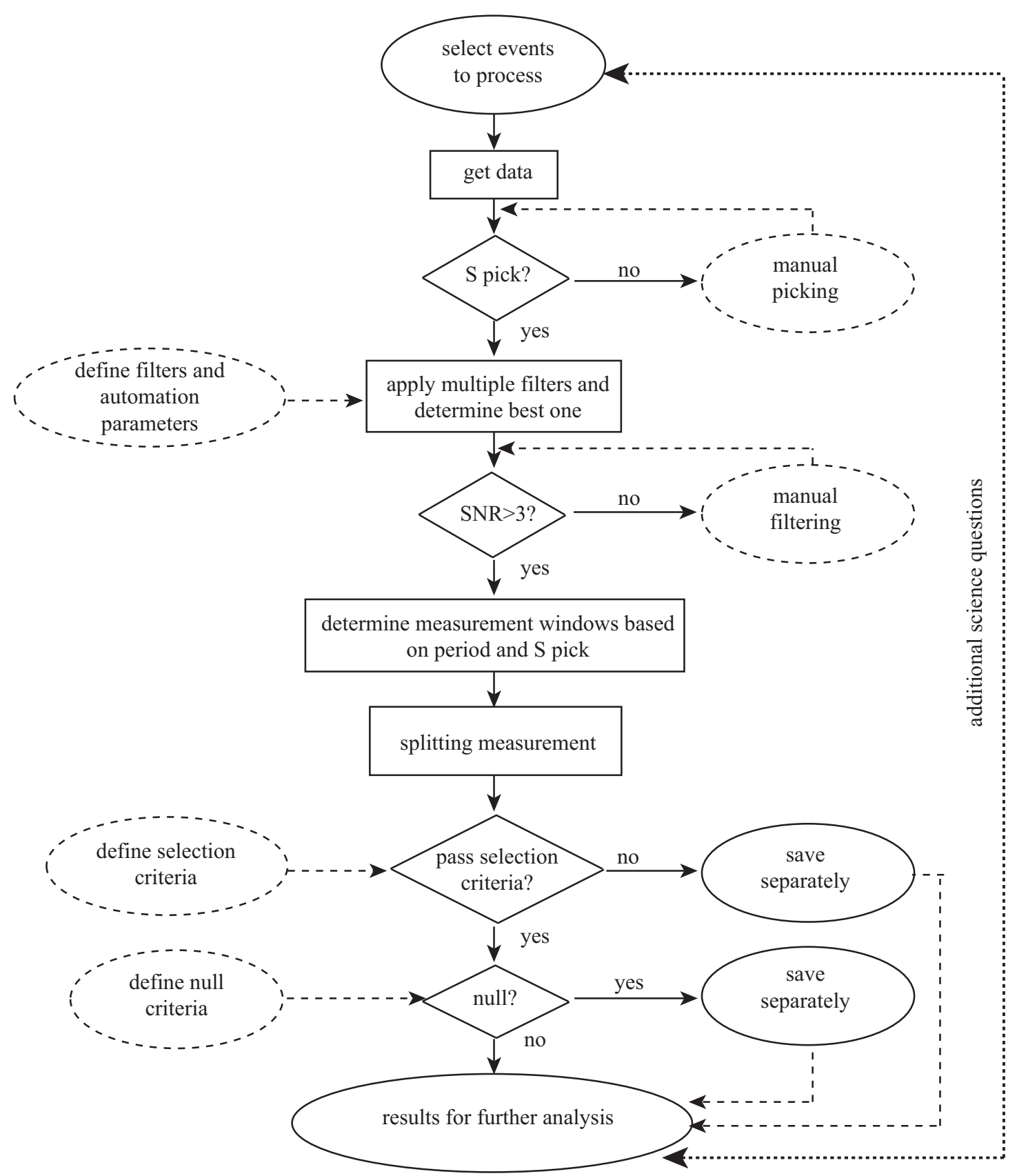

Figure 3.11. Flowchart of data processing steps for MFAST [after Savage et al., 2010b]. Dashed lines indicate optional steps. 


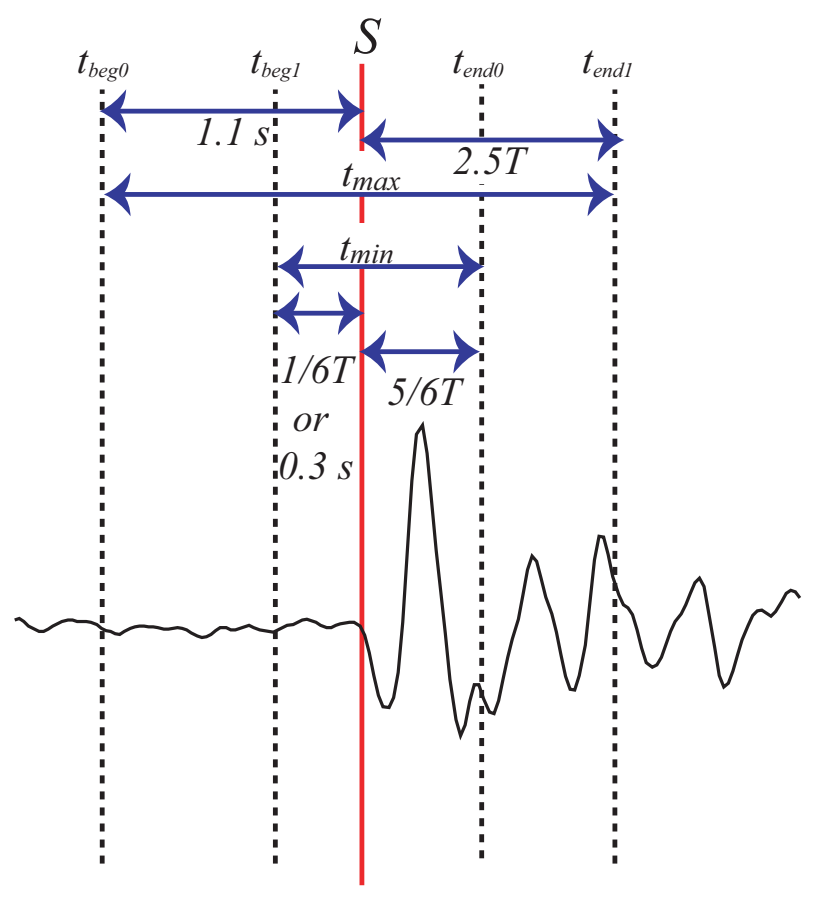

Figure 3.12. Schematic illustration of time windows around S arrival for cluster analysis.

The best filter is defined by the maximum product of the filter bandwidth (in octaves) and the SNR. The SNR is calculated by finding the mean of the amplitude of the fast Fourier transform (fft) of the signal $(0.05-3.05 \mathrm{~s}$ after the $\mathrm{S}$ wave arrival) to the fft of the noise $(0.05-3.05 \mathrm{~s}$ before the $\mathrm{S}$ wave arrival). The three best filters (fb1, fb2 and $\mathrm{fb} 3$ ) are applied to the data and advanced to the next step and those with SNR $<3$ result in the event being rejected from the analysis.

The choice of window extremes defined in this section is carried out automatically by MFAST using the $\mathrm{P}$ and $\mathrm{S}$ arrival times and the dominant frequency $f_{d}$ of the waveform. Figure 3.12 illustrates the window choices. The dominant frequency is calculated from the peak of an fft of the same three second window that was used to calculate the SNR, but is limited to $0.3 \leq f_{d} \leq 8 \mathrm{~Hz}$. The minimum window is one period $\left(1 T=1 / f_{d}\right)$ long and the maximum window is more than $2.5 T$ long. There are five start times between $t_{\text {beg } 0}$ and $t_{\text {beg } 1}$, which are usually 0.2 $\mathrm{s}$ apart so that $t_{b e g}=-0.3,-0.5,-0.7,-0.9,-1.1 \mathrm{~s}$. However, if the $\mathrm{P}-\mathrm{S}$ arrival time $\left(t_{S}-t_{P}\right)$ is less than $2.2 \mathrm{~s}, t_{\text {beg } 1}$ is set to $-0.1 \mathrm{~s}$ and $t_{\text {beg } 0}$ is $-\left(t_{S}-t_{P}\right) / 2$. There are between 15 and 25 end times between $t_{\text {end } 0}$ and $t_{\text {end } 1}$, which are between $t_{\text {end } 0}=5 / 6 t T$ and $t_{\text {end } 1}=2.5 T$. There are therefore between 75 and 125 windows for each filtered seismogram. The best window is chosen according to Teanby et al. [2004b] (above). 


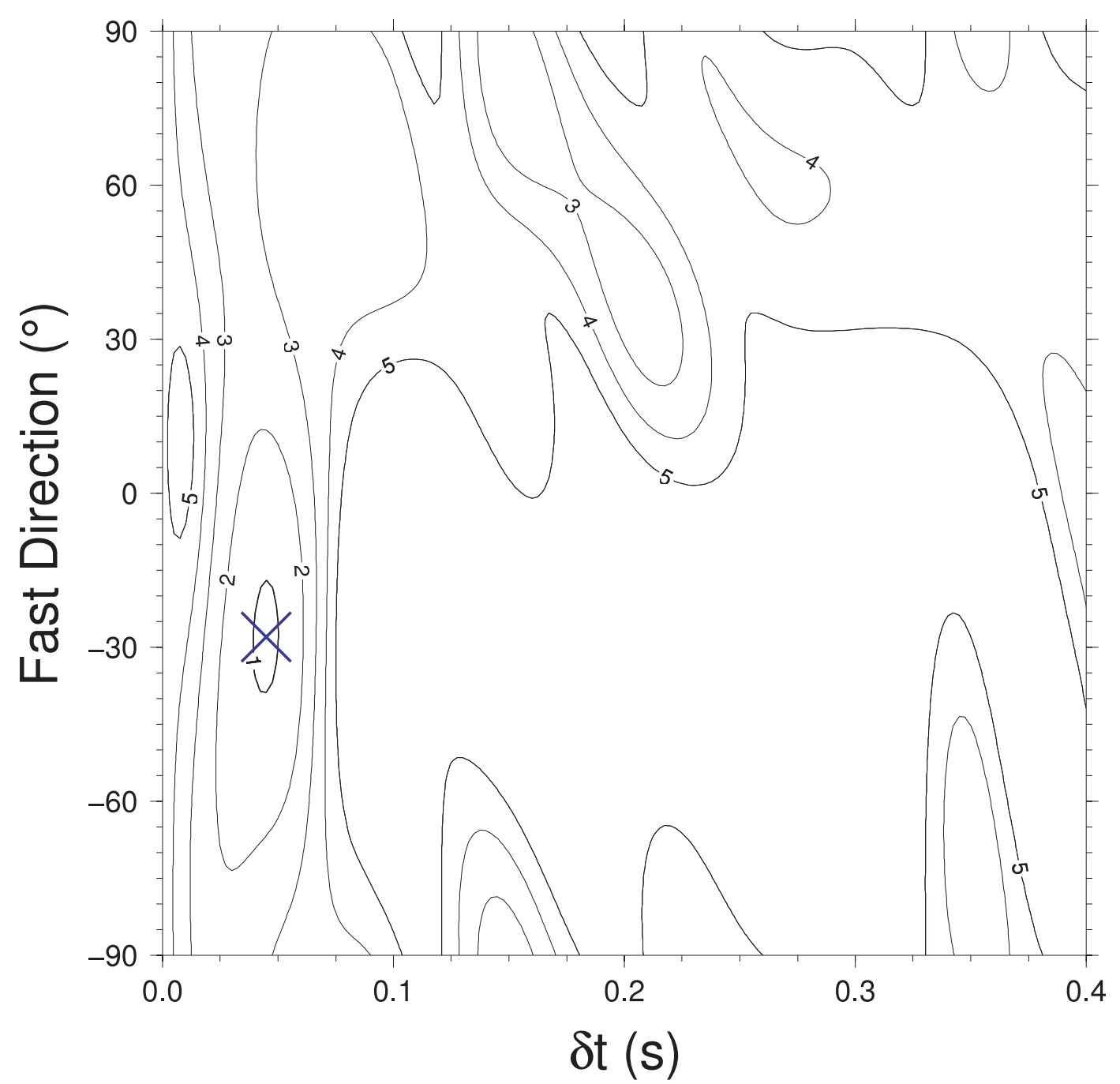

Figure 3.13. Eigenvalue contour plot from shear wave spitting analysis of earthquake 2241298 recorded at staion MOVZ. Contours indicate multiples of the standard errors.

In addition, MFAST automatically grades the results in order to reduce the effect of cycle skipping and to ensure low-quality measurements do not contaminate the results. To ensure high-quality measurements, grades are given according to the confidence interval, the SNR and the formal error bars on $\phi$ and $\delta$ t. An additional criterion is based on the area around the best result on the eigenvalue contour plot (Figure 3.13). Appendix E.6 summarises the grading. To reduce the risk of cycle skipping giving eroneous results, other clusters that are above the threshold defined in Section 3.2.3 are compared to the chosen cluster and those measurements that have multiple clusters with similar quality but different $\phi$ and $\delta \mathrm{t}$ are rejected. It is also possible to further grade the results from different filters using the same cluster grading and rejecting the results if they are too different, however we generally used only the best chosen filter (fb1) in order to minimise the processing time. 


\subsubsection{Circular statistics}

Conventional statistics of mean and error analyses cannot be used when dealing with directional data. This is because $0^{\circ}$ is the same as $360^{\circ}$ (the data are periodic) and so special statistical methods are required for the analysis of directional data. The following section outlines the treatment of directional data and follows the methods of Davis [1986]. Other tests such as the test of goodness-of fit and test of equality of two sets of data have been used in subsequent chapters where appropriate but are not described here.

\section{$180^{\circ}$ Ambiguity}

Shear wave splitting data represents orientation data rather than directional data; in other words $0^{\circ}$ is the same as $180^{\circ}$. The same statistics can be used as with directional data but the angles have to be doubled first $(\theta=2 \times \phi)$. This effectively transforms the orientation data into directional data and the mean, standard deviation and standard error can be calculated as below, as long as the result of the calculations is halved afterwards.

\section{Obtaining the mean of directional measurements}

The mean value of directional data can also be expressed as the dominant direction and can be found by computing the vector resultant $R$. Each measurement is treated as a normalised unit vector with direction $\theta_{i}(i=1, \ldots, n)$. The coordinates of the end point of the unit vector is given by

$$
\begin{aligned}
X_{i} & =\cos \theta_{i} \\
Y_{i} & =\sin \theta_{i}
\end{aligned}
$$

and $R$ can be obtained by summing the sines and cosines of the individual vectors so that the end position is

$$
\begin{aligned}
& X_{r}=\sum_{i=1}^{n} \cos \theta_{i} \\
& Y_{r}=\sum_{i=1}^{n} \sin \theta_{i}
\end{aligned}
$$




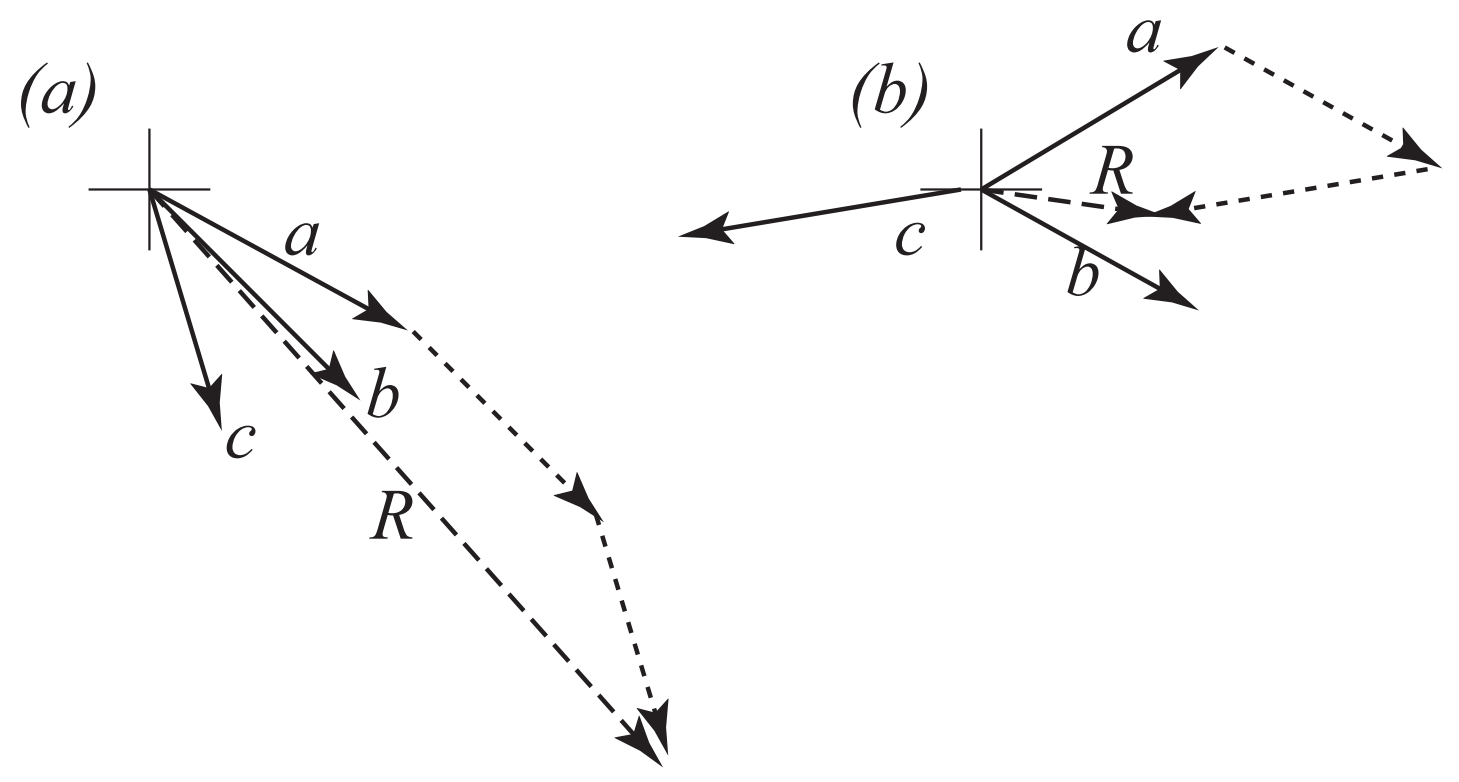

Figure 3.14. The mean direction of a set of unit vectors can be determined from the direction of the vector resultant $R$, obtained by combining the three unit vectors. The length of the resultant can be used to express the dispersion of the set of unit vectors. (a) Three vectors that are tightly clustered around a common direction result in a long $R$ that approaches the value of $n$. (b) Three widely dispersed vectors result in $R$ less than one [after Davis, 1986].

and the direction of $R$, which is the mean direction $\bar{\theta}$ is

$$
\begin{aligned}
\bar{\theta} & =\tan ^{-1}\left(\frac{Y_{r}}{X_{r}}\right) \\
& =\tan ^{-1}\left(\sum_{i=1}^{n} \sin \theta_{i} / \sum_{i=1}^{n} \cos \theta_{i}\right),
\end{aligned}
$$

and must be divided by two to yield the fast direction $\phi$. Figure 3.14 illustrates this concept. The length of the resultant depends on the dispersion of the vectors and so the length of $R$ can be used to obtain a measure of the spread

$$
\begin{aligned}
\bar{R} & =\sqrt{X_{r}^{2}+Y_{r}^{2}} / n \\
& =\sqrt{\left(\sum_{i=1}^{n} \cos \theta_{i}\right)^{2}+\left(\sum_{i=1}^{n} \sin \theta_{i}\right)^{2}} / n .
\end{aligned}
$$

The quantity $\bar{R}$ is the mean resultant length and is analogous (but reversed) to the variance, so that a large $\bar{R}$ has a small spread and vice versa. It is therefore sometimes expressed as its complement, which is the circular variance

$$
s_{0}^{2}=1-\bar{R}
$$


From the circular variance we can obtain the circular standard deviation and the angular standard deviation. The circular standard deviation is described as

$$
s_{\text {circular }}=\sqrt{-2 \ln (\bar{R})} \text {. }
$$

with values between 0 and infinity. This definition of the standard deviation is useful because, for a wrapped normal distribution, it is an estimator of the standard deviation of the underlying normal distribution. It will therefore allow the circular distribution to be standardised as in the linear case. For a measure between 1 and $180^{\circ}$ the angular standard deviation is used, which is a simple square root of the variance

$$
s_{\text {angular }}=\sqrt{1-\bar{R}} .
$$

\section{The von Mises distribution and Rayleigh's test for non-randomness}

In order to obtain information about the errors and deviations of circularly distributed data we must have a probability model of known characteristics against which we can test. The von Mises distribution is a circular equivalent of the normal distribution and similarly is characterised by two parameters, a mean direction $\bar{\theta}$ and a concentration parameter $\kappa$. If $\kappa$ is zero then all directions are equally probable and the distribution is circularly uniform. It is difficult to calculate $\kappa$ directly but tables can be used to obtain the value of $\kappa$ from $\bar{R}$. A test can then be done to see whether there is a preferred orientation. This is Rayleigh's test and it is carried out by seeing whether the value of $\bar{R}$ exceeds a certain threshold based on the number of observations $n$ and the significance level $\alpha$. Tables of critical values of $\bar{R}$ can be found in Davis [1986].

The confidence angle or standard error $s_{e}$ of the estimate of the mean direction $\bar{\theta}$ is given by (in radians)

$$
s_{e}=1 / \sqrt{n \bar{R} \kappa} .
$$

In general both the standard deviation and the standard error are useful measures. The standard deviation describes the spread of values in the sample and the standard error describes the sample mean's accuracy as an estimate of the population mean. 


\subsubsection{Anisotropy analysis}

In order to constrain the locations of high anisotropy, a two-dimensional tomographic inversion was conducted on the delay time $(\delta \mathrm{t})$ estimates from the shear wave splitting analysis to obtain the locations of strong anisotropy. We then produced spatial averages of splitting parameters as a first-order approximation to the heterogeneous anisotropic structure. These methods are described fully in Section 4.3.2 but an overview of the steps taken is presented here. Calculations for the resolution of the tomography are also included in Section 4.3.2.

\section{Two-dimensional delay-time tomography}

The following steps outline the two-dimensional delay-time tomography method.

The station and event locations are first converted from latitude and longitude into New Zealand Map Grid (NZMG) coordinates and the splitting data $(\phi, \delta$ t and the respective error margins) is read into MATLAB. Once these parameters are loaded, stations and events that are within the limits of the grid area (Figure 3.15 a) are identified. The following grid parameters are also defined:

- minimum box size for the checkerboard minsize $C B=4000$;

- maximum number of rays passing through a box maxnumber $=65$;

- minimum number of rays passing through a box minnumber $=20$;

- minimum box size minsize $=4000$.

The checkerboard model $\mathbf{m}_{C B}$ (Figure $3.15 \mathrm{~b}$ ) is constructed using the limits of the grid area and minsize $C B$. The design matrix $\mathbf{G}_{C B}$ for the checkerboard grid is built by finding where the rays cross the gridlines and calculating the distance that each ray crosses each grid box (Figure $3.15 \mathrm{c}$ ). The synthetic data for the checkerboard can then be calculated:

$$
\mathbf{d}_{C B}=\mathbf{G}_{C B} \mathbf{m}_{C B} .
$$

Boxes for the inversion are found by using quadtree gridding (Figure $3.15 \mathrm{~d}$ ). This method counts how many rays are in the current grid box. If there are more than maxnumber rays in the grid box then the gridbox is divided into four new equal sized boxes, as long as the new boxes are not smaller than minsize. If there are less than minnumber rays in the grid box then the box is flagged so that it is no longer 
included in the analysis. These steps are carried out for every box until there is either between maxnumber and minnumber rays in each box, the box is excluded or the box size reaches minsize.

The design matrix for the quadtree grid $\mathbf{G}$ is constructed in the same way as $\mathbf{G}_{C B}$. The synthetic checkerboard data are then weighted according to the error margins on the real measurements:

$$
\begin{aligned}
\mathbf{G}_{w} & =\mathbf{W} \times \mathbf{G} \\
\mathbf{d}_{C B w} & =\mathbf{W} \times \mathbf{d}_{C B}
\end{aligned}
$$

where

$$
\mathbf{W}=\operatorname{diag}\left(\frac{1 / \Delta \delta \mathrm{t}}{\max (1 / \Delta \delta \mathrm{t})}\right)
$$

The inversion of the weighted checkerboard data (Figure 3.15 e) is carried out using a medium-scale optimisation inversion function (lsqlin) in MATLAB. Constraints are set so that the minimum anisotropy strength can not be below $0 \mathrm{~s} / \mathrm{km}$ and the maximum can not exceed the maximum $\delta$ t observed for a ray path applied to one block length, i.e. $\delta \mathrm{t}_{\max } / L_{b}$ where $L_{b}$ is the width of the smallest block,

The inversion is then carried out on the real data $(\mathbf{d}=\delta \mathrm{t})$ by weighting and inverting them in the same way as the checkerboard data (Figure $3.15 \mathrm{f}$ ).

\section{Spatial Averaging}

The fast direction of shear wave anisotropy for each grid block is found through the following steps. First a weighting profile along each ray $w_{r}$ is constructed. The weighting profile was tested using four different definitions:

- the anisotropy strength $s$ of each block $b\left(s_{b}\right)$ from the 2-D delay time tomography along the ray path $r$ is normalised by the measured delay time $\delta \mathrm{t}_{r}$ for the ray and used as the weighting function $w_{r b}=s_{b} / \delta \mathrm{t}_{r}$,

- the weighting profile is a function of distance $d$ from the station so that the weight for each block along the raypath is $w_{r b}=1 / d_{b-s t a t i o n}$,

- the weighting profile is a function of distance $d$ from the station so that the weight for each block along the raypath is $w_{r b}=1 / d_{b-s t a t i o n}^{2}$,

- there is no weighting profile, each ray is weighted as one. 


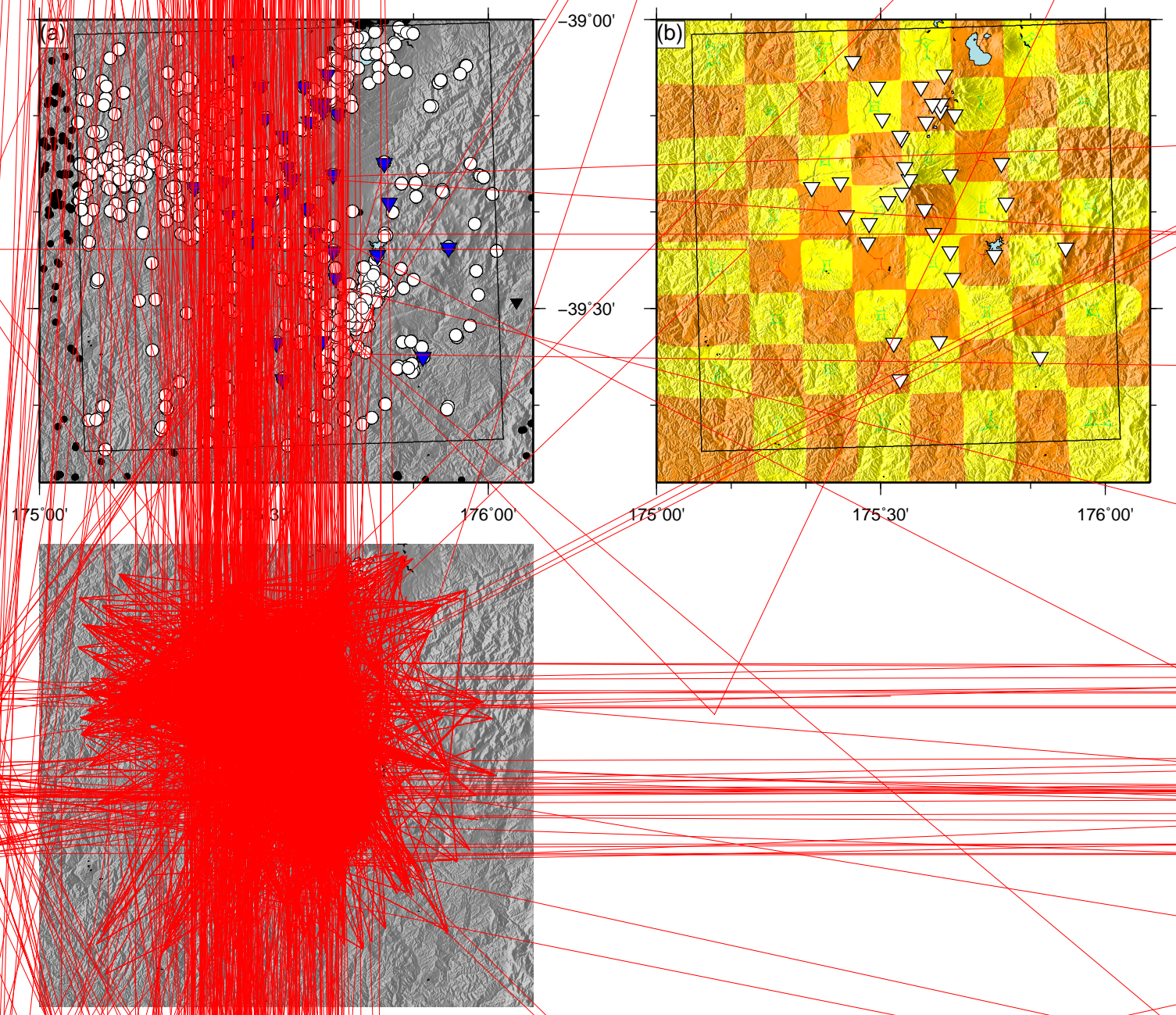

Figure 3.15. Examples of output from 2-D delay time tomography steps. See text in Section 3.2.5 for details. 
The $\phi$ value for each ray is then appointed to each block that the ray passes through and multiplied by the weighting for that ray and that block $\phi_{r b}=\phi_{r} w_{r b}$. All $\phi_{w}$ for each block are grouped together, and the average $\phi$, standard deviation and standard error for each grid block are calculated.

\subsubsection{Stress analysis}

\section{Coulomb stress}

Stress modelling in this project has been carried out using the Coulomb 3.1 package [Lin and Stein, 2004; Toda et al., 2005], which is available from [USGS, Last accessed 22 Feb 2011, http://earthquake.usgs.gov/research/modeling/coulomb/ download.php]. It is designed to calculate static displacements, strains, and stresses caused by fault slip, magmatic intrusion or dike expansion/contraction.

The Coulomb stress transfer is calculated, in its simplest form, by the equation

$$
\Delta \sigma_{\phi}=\Delta \tau+\mu^{\prime} \Delta \sigma_{\nu}
$$

$\Delta \sigma_{\phi}$ is the change in failure stress on the receiver fault caused by slip on the source faults, $\Delta \tau$ is the change in shear stress (positive in the direction of receiver fault slip), $\Delta \sigma_{\nu}$ is change in normal stress, and $\mu^{\prime}$ is the effective fault friction coefficient on the receiver fault. Failure is promoted when the Coulomb stress increases.

Coulomb 3.1 does not take into account factors such as dynamic stresses, pore fluid diffusion, and viscoelastic rebound, nor does it facilitate the inclusion of gravitational loading of topography. However, it is a simple tool that permits exploration of stress changes and can be used, as it has been in this project, to model inflation and deflation of an idealised magma reservoir.

To implement Coulomb 3.1, several parameters should first be defined or estimated (Appendix E.7). Here we assume the modelling is being carried out in order to analyse shear wave splitting results at a volcano. Other parameters should be used when modelling fault slip.

Coulomb 3.1 permits the application of a uniform regional or tectonic stress. The azimuths of the three principal stress directions should be mutually orthogonal and estimated using a priori information. In a normal faulting regime the plunge of S1 will be $90^{\circ}$ (vertical) and in a reverse faulting regime it will be zero (horizontal). 
The stress at the surface will usually be zero but there will be a vertical gradient. A value of $100 \mathrm{bars} / \mathrm{km}$ is recommended in the manual. We calculated the vertical gradient using the expressions presented by Zoback and Townend [2001].

Coulomb 3.1 calculates the stress tensor at locations on a model grid given the parameters listed in Appendix E.7. In order to calculate the direction of maximum horizontal stress, we use a variation of a script originally written by Sonja Greve and based on the method of Lund and Townend [2007].

The fit of the modelled maximum horizontal stress to the shear wave splitting fast direction results is calculated with an additional script. The fit is calculated by interpolating the model so that there are estimates of $S_{H \max }$ at the same locations as the measurements of $\phi$ and calculating the cosines of the difference of the angles. Fit values are given as the sum of the cosines (higher numbers equate to a better fit), and the normalised cosines, therefore a fit of 1 is a perfect match, 0 is exactly perpendicular at each point. 


\section{Distinguishing between stress-induced and structural anisotropy at Mount Ruapehu volcano, New Zealand}

Johnson, J. H., M. K. Savage, and J. Townend, (2011) Distinguishing between Stress-induced and Structural Anisotropy at Mount Ruapehu Volcano, New Zealand. J. Geophys. Res., Resubmitted after minor revisions 4 May 2011, Paper \# 2011JB008308

\section{Abstract}

We have created a benchmark of spatial variations in shear wave anisotropy around Mount Ruapehu, New Zealand, against which to measure future temporal changes. Anisotropy in the crust is often assumed to be caused by stress-aligned microcracks, and the polarisation of the fast quasi-shear wave $(\phi)$ is thus interpreted to indicate the direction of maximum horizontal stress, but can also be due to aligned minerals or macroscopic fractures. Changes in seismic anisotropy have been observed following a major eruption in 1995/96 and were attributed to changes in stress from the depressurisation of the magmatic system. Three-component broadband seismometers have been deployed to complement the permanent stations that surround Ruapehu, creating a combined network of 34 three-component seismometers. This denser observational network improves the resolution with which spatial variations in seismic anisotropy can be examined. Using an automated shear wave splitting analysis, we examine local earthquakes in 2008. We observe a strong azimuthal dependence of $\phi$ and so introduce a spatial averaging technique and two-dimensional tomography of recorded delay times. The anisotropy can be divided into regions in 
which $\phi$ agrees with stress estimations from focal mechanism inversions, suggesting stress-induced anisotropy, and those in which $\phi$ is aligned with structural features such as faults, suggesting structural anisotropy. The pattern of anisotropy that is inferred to be stress related cannot be modelled adequately using Coulomb modelling with a dike-like inflation source. We suggest that the stress-induced anisotropy is affected by the load of the volcano and a lithospheric discontinuity.

\subsection{Introduction}

\subsubsection{Overview}

In this paper we present shear wave splitting results from a combined network of 34 broadband and short-period three-component seismometers. This combined network is denser than those used in previous studies and so yields results with higher resolution of spatial variations in anisotropy. We use both shallow $(<30 \mathrm{~km})$ and deep $(>50 \mathrm{~km}$ ) earthquakes that occurred throughout 2008 near Mt. Ruapehu and the automated shear wave splitting method of Savage et al. [2010b] to measure anisotropy parameters. We employ a spatial averaging technique similar to that of Audoine et al. [2004] combined with a new method of two-dimensional tomography to analyse spatial variations in anisotropy and relate these to stress and structure in the region. The Coulomb stress package of Lin and Stein [2004] and Toda et al. [2005] is used to predict stress orientations consistent with a putative magma reservoir model and determine whether they are consistent with the anisotropy in regions where stress dominates. The results of this study will be used to detect future changes in anisotropy and to identify regions of past changes in anisotropy with more confidence.

Mount Ruapehu is a 2797 m-high andesitic stratovolcano and the highest active volcano in New Zealand (Figure 4.1). It is the southernmost of the large active volcanoes on the North Island, which make up the Taupo Volcanic Zone (TVZ). The TVZ is a spreading segment of the Earth's crust resulting from the subduction of the Pacific Plate beneath the Australian Plate at the obliquely-westward dipping Hikurangi subduction zone. Subsidence in the central axis of the TVZ at the southern termination has led to prominent active faults developing to the east and west of Mt. Ruapehu, which are down-thrown towards the mountain [Villamor and Berryman, 2006]. The ring plain is composed of laharic, fluvial, pyroclastic 
and fall deposits [Houghton et al., 1987], indicating that Mt. Ruapehu produces different styles of eruptions, some of which have the potential to be devastating to the surrounding area [Neall et al., 1999]. The principal road and rail routes of the central North Island cross the ring plain east and west of the volcano and there are three ski fields on the mountain, all of which are in high risk zones [Houghton et al., 1987]. Major magmatic eruptions occurred in 1945 and 1995/1996; the latter was the largest historical eruption of Mt. Ruapehu, producing a $12 \mathrm{~km}$-high volcanic ash plume and lahars on the flanks of the volcano [Bryan and Sherburn, 1999]. Mt. Ruapehu frequently experiences phreatic and phreato-magmatic eruptions [Hurst et al., 2004], which also threaten lives and property [Johnston et al., 2000].

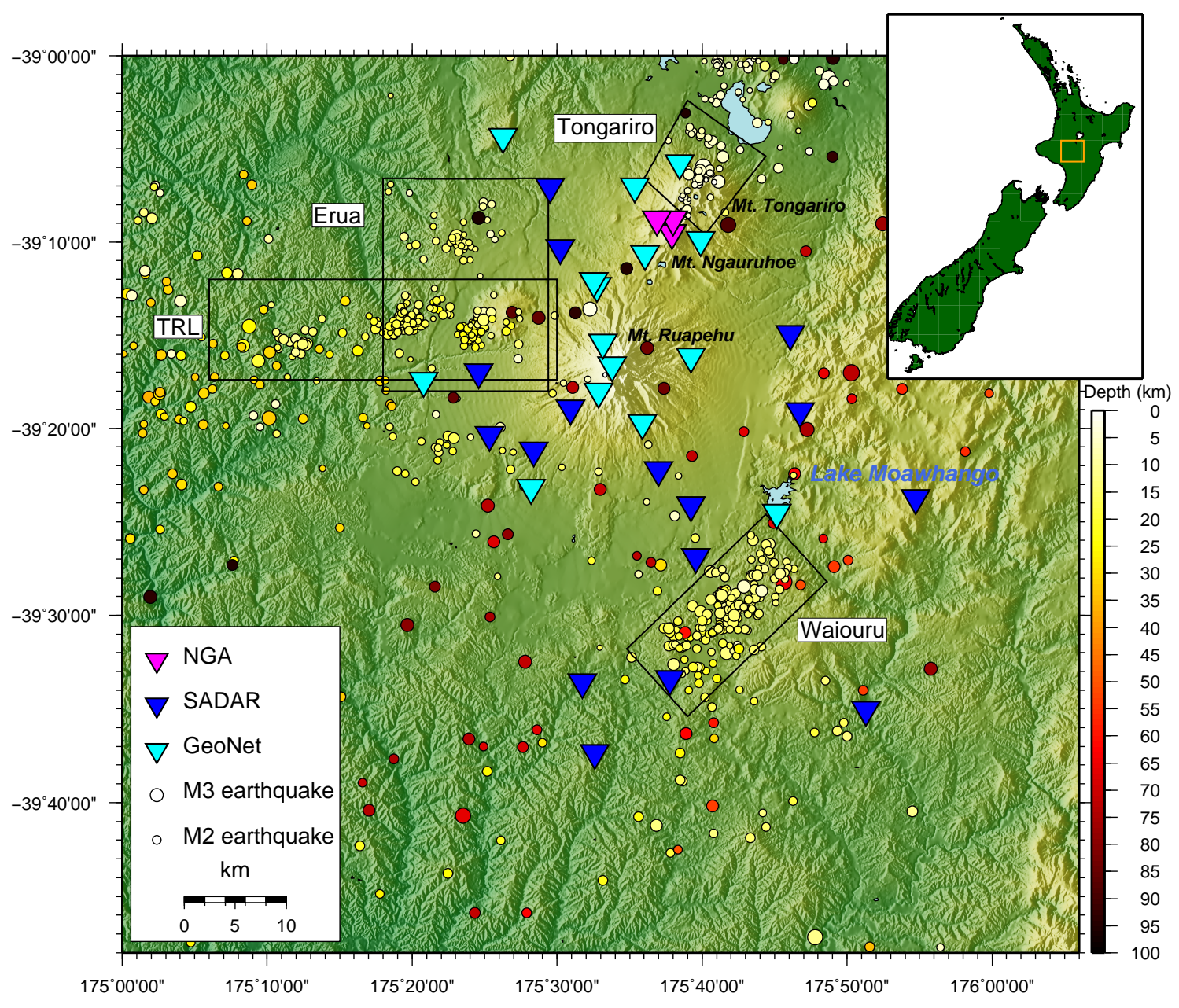

Figure 4.1. Map of the area around Mount Ruapehu (central peak). The circles indicate the catalogue locations of the earthquakes in 2008, used in this study, scaled by magnitude and colourcoded by depth. The inverted triangles represent three-component seismometers; the cyan are permanent GeoNet stations, the blue are temporary SADAR stations and the purple are temporary Ngauruhoe stations. Boxes represent clusters of earthquakes, see text for a discussion.

Eruptions of Mt. Ruapehu often occur with few or no detectable precursors, making prediction difficult [Hurst et al., 2004]. For this reason Mt. Ruapehu volcano has, 
in recent years, been subject to several studies of crustal seismic anisotropy using shear wave splitting analysis [Miller and Savage, 2001; Gerst and Savage, 2004] in an attempt to characterise the local stress regime.

\subsubsection{Previous studies of seismic anisotropy}

Miller and Savage [2001] measured shear wave splitting from shallow $(<30 \mathrm{~km})$ and deep (> $50 \mathrm{~km}$ ) earthquakes in 1994 and 1998 and observed a change in the dominant azimuth of fast polarisation $(\phi)$ spanning the magmatic eruption of 1995/1996. That study was extended by Gerst and Savage [2004], who used the same techniques and an additional deployment of three-component seismometers in 2002 to observe further changes in $\phi$.

The changes in $\phi$ in both studies were interpreted as being caused by a dike-shaped magma reservoir, or system of dikes, trending NE-SW. According to this model, the magma reservoir was pressurised before the eruption, producing a local stress field different from the regional stress field. Following the eruption the reservoir was less full and correspondingly less pressurised so the local stress returned to that of the surrounding region. The Gerst and Savage [2004] study suggested that the later changes in $\phi$ were due to repressurising of the reservoir in response to an increase of magma in the system. The stress in the surrounding crust caused by the pressurised magma reservoir is thought to preferentially align randomly oriented fluid-filled microcracks and cause seismic anisotropy that is detected through shear wave splitting (e.g. Crampin [1994]; Hatchell and Bourne [2005]). While aligned, these microcracks are anisotropic structures but they are transient with the changing stress and so we refer to this cause of anisotropy as stress-induced anisotropy. Crustal media with prominent structures such as layers, parallel fractures or lineated fabric can also be anisotropic (e.g. Kaneshima [1990]; Babuška and Cara [1991]; Boness and Zoback [2006a]). When the structures exhibit horizontal axes of symmetry, a vertically propagating shear wave will also be split and we refer to this intrinsic anisotropy as structural anisotropy. This complicates interpretation of shear wave splitting measurements because the possibility of both structural anisotropy and stress-induced anisotropy must be considered.

Kaneshima [1990] examined fast shear wave polarisation throughout Japan and attributed the observations to three mechanisms: stress-induced microcracks; cracks or fractures in the vicinity of active faults; and intrinsic rock properties from preferred orientation of minerals. Zinke and Zoback [2000] detected both stress-induced 
and structure related anisotropy at the same station in central California and distinguished between the two mechanisms by observing that different clusters of earthquakes gave different fast polarisations, thereby showing in that case that the shallow crust beneath the station did not influence the shear wave splitting. Boness and Zoback [2006a] measured shear wave anisotropy at stations on and around the San Andreas Fault and found by interpreting $\phi$ orientations in conjunction with the fault strikes and regional maximum horizontal compressive stress from borehole breakouts, that separate regions of structural and stress-induced anisotropy could be identified.

If we can differentiate between stress-induced and structural anisotropy then shear wave splitting analysis has the potential to be developed into a near-real time stressmonitoring tool. This could be a valuable addition to the monitoring arsenal at Mt. Ruapehu and other volcanoes that exhibit little precursory activity before eruptions.

\subsubsection{Regional Geology}

The basement greywacke in the Ruapehu region consists of the Torlesse and Waipapa terranes to the west and east respectively [Adams et al., 1998; Mortimer, 2004]. The geological textures of the basement rocks around Mt. Ruapehu were investigated by Beetham and Watters [1985] during the Tongariro Power development project. They found no discernible internal structure in the basement greywacke except a couple of small areas that display textural zone $2 \mathrm{~B}$, semi-schistose rocks (on the metamorphic sequence described by Turnbull et al. [2001]); these were to the north of the Kaimanawa Range (which is outside our study area) and around Lake Moawhango (Figure 4.1). Mortimer [1993] identifies these areas as Kaimanawa schist, which is a continuation of the Haast schist. The schist around Lake Moawhango is therefore the only region in which we expect to observe anisotropy related to the metamorphic texture. Anisotropy from the semi-schistose rocks would have $\phi$ oriented in the same direction as the strike of the fabric, that is $035-040^{\circ}$ [Beetham and Watters, 1985].

Figure 4.2 (a) displays four zones identified by Villamor and Berryman [2006] as having different fault strikes. The area of most dense faulting lies to the NE of Mt. 


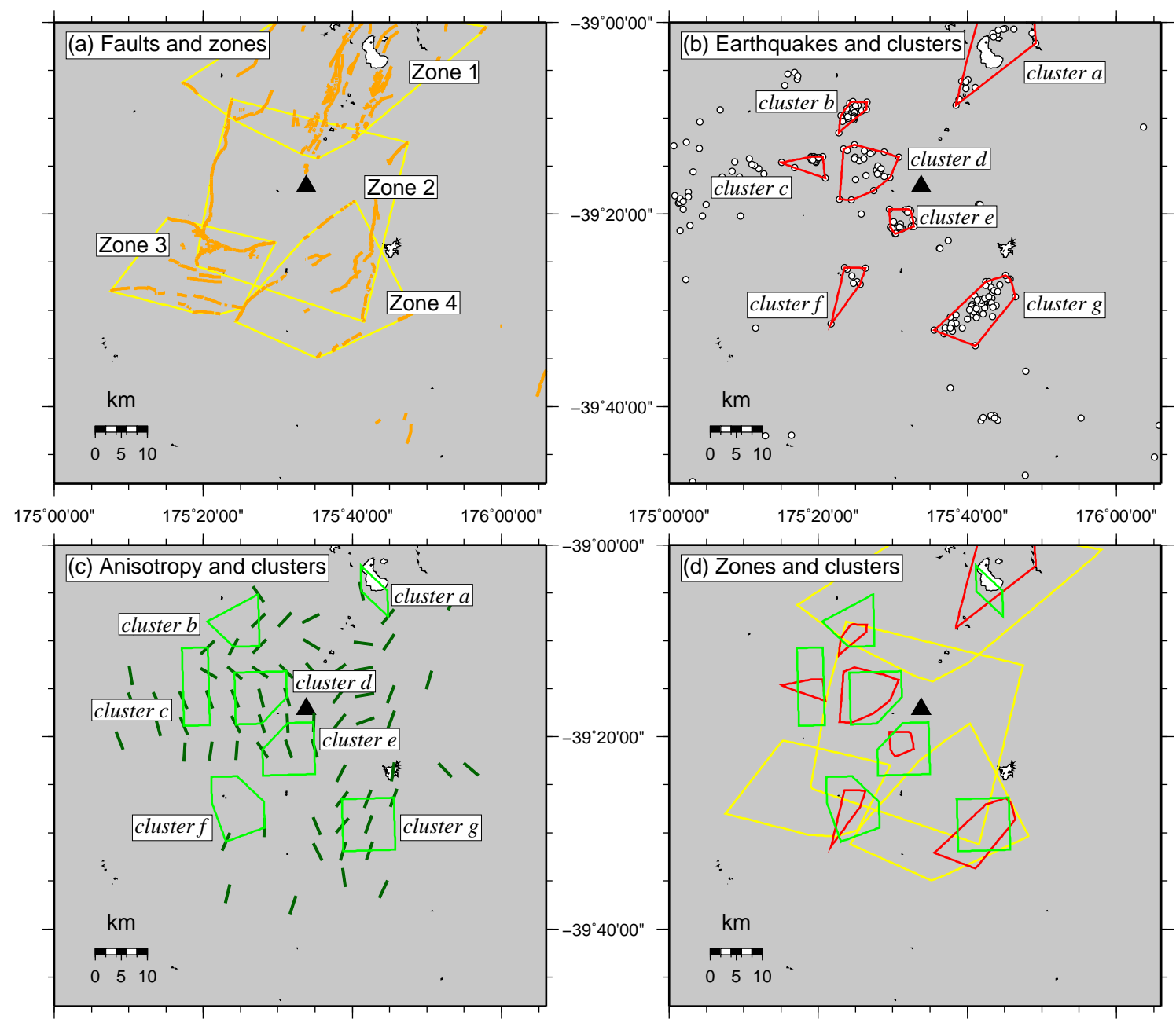

Figure 4.2. Zones and clusters for comparison of parameters. Black triangles indicate the summit of Mt. Ruapehu. (a) Orange lines are known faults (from the NZ Active Faults Database of GNS), yellow boxes group faults with similar strikes into four zones [Villamor and Berryman, 2006]. Zone 1 contains the Tongariro graben, zone 2 contains the Ruapehu Graben, zone 3 contains the Ohakune-Raetihi fault-set and zone 4 contains the Karioi fault-set. (b) White dots show epicentres of earthquakes with focal mechanism information from Sherburn et al. [2009], red boxes group the earthquakes into seven clusters. Clusters a, b, c and d are labelled for reference in the text to those clusters in zone 2. (c) Dark green bars show fast polarisation results from spatial averaging in section 4.4.2, green boxes show groups of data within $10 \mathrm{~km}$ of earthquake cluster centroid in (b). (d) Combination of all zone and cluster outlines.

Ruapehu (zone 1), around Mt. Ngauruhoe and Mt. Tongariro. The average strike of zone 1 faults is $030^{\circ}$ [Rowland and Sibson, 2001]. The strike of zone 2 faults of $010^{\circ}$ corresponds to the axis of the Ruapehu graben. Faults in zone 2 cross-cut the faults of zones 3 to the west and zone 4 to the east. Zone 4 faults strike NNE-SSW and the zone incorporates the area of the more pervasive metamorphic fabric, which is sub-parallel to the fault strikes. Zone 3 faults strike WNW-ESE in an area of Tertiary marine deposits and Quaternary volcanics, however there is no information about deeper basement fabric for this zone. 


\subsubsection{Stress Estimates}

Villamor and Berryman [2006] noted that all of the Tertiary faults in the area are normal, suggesting that the maximum compressive stress, $S_{1}$, is close to vertical. In a normal faulting regime the minimum compressive stress $\left(S_{3}\right)$ corresponds to the minimum horizontal compressive stress $\left(S_{H \min }\right)$ and for Andersonian fault orientations, is orthogonal to the fault strike. Conversely, the maximum horizontal compressive stress $\left(S_{H \max }\right)$ is parallel to fault strike, in which case we would expect $\phi$ to also be parallel to fault strike regardless of whether stress-induced or structural anisotropy due to fault zones were dominant. The sense of motion and the crosscutting of contemporary fault sets with three different strikes suggests that the minimum and intermediate principal values of the stress tensor are similar in magnitude, in which case small changes in horizontal stress magnitudes might rotate $S_{H \max }\left[\right.$ Villamor and Berryman, 2006]. Further evidence that $\left|S_{2}\right| \approx\left|S_{3}\right|$ in the Ruapehu region comes from Wallace et al. [2004], who modelled GPS observations with block rotation that allows opening of the Taupo rift and implies a change in stress orientation over a short distance due to compression at the southern termination of the TVZ. The temporal changes in shear wave splitting in the region observed by Miller and Savage [2001] and Gerst and Savage [2004] are also in accordance with this hypothesis. 


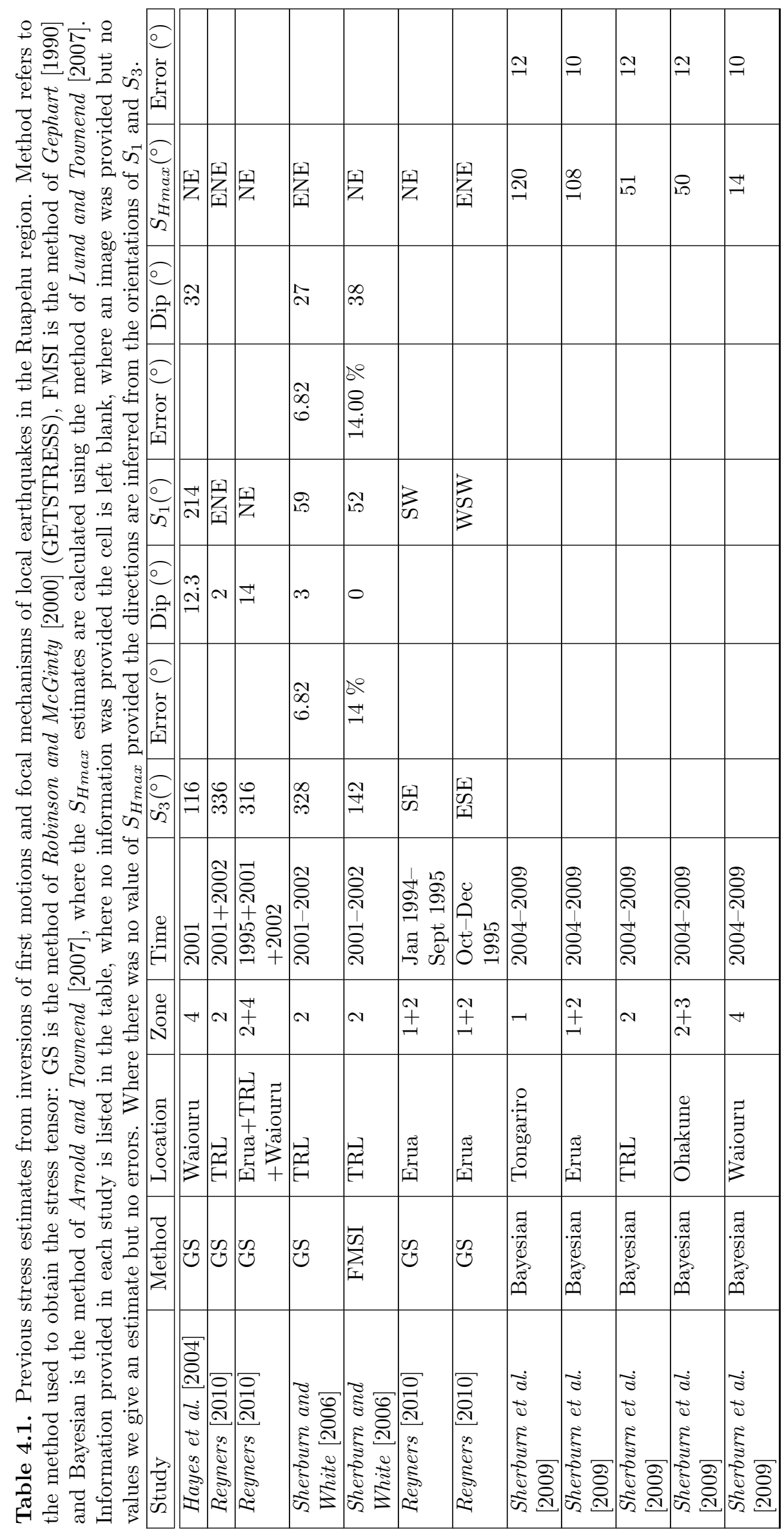


Estimates of local stress parameters have been calculated by inverting first motions and focal mechanisms of local earthquakes. A summary of these stress estimates can be found in Table 4.1. These calculated or inferred maximum horizontal compressive stresses will subsequently be referred to as $S_{\text {Hmax }}^{\text {focal }}$.
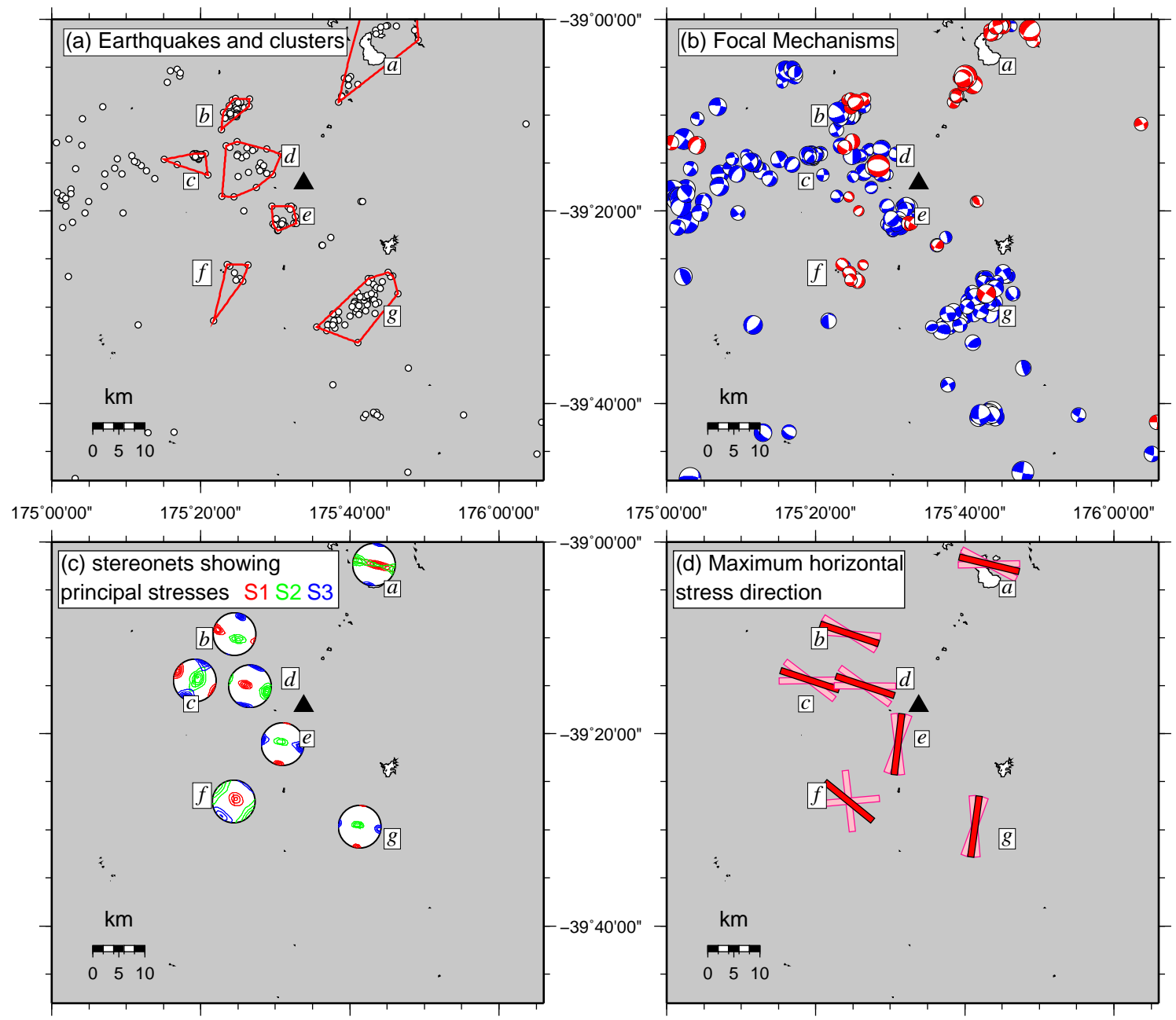

Figure 4.3. Steps to calculate $S_{H \max }$ from focal mechanism inversions using the method of Arnold and Townend [2007]. Black triangles indicate the summit of Mt. Ruapehu. (a) White dots show epicentres of earthquakes that have focal mechanism information, red boxes group the earthquakes into seven clusters. (b) Focal mechanisms used in the inversions, after Sherburn et al. [2009]. Blue indicates hypocentre is deeper than $8 \mathrm{~km}$ and red shallower than $8 \mathrm{~km}$. (c) Stereonets showing principal stresses with $S_{1}$ in red, $S_{2}$ in green and $S_{3}$ in blue. (d) $S_{H \max }$ estimations with $80 \%$ confidence interval in pink.

We carried out similar stress inversions of focal mechanisms to Sherburn et al. [2009] using the Bayesian method of Arnold and Townend [2007] but using slightly different clusters of events in order to better compare stress orientations to the zones of similar fault strikes previously described in Section 4.1.3 and displayed in Figure 4.2. The focal mechanisms used in the inversions are those calculated using first motions and the FPFIT method [Reasenberg and Oppenheimer, 1985] by Sherburn et al. [2009] for 
well-recorded earthquakes using parameters from the GeoNet earthquake database. Figure 4.3 (a) displays the epicentres of those earthquakes with calculated focal mechanisms, and the focal mechanisms are displayed in Figure 4.3 (b). The focal mechanism inversion method allows fault plane ambiguities, observational errors, and weights associated with individual focal mechanisms to be incorporated into the analysis. The clustering and resulting principal stresses can be seen in Figure 4.3. For cluster $a$ we used earthquakes that were all within our study area and fault zone 1 (Figure 4.2), which resulted in a smaller subset than that used by Sherburn et al. [2009], but we get stress tensor results that are in good agreement, with $S_{\text {Hmax }}^{\text {focal }}$ of $103 \pm 15^{\circ}$. We created four clusters of earthquakes from zone 2 , all of which were to the west of Mt. Ruapehu. The three from within the Erua cluster $(b, c$ and $d)$ give the same $S_{\text {Hmax }}^{\text {focal }}$ orientation of $108 \pm 19^{\circ}$ but the one closer to the mountain $(d)$ gave a normal faulting regime $\left(S_{1}\right.$ vertical) whereas the other two gave strike-slip $\left(S_{2}\right.$ vertical). The northernmost of these three clusters $(b)$ is in the overlap between zones 1 and 2 (Figure 4.2). The cluster that Sherburn et al. [2009] used to the southwest of Mt. Ruapehu was split into two, the northernmost of which was in our zone 2 (cluster $e$ ) and gave $S_{\text {Hmax }}^{\text {focal }}=007 \pm 14^{\circ}$. The resulting cluster in zone 3 (cluster $f$ ) displays a normal faulting regime with $S_{\text {Hmax }}^{\text {focal }}$ of $129 \pm 44^{\circ}$, which is sub-parallel to the faults in this zone (Figure 4.2 (a)), although only eight focal mechanisms were used in the inversion and the errors are large (Table 4.2). There were enough earthquakes in cluster $g$ (zone 4, the Waiouru swarm) to subdivide the cluster, however all subsets of earthquakes (divided by time, depth, spatially and randomly) yielded the same result to within errors; $S_{H \text { max }}^{\text {focal }}$ of $008 \pm 12^{\circ}$, which was very similar to that of cluster $e$ in zone 2 . 


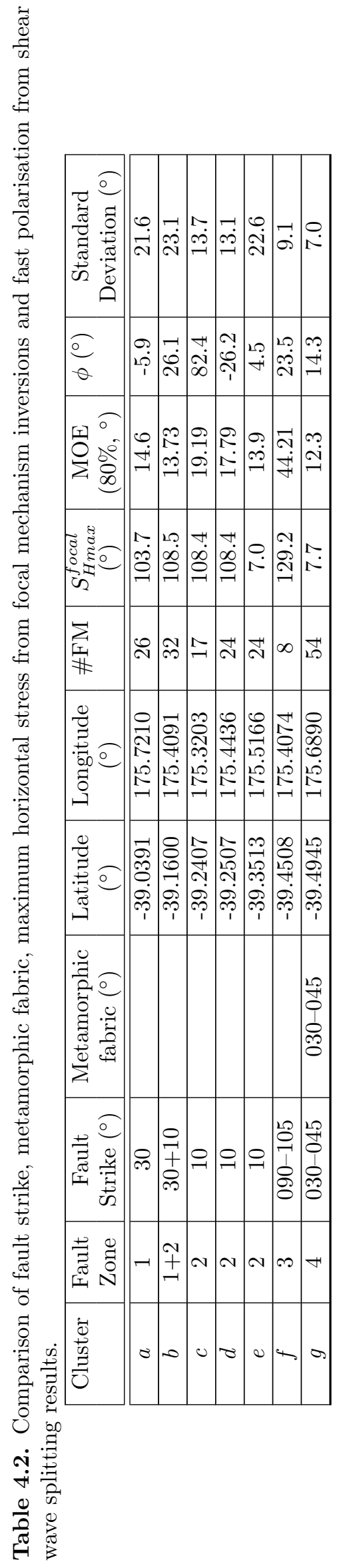




\subsection{Data}

The Spatial Anisotropy Deployment At Ruapehu (SADAR) project consisted of 13 broadband and three short-period temporary three-component seismometers, deployed around Mt. Ruapehu to complement the permanent network of three broadband and 12 short-period three-component seismometers [GeoNet, Last accessed 17 April 2011, http://www.geonet.org.nz] (Figure 4.1). A separate temporary deployment of three three-component broadband seismometers around Mt. Ngauruhoe took place in January and February 2008 [Jolly et al., 2011], the data from which are included in the combined data set with data from the permanent network and the SADAR stations. The SADAR stations were deployed throughout 2008 and during that time recorded 1978 local earthquakes, as small as magnitude 1.0, that were also recorded at the permanent sites and routinely located for the national New Zealand catalogue. Of these, 929 had impulsive $\mathrm{S}$ wave arrivals and displayed shear wave splitting at at least one station. More details about the seismic stations used in this analysis are listed in Appendix B.

Figure 4.1 shows the catalogue hypocenters of the recorded seismicity [GeoNet, Last accessed 17 April 2011, http://www.geonet.org.nz]. Hypocenters are routinely calculated by GeoNet and have typical formal errors on the order of $0.01^{\circ}$ horizontally and $2 \mathrm{~km}$ vertically. The earthquakes tend to occur in three main clusters. The majority of the earthquakes occur to the southeast of Mt. Ruapehu, around the village of Waiouru and this group of earthquakes is referred to hereafter as the Waiouru swarm. Many earthquakes also occur to the northwest of the mountain: these have been referred to in previous studies by several names including the National Park swarm [Sherburn and White, 2006], Raurimu swarm [Reyners, 2010] and Erua swarm [Keats et al., 2011] after features and villages local to the earthquakes. Here we adopt the term Erua swarm as Erua village is directly over the center of the swarm and, even though the Raurimu fault passes through the swarm, we find no evidence that the earthquakes are directly related to the fault. The cluster of earthquakes to the northwest continues in a line to the west. This is the so-called Taranaki-Ruapehu Line (TRL) [Sherburn and White, 2006; Stern, 2009]. The third cluster near Mt. Tongariro to the northeast is smaller and somewhat shallower $(<8$ $\mathrm{km}$ ) than the other two but nevertheless is important in this study and is referred to as the Tongariro swarm. Deep (>50 km) earthquakes originate from the subducted slab and Figure 4.1 illustrates the deepening of these earthquakes, and hence the subducted slab, to the northwest. 
Figure 4.4 displays map and profile views of the catalogue locations of the shallow $(<40 \mathrm{~km})$ earthquakes (grey) and the relocations (black) calculated using HypoDD (see Section 3.2.2 for details).
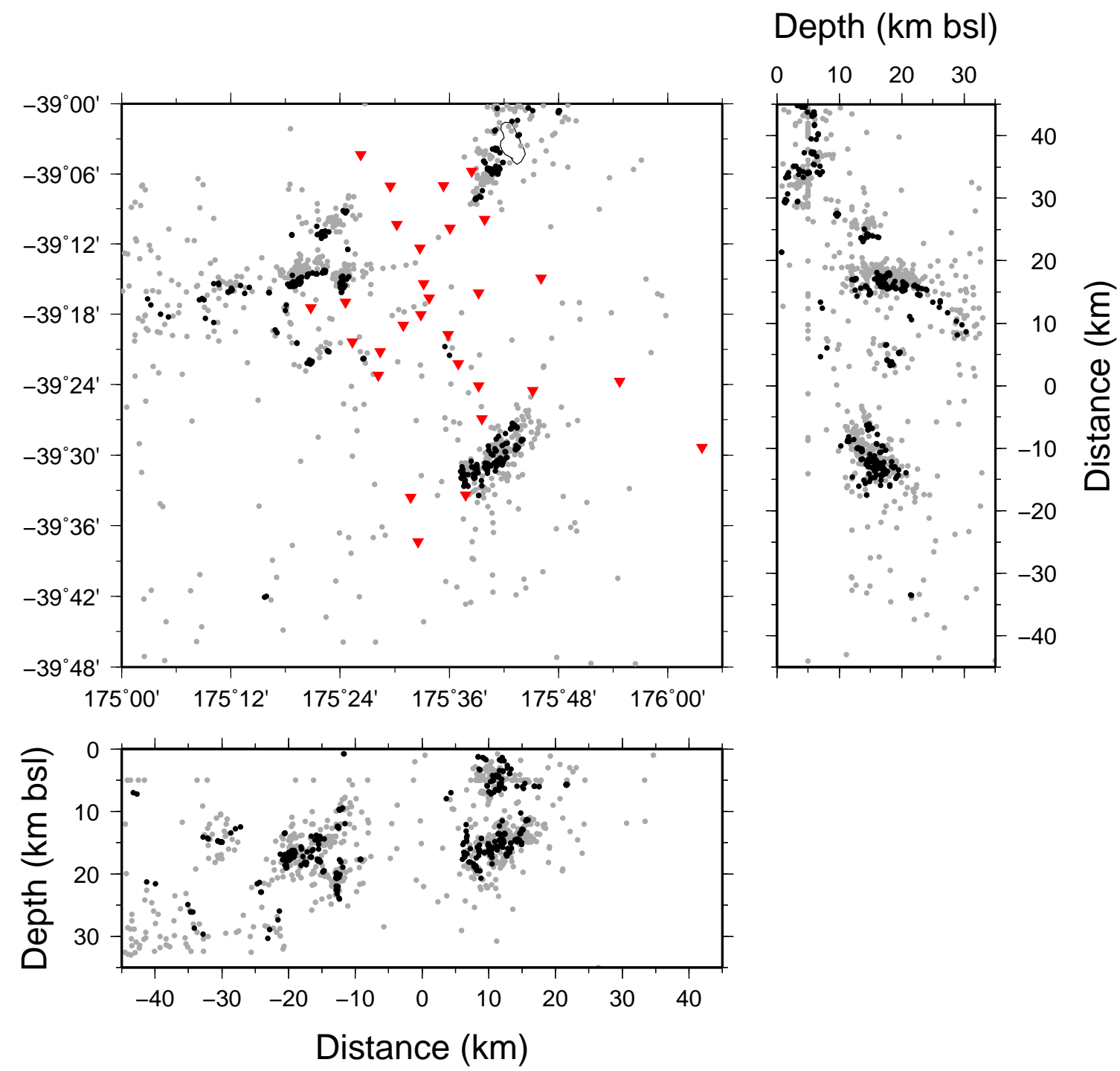

Figure 4.4. Map and depth profiles of relocated hypocentres of $60 \%$ of the shallow $(<40 \mathrm{~km}) 2008$ catalogue. Grey dots are catalogue locations and black are relocations using HypoDD [Waldhauser and Ellsworth, 2000]. (a) Map of catalogue and relocated earthquakes, red inverted triangles indicate seismic stations. (b) N-S depth profile. (c) W-E depth profile. 


\subsection{Method}

\subsubsection{Shear wave splitting}

We use the automatic shear wave splitting method of Savage et al. [2010b], which incorporates the Silver and Chan [1991] algorithm for calculating shear wave splitting using a grid-search inversion over the azimuth of the fast polarisation direction $\phi$ and delay time $\delta$ t, for a given time window, and Teanby et al. [2004a]'s SPLIT code, which conducts cluster analysis over a range of time windows to find the most stable result.

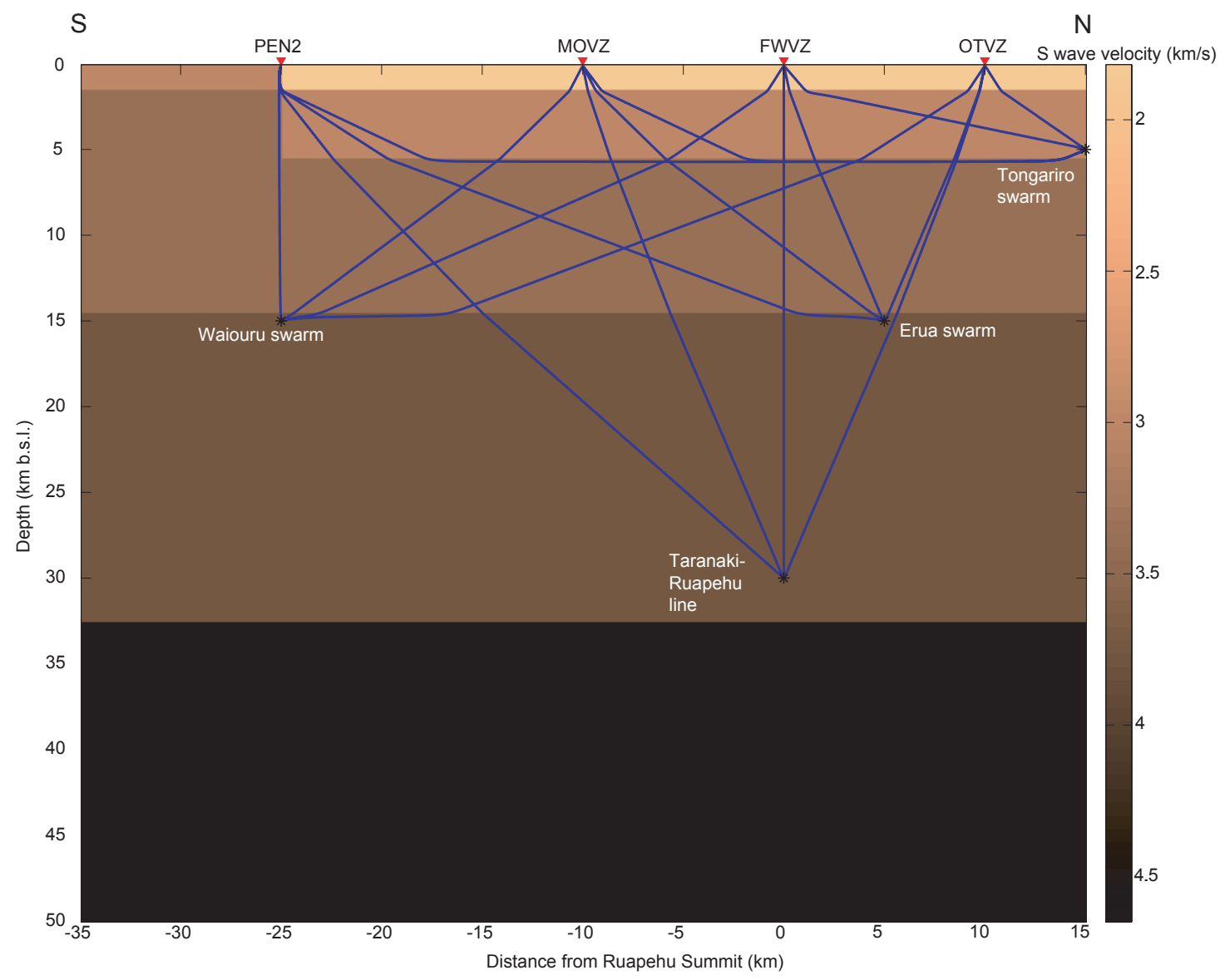

Figure 4.5. Modelled raypaths from reprasentative earthquakes to reprasentative stations in a $\mathrm{N}-\mathrm{S}$ transect. Earthquakes are modelled in the centre of each main cluster. Velocity model is from Latter [1981a]. Raypaths were calculated using the method of Kroon [2009].

The optimum filter is identified based on a product of the signal-to-noise ratio and bandwidth, and time window extremes are based on the dominant frequency of the first three seconds of the S waveform. In addition, the Savage et al. [2010b] method grades or rejects the splitting parameters chosen by the cluster analysis based on the 
distribution of clusters in order to mitigate cycle skipping and ambiguous results. Uncertainties $\left(\sigma_{d}\right)$ are calculated by finding the $95 \%$ confidence interval for the optimum values of $\phi$ and $\delta$ t after conducting an $F$ test for the chosen time window [Silver and Chan, 1991]. The method requires no input parameters, and the only manual step is determining the $\mathrm{S}$ arrival time. The incidence angle of each ray at each station is calculated using the TauP Toolkit [Crotwell et al., 1999] with the 1-D velocity model of Latter [1981a] for the Ruapehu region. Rays with incidence angles greater than $35^{\circ}$ from vertical are not included in the analyses as these lie outside the shear wave window $[N u t t l i, 1961]$ and S-P conversions at the surface could contaminate the waveforms. We observe that the steep velocity gradient in the top $0.5 \mathrm{~km}$ ensures that the majority of the local earthquakes recorded fulfill this criteria. Figure 4.5 displays the modelled raypaths from reprasentative earthquakes to reprasentative stations in a N-S transect. The initial polarisations of the incoming waves are calculated as part of the algorithm, such that applying the calculated splitting parameters returns the waves to their unsplit state. Results from the shear wave splitting analysis that gave $\phi$ to within $20^{\circ}$ of the polarisation of the incoming wave were considered null results [Peng and Ben-Zion, 2004] and not included in the interpretation. Nulls signify that no splitting was reliably detected [Silver and Chan, 1991], rendering the corresponding value of $\delta$ t meaningless and giving $\phi$ with a $90^{\circ}$ ambiguity. The $\phi$ results are often plotted as rose diagrams (circular histograms) at the station where the measurements were recorded (e.g. Figure 4.6).

Table 4.3. Station name with number of shear wave splitting measurements selected to be class A and B, with short $\delta \mathrm{t}(<0.5 \mathrm{sec})$ from the method of Savage et al. [2010b].

\begin{tabular}{|l|l|l|l|l|l|l|l|}
\hline $\begin{array}{l}\text { Station } \\
\text { Name }\end{array}$ & $\begin{array}{l}\text { SWS } \\
\text { measurements }\end{array}$ & $\begin{array}{l}\text { Mean } \\
\phi\left(^{\circ}\right)\end{array}$ & $\begin{array}{l}\text { Standard } \\
\text { Error }\left(^{\circ}\right)\end{array}$ & $\begin{array}{l}\text { Standard } \\
\text { Deviation } \\
\left({ }^{\circ}\right)\end{array}$ & $\begin{array}{l}\text { Mean } \\
\text { t } \\
(\mathrm{sec})\end{array}$ & $\begin{array}{l}\text { Standard } \\
\text { Deviation } \\
(\mathrm{sec})\end{array}$ \\
\hline \hline ALL & all & 5129 & 9.6 & 35.6 & 61.7 & 0.13 & 0.09 \\
& shallow & 3689 & 3.8 & inf & 64.7 & 0.13 & 0.09 \\
& deep & 873 & 15.8 & 36.5 & 49.2 & 0.13 & 0.07 \\
\hline \hline ABUR & all & 0 & & & & & \\
& shallow & 0 & & & & & \\
& deep & 0 & & & & & \\
\hline ASHAW & all & 97 & 32.1 & 15.8 & 37.4 & 0.15 & 0.09 \\
& shallow & 76 & 33.0 & 19.3 & 40.3 & 0.14 & 0.10 \\
& deep & 16 & 25.8 & 26.6 & 28.3 & 0.19 & 0.03 \\
\hline ATKR & all & 71 & -62.8 & inf & 49.2 & 0.07 & 0.03 \\
& shallow & 66 & -58.9 & 26.1 & 44.1 & 0.07 & 0.02 \\
& deep & 2 & 42.0 & 0.0 & 0.0 & 0.15 & 0.02 \\
\hline BALD & all & 51 & 15.1 & 28.4 & 43.8 & 0.13 & 0.06 \\
& shallow & 40 & 9.1 & 24.3 & 39.4 & 0.14 & 0.06 \\
& deep & 9 & 46.5 & inf & 46.0 & 0.05 & 0.00 \\
\hline BLYT & all & 96 & -14.6 & 13.8 & 31.6 & 0.05 & 0.03 \\
\hline
\end{tabular}


Table 4.3 - continued from previous page

\begin{tabular}{|c|c|c|c|c|c|c|c|}
\hline $\begin{array}{l}\text { Station } \\
\text { Name }\end{array}$ & & $\begin{array}{l}\text { SWS } \\
\text { measurements }\end{array}$ & $\begin{array}{l}\text { Mean } \\
\phi\left(^{\circ}\right)\end{array}$ & $\begin{array}{l}\text { Standard } \\
\text { Error }\left(^{\circ}\right)\end{array}$ & $\begin{array}{l}\text { Standard } \\
\text { Deviation } \\
\left(^{\circ}\right)\end{array}$ & $\begin{array}{l}\text { Mean } \\
\delta \mathrm{t} \\
(\mathrm{sec})\end{array}$ & $\begin{array}{l}\text { Standard } \\
\text { Deviation } \\
\text { (sec) }\end{array}$ \\
\hline & $\begin{array}{l}\text { shallow } \\
\text { deep }\end{array}$ & $\begin{array}{l}76 \\
17\end{array}$ & $\begin{array}{l}-14.5 \\
-7.9\end{array}$ & $\begin{array}{l}13.3 \\
\text { inf }\end{array}$ & $\begin{array}{l}28.4 \\
49.0\end{array}$ & $\begin{array}{l}0.05 \\
0.09\end{array}$ & $\begin{array}{l}0.03 \\
0.00\end{array}$ \\
\hline HOR2 & $\begin{array}{l}\text { all } \\
\text { shallow } \\
\text { deep }\end{array}$ & $\begin{array}{l}101 \\
82 \\
10\end{array}$ & $\begin{array}{l}2.6 \\
4.3 \\
-7.6\end{array}$ & $\begin{array}{l}18.4 \\
19.4 \\
\text { inf }\end{array}$ & $\begin{array}{l}32.4 \\
31.7 \\
31.7\end{array}$ & $\begin{array}{l}0.13 \\
0.13 \\
0.10\end{array}$ & $\begin{array}{l}0.05 \\
0.05 \\
0.00\end{array}$ \\
\hline KBEL & $\begin{array}{l}\text { all } \\
\text { shallow } \\
\text { deep }\end{array}$ & $\begin{array}{l}1 \\
0 \\
1\end{array}$ & $\begin{array}{l}48.0 \\
48.0\end{array}$ & $\begin{array}{l}0.0 \\
0.0\end{array}$ & $\begin{array}{l}0.0 \\
0.0\end{array}$ & $\begin{array}{l}0.00 \\
0.00\end{array}$ & $\begin{array}{l}0.00 \\
0.00\end{array}$ \\
\hline MAKA & $\begin{array}{l}\text { all } \\
\text { shallow } \\
\text { deep }\end{array}$ & $\begin{array}{l}0 \\
0 \\
0\end{array}$ & & & & & \\
\hline MASHUT & $\begin{array}{l}\text { all } \\
\text { shallow } \\
\text { deep }\end{array}$ & $\begin{array}{l}0 \\
0 \\
0\end{array}$ & & & & & \\
\hline MOA & $\begin{array}{l}\text { all } \\
\text { shallow } \\
\text { deep }\end{array}$ & $\begin{array}{l}4 \\
3 \\
1\end{array}$ & $\begin{array}{l}39.9 \\
40.2 \\
39.0\end{array}$ & $\begin{array}{l}\text { inf } \\
\text { inf } \\
\text { inf }\end{array}$ & $\begin{array}{l}31.7 \\
35.3\end{array}$ & $\begin{array}{l}0.07 \\
0.07 \\
0.07\end{array}$ & $\begin{array}{l}0.00 \\
0.00\end{array}$ \\
\hline MOUNDS & $\begin{array}{l}\text { all } \\
\text { shallow } \\
\text { deep }\end{array}$ & $\begin{array}{l}98 \\
85 \\
8\end{array}$ & $\begin{array}{l}83.4 \\
80.8 \\
-23.6\end{array}$ & $\begin{array}{l}25.2 \\
10.9 \\
\text { inf }\end{array}$ & $\begin{array}{l}31.6 \\
29.2 \\
42.5\end{array}$ & $\begin{array}{l}0.07 \\
0.05 \\
0.15\end{array}$ & $\begin{array}{l}0.06 \\
0.03 \\
0.00\end{array}$ \\
\hline PEN2 & $\begin{array}{l}\text { all } \\
\text { shallow } \\
\text { deep }\end{array}$ & $\begin{array}{l}175 \\
121 \\
37\end{array}$ & $\begin{array}{l}-13.0 \\
-11.5 \\
-17.5\end{array}$ & $\begin{array}{l}12.7 \\
13.6 \\
32.8\end{array}$ & $\begin{array}{l}39.4 \\
36.7 \\
40.3\end{array}$ & $\begin{array}{l}0.12 \\
0.13 \\
0.14\end{array}$ & $\begin{array}{l}0.06 \\
0.05 \\
0.03\end{array}$ \\
\hline TONG & $\begin{array}{l}\text { all } \\
\text { shallow } \\
\text { deep }\end{array}$ & $\begin{array}{l}84 \\
67 \\
10\end{array}$ & $\begin{array}{l}21.1 \\
20.1 \\
28.0\end{array}$ & $\begin{array}{l}12.0 \\
14.4 \\
27.6\end{array}$ & $\begin{array}{l}34.1 \\
35.2 \\
25.4\end{array}$ & $\begin{array}{l}0.20 \\
0.18 \\
0.31\end{array}$ & $\begin{array}{l}0.08 \\
0.09 \\
0.03\end{array}$ \\
\hline UP48 & $\begin{array}{l}\text { all } \\
\text { shallow } \\
\text { deep }\end{array}$ & $\begin{array}{l}126 \\
98 \\
17\end{array}$ & $\begin{array}{l}52.4 \\
64.5 \\
37.0\end{array}$ & $\begin{array}{l}41.7 \\
\text { inf } \\
18.0\end{array}$ & $\begin{array}{l}50.7 \\
52.7 \\
26.3\end{array}$ & $\begin{array}{l}0.12 \\
0.12 \\
0.06\end{array}$ & $\begin{array}{l}0.06 \\
0.06 \\
0.00\end{array}$ \\
\hline WAHI & $\begin{array}{l}\text { all } \\
\text { shallow } \\
\text { deep }\end{array}$ & $\begin{array}{l}174 \\
132 \\
29\end{array}$ & $\begin{array}{l}36.5 \\
35.8 \\
39.1\end{array}$ & $\begin{array}{l}30.4 \\
\text { inf } \\
16.5\end{array}$ & $\begin{array}{l}49.3 \\
61.6 \\
23.6\end{array}$ & $\begin{array}{l}0.17 \\
0.16 \\
0.24\end{array}$ & $\begin{array}{l}0.09 \\
0.08 \\
0.01\end{array}$ \\
\hline WHAKIN & $\begin{array}{l}\text { all } \\
\text { shallow } \\
\text { deep }\end{array}$ & $\begin{array}{l}96 \\
80 \\
10 \\
\end{array}$ & $\begin{array}{l}14.1 \\
11.5 \\
40.7 \\
\end{array}$ & $\begin{array}{l}24.7 \\
28.1\end{array}$ & $\begin{array}{l}42.5 \\
42.5 \\
45.8 \\
\end{array}$ & $\begin{array}{l}0.15 \\
0.15 \\
0.19 \\
\end{array}$ & $\begin{array}{l}0.09 \\
0.09 \\
0.11 \\
\end{array}$ \\
\hline$\overline{\mathrm{COVZ}}$ & $\begin{array}{l}\text { all } \\
\text { shallow } \\
\text { deep }\end{array}$ & $\begin{array}{l}0 \\
0 \\
0\end{array}$ & & & & & \\
\hline DRZ & $\begin{array}{l}\text { all } \\
\text { shallow } \\
\text { deep }\end{array}$ & $\begin{array}{l}80 \\
57 \\
13\end{array}$ & $\begin{array}{l}38.9 \\
43.8 \\
39.1\end{array}$ & $\begin{array}{l}\text { inf } \\
\text { inf } \\
\text { inf }\end{array}$ & $\begin{array}{l}53.7 \\
56.7 \\
36.6\end{array}$ & $\begin{array}{l}0.21 \\
0.19 \\
0.21\end{array}$ & $\begin{array}{l}0.00 \\
0.06 \\
0.00\end{array}$ \\
\hline FWVZ & $\begin{array}{l}\text { all } \\
\text { shallow } \\
\text { deep }\end{array}$ & $\begin{array}{l}284 \\
196 \\
41\end{array}$ & $\begin{array}{l}-18.5 \\
-29.4 \\
44.0\end{array}$ & $\begin{array}{l}22.1 \\
19.5 \\
\text { inf }\end{array}$ & $\begin{array}{l}51.6 \\
47.1 \\
52.9\end{array}$ & $\begin{array}{l}0.12 \\
0.12 \\
0.15\end{array}$ & $\begin{array}{l}0.08 \\
0.08 \\
0.07\end{array}$ \\
\hline KRVZ & $\begin{array}{l}\text { all } \\
\text { shallow } \\
\text { deep }\end{array}$ & $\begin{array}{l}158 \\
130 \\
13\end{array}$ & $\begin{array}{l}-37.6 \\
-41.4 \\
-7.8\end{array}$ & $\begin{array}{l}\text { inf } \\
\text { inf } \\
\text { inf }\end{array}$ & $\begin{array}{l}64.6 \\
59.7 \\
41.6\end{array}$ & $\begin{array}{l}0.35 \\
0.35 \\
0.32\end{array}$ & $\begin{array}{l}0.05 \\
0.05 \\
0.09\end{array}$ \\
\hline MOVZ & $\begin{array}{l}\text { all } \\
\text { shallow } \\
\text { deep }\end{array}$ & $\begin{array}{l}262 \\
143 \\
74\end{array}$ & $\begin{array}{l}6.2 \\
7.2 \\
1.1\end{array}$ & $\begin{array}{l}9.5 \\
11.7 \\
9.1\end{array}$ & $\begin{array}{l}34.3 \\
35.1 \\
20.1\end{array}$ & $\begin{array}{l}0.13 \\
0.15 \\
0.07\end{array}$ & $\begin{array}{l}0.08 \\
0.09 \\
0.01\end{array}$ \\
\hline \multicolumn{8}{|c|}{ Continued on next page } \\
\hline
\end{tabular}


Table 4.3 - continued from previous page

\begin{tabular}{|c|c|c|c|c|c|c|c|}
\hline $\begin{array}{l}\text { Station } \\
\text { Name }\end{array}$ & & $\begin{array}{l}\text { SWS } \\
\text { measurements }\end{array}$ & $\begin{array}{l}\text { Mean } \\
\phi\left(^{\circ}\right)\end{array}$ & $\begin{array}{l}\text { Standard } \\
\text { Error }\left(^{\circ}\right)\end{array}$ & $\begin{array}{l}\text { Standard } \\
\text { Deviation } \\
\left({ }^{\circ}\right)\end{array}$ & $\begin{array}{l}\text { Mean } \\
\delta \mathrm{t} \\
(\mathrm{sec})\end{array}$ & $\begin{array}{l}\text { Standard } \\
\text { Deviation } \\
(\mathrm{sec})\end{array}$ \\
\hline MTVZ & $\begin{array}{l}\text { all } \\
\text { shallow } \\
\text { deep }\end{array}$ & $\begin{array}{l}275 \\
185 \\
43\end{array}$ & $\begin{array}{l}-80.1 \\
-71.3 \\
22.4\end{array}$ & $\begin{array}{l}35.5 \\
28.7 \\
\text { inf }\end{array}$ & $\begin{array}{l}54.7 \\
49.8 \\
54.3\end{array}$ & $\begin{array}{l}0.11 \\
0.10 \\
0.11\end{array}$ & $\begin{array}{l}0.02 \\
0.03 \\
0.01\end{array}$ \\
\hline NGZ & $\begin{array}{l}\text { all } \\
\text { shallow } \\
\text { deep }\end{array}$ & $\begin{array}{l}230 \\
154 \\
33 \\
\end{array}$ & $\begin{array}{l}-25.1 \\
-57.9 \\
-7.4\end{array}$ & $\begin{array}{l}36.9 \\
\text { inf } \\
\text { inf }\end{array}$ & $\begin{array}{l}52.2 \\
65.3 \\
49.6 \\
\end{array}$ & $\begin{array}{l}0.18 \\
0.20 \\
0.17\end{array}$ & $\begin{array}{l}0.11 \\
0.13 \\
0.00\end{array}$ \\
\hline OTVZ & $\begin{array}{l}\text { all } \\
\text { shallow } \\
\text { deep }\end{array}$ & $\begin{array}{l}353 \\
262 \\
44 \\
\end{array}$ & $\begin{array}{l}-81.0 \\
-75.7 \\
84.3 \\
\end{array}$ & $\begin{array}{l}7.3 \\
8.1 \\
21.9 \\
\end{array}$ & $\begin{array}{l}34.9 \\
33.8 \\
36.4 \\
\end{array}$ & $\begin{array}{l}0.14 \\
0.14 \\
0.11 \\
\end{array}$ & $\begin{array}{l}0.04 \\
0.04 \\
0.00 \\
\end{array}$ \\
\hline PKVZ & $\begin{array}{l}\text { all } \\
\text { shallow } \\
\text { deep }\end{array}$ & $\begin{array}{l}172 \\
100 \\
53\end{array}$ & $\begin{array}{l}63.5 \\
72.2 \\
40.4\end{array}$ & $\begin{array}{l}11.9 \\
12.7 \\
27.3\end{array}$ & $\begin{array}{l}38.6 \\
34.3 \\
40.4\end{array}$ & $\begin{array}{l}0.11 \\
0.14 \\
0.17\end{array}$ & $\begin{array}{l}0.07 \\
0.07 \\
0.06\end{array}$ \\
\hline TRVZ & $\begin{array}{l}\text { all } \\
\text { shallow } \\
\text { deep }\end{array}$ & $\begin{array}{l}205 \\
134 \\
37 \\
\end{array}$ & $\begin{array}{l}-2.5 \\
-2.4 \\
-4.8 \\
\end{array}$ & $\begin{array}{l}10.1 \\
11.3 \\
\text { inf } \\
\end{array}$ & $\begin{array}{l}39.3 \\
37.7 \\
48.7 \\
\end{array}$ & $\begin{array}{l}0.21 \\
0.21 \\
0.21\end{array}$ & $\begin{array}{l}0.15 \\
0.15 \\
0.07\end{array}$ \\
\hline TUVZ & $\begin{array}{l}\text { all } \\
\text { shallow } \\
\text { deep }\end{array}$ & $\begin{array}{l}173 \\
120 \\
27\end{array}$ & $\begin{array}{l}36.0 \\
32.1 \\
37.3\end{array}$ & $\begin{array}{l}14.3 \\
16.8 \\
27.3\end{array}$ & $\begin{array}{l}39.3 \\
39.5 \\
35.6\end{array}$ & $\begin{array}{l}0.21 \\
0.23 \\
0.18\end{array}$ & $\begin{array}{l}0.08 \\
0.09 \\
0.06\end{array}$ \\
\hline TWVZ & $\begin{array}{l}\text { all } \\
\text { shallow } \\
\text { deep }\end{array}$ & $\begin{array}{l}186 \\
121 \\
50 \\
\end{array}$ & $\begin{array}{l}-26.3 \\
-34.3 \\
-16.2 \\
\end{array}$ & $\begin{array}{l}24.8 \\
\text { inf } \\
23.9 \\
\end{array}$ & $\begin{array}{l}49.5 \\
54.0 \\
37.5 \\
\end{array}$ & $\begin{array}{l}0.10 \\
0.04 \\
0.09 \\
\end{array}$ & $\begin{array}{l}0.09 \\
0.02 \\
0.00 \\
\end{array}$ \\
\hline WNVZ & $\begin{array}{l}\text { all } \\
\text { shallow } \\
\text { deep }\end{array}$ & $\begin{array}{l}238 \\
161 \\
44 \\
\end{array}$ & $\begin{array}{l}1.5 \\
0.8 \\
5.1\end{array}$ & $\begin{array}{l}10.4 \\
9.8 \\
30.8 \\
\end{array}$ & $\begin{array}{l}39.1 \\
37.2 \\
40.7\end{array}$ & $\begin{array}{l}0.10 \\
0.10 \\
0.21\end{array}$ & $\begin{array}{l}0.04 \\
0.04 \\
0.09\end{array}$ \\
\hline WPVZ & $\begin{array}{l}\text { all } \\
\text { shallow } \\
\text { deep }\end{array}$ & $\begin{array}{l}352 \\
266 \\
36\end{array}$ & $\begin{array}{l}-20.9 \\
-21.1 \\
-25.1\end{array}$ & $\begin{array}{l}40.5 \\
\text { inf } \\
24.5\end{array}$ & $\begin{array}{l}41.7 \\
40.1 \\
37.4\end{array}$ & $\begin{array}{l}0.14 \\
0.14 \\
0.12\end{array}$ & $\begin{array}{l}0.08 \\
0.08 \\
0.03\end{array}$ \\
\hline WTVZ & $\begin{array}{l}\text { all } \\
\text { shallow } \\
\text { deep }\end{array}$ & $\begin{array}{l}357 \\
284 \\
50\end{array}$ & $\begin{array}{l}63.0 \\
70.0 \\
9.6\end{array}$ & $\begin{array}{l}30.7 \\
29.0 \\
31.1\end{array}$ & $\begin{array}{l}50.4 \\
48.0 \\
43.6\end{array}$ & $\begin{array}{l}0.20 \\
0.20 \\
0.22\end{array}$ & $\begin{array}{l}0.09 \\
0.09 \\
0.07\end{array}$ \\
\hline NGA4 & $\begin{array}{l}\text { all } \\
\text { shallow } \\
\text { deep }\end{array}$ & $\begin{array}{l}16 \\
16 \\
0\end{array}$ & $\begin{array}{l}-4.5 \\
-4.5\end{array}$ & $\begin{array}{l}\text { inf } \\
\text { inf }\end{array}$ & $\begin{array}{l}38.5 \\
38.5\end{array}$ & $\begin{array}{l}0.27 \\
0.27\end{array}$ & $\begin{array}{l}0.00 \\
0.00\end{array}$ \\
\hline NGA5 & $\begin{array}{l}\text { all } \\
\text { shallow } \\
\text { deep }\end{array}$ & $\begin{array}{l}14 \\
10 \\
2 \\
\end{array}$ & $\begin{array}{l}-17.2 \\
-18.9 \\
19.2 \\
\end{array}$ & $\begin{array}{l}29.7 \\
44.5 \\
\text { inf } \\
\end{array}$ & $\begin{array}{l}28.4 \\
28.9 \\
20.2 \\
\end{array}$ & $\begin{array}{l}0.27 \\
0.27 \\
0.30 \\
\end{array}$ & $\begin{array}{l}0.14 \\
0.15 \\
0.01 \\
\end{array}$ \\
\hline NGA6 & $\begin{array}{l}\text { all } \\
\text { shallow } \\
\text { deep }\end{array}$ & $\begin{array}{l}28 \\
23 \\
4\end{array}$ & $\begin{array}{l}-18.7 \\
-10.6 \\
-33.8\end{array}$ & $\begin{array}{l}\text { inf } \\
\text { inf } \\
\text { inf }\end{array}$ & $\begin{array}{l}44.7 \\
45.0 \\
43.2\end{array}$ & $\begin{array}{l}0.12 \\
0.12 \\
0.05\end{array}$ & $\begin{array}{l}0.10 \\
0.10 \\
0.04\end{array}$ \\
\hline
\end{tabular}

\subsubsection{Delay Time Tomography}

In order to constrain the locations of high anisotropy, a two-dimensional tomographic inversion was conducted on the delay time $(\delta \mathrm{t})$ estimates from the shear wave splitting analysis to obtain the strength of anisotropy (the amount of splitting in seconds, measured per $\mathrm{km}$ ) in each of the grid blocks. We assume that the shear 


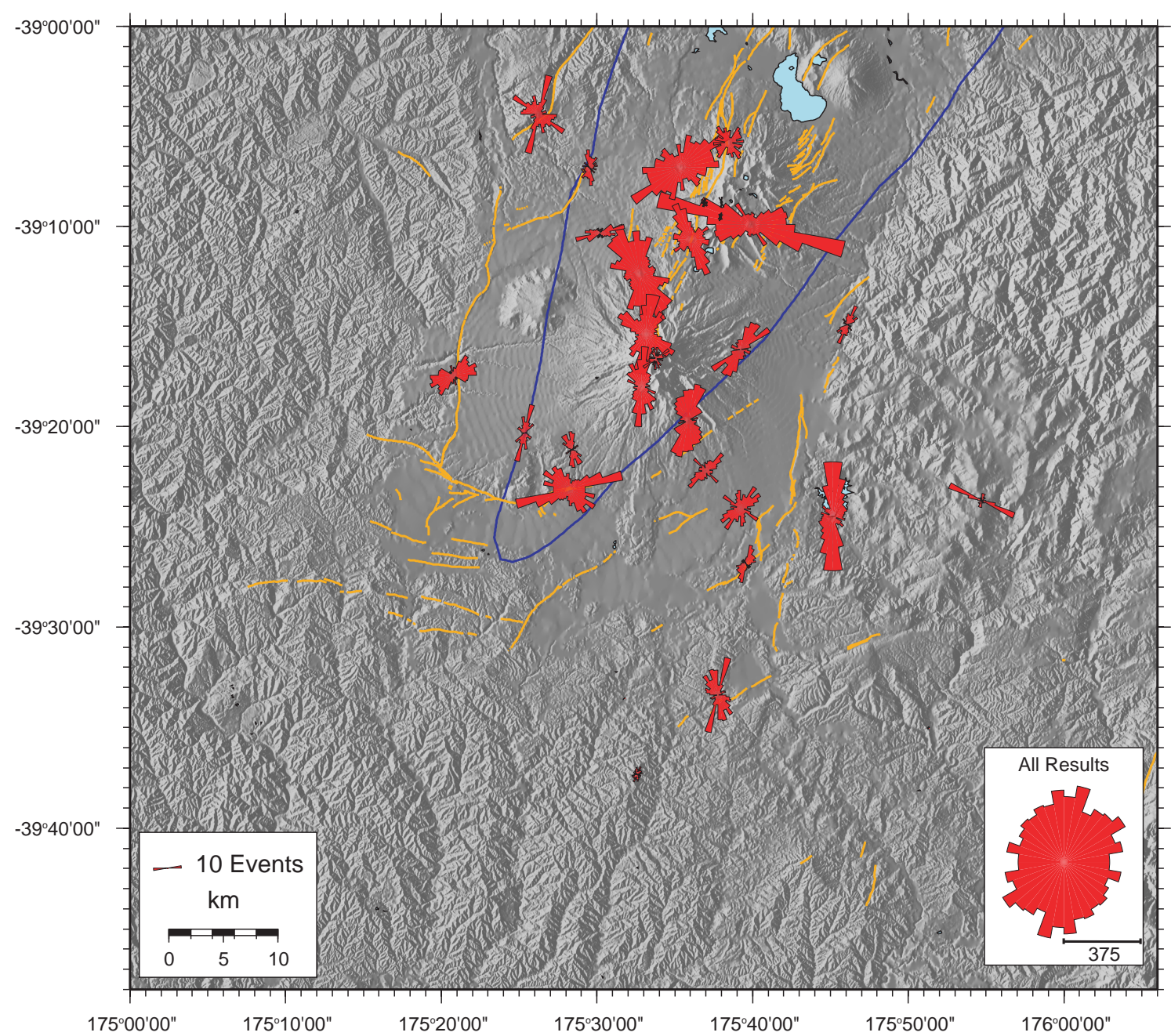

Figure 4.6. Rose diagrams of fast polarisation of split shear waves from automated method [Savage et al., 2010b]. Rose diagrams are plotted on the station at which the observations were made and are scaled according to the number of measurements so that the area of each bar, rather than the length, is proportional to the number of measurements. Numbers of measurements and statistical analyses are listed in Table 4.3. Note the bimodal forms of some of the rose diagrams. Orange lines are known faults (from the NZ Active Faults Database of GNS, after Villamor and Berryman [2006]), blue line is the outline of the TVZ (after Wilson et al. [1995]).

wave splitting delay times are accumulated along the raypath [e.g. Crampin, 1991; Zhang et al., 2007] and that the total delay time is simply the sum of the delay times for each grid block that the ray traverses.

$$
\delta \mathrm{t}_{r}=\sum_{b=1}^{n}\left(s_{b} \times L_{r b}\right)
$$

Here $\delta \mathrm{t}_{r}$ is the measured delay time of the ray $r, s_{b}$ is the strength of anisotropy per grid block, and $L_{r b}$ is the length of the raypath within each of the $n$ blocks. The assumption that $\delta \mathrm{t}$ is simply additive is a simplification of a non-linear relationship between heterogeneous anisotropy and the observed apparent $\delta \mathrm{t}$ at the surface. How- 
ever, it gives a first-order approximation to the heterogeneous anisotropic structure of regions of strong and weak anisotropy.

Other authors [Crampin, 1994; Sayers and Kachanov, 1995] have calculated crack density to characterise the strength of anisotropy, but the possibility of anisotropy in our study area being caused by effects other than fluid-filled cracks means that the anisotropy cannot be interpreted solely in terms of an idealised crack model. To accomplish the inversion, we first gridded the study region using a quadtree gridding system [Townend and Zoback, 2001, 2004] and a minimum block size of $5 \mathrm{~km}$ square, with each grid block having between 20 and 65 ray paths passing through it. These parameters were chosen to minimise the residuals from the tomographic inversion, while ensuring that each block contained enough data to give a reliable mean when the $\phi$ measurements of the rays intersecting it were averaged for the analysis in Section 4.3.3. The results obtained using variations of these parameters are listed in Table 4.4. The quadtree gridding method iteratively analyses each block; more than 65 ray paths passing through a block results in the block being subdivided further, fewer than 20 ray paths means that the block is excluded from the analysis. The gridding continues until all of the blocks fulfill these criteria by either having between 20 and 65 rays passing through them, or by having been divided until it has the minimum size of $5 \mathrm{~km}$ square. Figure 4.7 displays the rays and 145 grid blocks, 121 of which were used for the analysis. 


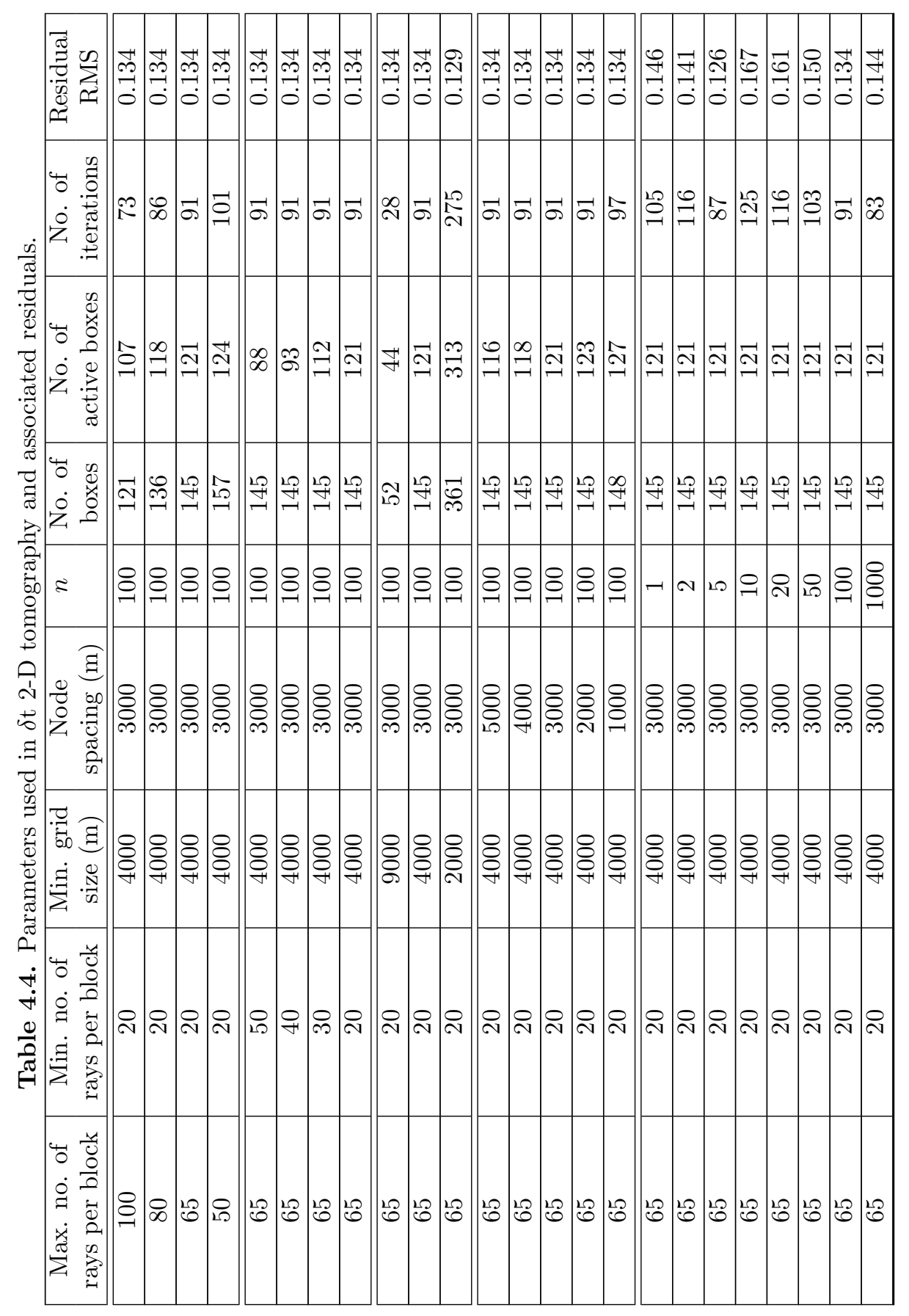




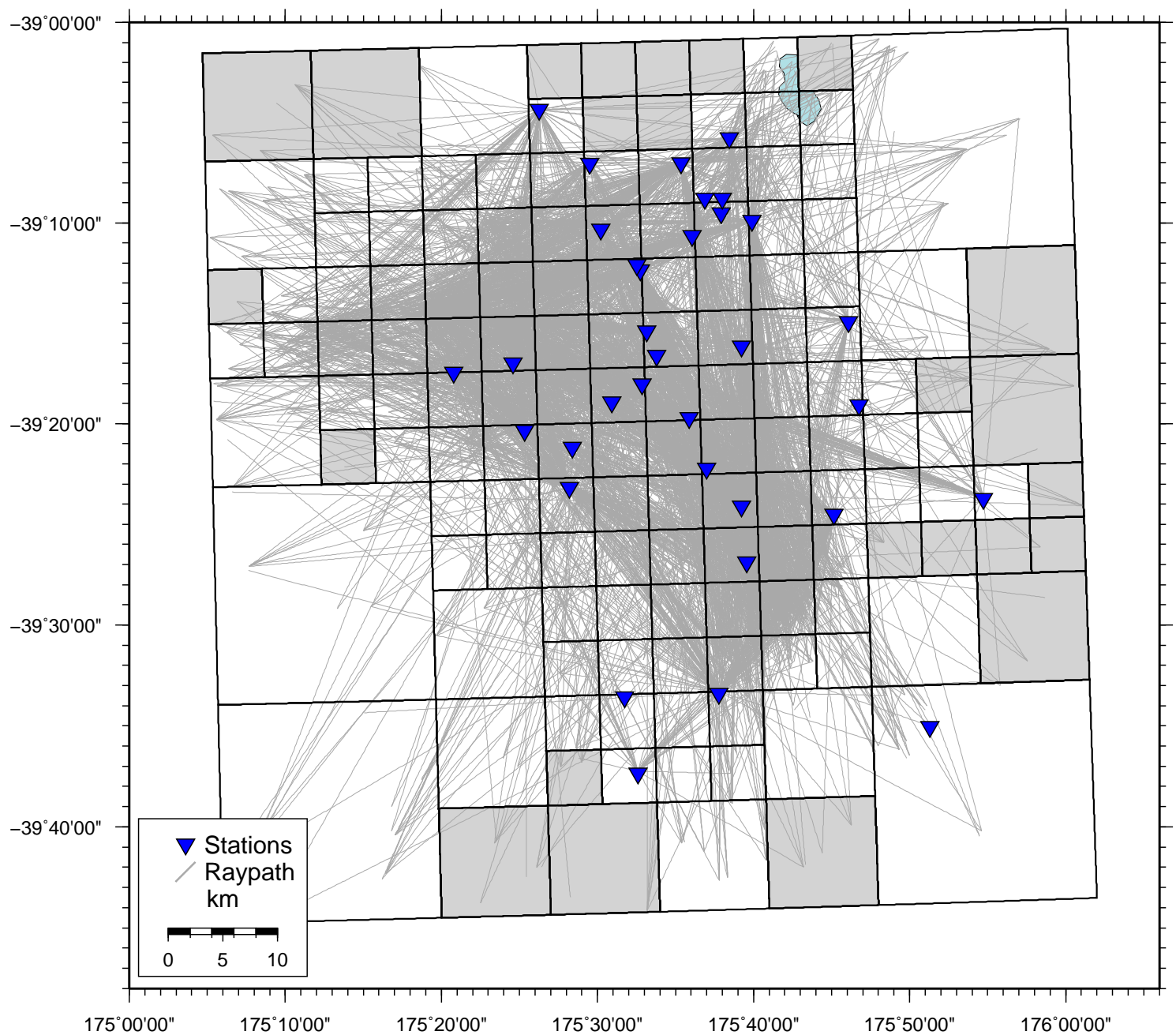

Figure 4.7. Region of analysis divided into grid blocks using quadtree gridding. Grey lines show raypaths, blue inverted triangles are seismic stations. Criteria for gridding were that there be between 65 and 20 raypaths passing through each box, with a minimum box size of $5 \mathrm{~km}$. This resulted in 149 blocks, 121 of which are used in the analysis. Shaded boxes show those not used. The grid is oriented at a slight angle from north because the grid was constructed using the New Zealand Map Grid (NZMG) coordinate system and plotted in latitude and longitude.

We used a medium-scale optimisation inversion function (lsqlin) in MATLAB, which uses an active set method similar to that described by Gill et al. [1981]. This algorithm determines a feasible initial solution by first solving the linear least-square problem, then converges on a final solution iteratively subject to bounding constraints. The active set refers to the elements that remain within the boundary constraints with each iteration. The constraints were set so that the minimum strength could not be below $0 \mathrm{~s} / \mathrm{km}$ and that the maximum could not exceed the maximum $\delta$ t observed for a ray path applied to one block length, i.e. $\delta \mathrm{t}_{\max } / L_{\min (b)}$ where $L_{\min (b)}$ is the width of the smallest block from the quadtree gridding. The 
data were weighted so that the problem becomes (in matrix notation)

$$
\mathbf{G}^{T} \mathbf{C}^{-1} \underline{d}=\left(\mathbf{G}^{T} \mathbf{C}^{-1} \mathbf{G}\right) \underline{m}
$$

Here $\mathbf{G}$ is the design matrix constructed by finding the distance that each ray traverses each block, i.e. from $L_{r b} ; \underline{d}$ is a vector containing the $\delta$ t measurements of each ray; $\underline{m}$ is the model solution containing the strength of anisotropy (the amount of splitting) per block. The errors can be split into the measurement errors and

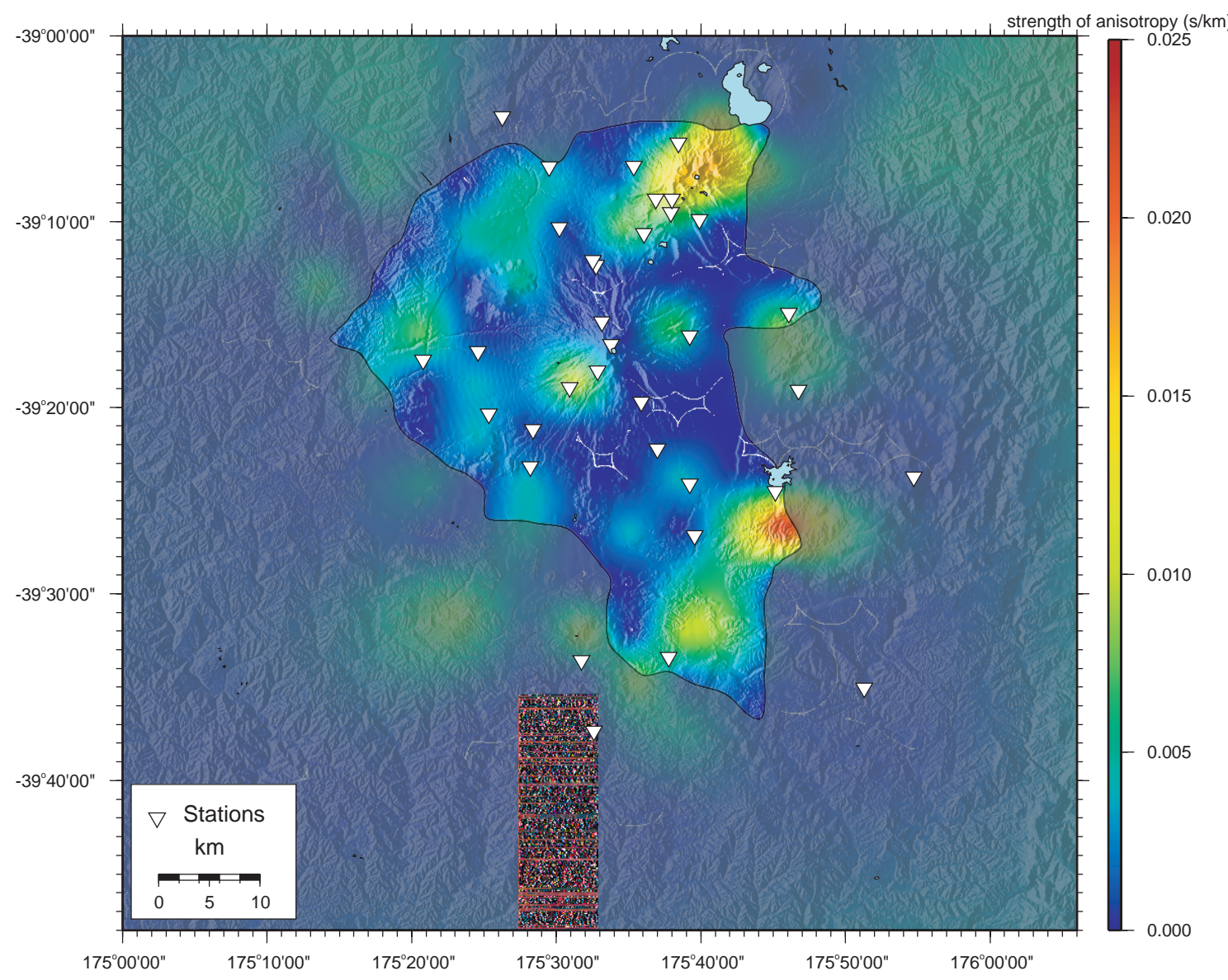

Figure 4.8. Delay time tomography from the inversion of data. Warm colours indicate strong anisotropy, shaded area shows the limit of statistical significance calculated from the model variance matrix, white inverted triangles are seismic stations.

the inversion errors, which originate from the scale of the strength variation of the model. 


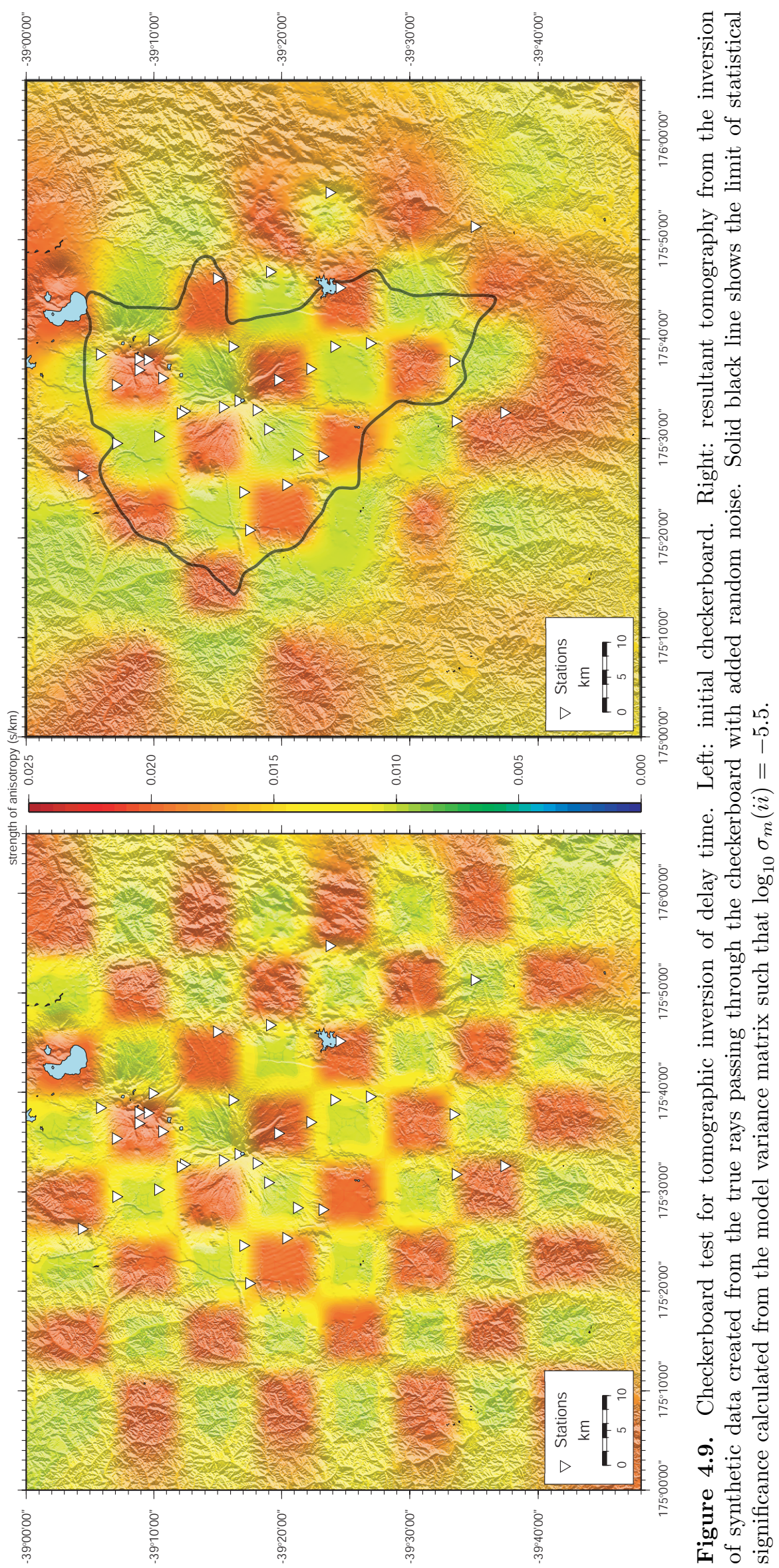


We therefore construct the error covariance matrix, $\mathbf{C}$, with diagonal components

$$
C_{i i}=\sigma_{d}^{2}(i)+L_{b}^{2} \times \tilde{\sigma}_{m}^{2} \times n_{b}(i) / n
$$

and constant off-diagonals with values:

$$
C_{i j}=\tilde{\sigma}_{m}^{2} \times L_{b}^{2} / n^{2}
$$

Here $\sigma_{d}^{2}(i)$ is the squared standard error of the $i^{t h}$ measurement, $L_{b}$ is the length scale of the boxes, $n_{b}(i)$ is the number of boxes the $i^{\text {th }}$ ray passes through and $\tilde{\sigma}_{m}^{2}$ is the mean of the variance of the result when the data are weighted as $1 / \sigma_{d}(i)$. To test the scale of the effect that each section of the ray has on other sections, $n$ is an arbitrary number of sections to further divide the portions of each ray in each box. We conducted the inversion with various values of $n$, ranging from 1 to 1000 and found the inversion results to be relatively insensitive to the weighting. The results are presented using $n=100$ and output parameters for different values of $n$ are listed in Table 4.4. We find that typical magnitudes of $\sigma_{d}(i)$ are approximately $10^{-2}-10^{-3}$ and typical magnitudes of $\sigma_{m}(i)$ are approximately $10^{-5}$. The error is therefore dominated my the measurement uncertainties, as expected. We performed a checkerboard test to assess where structures may be retrieved. The checkerboard test was carried out by constructing the checkerboard model vector $\underline{m}_{C B}$ using a regular grid with blocks given alternating anisotropy strength values of $0.01 \mathrm{~km} / \mathrm{s}$ and $0.02 \mathrm{~km} / \mathrm{s}$, and adding random noise drawn from a standard normal distribution. Synthetic $\delta$ t measurements were calculated by the linear multiplication:

$$
\underline{d}_{C B}=\mathbf{G}_{C B} \underline{m}_{C B}
$$

The inversion was then carried out using the quadtree grid as described above. Figure 4.8 displays the inversion results and Figure 4.9 displays the results from a checkerboard test.

The resolution matrix was found to be the identity matrix because the problem is over-constrained due to the design of the grid, namely that each block should have more than 20 rays passing through it in order to obtain a reliable average $\phi$ per block (see Section 4.3.3 below). The limit of statistical significance illustrated in Figure 4.8 and Figure 4.9 was constructed by finding the contour where the log of the standard deviation (square-root of the diagonal elements of the model variance matrix) was -5.5 (see Figure 4.10 for contours of $\sigma_{m}$ ). The value of $\log _{10} \sigma_{m}(i i)=-5.5$ was chosen because it encompassed most of the grid blocks with more than 20 rays, and 
included the features from the checkerboard test that were best reconstructed. It can be seen in Figure 4.10 that a different choice of cut-off would give a different limit of statistical significance and that the choice given here is a conservative estimate in that there are features of the checkerboard that are recreated outside of this limit.

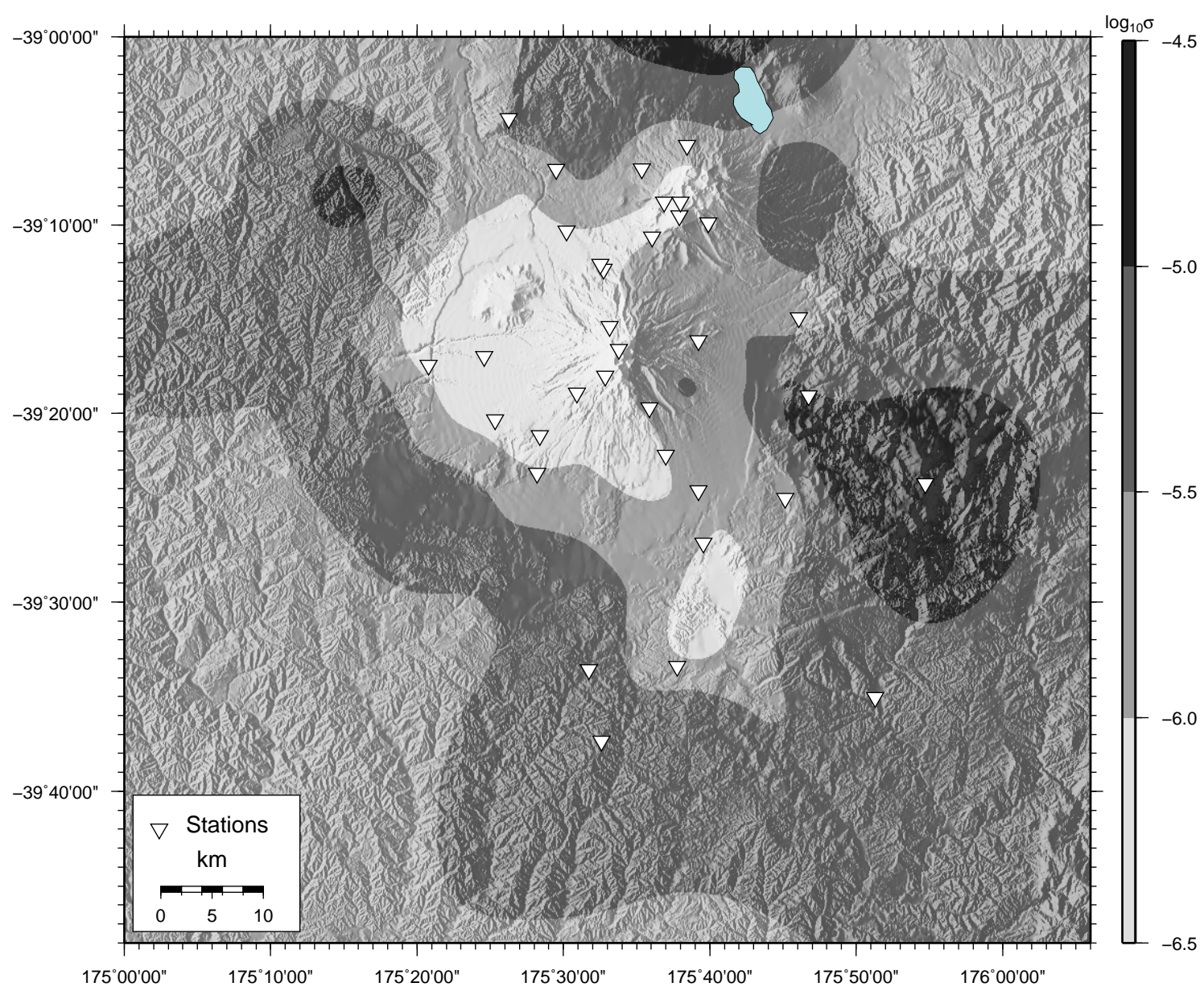

Figure 4.10. Contours of the square-root of the diagonal elements of the model variance matrix $\log _{10} \sigma_{m}(i i)$.

\subsubsection{Spatial Averaging}

We produced spatial averages of splitting parameters as a first-order approximation to the heterogeneous anisotropic structure [Audoine et al., 2004].

To construct the map of spatial averages in $\phi$, the $\phi$ values were weighted and assigned to each grid block that each ray passed through using the quadtree gridding from Section 4.3.2. The rose diagrams of the $\phi$ values are plotted and the mean direction (computed using circular statistics) from each grid block is plotted only when the standard deviation of the data is less than $30^{\circ}$ and the standard error of the 


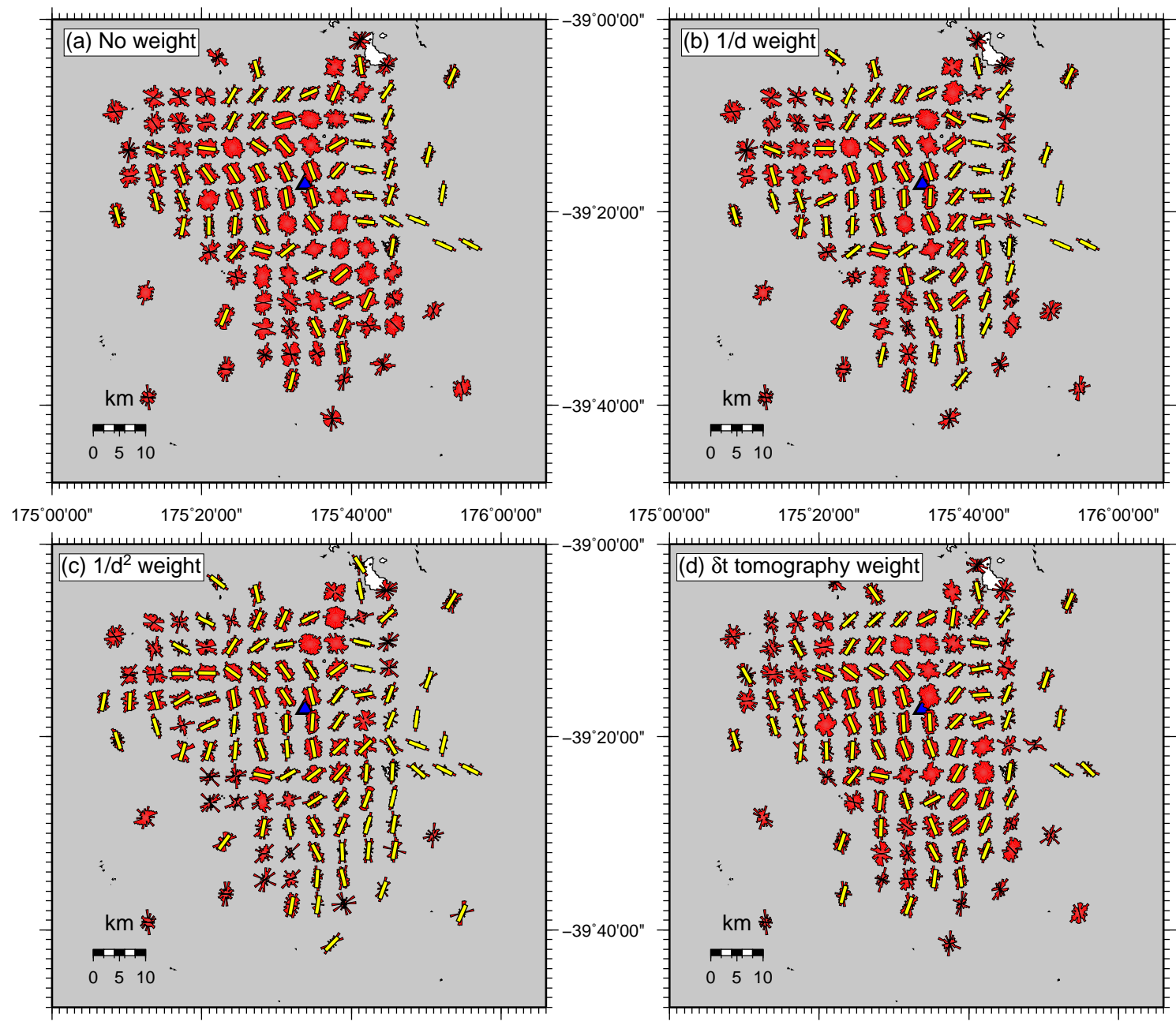

Figure 4.11. Spatial averages of fast shear wave polarisations from shear wave splitting. Red rose diagrams are normalised, yellow bars show mean polarisation. Black triangles indicate the summit of Mt. Ruapehu and yellow polygons show zones from Figure 4.2. Rose diagrams are plotted in the centre of each grid block and those grid blocks with less data than the threshold are not plotted. Grid blocks with a standard deviation larger than $30^{\circ}$ and standard error larger than $10^{\circ}$ do not have the mean plotted. (a) Spatial Average with no weighting function applied. (b) Spatial averaging with weighting inversely proportional to distance from the station. (c) Spatial averaging with weighting inversely proportional to the square of the distance from the station. (d) Spatial averaging with weighting functions along the raypaths taken from the two-dimensional tomographic inversion of delay time.

mean is less than $10^{\circ}$. These criteria enable us to eliminate those blocks exhibiting large scatter or more than one mode. Several weighting schemes were tested and are displayed in Figure 4.11; (a) corresponds to no weighting function, and (b) and (c) to the situations in which the $\phi$ values are weighted by $1 / d$ and $1 / d^{2}$, where $d$ is the distance of the grid block from the station [Audoine et al., 2004]. This weighting scheme was designed to account for the fact that the value of $\phi$ will be influenced by a greater amount by anisotropic media later in the path [Rumpker and Silver, 2000; Nistala and McMechan, 2005]. Figure 4.11 (d) displays weighting for the regions of 
high anisotropy determined by $\delta$ t tomography, described in Section 4.3.2. To obtain this weighting the anisotropy strength profile along each ray was normalised by the $\delta$ t measurement for the particular ray-path and used as the weighting function, $w$ :

$$
w_{r b}=\frac{s_{b}}{\delta t_{r}}
$$

Here $r$ signifies the passing ray and $b$ signifies the block in question so that the average fast direction for $n$ rays passing through the block, $\bar{\phi}_{b}$, is:

$$
\bar{\phi}_{b}=\tan ^{-1} 2\left(\frac{\sum_{r=1}^{n} \sin \phi_{r} \times w_{r b}}{\sum_{r=1}^{n} w_{r b}}, \frac{\sum_{r=1}^{n} \cos \phi_{r} \times w_{r b}}{\sum_{r=1}^{n} w_{r b}}\right)
$$

The spatial averaging using $1 / \mathrm{d}^{2}$ weighting (Figure 4.11 (c)) produces the least scatter and the most means with standard error and standard deviation to within the threshold values stated in Section 4.3.3. Spatial averaging using $1 / d^{2}$ weighting fuctions yielded 88 measurements within the thresholds, whereas 64 measurements were produced by averaging with no weighting function, 75 were produced with $1 / d$, and 73 were produced using the tomography weighting functions.. Therefore we will subsequently analyse the map of spatial averaging using $1 / \mathrm{d}^{2}$ weighting functions (Figure 4.12).

\subsection{Results}

\subsubsection{Shear wave splitting}

Figure 4.6 displays rose diagrams of the fast polarisations for earthquakes recorded in 2008, plotted at the station at which they were recorded and scaled according to how many measurements were obtained. Table 4.3 includes information about how many events were used at each station and Figure 4.13 displays results from just the shallow $(<30 \mathrm{~km})$ and deep $(>50 \mathrm{~km})$ earthquakes separately. The vast majority of the earthquakes fall into the shallow category and, while there are small differences between the results from the shallow and deep events at some stations, these results don't influence the overall analysis. The automatic shear wave splitting algorithm chooses a filter based on the dominant frequency of the waveforms and the bandwidth. We do not observe a systematic difference in the chosen filter as 


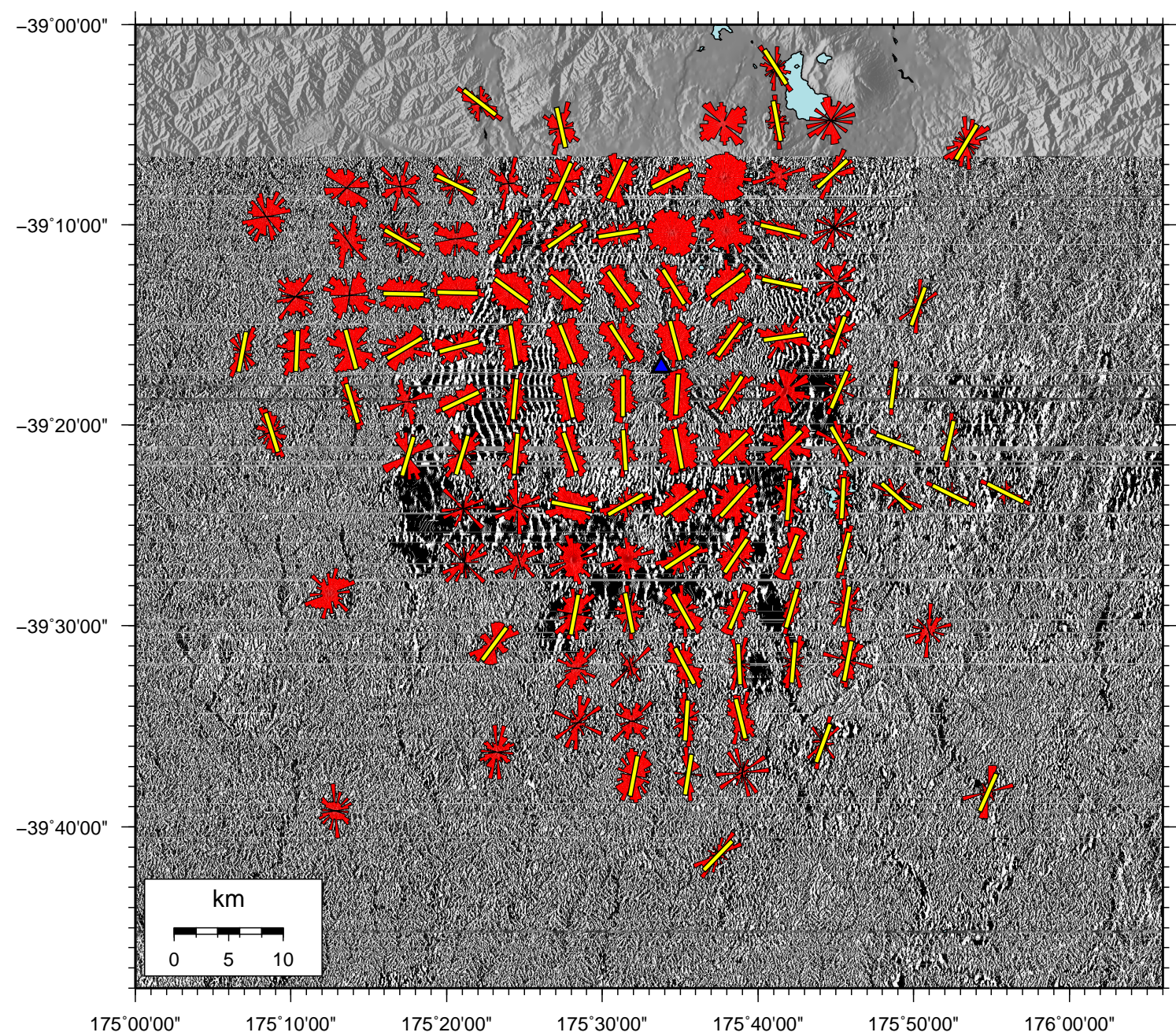

Figure 4.12. Spatial averages of fast shear wave polarisations from shear wave splitting with weighting inversely proportional to the square of the distance from the station. Red rose diagrams are normalised, yellow bars show mean polarisation. Blue triangle indicates the summit of Mt. Ruapehu. Rose diagrams are plotted in the centre of each grid block and those grid blocks with less data than the threshold are not plotted. Grid blocks with a standard deviation larger than $30^{\circ}$ and standard error larger than $10^{\circ}$ do not have the mean plotted.

a function of depth. We also do not see a systematic increase in delay time for the deep events (Figure 4.14). These observations suggest that at the frequencies chosen (predominantly 1-8 Hz) the anisotropy that we are measuring is crustal in origin, although upper mantle anisotropy could be affecting the waveforms at lower frequencies. We conclude that the following analysis benefits more from the inclusion of all of the data, thus increasing the data set, than it would from the exclusion of deep earthquakes. Therefore both deep and shallow earthquakes have been included throughout.

In order to ensure that there were no strong temporal changes in anisotropy during the SADAR experiment, we performed a temporal averaging analysis similar to that 

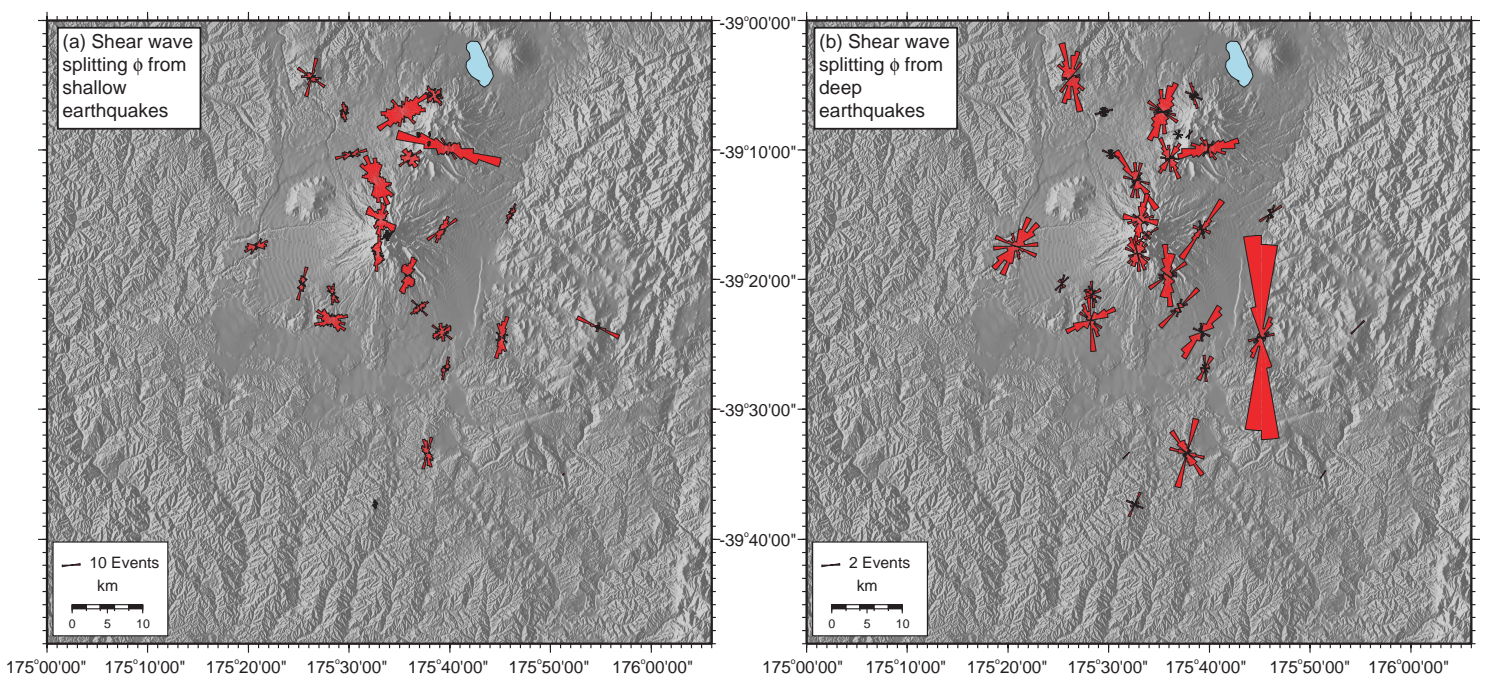

Figure 4.13. Rose diagrams of fast polarisation of split shear waves from automated method on earthquakes with hypocentres shallower than $30 \mathrm{~km}$ (a) and deeper than $50 \mathrm{~km}$ (b). Rose diagrams are plotted on the station at which the observations were made and are scaled according to the number of measurements. Note the different scales to Figure 4.6.

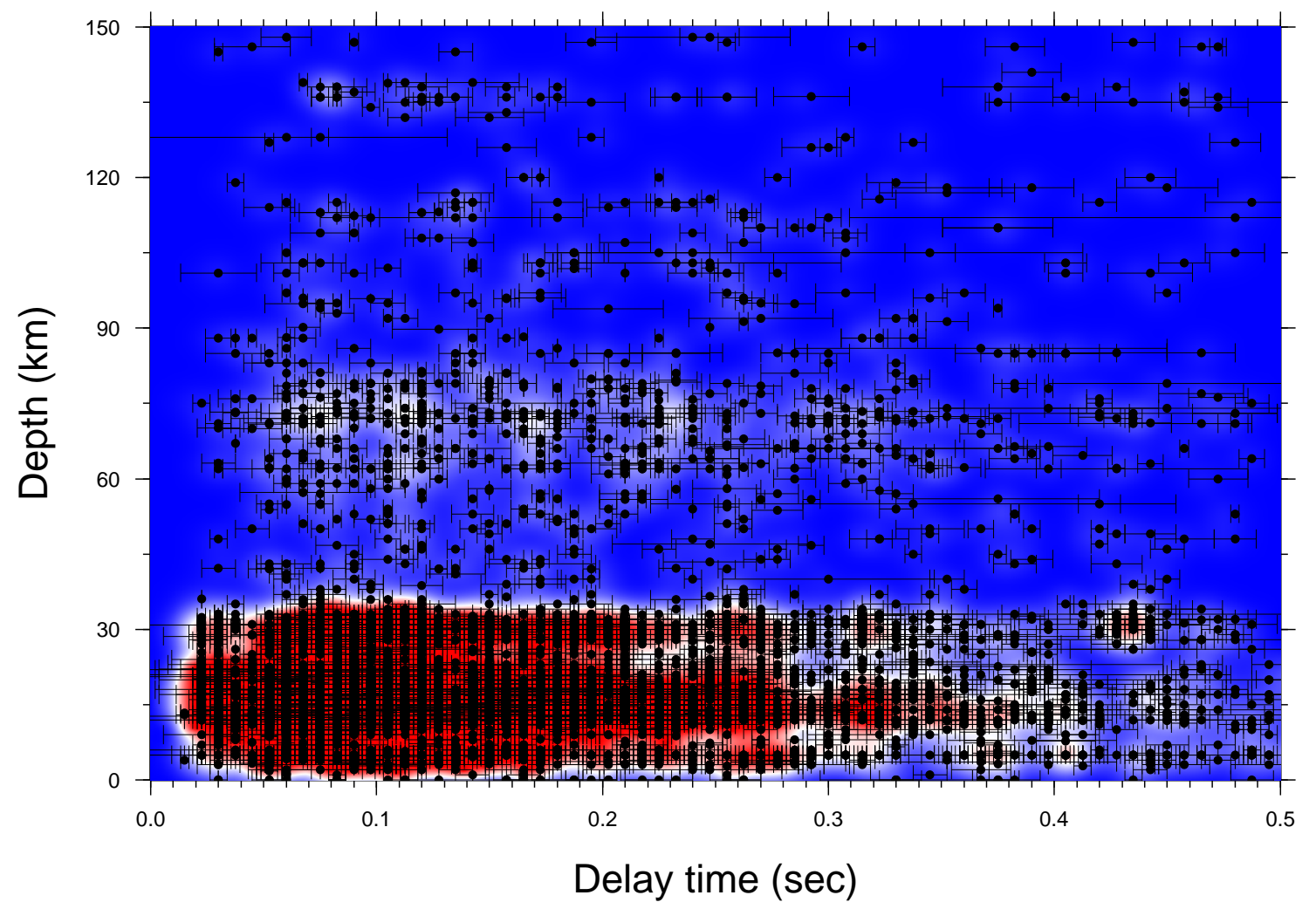

Figure 4.14. Analysis of earthquake depth with $\delta \mathrm{t}$ using all available data.

used by Savage et al. [2010a]. Figure 4.15 shows $\phi$ and $\delta$ t results throughout 2008 for a subset of the stations. Station TUVZ is on the east flank of Mt. Ruapehu and has been used in previous studies to demonstrate temporal changes in anisotropy [Gerst and Savage, 2004], in attenuation [Titzschkau et al., 2010] and in cross-correlation 


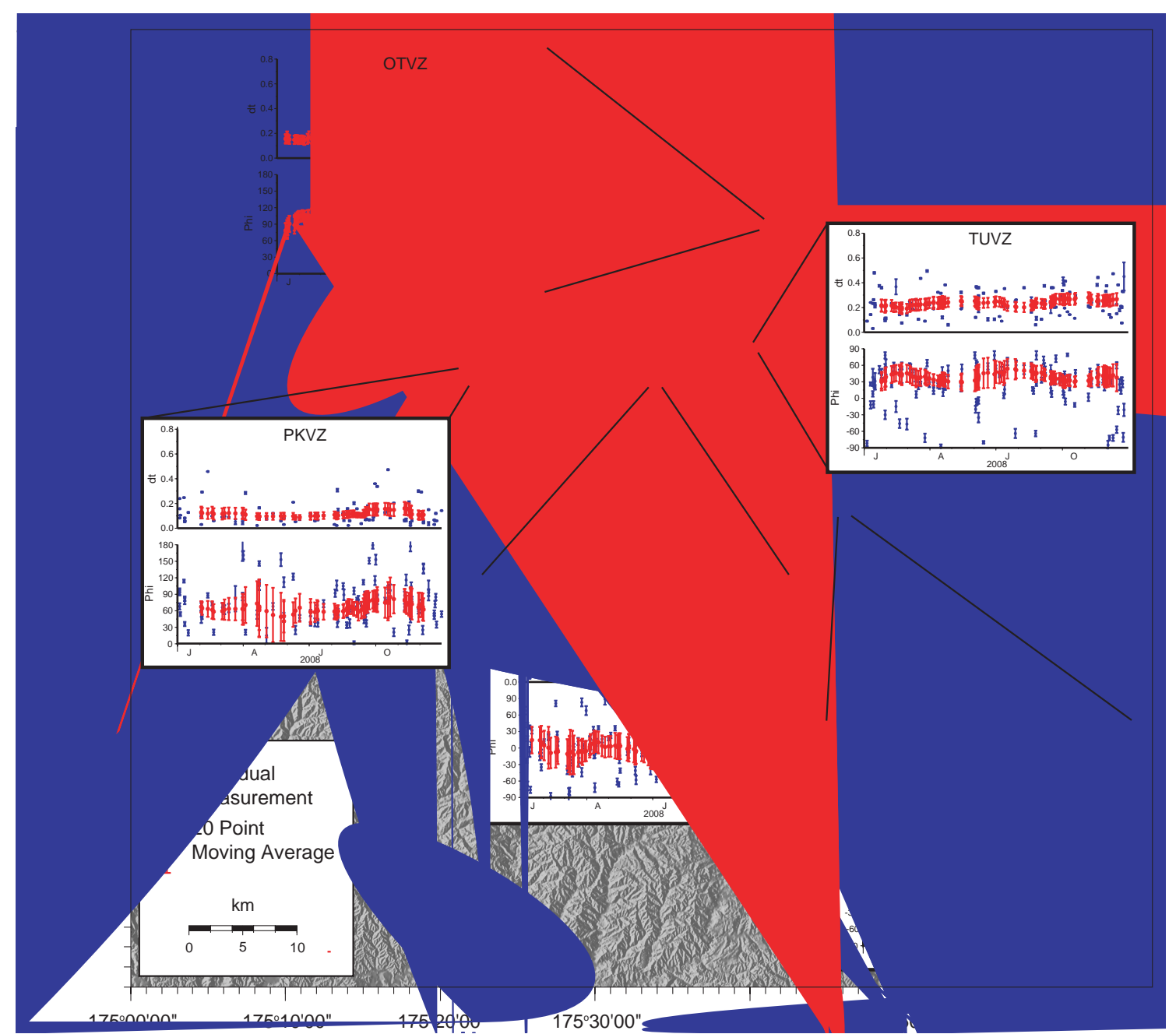

Figure 4.15. Shear wave splitting data for earthquakes throughout 2008 recorded at five stations. Scattergraphs show individual measurements (blue) with error bars indicating the $95 \%$ confidence interval for each measurement and a 20 point moving average (red) with error bars indicating the standard error of the mean. The top graph of each panel shows delay time results and the bottom graphs show fast orientation results.

functions from ambient seismic noise [Mordret et al., 2010]. Station TRVZ is on the southwest flank of the volcano, close to the summit and was also used by Gerst and Savage [2004]. Stations OTVZ, MOVZ and PKVZ are further away from the summit of Mt. Ruapehu at different azimuths from the volcano. Each of these stations is close to one of the main clusters of earthquakes displayed in Figure 4.1 but each records earthquakes from all three clusters. Figure 4.15 shows that there are small, insignificant variations in the $\phi$ and $\delta$ t averages throughout 2008 at some of the stations but that these variations are uncorrelated. We therefore treat all the data as time independent.

Some of the rose diagrams in Figure 4.6 display directions with very little scatter and one strong modal orientation. Others, however, display more scatter or bimodal 


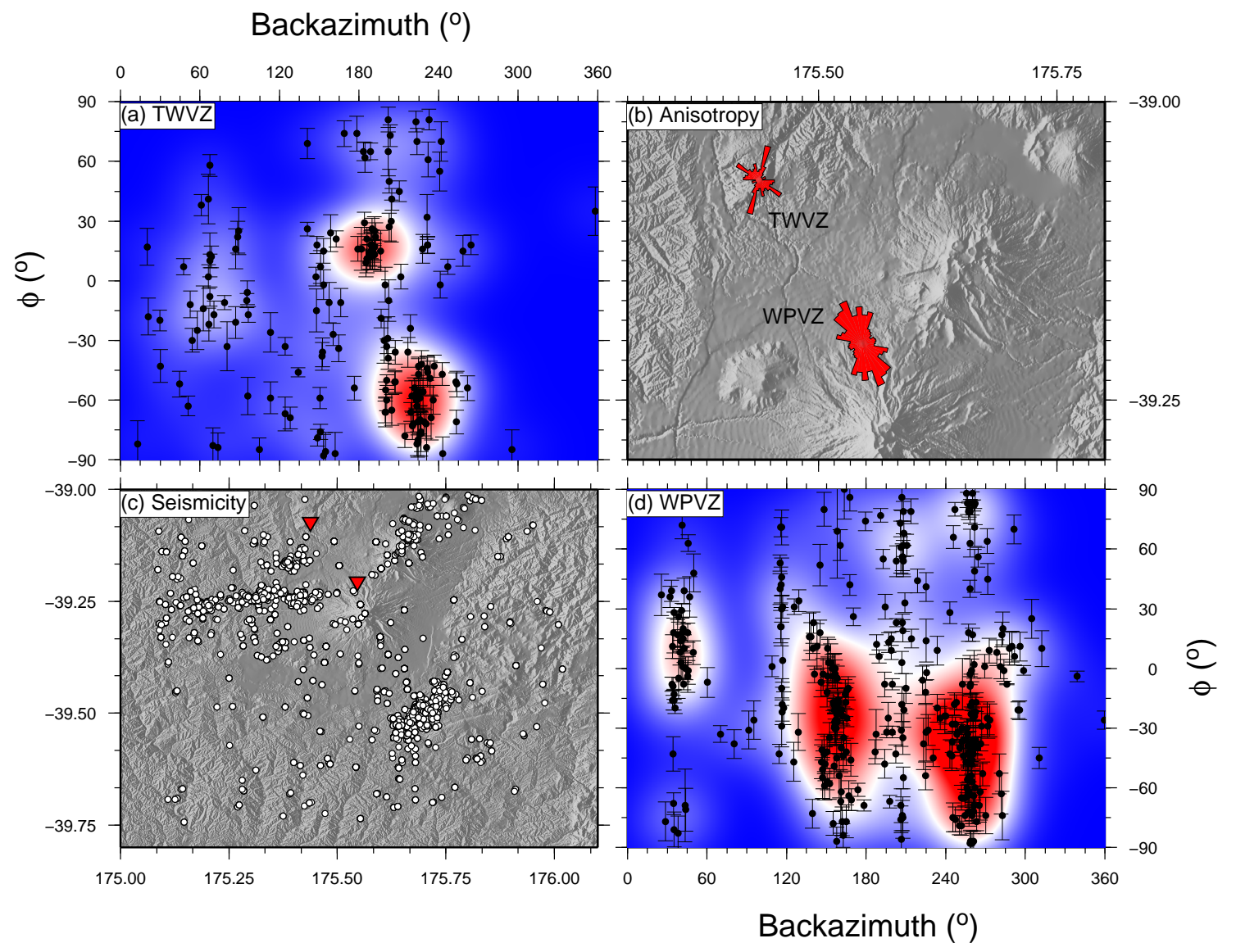

Figure 4.16. Azimuthal analysis of fast polarisation data for stations TWVZ and WPVZ. (a) Backazimuth versus fast polarisation plot for TWVZ showing three strong backazimuths giving distinct fast polarisations. Colours represent density of data points. (b) Zoom in on rose diagrams from Figure 4.6 for stations TWVZ and WPVZ, showing one bimodal and one linear rose diagram. (b) Map of the area around Mount Ruapehu (from Figure 4.1). The white circles indicate the catalogue locations of the earthquakes in 2008, used in this study. The inverted triangles represent seismometers. (d) Backazimuth versus fast polarisation plot for WPVZ showing three strong backazimuths again giving distinct fast polarisations even though these are not obvious from the rose diagram alone.

forms. Further analysis of the shear wave splitting results from station TWVZ (Figure 4.16 (a)) suggests that the two main modes of $\phi$ are caused by measurements from earthquakes with two dominant backazimuths. The two backazimuths are consistent with the distribution of seismicity displayed in Figure 4.1. There is also a third backazimuth associated with the third cluster of earthquakes that gives a discrete $\phi$, although this isn't obvious from the rose diagram. The two backazimuths from the strong orientations of $\phi$ are less then $050^{\circ}$ apart, suggesting that as the rays approach the station, they traverse similar paths. The path lengths and depths sampled by the rays further from the station are quite different, with more distant earthquakes sampling deeper crust. The two strong orientations of $\phi$ associated with these backazimuths are about $80^{\circ}$ different, suggesting the splitting occurred in the 
early part of the ray-path, further from the station. The same analysis of shear wave splitting results from station WPVZ (Figure 4.16 (d)) shows that, although the rose diagram doesn't show three clear modes, there is more than one value of $\phi$ caused by more than one backazimuth. This backazimuthal dependence suggests that the $\phi$ value obtained from shear wave splitting analysis is highly dependent upon the path that the ray takes, which has also been found in other regions by Zinke and Zoback [2000] and Liu et al. [2004]. Furthermore, this suggests that the anisotropy is not uniform throughout the crust in the study region, as is observed for mantle anisotropy [Greve et al., 2008] and that averaging $\phi$ over the whole region [Gerst and Savage, 2004] may not be appropriate. Comparison of $\phi$ and backazimuth with $\delta$ t data (Figure 4.17) indicates that the splitting delay times are also influenced by the backazimuth, suggesting that not only does the fast direction vary spatially, but the strength of anisotropy is also heterogeneous. Azimuthal analysis of $\phi$ data from all of the stations is in Figures 4.18 and 4.19. The majority of the stations display a similar pattern of $\phi$ dependence with backazimuth but some show a range of $\phi$ for a single backazimuth (e.g. at a backazimuth of $\sim 300^{\circ}$ at station ASHAW, Figure 4.18). All of the backazimuths that display this behaviour relate to seismicity from the TRL. The TRL seismicity ranges in depth from $15-35 \mathrm{~km}$ [Sherburn and White, 2005], suggesting that there is some depth dependence of shear wave splitting parameters, at least to the west of Mt. Ruapehu, that our analyses do not account for. However, the spatial averages of splitting parameters are a good firstorder approximation to the heterogeneous anisotropic structure and account for the majority of the spatial variations in observed $\phi$.

\subsubsection{Spatial Averaging}

There are some strong, but complex, patterns that are visible in the mean $\phi$ values in Figure 4.12. When all of the measurements are combined they have a mean of $013^{\circ}$ (from Figure 4.6), which correlates with most $S_{H \max }^{\text {foca }}$ estimations for the Ruapehu region (Table 4.1) and also regional anisotropy from SKS (007-066 ${ }^{\circ}$ ) and Pn studies [Greve et al., 2008; Seward et al., 2009]. This direction is sub-parallel to the trench caused by the Pacific Plate subducting beneath the Australian Plate striking at $015^{\circ}$ [Wood and Davy, 1994], and the strike of structures in the region such as the line of volcanoes that make up the TVZ and bounding faults $\left(010-030^{\circ}\right.$ Rowland and Sibson [2001]; Villamor and Berryman [2006]). However, the overall distribution does not have a single strong mode, and we observe a greater degree of heterogeneity when we examine the smaller scale detail. Only to the southeast 


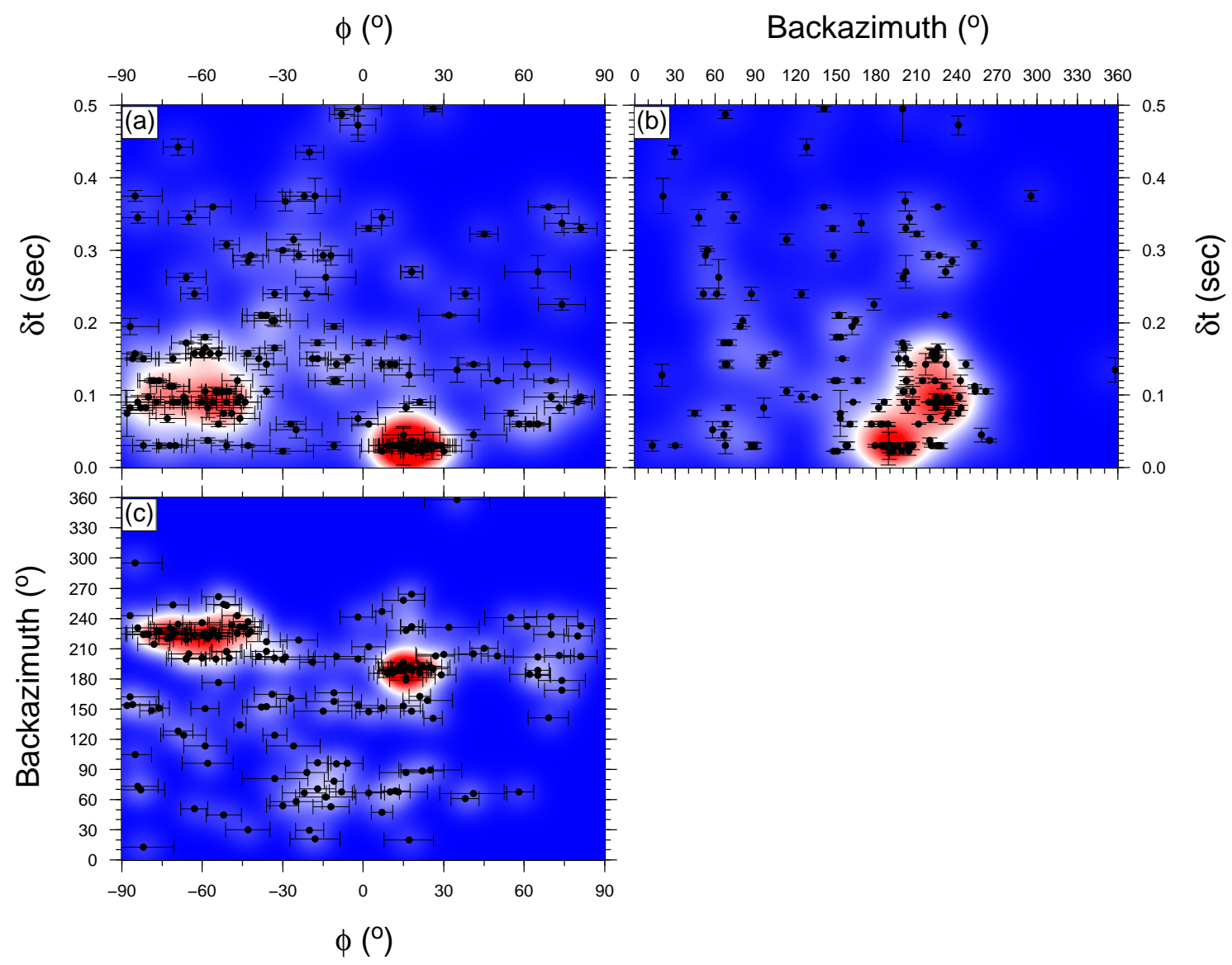

Figure 4.17. Azimuthal analysis of fast polarisation and delay time data for station TWVZ. (a) Delay time versus fast polarisation plot showing two strong delay times giving distinct fast polarisations. Colours represent density of data points. (b) Backazimuth versus delay time plot showing the two distinct delay time measurements with different backazimuths. (c) Backazimuth versus fast polarisation plot showing the same fast polarisations and backazimuths.

and northeast of the study region is the NNE-SSW trend dominant. South of Mt. Ruapehu (around $-175^{\circ} 30^{\prime}-39^{\circ} 20^{\prime}$ ) the trend is more north-south, and to the northwest of the volcano (around $-175^{\circ} 20^{\prime}-39^{\circ} 10^{\prime}$ ) the fast polarisations turn to a more east-west orientation.

\subsubsection{Delay Time Tomography}

Figure 4.8 displays the inversion results with several interesting features. There is a region of high anisotropy to the northeast of Mt. Ruapehu, which coincides with the location of other volcanoes: Mt. Ngauruhoe and Mt. Tongariro. The region immediately surrounding Mt. Ruapehu does not exhibit high anisotropy. There is a region of high anisotropy to the southeast of the study area, which corresponds to the persistently active Waiouru swarm (Figure 4.1) [Hayes et al., 2004]. The Waiouru 


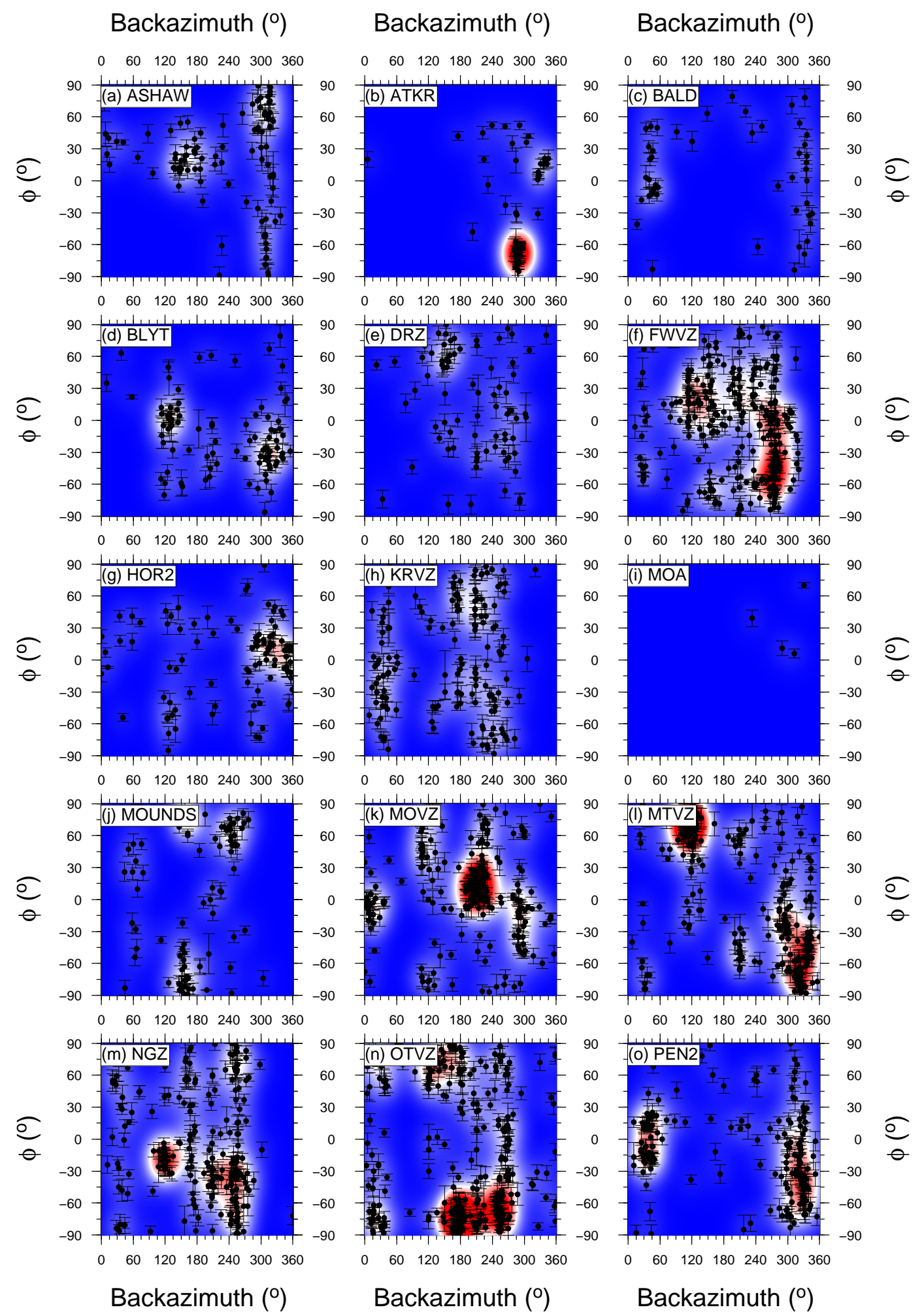

Figure 4.18. Azimuthal analysis of fast polarisation data for all stations. Colours represent density of data points.

swarm is a linear feature, which does not have many seismometers, nor earthquakes on its southeast side, resulting in the blocks encompassing the Waiouru swarm ap- 


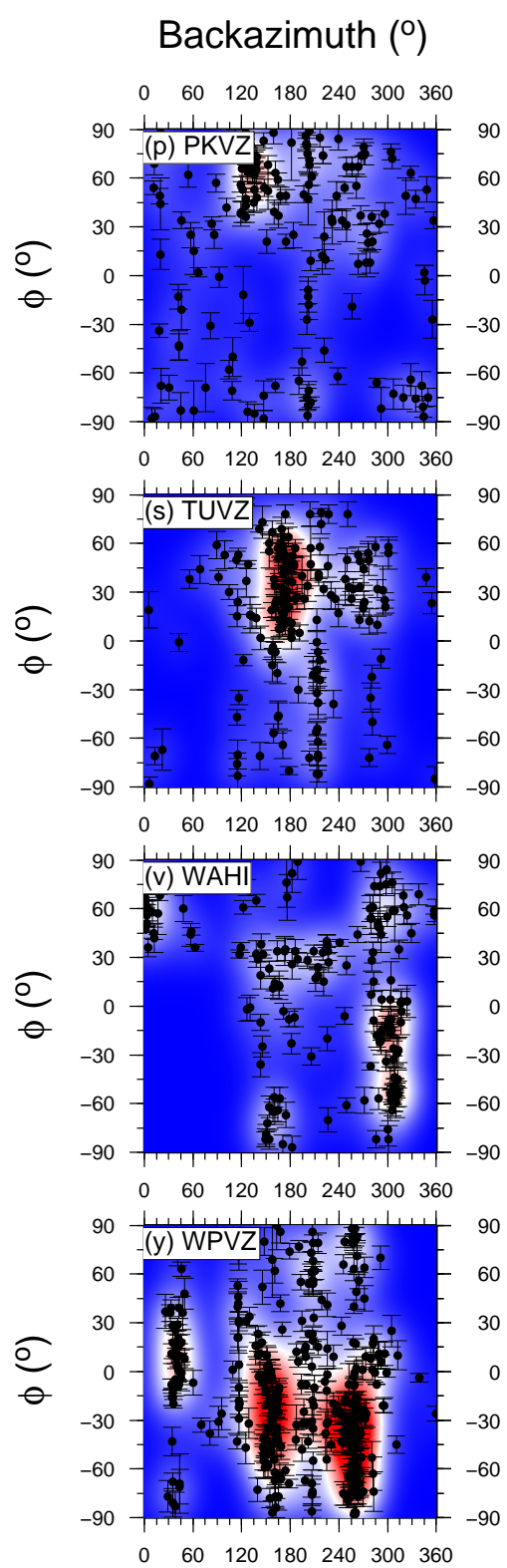

Backazimuth $\left({ }^{\circ}\right)$
Backazimuth $\left({ }^{\circ}\right)$

$\begin{array}{lllllll}0 & 60 & 120 & 180 & 240 & 300 & 360\end{array}$
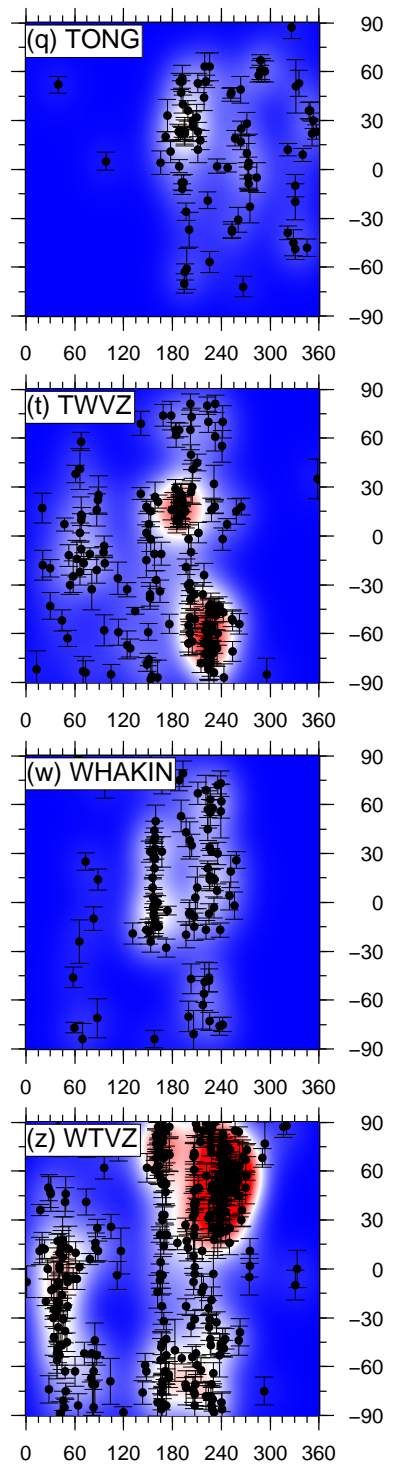

Backazimuth $\left({ }^{\circ}\right)$
Backazimuth $\left({ }^{\circ}\right)$
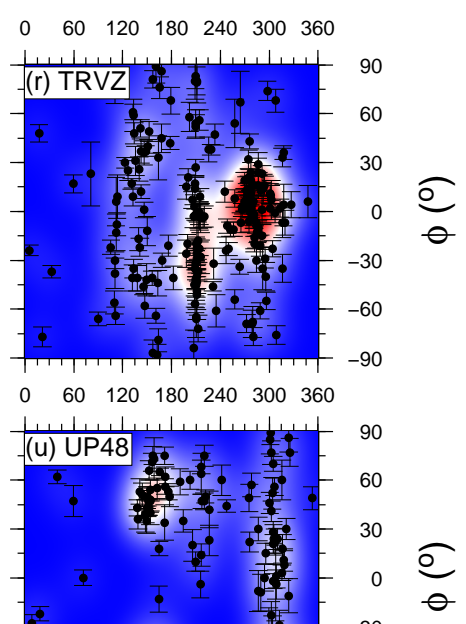

o

Figure 4.19. Azimuthal analysis of fast polarisation data for all stations. Colours represent density of data points.

pearing less populated with rays to the southeast (Figure 4.7). However, there are still many crossing rays and rays originating in these blocks so that the results of the checkerboard test (Figure 4.9) suggest that the region is well resolved.

To test the significance of the improvement of the model of anisotropy strength compared to the null case we performed an F test. The null case was calculated as a uniform anisotropy strength with a value equal to the mean anisotropy strength of the best model. The sum of squares of the weighted residuals for the null case was compared to the sum of squares of the weighted residuals for the best model to get 
an F statistic of 1.40. This exceeds the critical value of 1.06 and so the hypothesis that the models are the same to within errors can be rejected and the best model can be accepted to provide a significantly better fit to the data.

\subsection{Interpretation}

It is important to differentiate between stress-induced and structural anisotropy before interpreting shear wave splitting parameters and any time variations observed. Kaneshima [1990], Zinke and Zoback [2000] and Boness and Zoback [2006a] were able to distinguish between stress-induced and structure related anisotropy in regions where both the regional stress field and local structures were well known. These are examples where individual shear wave splitting measurements display either stressinduced or structure related anisotropy. The Boness and Zoback [2006a] study also found that some stations displayed a mix of polarisations, which could have been from a mix of anisotropy mechanisms or an average of the two.

As crustal stresses change, either locally due to earthquakes, fluid movement, and/or volcanic activity, or regionally due to tectonic movement, the contribution of stress induced microcracks to the anisotropy will change. If the effect of stress induced microcracks on shear wave splitting becomes stronger than that of the structural influences, and the direction of $S_{H \max }$ is different to the strike of the structure, then a rotation of $\phi$ may be observed. This temporal rotation of $\phi$ indicates a change in $S_{H \max }$, and therefore the stress tensor, that could be either directional or magnitudinal. We do not observe a temporal variation in shear wave splitting parameters during this study, however to aid the interpretation of past changes and possible future changes, it is important to create a benchmark.

To determine whether the anisotropy that was observed in 2008 is governed by stress-induced microcracks or structural influences, we use the zones and clusters defined in Section 4.1.3 and Figure 4.2 and compare the horizontal direction of metamorphic fabric, surficial fault strikes, $S_{\text {Hmax }}^{\text {focal }}$ and $\phi$. The results are listed in Table 4.2. These different observations all sample different levels within the crust; surficial faults give estimates of $S_{H \max }$ at the surface at the time of faulting and a possible source of anisotropy around the fault plane, seismic source observations give estimates of $S_{\text {Hax }}$ at the depth of the earthquake and anisotropy samples the path between the seismic source and the surface. Therefore this 2-D analysis is a first order approximation and more in depth interpretation could be done with the 

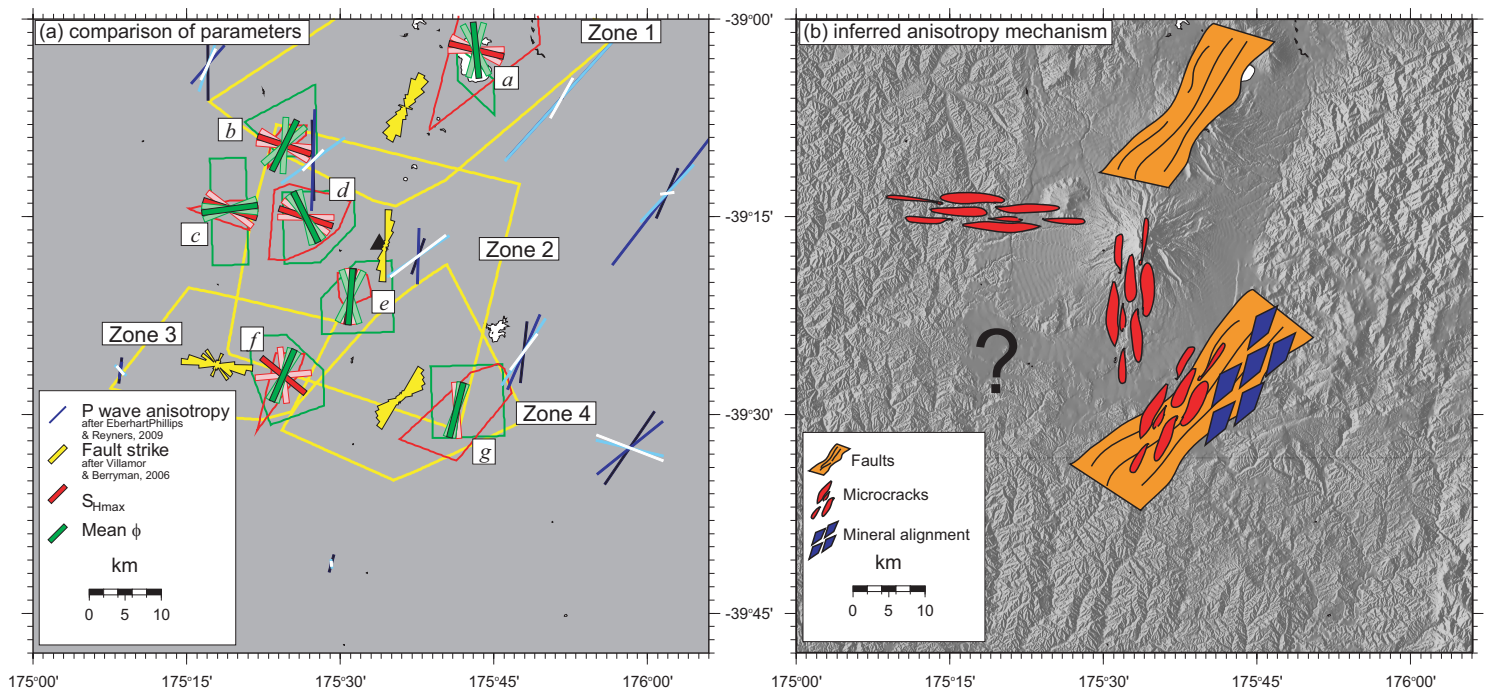

Figure 4.20. (a) Comparison of fault strikes, $\phi, S_{H \max }$ and fast direction from P anisotropy tomography. Black triangle indicates the summit of Mt. Ruapehu. Red bars show direction of $S_{H \max }$ from focal mechanism inversions with $80 \%$ confidence interval and red polygons show clusters of earthquakes used. Green bars show $\phi$ direction with standard error and green polygons show areas of anisotropy measurements used. Yellow rose diagrams show fault strikes after Villamor and Berryman [2006] and yellow polygons show zones from Figure 4.2. Blue bars show $\mathrm{P}$ wave anisotropy tomography after Eberhart-Phillips and Reyners [2009]. Length of bar indicates strength of anisotropy and colour represents depth of modelled anisotropy: white is at 0 $\mathrm{km}$, getting progressively darker through 4, 11 and $18 \mathrm{~km}$ depth (black). (b) Inferred anisotropy mechanisms around Mount Ruapehu. Anisotropy to the north of Mt. Ruapehu is thought to be controlled by fractures and faults. Anisotropy in to the west and south is thought to be caused by stress-induced microcracks, even though the direction changes. The area to the southwest does not contain enough data to draw conclusions about the mechanism of anisotropy, and anisotropy to the southeast is probably caused by a combination of mineral alignment, stress-induced microcrack alignment, and fractures.

use of a 3-D tomographic inversion of the shear wave splitting parameters. Figure 4.20 (a) also shows anisotropy fast azimuth and strength from the $\mathrm{P}$ wave anisotropy tomography of Eberhart-Phillips and Reyners [2009]. The estimates are plotted on the node of the inversion and vary with depth. Comparison of this $\mathrm{P}$ wave anisotropy with the stress, structure and shear wave splitting is difficult to quantify due to the coarse grid, however the general agreement is good and we suggest that the $\mathrm{P}$ wave anisotropy fits our data best shallower than about $11 \mathrm{~km}$ depth (light blue Figure 4.20 (a)). This mid-upper-crustal depth fits with the majority of the ray-paths, however, it is deeper than hypothesised models for magma reservoirs [Price et al., 2007] and so anisotropy at depth would probably not be affected by magma induced stresses. Eberhart-Phillips and Reyners [2009] attribute the crustal anisotropy to foliation in schists (east of study area), crustal stresses (southwest of study area) and fracture zones (north of study area). 


\subsubsection{Stress and Structure}

Comparison of stress estimates from our focal mechanism inversions (Section 4.1.4, Figure 4.3) and those of Hayes et al. [2004], Sherburn et al. [2009] and Reyners [2010] with Quaternary fault analysis of Villamor and Berryman [2006] (Figure 4.20) shows that active $S_{H \max }^{\text {focal }}$ often differs from those inferred from surface faults. Clusters $a$, $b, c$ and $d$ display $S_{\text {Hmax }}^{\text {focal }}$ that is sub-perpendicular to surface faults, whereas $S_{\text {Hmax }}^{\text {focal }}$ for clusters $e, f$ and $g$ align with surface faults.

\subsubsection{Anisotropy, Stress and Structure}

To easily compare $\phi$ to $S_{\text {Hmax }}^{\text {focal }}$ and structural influences, we take an average of the shear wave splitting results within each cluster indicated in Figure 4.2 (c). These estimates for average $\phi$ were calculated by taking results from the grid blocks within $10 \mathrm{~km}$ of the earthquake cluster centroid (Figure 4.2 (b)). We have carried out this analysis for the spatial averaging results using $1 / \mathrm{d}^{2}$ weighting functions and the comparisons are displayed in Figure 4.20 (a) with reference to the clusters defined by earthquake locations, and to the zones of similar fault strike determined by Villamor and Berryman [2006]. A cartoon summary of our interpretations is presented in Figure 4.20 (b).

\section{Cluster a}

In the region of dense faulting in zone 1 the observed shear wave splitting direction is sub-parallel to the fault strikes (Figure 4.20). The estimate of $S_{\text {Hmax }}^{\text {focal }}$, which uses earthquakes from the Tongariro swarm, is nearly perpendicular to $\phi$. Therefore in zone 1 (around cluster $a$ ), the observed shear wave splitting is most likely caused by anisotropy from the fault zones rather than stress induced anisotropy.

\section{Cluster b}

Cluster $b$, at the southwest edge of zone 1 , has similar $\phi$ and $S_{\text {Hmax }}^{\text {focal }}$ to cluster $a$. Even though the faulting is not as dense near cluster $b$, there are still faults with the same orientation (Figure 4.2) and therefore the inference of structural anisotropy being dominant holds for all of zone 1 (Figure 4.20 (b)). 


\section{Clusters $\mathrm{c}$ and $\mathrm{d}$}

$S_{\text {Hmax }}^{\text {focal }}$ for clusters $c$ and $d$ (in the west of zone 2) is approximately perpendicular to the general strike of the structure and $\phi$ correlates well with $S_{\text {Hmax }}^{\text {focal }}$, suggesting that stress induced anisotropy is the cause of shear wave splitting in this region (Figure $4.20(\mathrm{~b})$ ). Zone 2 is a region of weak fabric and few mapped faults (Figure 4.2 (a)), so it is not surprising that $\phi$ does not line up well with the structure (Figure 4.20 (a)).

\section{Cluster e}

Cluster $e$ is located closer to the centre of zone 2. In this case $S_{H \text { max }}^{\text {focal }}$ and $\phi$ align with fault strikes for the zone although there are few surficial faults close to the cluster, suggesting that stress is the governing anisotropy mechanism in this part of zone 2 as well (Figure $4.20(\mathrm{~b})$ ).

\section{Cluster $\mathbf{f}$}

Neither fault strikes, nor the estimate of $S_{H \max }^{\text {focal }}$ in zone 3 correlate with $\phi$ (Figure 4.20). However, this location is at the edge of our limit of statistical significance described in Section 4.3.2, and there is no information about basement fabric. While $S_{\text {Hmax }}^{\text {focal }}$ from earthquakes and surface fault strikes are well aligned in zone 3 , the errors on $S_{\text {Hmax }}^{\text {focal }}$ are large. We cannot draw conclusions about the cause of anisotropy in zone 3 as there is not enough data.

\section{Cluster g}

Our estimate of $S_{\text {Hmax }}^{\text {focal }}$ using this cluster (the Waiouru swarm) aligns well with estimates of $\phi$. There is also higher-grade schist [Beetham and Watters, 1985; Mortimer, 1993; Lin et al., 2007] striking in the same direction as mapped faults, as mentioned in Section 4.1.3, which also correlates with $\phi$ (Figure 4.20 (a)). In this case we are unable to distinguish the cause of anisotropy just by looking at the 2008 data as there were no evident changes in stress. If the anisotropy in this region is governed by stress-induced microcracks and there was a rotation in $S_{H \max }$ then a rotation of $\phi$ would be observed. Even if the anisotropy in 2008 was governed by structural influences, if there was a rotation of $S_{H \text { max }}$, there would likely be a 
change in magnitude of anisotropy as well. If this change in magnitude was large enough to effect the overall anisotropy then a rotation of $\phi$ would still be observed. Past estimates of $S_{\text {Hmax }}^{\text {focal }}$ for the Waiouru swarm [Hayes et al., 2004; Sherburn et al., 2009] have all been of similar strike so it is unlikely that temporal analysis of shear wave splitting will assist in the identification of the cause of anisotropy. The amount of shear wave splitting that we observe in this area is comparible with the values obtained by Godfrey et al. [2000], who used laboratory experiments to show that Haast schist can have as much as $0.2 \mathrm{sec}$ of splitting for a $5 \mathrm{~km}$ thick layer.

\subsubsection{Delay Time Tomography}

The interpretation of the delay time tomography must be considered in conjunction with the limitations of the method. It is designed to create a first-order approximation for regions of strong and weak anisotropy and will therefore not provide accurate absolute values of anisotropy strength. For example, discrete layers of anisotropy can cause the leading shear wave to resplit [e.g. Yardley and Crampin, 1991] and so the observed $\delta \mathrm{t}$ will be that of the last layer; a $90^{\circ}$ change of fast direction between discrete layers can result in destructive interference and an observed $\delta$ t smaller than that for either layer; or a layer of anisotropy that has its fast direction parallel with the polarisation of the incoming wave will not be split, resulting in zero delay time. However, the large volume of data and crossing rays used in this study allow us to interpret the inversion results with reference to the regions of strong and weak anisotropy.

To interpret the tomography of $\delta$ t and the regions of high anisotropy to the northeast and southeast of Mt. Ruapehu (Figure 4.8), we examined other evidence in these regions, as with the spatial averaging above. The region of strong anisotropy to the northeast of Mt. Ruapehu is strongest on the northeast flank of Mt. Tongariro, which is the location of the active Ketetahi geothermal field [Risk et al., 2002]. High porefluid pressures are likely at geothermal fields and there may be some hydrothermal alteration, creating more cracks in the rocks and therefore also higher percentage anisotropy [Lees and $W u, 1999]$. However, in Section 4.5.2 we saw that $S_{\text {Hmax }}^{\text {focal }}$ was not aligned with $\phi$ in this region and that the area is densely faulted. Therefore, high pore-fluid pressures probably do contribute to the high anisotropy by increasing the fractures, but the regional stress is not strong enough to overprint the structural effect. 
There is no evidence for high heat-flow in the Waiouru region, but the consistently active seismicity suggests fluid movement in a critically loaded fault zone [Hayes et al., 2004]. Therefore, high pore-fluid pressures at depth could be creating a strong stress induced anisotropy. The fault zone itself could be contributing to the anisotropy because even though there are fewer mapped faults in this region than in zone 1, the persistent seismicity suggests highly fractured rock at depth. Close to Waiouru Beetham and Watters [1985] also identified a region of higher-grade metamorphic (semi-schistose) rock, which could also be a cause of anisotropy. The combination of these three mechanisms could combine to create a stronger anisotropy (Figure $4.20(\mathrm{~b})$ ).

\subsubsection{Modelling}

Petrologic evidence for Mt. Ruapehu suggests that rather than having one main magma reservoir, magma is stored in evolving dikes and sills dispersed throughout the crust [Price et al., 2005]. If these magma pockets are connected then it is possible that magma injection into the system of small chambers could be modelled as a single expanding source, although it is unlikely to completely describe the system. Bryan and Sherburn [1999] suggested that the relatively quiet seismic scenario of the 1995/1996 eruptions reflected an open vent system, high heat flux and a small volume of magma involved in the eruption. An open system and low magma input are not conducive to detecting stress changes through shear wave splitting analysis at stations tens of kilometers away. However, Miller and Savage [2001] and Gerst and Savage [2004] found that shear wave splitting results changed over time, suggesting that the anisotropy was caused by microcracks aligned with time-varying stress, rather than static structure. Therefore, we use the Coulomb stress modelling package of Lin and Stein [2004] and Toda et al. [2005] to model the maximum horizontal stress caused by inflation sources to gauge whether the observed anisotropy could be caused by stress from one or more pressurised magma reservoirs. We carry out the Coulomb modelling with no regional stress as the evidence outlined in Section 4.1.4 suggests that $\left|S_{1}\right|($ vertical $)>\left|S_{2}\right| \approx\left|S_{3}\right|$ and $S_{H \max }$ is only sensitive to differences between $\left|S_{2}\right|$ and $\left|S_{3}\right|$. We use a coefficient of friction of 0.8 , Poisson's ratio of 0.25 , Young's modulus of $8 \mathrm{GPa}$, and calculate all models at $2 \mathrm{~km}$ depth. We used dike geometry based on the dike-like feature of low resistivity identified by Ingham et al. [2009], a point source similar to that modelled by Mordret et al. [2010], and a conduit-like source both centred on the volcano. The volume of the sources used was based on the maximum and minimum estimates of eruptive products of the 
1995/1996 eruptions, which are $0.005 \mathrm{~km}^{3}$ and $0.01 \mathrm{~km}^{3}$ [Bryan and Sherburn, 1999]. We tested different models (Table 4.5) because the geometry of the magma reservoir are currently unknown and to possibly constrain the geometry and volume of the subsurface magma.

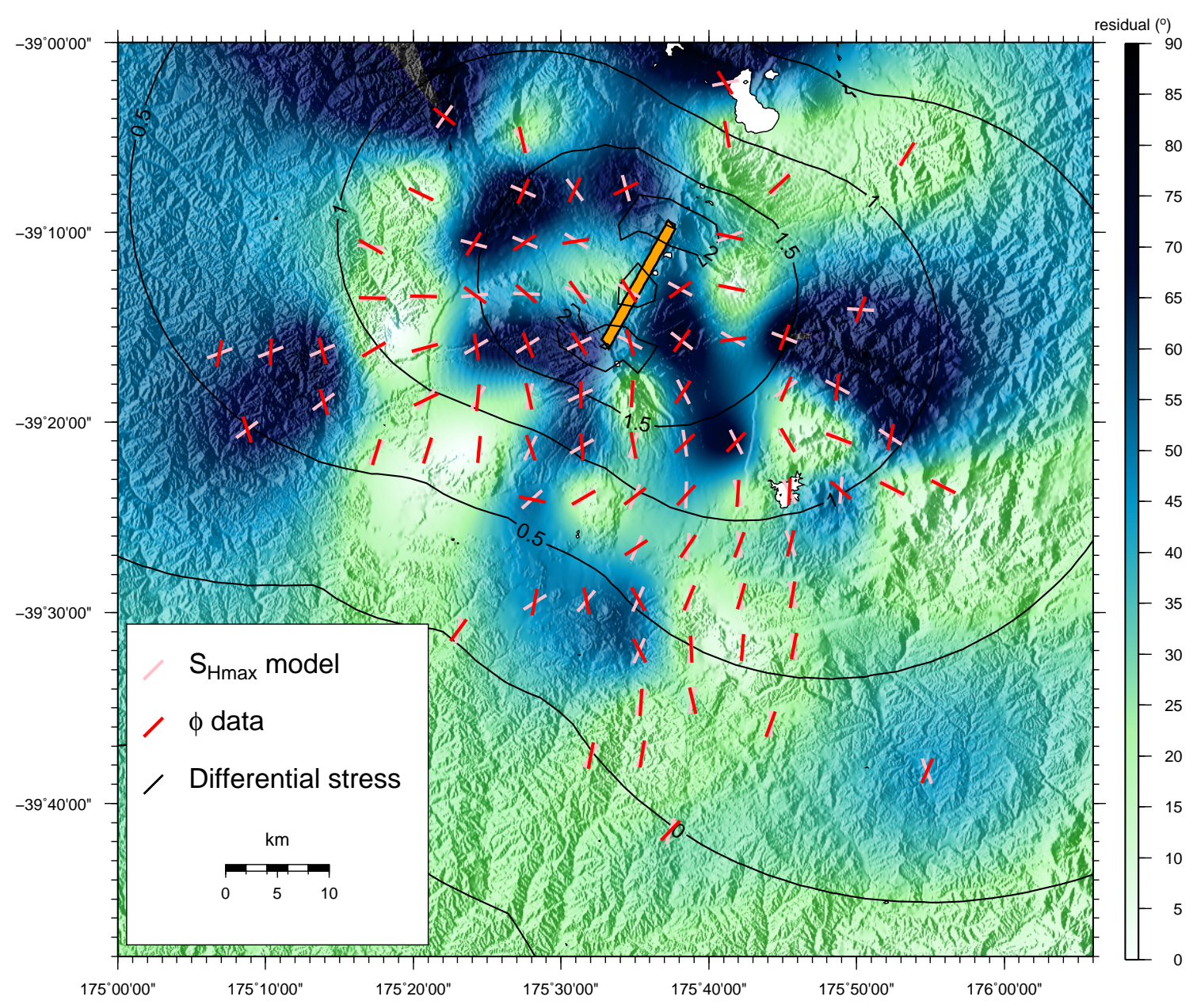

Figure 4.21. Results from Coulomb modelling of a dike (orange bar) with $0.5 \mathrm{~m}$ of expansion (not to scale). Mean $\phi$ from spatial averaging using $1 / d^{2}$ weighting in red, maximum horizontal stress direction as calculated using Coulomb modelling in pink, contours indicate the strength of the differential stress away from the source, background colours represent residual between data and model, with dark colours showing higher misfit.

Figure 4.21 displays the modelled maximum horizontal stress calculated using the method of Lund and Townend [2007], and the mean $\phi$ in each block from the spatial averaging described in Section 4.4.2, with the residuals shown by the coloured background. The pattern of anisotropy, while described well in places by the stress caused by a dike-like magma reservoir, is not consistent in other areas. There could be a major effect from other stress such as that from the topographic loading of the volcanic edifice [Horspool, 2003] and those related to the extension of the TVZ [Villamor and Berryman, 2006; Wallace et al., 2004] that we are unable to account 
for in this simple model. Table 4.5 lists the mean fit, calculated by finding the L1 norm, between the predicted maximum horizontal stress from the different models and the measured $\phi$. The fit is given for the whole study area rather than for specific areas as fault zone 2 includes the majority of the measurements and overlaps with the other zones, and zone 2 is the zone in which stress is inferred to be governing the anisotropy. The dominant feature in the misfit between the data and the model (Figure 4.21) is an E-W trending line to the northwest of Mt. Ruapehu. This line coincides well with the Taranaki-Ruapehu line (TRL), which is a discontinuity observed in electrical resistivity, gravity, seismic attenuation and receiver functions [Stern et al., 2006]. There is also a high rate of crustal seismicity along the line [Sherburn and White, 2005] although there are no mapped surficial features. We suggest that the TRL, as the transition point between different physical rock properties and a gravitational instability, harbors a high stress and possible crustal flow along the line could cause the development of crustal fabrics. Therefore, we attribute the orientation of $\phi$ in the region (Figure 4.20), and hence the misfit in Figure 4.21, to the TRL. 


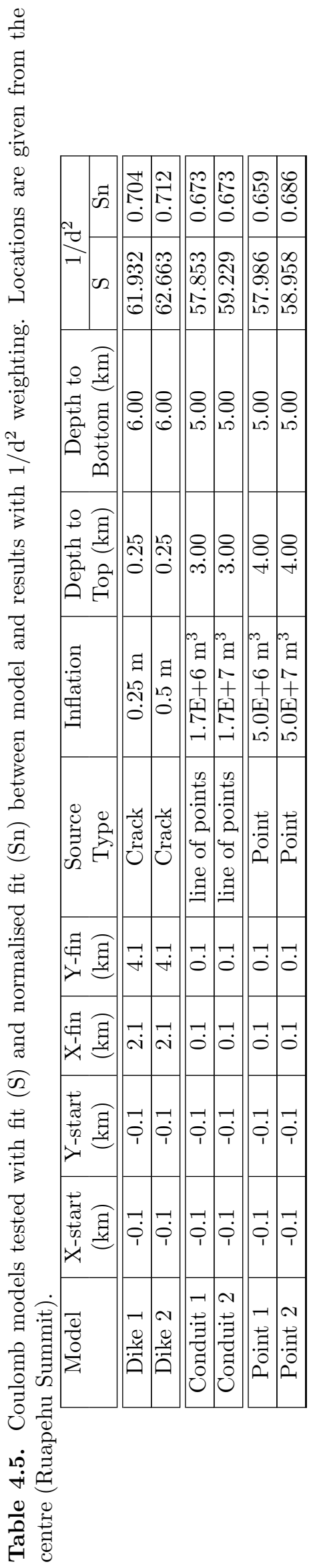


Finite element modelling including topography will illuminate the proportion of the stress causing the shear wave splitting observations that originates from topography and what stress effects are from different sources.

\subsection{Conclusions}

In this paper we have presented shear wave splitting results from a combined data set of two temporary deployments of three-component seismometers and the permanent network. The combined network was denser than any previous studies and so yields a result with a higher resolution of spatial variations in anisotropy. We have used earthquakes that occurred throughout 2008 near Mount Ruapehu and the automated shear wave splitting method of Savage et al. [2010b] to determine anisotropy parameters. We then used a spatial averaging technique combined with a new method of simplified two-dimensional tomography to analyse spatial variations in anisotropy and relate these to stress and structure in the region. Detailed explanations of the methods are provided in the manual discussed in Sections 3.2.5 and 4.7. Comparison of shear wave splitting results to stress estimates and local structures let us distinguish the regions in which shear wave splitting is governed by structural anisotropy and those in which stress-induced microcracks are the main cause. Our interpretations agree well with those of Eberhart-Phillips and Reyners [2009] derived from P wave anisotropy tomography. However, our different observations all sample different levels within the crust. Therefore this $2-\mathrm{D}$ analysis is a first order approximation and more in depth interpretation could be done with the use of a 3-D tomographic inversion of the shear wave splitting parameters.

Coulomb modelling with a dike-like magma reservoir oriented approximately NESW was used to calculate the maximum horizontal stress. The pattern of anisotropy, while described well in places by the stress caused by a dike-like magma reservoir and structural effects, could also have a major effect from the load of the volcanic edifice or other structures such as the Taranaki-Ruapehu line. Finite element modelling, including topography, will illuminate the proportion of the stress causing the shear wave splitting observations that originates from these different sources. The results of this study will be used to measure future changes in anisotropy and to identify the regions of past changes in anisotropy with more confidence. 


\subsection{Obtaining and Using the Programs}

The codes for this method have mainly been written in Matlab and GMT [Wessel and Smith, 1991]. The programs and a detailed technical manual can be found online (https://sites.google.com/site/jessicahelenjohnson/tessa). 


\section{Multiplets and multiple projects at Mount Ruapehu: tools for the detection of temporal variations in seismic anisotropy}

\section{Abstract}

The temporal variation of shear wave splitting and its interpretation is highly controversial. One of the main problems with the interpretation of temporal changes in shear wave splitting is the possibility of spatial variations being measured along differing ray paths and being interpreted as temporal variation. In this chapter we employ three different techniques in order to identify and minimise the uncertainties associated with the observations of temporally varying shear wave anisotropy at Mt. Ruapehu. The first approach applies a simplified two-dimensional tomographic inversion of recorded delay times of shear wave splitting $(\delta \mathrm{t})$ and a spatial averaging of fast direction of anisotropy $(\phi)$ to data from temporary seismic deployments in the Tongariro Volcanic Centre at Mt. Ruapehu in order to identify regions of changing seismic anisotropy. We observe a region of strong anisotropy (>0.025 s/km greater than the surrounding area) centred on Mt. Ruapehu in 1995, which was the time of a major magmatic eruption. This is interpreted to be due to high pressure, fluid and increased fracturing during the time of the eruption. We also observe strong anisotropy at Mt. Tongariro in $2008(\delta \mathrm{t} \sim 0.018 \mathrm{~s} / \mathrm{km}$ greater than the surrounding area) and a change in $\phi\left(\sim 80^{\circ}\right)$ and examine the temporal evolution of this anomaly using clusters of earthquakes and permanent seismic stations in operation since 2004. This anomaly is attributed to a change in the geothermal system. The second approach uses clusters of earthquakes and permanent seismic stations to analyse the temporal variation of shear wave splitting along similar paths. Stations close to the 
Waiouru swarm do not display changes in shear wave splitting over time, suggesting that the source of local anisotropy is unchanging. The third approach uses families of similar earthquakes occurring at Waiouru. Each multiplet is processed the same way to avoid changes in splitting parameters that are actually artifacts of time window or filter choice. We observe a change in the $\mathrm{S}$ coda of multiplet earthquakes around the time of the 2006 and 2007 phreatomagmatic eruptions of Mt. Ruapehu, which we attribute to altered scatterers. These scatterers inhibit the observation of subtle changes in the $\mathrm{S}$ waveforms that could cause changes in shear wave splitting, but have implications for inferring other changes along the path.

\subsection{Introduction}

Temporal variation of seismic anisotropy measured via shear wave splitting and its interpretation is highly controversial. Among the discussions there are accusations of observer bias in data selection [Aster et al., 1990], unsound statistical analyses [Seher and Main, 2004], misinterpretation of spatial variation [Liu et al., 2004] and lack of correlation with other stress determining factors/correlation with structural evidence [do Nascimento et al., 2004]. Most of the discussion is based around changes associated with large earthquakes but there have also been other studies conducted on shear wave splitting around volcanoes [e.g. Volti and Crampin, 2003; Bianco and Zaccarelli, 2009; Savage et al., 2010a; Roman et al., 2011; Keats et al., 2011]. Interpretation at volcanoes is often difficult due to the generally noisy waveforms and complicated interpretation of such observations when taking into account heterogeneity and complex stress regimes. Studies are therefore often coupled with other stress or strain indicators in order to minimise the ambiguity in the interpretation of shear wave splitting parameters.

The Tongariro Volcanic Centre (TVC), in the central North Island, New Zealand (Figure 5.1), consists of three large, historically active andesite volcanoes: Ruapehu, Ngauruhoe and Tongariro. These volcanoes are surrounded by an extensive ring plain made of stream, debris flow, lahar, lava, and ashflow deposits. Mount Ruapehu is a 2797 m-high andesitic stratovolcano and the highest active volcano in New Zealand. It is the southernmost of the large active volcanoes on the North Island, which make up the Taupo Volcanic Zone (TVZ), an extending continental back-arc system resulting from the subduction of the Pacific Plate beneath the Australian Plate at the obliquely-westward dipping Hikurangi subduction zone. Major 
magmatic eruptions occurred in 1945 and 1995/1996; the latter was the largest historical eruption of Mt. Ruapehu, producing a $12 \mathrm{~km}$-high volcanic ash plume and lahars on the flanks of the volcano [Bryan and Sherburn, 1999]. Mt. Ruapehu frequently experiences phreatic and phreato-magmatic eruptions [Hurst et al., 2004], which also threaten lives and property [Johnston et al., 2000]. The most recent phreatic eruptions occurred on the 4th of October 2006 and on the 25th of September 2007 [Jolly et al., 2010; Mordret et al., 2010]. Mt. Ngauruhoe is also an andesite stratovolcano, and most recently erupted in 1974 and 1975, when avalanches of hot pyroclastic debris were generated reaching the base of the $900 \mathrm{~m}$-high cone [Nairn and Self , 1978]. Volcanic earthquakes, which suggest current active fluid movement, are frequently observed at Mt. Ngauruhoe and Mt. Tongariro [Hagerty and Benites, 2003; Jolly et al., 2011].

Eruptions of Mt. Ruapehu often occur with few or no detectable precursors, making prediction difficult [Hurst et al., 2004]. For this reason Mt. Ruapehu volcano has, in recent years, been subject to several studies of crustal seismic anisotropy using shear wave splitting analysis [Miller and Savage, 2001; Gerst and Savage, 2004] in an attempt to characterise the local stress regime. Miller and Savage [2001] measured shear wave splitting from shallow $(<30 \mathrm{~km})$ and deep $(>50 \mathrm{~km})$ earthquakes in 1994 and 1998 and observed a change in the dominant azimuth of fast polarisation $(\phi)$ spanning the magmatic eruption of 1995/1996. That study was extended by Gerst and Savage [2004], who used the same techniques and an additional deployment of three-component seismometers in 2002 to observe further changes in $\phi$.

The changes in $\phi$ in both studies were interpreted as being caused by a dike-shaped magma reservoir, or system of dikes, trending NE-SW. According to this model, the magma reservoir was pressurised before the eruption, producing a local stress field different from the regional stress field. Following the eruption the reservoir was less full and correspondingly less pressurised so the local stress returned to that of the surrounding region. The Gerst and Savage [2004] study suggested that the later changes in $\phi$ were due to repressurising of the reservoir in response to an increase of magma in the system. The stress in the surrounding crust caused by the pressurised magma reservoir is thought to preferentially align randomly oriented fluid-filled microcracks and cause seismic anisotropy that is detected through shear wave splitting [e.g. Crampin, 1994; Hatchell and Bourne, 2005].

In this chapter we employ three different techniques in order to identify and minimise the uncertainties associated with the observations of temporally varying shear wave anisotropy at Mt. Ruapehu. We discuss the results and merits of each method and 


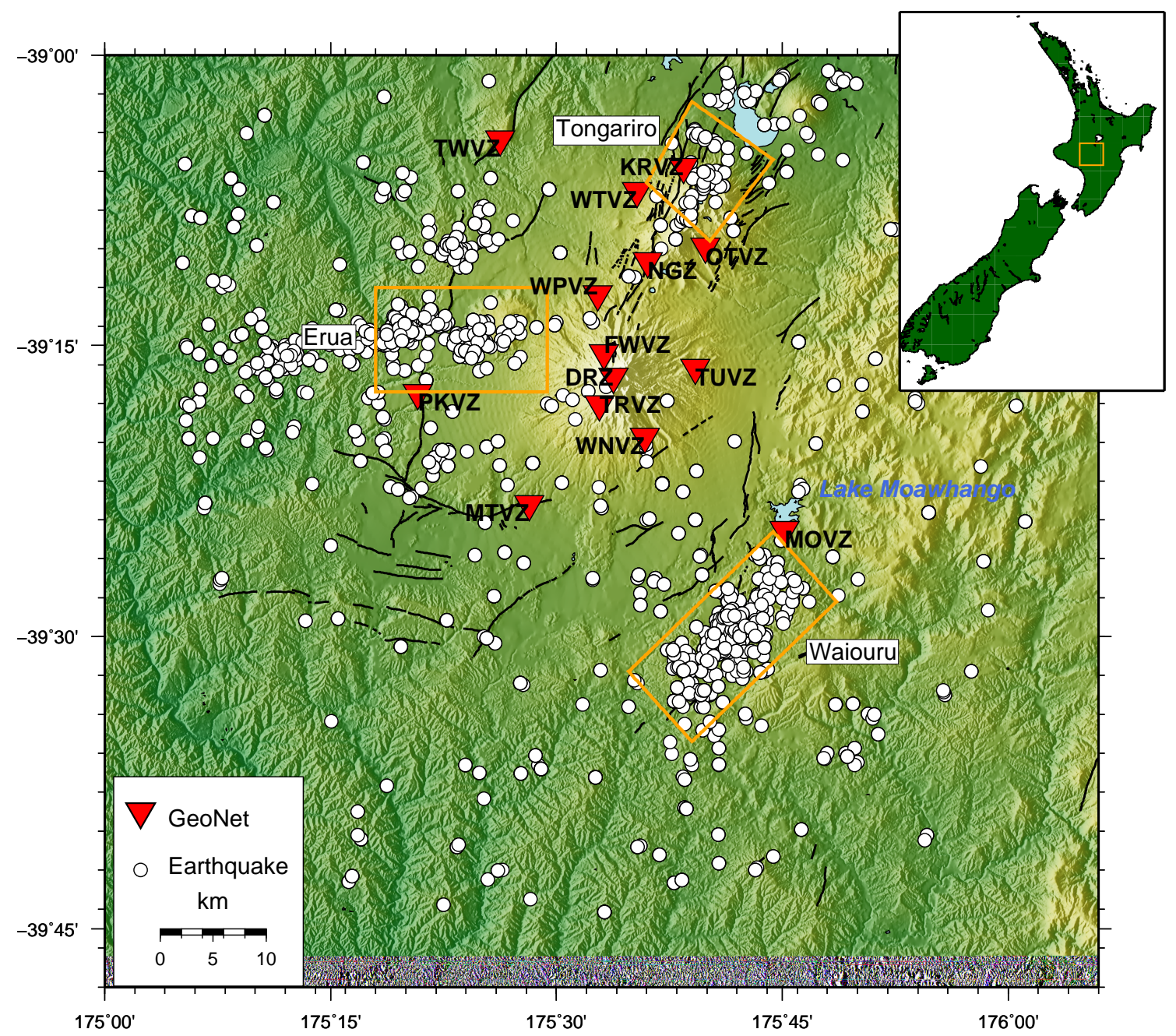

Figure 5.1. Map of the Tongariro Volcanic Centre showing seismicity during 2008. Boxes indicate clusters of earthquakes, faults (black) are from the NZ active fault database [GNS Science, 2011]. Red inverted triangles show locations of permanent GeoNet seismic stations [GeoNet, Last accessed 17 April 2011]. Inset shows study region (orange box) in the central North Island, New Zealand.

finally make a recommendation for the best method of monitoring anisotropy at Mt. Ruapehu.

The first approach uses temporary deployments of three component seismometers around Mt. Ruapehu in 1994, 1995, 1998, 2001, 2002 and 2008. Data from these deployments have been repicked and re-analysed for shear wave splitting using the automatic algorithm of Savage et al. [2010b]; MFAST. The shear wave splitting results are then inverted using two-dimensional delay time tomography and a spatial averaging of fast directions is applied using the methods described in Chapter 4. This analysis takes into account the differing earthquake and sensor locations during each of the deployments and so data from each time period can be compared. 
The second technique uses clusters of earthquakes and permanent seismic stations to analyse the temporal variation of shear wave splitting along similar paths. This method also uses MFAST to calculate the shear wave splitting parameters and the results are compared with the benchmark of anisotropy from Chapter 4 in order to refine the location of changing anisotropy.

The third approach uses families of similar earthquakes, as shear wave splitting can be highly dependent on both the raypath and the focal mechanism. These multiplets were identified from a swarm occurring at Waiouru using a cross-correlation method on data from permanent seismometers, some of which have been in operation since 1990. The earthquakes that are part of the multiplets were also identified on data from the temporary deployments and the permanent network as it was expanded. Each multiplet was then processed in exactly the same way, to avoid changes in splitting parameters that are actually artifacts of time window or filter choice, and can thus be used to monitor any changes in anisotropy around Mt. Ruapehu.

\subsection{Data}

The three techniques described in this chapter utilise most of the available seismic data that have been recorded around Mt. Ruapehu. These data come from seven temporary deployments and the permanent seismic network. Single component and three-component data from the permanent seismic network around Mt. Ruapehu were provided by GeoNet [GeoNet, Last accessed 17 April 2011, http://www . geonet.org.nz].

Fourteen three-component seismometers were positioned around Mt. Ruapehu between 28 January and 13 March 1994 by Leeds University, the University of Memphis and IGNS (Institute of Geophysical and Nuclear Science, now called GNS Science). The purpose of this deployment was to characterise the seismicity beneath Crater Lake [Hurst, 1998].

Twelve three-component seismometers were installed around Mt. Ruapehu between September and December 1995 by IGNS to observe the 1995 eruption sequence and to act as a backup in case the permanent stations were destroyed.

Three three-component seismometers were deployed around Mt. Ruapehu between February and July 1998 by Leeds University and IGNS. The purpose of this exper- 
iment was to characterise the post-eruption background seismicity [Sherburn et al., 1999].

The START experiment was carried out between January and June 2001 by the University of Cambridge. It was designed to create even coverage over the central and northern Tongariro volcanic centre with 28 three-component seismometers for use in seismic tomography [Rowlands et al., 2005].

In 2001, seismometers around Waiouru were set up by the University of Leeds in order to characterise earthquakes in the Waiouru swarm [Hayes et al., 2004]. This deployment was a smaller part of CNIPSE (Central North Island Passive Seismic Experiment), of which we use 10 three-component seismometers.

The CHanging Anisotropy at Ruapehu Mountain (CHARM) experiment was carried out by Victoria University of Wellington (VUW) between January and July 2002. It was designed to reoccupy the stations from the 1994 and 1998 deployments to further investigate the changes in shear wave splitting around Mt. Ruapehu [Gerst and Savage, 2004], and consisted of nine three-component seismometers.

The Spatial Anisotropy Deployment At Ruapehu (SADAR) was part of this VUW project to investigate seismic anisotropy at Mt. Ruapehu. SADAR consisted of sixteen temporary three-component seismometers, positioned around Mt. Ruapehu during 2008 to complement the permanent (GeoNet) network of fifteen threecomponent seismometers.

$\mathrm{P}$ and $\mathrm{S}$ phases were picked for earthquakes within $100 \mathrm{~km}$ of the summit of Mt. Ruapehu and shear wave splitting analysis was carried out using the automated method of Savage et al. [2010b]. Figure 5.2 displays the locations of seismometers for each of these time periods and the raypaths of the earthquakes that were recorded in the Ruapehu region. Station details are listed in Appendix B.2.

\subsection{Method}

\subsubsection{Delay time tomography and spatial averaging of $\phi$}

We used the method of two-dimensional $\delta$ t tomography and spatial averaging described in Chapter 4, and applied it to each of the temporary deployments. The assumption used in this inversion, that $\delta \mathrm{t}$ is simply additive, is a simplification of a 
Table 5.1. Parameters used in delay time tomography and fast direction spatial averaging.

\begin{tabular}{|l|l|}
\hline Parameter & Value \\
\hline \hline West Longitude $\left(^{\circ}\right)$ & 174.842 \\
\hline East Longitude $\left(^{\circ}\right)$ & 176.275 \\
\hline North Latitude $\left(^{\circ}\right)$ & -38.819 \\
\hline South Latitude $\left(^{\circ}\right)$ & -39.930 \\
\hline Checkerboard grid size $(\mathrm{km})$ & 9 \\
\hline Node spacing $(\mathrm{km})$ & 3 \\
\hline Minimum number of rays & 10 \\
\hline Minimum grid size $(\mathrm{km})$ & 4 \\
\hline
\end{tabular}

non-linear relationship between heterogeneous anisotropy and the observed apparent $\delta \mathrm{t}$ at the surface. It is designed to create a first-order approximation for regions of strong and weak anisotropy and will therefore not provide accurate absolute values of anisotropy strength, but will indicate relative strengths.

As most of the deployments were not as densely populated, nor run for as long as the 2008 deployment that was used in Chapter 4, some of the parameters had to be changed. We used the same parameters for each deployment. The parameters used are listed in Table 5.1.

We chose to expand the whole grid in order to include earthquakes from further away from Mt. Ruapehu because some of the deployments would have too few earthquakes otherwise. We also lowered the minimum number of raypaths that pass through a grid square to ensure that the majority of the grid squares are included in the analysis. We chose to keep the minimum grid size the same in order to achieve higher resolution where the data permitted and we chose to use a regular grid, rather than the quadtree gridding, so that the deployments could be compared. Use of a uniform grid enables the use of the resolution matrices to define the regions in which the the data model is well resolved, rather than using the variance as in Chapter 4. The grids and rays are displayed in Figure 5.2.

\subsubsection{Shear wave splitting using clusters}

Keats et al. [2011] (Appendix G) carried out shear wave splitting analysis on the Erua cluster (Figure 5.1) and identified a significant change in shear wave splitting parameters around the time of the 2006 and 2007 phreatomagmatic eruptions of 


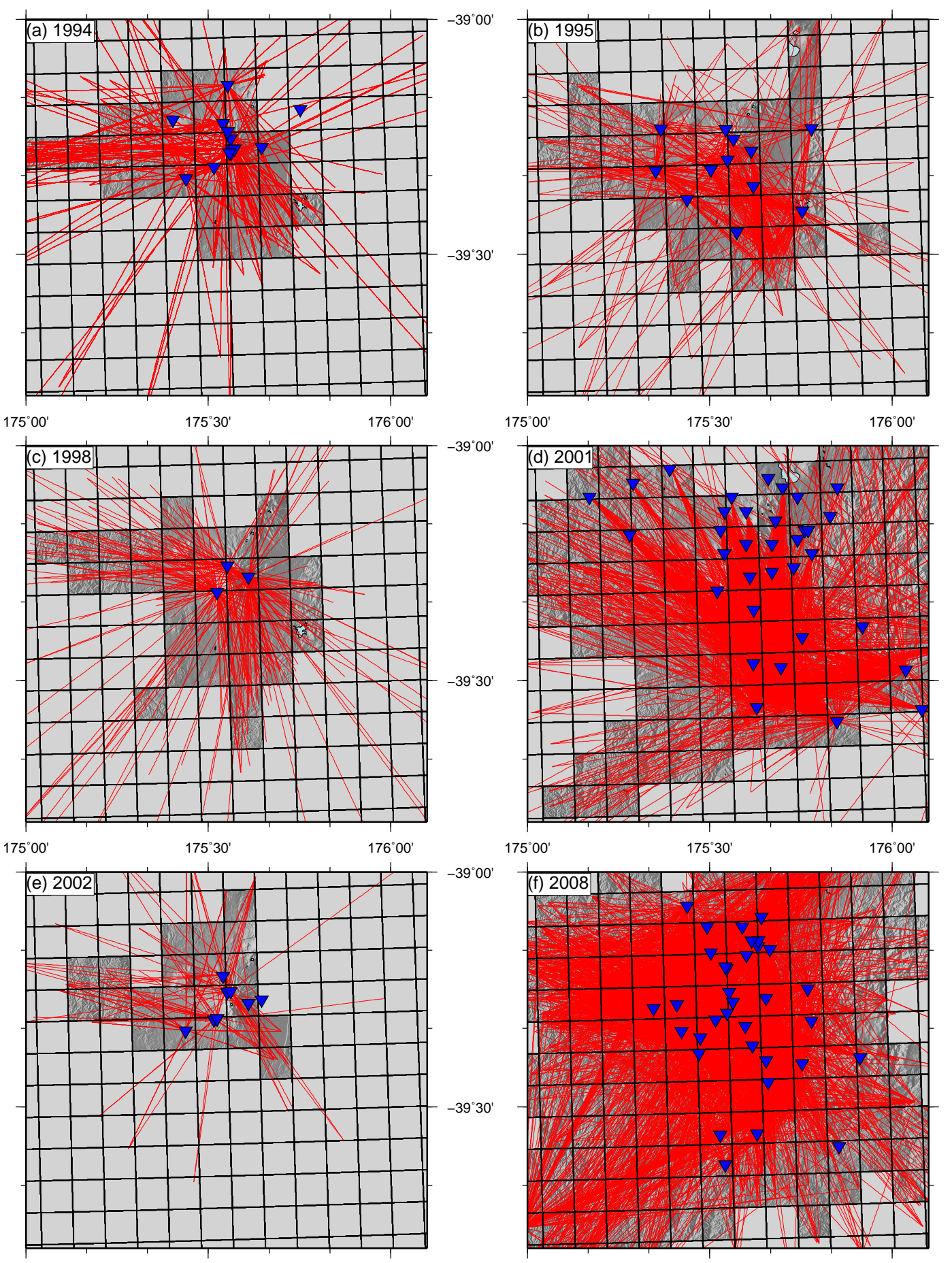

Figure 5.2. Maps of the stations (blue inverted triangles), grids and rays (red lines) used in the inversions and spatial averaging from the temporary deployment data in 1994, 1995, 1998, 2001 (START and CNIPSE), 2002 (CHARM) and 2008 (SADAR). Light grey boxes represent grid squares intersected by fewer than 10 rays and are therefore excluded from the analysis.

Mt. Ruapehu. These were attributed to an increase in pore-fluid pressure close to the earthquake swarm because of the orientations of $\phi$, the decrease in $\delta \mathrm{t}$, and also because a change in $b$-value was observed. This interpretation agrees with 
the conclusions of Chapter 4: that anisotropy in this region is stress controlled. We used another of the clusters of earthquakes identified by Latter [1981a]: the Waiouru swarm [Hayes et al., 2004] (Figure 5.1). Using MFAST, which is the same automatic shear wave splitting method as used by Keats et al. [2011], we examine shear wave splitting parameters at four permanent stations and plot the results using a moving average plot similar to that used by Savage et al. [2010a].

\subsubsection{Identification of multiplets}

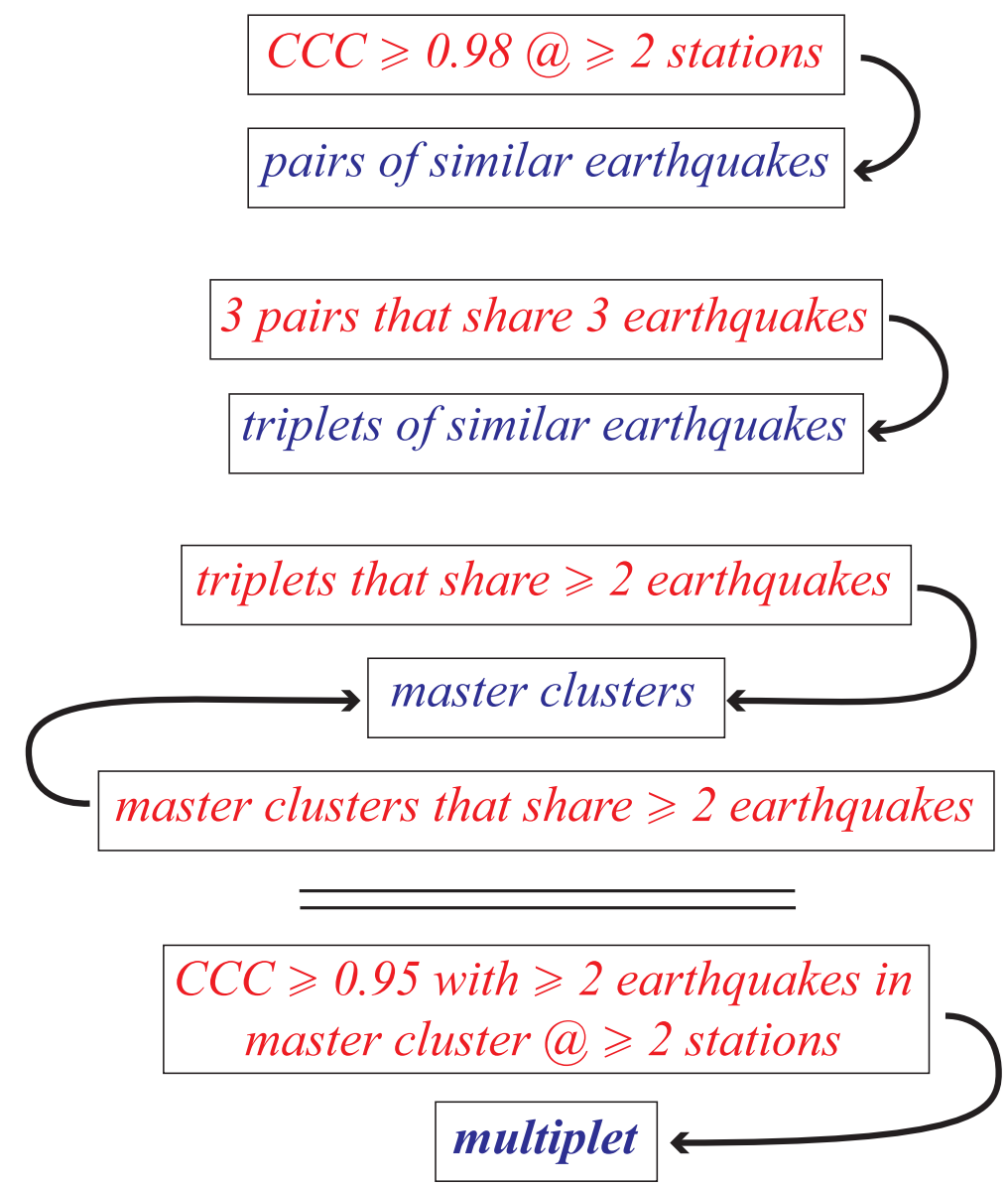

Figure 5.3. Flowchart of the steps taken to identify multiplets of repeating earthquakes based on the cross-correlation coefficient $(C C C)$.

In order to identify repeating earthquakes, we obtained single-component data from four permanent stations (CNZ, DRZ, NGZ and TUVZ), and three-component data from four permanent stations (FWVZ, MOVZ, MTVZ and WNVZ) for earthquakes within the Waiouru swarm $\left(-39.40^{\circ}\right.$ to $-39.60^{\circ}, 175.50^{\circ}$ to $\left.175.85^{\circ}\right)$. We resampled the data to $100 \mathrm{~Hz}$ and cross-correlated all of the earthquakes using BCSEIS [Du 
et al., 2004a]. The window around the $\mathrm{P}$ wave consisted of 254 sample points, 30 of them before the $\mathrm{P}$ arrival. A longer time window around the $\mathrm{S}$ wave was used, with 382 sample points, 50 of them before the picked $\mathrm{S}$ arrival. To identify families of repeating earthquakes we used a clustering method similar to the equivalence class method [Press et al., 1986] but with some additional constraints. We first identified earthquakes that have a cross-correlation coefficient $(C C C)$ of greater than 0.98 at at least two stations. These are the so-called master clusters. For the master clusters, each earthquake had to pass the criterion $(C C C \geq 0.98)$ with at least two other earthquakes in the master cluster, therefore creating master clusters with at least three earthquakes. We then allowed earthquakes that have a $C C C$ of greater than 0.95 at at least two stations with at least two of the earthquakes in the master cluster to make up the multiplets. These steps are displayed in the flow diagram in Figure 5.3. This resulted in 29 multiplets with between three and 44 earthquakes in them. Table 5.2 lists the earthquakes in each multiplet.

Table 5.2. Multiplet earthquakes. Bold type signifies a master event.

\begin{tabular}{|c|c|c|c|c|c|c|c|}
\hline Multiplet & cusp IDs & & & & & & \\
\hline 1 & 713435 & 717681 & 731389 & 740513 & 745014 & 745752 & 751102 \\
\hline & 751686 & 780799 & 823836 & 869045 & & & \\
\hline 2 & 717681 & 731389 & 773618 & 774660 & 783667 & 798565 & 863370 \\
\hline & 863381 & 863504 & 864062 & 865319 & 866401 & 867601 & 868322 \\
\hline & 868424 & 868430 & 868433 & 873893 & 876979 & 880548 & 880559 \\
\hline & 880563 & 880566 & 880737 & 880744 & & & \\
\hline 3 & 774660 & 780799 & 849235 & 854166 & 860382 & 861136 & 862134 \\
\hline & 864062 & 868322 & 868424 & 868433 & 869464 & 870108 & 873893 \\
\hline & 875217 & 876379 & 880827 & & & & \\
\hline 4 & 1320199 & 1407505 & 1530126 & & & & \\
\hline 5 & 1630239 & 1837466 & 1837469 & 1837485 & 1838463 & 1844136 & 1844141 \\
\hline & 1844285 & 1844342 & 1866471 & 1866993 & & & \\
\hline 6 & 1856497 & 1856512 & 1856565 & 1858659 & 1997935 & & \\
\hline 7 & 1856579 & 1856581 & 1857554 & 1857709 & 1858650 & & \\
\hline 8 & 1915931 & 2010897 & 2010899 & 2010902 & 2056035 & 2093879 & 2100212 \\
\hline & 2128942 & 2131174 & 2175922 & 2177432 & & & \\
\hline 9 & 2267528 & 2539392 & 2548822 & 2548988 & 2550123 & 2557082 & 2560748 \\
\hline & 2561051 & 2561411 & 2565068 & 2701496 & & & \\
\hline 10 & 2353070 & 2355161 & 2355194 & 2355203 & 2355592 & 2355616 & 2359582 \\
\hline & 2566751 & & & & & & \\
\hline 11 & 2368688 & 2563550 & 2677553 & 2689944 & 2859875 & 2859887 & 3100667 \\
\hline & 3216444 & 3288154 & 3288881 & & & & \\
\hline 12 & 2442078 & 2442299 & 2442300 & 2442302 & 2442303 & 2442304 & 2442405 \\
\hline 13 & 2458279 & 2484722 & 2501538 & 2501539 & 2502953 & 2526030 & 2526035 \\
\hline & 2538312 & 2538404 & 2689944 & 2690039 & 2693049 & 2693051 & 2693060 \\
\hline & 2693302 & 2771194 & 3274673 & 3288154 & & & \\
\hline 14 & 2459523 & 2460165 & 2464351 & 2530772 & 2531389 & 2531931 & 2532721 \\
\hline & 2537329 & 2538507 & 2538717 & 2538822 & 2538823 & 2538975 & 2539308 \\
\hline & & & & & & ntinued o & next page \\
\hline
\end{tabular}


Table 5.2 - continued from previous page

\begin{tabular}{|c|c|c|c|c|c|c|c|}
\hline \multirow[t]{2}{*}{ Multiplet } & \multicolumn{7}{|l|}{ cusp IDs } \\
\hline & 2539652 & 2539694 & 2539707 & 2546304 & 3077131 & 3229833 & \\
\hline \multirow[t]{6}{*}{15} & 2499114 & 2918465 & 2920005 & 2965285 & 2986978 & 3070830 & 3070885 \\
\hline & 3070953 & 3071154 & 3073848 & 3074564 & 3074577 & 3075075 & 3075080 \\
\hline & 3075120 & 3075569 & 3075611 & 3076773 & 3077946 & 3077961 & 3079448 \\
\hline & 3079478 & 3079945 & 3080076 & 3084258 & 3084347 & 3086027 & 3088192 \\
\hline & 3088218 & 3102981 & 3150718 & 3277992 & 3289146 & 3289205 & 3289353 \\
\hline & 3293999 & 3294043 & & & & & \\
\hline 16 & 2520987 & 2521149 & 2539885 & 2539988 & 2540062 & & \\
\hline \multirow[t]{3}{*}{17} & 2530772 & 2531931 & 2537329 & 2537985 & 2538249 & 2538507 & 2538717 \\
\hline & 2538822 & 2538823 & 2538975 & 2539308 & 2539652 & 2539707 & 2540419 \\
\hline & 2540868 & 2546304 & 2549525 & & & & \\
\hline \multirow[t]{2}{*}{18} & 2563550 & 2693049 & 2693051 & 2720628 & 2859875 & 2859887 & 3288154 \\
\hline & 3288881 & 3291353 & & & & & \\
\hline \multirow[t]{3}{*}{19} & 2676041 & 2777691 & 3133832 & 3133964 & 3134027 & 3134081 & 3135682 \\
\hline & 3136017 & 3137898 & 3142533 & 3143038 & 3153938 & 3156482 & 3175137 \\
\hline & 3178427 & 3202538 & 3207543 & & & & \\
\hline \multirow[t]{3}{*}{20} & 2676790 & 2725573 & 2725574 & 2747440 & 2765758 & 2772399 & 2772488 \\
\hline & 2777691 & 2797559 & 3027639 & 3057766 & 3081493 & 3109682 & 3129968 \\
\hline & 3280642 & & & & & & \\
\hline 21 & 2907489 & 2917284 & 2917957 & 2918465 & 2920005 & 2937239 & \\
\hline \multirow[t]{2}{*}{22} & 2938861 & 2961110 & 2982487 & 2996638 & 3021652 & 3045008 & 3103243 \\
\hline & 3103261 & 3104845 & 3109934 & 3113282 & & & \\
\hline 23 & $\begin{array}{c}\mathbf{2 9 6 7 7 2 6} \\
2976722\end{array}$ & 2969232 & 2969612 & 2969636 & 2969678 & 2970933 & 2971906 \\
\hline \multirow[t]{2}{*}{24} & 3005858 & 3017516 & 3018185 & 3018248 & 3019564 & 3021594 & 3021597 \\
\hline & 3023710 & 3041236 & 3051626 & 3067506 & & & \\
\hline 25 & 3066061 & 3066066 & 3066710 & 3066741 & 3067057 & 3068795 & \\
\hline \multirow[t]{7}{*}{26} & 3070830 & 3070885 & 3070953 & 3071154 & 3071184 & 3073848 & 3073965 \\
\hline & 3074012 & 3074017 & 3074564 & 3074577 & 3075075 & 3075120 & 3075123 \\
\hline & 3075286 & 3075569 & 3075611 & 3075615 & 3075970 & 3076773 & 3077946 \\
\hline & 3077961 & 3078277 & 3079448 & 3079478 & 3079945 & 3080076 & 3084258 \\
\hline & 3084347 & 3086027 & 3088192 & 3088218 & 3088804 & 3089557 & 3089565 \\
\hline & 3095407 & 3102758 & 3104712 & 3107545 & 3150718 & 3289146 & 3289205 \\
\hline & 3293999 & 3294043 & & & & & \\
\hline 27 & 3094273 & 3094593 & 3094604 & 3095542 & 3278505 & & \\
\hline \multirow[t]{4}{*}{28} & 3125573 & 3126350 & 3129628 & 3131840 & 3131857 & 3131947 & 3132508 \\
\hline & 3133832 & 3133964 & 3133970 & 3134027 & 3134081 & 3134255 & 3135682 \\
\hline & 3136017 & 3137898 & 3138498 & 3140333 & 3142533 & 3143038 & 3153938 \\
\hline & 3156482 & 3175137 & 3178427 & 3202538 & 3207543 & & \\
\hline \multirow[t]{2}{*}{29} & 3244745 & 3276886 & 3277040 & 3277055 & 3277314 & 3277472 & 3280580 \\
\hline & 3280586 & 3281359 & 3285055 & & & & \\
\hline
\end{tabular}

Examination of the multiplets showed that there were several multiplets that shared more than 10 earthquakes. These were 14 and 17, 15 and 26, and 19 and 28. These multiplets were therefore combined, resulting in 26 distinct multiplets. We also investigated the behavior of the multiplets over the times in which the instrumentation changed in order to link multiplets so that they spanned a larger time interval. 
To link the multiplets we first sought earthquakes that appeared in more than one multiplet and occurred at times in the overlap period between instrument changes. We then broadened the search to allow earthquakes that occurred in the overlap period to only fulfill the $C C C$ criterion at a single station. However, this did not result in any early multiplets being linked with later ones. Figure 5.4 shows that more distinct multiplets were identified when more stations were operational and there were few earthquakes during the instrument overlap periods.
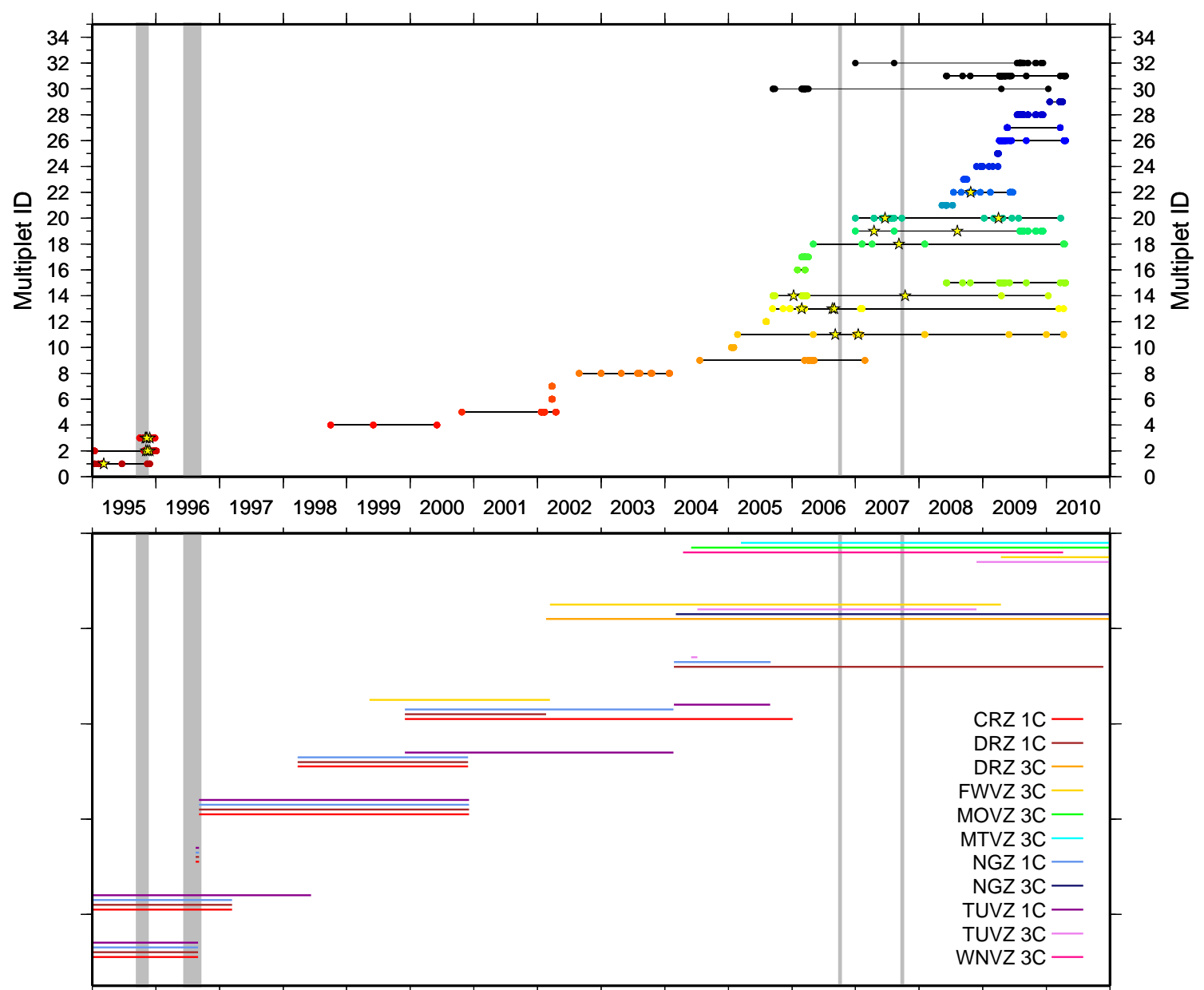

Figure 5.4. Top: Temporal evolution of multiplets. Coloured dots are earthquakes, where the colour represents the multiplet ID, black dots are combined multiplet earthquakes, yellow stars show time of coda changes. Multiplets are connected by solid black lines. Bottom: Instrument operation times. Colours represent stations. $1 \mathrm{C}$ and $3 \mathrm{C}$ indicate single-component and threecomponent instruments respectively. Grey bars indicate times of eruptions.

Once the multiplets had been identified we examined the automatic splitting parameters for the multiplet earthquakes and defined an optimum filter and time window based on those automatically chosen. The multiplet earthquakes were repicked according to the cross-correlation lag times and the shear wave splitting was analysed again using the optimum parameters. 


\subsection{Results and discussion}

\subsubsection{Delay time tomography}

The results of the delay time tomographic inversions for the six temporary deployments are displayed in Figure 5.5. The shaded regions show the area outside the resolution as defined by the diagonals of the resolution matrices.

The stability of these results were tested by 'jackknife' tests: removing random selections of earthquakes (Figure 5.6), and checkerboard tests (Figure 5.7). The regions of good resolution are conservative estimates based on the resolution matrices and the recreated features of the checkerboard tests. The main features of strong anisotropy were found to be stable and robust following jackknife inversions using 2 sets of independent data. The deployments in 1994, 1998 and 2002 had very small areas of resolution; we therefore concentrate on the inversions of the 1995, 2001 and 2008 deployments.

The feature of high anisotropy strength just southwest of lake Moawhango, in the Waiouru region (marked with W), appears in 1995, 2001 and 2008. This is the region that was interpreted to be caused in part by schistose mineral alignment and aligned fractured fault zones in Chapter 4 and would therefore be unlikely to change over time.

There are other prominent features of high anisotropy in each of the three inversions. In 2008 the main feature of high anisotropy, other than at Waiouru, is close to Mt. Tongariro (marked with T). It is not visible in 2001, despite good resolution. This was interpreted as being a highly fractured geothermal area in Chapter 4. In 2001 the main feature of high anisotropy, other than Waiouru, is near the Erua swarm (marked with E). Keats [2010] investigated the $b$-value and seismicity rate of this cluster over time and found that in 2001 there was a spike in seismicity. This was originally thought to be due to the CNIPSE deployment allowing a higher sensitivity, however the magnitude of completeness did not go down at this time and the $b$-value decreased, which is contrary to what would be expected if the anomaly was due to better detection. Hence, it seems that there was a change in the characteristics of the Erua swarm in 2001 and this is reflected in the anisotropy tomography. The main feature of high anisotropy, which is the highest out of all of the inversions at $>0.025 \mathrm{~s} / \mathrm{km}$ splitting, is centred just to the west of Ruapehu summit in 1995, which is during the major eruption. The high anisotropy at this time is probably caused by 

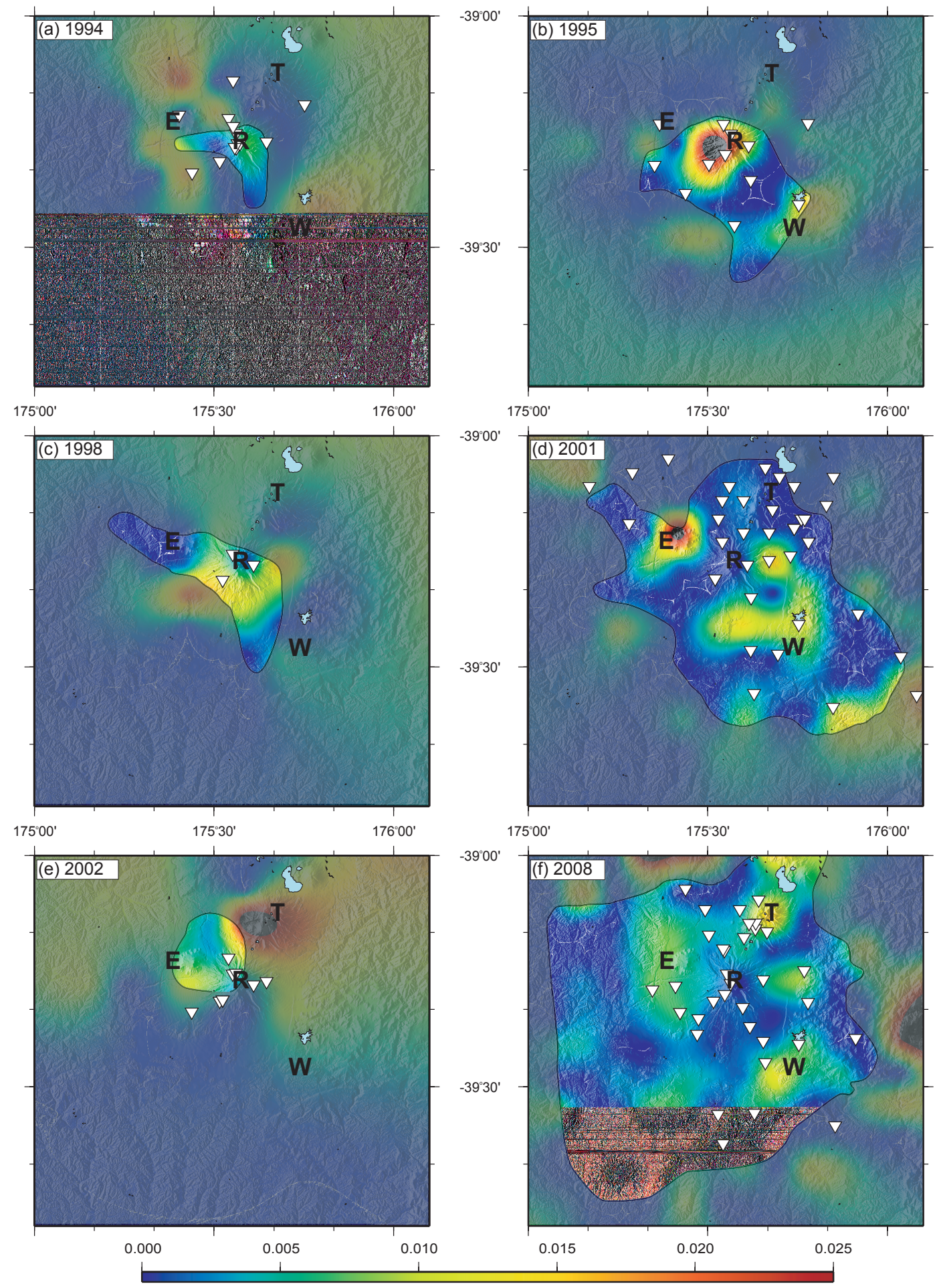

strength of anisotropy $(\mathrm{s} / \mathrm{km})$

Figure 5.5. Maps showing the results of delay time tomography for the six temporary deployments. Shaded areas indicate the estimated limit of resolution. $\mathbf{W}$ indicates Waiouru region, $\mathbf{R}$ is Ruapehu, $\mathbf{T}$ is Tongariro, and $\mathbf{E}$ is Erua for reference. 


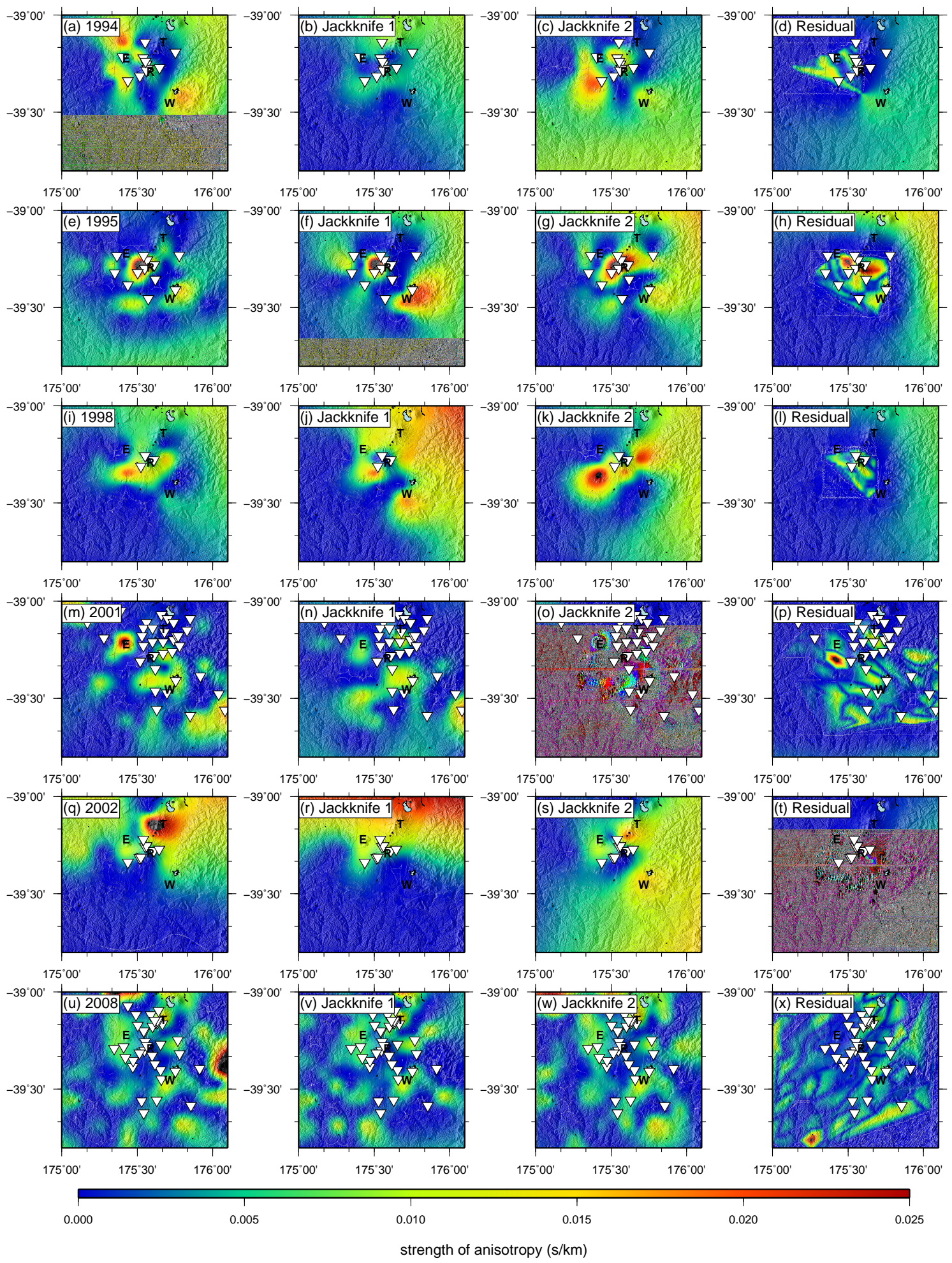

Figure 5.6. Results from the delay time tomography (left column, from Figure 5.5), two examples of jackknife tests and the difference between the two jackknifes (right column). Jackknife 1 was carried out using exactly half of the data chosen randomly, jackknife 2 was carried out with the other half so that jackknife 1 and 2 are mutually independent. $\mathbf{W}$ indicates Waiouru region, $\mathbf{R}$ is Ruapehu, $\mathbf{T}$ is Tongariro, and $\mathbf{E}$ is Erua for reference. 
high pressure, fluid and increased fracturing during the time of the eruption. This area may also be higher than average in 1994 and 1998, although the resolution is not good. However, it is an area of low anisotropy in 2001 and 2008. 

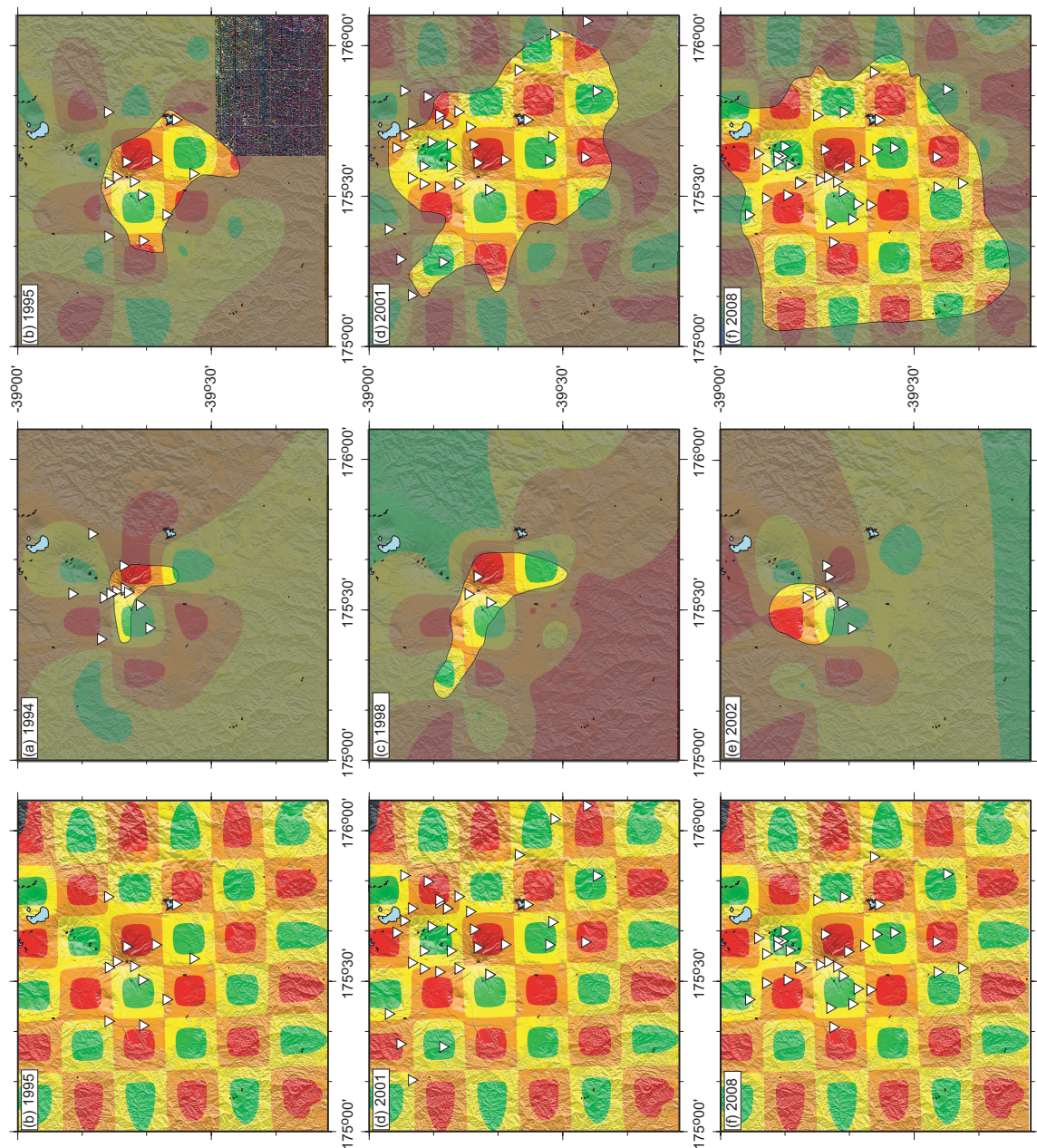

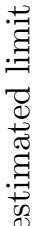

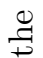

$\stackrel{\overbrace{}}{\leftrightarrows}$

:

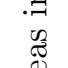

๘ँౖ

$\frac{\pi}{\pi}$
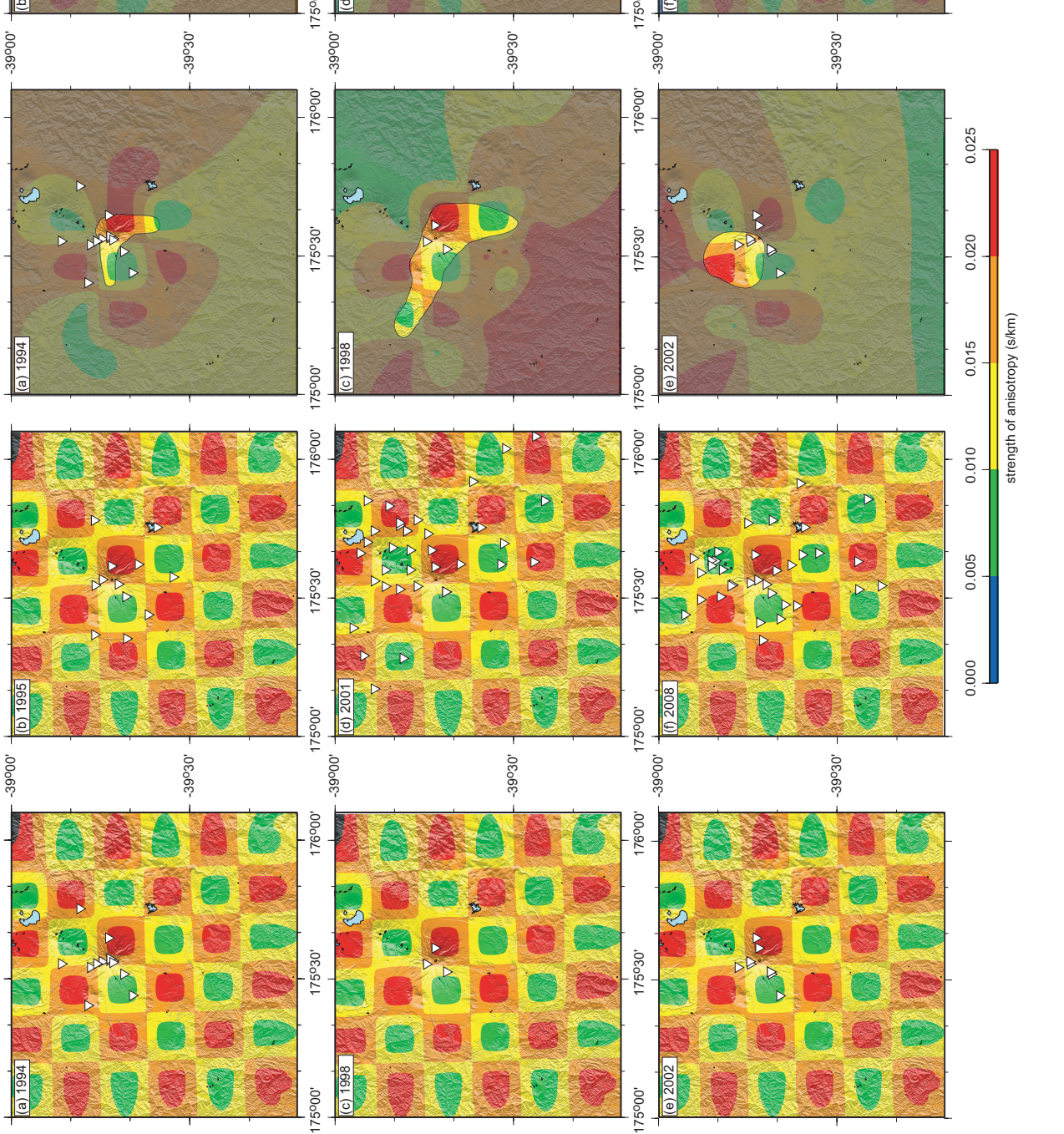


\subsubsection{Spatial averaging of $\phi$}

The results of the spatial averaging of $\phi$ for the six temporary deployments display fairly good continuity between deployments (Figure 5.8). Figure 5.9 displays the comparison of each deployment with the 2008 deployment (Figure $5.8 \mathrm{f}$ ). Most of the average fast directions fit well, with the $\mathrm{L} 1$ norm fit $(\mathrm{Sn})$ above 0.7 , where

$$
\mathrm{Sn}=\frac{\sum\left|\cos \left(\phi_{2008}-\phi\right)\right|}{N} \text {. }
$$

The anomaly seems to be the 1994 deployment (Figure 5.9 a), in which all of the average fast directions have a NNW orientation. This agrees with the results of Miller and Savage [2001], even though the data were completely reprocessed and included more stations and earthquakes in this study.

All of the deployments that have data near Waiouru show a good fit in that region. Both the 1995 and 2001 spatial average maps show high differences from SADAR near Mt. Tongariro, although there is evidence of an E-W trend just west of Tongariro in 2001. This may be because the high anisotropy near Mt. Tongariro that was observed in 2008 was anomalous. In this case the fast direction near Mt. Tongariro in 2008 may also have been anomalous. We examine this anomaly in more detail in the following section.

\subsubsection{Shear wave splitting using clusters}

Figure 5.10 displays rose diagrams of shear wave splitting results from the Waiouru swarm and moving window time averages. The stations close to the Waiouru swarm (MOVZ and MTVZ) display very little scatter. This is evidence that the automatic method yields stable results. 

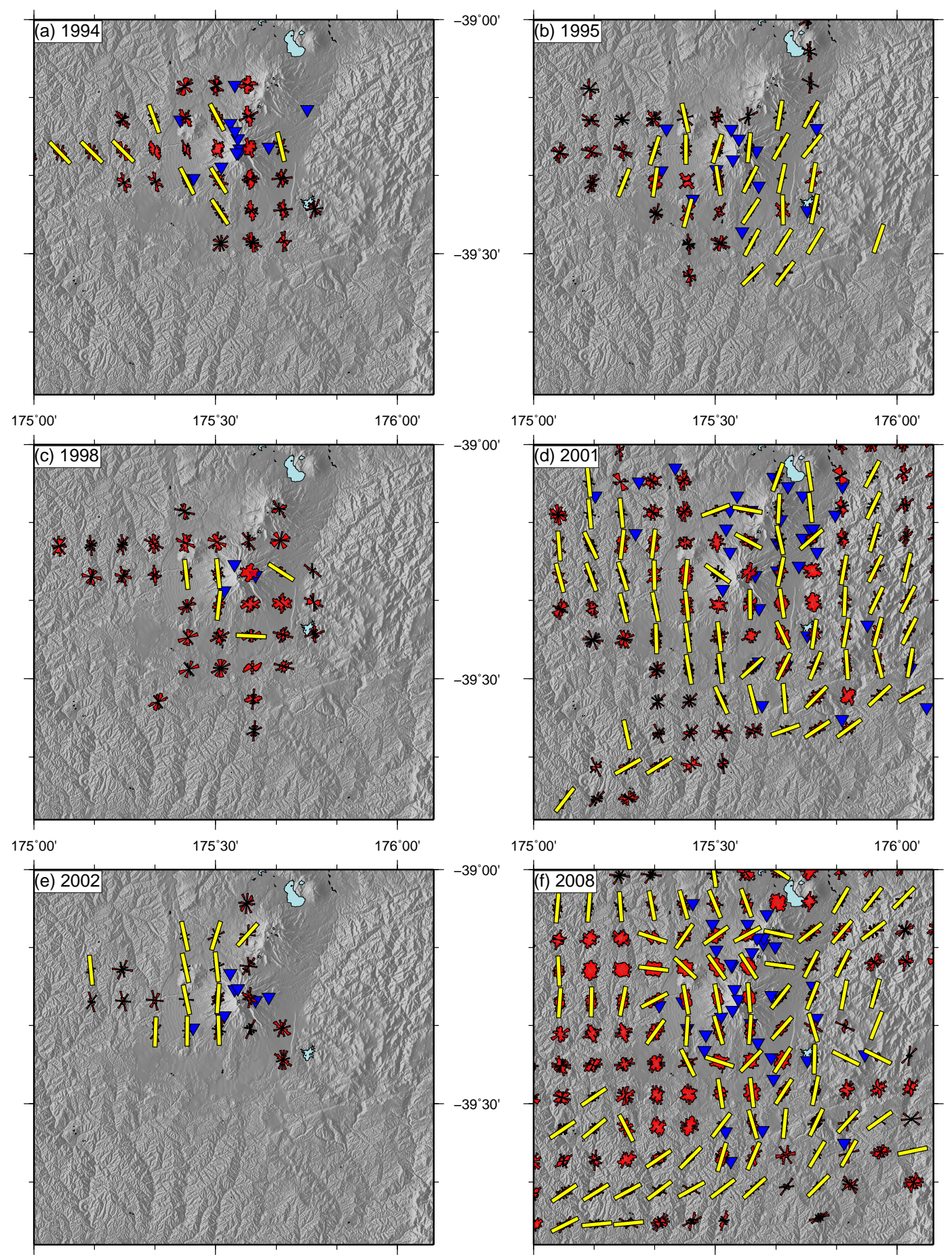

Figure 5.8. Maps of the spatial averaging of fast direction for the six temporary deployments. Red rose diagrams show all measurements in grid squares that contain more than 10 passing rays and yellow bars indicate the mean fast direction in each grid square when the standard deviation is less than $30^{\circ}$ and the standard error of the mean is less than $10^{\circ}$. 

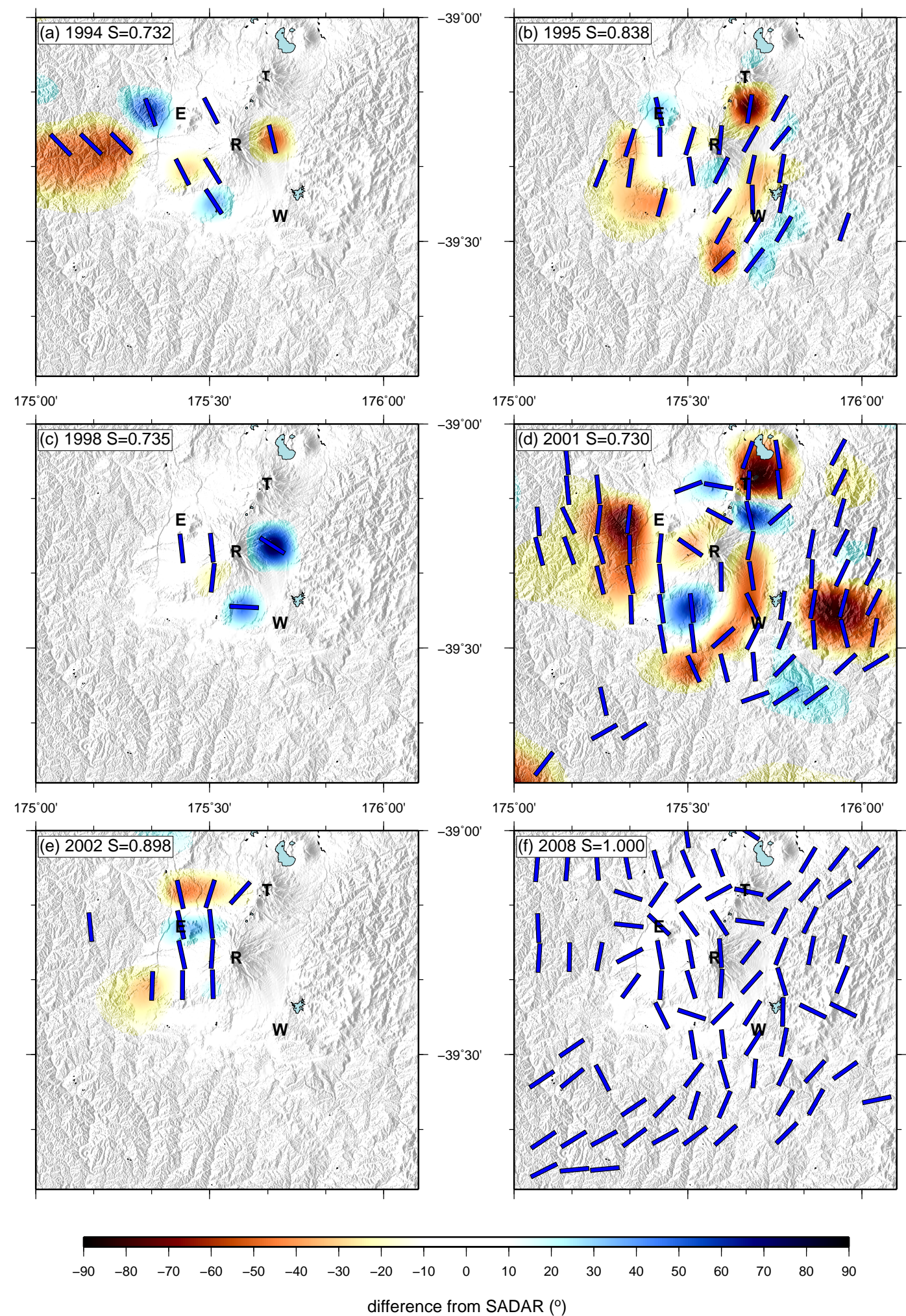

Figure 5.9. Maps of the spatial averaging of fast direction for the six temporary deployments compared to the 2008 deployment. Blue bars show the fast direction for the grid squares in each time period and the background colours represent the difference between that result and the results in 2008. The S value indicates the L1 norm fit between the two deployments. 

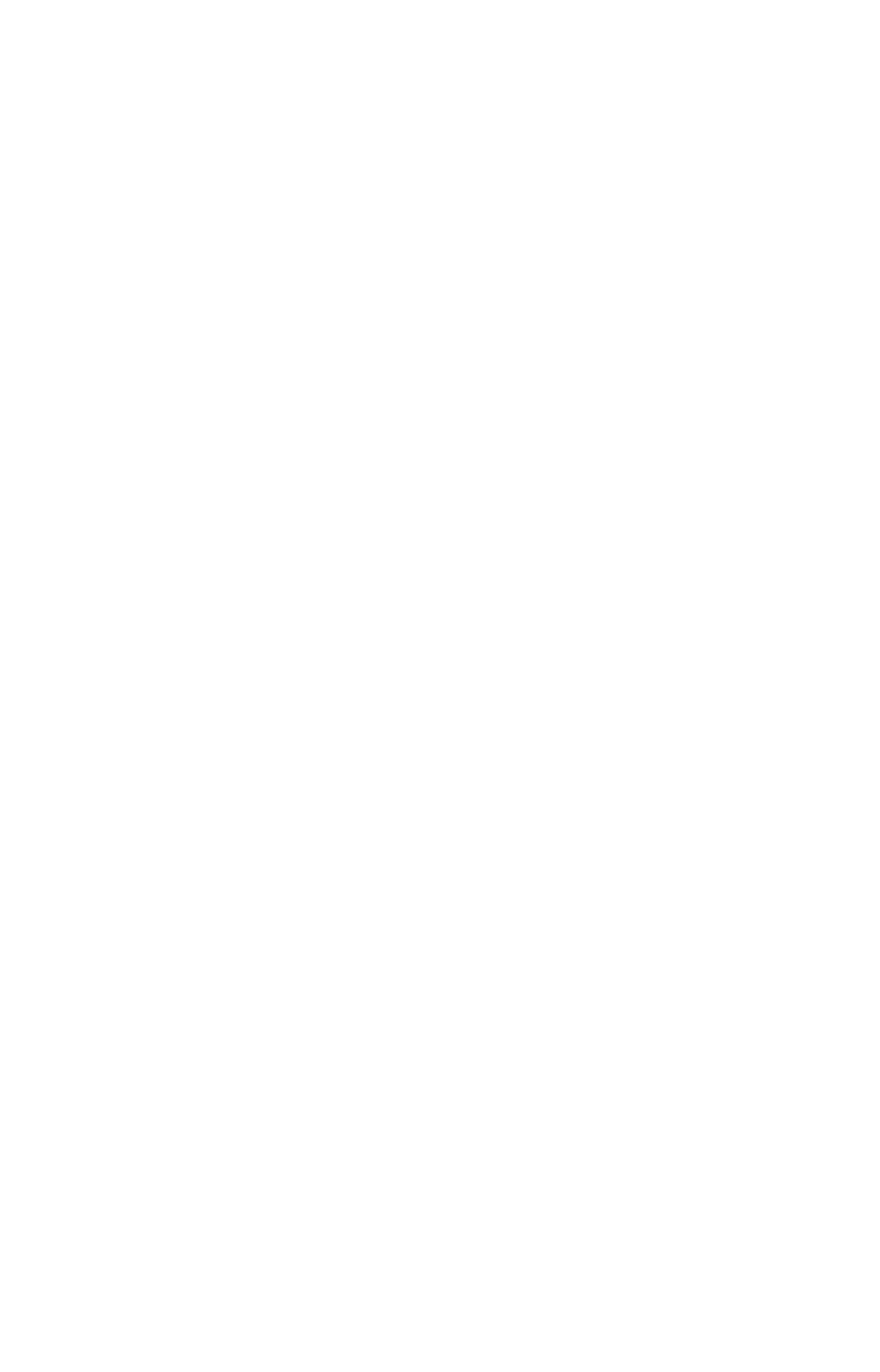

$\stackrel{\infty}{: 0}$

ज्ञ

in 0

$\sum_{\substack{\infty \\ \infty}}^{\infty}$

$\stackrel{\infty}{\Xi}$.

离

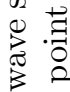

สี กิ

$\frac{-1}{n}$

앙

$\bigcup_{0}^{2}$,

80

宽

$\infty 0$ స

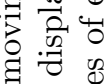

票寻

뇽

묨

$\bar{\theta}$

$\sigma$

is 운

贻

ฮิ ฮี

范

\%

㟧

可 풍

沓:

$\Xi$

$\dot{1}$.

s.

․ㅓㅇ

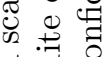

可

$\theta$ of 2

$\infty$

恶

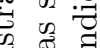

$\exists$ on

की สี

苛

๘ี

$\circ$ :

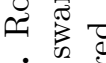

$\dot{0} \Xi$

10

要

.0. 
Figure 5.11. Cross-sectional schematic map of Tongariro Volcano geothermal system derived from magnetotelluric and DC resistivity measurements [Walsh et al., 1998]. Star indicates probable depths of low-frequency earthquakes, after Hagerty and Benites [2003].

Station FWVZ, which is the station that displayed the most significant variation using the Erua swarm [Keats et al., 2011], doesn't display any significant variation at the time of the eruptions using the Waiouru swarm. This observation agrees with the interpretation that the variations detected by Keats et al. [2011] were due to near-source effects and with the interpretation in Chapter 4, that the main region of anisotropy near the Waiouru swarm is governed by structural effects such as schistose mineral alignment and aligned fractures. The stations close to Mt. Tongariro also do not display significant variation with time, although the $\phi$ results are very scattered. The tomography results from Section 5.4.1 suggested that there was a difference in anisotropy here in 2008 but there is no evidence for such a change when using the continuous data since 2004. The fact that the stations around Mt. Tongariro do not show significant variation during the time that they have been in operation suggests that there was a change in anisotropy before 2004 .

Hagerty and Benites [2003] identified unusual low-frequency seismic events beneath Mt. Tongariro beginning in 2001. The location of the low frequency events was found to be related to the geothermal reservoir beneath Mt. Tongariro (Figure 5.11), suggesting that there was a change in the geothermal system at this time. This change in a system with high temperature $\left(>250^{\circ} \mathrm{C}\right)$ and pressure $(>35$ bar $)$ fluid is likely to have affected the anisotropy here.

\subsubsection{Shear wave splitting using multiplets}

The application of MFAST to the multiplets yields very little scatter in shear wave splitting parameters. However, the variation in noise content prompts the algorithm to select different filters for some of the earthquakes. This sometimes leads to a different splitting result. In general earthquakes in the same multiplet yield the same shear wave splitting parameters within uncertainties when the same filter is applied, regardless of the time window and S wave arrival pick (Figure 5.12). The results using the multiplets generally mirror the prominent direction gained by using the whole Waiouru cluster (Figure 5.10); there are exceptions however. In particular, the multiplets display two distinct modes at FWVZ, both of which agree with the modes 


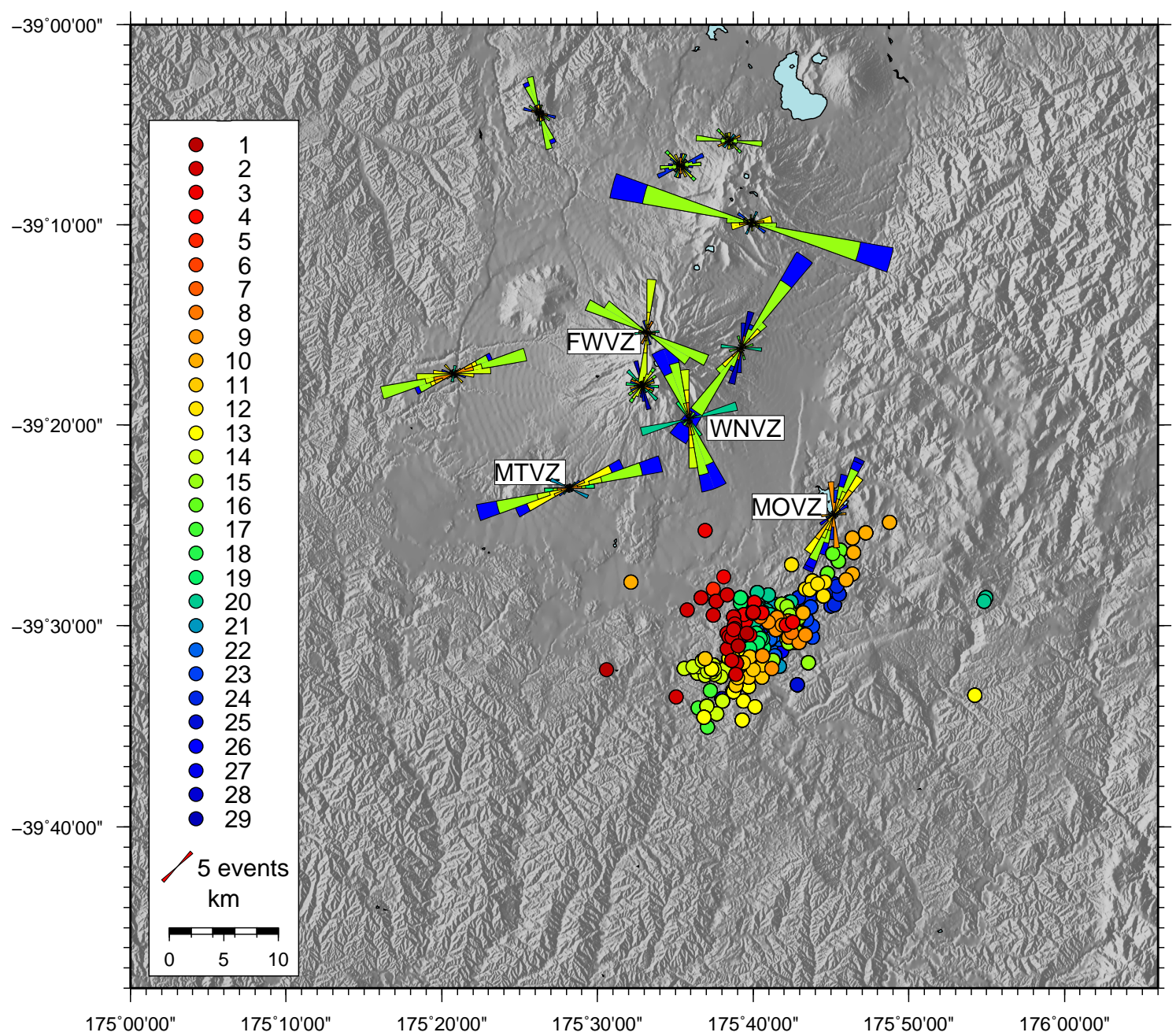

Figure 5.12. Rose diagrams showing shear wave splitting fast direction results using different multiplets (coloured dots) filtered at 1-5 Hz.

seen in the rose diagram in Figure 5.10, but neither of which shows a dominance, which is contrary to the results using the whole cluster. The two different modes could be due to a difference in source location, and hence raypath, or could be a result of a different source mechanism. Appendix F.1 lists the results displayed in Figures 5.12 and 5.13 .

The incoming polarisation is consistent for each multiplet at each station (Figure 5.13). This suggests either that there are two anisotropic layers, or simply that the mechanism of each earthquake within each multiplet is consistent. The mechanism of each earthquake within each multiplet is expected to be consistent as the criteria used to identify multiplets requires similarity of first motions. This, and the fact that there is no consistency between multiplets at the same stations, leads us to the conclusion that it is the latter of these; i.e. that each multiplet has a consistent 


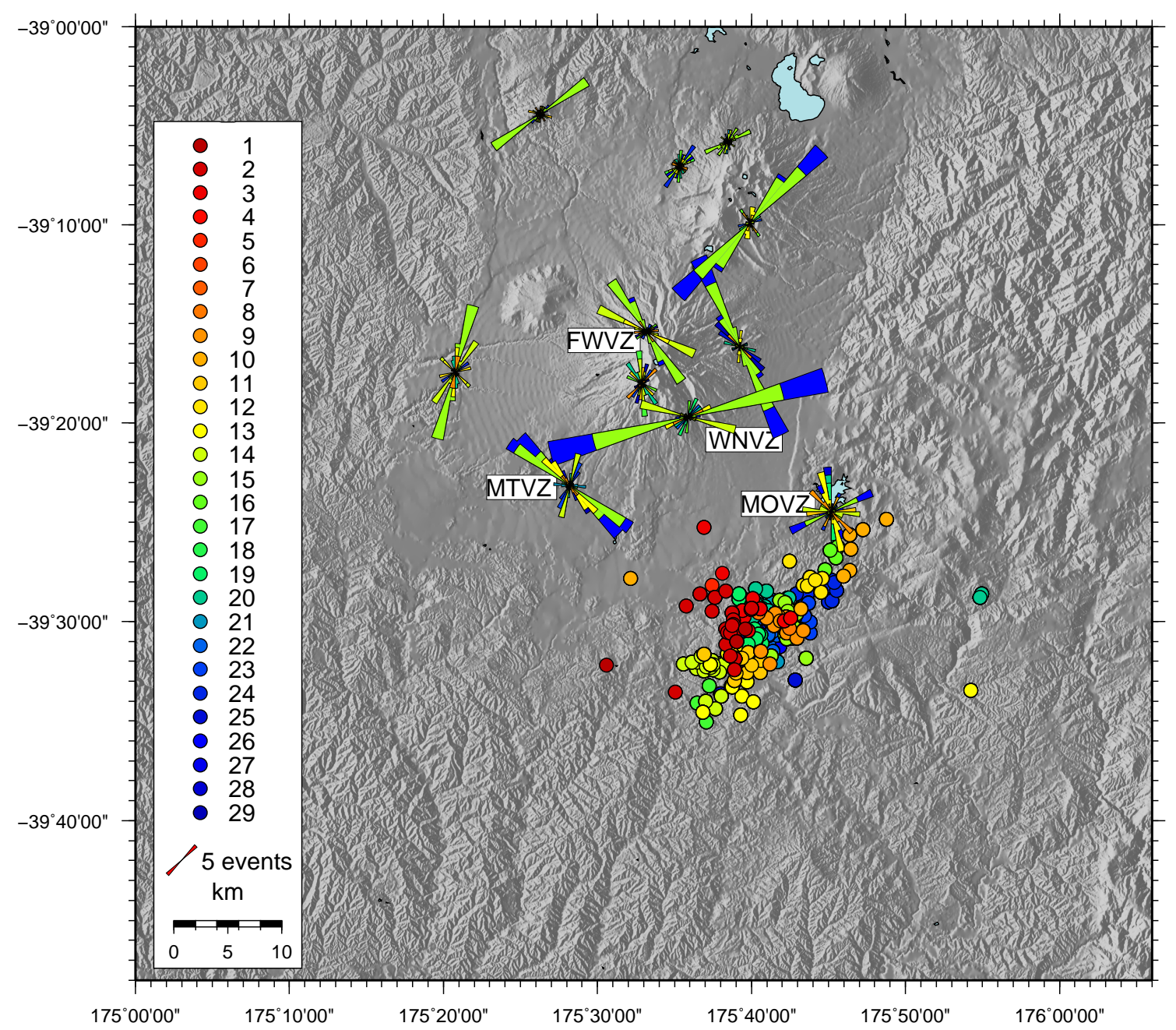

Figure 5.13. Rose diagrams showing shear wave splitting incoming polarisation of $\mathrm{S}$ waves produced by different multiplets (coloured dots).

earthquake mechanism and that there is one layer of anisotropy.

Figures 5.12 and 5.13 also show the catalogue locations of the multiplet earthquake epicentres. Some of the multiplets show up to $30 \mathrm{~km}$ of scatter from the mean cluster centroid. However, the multiplets were defined using a CCC of at least 0.95, meaning that we can assume the multiplet earthquakes are actually separated by no more than $\lambda / 4$ [Geller and Mueller, 1980] (see Section 2.4.2 for an explanation). We can therefore infer that the multiplet earthquakes are no more than $2 \mathrm{~km}$ apart, based on a lowest frequency of $1 \mathrm{~Hz}$, and maximum seismic velocity of $8 \mathrm{~km} / \mathrm{s}$. Earthquake relocation using the double-difference technique may be used to confirm this inference, however the relocation technique can generally relocate about $60-80 \%$ of events. This means that several earthquakes might be rejected.

Figure 5.14 displays the same data as Figure 5.12, plotted over time. Even though 
the fast directions are fairly stable through time, there are some steps in the results, particularly in the delay times.

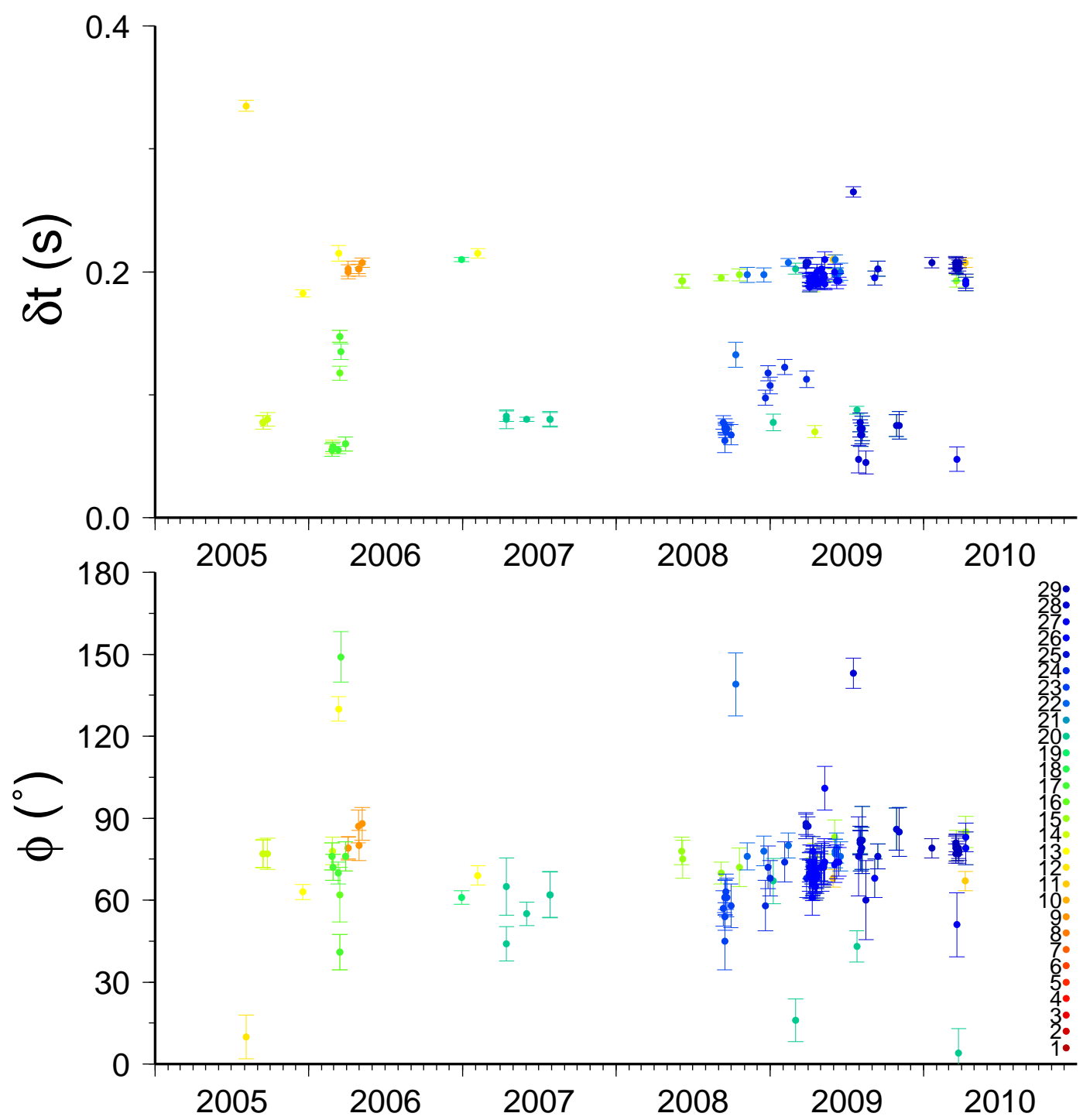

Figure 5.14. Fast direction $(\phi)$ and delay time $(\delta \mathrm{t})$ at station MTVZ over time. Results are colour coded by multiplet ID, error bars show $95 \%$ confidence interval. Results from $1-5 \mathrm{~Hz}$ filtered data.

\subsubsection{Scatterers}

Examination of the waveforms revealed that some of the multiplets displayed a change later in the coda. Figure 5.15 shows one such example using multiplet 14. The other multiplets are displayed in Appendix F. Earthquakes were selected as the same multiplet based of the similarity of the $\mathrm{P}$ coda. The $\mathrm{S}$ coda is markedly different between earthquakes 3 and 4 even though the $\mathrm{P}$ coda is very similar and the relative arrival times of the two waves is the same. The cross-correlation matrix 


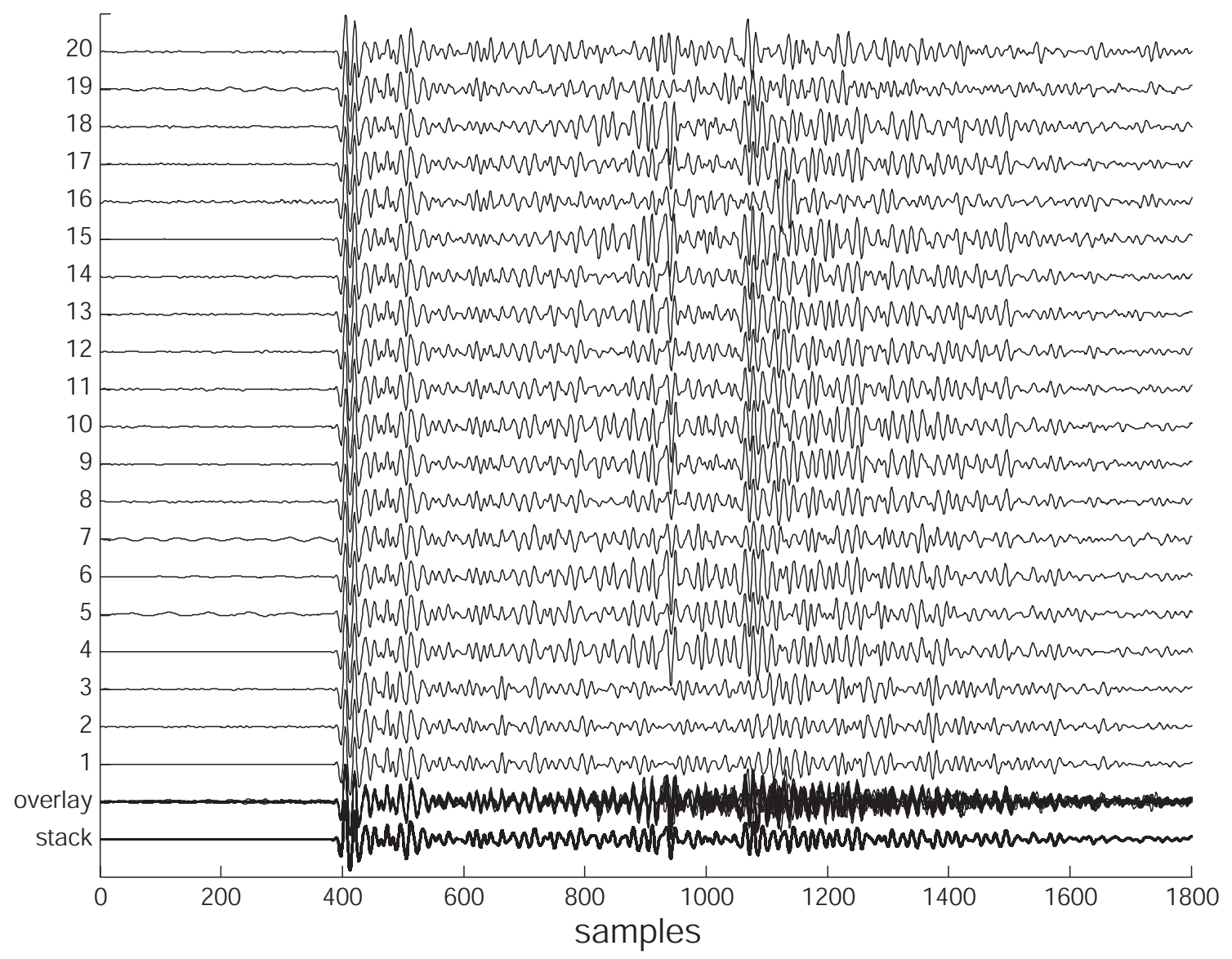

Figure 5.15. Waveforms of earthquakes belonging to multiplet 14, recorded on the vertical component at station TUVZ sampled at $100 \mathrm{~Hz}$ and filtered 1-10 Hz. Bottom trace shows a stack of all of the traces and the second to last trace is all other traces overlaid. A change in coda can be seen between events 3 and 4 . This is discussed in the text and is illustrated further in Figure 5.16 .

for the entire waveform (200 samples before to 2000 samples after the origin) is displayed in Figure 5.16, and confirms that the first three earthquakes differ from the others. The stars on Figure 5.4 display the times at which similar changes occur within other multiplets, where changes are defined as a cross-correlation coefficient of the whole waveform of 0.5 or less. The times are plotted at the mid-point between the times of the earthquakes that change, but the change could have occurred at any time between the earthquakes. All of the waveform changes occur around the times of eruptions.

We suggest that the change in the waveforms from the multiplet earthquakes was due to the alteration of a scatterer or scatterers associated with or caused by the eruptions. Similar observations have been made by Taira et al. [2008] using a well know repeating earthquake source in California. The arrivals associated with the scatterers that we observe often arrive at the stations at a similar time to the $\mathrm{S}$ wave 


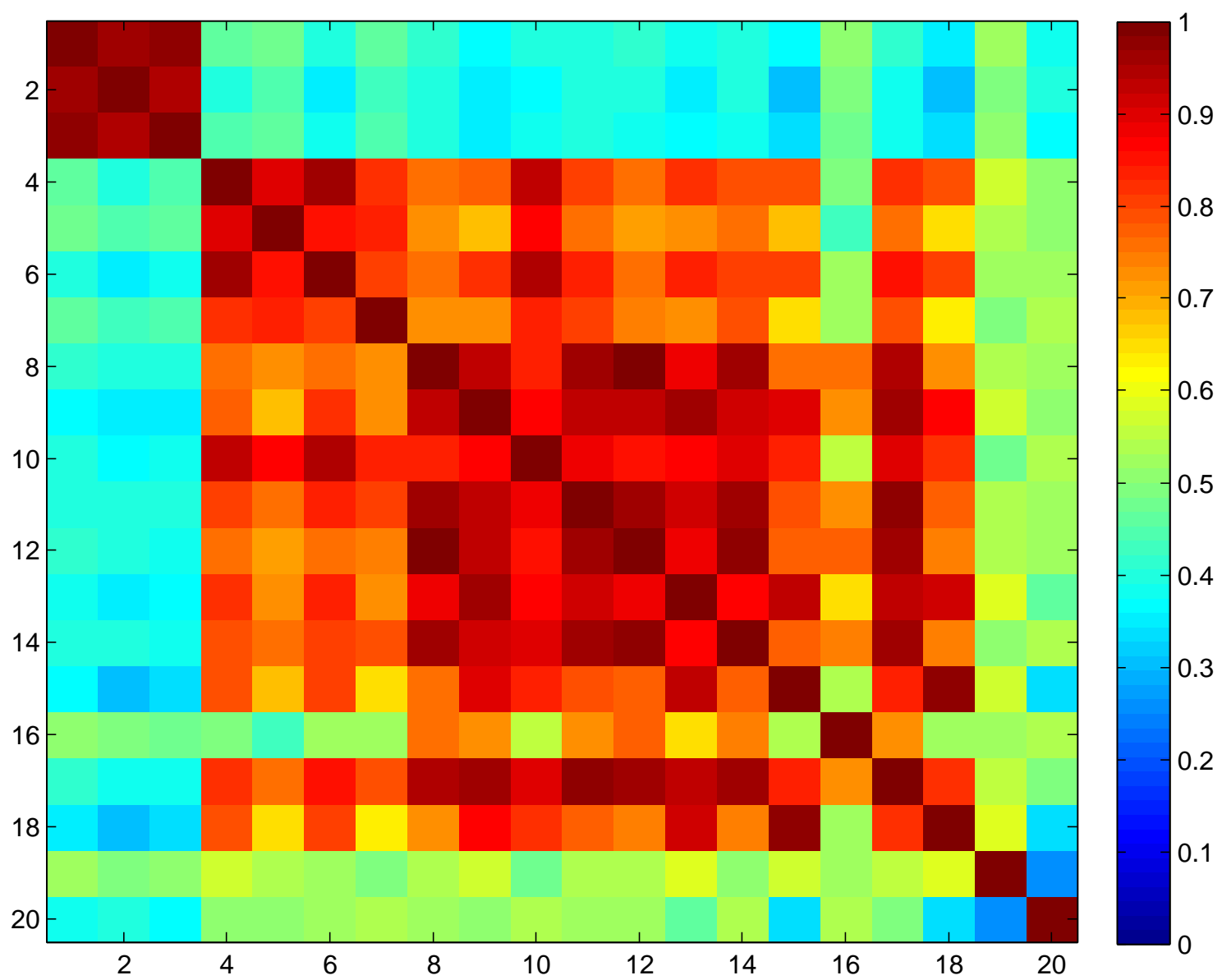

Figure 5.16. Cross-correlation matrix for multiplet 14 using the whole waveform, recorded on the vertical component at station TUVZ and filtered 1-10 Hz. Colours represent cross-correlation coefficient $(C C C)$. $C C C$ for the portion of the coda used to identify the multiplets would result in a solid red block, as they are all above 0.95. A change in $C C C$ in this plot using the whole waveform can be seen between events 3 and 4, which can also be seen in Figure 5.15, and is discussed in the text.

arrival, and therefore change the $\mathrm{S}$ coda, causing the automatic algorithm to select different shear wave splitting parameters. In Chapter 6 we see a similar behaviour of a scattered phase using a multiplet at Okmok volcano, Alaska. It was found that there were two populations within the multiplet, both giving the same fast direction, but the delay times fell into two clusters. Examination of the waveforms revealed an extra pulse of energy preceding the $\mathrm{S}$ wave arrival seen when the components were rotated into fast and slow directions. The pulse was interpreted to originate from a $\mathrm{P}$ to $\mathrm{S}$ conversion due to a scatterer along the raypath. Other multiplet cross-correlation matrices (e.g. Appendix F, multiplets 5, 9 and 19) potentially show migration or healing as the waveforms become more, or less similar over time. Further work, including tracking individual scatterers, could help to understand the evolution of these multiplets. 


\subsection{Conclusions}

We have shown that carrying out spatial averaging of shear wave splitting parameters can minimise the problem of misinterpretation of spatial variation as temporal variation. While this method can be used on sparse networks, the uncertainties associated with it grow larger due to a lack of crossing rays and so it is not practical unless there is a dense permanent network. This method would also not be employable as a real time monitoring tool because the inversion requires raypaths over the whole area of investigation. Therefore the results are averaged over the time of the deployment. A potential near-real time technique could use three-month moving windows, as long as the permanent network is well populated.

Investigation of shear wave splitting using clusters of earthquakes has been shown to be a robust way of monitoring temporal changes. This method is more adaptable to near-real time monitoring because as long as there are earthquakes in the cluster, measurements can be carried out. However, using a cluster of earthquakes creates some scatter in the shear wave splitting parameters, which could be because of the slight variation in earthquake location and source mechanism. Therefore statistical tests would have to be carried out to ensure the significance of any changes observed, which may result in a delayed diagnosis.

Multiplets ensure that changes in shear wave splitting parameters are not an artifact of varying source mechanism or location. The use of multiplets as a tool for monitoring temporal changes in anisotropy is complex. An understanding of the whole wavetrain is needed so that scatterers can be identified and not erroneously matched in the shear wave splitting algorithm. Understanding the scatterers themselves, and their temporal evolution, could be useful for the monitoring of volcanoes, but in shear wave splitting analysis they are unwanted noise. A gradual stretching or compression of the multiplet wavetrain, such as was seen by Schaff and Beroza [2004] in California, may yield a gradual change in anisotropy although we do not observe that type of behavior at Mt. Ruapehu. Baisch and Bokelmann [2001] observed reduced correlation in multiplets immediately following the Loma Prieta earthquake in California, and a healing over 5 years. Further work will investigate whether the scatterers that we observe associated with the 2006 and 2007 eruptions exhibit similar behavior, and whether healing can be observed in a gradual change in shear wave splitting parameters. We conclude that the use of multiplets has potential for both detection of temporal changes in anisotropy, and for monitoring the evolution of scatterers, but more work is needed to understand the whole wavetrain. 
The occurrence of earthquakes that belong to multiplets, and the existence of multiplets at all cannot be guaranteed at volcanoes. This method could be used in hindsight to observe stress changes but would be difficult to employ as a real-time monitoring tool unless the multiplet earthquakes were well understood [e.g. Rubinstein and Ellsworth, 2010].

Currently, the recommendation for the best method for tracking changes in anisotropy at Mt. Ruapehu is to use the anisotropy tomography estimation and spatial averaging with three-month moving windows. These windows could be adjusted and the method could be updated so that a test for a minimum number of crossing rays or area of resolution could be performed before each inversion. When combined with the monitoring of anisotropy parameters using clusters of earthquakes, this method may provide a mid-term monitoring tool. 



\section{Anisotropy, repeating earthquakes and seismicity associated with the 2008 eruption of Okmok volcano, Alaska}

Johnson, J. H., S. Prejean, M. K. Savage \& J. Townend (2010) Anisotropy, repeating earthquakes, and seismicity associated with the 2008 eruption of Okmok volcano, Alaska. J. Geophys. Res., 115, B00B04, doi:10.1029/2009JB006991.

\section{Abstract}

We use shear wave splitting (SWS) analysis and double-difference relocation to examine temporal variations in seismic properties prior to and accompanying magmatic activity associated with the 2008 eruption of Okmok volcano, Alaska. Using bispectrum cross-correlation, a multiplet of 25 earthquakes is identified spanning five years leading up to the eruption, each event having first motions compatible with a normal fault striking NE-SW. Cross-correlation differential times are used to relocate earthquakes occurring between January 2003 and February 2009. The bulk of the seismicity prior to the onset of the eruption on 12 July 2008 occurred southwest of the caldera beneath a geothermal field. Earthquakes associated with the onset of the eruption occurred beneath the northern portion of the caldera and started as deep as $13 \mathrm{~km}$. Subsequent earthquakes occurred predominantly at 3 $\mathrm{km}$ depths, coinciding with the depth at which the magma body has been modelled using geodetic data. Automated SWS analysis of the Okmok catalogue reveals radial polarisation outside the caldera and a northwest-southeast polarisation within. We interpret these polarisations in terms of a magma reservoir near the centre of the caldera, which we model with a Mogi point source. SWS analysis using the 
same input processing parameters for each event in the multiplet reveals no temporal changes in anisotropy over the duration of the multiplet, suggesting either a short-term or small increase in stress just before the eruption that was not detected by GPS, or eruption triggering by a mechanism other than a change of stress in the system.

\subsection{Introduction}

Modern geophysical techniques have been used successfully to observe changes at some volcanoes before magmatic eruptions. Observations include increases in seismicity [McNutt, 1996], long-period and very-long-period earthquakes and tremor [Chouet, 1996; Neuberg et al., 2000], changes in tremor frequency [Powell and Neuberg, 2003] and attenuation [Del Pezzo et al., 2004], migrating earthquake sources [Roman et al., 2006] and ground deformation [Lu et al., 2005; Wadge et al., 2006; Palano et al., 2008]. There is commonly ambiguity in the interpretation of these observations, and at other volcanoes these phenomena have not yet been seen at all. There is therefore a need for multiple techniques, measuring different physical attributes, in order to monitor changes in the Earth's crust linked to eruption processes. Any overpressured magma storage system, be it a system of dikes, sills, conduits, volumetric chamber or a combination of these, will exert a stress on the surrounding country rock that may or may not be manifested as observable surface strain [Gudmundsson et al., 2009]. Determining and understanding the local state of stress may be key to predicting if and when a volcano will erupt.

Shear wave splitting (SWS) analysis around volcanoes can in principle be a useful indicator of maximum horizontal stress direction in the crust and, hence, the pressure induced by magma movement [Bianco et al., 1998], but spatial variations can be misinterpreted as temporal changes [Peng and Ben-Zion, 2004; Townend and Zoback, 2006]. For this reason, we introduce a method of detecting temporal variations in seismic anisotropy at active volcanoes by analysing highly correlated repeating earthquakes. This approach ensures that any changes in splitting parameters can be interpreted to stem from changing properties of the medium over time rather than changes in the source or the path geometry and can be compared to surface strain measurements if suitable data are available. Okmok volcano on Umnak Island, Alaska (Figure 6.1), was chosen as a case study because there is a network of three component seismometers, a good time series of the deformation throughout the eruptive cycle [Lu and Dzurisin, 2010; Freymueller and Kaufman, 2010] and, 
upon further inspection, repeating earthquakes with impulsive phase arrivals. The bispectrum cross-correlation technique of Du et al. [2004a] with high correlation thresholds is used here to identify sequences of repeating earthquakes at Okmok volcano spanning six years (from January 2003 to February 2009) prior to and just after the recent eruption near Cone D in the caldera. We use double-difference relocation [Waldhauser and Ellsworth, 2000] to determine the hypocentres of the earthquakes and to elucidate the structure in the co-eruptive seismicity throughout the caldera. An automated shear wave splitting analysis [Savage et al., 2010b] is then applied to the Alaska Volcano Observatory (AVO) Okmok earthquake catalogue to obtain a general overview of the seismic anisotropy in the region. Finally, fixed input parameters are used on the repeating events for the shear wave splitting analysis in order to treat each event as the same source and estimate bounds on changes in anisotropy over the time period [Liu et al., 2008].

\subsubsection{Background}

Okmok volcano (Figure 6.1) is part of the Aleutian Volcanic Arc, a chain of more than 40 active volcanoes representing the surface magmatic expression of the subduction of the Pacific Plate as it moves northward beneath the North American Plate [Packer et al., 1975]. Since written records began 250 years ago, Okmok has been one of the most active caldera systems in North America with eruptions occurring every 10-30 years [Miller et al., 1998]. Geologic evidence suggests that there have been multiple explosive phreatomagmatic eruptions since the last caldera-forming eruption 2050 years ago [Begét et al., 2005].

The most recent eruption of Okmok volcano, which occurred just north of Cone D (Figure 6.1), commenced on 12 July 2008. This 2008 eruption had a notable lack of geophysical precursors. Geodetic data indicate that the caldera inflated continuously between 1997 and 2005 [Fournier et al., 2009]. Subsequently, the caldera was geodetically quiet between 2005 and early 2008, when renewed inflation was detected. There was however no notable increase in inflation rate prior to eruption onset [Freymueller and Kaufman, 2010]. Similarly, there was no indication of the incipient eruption in seismic data other than a 5 hour precursory sequence of earthquakes (Figure 6.2). The first earthquake in the sequence, which was too small to be located, occurred at 14:36 UTC on 12 July 2008. During the following 3 hours, small detectable earthquakes occurred at a rate of 5-15 events per hour. At approximately 18:32 UTC, in the final hour preceding eruption, the rate of earthquakes 


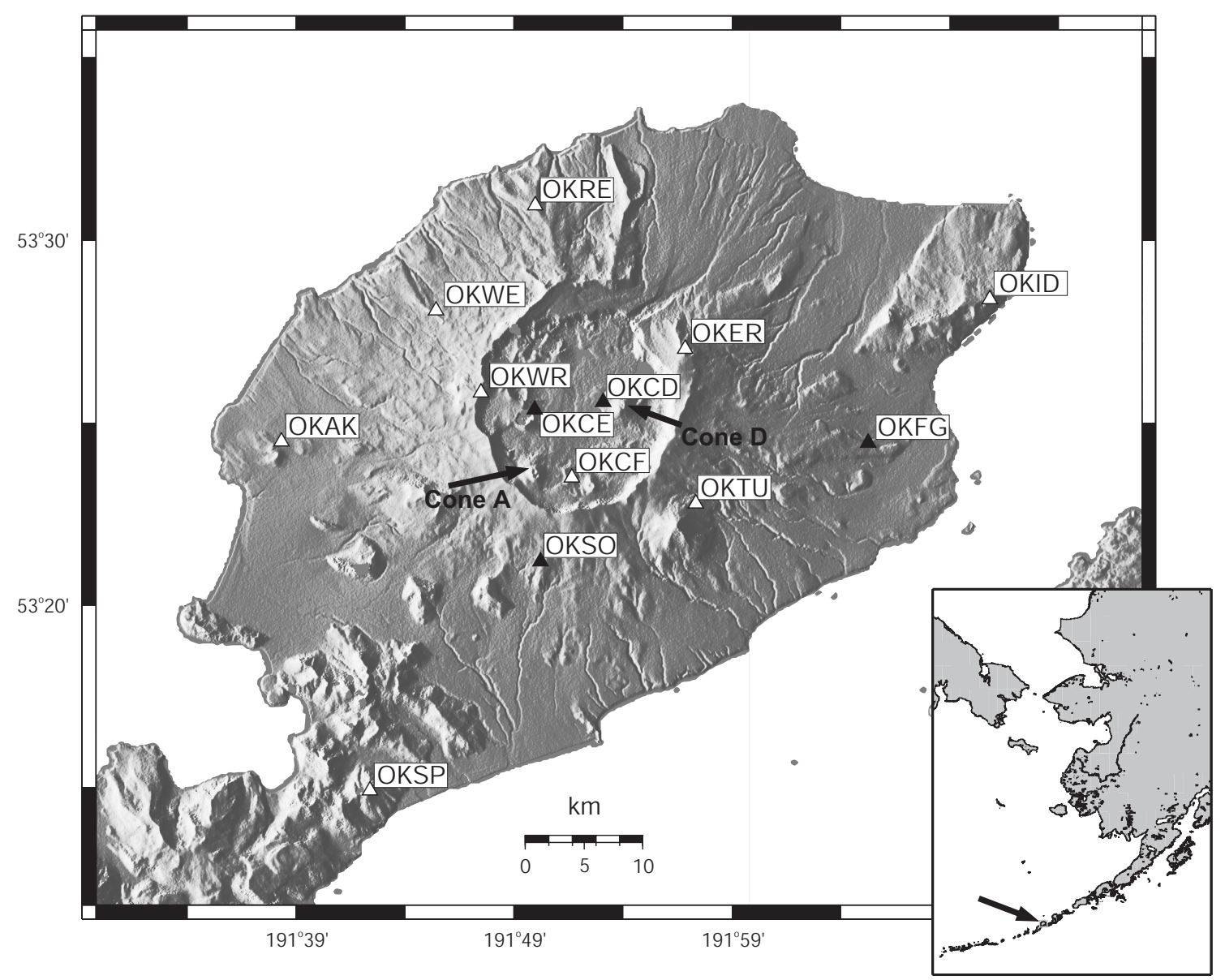

Figure 6.1. Digital elevation model from NASA's SRTM of the northeast of Umnak Island with Okmok caldera, showing vertical-component short-period seismometer stations (empty triangles) and 3-component broadband seismometer stations (filled triangles) of the Alaska Volcano Observatory network. Cone A is the location of previous historic eruptions. Cone $\mathrm{D}$ is close to the location of the 2008 eruption. Inset shows the location of Umnak Island in the Aleutian Arc, Alaska.

increased sharply, causing a rapid-fire sequence of events, or spasmodic burst, which merged into tremor at approximately 19:30 UTC as the eruption initiated. The entire pre-eruptive sequence consisted of 56 locatable earthquakes with a magnitude of greater than 1 [see AVO earthquake catalogue, Dixon and Stihler, 2009]. The largest earthquake was M 2.4 and occurred at 19:24 UTC on 12 July 2008. Spectra indicate that the pre-eruptive events were primarily brittle failure earthquakes rich in high frequency energy, though small numbers of long-period (LP) and very-longperiod (VLP) events also occurred. Although periodic episodes of volcanic tremor were commonly observed at Okmok in 2003-2005, no tremor was observed in 2008 prior to the eruption. The eruption itself was predominantly phreatomagmatic and lasted five weeks. 


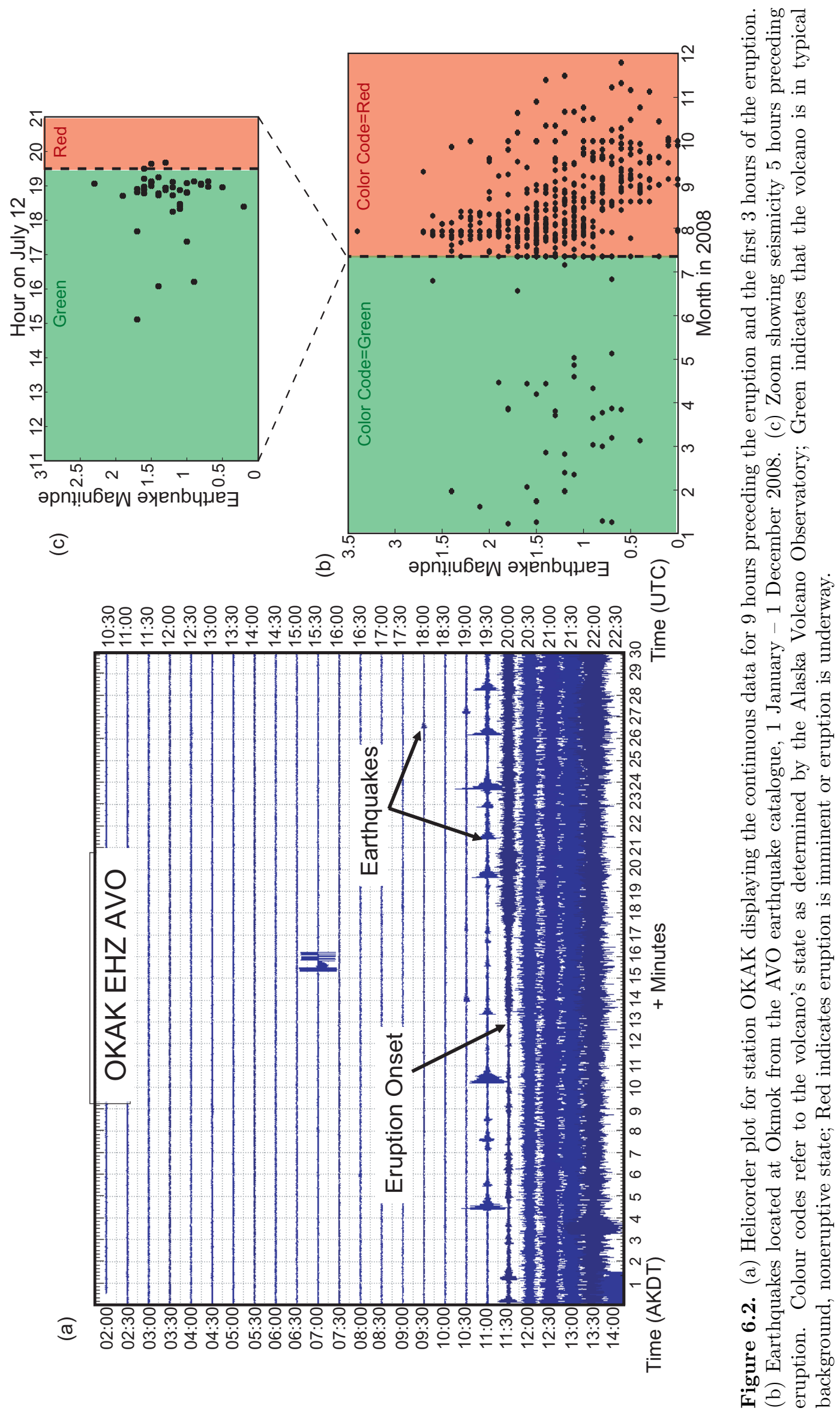


The initiation of the eruption was accompanied by more than 10 hours of strong continuous tremor, which masked smaller discrete earthquakes (Figure 6.2). The co-eruptive sequence consisted of 260 locatable earthquakes, of which $80 \%$ were located within the caldera. Haney [2010] has analysed the co-eruptive VLP tremor and found it to have occurred NNW of Cone D, close to the new cone built by the 2008 eruption.

The previous eruption occurred in 1997 when Cone A in the caldera was active for nearly four months, repeatedly erupting low ash clouds and producing a voluminous lava flow [Patrick et al., 2004]. Eruptions in 1945 and 1958 also occurred at Cone A [Larsen et al., 2009]. However re-evaluation of historical accounts of activity at Okmok combined with geologic mapping and tephra studies [Neal et al., 2003] suggest that other historic eruptions have been more complex, with more violent eruptions from other vents within the caldera affecting all flanks of the volcano.

Inflation prior to the 1997 eruption of Okmok had been ongoing since 1993, and has been modelled using Interferemetric Synthetic Aperture Radar (InSAR) data as a Mogi point source [Mogi, 1958] 2.7-3.2 km below sea level (bsl) in the centre of the caldera [Lu et al., 2000, 2005]. Lu et al. [2000, 2005] also modelled the deflation measured during the 1997 Cone A eruption as a Mogi source at similar depth, also beneath the centre of the caldera. They found the subsidence during the 1997 eruption to equate to a volume change in the magma reservoir of about $0.048 \mathrm{~km}^{3}$ [Lu et al., 2000, 2005].

Deformation since 1997 detected by GPS and InSAR showed an inflation that was rapid up to 2000, slowed from 2000 to 2001, and then increased in rate again until 2002 [Miyagi et al., 2004]. The deformation has been interpreted by Miyagi et al. [2004] as a charging magma body with varying magma flux, and has been modelled using a source similar to that of Lu et al. [2000, 2005]. Inflation is inferred from GPS data to have continued between 2001 and mid-2004, but there was little deformation between 2005 and 2007 [Fournier et al., 2009] or between 2007 and 2008 [Freymueller and Kaufman, 2010], which suggests that magma influx slowed. Masterlark et al. [2010] used ambient noise tomography to refine the location of the magma reservoir and reported a low-velocity zone, indicating the presence of a magma reservoir at greater than $4 \mathrm{~km}$ depth, which is significantly deeper than previous geodetic estimates. Masterlark et al. [2010] also constructed finite element models for the deformation to account for the complexity of the subsurface not included in conventional half-space models. This enabled them to reduce the magma reservoir's 
depressurisation to within lithostatic constraints while retaining the magnitude of deformation observed by InSAR.

In the period between January 2003 and February 2009, 991 earthquakes near Okmok were recorded on seismic stations of the AVO and the Alaska Earthquake Information Center (AEIC) networks along the Aleutian chain. Approximately half of the earthquakes recorded during the six years occurred between 12 July 2008 and 1 February 2009 and a third of all the seismicity was associated with the 2008 eruption. We have used local events for the SWS analysis so that there is less ambiguity in the origin of the anisotropy. Events deeper than about $20 \mathrm{~km}$ are not generally catalogued by the AVO and we have therefore focussed this study on local upper crustal events. The AVO Okmok network, installed in 2003, consists, at the time of writing, of four three-component broadband and nine vertical-component short-period seismometers spanning the caldera (Figure 6.1). The seismic stations are complemented by campaign GPS and four continuous GPS sites [Fournier et al., 2009] located at the three-component seismometer stations (Figure 6.1, filled triangles), and satellite coverage including InSAR.

\subsubsection{Seismic anisotropy}

Shear wave splitting occurs when a shear wave travels through a seismically anisotropic medium, i.e. one in which seismic waves travel faster in one direction or with one polarisation than another. In the Earth's upper crust, anisotropy is most likely to be caused either by horizontal stress closing microcracks perpendicular to the maximum compressive stress [Crampin, 1994] or by pervasive structural features. For a near-vertical propagation direction, the shear wave with the displacement in the plane of the open cracks will travel faster than that crossing the plane of the cracks, and so a fast shear wave with orientation $\phi$, and a slow shear wave orthogonal to $\phi$, separated by a delay time $\delta t$, will be observed [Babuška and Cara, 1991]. Crack-induced anisotropy has in some studies been considered a direct indicator of present-day stress [Nur, 1971; Crampin, 1994; Savage, 1999], with $\phi$ providing information about the orientation of maximum horizontal stress and $\delta t$ giving information about the strength of anisotropy and the amount of time that the wave spent traversing the anisotropic medium. Boness and Zoback [2006a] compared shear wave splitting measurements in California to results obtained using other methods of determining maximum horizontal stress such as focal mechanism 
inversions and borehole breakouts, and concluded that shear velocity anisotropy parameters from local earthquakes are generally valid proxies for maximum horizontal stress. Other studies that combine the aforementioned stress estimation methods with shear wave splitting results have found that strong geological fabric or aligned structures rather than the maximum stress can govern the observed $\phi$ in some situations [Balfour et al., 2005; Audoine et al., 2000; do Nascimento et al., 2004]. Zinke and Zoback [2000] detected both stress-induced and structure-induced anisotropy at a single station near the Quien Sabe fault system in California. Miller and Savage [2001] and Gerst and Savage [2004] observed a change in $\phi$ around Mt. Ruapehu volcano in New Zealand and interpreted it as stress changes due to a pressurised dike beneath the volcano. Savage et al. [2010a] observed strong correlations between shear wave splitting parameters and GPS baseline length changes at Asama volcano, Japan.

We consider azimuthal anisotropy caused by structure to be unlikely in the case of Okmok volcano. The entire edifice of Okmok is composed of massive, sub-horizontal, layered lava flows, which are tens of meters thick and within each flow the structure is chaotic [C. Neal, pers. comm.]. Thus, at the wavelengths of seismic waves $(\sim$ $1 \mathrm{~km}$ ), there are likely to be few consistent non-horizontal foliations. We have therefore assumed that crack-induced anisotropy is the dominant cause of shear wave splitting observations at Okmok. At Okmok the regional maximum horizontal stress is estimated to be approximately northwest-southeast [Nakamura and Uyeda, 1980] due to the convergence of the plates. A deviation from that orientation would suggest a different local stress field overprinting the regional one.

Liu et al. [2004] showed that spatial variations of anisotropy can be erroneously mapped into temporal changes. For this reason, we have used repeating earthquakes so that any changes in splitting parameters can be assumed to be the result of varying properties within the medium over time, rather than changes in the source or the path geometry [Liu et al., 2008]. Repeatable sources of seismic energy have been used to study changes in seismic velocity along common propagation paths in the Earth's crust for over a century [Reasenberg and Aki, 1974, and references therein]. Poupinet et al. [1984] used microearthquake doublets to monitor stress around the Calaveras Fault in California. They defined a doublet as a pair of earthquakes having nearly identical waveforms and the same hypocentre and magnitude but occurring at different times. Poupinet et al.'s [1984] decision to use doublets was influenced by the fact that earthquake sources are richer in shear wave energy than explosions and it is this same property that makes repeating earthquakes attractive in this 
study. Having two earthquakes with seismograms that are highly similar requires very similar source parameters (hypocentre and moment tensor) and near-uniform seismic properties along the propagation path [Poupinet et al., 1984]. Doublets or multiplets (more than two events) are typically identified on the basis of waveform similarity, determined by cross-correlation [Schaff and Richards, 2004]. Another hurdle that must be overcome in shear wave splitting analysis is that the results can be heavily frequency-dependent [e.g. Marson-Pidgeon and Savage, 1997]. Boness and Zoback [2006b] also showed that the polarisation of the shear waves and the amount of anisotropy recorded are strongly dependent on the frequency and length scale of investigation. Using repeating earthquakes ensures similar raypath lengths and using the same filter on each repeated earthquake ensures that we are sampling the same features with each event.

\subsection{Cross-correlation}

\subsubsection{Method}

Cross-correlation using the Bispectrum Cross-correlation package for SEISmic events (BCSEIS, Du et al. [2004a], see below) has been performed in this study to identify multiplets and to assist in the relocation of hypocentres (see Section 6.3.1). BCSEIS cross-correlates both raw and band-pass filtered waveforms and then verifies (selects or rejects) the estimated time delay by comparing the result to that from cross-correlation in the third-order spectral domain. This makes the method less sensitive to Gaussian noise than cross-correlation delay time estimates alone $[D u$ et al., 2004a].

A Hanning tapered band-pass filter between 1 and $10 \mathrm{~Hz}$ was used on all 991 events in our data set. Our results agree with observations made by Du et al. [2004a]; choosing a different filter in the range $0.5-5 \mathrm{~Hz}$ to $5-20 \mathrm{~Hz}$ was not found to change the final results appreciably.

The BCSEIS algorithm gives the maximum cross-correlation coefficient and the corresponding time delays of event pairs at each station (absolute cross-correlation coefficients are given between 0 and 1, 1 being perfect correlation). In this study multiplets were identified by having a $\mathrm{P}$ wave cross-correlation coefficient of more than 0.95 at three or more stations; if one or more of the stations showed a crosscorrelation coefficient of more than 0.98 then the threshold at the other stations was 
lowered to 0.85. These criteria have been adapted based on studies by Shearer [1997] and Du et al. [2004a], although the thresholds are higher in this study. Choosing only those events with a high cross-correlation coefficient ensures extremely similar waveforms [Hemmann et al., 2003; Igarashi et al., 2003; Du et al., 2004b; Pandolfi et al., 2006]. A higher correlation in the $\mathrm{P}$ waveforms than in the $\mathrm{S}$ waveforms was sought because the nature of this study required the inclusion of $\mathrm{S}$ waveforms that change over time. The cross-correlation analysis used the vertical-component because the temporal changes observed in shear wave splitting were expected to be more pronounced in the horizontal components of the $\mathrm{S}$ waveform.

Schaff et al. [2004] tested a variety of window lengths and found that although smaller windows generally produced higher-similarity measurements than longer ones they also produced larger residuals after double-difference relocation. We used a window of $2.54 \mathrm{~s}$ around the $\mathrm{P}$ wave, making a total of 254 sample points with 30 of them before the $\mathrm{P}$ arrival. This window was large enough to contain several cycles of the seismic signal, producing precise delay time measurements, and to yield events with highly similar waveforms (Figure 6.3). A longer time window of $3.82 \mathrm{~s}$ was used for the $\mathrm{S}$ wave calculation to accommodate the uncertainty associated with the catalogue $\mathrm{S}$ arrival picks and the longer periods of the $\mathrm{S}$ waves. This corresponded to 382 sample points, 50 of them before the picked $\mathrm{S}$ arrival. Where there were no catalogue picks, we used the TauP toolkit [Crotwell et al., 1999] with a fixed P to S wave velocity ratio of 1.78 [Masterlark et al., 2010] to estimate the phase arrivals before performing cross-correlation. Estimating $\mathrm{P}$ and $\mathrm{S}$ picks should enable the true arrivals to be identified by cross-correlation if the true pick is within the crosscorrelation time window, otherwise the resulting low cross-correlation coefficient should ensure that the estimated pick is not included in further analyses. However, the vertical component of the waveforms sometimes include spurious phases such as S-P conversions (a discussion of which follows in Section 6.5.3) creating erroneous picks. The erroneous picks were found to bias the relocation results and therefore only the catalogue picks were included in the analyses. Our cross-correlation analysis produced a total of 3,721,517 $\mathrm{P}$ wave waveform-based differential times.

\subsubsection{Results}

The cross-correlation analysis revealed three distinct multiplets and approximately 50 doublets with cross-correlation coefficients exceeding the thresholds stated in Section 6.2.1. After examination of the multiplets, the largest was chosen for further 

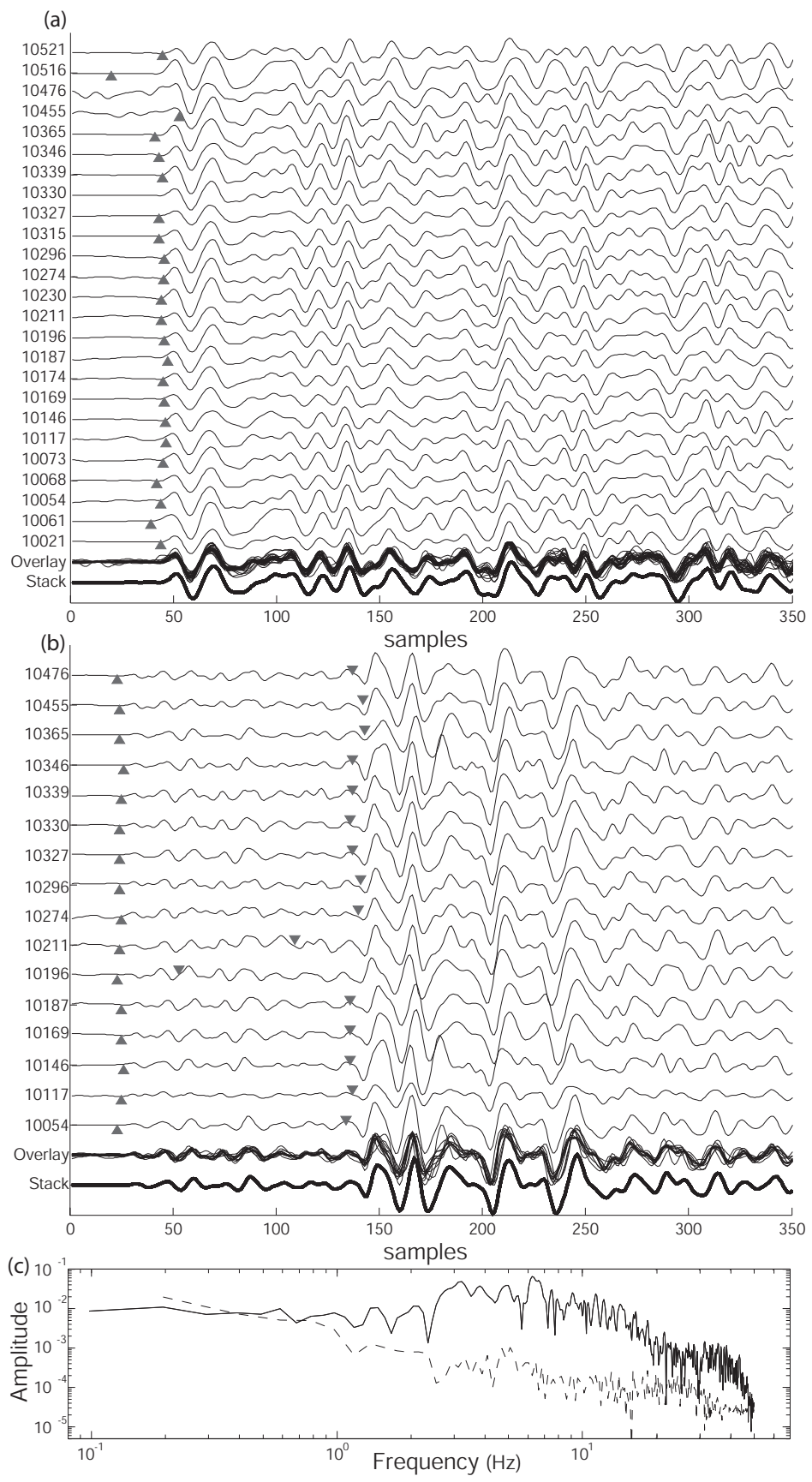

Figure 6.3. (a) 25 events belonging to the multiplet beneath Cone A, recorded at station OKWE, vertical component, filtered $1-10 \mathrm{~Hz}$, normalised and aligned according to the time shift from crosscorrelation analysis. (b) Multiplet traces recorded at station OKSO, east component, filtered 1-10 $\mathrm{Hz}$, normalised and aligned according to the time shift from cross-correlation analysis. Traces are in chronological order with the most recent at the top. Bottom traces show the time shifted stack of all events and all events overlayed. Triangles show catalogue P picks. Inverted triangles show catalogue S picks. (c) Frequency spectrum of the unfiltered stacked waveform of all of the recorded multiplet events at OKSO, which is one of the broadband seismometers. Dashed line shows frequency spectrum representative of the noise, constructed from the 4 seconds before the $\mathrm{P}$ arrival. 
analysis, since the others contained too few earthquakes or were sequenced over short time periods. One of the multiplets not chosen for this study also occurred beneath Cone A but was not recorded well at the three-component stations and the other occurred near Cone D over three hours during the 2008 eruption. The chosen multiplet consisted of 25 events (Table 6.1) occurring sporadically over the five years from 2004 to 2008. The events in the multiplet do not appear to have continued after the onset of the eruption on 12 July 2008 and not all events were recorded at all of the stations.

Figure 6.3 shows the vertical component of all 25 events recorded at station OKWE and the east component of the 15 events recorded at station OKSO. The catalogue $\mathrm{P}$ picks are close together. There are no $\mathrm{S}$ picks on the OKWE traces and the $\mathrm{S}$ picks are not well aligned on the OKSO traces. This is because the $\mathrm{S}$ arrivals are usually more difficult to identify when picking, especially at stations with only one component, and caution must be taken when conducting the relocation and shear wave splitting analysis because the catalogue $\mathrm{S}$ picks, if present, may not be accurate. For this reason the cross correlation results, which produce highly accurate relative $\mathrm{P}$ and $\mathrm{S}$ times between earthquakes, are heavily weighted during the relocation in Section 6.3.1 and we have used the results from BCSEIS to repick the S phase arrivals on relevant records of the multiplet before the shear wave splitting analysis in Section 6.4.1.

\subsection{Relocation}

\subsubsection{Method}

We have performed hypocentre relocation using hypoDD, the double-differencing algorithm of Waldhauser and Ellsworth [2000] and Waldhauser [2001], applied to catalogue phase data and differential times from the cross-correlation analysis. The double-difference residuals (the residual between the observed and calculated traveltime difference between two events at a common station) at each station were minimised initially by a weighted least squares inversion using the conjugate gradients method, followed by the method of Singular Value Decomposition (SVD) on the multiplet identified in Section 6.2.2.

Using the hypoDD technique, approximately $65 \%$ of the 991 earthquakes were relocated with data from the 13 stations of the AVO Okmok network. Only the Okmok 
Table 6.1. Catalogue details of the events belonging to the multiplet

\begin{tabular}{|c|c|c|c|c|c|c|c|c|c|c|}
\hline ID & Year & Month & Day & Hour & Minute & Second & $\begin{array}{c}\text { Lat } \\
\left({ }^{\circ}\right.\end{array}$ & $\begin{array}{c}\text { Long } \\
\left({ }^{\circ}\right.\end{array}$ & $\begin{array}{c}\text { Depth } \\
(\mathrm{km})\end{array}$ & Mag \\
\hline \hline 10021 & 2004 & 02 & 13 & 00 & 31 & 37.74 & 53.391 & -168.221 & -2.5 & 1.1 \\
\hline 10054 & 2004 & 11 & 12 & 16 & 57 & 30.82 & 53.442 & -168.240 & 14.0 & 2.3 \\
\hline 10061 & 2005 & 02 & 14 & 10 & 41 & 28.76 & 53.374 & -168.236 & -0.1 & 0.7 \\
\hline 10068 & 2005 & 04 & 21 & 12 & 32 & 28.60 & 53.398 & -168.236 & 1.5 & 1.2 \\
\hline 10073 & 2005 & 06 & 16 & 13 & 36 & 46.95 & 53.399 & -168.185 & 8.2 & 1.1 \\
\hline 10117 & 2005 & 08 & 22 & 13 & 08 & 28.75 & 53.402 & -168.188 & 5.0 & 0.7 \\
\hline 10146 & 2005 & 11 & 13 & 04 & 59 & 48.51 & 53.427 & -168.175 & 3.9 & 1.1 \\
\hline 10169 & 2006 & 01 & 29 & 17 & 24 & 48.89 & 53.425 & -168.145 & 5.7 & 1.3 \\
\hline 10174 & 2006 & 03 & 10 & 08 & 57 & 03.51 & 53.404 & -168.183 & 3.0 & 0.8 \\
\hline 10187 & 2006 & 04 & 14 & 18 & 30 & 19.89 & 53.407 & -168.179 & 2.9 & 0.6 \\
\hline 10196 & 2006 & 05 & 14 & 11 & 52 & 51.95 & 53.396 & -168.181 & 1.3 & 0.7 \\
\hline 10211 & 2006 & 06 & 06 & 21 & 08 & 31.16 & 53.406 & -168.176 & 1.1 & 0.1 \\
\hline 10230 & 2006 & 07 & 29 & 21 & 41 & 03.21 & 53.403 & -168.183 & 3.0 & 0.3 \\
\hline 10274 & 2006 & 09 & 20 & 07 & 50 & 43.50 & 53.406 & -168.180 & 0.8 & 0.1 \\
\hline 10296 & 2006 & 11 & 23 & 15 & 21 & 34.48 & 53.402 & -168.185 & 0.2 & 0.3 \\
\hline 10315 & 2007 & 02 & 22 & 10 & 51 & 15.56 & 53.384 & -168.200 & -3.0 & 1.3 \\
\hline 10327 & 2007 & 04 & 27 & 08 & 52 & 19.67 & 53.402 & -168.188 & 2.4 & 0.9 \\
\hline 10330 & 2007 & 05 & 18 & 10 & 26 & 12.54 & 53.400 & -168.205 & 1.8 & 1.1 \\
\hline 10339 & 2007 & 07 & 01 & 14 & 52 & 06.30 & 53.394 & -168.216 & 1.8 & 0.7 \\
\hline 10346 & 2007 & 07 & 15 & 02 & 50 & 38.24 & 53.400 & -168.190 & 2.2 & 1.3 \\
\hline 10365 & 2007 & 08 & 17 & 12 & 18 & 46.93 & 53.406 & -168.184 & 2.8 & 1.7 \\
\hline 10455 & 2007 & 09 & 28 & 06 & 34 & 20.58 & 53.402 & -168.193 & 0.6 & 0.9 \\
\hline 10476 & 2007 & 11 & 05 & 04 & 00 & 37.50 & 53.401 & -168.190 & 3.9 & 0.5 \\
\hline 10516 & 2008 & 04 & 15 & 12 & 15 & 47.76 & 53.402 & -168.185 & 2.3 & 1.9 \\
\hline 10521 & 2008 & 05 & 05 & 01 & 32 & 12.34 & 53.407 & -168.180 & 5.1 & 0.7 \\
\hline
\end{tabular}

network was used because the stations on other islands in the chain may bias the results by not giving full azimuthal coverage. The data consisted of 15,412 $\mathrm{P}$ and $3099 \mathrm{~S}$ catalogue differential times. Applying a cross-correlation coefficient threshold of 0.7 to the BCSEIS cross-correlation results yielded $13528 \mathrm{P}$ and $1387 \mathrm{~S}$ crosscorrelation differential times. There were five sets of iterations with ten iterations per set. The weightings were chosen so that in the initial iterations the catalogue $\mathrm{P}$ and $\mathrm{S}$ picks were used almost exclusively, then the cross-correlations were phased in such that in later iterations the cross-correlation relative times were used almost exclusively (see Appendix E.4). This weighting scheme was adopted to ensure that the catalogue data mainly constrained the absolute positions of events without sacrificing the highly accurate cross-correlation data [Waldhauser and Ellsworth, 2000; Waldhauser, 2001]. All of the seismicity was treated as a single cluster and the initial location of the centre of the cluster was taken from the catalogue locations. The velocity model entered into hypoDD was that of Masterlark et al. [2010]. We adapted data selection parameters based mainly on those used by Waldhauser and Ellsworth [2000] in California, where there is excellent station coverage. Therefore, 
we reduced both the number of links required and the linking distance (where a link is defined as an event pair with travel time information at a common station) and kept the other parameters the same as those suggested by Waldhauser and Ellsworth [2000]. The maximum event-station pair distance was set as $400 \mathrm{~km}$ in order to include all of the events; the maximum hypocentral separation was set as $4 \mathrm{~km}$; the maximum number of neighbors per event was four; the minimum number of links required to define a neighbor was five; the minimum number of links per pair saved was five and the maximum number of links per pair saved was 20 .

The results from hypoDD were validated by relocating smaller subsets of events using the SVD method included in the hypoDD package, and by relocating the whole catalogue using different station distributions, varying linking parameters, and making small adjustments to the velocity model. The output did not change significantly (the cluster centroid stayed within $0.01^{\circ}$ horizontally and $0.2 \mathrm{~km}$ vertically of the best result), confirming the stability of the results. The multiplet identified using BCSEIS (Section 6.2.1) was verified by visually inspecting the relocated hypocentres and by relocating them using the SVD method. There were no obvious outliers to be rejected from the group.

\subsubsection{Results}

Figure 6.4 shows the relocations of all of the earthquake hypocentres colour-coded according to time and Figure 6.5 displays the catalogue and relocated locations of the multiplet. Typical errors for the catalogue locations of the multiplet events are reported to be of the order of $0.5 \mathrm{~km}$ horizontally and $1 \mathrm{~km}$ vertically. The catalogue errors are based on the uncertainty in the location calculation using catalogue picks and do not take into account the uncertainty in the velocity model. Following double-difference relocation using the SVD inversion method, the relative errors are found to be two orders of magnitude smaller, suggesting that the catalogue errors are in fact larger than those reported. The multiplet event hypocentre locations are now within $300 \mathrm{~m}$ vertically and $150 \mathrm{~m}$ horizontally of each other. The latitude, longitude and depth of the relocated multiplet are $53.403^{\circ},-168.186^{\circ}$ and $3.6 \mathrm{~km}$ bsl respectively. 

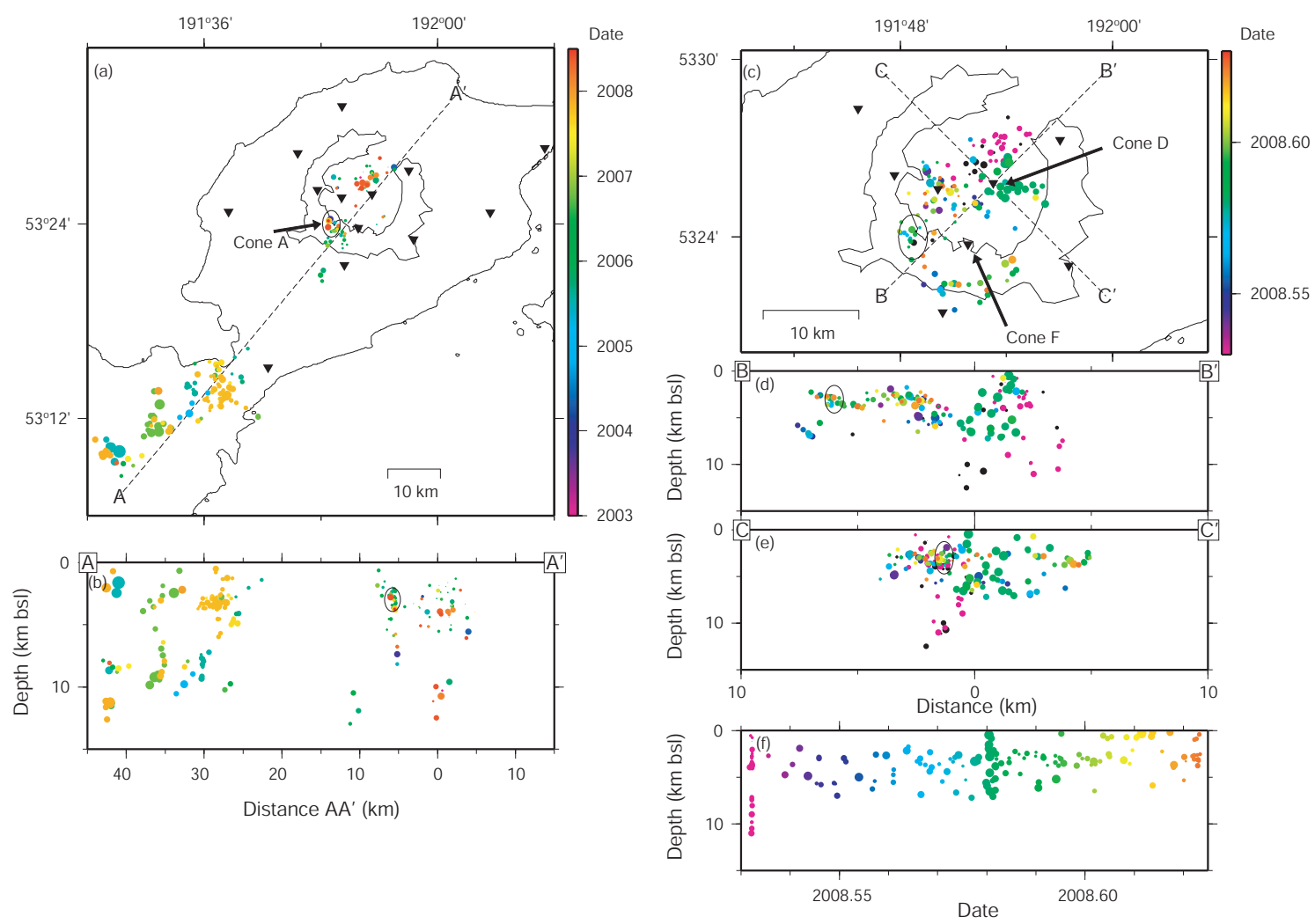

Figure 6.4. Relocated hypocentres of $65 \%$ of the AVO Okmok catalogue colour-coded according to time. Left panels show all relocated seismicity up to eruption onset on 12 July 2008 (2008.53). (a) Map view. (b) Depth section of the profile A-A', southwest-northeast with zero at Cone D. Right panels show relocated co-eruptive seismicity; 11 July 2008 to 15 August 2008. (c) Map view. (d) Depth section along the profile B-B', southwest-northeast with zero at Cone D. (e) Depth section along the profile $\mathrm{C}-\mathrm{C}^{\prime}$, northwest-southeast with zero at Cone D. Black ellipses show the location of the cluster of seismicity that contains the multiplet. (f) Depth versus time for the duration of the eruption.

\subsection{Shear wave splitting}

\subsubsection{Method}

The Silver and Chan [1991] algorithm for calculating shear wave splitting uses a grid-search inversion over the azimuth of the fast polarisation direction $\phi$ and delay time $\delta \mathrm{t}$, for a given time window. It has been incorporated into Teanby et al. [2004a]'s SPLIT code, which conducts cluster analysis over a range of time windows to find the most stable result. Savage et al.'s [2010b] automated method calculates the optimum three filters to apply to the data on the basis of signal-to-noise ratios and then determines the maximum and minimum time window around the $\mathrm{S}$ arrival for use in the Teanby et al. [2004a] code. These time window extremes are based on the dominant frequency of the first three seconds of the $\mathrm{S}$ waveform. Errors are 

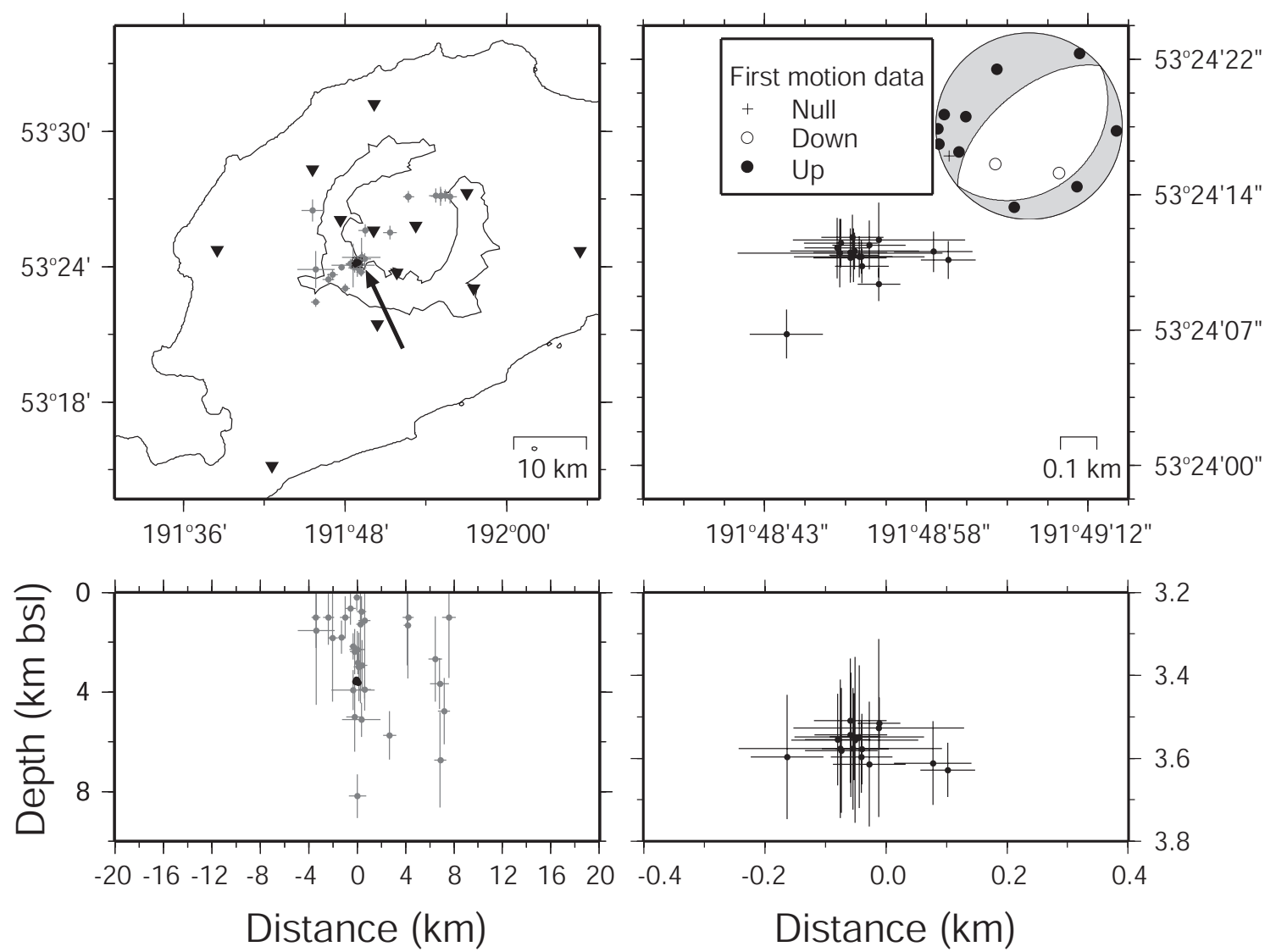

Figure 6.5. Left: Catalogue locations of events in the identified multiplet in grey, relocated hypocentres in black. Right: Zoom of relocated hypocentres of multiplet events. Depth sections are centred on the multiplet. Inset is a composite focal mechanism, constructed with first motions of all multiplet earthquakes.

calculated by finding the $95 \%$ confidence interval for the optimum values of $\phi$ and $\delta$ t after conducting an $F$ test for the chosen time window [Silver and Chan, 1991]. We conducted the shear wave splitting analysis on all three best filters as well as on the raw data for the 991 events. Output parameters from all three filters were included in the results in order to minimise the effects of frequency-dependent shear wave splitting. If all frequencies yielded the same results, then these were combined and assigned a larger weighting than if there were discrepancies, in which case the results cancelled each other out and were therefore assigned a smaller weight.

Results from the shear wave splitting analysis that gave $\phi$ to within $20^{\circ}$ of the polarisation of the incoming wave were considered null results [Peng and Ben-Zion, 2004] and not included in the interpretation. Nulls signify that no splitting was reliably detected [Silver and Chan, 1991], rendering the corresponding value of $\delta \mathrm{t}$ meaningless and giving $\phi$ with a $90^{\circ}$ ambiguity. 


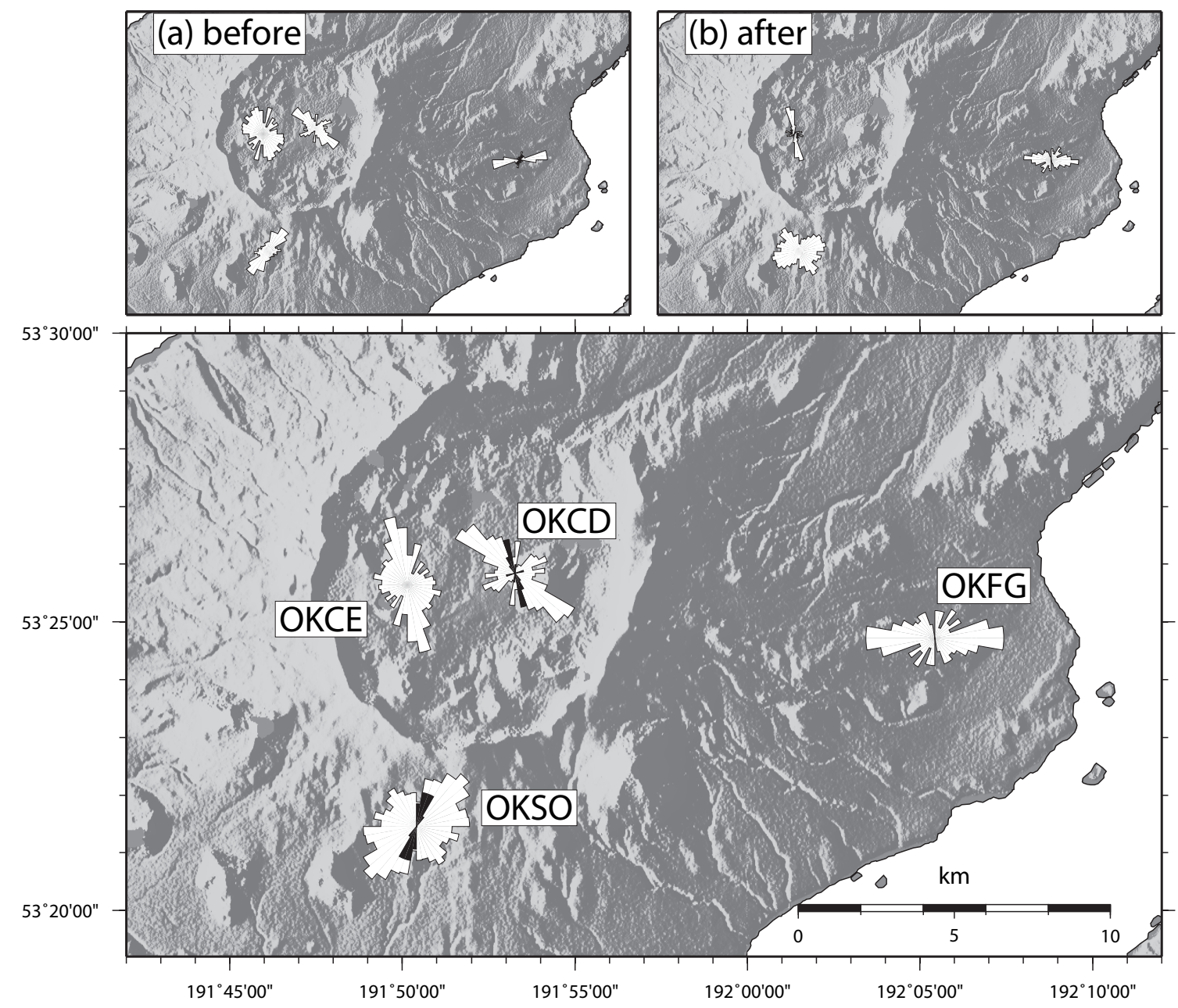

Figure 6.6. Rose diagrams showing frequency of fast shear wave polarisation measurements at the four 3-component seismometers from automated shear wave splitting analysis of the whole AVO Okmok catalogue. Black overlayed rose diagrams in large plot show multiplet fast polarisations. Smaller plots show (a) polarisations before the onset of the eruption on 12 July 2008; (b) polarisations after the onset of the eruption; 12 July 2008 to 28 February 2009. Table 6.2 displays the number of earthquakes used for each rose diagram. Station OKCD was destroyed in the eruption.

Valid shear wave splitting measurements require the angle of incidence $(i)$ to be within the shear wave window of $35^{\circ}-45^{\circ}$ [Nuttli, 1961; Booth and Crampin, 1985], outside which S-P reflections at the surface disturb the seismic record and shear wave splitting measurements give erroneous results. The critical angle $i_{c}$ is calculated from wave velocities $v_{S}$ and $v_{P}$ as $i_{c}=\arcsin \left(v_{S} / v_{P}\right) \approx 45^{\circ}$ if $v_{P} / v_{S} \approx \sqrt{2}$ or $i_{c}=\arcsin \left(v_{S} / v_{P}\right) \approx 35^{\circ}$ if $v_{P} / v_{S} \approx \sqrt{3}[N u t t l i, 1961]$, but a low-velocity layer at the surface has the effect of increasing the number of events that arrive inside the shearwave window by reducing $i$ [Bernard and Zollo, 1989]. The 3D velocity model of Masterlark et al. [2010] obtained by ambient seismic noise tomography confirms that the velocity gradient at Okmok is high near the surface and the $\mathrm{S}$ wave speed is low $(\sim 1.8 \mathrm{~km} / \mathrm{s})$. This, in combination with the fact that most of the earthquakes 
Table 6.2. Number of events used in different time periods for the shear wave splitting anaysis and displayed in Figure 6.6

\begin{tabular}{|c|c|c|c|}
\hline Station & $\begin{array}{c}\text { 1st January 2003 - } \\
\text { 28th February 2009 }\end{array}$ & $\begin{array}{c}\text { 1st January 2003 - } \\
\text { 11th July 2008 }\end{array}$ & $\begin{array}{c}\text { 12th July 2008 - } \\
\text { 28th February 2009 }\end{array}$ \\
\hline \hline OKCD & 238 & 238 & 0 \\
\hline OKCE & 284 & 201 & 84 \\
\hline OKFG & 172 & 26 & 146 \\
\hline OKSO & 646 & 285 & 361 \\
\hline
\end{tabular}

have depths similar to or greater than their lateral distance from the seismometers, means that we can reasonably assume that angles of incidence from all of the events were within the shear wave window.

Shear wave splitting analysis of the multiplet was conducted using the optimum filter and time window for each event-station pair. A set of parameters were chosen after inspection of the results from the automated analysis and then applied to each event in the multiplet.

\subsubsection{Results}

Figure 6.6 shows rose diagrams of the fast polarisations for earthquakes between January 2003 and February 2009 at the four three-component stations (Table 6.2). The diagrams show results obtained using Savage et al.'s [2010b] automated shear wave splitting analysis performed on all local events. The stations outside the caldera walls (OKSO and OKFG) have fast polarisations that are radial to the caldera, and the two inside the caldera have northwest-southeast polarisations. Stations OKCE and OKFG do not display a significant change in general $\phi$ orientation coinciding with the onset of the eruption on 12 July 2008 (Figure 6.6 inset). There is more scatter after the onset of the eruption in the case of OKFG and less scatter after the onset in the case of OKCE. The OKSO results contain more scatter after the onset of the eruption and display a bimodal distribution with two dominant fast polarisations, one of approximately $70^{\circ}$ and the other of approximately $-45^{\circ}$. Station OKCD does not have any post-eruption data as it was destroyed by the initial explosions of the eruption. No significant change in $\phi$ or $\delta$ t is seen immediately prior to the eruption using any of the catalogues.

Looking specifically at the location of the multiplet described in Sections 6.2.2 and 6.3.2 and Table 6.1, and the velocity model of Masterlark et al. [2010] with an S wave 

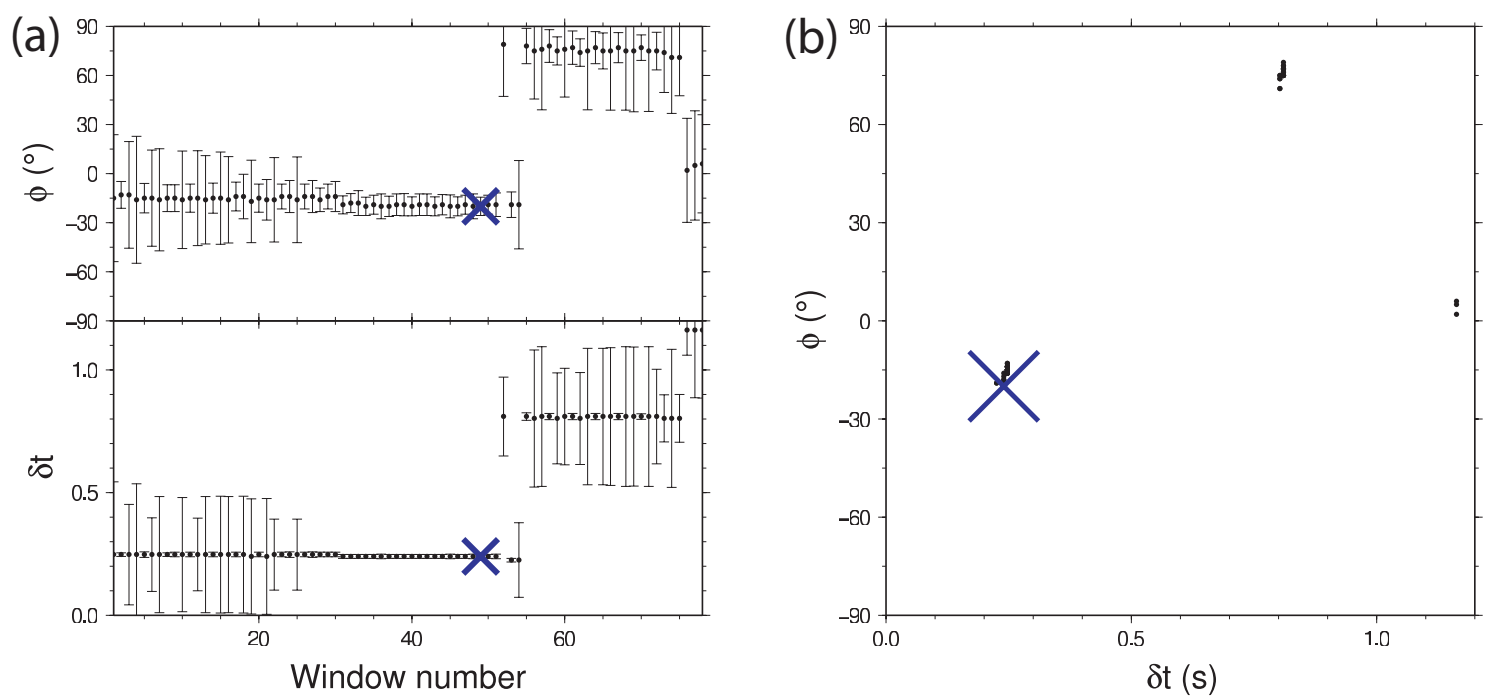

Figure 6.7. Results from the cluster analysis using Teanby et al. [2004a]'s algorithm of event 10339 at station OKCD. 78 windows with start times between 0.7 and 0.3 seconds before the S pick and between 0.63 and 1.54 seconds after the S pick. Plot (a) shows results of $\phi$ and $\delta \mathrm{t}$ for each window number and the errors associated with them. Plot (b) shows the same results with $\phi$ against $\delta$ t. Crosses represent optimum result. Note existence of three different clusters of splitting parameters.

velocity of $1.8 \mathrm{~km} / \mathrm{s}$ in the top few kms of the crust from Masterlark et al.'s [2010] tomography result, the angle of incidence of the body waves from the multiplet can be calculated using the two-point ray tracing method of Kim and Baag [2002]. The incidence angles are found to be $45^{\circ}$ at station $\mathrm{OKCD}$ and $44^{\circ}$ at station $\mathrm{OKSO}$, which are within the shear wave window.

Two of the four three-component seismometers surrounding Okmok volcano (OKSO and OKCD, Figure 6.1) produced enough waveforms from the multiplet to be used in the temporal shear wave splitting analysis. After applying the automated shear wave splitting analysis to the waveforms and inspecting the results, the input processing parameters displayed in Table 6.3 were adopted for the multiplet as a whole. An example of the cluster analysis is displayed in Figure 6.7. Each point represents a different analysis window and the corresponding splitting parameters. Three clusters of $\phi$ and $\delta$ t combinations are produced by different windows. One of these

Table 6.3. Parameters applied to the multiplet during the shear wave splitting analysis

\begin{tabular}{|c|c|c|c|}
\hline Station & Filter & \multicolumn{2}{|c|}{ window parameters } \\
\cline { 3 - 4 } & $(\mathrm{Hz})$ & seconds before S arrival & seconds after S arrival \\
\hline \hline OKCD & $1-5$ & 0.8 & 0.8 \\
\hline OKSO & $1-3$ & 0.8 & 0.8 \\
\hline
\end{tabular}



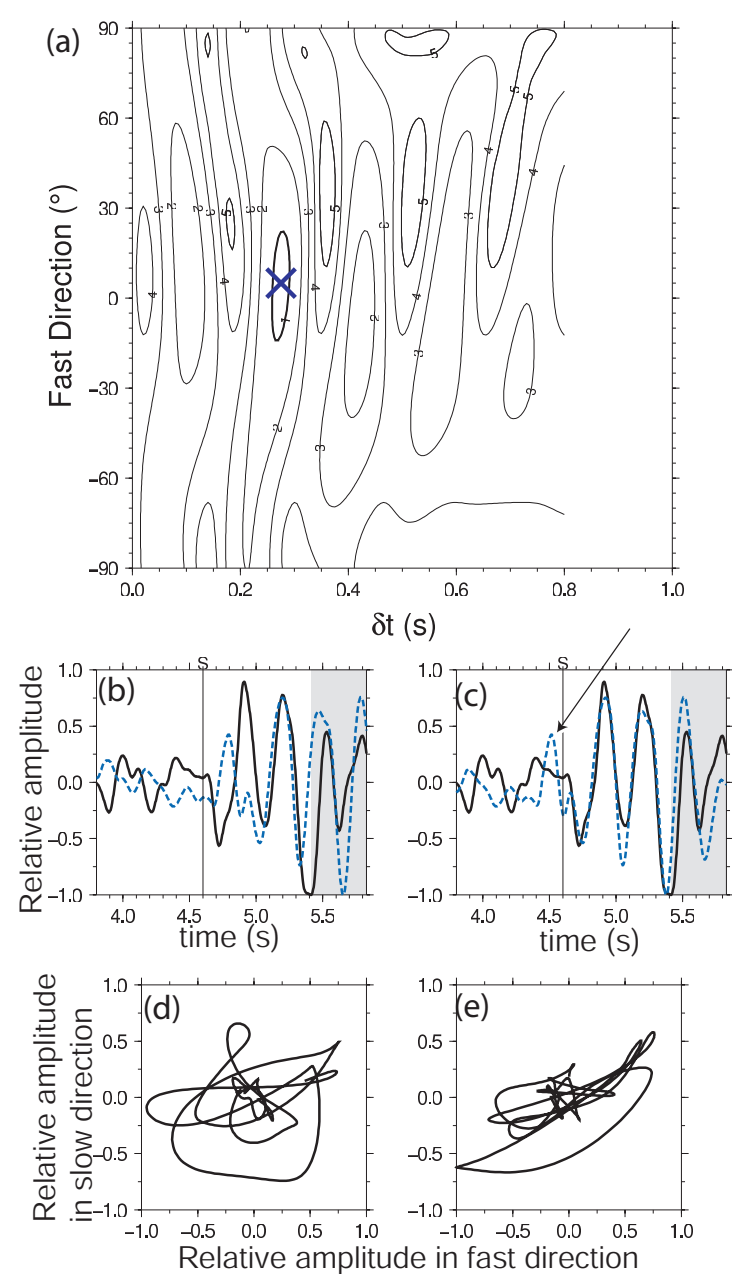
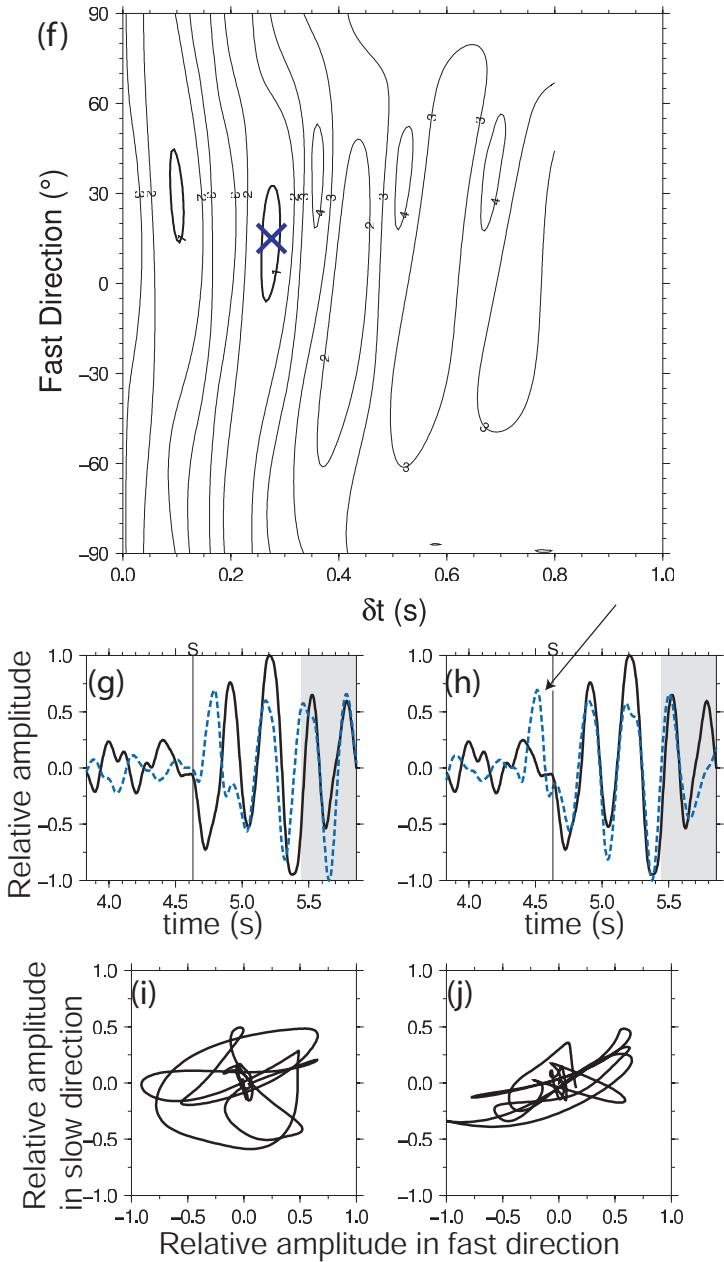

Figure 6.8. Shear wave splitting analysis results from station OKSO. Left panels show results from event 10196, right panels are from event 10327. Plots (a) and (f) display error surfaces of fast polarisation against delay time. Thick line shows $95 \%$ confidence interval, cross shows chosen values. Plots (b) and (g) display the horizontal components of the seismograms around the S picks, rotated to the fast and slow orientations. Plots (d) and (i) show the elliptical particle motion. Plots (c) and (h) display the same seismograms shifted in time by $\delta$ t so that the waveforms correlate. Plots (e) and (j) show the more linear particle motion of the corrected waves. Note the pulse of energy on the slow (dashed) trace before the $\mathrm{S}$ arrival (arrows). It has different amplitudes on the seismograms from different events and can influence the result from the shear wave splitting algorithm if its amplitude is big enough.

combinations (the one chosen by the Teanby et al. [2004a] algorithm, shown by the cross) is measured by the majority of the windows and gives a realistic result. The windows that choose a larger $\delta$ t likely include cycle skipping. In general, narrower frequency bands are more likely to produce cycle skipping by creating more monochromatic waveforms. Cycle skipping can sometimes be identified by clusters with $\delta$ t separated by a multiple of the dominant period, or by $\delta$ t equivalent to an unusually high percentage of anisotropy. Identification of cycle skipping is best carried out by viewing the waveforms and determining the quality of the result by eye. 
A random subsample of results was viewed in this experiment as a quality control and an example of the results can be seen in Figure 6.8.

The choice of parameters used on the multiplet was based on a combination of the dominant frequencies contained in the waveform, the time between the $\mathrm{P}$ and $\mathrm{S}$ arrivals, and the most stable results from the automated analysis and are displayed in Table 6.3. The frequency bands were chosen to obtain the best signal-to-noise ratio while encompassing the dominant frequencies. Broad frequency bands and long windows before the $\mathrm{S}$ arrival were chosen to minimise the risk of cycle skipping; the $\mathrm{P}$ coda is minimal on the horizontal components so the impulsive $\mathrm{S}$ arrival can be matched (Figure 6.3). The length of the window after the $\mathrm{S}$ arrival ensured at least one period of the $\mathrm{S}$ wave was included while avoiding spurious secondary phases in later parts of the waveform, which could degrade the splitting estimates [Teanby et al., 2004a].

We have applied the same parameters to each event using the $\mathrm{S}$ arrival pick from the BCSEIS analysis. The results are plotted in Figures 6.6 (black overlay) and 6.9 and the statistical analysis of the results is summarised in Table 6.4 and discussed further in Section 6.5.

Different input processing parameters were used in an attempt to gain more information from the multiplet analysis and the earthquakes surrounding the multiplet; namely using a lower cross-correlation coefficient threshold and signal-to-noise ratio. However, while yielding slightly more data, this resulted in a poorer quality data set overall with more scatter in results and no other conclusions could be drawn. 

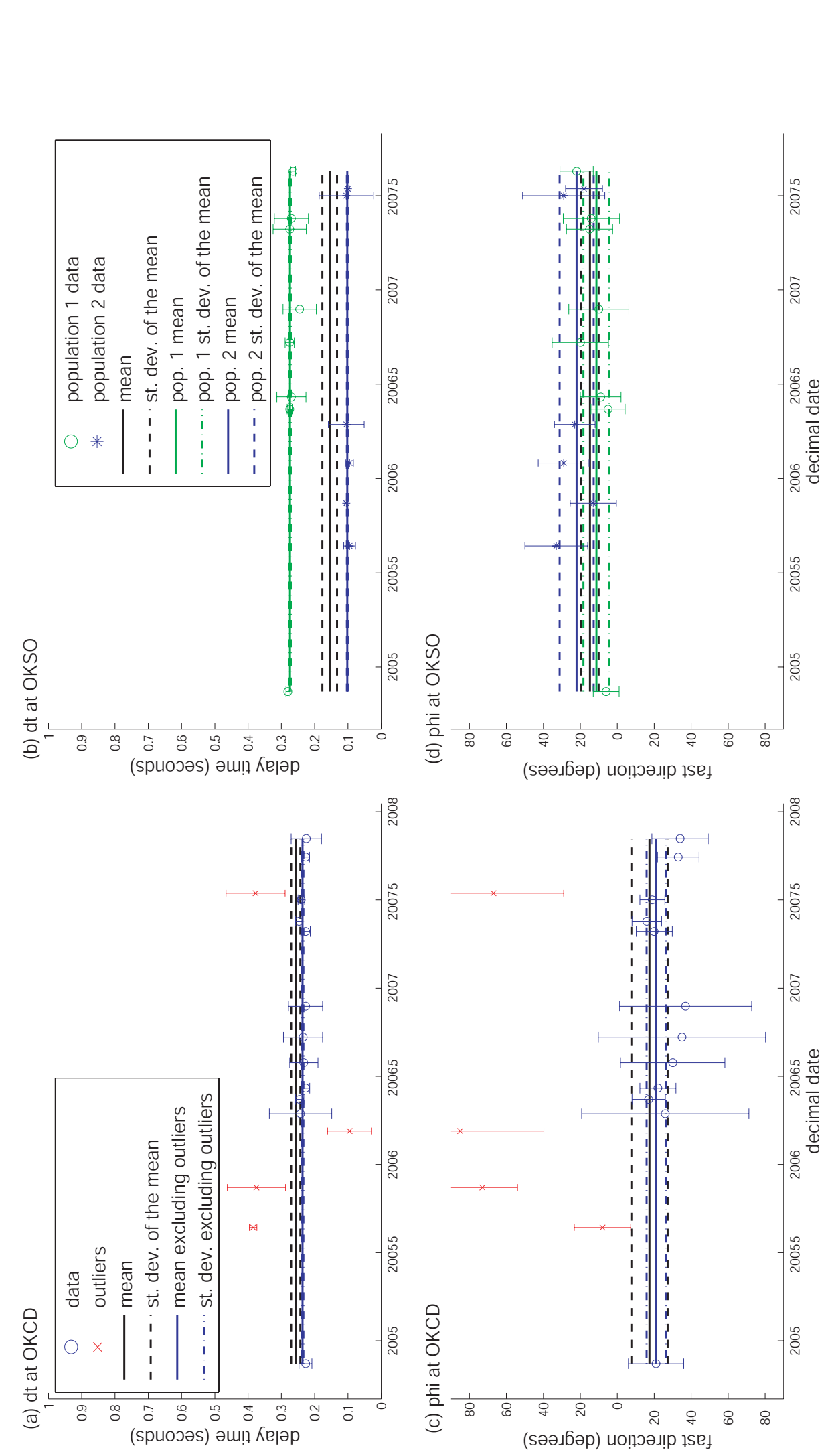

总 光 름 용 월

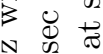

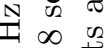
is $\dot{0} \div$ $-3$

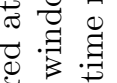
昰 a 氙 定 3 出 궁

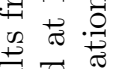
要跑语 娄 它高焉 结它

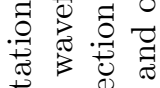
क

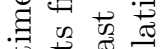

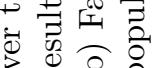
वे 药

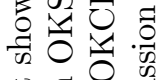
닝. 要 的施 需范 जी

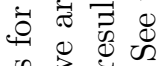
की

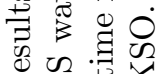
So 00

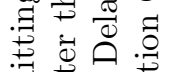

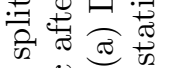
苾昆䒕 30.

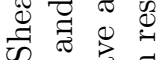
क. 0 $0 . \overline{0}$

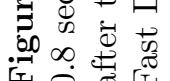




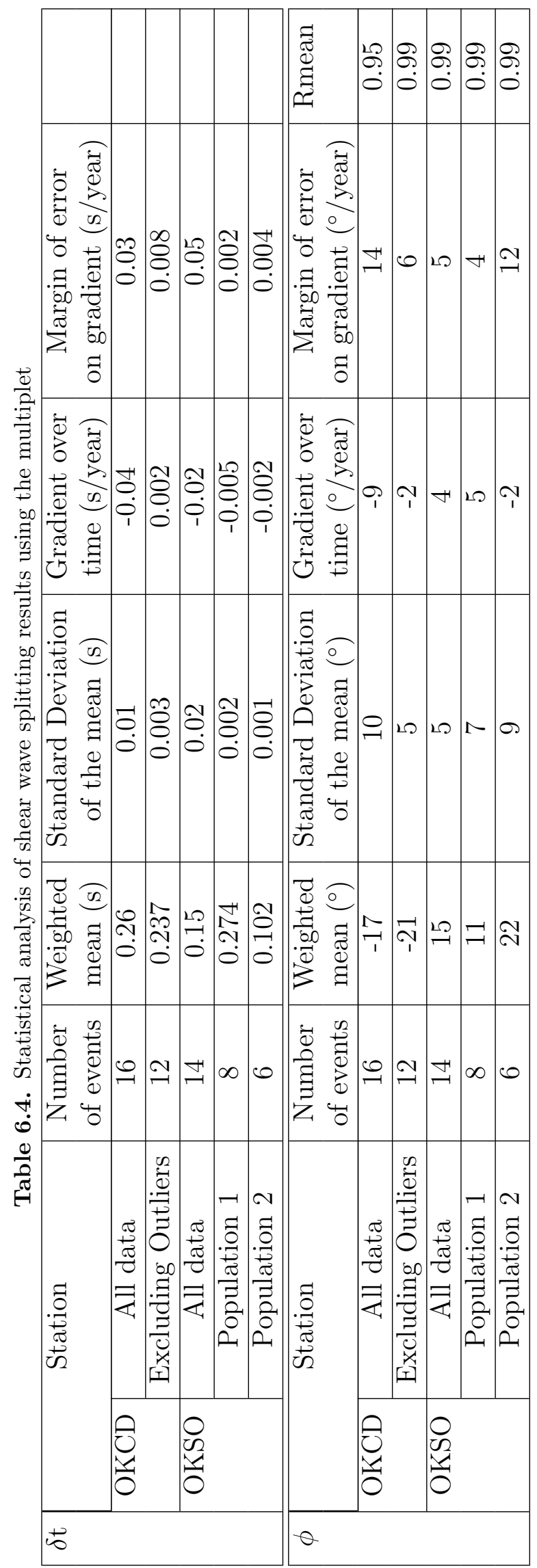




\subsection{Discussion and interpretation}

\subsubsection{The multiplet}

The presence of repeating earthquakes underneath Cone A (Figures 6.1, 6.4 and 6.5) shows that there was some ongoing process that continued unchanged throughout the period February 2004 to May 2008. The occurrence of individual earthquakes within the multiplet does not appear to correlate with any notable deformation events. A few of the repeating earthquakes occurred at the same time as tremor in 2004 and 2005, when periodic episodes of volcanic tremor were commonly observed at Okmok [C.Reyes, pers. comm]. There does not appear to be a correlation between their times of occurrence however. Multiplet earthquakes do not appear to have continued after the onset of the 2008 eruption in spite of seismicity contining in the same area. This is likely because the structure over the path was changed by the eruption, changing the waveforms and so producing lower cross-correlation coefficients, rather than because the earthquake mechanism and location necessarily altered.

The impulsive $\mathrm{P}$ and $\mathrm{S}$ arrivals in the records from the multiplet earthquakes suggest that they were caused by brittle failure [Latter, 1981a; McNutt, 1996]. Figure 6.3, panel c, displays the frequency spectrum for the unfiltered stacked waveform of the multiplet recorded at OKSO. The spectrum is representative of the broadband stations and displays a form that is typical of a brittle fracture volcano-tectonic (VT) earthquake [Brune, 1970; Latter, 1981a], although with a slight deficit of long-period (LP) energy in the band $0.1-1 \mathrm{~Hz}$. The first motions were repicked and found to be consistent for all of the events in the multiplet at each station. The focal mechanism constructed using the first motions is compatible with a normal fault striking at approximately NE-SW (Figure 6.5 inset). Repeating earthquakes at volcanoes usually contain lower frequencies [Neuberg, 2000; McNutt, 1996] because they involve fluid-pressurisation processes in their mechanisms. The lack of LP energy in these spectra suggests that fluid did not play a significant role in the source mechanism of the multiplet earthquakes [Latter, 1981a; McNutt, 1996]. Future work could include a full focal mechanism inversion and waveform analysis to gain more information about the source mechanisms and the raypath properties. Focal mechanisms from other events could also be used to gain more insight into the local stresses and processes. 


\subsubsection{Relocation}

The relocation results indicate that patch of events surrounding the identified multiplet beneath Cone A remained stationary over the time that the network was active (Figure 6.4). Earthquakes persisted in this area throughout the course of the eruption.

Relocated seismicity prior to the onset of the eruption on 12 July 2008 (Figure 6.4, left panel) was predominantly located to the southwest of the caldera where there are pre-Holocene volcanic centres and a geothermal field [Motyka et al., 1994]. The relocated hypocentres of earthquakes associated with the onset of the eruption (mid2008, right panel of Figure 6.4), which presumably opened the pathway for magma ascent, occurred in the northern portion of the caldera and started as deep as 13 $\mathrm{km}$. Due to the close network geometry the structure of the seismicity at depth is not well defined. Earthquakes occurring during the middle and later portions of the eruption occurred mostly at depths of about $3 \mathrm{~km}$ bsl but none were deeper than about $7 \mathrm{~km}$. Toward the end of the eruption, many relocated earthquakes occurred at shallower depths, and there are only a few relocated events as deep as $5 \mathrm{~km}$. The relocated co-eruptive seismicity was well distributed throughout the caldera, which is consistent with being caused by small scale stress changes from increased pore pressures as the magma near the surface exsolved fluid and gas heated existing groundwater. Some of the earthquakes throughout the caldera may have been from reactivated faults accommodating the caldera-wide deformation observed during the eruption [Freymueller and Kaufman, 2010]. The depth of the majority of the relocated co-eruptive earthquakes coincided with the depth at which the centre of deformation is modelled and interpreted to be the magma body [Lu et al., 2005], suggesting they are causally related. The relocated earthquakes reached the surface on the eastern edge of the cluster of seismicity just northeast of Cone D, the location of the 2008 eruptive vent. There was a ring of relocated seismicity around Cone F with a lack of earthquakes within it at all depths. We speculate that the pattern of seismicity around Cone F could represent a ring fault that is a weak part of the volcanic structure and has been reactivated or is simply an easy pathway for fluids. If this is the case, the lack of seismicity near Cone F may be due to local stresses by being accommodated by the surrounding ring fault. 


\subsubsection{Shear wave splitting}

The difference in $\phi$ at different stations suggests that the observed anisotropy is close to each station (Figure 6.6). The average orientation of $\phi$ at stations OKFG and OKSO is approximately radial with respect to the centre of the caldera. Assuming that anisotropy in the crust is caused by the maximum horizontal stress preferentially aligning microcracks [Crampin, 1994], the radial orientation agrees with $L u$ et al. [2000]'s model of a charged magma body in the centre of the caldera (see Section 6.1.1). Visual examination of the rose diagrams indicates that station OKSO experienced a change in fast polarisation after the onset of the eruption (Figure $6.6 \mathrm{~b}$ ). This could be interpreted as a change in the maximum horizontal stress due to the eruption releasing pressure within the magma reservoir, particularly as one of the directions is consistent with the stations within the caldera (OKCD and OKCE). There was also a change in the amount of scatter, and in the standard deviation, before and after the onset of the eruption at all stations. However, the location of the majority of the seismicity shifted from the western geothermal areas to be predominantly scattered around the caldera. This shift in epicentres changed the dominant backazimuth and so the observed variation in $\phi$ is not necessarily a temporal one.

The two three-component stations inside the caldera (OKCD and OKCE) showed a northwest-southeast orientation of $\phi$ (Figure 6.6). We interpret this to be a result of the regional maximum horizontal stress due to the subduction of the Pacific Plate beneath the North American Plate. The pressurised magma body beneath the centre of the caldera is inferred to have produced an isotropic stress on the surrounding rock. According to this model, directly above the magma body the maximum local stress was vertical and had less influence on the direction of the maximum horizontal stress. The geographical locations of OKCD and OKCE are such that the maximum horizontal stress was not altered significantly from the background. Haney [2010]'s results from modelling of the VLP tremor agree with this hypothesis. Figure 6.10 displays the results from Coulomb stress modelling [Lin and Stein, 2004; Toda et al., 2005]. The source of inflation used here is similar to that modelled by Lu and Dzurisin [2010], namely a Mogi point source centred at 2.9 $\mathrm{km}$ bsl but with a potency of $2 \times 10^{9} \mathrm{~m}^{3}$. This potency is 1.5 orders of magnitude larger than the volume change modelled by Lu et al. [2000, 2003, 2005] and Fournier et al. [2009] because we are concentrating on a deviation from the regional stress rather than from a post-eruption reference, where there is magma left in the system. The tectonic stress is estimated using the methods of Zoback and Townend [2001]. 
We assume a normal faulting regime and a coefficient of friction of 0.8 , meaning that the maximum stress $\left(\mathrm{S}_{1}\right)$ is vertical and has a vertical gradient of $26 \mathrm{MPa} / \mathrm{km}$. We assume the orientation of the maximum horizontal stress $\left(\mathrm{S}_{2}\right)$ to be $135^{\circ}$ [Nakamura and Uyeda, 1980] and to have a vertical gradient of $20 \mathrm{MPa} / \mathrm{km}$, which is an average of the maximum and minimum stress gradients.
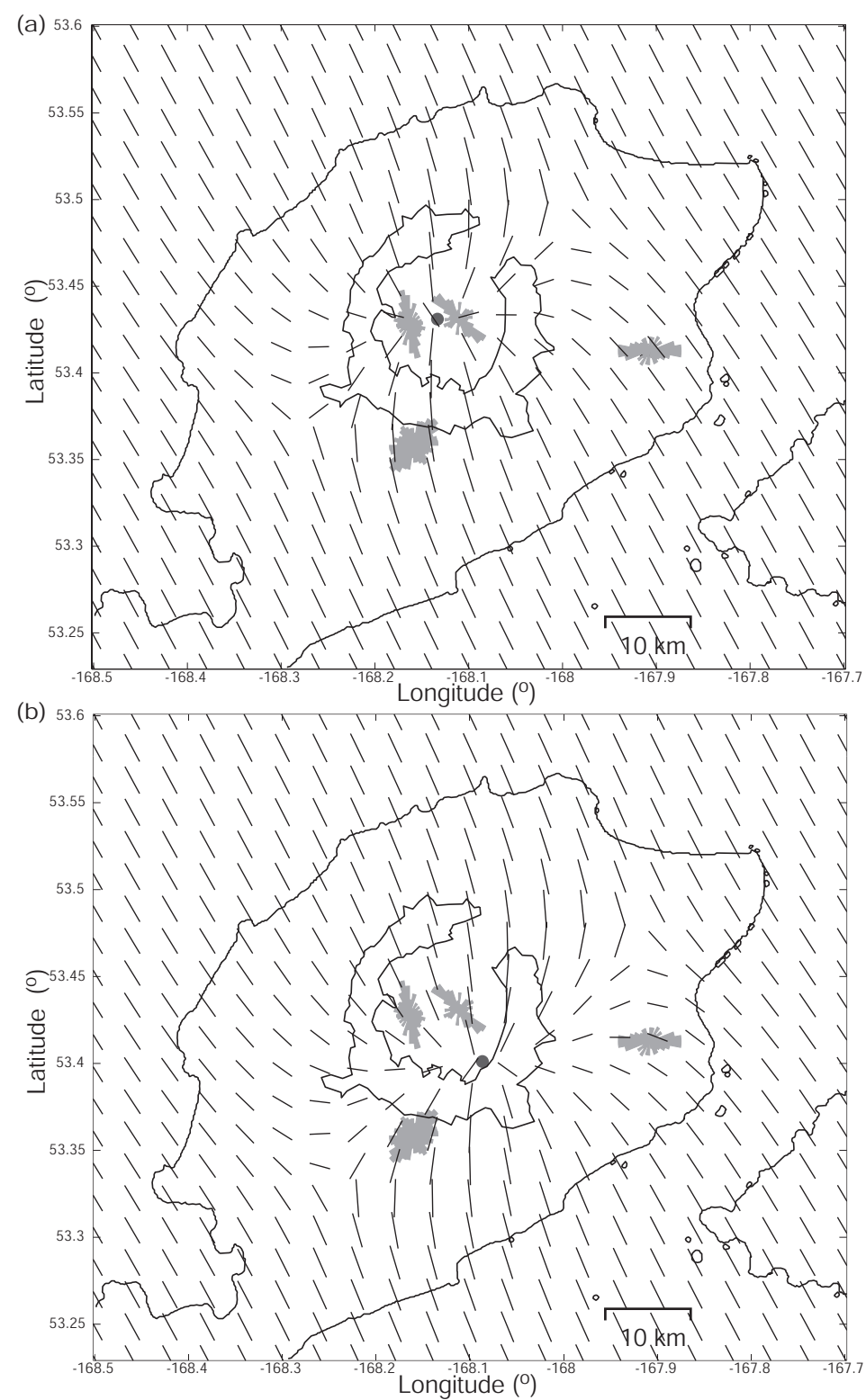

Figure 6.10. Maximum horizontal stress from a Mogi point-source of inflation at $2.9 \mathrm{~km} \mathrm{bsl}$ with potency of $2 \times 10^{9} \mathrm{~m}^{3}$, indicated with grey circle. The tectonic stress is estimated using the methods of Zoback and Townend [2001]. The black lines indicate the direction of maximum horizontal stress at $1.5 \mathrm{~km}$ depth calculated using the technique of Lund and Townend [2007]. The grey rose diagrams display the shear wave splitting fast polarisation at the four 3-component stations. (a) Source location in the centre of the caldera. (b) Preferred source location. 
Stress was modelled at $1.5 \mathrm{~km}$ depth, which is mid-way between the source of inflation and the surface and captures a portion of most of the earthquake raypaths at a location where the anisotropy is inferred to be present. The top plot of Figure 6.10 shows the results when the source of inflation is located in the centre of the caldera, as modelled by Lu et al. [2000, 2003, 2005]. The bottom plot displays the results when the source of inflation is moved to best fit the fast polarisation results, while keeping the other parameters the same. This modelling suggests that our interpretation of the fast polarisation results is plausible. The location that best fits the splitting results lies to the SSE of the centre of the caldera. Anisotropy caused by stress at depth may not correlate perfectly with strain observed at the surface because the subsurface rocks are heterogeneous, with contrasting mechanical stiffnesses accommodating stress differently [Kinvig et al., 2009]. Furthermore, the shear wave splitting method gives a measure of anisotropy intergrated over the whole raypath, while the model calculates the stress at a single depth. Therefore, a discrepency between the estimation of the source using these different methods is inevitable. The best-fitting location of the source also falls within the seismically quiet zone below Cone F, identified on Figure $6.4 \mathrm{~b}$, and is in the same section of the caldera in which Fournier et al. [2009] modelled the inflation source.

Statistical analysis of the multiplet shear wave splitting results is summarised in Table 6.4 and Figure 6.9. Weighting proportional to the errors defined in Section 6.4.1 was applied to all calculations. A Rayleigh test [Davis, 1986] reveals that both stations OKCD and OKSO exhibited significant mean $\phi$ values despite the scatter. Using weighted least squares regression of $\phi$ with time, $\phi$ at both stations is found to be unchanging within $95 \%$ confidence. Both stations also exhibited unchanging $\delta$ t within the same margins of error.

Station OKCD displayed a mean $\phi$ of $-17^{\circ}$ and $\delta \mathrm{t}$ of $0.26 \mathrm{~s}$. If there were no changes in splitting parameters, we would expect all points to be drawn from a common $\chi^{2}$ distribution. A Kolmogorov-Smirnov test enables us to reject this hypothesis. Therefore the outliers (denoted by crosses in Figure 6.9) were caused by a process not described by a Gaussian distribution and are excluded in the following analyses. This other process could be a change in shear wave splitting or simply a lower quality of data. After inspection of the splitting results, we found that these outliers yield measurements of lower quality, confirming their rejection from the analysis. Removing these outliers from the calculations reduced the standard deviation and changed the mean values to $\phi$ of $-21^{\circ}$ and $\delta$ t of $0.24 \mathrm{~s}$. This $\delta$ t value equates to anisotropy of $4.6 \%$, assuming that the anisotropy is constant along the whole 
raypath. This is within the range of percentages observed for volcanic environments similar to Okmok volcano [Savage et al., 1989]. However, as the raypath did not change during this analysis, we are unable to tell whether the anisotropy changes along the path or where along the path it changes. A Student's $t$ test produces a twotailed $P$ value of 0.43 , confirming that the orientation of $\phi$ from this multiplet is not statistically different from the average orientation of the data from all back azimuths displayed in Figure 6.6, and suggesting that the region of anisotropy extended close to the station.

Station OKSO displayed a mean $\phi$ of $15^{\circ}$ and $\delta$ t of $0.15 \mathrm{~s}$. The delay times indicated two populations; one of $0.10 \mathrm{~s}$ and one of $0.27 \mathrm{~s}$. However, a $t$ test showed that these two populations' average $\phi$ values were not significantly different, with a two-tailed $P$ value of 0.36 . In other words, some process changed the apparent percentage of observed anisotropy, of between $5.6 \%$ and $2.2 \%$, but not its polarisation. This change in the apparent percent anisotropy could be attributed to an artifact of the processing such as cycle skipping. However, visual inspection of the waveforms indicated skipping has not occurred (see Figure 6.8 for examples). The difference in $\delta$ t corresponds to a difference of less than a quarter of the dominant wavelength, also indicating that cycle skipping was not the cause. Some of the waveforms displayed a small pulse of energy before the $\mathrm{S}$ wave arrival (indicated in Figure 6.8), which influenced the selection of parameters made by the shear wave splitting algorithm. The multiplet was identified by cross correlation coefficients of greater than 0.95 , therefore a small amount of dissimilarity within the window of investigation is to be expected and is unavoidable. The use of a higher threshold for cross correlation yields too few events in the multiplet to carry out this analysis. We have not studied the full receiver functions of these earthquakes and so cannot say exactly what the extra pulse represents, but assume that it must be due to a slight difference in the location of the earthquake and therefore the intersection of the ray with a scatterer that the other event rays do not encounter. This pulse is thus interpreted as an S-P conversion of the wave coming into contact with a structure, possibly part of the magma reservoir or another heterogeneity. The average $\phi$ of $15^{\circ}$, when compared using a $t$ test to that observed at station OKSO for events with all back azimuths, gave a two-tailed $P$ value of 0.56 . This indicated that they were not statistically different and also suggested a source of anisotropy extending to the station.

Modelling of the magma influx using GPS suggests that there was major injection beneath Okmok from 2002 to 2004 but after that time there was quiescence [Fournier et al., 2009], except for notable inflation in early 2008 [Larsen et al., 2009]. 
The 2008 episode occurred after the time of the last earthquake in the multiplet. Therefore, the average $\phi$ value that deviates from the regional maximum horizontal stress was probably established before we started to observe the multiplet. The lack of significant change in $\phi$ between 2004 and the last member of the multiplet before the 2008 eruption of Okmok is thus consistent with geodetic results. An increase in the pressure of the magma reservoir, causing an increase in radial stress would have increased the percentage of anisotropy and therefore the delay times between the fast and slow shear waves [Crampin, 1994]. No increase in delay times was observed using the multiplet. This suggests that there was little change in the magnitude of the stress during the occurrence of the multiplet, which is also consistent with geodetic results. However, the analysis using the multiplet cannot resolve any changes in stress that may have occurred immediately preceding the eruption and in particular the increase in seismicity in the 5 hours before the eruption onset (Figure 6.2).

Although our study cannot resolve the physical process that triggered the eruption uniquely, we speculate on the triggering process based on constraints from our results. When considering possible triggers for the eruption we take into account that: 1) the caldera was inflating, but not at an increased rate at the time of the eruption onset [Freymueller and Kaufman, 2010]; 2) magma was shallow as intrusion continued [Masterlark et al., 2010]; 3) there was not a large stress change in the 4 years before the eruption; 4) no regional earthquake activity occurred at the time [Dixon and Stihler, 2009]; 5) in the 4-5 years prior to eruption, there was little seismicity, but significant tremor; 6) most recent eruptions have not been particularly explosive [Neal et al., 2003]; and 7) water was available and the eruption was phreatomagmatic in nature [Larsen et al., 2009]. These things together lead us to the conclusion that the system was open and able to passively degass without significant overpressure developing. The hot, shallow open system led to seismic quiescence. The deep earthquakes could suggest some increase in recharge rate, though if that was true it wasn't reflected in the GPS. This may have increased the stress on a shorter time scale than we are able to resolve using the multiplet study or for a small region around the reservoir, which is not sampled by the raypath. Ongoing intrusion may have simply reached a point were it overcame the overburden stress so the stress change was smaller than can be resolved with this method. Contact with water could also have been a trigger, though we cannot substantiate that. 


\subsection{Conclusions}

A multiplet of 25 earthquakes has been identified using a bispectrum crosscorrelation technique applied to the Okmok volcano AVO catalogue. The events in the multiplet have cross-correlation coefficients of more than 0.95 at three or more stations or more than 0.98 at one station. The presence of a multiplet implies that there was some ongoing process beneath the Cone A that was unchanged over the period February 2004 to May 2008. However, the occurrence of multiplet earthquakes does not correlate with notable deformation events, nor with tremor.

Double-difference relocation of the entire Okmok catalogue confirms that earthquakes in the multiplet occurred at the same location to within $300 \mathrm{~m}$ vertically and $150 \mathrm{~m}$ horizontally. There has been seismicity around this location for the whole time that the seismic network has been deployed and it persisted throughout the eruption, however events belonging to the multiplet could not be identified after the onset of the eruption. Relocation of hypocentres revealed structure in the co-eruptive seismicity of the recent 2008 eruption just north of Cone D. Seismicity started at between $13 \mathrm{~km}$ depth bsl and the surface then continued at about $3 \mathrm{~km}$ depth beneath the centre of the caldera, where the majority of the seismicity occurred. This is also the location of the centre of deformation as modelled by GPS and InSAR. Co-eruptive earthquakes were mostly scattered throughout the caldera, possibly accommodating local stress changes due to increased pore pressures. The relocation shows the shallowest earthquakes close to Cone D, which was the location of the 2008 eruptive vent.

Shear wave splitting analysis of the whole catalogue using an automated method yields a fast splitting polarisation that is radial to the caldera at the seismometers outside the caldera. This is interpreted as the direction of maximum horizontal stress, caused by the charging magma body preferentially aligning microcracks and causing anisotropy in the surrounding rock. The two seismometers inside the caldera displayed a fast splitting direction approximately northwest-southeast, which we interpret to be the regional stress caused by the tectonic regime. This orientation was observable because the stations lie directly above the presumed magma body so that the vertical stress it exerts does not significantly affect the maximum horizontal stress between the station and the magma body.

A separate shear wave splitting analysis was applied to the events in the multiplet using fixed input parameters in order to treat each event in the same manner. This way any changes in output parameters could be assumed to be the cause of changing 
properties of the medium over time rather than changes in the source, or the path, or the analysis input parameters. We observe no significant change in shear wave splitting from the multiplet between November 2004 and September 2007, for the frequency band 1-5 Hz, along the path between the multiplet and the stations. This is unsurprising as the GPS measurements show little variation in the inferred volume change over the same time period. The fast polarisation of the shear wave splitting in the multiplet was the same as that found from the analysis of the whole catalogue at each station. However, each station displayed a different $\phi$ value to the others, suggesting that the source of anisotropy was local to the stations. The delay times gave a percent anisotropy of $4-6 \%$, which is typical of volcanic regions.

We speculate that the eruption may have been triggered by ongoing intrusion simply reaching a point were the overburden stress was overcome. However, our results show no stress change in the 4 years before the eruption Therefore, the stress may have increased on a shorter time scale than we are able to resolve using the multiplet study or for a small region around the reservoir, which is not sampled by the raypath. A third possibility is that the system was in a state of criticality and the stress change that triggered the eruption was too small to be resolved by this technique. Continued analysis of the seismicity of Okmok volcano will show whether there has been a change in shear wave anisotropy since the 2008 eruption to accompany the deflation of the volcanic edifice inferred from geodetic modelling [ $L u$ and Dzurisin, 2010]. 


\section{Synthesis and conclusions}

The principal focus of this thesis was to determine whether anisotropy measured from shear wave splitting changes around a volcano prior to and during magmatic eruptions.

The temporal variation of shear wave splitting and its interpretation is highly controversial. One of the main problems with the interpretation of temporal changes is the possibility of spatial variations being sampled along differing raypaths and being interpreted as temporal changes. We have taken two approaches to mitigate this problem. The first is to understand where the anisotropy is acting so that even if the raypaths differ, changes can be inferred. The second is to keep the raypath constant so that, even if the exact location of the anisotropy is not known, any changes observed can be confidently interpreted to be temporal. The combination of these two investigations has led to a more thorough understanding of the anisotropy in the two study regions.

In Chapter 4, we presented shear wave splitting results at Mt. Ruapehu from a combined data set of two temporary deployments of three-component seismometers and the permanent network. We used earthquakes that occurred throughout 2008 near Mt. Ruapehu and the automated shear wave splitting method of Savage et al. [2010b] to determine anisotropy parameters. A two-dimensional tomographic inversion was applied to the shear wave splitting delay times in order to locate regions of strong anisotropy. In 2008, regions of strong anisotropy were identified in the Waiouru area and near Mt. Tongariro. We then used a spatial averaging technique to analyse spatial variations in the fast polarisation direction. Comparison of shear wave splitting results to stress estimates and local structures let us distinguish regions in which shear wave splitting is governed by structural anisotropy and those in which stress-induced microcracks were the main cause. Anisotropy in the Waiouru region is inferred to be caused in part by schistose mineral alignment, and the anisotropy near Mt. Tongariro was interpreted to be caused by macroscopic fractures. The region to the west of Mt. Ruapehu exhibits weaker anisotropy than 
the previously mentioned areas, but still displayed detectable anisotropy, which was inferred to be caused by stress-induced microcracks. These interpretations enable us to focus investigations on the regions that are more likely to display temporal changes in shear wave splitting related to volcanic activity.

In Chapter 5, the techniques that were developed for the analysis of the 2008 data were also applied to other past deployments around Mt. Ruapehu. We found that the region of high anisotropy near the Waiouru swarm was stable in time in terms of both delay time and fast polarisation measurements. This agrees with the interpretation that the anisotropy in this region is controlled by schistose mineral alignment and faulting. A key observation was a region of strong anisotropy detected using the 1995 data and centred just to the west of Mt. Ruapehu. This feature occurred in the region of anisotropy that was identified as stress-controlled and was present at the time of a large magmatic eruption, and hence is interpreted to be due to increased pore-fluid pressure and fractures from the eruption. The application of these techniques to temporally varying data has also uncovered the possibility that the high anisotropy area near Mt. Tongariro in 2008 was a temporary feature.

Another technique employed in Chapter 5 to mitigate the problem of spatial variations of anisotropy being interpreted as temporal variations was the application of the automated shear wave splitting method to ongoing swarms of earthquakes. The two swarms that we used were the Waiouru swarm and the Erua swarm, which are both approximately $20 \mathrm{~km}$ away from Mt. Ruapehu. We observed changes in shear wave splitting parameters associated with the 2006 and 2007 phreatomagmatic eruptions using earthquakes from the Erua swarm, but such changes were not observed using earthquakes from the Waiouru swarm. Changes have been observed in the character of these earthquake swarms associated with volcanic eruptions, and so the character of the Erua swarm over the 2006-2007 period was investigated further. It was found that the $b$-value for the Erua swarm increased at the time of the change in shear wave splitting parameters, although this was the only parameter related to the seismicity to vary significantly. The conclusion is that the anisotropy is usually governed by stress-induced microcracks but the change was near-source and due to an increase in pore-fluid pressure associated with the eruptions, making the medium in the source region more isotropic, and allowing the structure in the region to govern the weaker anisotropy. The consistent shear wave splitting parameters obtained from the Waiouru earthquakes matches the results from the spatial averaging and delay time tomography, and agrees with the interpretation of a structural mechanism of anisotropy. 
In Chapter 5, we also investigated the occurrence of repeating earthquakes, and their use in monitoring shear wave splitting. At Mt. Ruapehu, repeating earthquakes were not identified in the Erua region, but were identified in the Waiouru swarm. These multiplets were used to demonstrate the validity of assuming a single layer of anisotropy. Each earthquake within a multiplet yielded the same shear wave splitting results to within errors but no variations over time were observed. This may have been due to the multiplets not spanning the time of the large eruptions. It may also have been due to the source region having a strong intrinsic anisotropy such that subtle changes in stress would not affect the anisotropy. An interesting observation related to the 2006 and 2007 eruptions of Mt. Ruapehu was the alteration of scatterers at the times of the eruptions. These scatterers inhibit the observation of subtle changes in the $\mathrm{S}$ waveforms that could affect shear wave splitting, but have implications for inferring other changes along the path.

We have shown that anisotropy at Mt. Ruapehu is not completely governed by stress from the magma plumbing system, and that even in the regions where the shear wave splitting is inferred to be stress-induced, the stresses are complex. Therefore, we cannot rule out any of the models of the magma plumbing system from previous studies described in Chapter 2, but suggest that the system is acting on several scales. A deep magma reservoir may affect the wider stress regime but is masked in areas by more localised sources. Changes in time seem to be due to variations in pore-fluid pressure, which are linked to volcanic activity, but the relationship of shear wave splitting to volcanic activity cannot simply be modelled with Coulomb stresses. This suggests that a highly localised investigation of shear wave splitting close to the summit of Mt. Ruapehu may reveal detectable changes preceding small eruptions because the changes that have been observed on this scale have been attributed to increased pressure [e.g. Otway, 1979; Mordret et al., 2010; Jolly et al., 2010].

In Chapter 6 we applied some of these methods to Okmok volcano, Alaska. At Okmok, we found a difference in the dominant fast polarisations of anisotropy from the local seismicity before the major eruption in 2008 when compared to that from the co-eruptive seismicity. The observed change was likely due to a variation in raypath as most of the pre-eruptive seismicity originated in the geothermal fields to the southeast of the caldera, whereas the co-eruptive seismicity occurred mainly within the northern part of the caldera. In order to identify whether the changes in shear wave splitting were due to temporal changes, a multiplet of 25 earthquakes was identified and used to investigate the anisotropy over time. 
Shear wave splitting analysis using the multiplet at Okmok did not reveal any systematic changes over time associated with the 2008 eruption, but did yield other interesting observations. We found that there were two populations within the multiplet. Both gave the same fast direction, but the delay time fell into two clusters. Examination of the waveforms revealed an extra pulse of energy preceding the $\mathrm{S}$ wave arrival seen when the components were rotated into fast and slow directions. The pulse was interpreted to originate from a $\mathrm{P}$ to $\mathrm{S}$ conversion due to a scatterer along the raypath. Similar relationships have been seen using repeating earthquakes at Piton de la Fornaise volcano in La Reunion [Savage et. al, in prep.] and at Ruapehu (Chapter 5).

The use of multiplets as a tool for monitoring temporal changes in anisotropy has been revealed to have potential, but requires careful analysis. An understanding of the whole wavetrain is needed so that scatterers can be identified and not erroneously matched in the shear wave splitting algorithm. Understanding the scatterers themselves, and their temporal evolution, could be useful for the monitoring of volcanoes, but in shear wave splitting analysis they are unwanted noise. Analysis of the individual scatterers is beyond the scope of this thesis. A gradual stretching or compression of the multiplet wavetrain may yield a gradual change in anisotropy although we do not observe that type of behaviour at either of the volcanoes in this study. We conclude that the use of multiplets has potential for both detection of temporal changes in anisotropy, and for monitoring the evolution of scatterers, but more work is needed to understand the whole wavetrain.

This thesis prompts several avenues of further investigation. The 2-D delay time tomography and spatial averaging of fast polarisations provides a first order approximation for anisotropy structure, but full 3-D tomography of the shear wave splitting results will enable us to gain even better resolution of the regions of anisotropy. Finite element modelling, including topography, will illuminate the proportion of the stress causing the shear wave splitting observations that originates from sources other than a simple inflation of the magmatic system. Application of 2-D delay time tomography and spatial averaging of fast polarisations to past deployments has uncovered the possibility that the high anisotropy area near Mt. Tongariro in 2008 was a temporary feature. This could be investigated further by examining the temporal variations in shear wave splitting at stations close to Mt. Tongariro. Finally, analysis of the scatterers seen in the coda of repeating earthquakes, their relationship with volcanic activity, and their effect on shear wave splitting parameters could provide important information for the development of the methods presented 
in this thesis. 



\section{Bibliography}

Abt, D. L., and K. M. Fischer (2008), Resolving three-dimensional anisotropic structure with shear wave splitting tomography, Geophysical Journal International, $173(3), 859-886$.

Adams, C. J., H. J. Campbell, I. J. Graham, and N. Mortimer (1998), Torlesse, Waipapa and Caples suspect terranes of New Zealand: Integrated studies of their geological history in relation to neighbouring terranes, Episodes, 21 (4), 235-240.

Alaska Volcano Observatory (Last accessed 22 Feb 2011), https://www.avo. alaska.edu/.

Allmann, B. P., and P. M. Shearer (2007), Spatial and temporal stress drop variations in small earthquakes near Parkfield, California, Journal Of Geophysical Research-Solid Earth, 112(B4), doi:DOI:10.1029/2006JB004395.

Alparone, S., and S. Gambino (2003), High precision locations of multiplets on south-eastern flank of Mt. Etna (Italy): reconstruction of fault plane geometry, Physics Of The Earth And Planetary Interiors, 135(4), 281-289.

Antolik, M., R. M. Nadeau, R. C. Aster, and T. V. McEvilly (1996), Differential analysis of coda Q using similar microearthquakes in seismic gaps. 2. Application to seismograms recorded by the Parkfield high resolution seismic network, Bulletin Of The Seismological Society Of America, 86(3), 890-910.

Arnold, R., and J. Townend (2007), A Bayesian approach to estimating tectonic stress from seismological data, Geophysical Journal International, 170(3), 13361356 .

Aster, R. C., and J. Scott (1993), Comprehensive characterization of wave-form similarity in microearthquake data sets, Bulletin Of The Seismological Society Of America, 83(4), 1307-1314.

Aster, R. C., P. M. Shearer, and J. Berger (1990), Quantitative measurements of shear-wave polarizations at the Anza seismic network, Southern California - implications for shear-wave splitting and earthquake prediction, Journal Of Geophysical Research-Solid Earth And Planets, 95(B8), 12,449-12,473.

Aster, R. C., G. Slad, J. Henton, and M. Antolik (1996), Differential analysis of coda Q using similar microearthquakes in Seismic gaps. 1. Techniques and application to seismograms recorded in the Anza Seismic gap, Bulletin Of The Seismological Society Of America, 86(3), 868-889. 
Audoine, E., M. K. Savage, and K. Gledhill (2000), Seismic anisotropy from local earthquakes in the transition region from a subduction to a strike-slip plate boundary, New Zealand, Journal Of Geophysical Research-Solid Earth, 105(B4), 8013-8033.

Audoine, E., M. K. Savage, and K. Gledhill (2004), Anisotropic structure under a back arc spreading region, the Taupo Volcanic Zone, New Zealand, J. Geophys. Res., 109, -, doi:10.1029/2003JB002932.

Babuška, V., and M. Cara (1991), Seismic Anisotropy in the Earth, vol. 10, Kluwer Academic Publishers.

Baisch, S., and G. H. R. Bokelmann (2001), Seismic waveform attributes before and after the Loma Prieta earthquake: Scattering change near the earthquake and temporal recovery, Journal Of Geophysical Research-Solid Earth, 106 (B8), $16,323-16,337$.

Baisch, S., and H. P. Harjes (2003), A model for fluid-injection-induced seismicity at the KTB, Germany, Geophysical Journal International, 152(1), 160-170.

Baisch, S., R. Weidler, R. Voros, D. Wyborn, and L. de Graaf (2006), Induced seismicity during the stimulation of a geothermal HFR reservoir in the Cooper Basin, Australia, Bulletin Of The Seismological Society Of America, 96(6), 22422256.

Baisch, S., L. Ceranna, and H. P. Harjes (2008), Earthquake cluster: What can we learn from waveform similarity?, Bulletin Of The Seismological Society Of America, 98(6), 2806-2814.

Balfour, N. J., M. K. Savage, and J. Townend (2005), Stress and crustal anisotropy in Marlborough, New Zealand: evidence for low fault strength and structurecontrolled anisotropy, Geophysical Journal International, 163(3), 1073-1086.

Barani, S., G. Ferretti, M. Massa, and D. Spallarossa (2007), The waveform similarity approach to identify dependent events in instrumental seismic catalogues, Geophysical Journal International, 168(1), 100-108.

Barisin, I., S. Leprince, and T. Wright (2009), Surface displacements in the september 2005 afar rifting event from satellite image matching: asymmetric uplift and faulting, Geophysical Research Letters, 36, L07,301, doi:10.1029/2008GL036431.

Battaglia, J., J. L. Got, and P. Okubo (2003), Location of long-period events below Kilauea Volcano using seismic amplitudes and accurate relative relocation, Journal Of Geophysical Research-Solid Earth, 108(B12), doi:10.1029/2003JB002517.

Beanland, S., and J. Haines (1998), The kinematics of active deformation in the North Island, New Zealand, determined from geological strain rates, New Zealand Journal Of Geology And Geophysics, 41(4), 311-323. 
Beetham, R. D., and W. A. Watters (1985), Geology of Torlesse and Waipapa terrane basement rocks encountered during the Tongariro power development project, North-Island, New-Zealand, New Zealand Journal Of Geology And Geophysics, 28(4), 575-594.

Begét, J. E., J. F. Larsen, C. A. Neal, C. J. Nye, and J. R. Schaefer (2005), Preliminary volcano-hazard assessment for Okmok Volcano, Umnak Island, Alaska, Report of investigations 2004-3, Alaska Department of Natural Resources, Division of Geological \& Geophysical Surveys.

Bernard, P., and A. Zollo (1989), Inversion of near-source S-polarization for parameters of double-couple point sources, Bulletin Of The Seismological Society Of America, 79 (6), 1779-1809.

Beroza, G. C., A. T. Cole, and W. L. Ellsworth (1995), Stability of coda wave attenuation during the Loma-Prieta, California, earthquake sequence, Journal of Geophysical Research-Solid Earth, 100(B3), 3977-3987.

Bianco, F., and L. Zaccarelli (2009), A reappraisal of shear wave splitting parameters from Italian active volcanic areas through a semiautomatic algorithm, Journal of Seismology, 13(2), 253-266.

Bianco, F., M. Castellano, G. Milano, and G. Vilardo (1996), Shear wave polarization alignment on the eastern flank of Mt. Etna Volcano (Sicily, Italy), Annali di Geofisica, XXXIX, 429-443.

Bianco, F., M. Castellano, G. Milano, G. Ventura, and G. Vilardo (1998), The Somma-Vesuvius stress field induced by regional tectonics: evidences from seismological and mesostructural data, Journal Of Volcanology And Geothermal Research, 82(1-4), 199-218.

Bianco, F., L. Scarfi, E. Del Pezzo, and D. Patane (2006), Shear wave splitting changes associated with the 2001 volcanic eruption on Mt Etna, Geophysical Journal International, 167(2), 959-967.

Bibby, H. M., T. G. Caldwell, F. J. Davey, and T. H. Webb (1995), Geophysical evidence on the structure of the Taupo Volcanic Zone and its hydrothermal circulation, Journal Of Volcanology And Geothermal Research, 68(1-3), 29-58.

Bokelmann, G. H. R., and G. C. Beroza (2000), Depth-dependent earthquake focal mechanism orientation: Evidence for a weak zone in the lower crust, J. Geophys. Res., 105,-, doi:10.1029/2000JB900205.

Bokelmann, G. H. R., and H.-P. Harjes (2000), Evidence for temporal variation of seismic velocity within the upper continental crust, J. Geophys. Res., 105,-, doi:10.1029/2000JB900207.

Boness, N. L., and M. D. Zoback (2006a), Mapping stress and structurally controlled crustal shear velocity anisotropy in California, Geology, 34(10), 825-828. 
Boness, N. L., and M. D. Zoback (2006b), A multiscale study of the mechanisms controlling shear velocity anisotropy in the San Andreas Fault Observatory at Depth, Geophysics, 71(5), F131-F146.

Booth, D. C., and S. Crampin (1985), Shear-wave polarizations on a curved wavefront at an isotropic free-surface, Geophysical Journal Of The Royal Astronomical Society, 83(1), 31-45.

Booth, D. C., M. Wyss, and D. Gillard (1992), Shear-wave polarization alignments recorded above the Kaoiki fault zone, Hawaii, Geophysical Research Letters, 19(11), 1141-1144.

Brancato, A., and S. Gresta (2003), High precision relocation of microearthquakes at Mt. Etna (1991-1993 eruption onset): a tool for better understanding the volcano seismicity, Journal Of Volcanology And Geothermal Research, 124(3-4), 219-239.

Brenguier, F., N. M. Shapiro, M. Campillo, V. Ferrazzini, Z. Duputel, O. Coutant, and A. Nercessian (2008), Towards forecasting volcanic eruptions using seismic noise, Nature Geoscience, 1(2), 126-130.

Brune, J. N. (1970), Tectonic stress and spectra of seismic shear waves from earthquakes, Journal of Geophysical Research, 75(26), 4997-\&.

Bryan, C. J., and S. Sherburn (1999), Seismicity associated with the 1995-1996 eruptions of Ruapehu volcano, New Zealand: narrative and insights into physical processes, Journal Of Volcanology And Geothermal Research, 90(1-2), 1-18.

Bryan, C. J., and S. Sherburn (2003), Eruption-induced modifications to volcanic seismicity at Ruapehu, New Zealand, and its implications for eruption forecasting, Bulletin Of Volcanology, 65(1), 30-42.

Carmona, E., D. Stich, J. M. Ibanez, and G. Saccorotti (2009), Multiplet focal mechanisms from polarities and relative locations: The Iznajar Swarm in Southern Spain, Bulletin Of The Seismological Society Of America, 99(6), 3421-3429.

Carrivick, J. L., V. Manville, and S. J. Cronin (2009), A fluid dynamics approach to modelling the 18th March 2007 lahar at Mt. Ruapehu, New Zealand, Bulletin Of Volcanology, $71(2), 153-169$.

Cassidy, J., M. Ingham, C. A. Locke, and H. Bibby (2009), Subsurface structure across the axis of the Tongariro Volcanic Centre, New Zealand, Journal Of Volcanology And Geothermal Research, 179(3-4), 233-240.

Cattaneo, M., P. Augliera, D. Spallarossa, and C. Eva (1997), Reconstruction of seismogenetic structures by multiplet analysis: An example of Western Liguria, Italy, Bulletin Of The Seismological Society Of America, 87(4), 971-986.

Cattaneo, M., P. Augliera, D. Spallarossa, and V. Lanza (1999), A waveform similarity approach to investigate seismicity patterns, Natural Hazards, 19(2-3), 123-138. 
Chapman, M. (2003), Frequency-dependent anisotropy due to meso-scale fractures in the presence of equant porosity, Geophysical Prospecting, 51(5), 369-379.

Chevrot, S. (2000), Multichannel analysis of shear wave splitting, Journal Of Geophysical Research-Solid Earth, 105(B9), 21,579-21,590.

Chouet, B. A. (1996), Long-period volcano seismicity: Its source and use in eruption forecasting, Nature, 380(6572), 309-316.

Christensen, N. I., and W. D. Mooney (1995), Seismic velocity structure and composition of the continental-crust - a global view, Journal Of Geophysical ResearchSolid Earth, 100(B6), 9761-9788.

Christenson, B. W. (1994), Convection and stratification in Ruapehu Crate Lake, New-Zealand - implications for lake nyos-type gas-release eruptions, Geochemical Journal, 28(3), 185-197.

Christenson, B. W., and C. P. Wood (1993), Evolution of a vent-hosted hydrothermal system beneath Ruapehu Crater Lake, New-Zealand, Bulletin Of Volcanology, $55(8), 547-565$.

Christenson, B. W., A. G. Reyes, R. Young, A. Moebis, S. Sherburn, J. Cole-Baker, and K. Britten (2010), Cyclic processes and factors leading to phreatic eruption events: Insights from the 25 September 2007 eruption through Ruapehu Crater Lake, New Zealand, Journal Of Volcanology And Geothermal Research, 191(1-2), $15-32$.

Chun, K. Y., Q. Y. Yuan, and G. A. Henderson (2010), Precursory Rise of P-Wave Attenuation before the 2004 Parkfield Earthquake, Bulletin Of The Seismological Society Of America, 100(2), 509-521.

Crampin, S. (1984), An introduction to wave-propagation in anisotropic media, Geophysical Journal Of The Royal Astronomical Society, 76(1), 17-28.

Crampin, S. (1985), Evaluation of anisotropy by shear-wave splitting, Geophysics, $50(1), 142-152$.

Crampin, S. (1986), Anisotropy and transverse isotropy, Geophysical Prospecting, $34(1), 94-99$.

Crampin, S. (1991), Wave propagation through fluid-filled inclusions of various shapes: Interpretation of extensive-dilatancy anisotropy, Geophysical Journal International, 10\%, 611-623.

Crampin, S. (1994), The fracture criticality of crustal rocks, Geophysical Journal International, 118(2), 428-438.

Crampin, S., and S. Peacock (2005), A review of shear-wave splitting in the compliant crack-critical anisotropic Earth, Wave Motion, 41(1), 59-77. 
Crampin, S., and S. Peacock (2008), A review of the current understanding of seismic shear-wave splitting in the Earth's crust and common fallacies in interpretation, Wave Motion, 45(6), 675-722.

Crampin, S., and S. V. Zatsepin (1997), Modelling the compliance of crustal rock .2. response to temporal changes before earthquakes, Geophysical Journal International, 129(3), 495-506.

Crampin, S., T. Volti, and R. Stefansson (1999), A successfully stress-forecast earthquake, Geophysical Journal International, 138(1), F1-F5.

Crampin, S., T. Volti, S. Chastin, A. Gudmundsson, and R. Stefansson (2002), Indication of high pore-fluid pressures in a seismically-active fault zone, Geophysical Journal International, 151 (2), F1-F5.

Crotwell, H. P., T. J. Owens, and J. Ritsema (1999), The TauP Toolkit: Flexible seismic travel-time and ray-path utilities, Seismological Research Letters, 70, 154160.

Currie, C. A., J. F. Cassidy, and R. D. Hyndman (2001), A regional study of shear wave splitting above the Cascadia subduction zone: Margin-parallel crustal stress, Geophysical Research Letters, 28(4), 659-662.

Davey, F. J., S. A. Henrys, and E. Lodolo (1995), Asymmetric rifting in a continental back-arc environment, North-Island, New-Zealand, Journal Of Volcanology And Geothermal Research, 68(1-3), 209-\&.

Davis, J. C. (1986), Statistics and Data Analysis in Geology, John Wiley \& Sons, Inc.

De Meersman, K., J. M. Kendall, and M. van der Baan (2009), The 1998 Valhall microseismic data set: An integrated study of relocated sources, seismic multiplets, and S-wave splitting, Geophysics, $74(5), \mathrm{B} 183-\mathrm{B} 195$.

Del Pezzo, E., F. Bianco, S. Petrosino, and G. Saccorotti (2004), Changes in the coda decay rate and shear-wave splitting parameters associated with seismic swarms at Mt. Vesuvius, Italy, Bulletin Of The Seismological Society Of America, 94(2), 439-452.

Dibble, R. R., J. Olsen, and B. A. Sissons (1985), A seismic travel-time graph to 25 $\mathrm{km}$ from explosions in Ruapehu Crater Lake, New Zealand volcanological record: Volcano and Geothermal Observations 1983, (13), 62-65.

Dixon, J., and S. Stihler (2009), Catalog of earthquake hypocenters at Alaskan volcanoes: January 1 through December 31, 2008, U.S. Geological Survey Data Series, $467,88$.

do Nascimento, A. F., F. H. R. Bezerra, and M. K. Takeya (2004), Ductile Precambrian fabric control of seismic anisotropy in the Açu dam area, northeastern Brazil, J. Geophys. Res., 109,-, doi:10.1029/2004JB003120. 
Donoghue, S. L., J. A. Gamble, A. S. Palmer, and R. B. Stewert (1995), Magma mingling in an andesite pyroclastic flow of the Pourahu member, Ruapehu Volcano, New-Zealand, Journal Of Volcanology And Geothermal Research, 68(1-3), $177-191$.

Dreger, D., R. M. Nadeau, and A. Chung (2007), Repeating earthquake finite source models: Strong asperities revealed on the San Andreas Fault, Geophysical Research Letters, $34(23)$, L23,302-1-5.

Du, W. X., C. H. Thurber, and D. Eberhart-Phillips (2004a), Earthquake relocation using cross-correlation time delay estimates verified with the bispectrum method, Bulletin Of The Seismological Society Of America, 94(3), 856-866.

Du, W. X., C. H. Thurber, M. Reyners, D. Eberhart-Phillips, and H. J. Zhang (2004b), New constraints on seismicity in the Wellington region of New Zealand from relocated earthquake hypocentres, Geophysical Journal International, 158(3), 1088-1102.

Eberhart-Phillips, D., and M. Reyners (2009), Three-dimensional distribution of seismic anisotropy in the Hikurangi subduction zone beneath the central North Island, New Zealand, Journal Of Geophysical Research-Solid Earth, 114, doi:10. 1029/2008JB005947.

Elkibbi, M., M. Yang, and J. A. Rial (2005), Crack-induced anisotropy models in The Geysers geothermal field, Geophysical Journal International, 162(3), 10361048 .

Evans, K. F., H. Moriya, H. Niitsuma, R. H. Jones, W. S. Phillips, A. Genter, J. Sausse, R. Jung, and R. Baria (2005), Microseismicity and permeability enhancement of hydrogeologic structures during massive fluid injections into granite at $3 \mathrm{~km}$ depth at the Soultz HDR site, Geophysical Journal International, 160(1), 388-412.

Everitt, B., S. Landau, and M. Leese (2001), Cluster Analysis, fourth ed., Arnold, London.

Fischer, T., and J. Michalek (2008), Post 2000-swarm microearthquake activity in the principal focal zone of West Bohemia/Vogtland: Space-time distribution and waveform similarity analysis, Studia Geophysica Et Geodaetica, 52(4), 493-511.

Fournier, T., J. Freymueller, and P. Cervelli (2009), Tracking magma volume recovery at Okmok volcano using GPS and an unscented Kalman filter, Journal of Geophysical Research-Solid Earth, 114, B02,405.

Freymueller, J. T., and A. M. Kaufman (2010), Changes in the magma system during the 2008 eruption of Okmok volcano, Alaska, based on GPS measurements, Journal Of Geophysical Research-Solid Earth, 115, B12,415, doi: 10.1029/2010JB007716. 
Fukao, Y. (1984), Evidence from core-reflected shear-waves for anisotropy in the Earth's mantle, Nature, 309 (5970), 695-698.

Gamble, J. A., C. P. Wood, R. C. Price, I. E. M. Smith, R. B. Stewart, and T. Waight (1999), A fifty year perspective of magmatic evolution on Ruapehu Volcano, New Zealand: verification of open system behaviour in an arc volcano, Earth And Planetary Science Letters, 170(3), 301-314.

Gamble, J. A., R. C. Price, I. E. M. Smith, W. C. McIntosh, and N. W. Dunbar (2003), Ar-40/Ar-39 geochronology of magmatic activity, magma flux and hazards at Ruapehu volcano, Taupo Volcanic Zone, New Zealand, Journal Of Volcanology And Geothermal Research, 120(3-4), 271-287.

Gao, Y., and S. Crampin (2004), Observations of stress relaxation before earthquakes, Geophysical Journal International, 157(2), 578-582.

Gardine, M., and D. C. Roman (2010), Investigating the pre- and post-eruptive stress regime at Redoubt volcano, Alaska, from 2008-2010 using seismic anisotropy and stress-tensor inversions, in Abstract V31E-06 presented at 2010 Fall Meeting, AGU, San Francisco, Calif., 13-17 Dec.

Geller, R. J., and C. S. Mueller (1980), 4 similar earthquakes in central California, Geophysical Research Letters, 7(10), 821-824.

GeoNet (Last accessed 17 April 2011), http://www.geonet.org.nz/.

Geophysics Program, University of Washington (1980), Eruption of mt. st. helens: Seismology, Nature, 285, 529-531.

Gephart, J. W. (1990), FMSI - a fortran program for inverting fault slickenside and earthquake focal mechanism data to obtain the regional stress tensor, Computers E Geosciences, 16(7), 953-989.

Gerst, A. (2003), Temporary changes in seismic anisotropy as a new eruption forecasting tool?, Master's thesis, Victoria University of Wellington.

Gerst, A., and M. K. Savage (2004), Seismic anisotropy beneath Ruapehu Volcano: A possible eruption forecasting tool, Science, 306(5701), 1543-1547.

Giggenbach, W. (1974), Chemistry of Crater Lake, Mt Ruapehu (New-Zealand) during and after 1971 active period, New Zealand Journal Of Science, 17(1), $33-45$.

Gill, P., W. Murray, and M. Wright (1981), Practical Optimization, Academic Press.

Gledhill, K. R. (1991), Evidence for shallow and pervasive seismic anisotropy in the Wellington region, New-Zealand, Journal Of Geophysical Research-Solid Earth, 96(B13), 21,503-21,516.

GNS Science (2011), GEONET NEWS, EXPLORING THE GEONET PROJECT, Issue 14 . 
Godfrey, N., N. Christensen, and D. Okaya (2000), Anisotropy of schists: Contribution of crustal anisotropy to active source seismic experiments and shear wave splitting observations, Journal of Geophysical Research, 105, 27,991-28,007.

Goldstein, P., and A. Snoke (2005), SAC Availability for the IRIS Community, Incorporated Institutions for Seismology Data Management Center Electronic Newsletter, available at http://www.iris.edu/news/newsletter/vol7no1/page1.htm.

Goldstein, P., D. Dodge, M. Firpo, and L. Minner (2003), SAC2000: Signal processing and analysis tools for seismologists and engineers, Invited contribution to "The IASPEI International Handbook of Earthquake and Engineering Seismology", Academic Press, London.

Got, J. L., and O. Coutant (1997), Anisotropic scattering and travel time delay analysis in Kilauea volcano, Hawaii, earthquake coda waves, Journal Of Geophysical Research-Solid Earth, 102(B4), 8397-8410.

Got, J. L., and J. Frechet (1993), Origins of amplitude variations in seismic doublets - source or attenuation process, Geophysical Journal International, 114(2), 325340 .

Got, J. L., G. Poupinet, and J. Frechet (1990), Changes in source and site effects compared to coda Q-1 temporal variations using microearthquakes doublets in California, Pure And Applied Geophysics, 134(2), 195-228.

Got, J. L., J. Frechet, and F. W. Klein (1994), Deep fault plane geometry inferred from multiplet relative relocation beneath the south flank of Kilauea, Journal of Geophysical Research-Solid Earth, 99 (B8), 15,375-15,386.

Got, J. L., P. Okubo, R. Machenbaum, and W. Tanigawa (2002), A real-time procedure for progressive multiplet relative relocation at the Hawaiian Volcano Observatory, Bulletin Of The Seismological Society Of America, 92(5), 2019-2026.

Graham, I. J., J. W. Cole, R. M. Briggs, J. A. Gamble, and I. E. M. Smith (1995), Petrology and petrogenesis of volcanic-rocks from the Taupo Volcanic Zone - a review, Journal Of Volcanology And Geothermal Research, 68(1-3), 59-87.

Green, D. N., and J. Neuberg (2006), Waveform classification of volcanic lowfrequency earthquake swarms and its implication at Soufrière Hills Volcano, Montserrat, Journal Of Volcanology And Geothermal Research, 153(1-2), 51-63.

Greve, S. M., M. K. Savage, and S. D. Hofmann (2008), Strong variations in seismic anisotropy across the Hikurangi subduction zone, North Island, New Zealand, Tectonophysics, 462(1-4), 7-21.

Gudmundsson, A., V. Acocella, and S. Vinciguerra (2009), Understanding stress and deformation in active volcanoes, Tectonophysics, 471(1-2), 1-3.

Hackett, W. R., and B. F. Houghton (1989), A facies model for a quaternary andesitic composite-volcano - Ruapehu, New-Zealand, Bulletin Of Volcanology, $51(1), 51-68$. 
Hagerty, M., and R. Benites (2003), Tornillos beneath Tongariro Volcano, New Zealand, Journal of Volcanology and Geothermal Research, 125, 151-169.

Haney, M. M. (2010), Location and mechanism of VLP tremor during the 2008 eruption of Okmok Volcano from inter-station arrival times, Journal of Geophysical Research-Solid Earth, 115, B00B05, doi:10.1029/2010JB007440.

Hatchell, P., and S. Bourne (2005), Rocks under strain: Strain-induced time-lapse time shifts are observed for depleting reservoirs, The Leading Edge, 24(12), 12221225.

Hayes, G., M. Reyners, and G. Stuart (2004), The Waiouru, New Zealand, earthquake swarm: Persistent mid crustal activity near an active volcano, Geophysical Research Letters, $31(19)$.

Healy, A. J. (1954), Contributions to a knowledge of the naturalized flora of New Zealand. no. 4, Trans Roy Soc New Zealand, 82(2), 263-269.

Hemmann, A., T. Meier, G. Jentzsch, and A. Ziegert (2003), Similarity of waveforms and relative relocalisation of the earthquake swarm 1997/1998 near Werdau, Journal Of Geodynamics, 35(1-2), 191-208.

Henrys, S., M. Reyners, and H. Bibby (2003), Exploring the plate boundary structure of the North Island, New Zealand, Eos Trans. AGU, 84, doi:10.1029/ $2003 \mathrm{EO} 310002$.

Hess, H. H. (1964), Seismic anisotropy of uppermost mantle under oceans, Nature, 203(494), 629-\&.

Hill, M. N. (1952), Seismic refraction shooting in an area of the eastern Atlantic, Philosophical Transactions Of The Royal Society Of London Series AMathematical And Physical Sciences, 244(890), 561-594.

Hochstein, M. P. (1995), Crustal heat-transfer in the Taupo Volcanic Zone (NewZealand) - comparison with other volcanic arcs and explanatory heat-source models, Journal Of Volcanology And Geothermal Research, 68(1-3), 117-151.

Hofmann, S. D. (2002), Seismic anisotropy in the crust and mantle: A study at the western edge of the Central Volcanic Region, New Zealand, Master's thesis, Victoria University of Wellington.

Horspool, N. A. (2003), Bending stress and faulting linked to the load of Ruapehu Volcano, Master's thesis, Victoria University of Wellington.

Houghton, B. F., J. H. Latter, and W. R. Hackett (1987), Volcanic Hazard assessment for Ruapehu composite volcano, Taupo Volcanic Zone, New Zealand, Bulletin of Volcanology, 49, 737-751.

Hurst, A. W. (1992), Stochastic simulation of volcanic tremor from Ruapehu, Journal of Volcanology and Geothermal Research, 51, 185-198. 
Hurst, A. W. (1998), Shallow seismicity beneath Ruapehu Crater Lake: results of a 1994 seismometer deployment, Bulletin Of Volcanology, 60(1), 1-9.

Hurst, A. W., and R. R. Dibble (1981), Bathymetry, heat output and convection in Ruapehu Crater Lake, New Zealand, Journal of Volcanology and Geothermal Research, 9, 215-236.

Hurst, A. W., and N. Fournier (2009), Near real-time volcano GPS, in Tongariro Day- a workshop to discuss scientific advances at the Tongariro National Park volcanoes.

Hurst, A. W., and P. J. McGinty (1996), Seismicity of Ruapehu Volcano recorded during the 1994 seismometer deployment, Science report 95/45. 13 p, Institute of Geological and Nuclear Sciences.

Hurst, A. W., and P. J. McGinty (1999), Earthquake swarms to the west of Mt Ruapehu preceding its 1995 eruption, Journal Of Volcanology And Geothermal Research, 90(1-2), 19-28.

Hurst, A. W., and S. Sherburn (1993), Volcanic tremor at Ruapehu - characteristics and implications for the resonant source, New Zealand Journal Of Geology And Geophysics, 36(4), 475-485.

Hurst, A. W., and J. Vandemeulebrouck (1995), Some notes on the last years of Ruapehu Crater Lake (1994-1995), Tech. rep., IGNS Science Contribution 1218.

Hurst, A. W., and J. Vandemeulebrouck (1996), Acoustic noise and temperature monitoring of the crater lake of Mount Ruapehu Volcano, Journal Of Volcanology And Geothermal Research, 71(1), 45-51.

Hurst, A. W., H. M. Bibby, B. J. Scott, and M. J. McGuinness (1991), The heatsource of Ruapehu Crater Lake - deductions from the energy and mass balances, Journal Of Volcanology And Geothermal Research, 46(1-2), 1-20.

Hurst, A. W., B. J. Scott, C. Werner, N. Stevens, and H. Cowan (2004), Monitoring New Zealand volcanoes, in Tephra, pp. 12-17.

Igarashi, T., T. Matsuzawa, and A. Hasegawa (2003), Repeating earthquakes and interplate aseismic slip in the northeastern Japan subduction zone, Journal of Geophysical Research-Solid Earth, 108(B5), ESE8-1-9.

Ingham, M. R., H. M. Bibby, W. Heise, K. A. Jones, P. Cairns, S. Dravitzki, S. L. Bennie, T. G. Caldwell, and Y. Ogawa (2009), A magnetotelluric study of Mount Ruapehu volcano, New Zealand, Geophysical Journal International, 179(2), 887904.

Jacobs, K. M., and S. R. McNutt (2010), The 2006 Eruption of Augustine Volcano, Alaska, chap. 3: Using seismic b-values to interpret seismicity rates and physical processes during the pre-eruptive earthquake swarm at Augustine Volcano 20052006, pp. 1-17, U.S. Geological Survey Professional Paper 1769. 
Johnston, D. M., and V. E. Neall (1995), Ruapehu awakens: The 1945 eruption of ruapehu, The Science Centre and Anawatu Museum Scietific Monograph No. 1.

Johnston, D. M., B. F. Houghton, V. E. Neall, K. R. Ronan, and D. Paton (2000), Impacts of the 1945 and 1995-1996 Ruapehu eruptions, New Zealand: An example of increasing societal vulnerability, Geological Society Of America Bulletin, 112(5), 720-726.

Jolly, A. D., S. Sherburn, P. Jousset, and G. Kilgour (2010), Eruption source processes derived from seismic and acoustic observations of the 25 September 2007 Ruapehu eruption-North Island, New Zealand, Journal Of Volcanology And Geothermal Research, 191 (1-2), 33-45.

Jolly, A. D., J. Neuberg, P. Jousset, and S. Sherburn (2011), A new source process for evolving repetitious earthquakes at Ngauruhoe volcano, New Zealand, in prep.

Jones, K. A., M. R. Ingham, and H. M. Bibby (2008), The hydrothermal vent system of Mount Ruapehu, New Zealand - a high frequency MT survey of the summit plateau, Journal Of Volcanology And Geothermal Research, 176(4), 591-600.

Jost, M. L., T. Busselberg, O. Jost, and H. P. Harjes (1998), Source parameters of injection-induced microearthquakes at $9 \mathrm{~km}$ depth at the KTB deep drilling site, Germany, Bulletin Of The Seismological Society Of America, 88(3), 815-832.

Joswig, M. (1995), Automated classification of local earthquake data in the bug small array, Geophysical Journal International, 120(2), 262-286.

Joyce, K. E., S. Samsonov, and G. Jolly (2009), Temperature, color and deformation monitoring of volcanic regions in New Zealand, 2009 IEEE International Geoscience And Remote Sensing Symposium, Vols 1-5, pp. 17-20.

Kaneshima, S. (1990), Origin of crustal anisotropy - shear-wave splitting studies in Japan, Journal Of Geophysical Research-Solid Earth And Planets, 95(B7), $11,121-11,133$.

Keats, B. (2010), The Erua earthquake cluster and seismic anisotropy in the Ruapehu region, Master's thesis, Victoria University of Wellington.

Keats, B. S., J. H. Johnson, and M. K. Savage (2011), The Erua earthquake cluster and seismic anisotropy in the Ruapehu region, New Zealand, Geophysical Research Letters, In prep.

Kendall, J. M., and P. G. Silver (1996), Constraints from seismic anisotropy on the nature of the lowermost mantle, Nature, 381 (6581), 409-412.

Kilgour, G., V. Manville, F. Della Pasqua, A. Graettinger, K. A. Hodgson, and G. E. Jolly (2010), The 25 September 2007 eruption of Mount Ruapehu, New Zealand: Directed ballistics, surtseyan jets, and ice-slurry lahars, Journal Of Volcanology And Geothermal Research, 191(1-2), 1-14. 
Kim, W., and C. E. Baag (2002), Rapid and accurate two-point ray tracing based on a quadratic equation of takeoff angle in layered media with constant or linearly varying velocity functions, Bulletin Of The Seismological Society Of America, 92(6), 2251-2263.

Kimura, H., K. Kasahara, and T. Takeda (2009), Subduction process of the Philippine Sea Plate off the Kanto district, central Japan, as revealed by plate structure and repeating earthquakes, Tectonophysics, 472(1-4), 18-27.

King, G. C. P., R. S. Stein, and J. Lin (1994), Static stress changes and the triggering of earthquakes, Bulletin of the Seismological Society of America, 84, 935-953.

Kinvig, H. S., A. Geyer, and J. Gottsmann (2009), On the effect of crustal layering on ring-fault initiation and the formation of collapse calderas, Journal of Volcanology and Geothermal Research, 186(3-4), 293-304.

Konstantinou, K. I., C. H. Lin, W. T. Liang, and Y. C. Chan (2009), Seismogenic stress field beneath the Tatun Volcano Group, northern Taiwan, Journal Of Volcanology And Geothermal Research, 187(3-4), 261-271.

Kroon, D.-J. (2009), Accurate Fast Marching, mathworks.

Kufner, S. (2010), Temporal variation of seismic anisotropy at Okmok Vaolcano (Alaska), Master's thesis, Victoria University of Wellington.

Lamarche, G., and J. F. Lebrun (2000), Transition from strike-slip faulting to oblique subduction: active tectonics at the Puysegur Margin, South New Zealand, Tectonophysics, 316(1-2), 67-89.

Larsen, J., et al. (2009), Eruption of Alaska volcano breaks historic pattern, Eos Trans. $A G U$, 90(20), 173.

Latter, J. H. (1981a), Volcanic earthquakes, and their relationship to eruptions at Ruapehu and Ngauruhoe volcanos, Journal Of Volcanology And Geothermal Research, 9(4), 293-309.

Latter, J. H. (1981b), Location of zones of anomalously high S-wave attenuation in the upper crust near Ruapehu and Ngauruhoe volcanos, New Zealand, Journal Of Volcanology And Geothermal Research, 10(1-3), 125-156.

Lees, J. M. (1998), Multiplet analysis at Coso geothermal, Bulletin Of The Seismological Society Of America, 88(5), 1127-1143.

Lees, J. M., and H. T. Wu (1999), P wave anisotropy, stress, and crack distribution at Coso geothermal field, California, Journal Of Geophysical Research-Solid Earth, 104 (B8), 17,955-17,973.

Li, L., Q. F. Chen, X. Cheng, and F. Niu (2007), Spatial clustering and repeating of seismic events observed along the 1976 Tangshan fault, north China, Geophysical Research Letters, 34(23), L23,309, doi:10.1029/2007GL031594. 
Li, L., Q. F. Chen, F. L. Niu, H. Fu, R. F. Liu, and Y. Y. Hou (2009), Slip rate along the Lijiang-Ninglang fault zone estimated from repeating microearthquakes, Chinese Science Bulletin, 54(3), 447-455.

Lin, F. C., M. H. Ritzwollerl, J. Townend, S. Bannister, and M. K. Savage (2007), Ambient noise Rayleigh wave tomography of New Zealand, Geophysical Journal International, 170(2), 649-666.

Lin, J., and R. S. Stein (2004), Stress triggering in thrust and subduction earthquakes and stress interaction between the southern San Andreas and nearby thrust and strike-slip faults, Journal Of Geophysical Research-Solid Earth, 109(B02303), doi:10.1029/2003JB002607.

Liu, E., J. H. Queen, X. Y. Li, M. Chapman, S. Maultzsch, H. B. Lynn, and E. M. Chesnokov (2003), Observation and analysis of frequency-dependent anisotropy from a multicomponent VSP at Bluebell-Altamont Field, Utah, Journal Of Applied Geophysics, 54(3-4), 319-333.

Liu, E. R., S. Crampin, J. H. Queen, and W. D. Rizer (1993), Behavior of shearwaves in rocks with 2 sets of parallel cracks, Geophysical Journal International, 113(2), 509-517.

Liu, Y., H. Zhang, C. Thurber, and S. Roecker (2008), Shear wave anisotropy in the crust around the San Andreas Fault near Parkfield: spatial and temporal analysis, Geophysical Journal International, 172(3), 957-970.

Liu, Y. F., T. L. Teng, and Y. Ben-Zion (2004), Systematic analysis of shear-wave splitting in the aftershock zone of the 1999 Chi-Chi, Taiwan, earthquake: Shallow crustal anisotropy and lack of precursory variations, Bulletin Of The Seismological Society Of America, 94 (6), 2330-2347.

Long, M. D., M. V. de Hoop, and R. D. van der Hilst (2008), Wave-equation shear wave splitting tomography, Geophysical Journal International, 172(1), 311-330.

Lu, Z., and D. Dzurisin (2010), Ground surface deformation patterns, magma supply, and magma storage at Okmok volcano, Alaska, from InSAR analysis: 2. Coeruptive deflation, July-August 2008, Journal of Geophysical Research, 115, B00B03, doi:10.1029/2009JB006970.

Lu, Z., D. Mann, J. T. Freymueller, and D. J. Meyer (2000), Synthetic aperture radar interferometry of Okmok volcano, Alaska: Radar observations, Journal Of Geophysical Research-Solid Earth, 105(B5), 10,791-10,806.

Lu, Z., T. Masterlark, D. Dzurisin, R. Rykhus, and C. Wicks (2003), Magma supply dynamics at Westdahl volcano, Alaska, modeled from satellite radar interferometry, Journal Of Geophysical Research-Solid Earth, 108(B7), 2354, doi: 10.1029/2002JB002311.

Lu, Z., T. Masterlark, and D. Dzurisin (2005), Interferometric synthetic aperture radar study of Okmok volcano, Alaska, 1992-2003: Magma supply dynamics and 
postemplacement lava flow deformation, Journal Of Geophysical Research-Solid Earth, 110(B02403), doi:10.1029/2004JB003148.

Lund, B., and J. Townend (2007), Calculating horizontal stress orientations with full or partial knowledge of the tectonic stress tensor, Geophysical Journal International, $170(3)$, 1328-1335.

Mainprice, D., and A. Nicolas (1989), Development of shape and lattice preferred orientations - application to the seismic anisotropy of the lower crust, Journal of Structural Geology, 11(1-2), 175-189.

Mainprice, D., A. Tommasi, H. Couvy, P. Cordier, and D. J. Frost (2005), Pressure sensitivity of olivine slip systems and seismic anisotropy of Earth's upper mantle, Nature, 433(7027), 731-733.

Manville, V., K. A. Hodgson, and I. A. Nairn (2007), A review of break-out floods from volcanogenic lakes in New Zealand, New Zealand Journal Of Geology And Geophysics, 50(2), 131-150.

Marone, C., J. E. Vidale, and W. L. Ellsworth (1995), Fault healing inferred from time-dependent variations in-source properties of repeating earthquakes, Geophysical Research Letters, 22(22), 3095-3098.

Marson-Pidgeon, K., and M. K. Savage (1997), Frequency-dependent anisotropy in Wellington, New Zealand, Geophysical Research Letters, 24(24), 3297-3300.

Martinez-Arevalo, C., F. Bianco, J. M. Ibanez, and E. Del Pezzo (2003), Shallow seismic attenuation and shear-wave splitting in the short period range of Deception Island volcano (Antarctica), Journal Of Volcanology And Geothermal Research, 128(1-3), 89-113.

Masterlark, T., M. Haney, H. Dickinson, T. Fournier, and C. Searcy (2010), Rheologic and structural controls on the deformation of Okmok volcano, Alaska: FEMs, InSAR and ambient noise tomography., Journal of Geophysical Research-Solid Earth, In Press.

Maultzsch, S., M. Chapman, E. R. Liu, and X. Y. Li (2003), Modelling frequencydependent seismic anisotropy in fluid-saturated rock with aligned fractures: implication of fracture size estimation from anisotropic measurements, Geophysical Prospecting, 51(5), 381-392.

Maurer, H., and N. Deichmann (1995), Microearthquake cluster detection based on wave-form similarities, with an application to the western Swiss Alps, Geophysical Journal International, 123(2), 588-600.

Mavko, G., T. Mukerji, and J. Dvorkin (1998), The rock physics handbook: A tool for seismic analysis in porous media, Cambridge University Press.

McNutt, S. R. (1996), Monitoring and Mitigation of Volcano Hazards., chap. Seismic Monitoring and Eruption Forecasting of Volcanoes: A Review of the State-of-theArt and Case Histories., pp. 99-146, Springer-Verlag Berlin Heidelberg. 
Miller, M., D. Paton, and D. M. Johnston (1999), Community vulnerability to volcanic hazard consequences, Disaster prevention and management, 8, 255-260.

Miller, T. P., R. G. McGimsey, D. H. Richter, J. R. Riehle, G. J. Nye, M. E. Yount, and J. A. Dumoulin (1998), Catalog of the historically active volcanoes of Alaska, Open-File Report OF 98-0582, 104 p, US Geol. Surv.

Miller, V., and M. Savage (2001), Changes in seismic anisotropy after volcanic eruptions: Evidence from Mount Ruapehu, Science, 293(5538), 2231-2233.

Miller, V., T. Hurst, and J. Beavan (2003), Feasibility study for geodetic monitoring of Mt Ruapehu volcano, New Zealand, using GPS, New Zealand Journal of Geology And Geophysics, 46(1), 41-46.

Milligan, G. W., and M. C. Cooper (1985), An examination of procedures for determining the number of clusters in a data set., Psychometrika, 50, 159-179.

Miyagi, Y., J. T. Freymueller, F. Kimata, T. Sato, and D. Mann (2004), Surface deformation caused by shallow magmatic activity at Okmok volcano, Alaska, detected by GPS campaigns 2000-2002, Earth Planets And Space, 56(12), E29-E32.

Mogi, K. (1958), Relations between the eruptions of various volcanoes and the deformations of the ground surfaces around them, Bulletin of the Earthquake Research Institute, Vol. 36, 99-134.

Montagner, J. P., and B. L. N. Kennett (1996), How to reconcile body-wave and normal-mode reference earth models, Geophysical Journal International, 125(1), $229-248$.

Mordret, A., A. D. Jolly, Z. Duputel, and N. Fournier (2010), Monitoring of phreatic eruptions using interferometry on retrieved cross-correlation function from ambient seismic noise: Results from Mt. Ruapehu, New Zealand, Journal Of Volcanology And Geothermal Research, 191(1-2), 46-59.

Moriya, H., H. Niitsuma, and R. Baria (2003), Multiplet-clustering analysis reveals structural details within the seismic cloud at the Soultz geothermal field, France, Bulletin Of The Seismological Society Of America, 93(4), 1606-1620.

Morley, A. M., G. W. Stuart, J. M. Kendall, and M. Reyners (2006), Mantle wedge anisotropy in the Hikurangi subduction zone, central North Island, New Zealand, Geophysical Research Letters, 33(5), L05,301, doi:10.1029/2005GL024569.

Mortimer, N. (1993), Metamorphic zones, terranes, and Cenozoic faults in the Marlborough Schist, New Zealand, New Zealand Journal of Geology and Geophysics, 36, 357-368.

Mortimer, N. (2004), New Zealand's geological foundations, Gondwana Research, 7(1), 261-272.

Motyka, R., S. Liss, C. Nye, and M. Moorman (1994), Geothermal resources of the Aleutian arc, Tech. rep., Alaska Division of Geological \& Geophysical Surveys. 
Munson, C. G., C. H. Thurber, and Y. P. Li (1993), Observations of shear-wave splitting on the southeast flank of Mauna-Loa Volcano, Hawaii, Geophysical Research Letters, 20(12), 1139-1142.

Munson, C. G., C. H. Thurber, Y. P. Li, and P. G. Okubo (1995), Crustal shearwave anisotropy in southern Hawaii - spatial and temporal analysis, Journal of Geophysical Research-Solid Earth, 100(B10), 20,367-20,377.

Musumeci, C., D. Patane, L. Scarfi, and S. Gresta (2005), Stress directions and shear-wave anisotropy: Observations from local earthquakes in southeastern Sicily, Italy, Bulletin Of The Seismological Society Of America, 95(4), 1359-1374.

Nadeau, R. M., and L. R. Johnson (1998), Seismological studies at Parkfield VI: Moment release rates and estimates of source parameters for small repeating earthquakes, Bulletin Of The Seismological Society Of America, 88(3), 790-814.

Nadeau, R. M., and T. V. McEvilly (1999), Fault slip rates at depth from recurrence intervals of repeating microearthquakes, Science, 285(5428), 718-721.

Nairn, I., and S. Self (1978), Explosive eruptions and pyroclastic avalanches from Ngauruhoe in February 1975, Journal of Volcanology and Geothermal Research, 3, 39-60.

Nairn, I. A., C. P. Wood, and C. A. Y. Hewson (1979), Phreatic eruptions of Ruapehu - April 1975, New Zealand Journal Of Geology And Geophysics, 22(2), $155-170$.

Nakagawa, M., K. Wada, T. Thordarson, C. P. Wood, and J. A. Gamble (1999), Petrologic investigations of the 1995 and 1996 eruptions of Ruapehu volcano, New Zealand: formation of discrete and small magma pockets and their intermittent discharge, Bulletin Of Volcanology, 61(1-2), 15-31.

Nakamura, K., and S. Uyeda (1980), Stress gradient in arc-back arc regions and plate subduction, J. Geophys. Res., 85(80B0618), 6419-6428.

Neal, C. A., J. E. Begét, D. Grey, and B. Wolfe (2003), The 1817 eruption of Okmok caldera, Umnak Island, Alaska: New insights into a complex historical eruption in the Eastern Aleutians, Eos Trans. AGU, 84(46), Fall Meet. Suppl., Abstract V42B-0346.

Neall, V. E., B. F. Houghton, S. J. Cronin, S. L. Donogue, K. A. Hodgson, D. M. Johnston, J. A. Lecointre, and A. R. Mitchell (1999), Volcanic hazards at Ruapehu volcano., Ministry of Civil Defence, Volcanic Hazards Information Series No. 8.

Neuberg, J. (2000), Characteristics and causes of shallow seismicity in andesite volcanoes, Philosophical Transactions Of The Royal Society Of London Series A-Mathematical Physical And Engineering Sciences, 358(1770), 1533-1546.

Neuberg, J., R. Luckett, B. Baptie, and K. Olsen (2000), Models of tremor and low-frequency earthquake swarms on Montserrat, Journal Of Volcanology And Geothermal Research, 101(1-2), 83-104. 
Nistala, S., and G. A. McMechan (2005), 3D Modeling of fracture-induced shearwave splitting in the Southern California basin, Bulletin Of The Seismological Society Of America, 95(3), 1090-1100.

Niu, F. L., P. G. Silver, R. M. Nadeau, and T. V. McEvilly (2003), Migration of seismic scatterers associated with the 1993 Parkfield aseismic transient event, Nature, 426 (6966), 544-548.

Nur, A. (1971), Viscous phase in rocks and low-velocity zone, Journal Of Geophysical Research, 76(5), 1270-\&.

Nuttli, O. (1961), The effect of the Earth's surface on the S wave particle motion, Bulletin of the Seismological Society of America, 51(2), 237-246.

Ocana, E., D. Stich, E. Carmona, F. Vidal, M. Breton, M. Navarro, and A. GarciaJerez (2008), Spatial analysis of the La Paca, SE Spain, 2005 seismic series through the relative location of multiplets and principal component analysis, Physics Of The Earth And Planetary Interiors, 166(3-4), 117-127.

Oppenheimer, C., and D. Stevenson (1989), Liquid sulfur lakes at Poas volcano, Nature, 342(6251), 790-793.

Otway, P. M. (1979), Ruapehu deformation surveys and the 1975 April eruption, New Zealand Journal Of Geology And Geophysics, 22(2), 170-173.

Packer, D. R., G. E. Brogae, and D. B. Stone (1975), New data on plate tectonics of Alaska, Tectonophysics, 29(1-4), 87 - 102, doi:10.1016/0040-1951(75)90135-3.

Paige, C. C., and M. A. Saunders (1982), LSQR - an algorithm for sparse linearequations and sparse least-squares, Acm Transactions On Mathematical Software, $8(1), 43-71$.

Palano, M., G. Pugisi, and S. Gresta (2008), Ground deformation patterns at Mt. Etna from 1993 to 2000 from joint use of InSAR and GPS techniques, Journal of Volcanology And Geothermal Research, 169(3-4), 99-120.

Pandolfi, D., C. J. Bean, and G. Saccorotti (2006), Coda wave interferometric detection of seismic velocity changes associated with the $1999 \mathrm{M}=3.6$ event at Mt. Vesuvius, Geophysical Research Letters, 33(6), L06,306, doi:10.1029/2005GL025355.

Patrick, M. R., J. Dehn, and K. Dean (2004), Numerical modeling of lava flow cooling applied to the 1997 Okmok eruption: Approach and analysis, Journal of Geophysical Research-Solid Earth, 109 (B3), doi:10.1029/2003JB002537.

Peng, Z. G., and Y. Ben-Zion (2004), Systematic analysis of crustal anisotropy along the Karadere-Duzce branch of the North Anatolian fault, Geophysical Journal International, $159(1), 253-274$.

Peng, Z. G., and Y. Ben-Zion (2005), Spatiotemporal variations of crustal anisotropy from similar events in aftershocks of the 1999 M7.4 Izmit and M7.1 Duzce, Turkey, earthquake sequences, Geophysical Journal International, 160(3), 1027-1043. 
Petersen, T. (2007), Swarms of repeating long-period earthquakes at Shishaldin Volcano, Alaska, 2001-2004, Journal Of Volcanology And Geothermal Research, 166, 177-192.

Poupinet, G., W. L. Ellsworth, and J. Frechet (1984), Monitoring velocity variations in the crust using earthquake doublets - an application to the Calaveras Fault, California, Journal Of Geophysical Research, 89(NB7), 5719-5731.

Powell, T. W., and J. Neuberg (2003), Time dependent features in tremor spectra, Journal Of Volcanology And Geothermal Research, 128(1-3), 177-185.

Press, W. H., B. P. Flannery, S. A. Teukolsky, and W. T. Vetterling (1986), Numerical Recipes: The Art of Scientific Computing, New York: Cambridge University Press.

Price, R. C., J. A. Gamble, I. E. M. Smith, R. B. Stewart, S. Eggins, and I. C. Wright (2005), An integrated model for the temporal evolution of andesites and rhyolites and crustal development in New Zealand's North Island, Journal Of Volcanology And Geothermal Research, 140(1-3), 1-24.

Price, R. C., R. George, J. A. Gamble, S. Turner, I. E. M. Smith, C. Cook, B. Hobden, and A. Dosseto (2007), U-Th-Ra fractionation during crustal-level andesite formation at Ruapehu volcano, New Zealand, Chemical Geology, 244, 437-451.

Ratdomopurbo, A., and G. Poupinet (1995), Monitoring a temporal change of seismic velocity in a volcano - application to the 1992 eruption of Mt-Merapi (Indonesia), Geophysical Research Letters, 22(7), 775-778.

Rau, R. J., K. H. Chen, and K. E. Ching (2007), Repeating earthquakes and seismic potential along the northern Longitudinal Valley fault of eastern Taiwan, Geophysical Research Letters, 34(24), L24,301, doi:10.1029/2007GL031622.

Rawlinson, Z., J. Townend, and S. Bannister (2010), Earthquake detection and location via waveform cross-correlation in the Rotokawa geothermal field., in Eccles, J.D., Grigor, M.R., Hoskin, P.W.O., Hikuroa, D.C.H. (eds). Abstract Volume, GeoNZ 2010 Conference, Auckland, New Zealand. Geoscience Society of New Zealand Miscellaneous Publication.

Reasenberg, P., and K. Aki (1974), Precise, continuous measurement of seismic velocity for monitoring in-situ stress, Journal Of Geophysical Research, 79(2), 399-406.

Reasenberg, P., and D. Oppenheimer (1985), FPFIT, FPPLOT and FPPAGE: Fortran computer programs for calculating and displaying earthquake fault-plane solutions, Tech. Rep. 109, U.S. Geol. Surv. Open-File Rept. 85-739.

Reyners, M. (1980), A micro-earthquake study of the plate boundary, North-Island, New-Zealand, Geophysical Journal Of The Royal Astronomical Society, 63(1), $1-22$. 
Reyners, M. (2010), Stress and strain from earthquakes at the southern termination of the Taupo Volcanic Zone, New Zealand, Journal Of Volcanology And Geothermal Research, 190(1-2), 82-88.

Rial, J. A., M. Elkibbi, and M. Yang (2005), Shear-wave splitting as a tool for the characterization of geothermal fractured reservoirs: lessons learned, Geothermics, $34(3), 365-385$.

Ribe, N. M. (1989), Seismic anisotropy and mantle flow, Journal Of Geophysical Research-Solid Earth And Planets, 94(B4), 4213-4223.

Risk, G. F., H. M. Bibby, C. J. Bromley, T. G. Caldwell, and S. L. Bennie (2002), Appraisal of the Tokaanu-Waihi geothermal field and its relationship with the Tongariro geothermal field, New Zealand, Geothermics, 31 (1), 45-68.

Robinson, R., and P. J. McGinty (2000), The enigma of the Arthur's Pass, New Zealand, earthquake 2. The aftershock distribution and its relation to regional and induced stress fields, Journal Of Geophysical Research-Solid Earth, 105(B7), $16,139-16,150$.

Roman, D. C., J. Neuberg, and R. R. Luckett (2006), Assessing the likelihood of volcanic eruption through analysis of volcanotectonic earthquake fault-plane solutions, Earth And Planetary Science Letters, 248(1-2), 244-252.

Roman, D. C., M. K. Savage, R. Arnold, J. L. Latchman, and S. De Angelis (2011), Analysis and forward modeling of seismic anisotropy during the ongoing eruption of the Soufrière Hills Volcano, Montserrat, 1996-2007, Journal Of Geophysical Research-Solid Earth, 116, B03,201, doi:10.1029/2010JB007667.

Rowland, J. V., and R. H. Sibson (2001), Extensional fault kinematics within the Taupo Volcanic Zone, New Zealand: soft-linked segmentation of a continental rift system, New Zealand Journal Of Geology And Geophysics, 44(2), 271-283.

Rowlands, D. P., R. S. White, and A. J. Haines (2005), Seismic tomography of the Tongariro Volcanic Centre, New Zealand, Geophysical Journal International, $163(3), 1180-1194$.

Ruapehu Surveillance Group (1996), Volcanic eruption at a New Zealand ski resort prompts reevaluation of hazards, Eos Trans. AGU, 77(20), 189-191.

Rubinstein, J. L., and W. L. Ellsworth (2010), Precise estimation of repeating earthquake moment: Example from Parkfield, California, Bulletin Of The Seismological Society Of America, 100(5A), 1952-1961.

Rumpker, G., and P. G. Silver (2000), Calculating splitting parameters for plumetype anisotropic structures of the upper mantle, Geophysical Journal International, $143(3), 507-520$.

Salmon, M., T. Stern, and M. K. Savage (2011), A $7 \mathrm{~km}$ step in the continental Moho and its geodynamic consequences: the Taranaki-Ruapehu line, New Zealand, Journal of Geophysical Research-Solid Earth, In Review. 
Savage, M. K. (1999), Seismic anisotropy and mantle deformation: What have we learned from shear wave splitting?, Reviews Of Geophysics, 37(1), 65-106.

Savage, M. K., X. R. Shih, R. P. Meyer, and R. C. Aster (1989), Shear-wave anisotropy of active tectonic regions via automated S-wave polarization analysis, Tectonophysics, 165(1-4), 279-292.

Savage, M. K., W. A. Peppin, and U. R. Vetter (1990), Shear-wave anisotropy and stress direction in and near Long Valley Caldera, California, 1979-1988, Journal Of Geophysical Research-Solid Earth And Planets, 95(B7), 11,165-11,177.

Savage, M. K., T. Ohminato, Y. Aoki, H. Tsuji, and S. M. Greve (2010a), Stress magnitude and its temporal variation at Mt. Asama Volcano, Japan, from seismic anisotropy and GPS, Earth And Planetary Science Letters, 290(3-4), 403-414.

Savage, M. K., A. Wessel, N. A. Teanby, and A. W. Hurst (2010b), Automatic measurement of shear wave splitting and applications to time varying anisotropy at Mount Ruapehu volcano, New Zealand, Journal Of Geophysical Research-Solid Earth, 115, B12,321, doi:10.1029/2010JB007722.

Sayers, C. M. (2007), Asymmetry in the time-lapse seismic response to injection and depletion, Geophysical Prospecting, 55(5), 699-705.

Sayers, C. M., and M. Kachanov (1995), Microcrack-induced elastic-wave anisotropy of brittle rocks, Journal Of Geophysical Research-Solid Earth, 100 (B3), 41494156.

Schaff, D. P., and G. C. Beroza (2004), Coseismic and postseismic velocity changes measured by repeating earthquakes, Journal Of Geophysical Research-Solid Earth, 109(B10), doi:10.1029/2004JB003011.

Schaff, D. P., and P. G. Richards (2004), Repeating seismic events in China, Science, 303 (5661), 1176-1178.

Schaff, D. P., G. H. R. Bokelmann, W. L. Ellsworth, E. Zanzerkia, F. Waldhauser, and G. C. Beroza (2004), Optimizing correlation techniques for improved earthquake location, Bulletin Of The Seismological Society Of America, 94 (2), 705-721.

Scott, B. J., and J. Travers (2009), Volcano monitoring in NZ and links to SW Pacific via the Wellington VAAC, Natural Hazards, 51(2), 263-273.

Seher, T., and I. G. Main (2004), A statistical evaluation of a 'stress-forecast' earthquake, Geophysical Journal International, 157(1), 187-193.

Seward, A. M., C. M. Henderson, and E. G. C. Smith (2009), Models of the upper mantle beneath the central North Island, New Zealand, from speeds and anisotropy of subhorizontal P waves (Pn), J. Geophys. Res., 114(B1), B01,301, doi:10.1029/2008JB005805. 
Shearer, P. M. (1997), Improving local earthquake locations using the L1 norm and waveform cross correlation: Application to the Whittier Narrows, California, aftershock sequence, Journal Of Geophysical Research-Solid Earth, 102(B4), 8269-8283.

Sherburn, S., and C. J. Bryan (1999), The eruption detection system: Mt. Ruapehu, New Zealand, Seismological Research Letters, 70(5), 505-511.

Sherburn, S., and R. S. White (2005), Crustal seismicity in Taranaki, New Zealand using accurate hypocentres from a dense network, Geophysical Journal International, 162(2), 494-506.

Sherburn, S., and R. S. White (2006), Tectonics of the Taranaki region, New Zealand: earthquake focal mechanisms and stress axes, New Zealand Journal of Geology And Geophysics, 49(2), 269-279.

Sherburn, S., C. J. Bryan, A. W. Hurst, J. H. Latter, and B. J. Scott (1999), Seismicity of Ruapehu volcano, New Zealand, 1971-1996: a review, Journal Of Volcanology And Geothermal Research, 88(4), 255-278.

Sherburn, S., J. Townend, R. Arnold, and L. Woods (2009), EQC Project 08/550 Establishing a Spatiotemporal Benchmark for Ongoing Crustal Stress Monitoring in the Southern Taupo Volcanic Zone, GNS Science Consultancy Report, 185, 44.

Shih, X. R., and R. P. Meyer (1990), Observation of shear-wave splitting from natural events - south moat of Long Valley Caldera, California, June 29 to August 12, 1982, Journal Of Geophysical Research-Solid Earth And Planets, 95(B7), 11,17911,195 .

Shih, X. R., R. P. Meyer, and J. F. Schneider (1989), An automated, analytical method to determine shear-wave splitting, Tectonophysics, 165(1-4), 271-278.

Shimizu, H., K. Umakoshi, N. Matsuwo, and K. Ohta (1992), Seismological observations of Unzen volcano before and during the 1990-1992 eruption, Kyushu University Press, Fukuoka, Japan.

Silver, P. G., and W. W. Chan (1991), Shear-wave splitting and subcontinental mantle deformation, Journal Of Geophysical Research-Solid Earth, 96(B10), 16,42916,454 .

Silver, P. G., and M. K. Savage (1994), The interpretation of shear-wave splitting parameters in the presence of 2 anisotropic layers, Geophysical Journal International, 119(3), 949-963.

Sissons, B. A., and R. R. Dibble (1981), A seismic refraction experiment southeast of Ruapehu volcano, New Zealand Journal of Geology and Geophysics, 24, 31-38.

Smalley, R. F., J. L. Chatelain, D. L. Tutcotte, and R. Prevot (1987), A fractal approach to the clustering of earthquakes - applications to the seismicity of the New Hebrides, Bulletin Of The Seismological Society Of America, 77(4), 13681381. 
Smith, W. D. (1981), A short history of New Zealand seismograph stations, Tech. Rep. 171, Department of Scientific and Industrial Research, New Zealand, Geophysics Division.

Song, X. D., and P. G. Richards (1996), Seismological evidence for differential rotation of the Earth's inner core, Nature, 382(6588), 221-224.

Spottiswoode, S. M., and A. M. Milev (1998), The use of waveform similarity to define planes of mining-induced seismic events, Tectonophysics, 289(1-3), 51-60.

Starostin, A. B., A. A. Barmin, and O. E. Melnik (2005), A transient model for explosive and phreatomagmatic eruptions, Journal Of Volcanology And Geothermal Research, 143(1-3), 133-151.

Stein, S., and M. Wysession (2003), An Introduction to Seismology, Earthquakes, and Earth Structure, Blackwell Publishing.

Stern, T., W. Stratford, A. Seward, M. Henderson, M. Savage, E. Smith, A. Benson, S. Greve, and M. Salmon (2010), Crust-mantle structure of the central North Island, New Zealand, based on seismological observations, Journal Of Volcanology And Geothermal Research, 190(1-2), 58-74.

Stern, T. A. (2009), Reconciling short- and long-term measures of extension in continental back arcs: heat flux, crustal structure and rotations within central North Island, New Zealand, Extending A Continent: Architecture, Rheology And Heat Budget, 321, 73-87.

Stern, T. A., W. R. Stratford, and M. L. Salmon (2006), Subduction evolution and mantle dynamics at a continental margin: Central North Island, New Zealand, Reviews Of Geophysics, 44(4), RG4002, doi:10.1029/2005RG000171.

Stewart, R. (2010), Andesites as magmatic liquids or liquid-crystal mixtures: Insights from Egmont and Ruapehu Volcanoes, New Zealand, Central European Journal of Geosciences, 2(3), 329-338.

Stich, D., G. Alguacil, and J. Morales (2001), The relative locations of multiplets in the vicinity of the Western Almeria (southern Spain) earthquake series of 19931994, Geophysical Journal International, 146(3), 801-812.

Sudo, Y., et al. (1998), Seismic activity and ground deformation associated with 1995 phreatic eruption of Kuju Volcano, Kyushu, Japan, Journal of volcanology and geothermal research, 81, 245.

Syuhada (2010), Seismic attenuation anisotropy in the southern part of the Taupo Volcanic Zone, North Island, New Zealand, Master's thesis, Victoria University of Wellington.

Taira, T., P. G. Silver, F. L. Niu, and R. M. Nadeau (2008), Detecting seismogenic stress evolution and constraining fault zone rheology in the San Andreas Fault following the 2004 Parkfield earthquake, Journal Of Geophysical Research-Solid Earth, 113(B3), B03,303, doi:10.1029/2007JB005151. 
Tang, C. H., J. A. Rial, and J. M. Lees (2005), Shear-wave splitting: A diagnostic tool to monitor fluid pressure in geothermal fields, Geophysical Research Letters, 32(21), L21,317, doi:10.1029/2005GL023551.

Tang, C. H., J. A. Rial, and J. M. Lees (2008), Seismic imaging of the geothermal field at Krafla, Iceland using shear-wave splitting, Journal Of Volcanology And Geothermal Research, 176 (2), 315-324.

Teanby, N., J. M. Kendall, R. H. Jones, and O. Barkved (2004a), Stress-induced temporal variations in seismic anisotropy observed in microseismic data, Geophysical Journal International, 156(3), 459-466.

Teanby, N. A., J. M. Kendall, and M. Van der Baan (2004b), Automation of shearwave splitting measurements using cluster analysis, Bulletin Of The Seismological Society Of America, 94 (2), 453-463.

Templeton, D. C., R. M. Nadeau, and R. Burgmann (2008), Behavior of repeating earthquake sequences in central California and the implications for subsurface fault creep, Bulletin Of The Seismological Society Of America, 98(1), 52-65.

Thelen, W., M. West, and S. Senyukoy (2010), Seismic characterization of the fall 2007 eruptive sequence at Bezymianny Volcano, Russia, Journal Of Volcanology And Geothermal Research, 194(4), 201-213.

Titzschkau, T., M. Savage, and T. Hurst (2010), Changes in attenuation related to eruptions of Mt. Ruapehu Volcano, New Zealand, Journal Of Volcanology And Geothermal Research, 190(1-2), 168-178.

Tod, S. R., and E. Liu (2002), Frequency-dependent anisotropy due to fluid flow in bed limited cracks, Geophysical Research Letters, 29(15).

Toda, S., R. S. Stein, K. Richards-Dinger, and S. B. Bozkurt (2005), Forecasting the evolution of seismicity in southern California: Animations built on earthquake stress transfer, Journal Of Geophysical Research-Solid Earth, 110(B5), doi:10. 1029/2004JB003415.

Townend, J., and M. D. Zoback (2001), Implications of earthquake focal mechanisms for the frictional strength of the San Andreas fault system, Geological Society, London, Special Publications, 186(1), 13-21, doi:10.1144/GSL.SP.2001.186.01.02.

Townend, J., and M. D. Zoback (2004), Regional tectonic stress near the San Andreas fault in central and southern California, Geophysical Research Letters, 31 (15), L15S11, doi:10.1029/2003GL018918.

Townend, J., and M. D. Zoback (2006), Stress, strain, and mountain building in central Japan, Journal of Geophysical Research-Part B-Solid Earth, 111(B3), B03,411, doi:10.1029/2005JB003759.

Turnbull, I. M., N. Mortimer, and D. Craw (2001), Textural zones in the Haast Schist - a reappraisal, New Zealand Journal Of Geology And Geophysics, 44(1), 171-183. 
Unglert, K., M. Savage, N. Fournier, and T. Ohkura (2010), Correlating variations in GPS and shear-wave splitting: Is there a common source?, in Abstract G21D-07 presented at 2010 Fall Meeting, AGU, San Francisco, Calif., 13-17 Dec.

USGS (Last accessed 22 Feb 2011), Coulomb 3 stress change modeling software, http://earthquake.usgs.gov/research/modeling/coulomb/download.php.

Villamor, P., and K. R. Berryman (2006), Late quaternary geometry and kinematics of faults at the southern termination of the taupo volcanic zone, new zealand, New Zealand Journal Of Geology And Geophysics, 49(1), 1-21.

Vlahovic, G., M. Elkibbi, and J. A. Rial (2003), Shear-wave splitting and reservoir crack characterization: the Coso geothermal field, Journal Of Volcanology And Geothermal Research, 120(1-2), 123-140.

Volti, T., and S. Crampin (2003), A four-year study of shear-wave splitting in Iceland: 1. Background and preliminary analysis, New Insights Into Structural Interpretation And Modelling, (212), 117-133.

Wadge, G., G. S. Mattioli, and R. A. Herd (2006), Ground deformation at Soufière Hills Volcano, Montserrat during 1998-2000 measured by radar interferometry and GPS, Journal Of Volcanology And Geothermal Research, 152(1-2), 157-173.

Walcott, R. I. (1987), Geodetic strain and the deformational history of the north island of new zealand during the late cainozoic, Philosophical Transactions of the Royal Society of London, 321, 163-181.

Waldhauser, F. (2001), hypoDD- a program to compute double-difference hypocenter locations, Open file report 01-113, U.S. Geol. Survey.

Waldhauser, F., and W. L. Ellsworth (2000), A double-difference earthquake location algorithm: Method and application to the northern Hayward fault, California, Bulletin Of The Seismological Society Of America, 90(6), 1353-1368.

Waldhauser, F., W. L. Ellsworth, D. P. Schaff, and A. Cole (2004), Streaks, multiplets, and holes: High-resolution spatio-temporal behavior of Parkfield seismicity, Geophysical Research Letters, 31(18), L18,608, doi:10.1029/2004GL020649.

Wallace, L. M., J. Beavan, R. McCaffrey, and D. Darby (2004), Subduction zone coupling and tectonic block rotations in the North Island, New Zealand, Journal of Geophysical Research, 109 (B12), B12,406, doi:10.1029/2004JB003241.

Walsh, F., M. Hochstein, and C. Bromley (1998), The Tongariro geothermal system (NZ): Review of geophysical data., in Proceedings 20th NZ Geothermal Workshop.

Wellman, H. W. (1971), Reference lines, fault classification, transform systems, and ocean-floor spreading, Tectonophysics, 12, 199-\&.

Wessel, P., and W. H. F. Smith (1991), Free software helps map and display data, Eos Trans. AGU, 72(441), 445. 
Wiemer, S. (2001), A software package to analyze seismicity: Zmap, Seismological Research Letters, 72, 373-382, doi:0.1785/gssrl.72.3.373.

Wiemer, S., and M. Wyss (2002), Mapping spatial variability of the frequencymagnitude distribution of earthquakes, Advances In Geophysics, Vol 45, 45, 259302.

Wilson, C. J. N., B. F. Houghton, M. O. McWilliams, M. A. Lanphere, S. D. Weaver, and R. M. Briggs (1995), Volcanic and structural evolution of Taupo Volcanic Zone, New-Zealand - a review, Journal Of Volcanology And Geothermal Research, $68(1-3), 1-28$.

Wong, D., D. Clifton, and L. Tarassenko (2009), An introduction to the bispectrum for eeg analysis, IEEE PGBIOMED, Oxford, UK.

Wood, R., and B. Davy (1994), The Hikurangi Plateau, Marine Geology, 118(1-2), $153-173$.

Wright, I. C. (1993), Pre-spread rifting and heterogenous volcanism in the southern Havre Trough back-arc basin, Marine Geology, 113(3-4), 179-200.

Yamawaki, T., T. Nishimura, and H. Hamaguchi (2004), Temporal change of seismic structure around Iwate volcano inferred from waveform correlation analysis of similar earthquakes, Geophysical Research Letters, 31(24), L24,616, doi:10.1029/ 2004GL021103.

Yardley, G. S., and S. Crampin (1991), Extensive-dilatancy anisotropy - relative information in vsps and reflection surveys, GEOPHYSICAL PROSPECTING, 39, 337-355, doi:10.1111/j.1365-2478.1991.tb00316.

Zaccarelli, L., D. Pandolfi, F. Bianco, G. Saccorotti, C. J. Bean, and E. Del Pezzo (2009), Temporal changes in seismic wave propagation towards the end of the 2002 Mt Etna eruption, Geophysical Journal International, 178(3), 1779-1788.

Zatsepin, S. V., and S. Crampin (1997), Modelling the compliance of crustal rock .1. response of shear-wave splitting to differential stress, Geophysical Journal International, 129(3), 477-494.

Zhang, H. J., Y. F. Liu, C. Thurber, and S. Roecker (2007), Three-dimensional shear-wave splitting tomography in the Parkfield, California, region, Geophysical Research Letters, 34(24), L24,308, doi:10.1029/2007GL031951.

Zhao, P., and Z. Peng (2009), Depth extent of damage zones around the central Calaveras fault from waveform analysis of repeating earthquakes, Geophysical Journal International, pp. 1817-30.

Zhao, P., and Z. G. Peng (2008), Velocity contrast along the Calaveras fault from analysis of fault zone head waves generated by repeating earthquakes, Geophysical Research Letters, 35 (1), L01,303, doi:10.1029/2007GL031810. 
Zinke, J. C., and M. D. Zoback (2000), Structure-related and stress-induced shearwave velocity anisotropy: Observations from microearthquakes near the Calaveras fault in central California, Bulletin Of The Seismological Society Of America, 90(5), 1305-1312.

Zoback, M. D., and J. Townend (2001), Implications of hydrostatic pore pressures and high crustal strength for the deformation of intraplate lithosphere, Tectonophysics, 336(1-4), 19-30. 



\section{A. Appendix: Eruptive activity at Ruapehu}




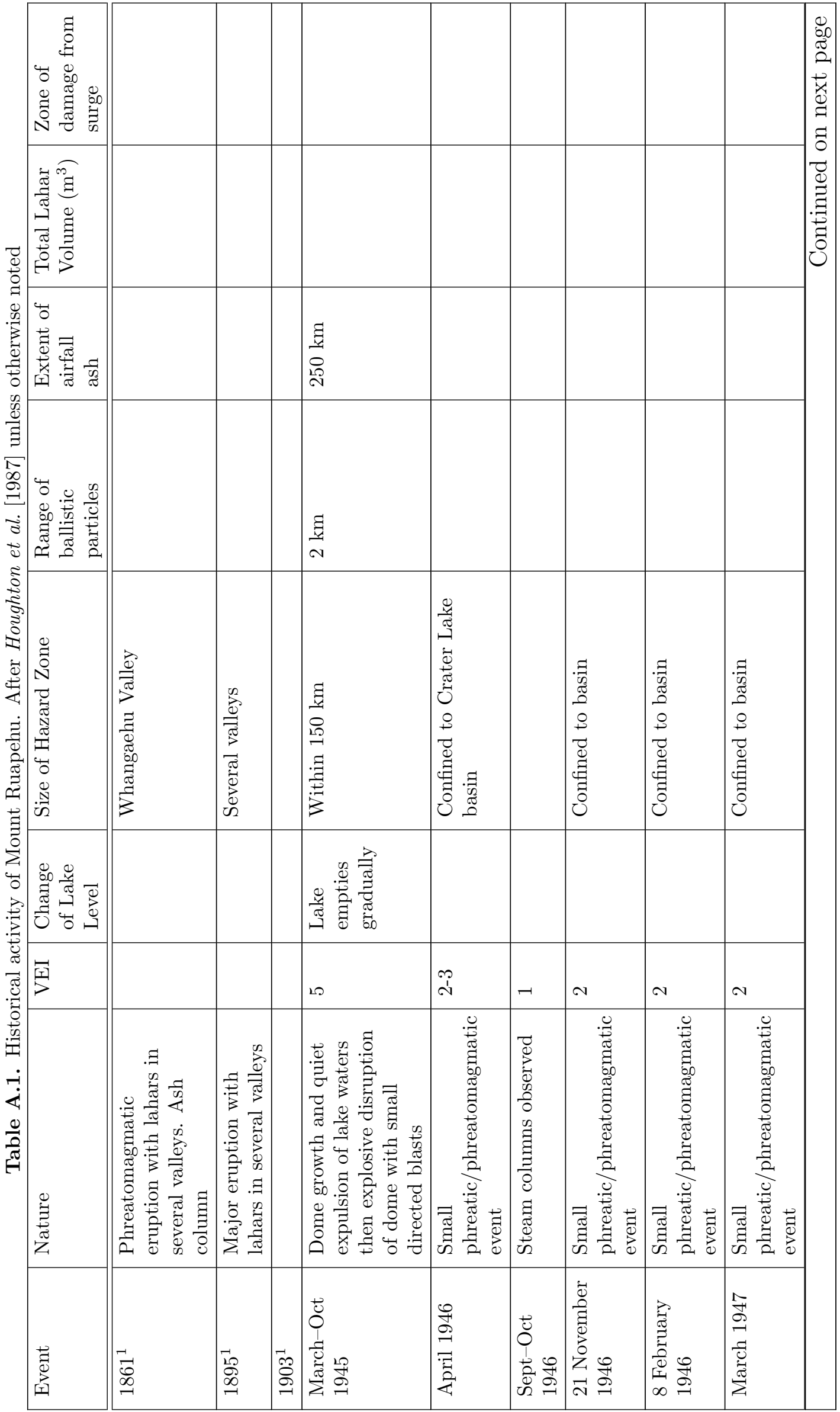




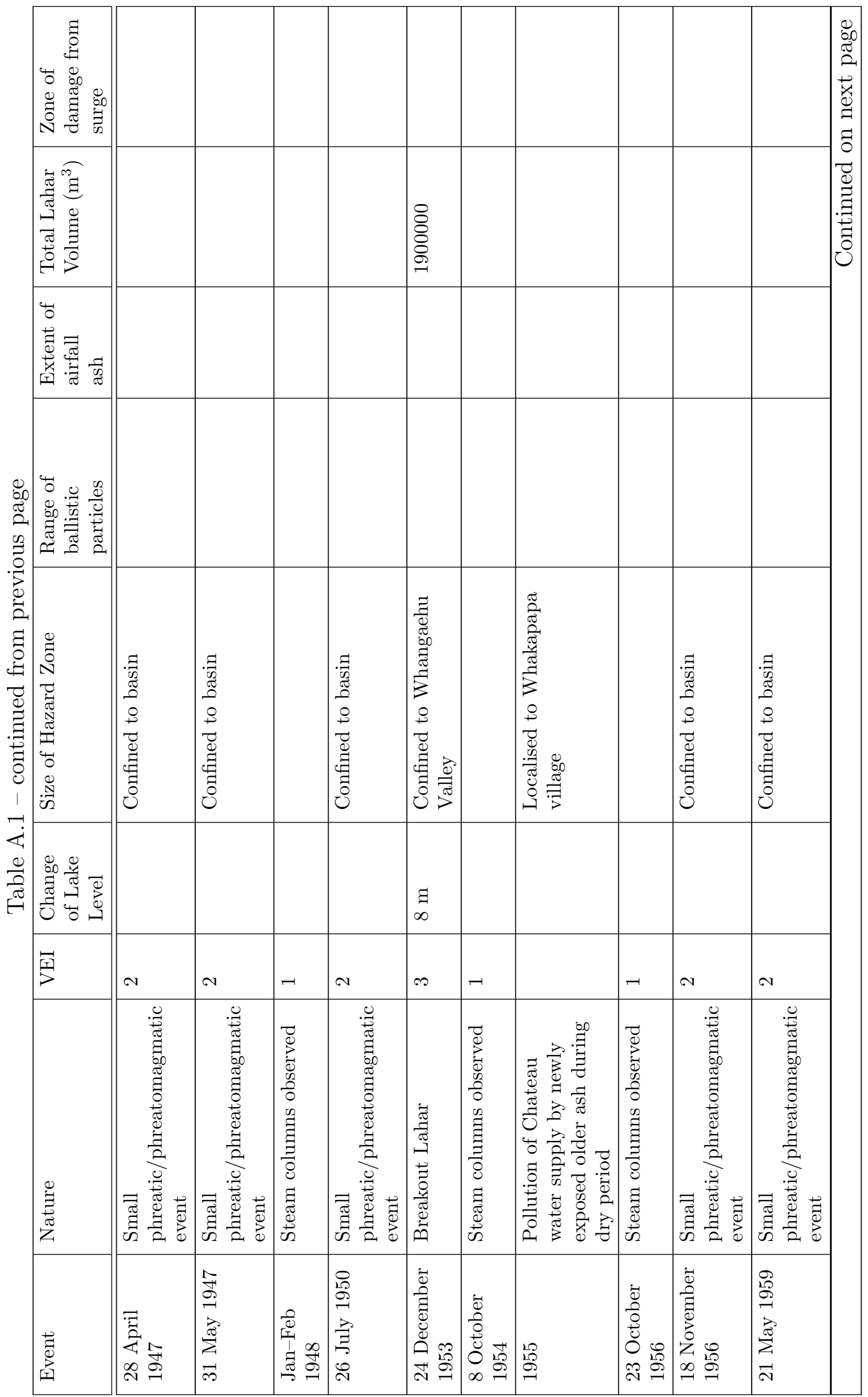




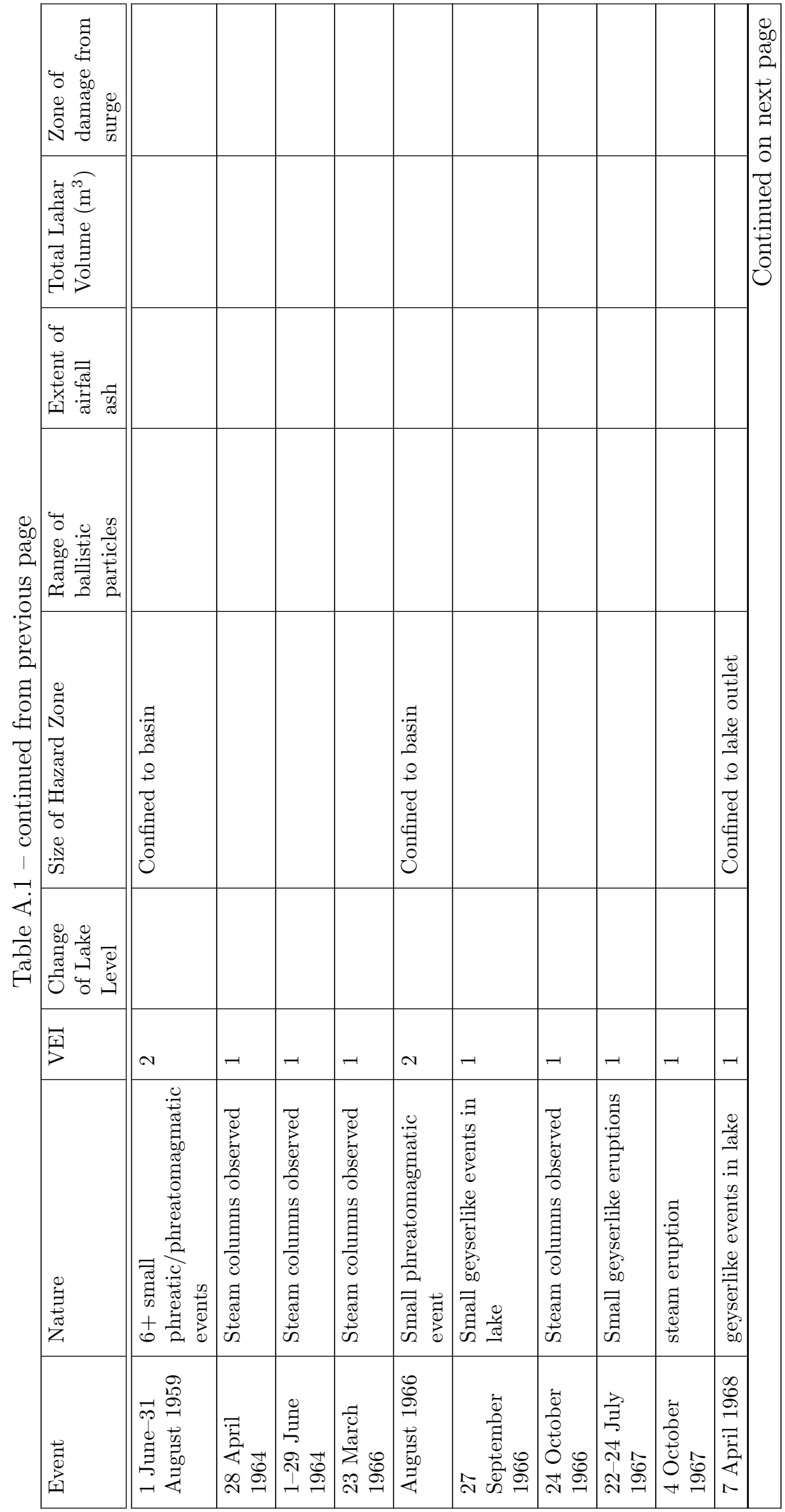




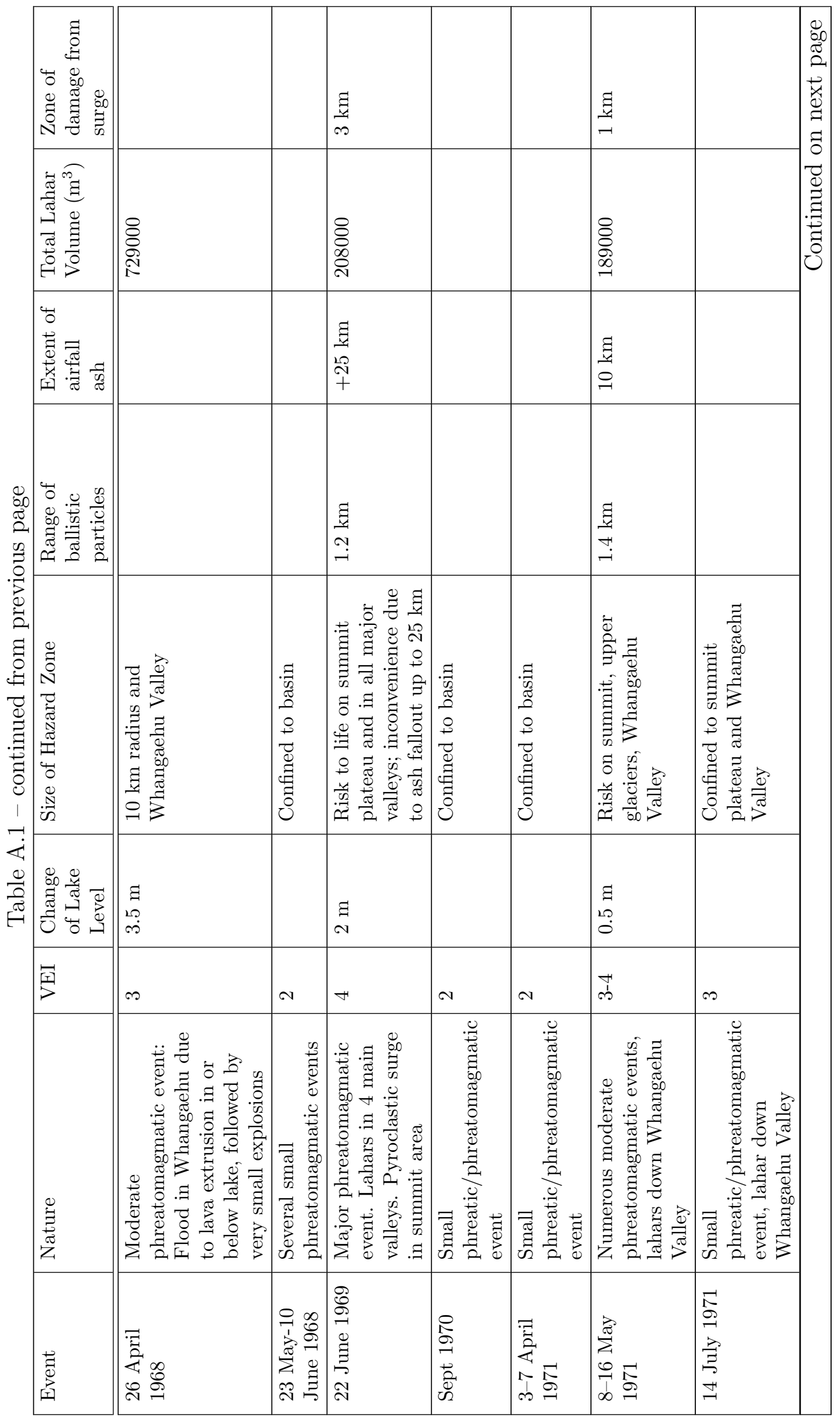




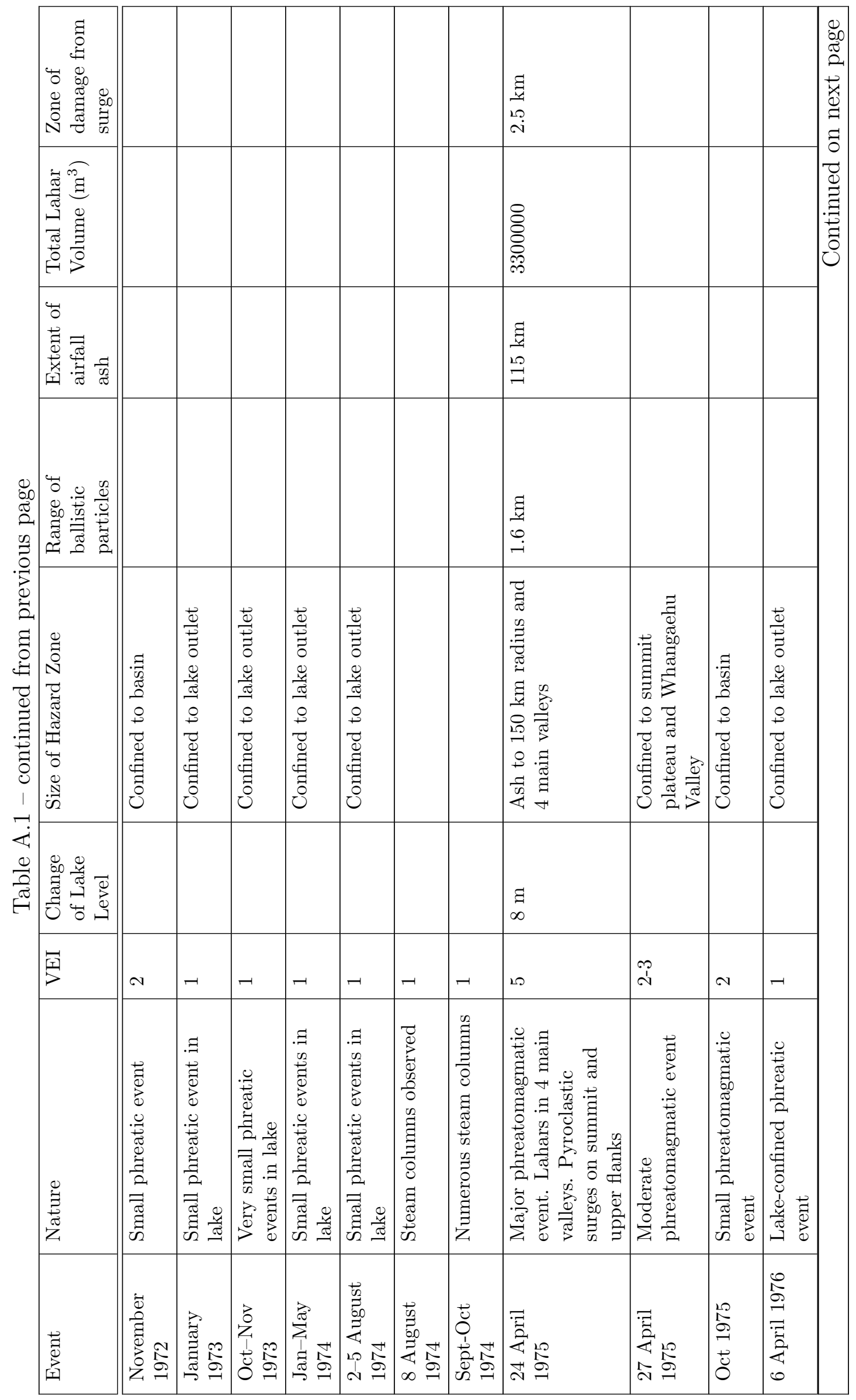




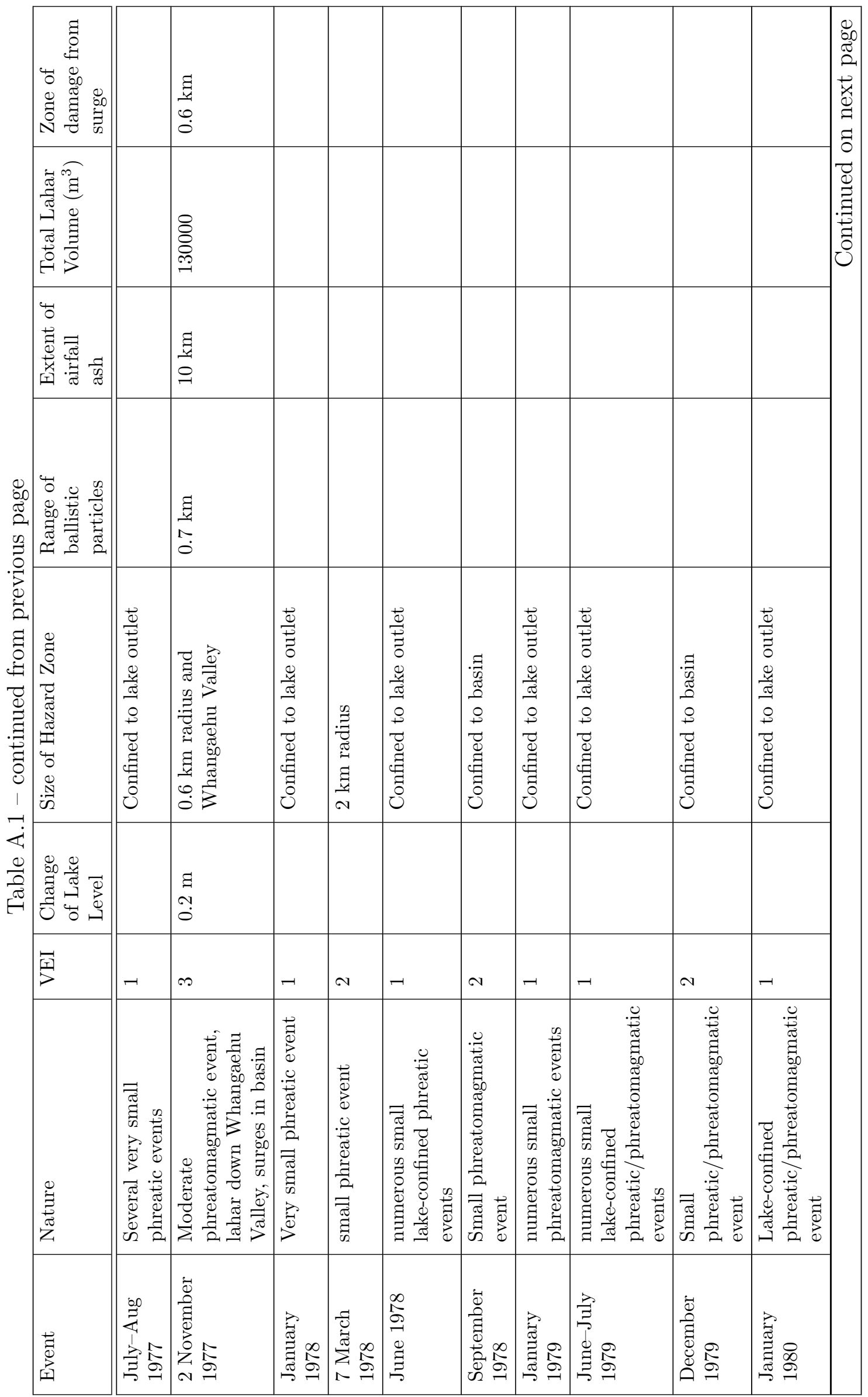




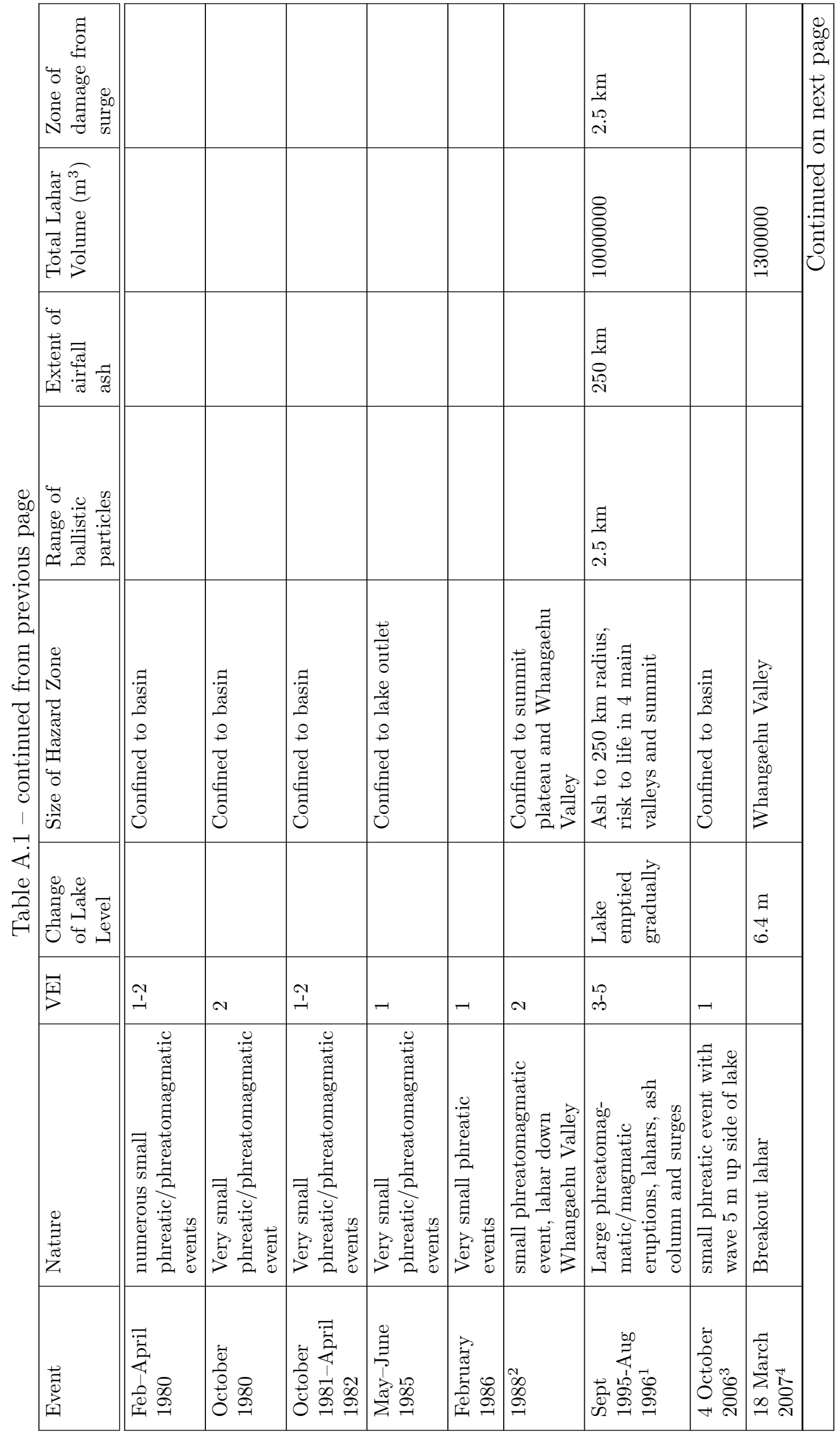



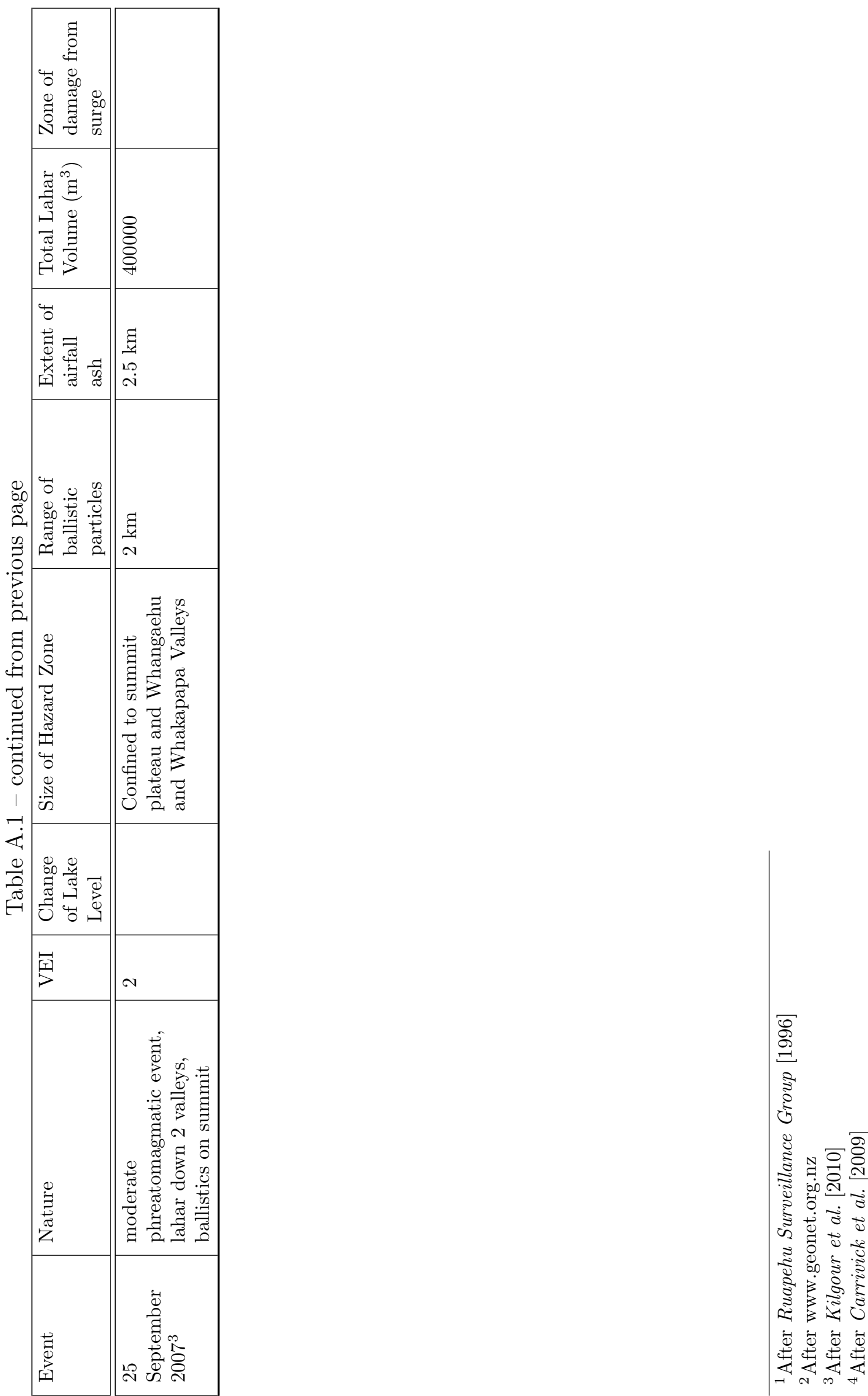
Table A.2. Phases of the 1995/1996 Ruapehu Eruption, after Bryan and Sherburn [1999]

\begin{tabular}{|c|c|}
\hline \multicolumn{2}{|l|}{ Pre-eruption } \\
\hline To Sept. 17,1995 & $\begin{array}{l}\text { Two and } 7 \mathrm{~Hz} \text { tremor independent; changes in Crater Lake } \\
\text { water temperature do not correlate with seismicity changes. }\end{array}$ \\
\hline \multicolumn{2}{|c|}{ Phase 1 (Sept. 17-30, 1995) } \\
\hline Sept. 17 & $\begin{array}{l}\text { Changes in the amplitude and frequency content of tremor } \\
\text { precede a moderate lahar-producing eruption through Crater } \\
\text { Lake, accompanied by a } M_{L} 3.6 \text { volcanic earthquake. }\end{array}$ \\
\hline Sept. $17-25$ & $\begin{array}{l}\text { Sub-1 Hz tremor common, and sometimes triggered by } \\
\text { volcano-tectonic earthquakes. }\end{array}$ \\
\hline Sept. 19 & $\begin{array}{l}\mathrm{M}_{L} 3.4 \text { volcanic earthquake accompanies a lahar-producing } \\
\text { eruption through Crater Lake. }\end{array}$ \\
\hline Sept. 23 & $\begin{array}{l}\mathrm{M}_{L} 3.2 \text { volcanic earthquake accompanies an eruption through } \\
\text { Crater Lake; three lahars generated. Sustained eruption begins }\end{array}$ \\
\hline Sept. $24-25$ & $\begin{array}{l}\text { Tremor and numerous volcanic earthquakes accompany a } 10 \mathrm{~km} \\
\text { high eruption column; sustained lahars. }\end{array}$ \\
\hline \multicolumn{2}{|c|}{ Phase 2 (Sept. 30-Oct. 7, 1995) } \\
\hline Sept. 30-Oct. 7 & $\begin{array}{l}\text { Tremor and volcanic earthquakes accompany moderate but } \\
\text { intermittent eruptions. }\end{array}$ \\
\hline \multicolumn{2}{|c|}{ Phase 3 (Oct. 7-14, 1995) } \\
\hline Oct. 7 & $\begin{array}{l}\mathrm{M}_{L}>3.6 \text { volcanic earthquake accompanies an ash eruption to } \\
7.5 \mathrm{~km} .\end{array}$ \\
\hline Oct. 11 & $\begin{array}{l}\text { An } 8 \text {-h episode of wideband tremor accompanies an eruption of } \\
\text { about } 0.02 \mathrm{~km}^{3} \text { of ash; plume to } 8-10 \mathrm{~km} \text {. Crater Lake emptied. }\end{array}$ \\
\hline Oct. 14 & $\begin{array}{l}\text { A } 5 \text {-h episode of wideband tremor accompanies an eruption of } \\
\text { about } 0.01 \mathrm{~km}^{3} \text { of ash; plume to } 11 \mathrm{~km} \text {. }\end{array}$ \\
\hline \multicolumn{2}{|c|}{ Phase 4 (Oct. 14-Nov. 9, 1995) } \\
\hline Oct. $14-31$ & $\begin{array}{l}\text { Small volcanic earthquakes accompany eruptions of ash to a few } \\
\text { hundred meters above the crater. }\end{array}$ \\
\hline \multicolumn{2}{|c|}{ Intra-eruption (Nov. 9, 1995-June 10, 1996) } \\
\hline $\begin{array}{l}\text { Nov. 1995-Feb. } \\
1996\end{array}$ & $\begin{array}{l}\text { Seven hertz tremor sustained at ; } 3 \mu \mathrm{m} / \mathrm{s} \text {; about } 20 \text { shallow } \\
\text { volcano-tectonic earthquakes per day. }\end{array}$ \\
\hline Feb.--June 1996 & $\begin{array}{l}\sim 50 \text { shallow volcano-tectonic earthquakes per day; a small lava } \\
\text { extrusion seen in Crater Lake. }\end{array}$ \\
\hline \multicolumn{2}{|c|}{ Phase 5 (June 10-16, 1996) } \\
\hline June 10 & Minor, intermittent tremor. \\
\hline June 15 & $\begin{array}{l}\text { Strong wideband tremor. Six small }\left(\mathrm{M}_{L}<2\right) \text { volcano-tectonic } \\
\text { earthquakes. }\end{array}$ \\
\hline \multicolumn{2}{|c|}{ Phase 6 (June 16-July 1, 1996) } \\
\hline June 16 & $\begin{array}{l}\text { Wideband volcanic earthquakes and tremor accompany an ash } \\
\text { eruption to several kilometers above the crater; a small lahar } \\
\text { empties Crater Lake. }\end{array}$ \\
\hline June $17-18$ & $\begin{array}{l}\text { Numerous volcanic earthquakes accompanied by ground-coupled } \\
\text { airwaves and a change to strombolian style eruptions; molten } \\
\text { material erupted to several hundred meters above the crater and } \\
\text { bombs to } 1.5 \mathrm{~km} \text {. }\end{array}$ \\
\hline & Continued on next page \\
\hline
\end{tabular}


Table A.2 - continued from previous page

\begin{tabular}{|l|l|}
\hline \multicolumn{1}{|c|}{ Phase 7 (July 1-15, 1996) } \\
\hline July 1-6 & $\begin{array}{l}\text { Many small wideband volcanic earthquakes and intermittent } \\
\text { tremor. }\end{array}$ \\
\hline July 7 & $\begin{array}{l}\text { Continuous tremor and volcanic earthquakes with } \\
\text { ground-coupled airwaves accompany the eruption of ash to 5 km } \\
\text { elevation. }\end{array}$ \\
\hline July 8-10 & $\begin{array}{l}\text { Minor tremor and volcanic earthquakes accompany intermittent } \\
\text { ash eruptions. }\end{array}$ \\
\hline \hline Phase 8 (July 15-19, 1996) \\
\hline July 15-16 & $\begin{array}{l}\text { Strong tremor. Ash eruption to an elevation of 7 km. Numerous } \\
\text { discrete volcanic earthquakes with ground-coupled airwaves. }\end{array}$ \\
\hline \hline Phase 9 (July 19-24, 1996) \\
\hline July 19-24 & Tremor and volcanic earthquakes accompany ash eruptions. \\
\hline \hline \multicolumn{2}{|c|}{ Phase 10 (July 24-Aug. 11, 1996) } \\
\hline July 24-Aug. 11 & $\begin{array}{l}\text { Intermittent, low-amplitude tremor and minor, low-elevation } \\
\text { eruptions. }\end{array}$ \\
\hline \hline \multicolumn{2}{|c|}{ New background and post-eruption } \\
\hline From Aug. 11 & $\begin{array}{l}\text { Seven hertz tremor stabilises at about 2 } \mu \mathrm{m} / \mathrm{s} ; \text { shallow } \\
\text { volcano-tectonic earthquakes become common. }\end{array}$ \\
\hline Sept. 9 & Last observed ash eruption. \\
\hline
\end{tabular}



B. Appendix: Station details 


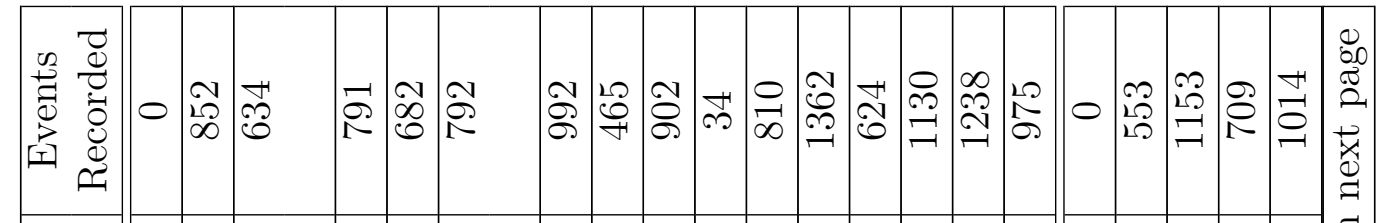

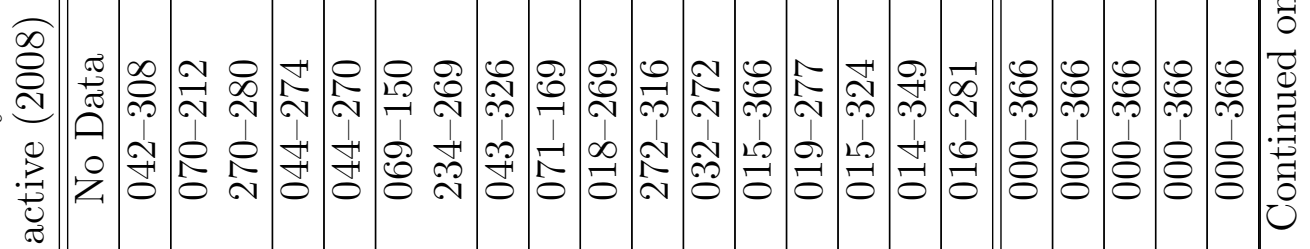

\begin{tabular}{|c|c|c|c|c|c|c|c|c|c|c|c|c|c|c|c|c|c|c|c|c|c|}
\hline 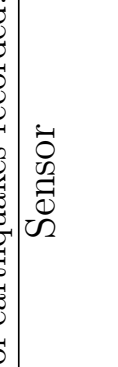 & 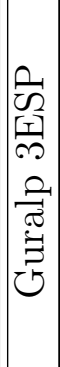 & 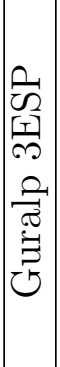 & 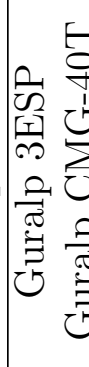 & 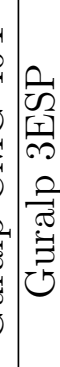 & 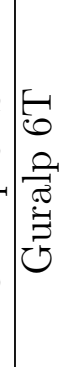 & 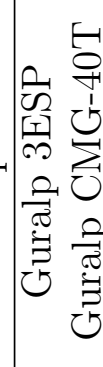 & 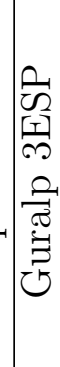 & 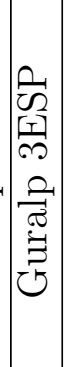 & 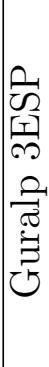 & 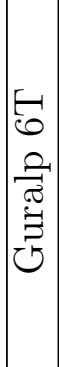 & 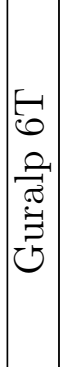 & 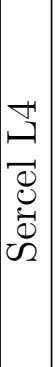 & 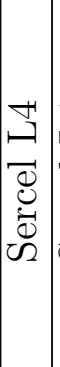 & 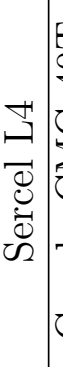 & 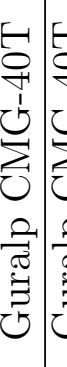 & 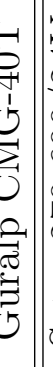 & 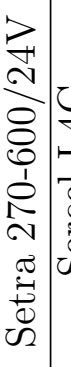 & ك & 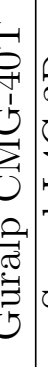 & بـ & \\
\hline 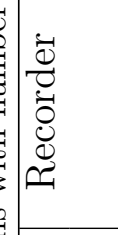 & 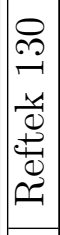 & 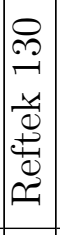 & 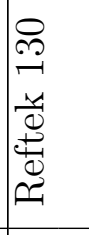 & 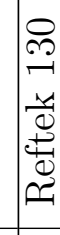 & 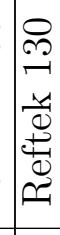 & 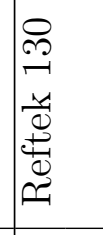 & 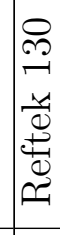 & 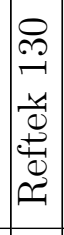 & 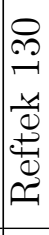 & 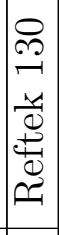 & 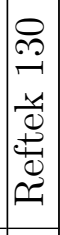 & 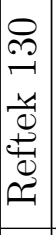 & 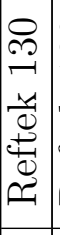 & 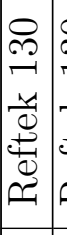 & 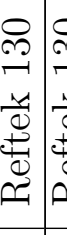 & 党 & 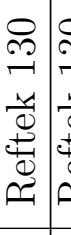 & 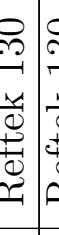 & 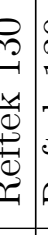 & 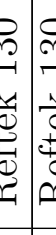 & \\
\hline 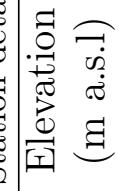 & 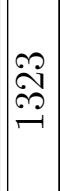 & $\frac{\Omega}{\infty}$ & $\begin{array}{l}\infty \\
\stackrel{\infty}{\infty} \\
\end{array}$ & $\begin{array}{l}\infty \\
\Re \\
i\end{array}$ & $\stackrel{\sigma}{\Omega}$ & $\underset{\sim}{\stackrel{N}{O}}$ & $\stackrel{\infty}{\not+}$ & 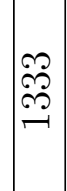 & 哽 & $\begin{array}{l}12 \\
8 \\
0\end{array}$ & $\stackrel{8}{\infty}$ & $\stackrel{f}{D}$ & $\vec{g}$ & $\begin{array}{ll}2 \mathscr{2} \\
\mathscr{S}\end{array}$ & 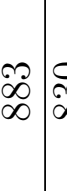 & & $\begin{array}{l}\infty \\
2 \\
= \\
=\end{array}$ & & & 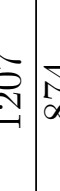 & \\
\hline 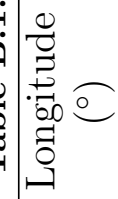 & $\mid \begin{array}{l}2 \\
2 \\
N \\
1 \\
20 \\
1 \\
-1\end{array}$ & $\begin{array}{l}1 \\
0 \\
10 \\
0 \\
10 \\
10 \\
-1\end{array}$ & 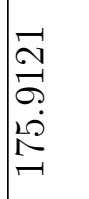 & 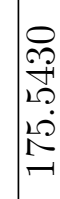 & 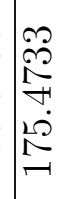 & $\begin{array}{l}\sqrt[1]{2} \\
\stackrel{N}{7} \\
\underset{1}{2} \\
\stackrel{-1}{1}\end{array}$ & $\begin{array}{l}2 \\
\infty \\
N \\
10 \\
10 \\
10 \\
1\end{array}$ & $\begin{array}{l}8 \\
\stackrel{8}{7} \\
\stackrel{1}{0} \\
\stackrel{1}{1}\end{array}$ & $\begin{array}{l}\frac{10}{10} \\
10 \\
10 \\
10 \\
10 \\
-1\end{array}$ & 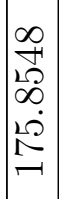 & 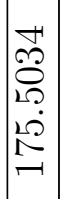 & 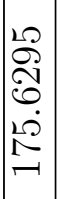 & $\begin{array}{l}0 \\
\infty \\
0 \\
2 \\
10 \\
10 \\
-1\end{array}$ & \begin{tabular}{c|c}
0 & 1 \\
0 & 1 \\
-0 & 1 \\
0 & 1 \\
$\dot{\rho}$ & 1 \\
1 & 1 \\
-1 & 1
\end{tabular} & 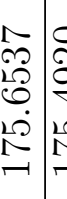 & 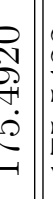 & 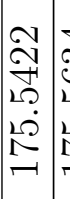 & 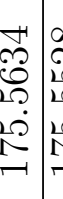 & 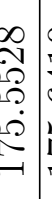 & 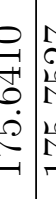 & \\
\hline : & $\begin{array}{l}\Omega \\
\stackrel{R}{n} \\
\tilde{n} \\
\infty \\
\infty \\
\infty \\
1\end{array}$ & $\begin{array}{c}\vec{\infty} \\
\stackrel{+}{+} \\
\overrightarrow{1} \\
\infty \\
\infty \\
1\end{array}$ & 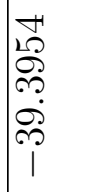 & 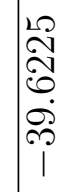 & 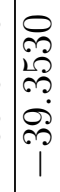 & 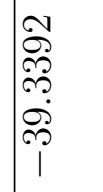 & $\begin{array}{l}\infty \\
\infty \\
20 \\
20 \\
\infty \\
\infty \\
1 \\
1\end{array}$ & $\begin{array}{l}\vec{\infty} \\
\tilde{\infty} \\
\sim \\
\sim \\
\infty \\
\infty \\
i\end{array}$ & $\begin{array}{l}0 \\
20 \\
\infty \\
\infty \\
\infty \\
\infty \\
\infty\end{array}$ & $\begin{array}{c}0 \\
\infty \\
\infty \\
2 \\
2 \\
\infty \\
\infty \\
\infty \\
1\end{array}$ & 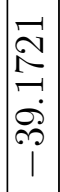 & $\mid \begin{array}{l}0 \\
0 \\
\infty \\
20 \\
20 \\
\infty \\
1 \\
1\end{array}$ & $\begin{array}{l}\infty \\
\infty \\
\infty \\
\sim \\
\sim \\
\infty \\
\infty \\
i\end{array}$ & 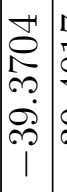 & 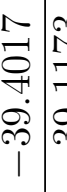 & '? & 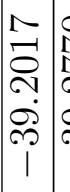 & 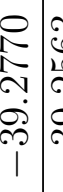 & 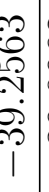 & 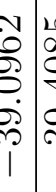 & \\
\hline 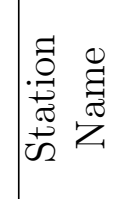 & 舅 & 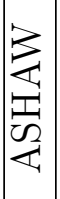 & 点 & $\underset{\oplus}{\stackrel{\Xi}{\rightleftarrows}}$ & $\mid \begin{array}{l}-\vec{E} \\
\vec{a} \\
\vec{n}\end{array}$ & 疍 & 四 & 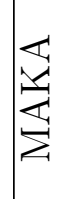 & 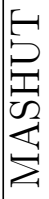 & $\underset{\Xi}{ٍ}$ & 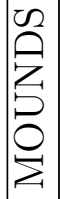 & 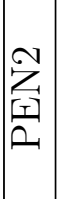 & $\begin{array}{l}0 \\
\vdots \\
0 \\
\uplus\end{array}$ & 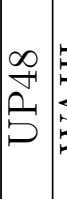 & $\begin{array}{l}3 \\
3\end{array}$ & $\begin{array}{l}\frac{3}{4} \\
\frac{1}{3} \\
\frac{1}{3} \\
\frac{1}{3}\end{array}$ & 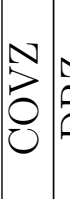 & 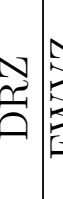 & $\frac{5}{I}$ & & \\
\hline
\end{tabular}




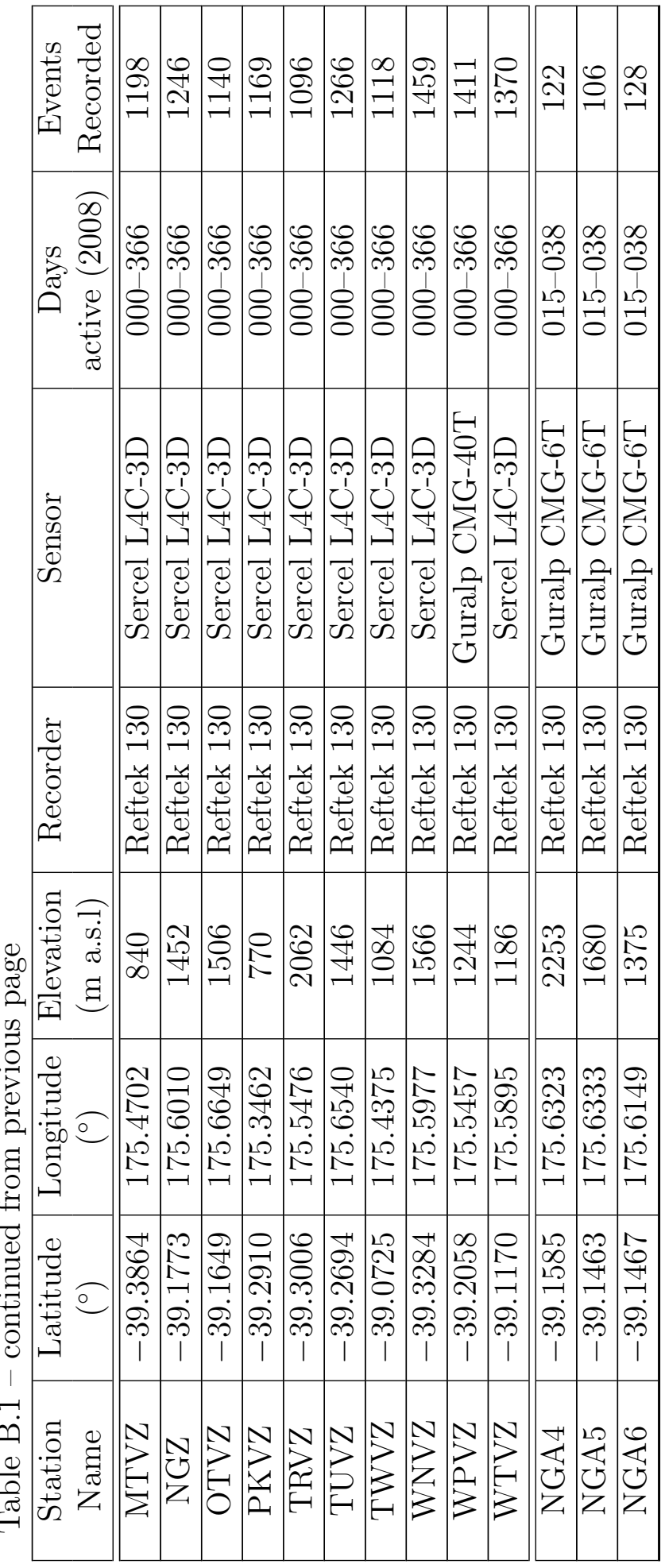




\begin{tabular}{|c|c|c|c|c|c|c|c|c|c|c|c|c|c|c|c|}
\hline 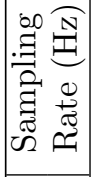 & $\mid \begin{array}{l} \\
20 \\
ن \\
0\end{array}$ & $\begin{array}{l}20 \\
\text { Dิ }\end{array}$ & $\mid$ & 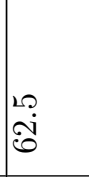 & $\begin{array}{l}20 \\
\\
0\end{array}$ & $\begin{array}{l}20 \\
\text { ปิ }\end{array}$ & 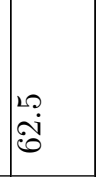 & 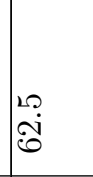 & 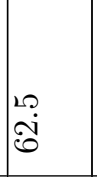 & 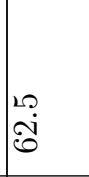 & 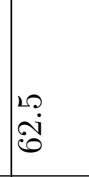 & $\begin{array}{l}20 \\
\text { ชิ }\end{array}$ & 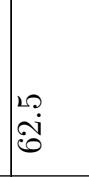 & $\begin{array}{l}20 \\
\text { ชิ } \\
0\end{array}$ & 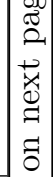 \\
\hline 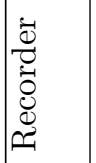 & 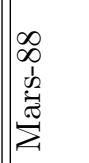 & 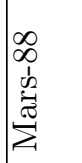 & 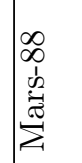 & 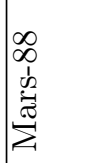 & 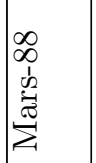 & 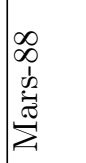 & 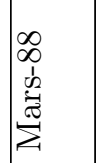 & 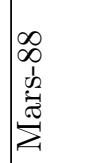 & 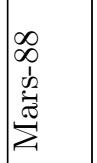 & 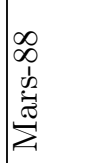 & 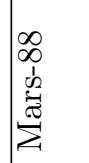 & 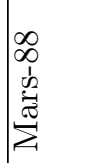 & 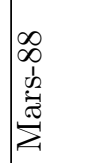 & 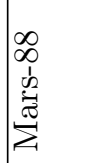 & $\mid$ \\
\hline 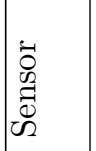 & 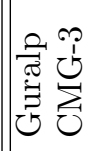 & 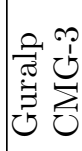 & 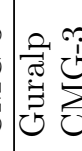 & 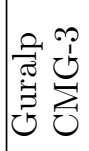 & 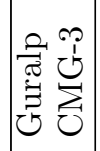 & 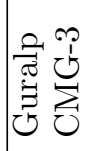 & 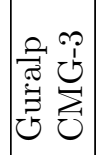 & 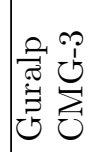 & 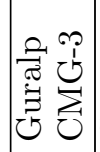 & 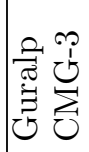 & 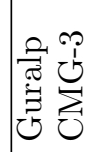 & 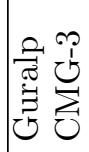 & 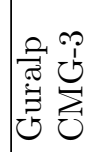 & 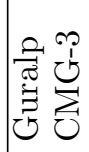 & \\
\hline \multirow{2}{*}{ 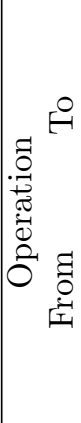 } & 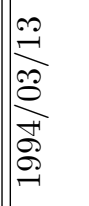 & 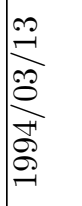 & 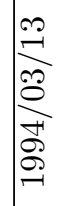 & 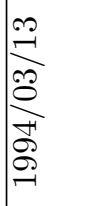 & 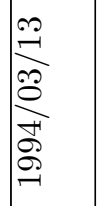 & 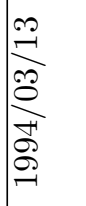 & 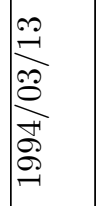 & 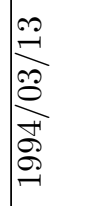 & 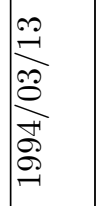 & 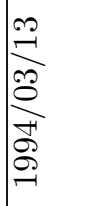 & 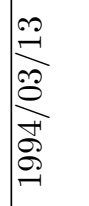 & 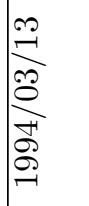 & 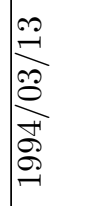 & 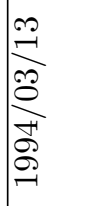 & \\
\hline & 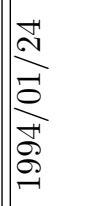 & 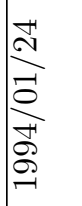 & 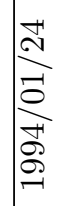 & 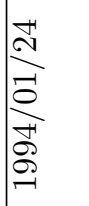 & 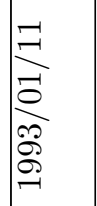 & 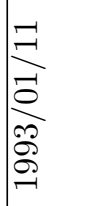 & 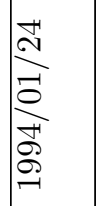 & 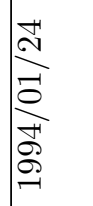 & 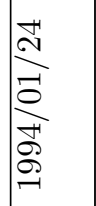 & 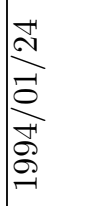 & 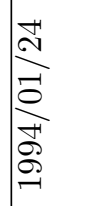 & 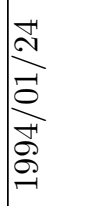 & 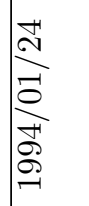 & 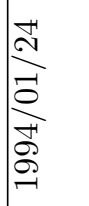 & \\
\hline 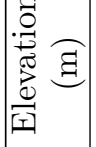 & 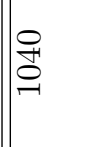 & 点 & ஓ্ট & $\underset{ }{\stackrel{g}{\sharp}}$ & & \&્ટ & 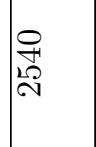 & 융 & ஜ̊ & $\underset{\substack{2 \\
i}}{\triangleright}$ & ஜ્ర్ర & $\underset{\stackrel{0}{10}}{\stackrel{10}{=}}$ & $\mid \begin{array}{l}8 \\
2 \\
13 \\
-1\end{array}$ & \& & \\
\hline 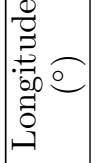 & 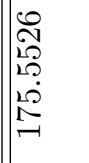 & $\begin{array}{l}8 \\
8 \\
20 \\
20 \\
120 \\
10 \\
-1\end{array}$ & 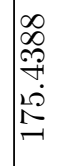 & 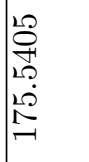 & 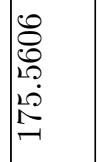 & 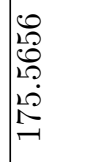 & $\begin{array}{l}\infty \\
0 \\
0 \\
10 \\
12 \\
12 \\
\\
-1\end{array}$ & 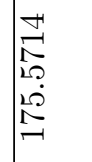 & $\begin{array}{l}\sqrt{2} \\
0 \\
0 \\
10 \\
10 \\
10 \\
10 \\
1 \\
-1\end{array}$ & 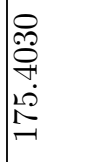 & 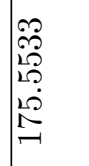 & \begin{tabular}{l}
$\infty$ \\
$\infty$ \\
\hdashline 0 \\
0 \\
10 \\
1 \\
-1
\end{tabular} & 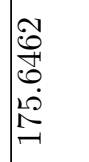 & 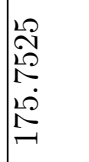 & \\
\hline 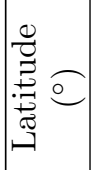 & $\mid$\begin{tabular}{l}
$\infty$ \\
0 \\
\hdashline \\
-1 \\
\hdashline \\
0 \\
$i$ \\
1
\end{tabular} & 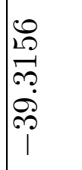 & 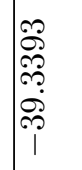 & 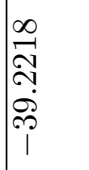 & 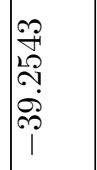 & 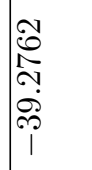 & 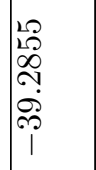 & 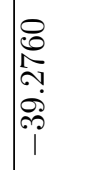 & $\begin{array}{l}\stackrel{19}{\infty} \\
\infty \\
\sim \\
0 \\
9 \\
1\end{array}$ & 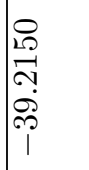 & 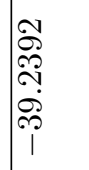 & 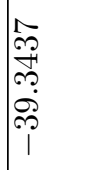 & 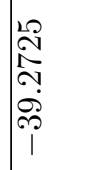 & 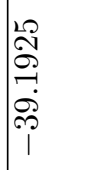 & \\
\hline 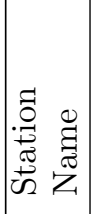 & 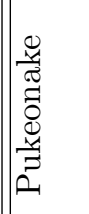 & 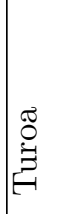 & 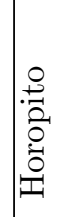 & 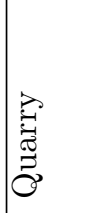 & $\begin{array}{l}\vec{z} \\
\vec{y} \\
\vec{y} \\
\tilde{w}\end{array}$ & ڤ̆̈̆ & 苞 & 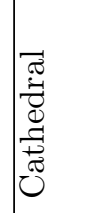 & 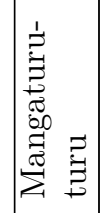 & 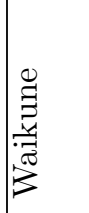 & 胥 & 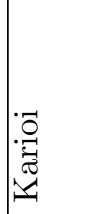 & 竭 & 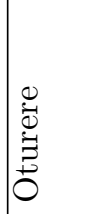 & \\
\hline 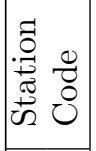 & 资 & 党 & 总 & 岕 & 且 & 苔 & 且 & 賕 & $\sum_{3}$ & 发 & $\varliminf_{\exists}$ & 吝 & 凷 & 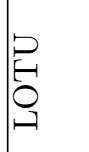 & \\
\hline & $\stackrel{\leftrightarrow}{\mathscr{S}}$ & 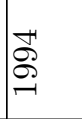 & $\stackrel{8}{\mathscr{O}}$ & 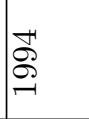 & 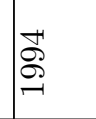 & 島 & $\stackrel{\vec{\sigma}}{\mathscr{\Xi}}$ & 苛 & 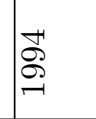 & 䓞 & $\stackrel{\vec{S}}{\mathscr{S}}$ & 莺 & 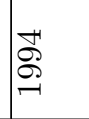 & 茑 & \\
\hline
\end{tabular}




\begin{tabular}{|c|c|c|c|c|c|c|c|c|c|c|c|c|c|c|c|c|}
\hline 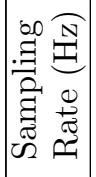 & 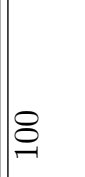 & 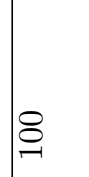 & 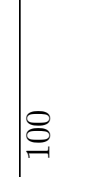 & 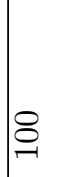 & $\stackrel{8}{\bigodot}$ & 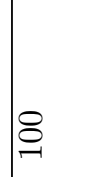 & $\stackrel{8}{\bigodot}$ & 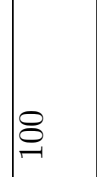 & 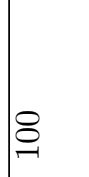 & 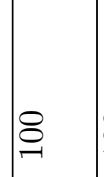 & 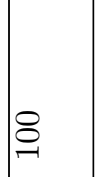 & 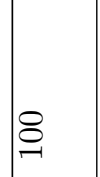 & 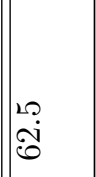 & 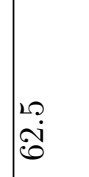 & 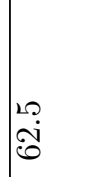 & 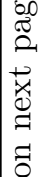 \\
\hline 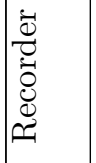 & & & & & & & & & & & & & 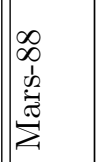 & 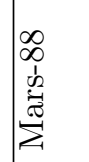 & 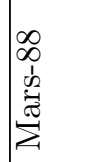 & ن \\
\hline 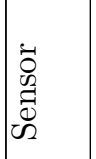 & 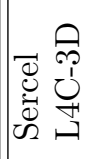 & 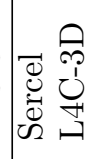 & 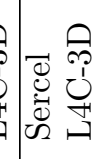 & 焉 & 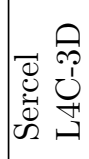 & 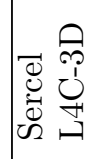 & 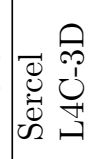 & 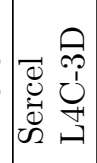 & 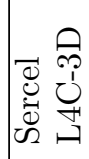 & 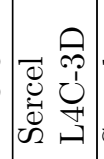 & 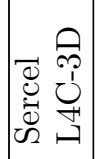 & 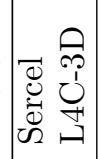 & 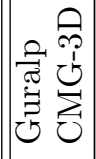 & 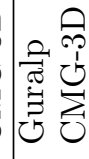 & 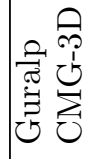 & \\
\hline${ }^{\ominus}$ & 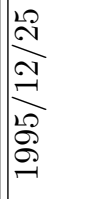 & 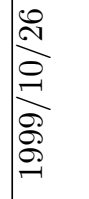 & 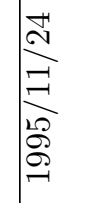 & 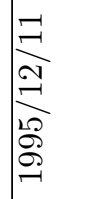 & 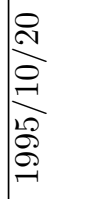 & 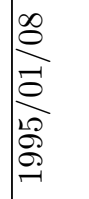 & 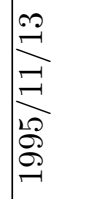 & 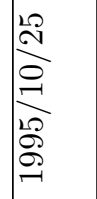 & 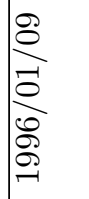 & 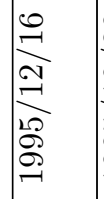 & 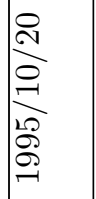 & 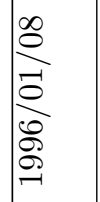 & 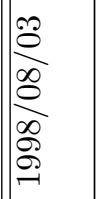 & $\begin{array}{l}\stackrel{N}{N} \\
\hat{N} \\
\stackrel{0}{\infty} \\
\stackrel{\infty}{\mathscr{D}} \\
\stackrel{D}{-}\end{array}$ & 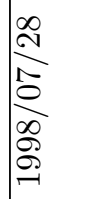 & \\
\hline 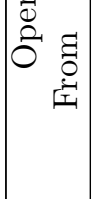 & 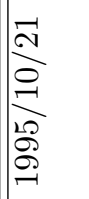 & 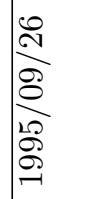 & 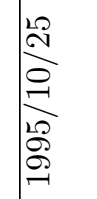 & 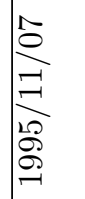 & 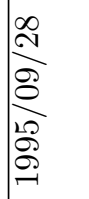 & 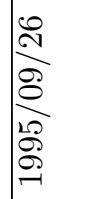 & 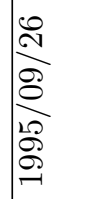 & 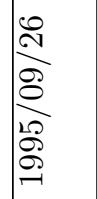 & 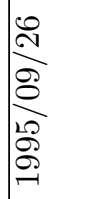 & 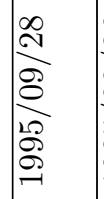 & 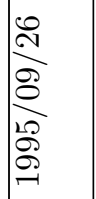 & $\begin{array}{l}-1 \\
0 \\
0 \\
-1 \\
20 \\
2 \\
2 \\
2 \\
-1\end{array}$ & 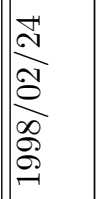 & 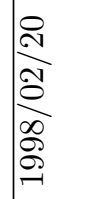 & 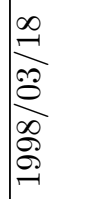 & \\
\hline 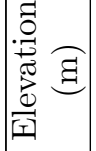 & $\stackrel{8}{8}$ & $\stackrel{一}{\bigotimes}$ & 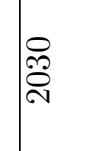 & 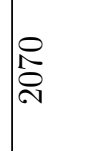 & 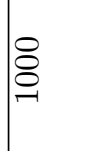 & $\underset{8}{8}$ & $\underset{్}{\stackrel{ి}{0}}$ & 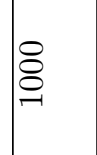 & $\stackrel{8}{\varrho}$ & $\underset{-}{\bigotimes}$ & $\underset{8}{8}$ & \&్రి & 尺े & $\begin{array}{l}0 \\
\text { I } \\
-1\end{array}$ & 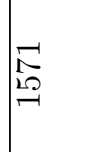 & \\
\hline 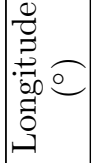 & 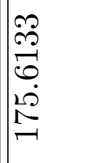 & 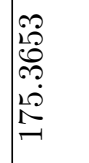 & $\begin{array}{l}1 \\
-40 \\
10 \\
10 \\
10 \\
1-1\end{array}$ & 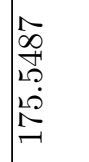 & 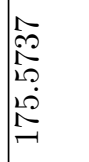 & 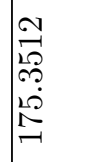 & 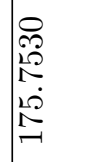 & 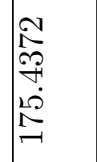 & $\begin{array}{l}20 \\
20 \\
0 \\
0 \\
10 \\
10 \\
-1 \\
-1\end{array}$ & 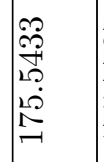 & $\mid \begin{array}{l}1 \\
\infty \\
0 \\
1 \\
1 \\
20 \\
1 \\
-1 \\
-1\end{array}$ & $\begin{array}{l}\infty \\
\stackrel{1}{0} \\
0 \\
\infty \\
20 \\
10 \\
\\
-1\end{array}$ & $\mid \begin{array}{l}\infty \\
\frac{\infty}{20} \\
20 \\
20 \\
20 \\
1 \\
-1\end{array}$ & $\begin{array}{l}1 \\
0 \\
0 \\
0 \\
10 \\
10 \\
-1\end{array}$ & 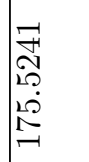 & \\
\hline 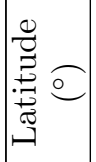 & 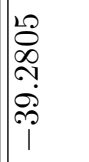 & 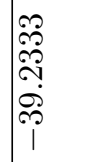 & 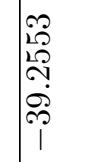 & 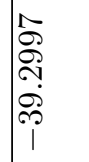 & 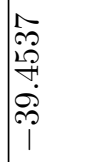 & 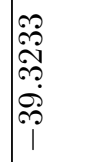 & 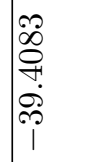 & 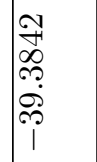 & 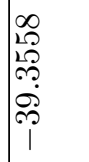 & 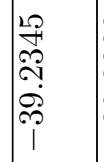 & 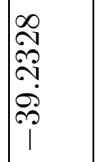 & 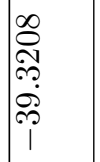 & 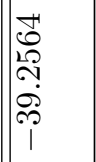 & 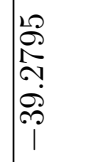 & 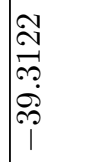 & \\
\hline 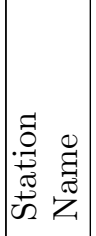 & 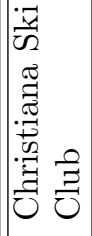 & 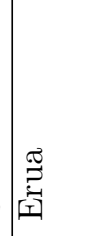 & 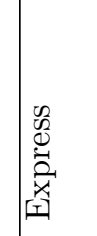 & 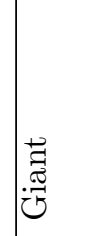 & 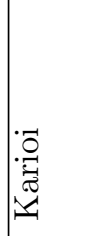 & 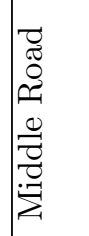 & 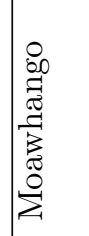 & 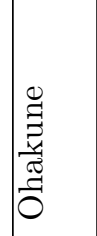 & 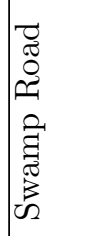 & 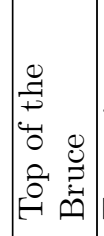 & 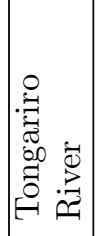 & 芯 & 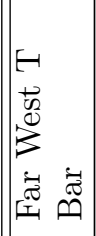 & 祬 & 总 & \\
\hline 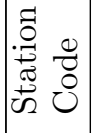 & U & $B^{3}$ & 炃 & 庐 & 焉 & 宝 & 空 & 党 & $\sum_{n}^{\infty}$ & $\vec{b}_{0}^{\vec{b}}$ & 苔 & 家 & 焦 & 武 & 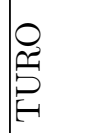 & \\
\hline & 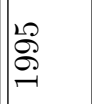 & $\begin{array}{l}\mathscr{2} \\
\stackrel{2}{9}\end{array}$ & $\stackrel{20}{\stackrel{2}{\mathscr{g}}}$ & 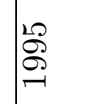 & $\begin{array}{l}\stackrel{2}{2} \\
\stackrel{9}{9}\end{array}$ & $\stackrel{20}{\mathscr{2}}$ & $\stackrel{20}{\mathscr{\Omega}}$ & $\begin{array}{l}\stackrel{2}{2} \\
\stackrel{9}{9}\end{array}$ & 㟶 & $\begin{array}{l}28 \\
\stackrel{2}{9} \\
\stackrel{9}{2}\end{array}$ & $\stackrel{2}{\mathscr{g}}$ & $\begin{array}{l}\mathscr{2} \\
\stackrel{2}{9} \\
\stackrel{9}{2}\end{array}$ & $\stackrel{\infty}{\mathscr{\infty}}$ & $\stackrel{\infty}{\mathscr{2}}$ & 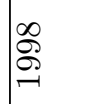 & \\
\hline
\end{tabular}




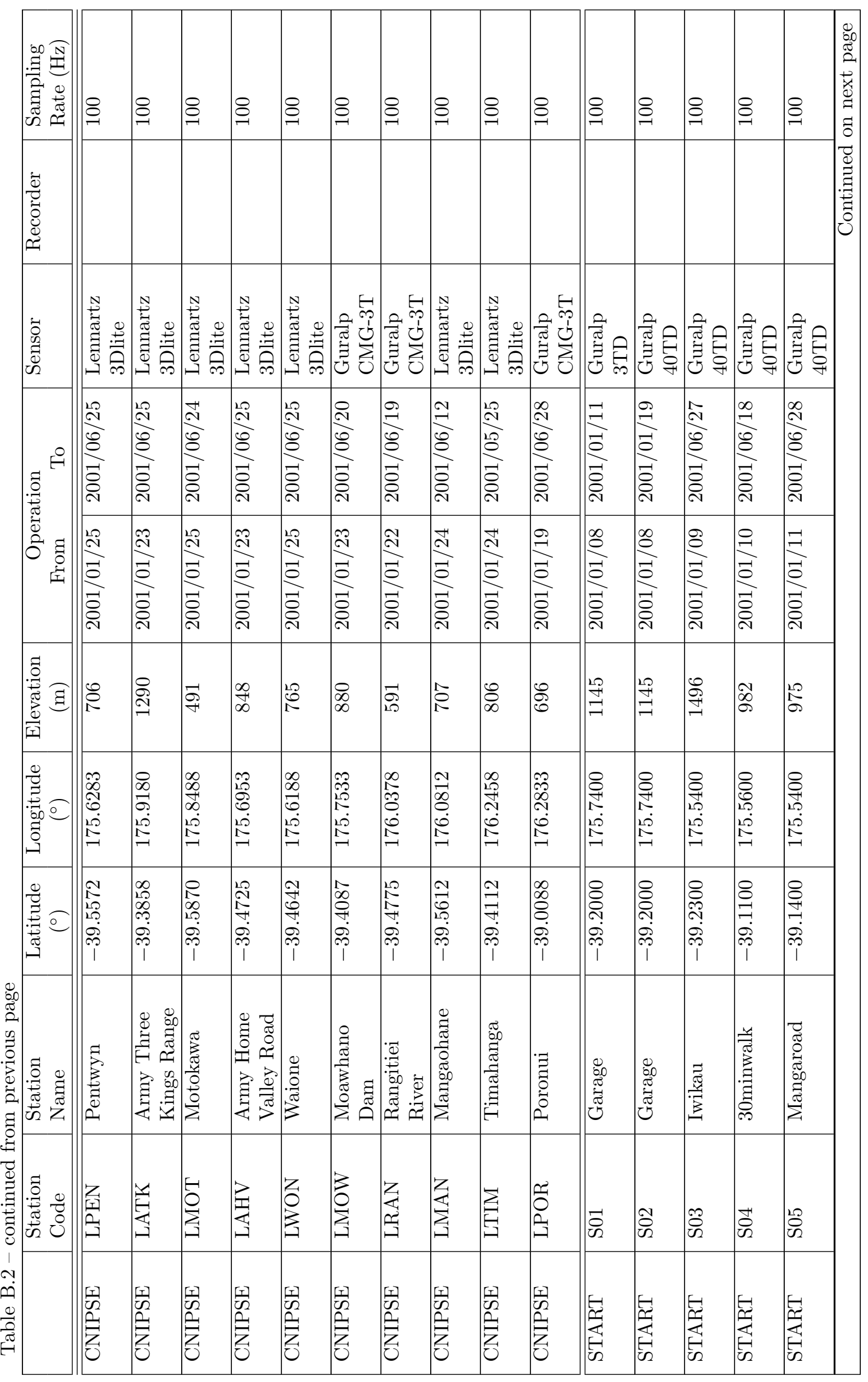




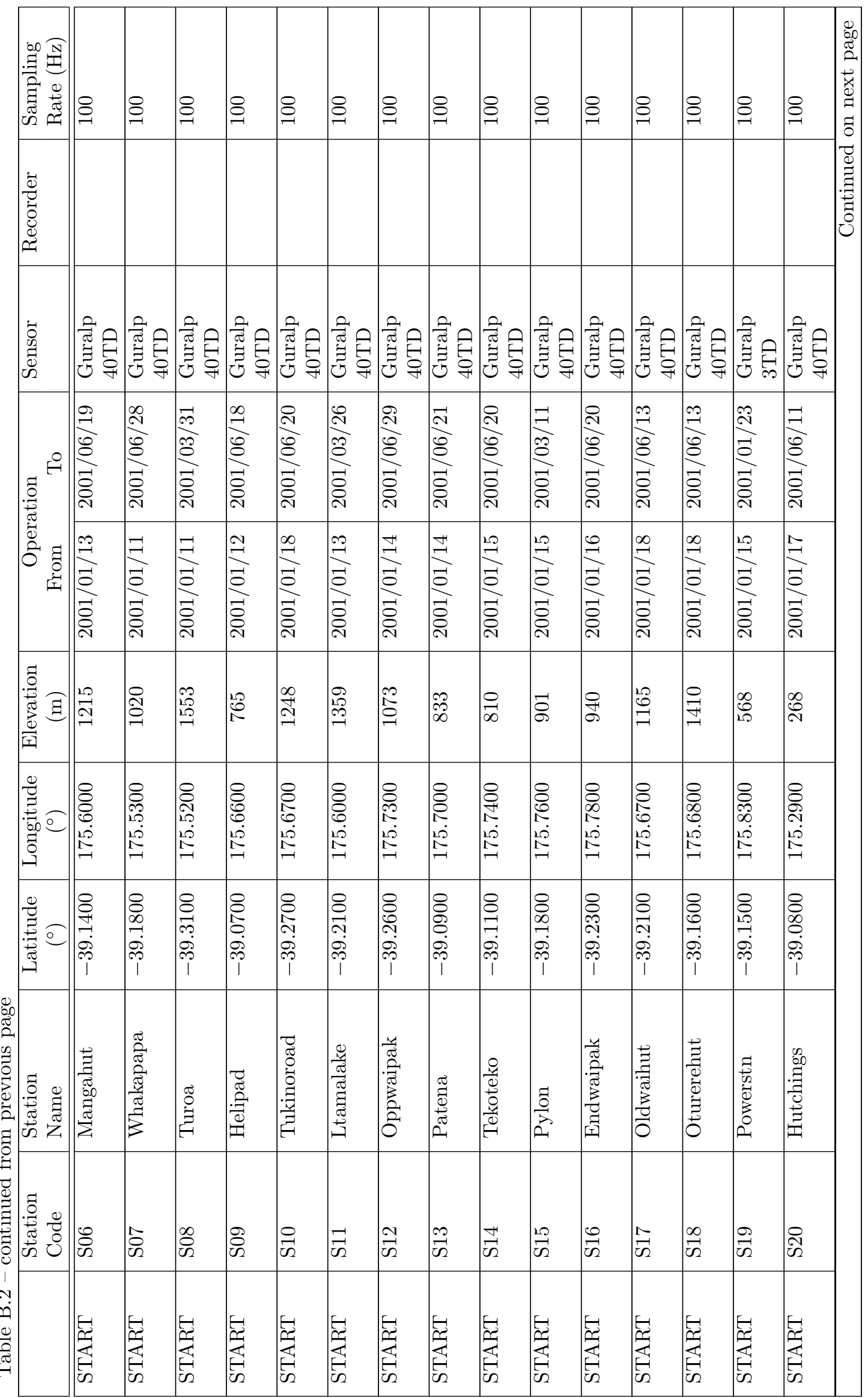




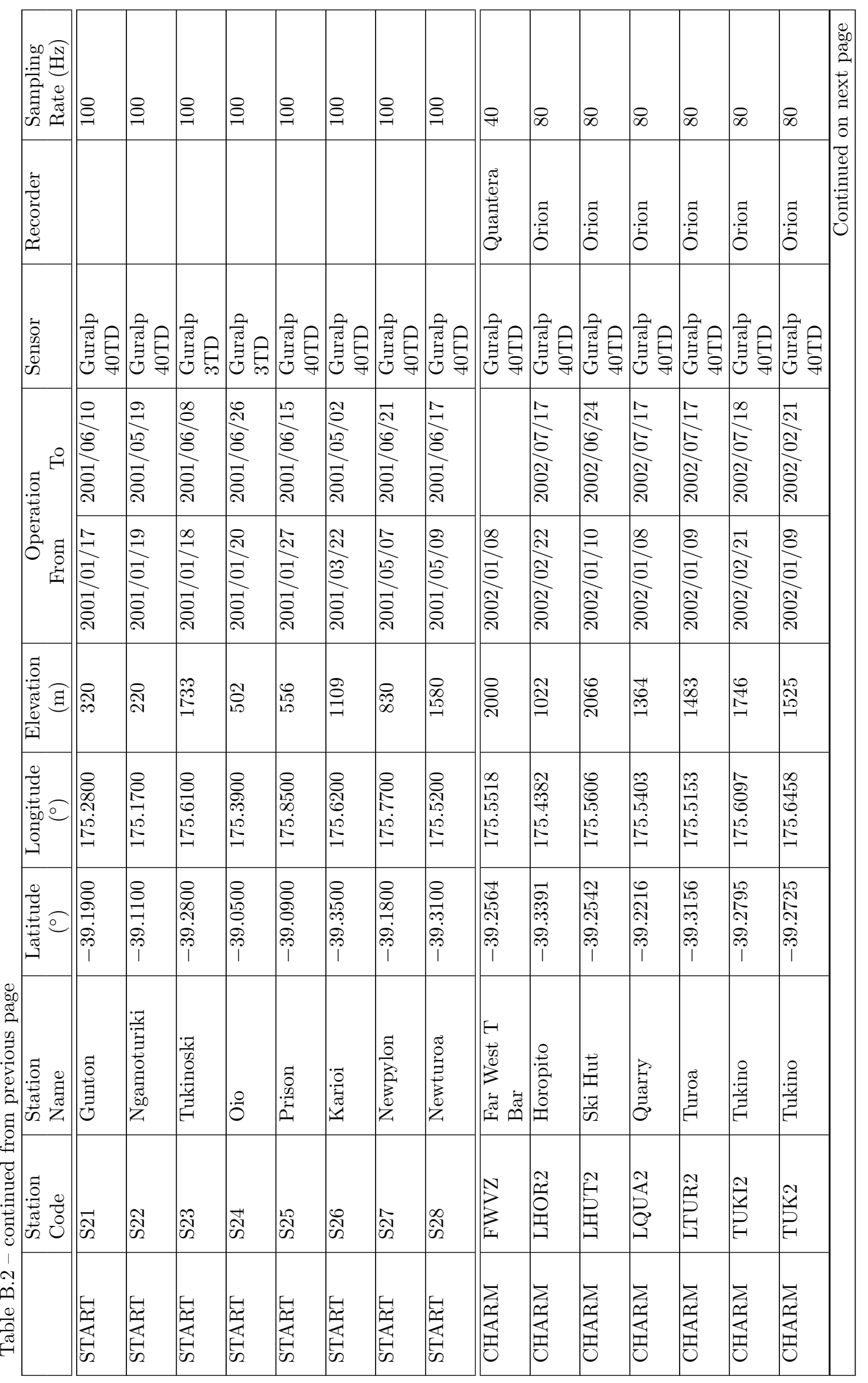




\begin{tabular}{|c|c|c|c|c|c|c|c|c|c|c|c|c|c|c|}
\hline 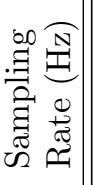 & $\infty$ & $\varnothing$ & $\stackrel{一}{\varrho}$ & 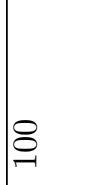 & $\underset{-}{\stackrel{-}{0}}$ & $\underset{-1}{\stackrel{-}{0}}$ & 8 & $\underset{-1}{\stackrel{-}{0}}$ & 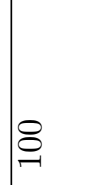 & 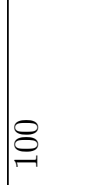 & 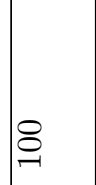 & $\underset{-1}{\stackrel{-}{8}}$ & $\stackrel{\ominus}{\stackrel{ }{2}}$ & 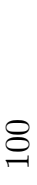 \\
\hline 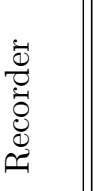 & 苛 & .0. & 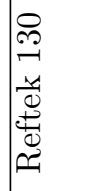 & 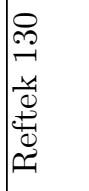 & 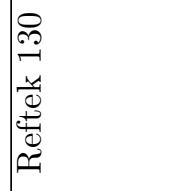 & 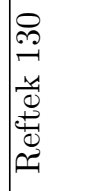 & 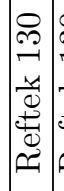 & 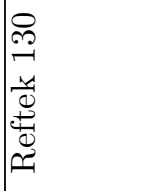 & 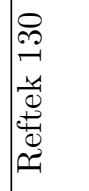 & 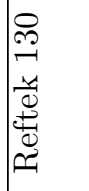 & 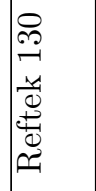 & 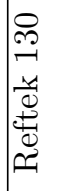 & 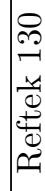 & 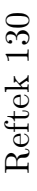 \\
\hline 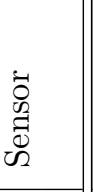 & 福是 & 襾 & 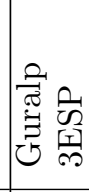 & 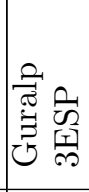 & 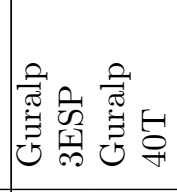 & 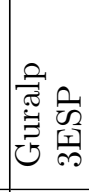 & 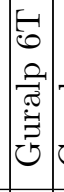 & 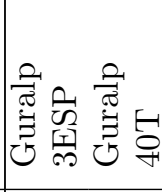 & 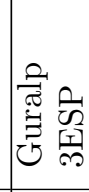 & 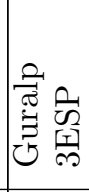 & 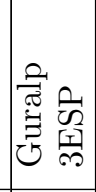 & 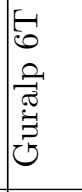 & 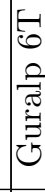 & 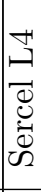 \\
\hline$\stackrel{0}{\oplus}$ & 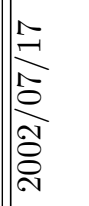 & 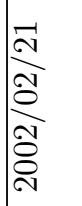 & & 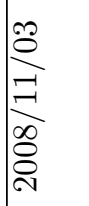 & 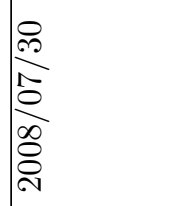 & 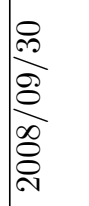 & 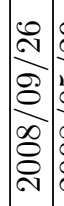 & 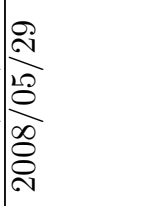 & 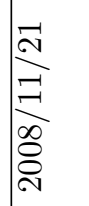 & 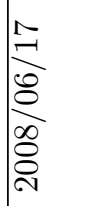 & 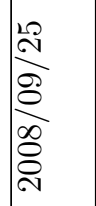 & 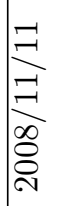 & 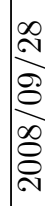 & 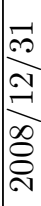 \\
\hline 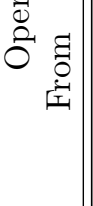 & 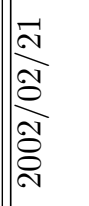 & 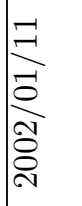 & 苟 & 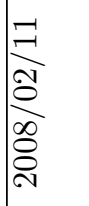 & 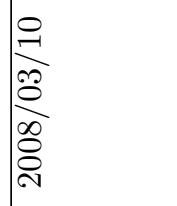 & 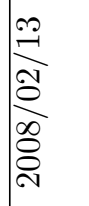 & 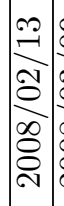 & 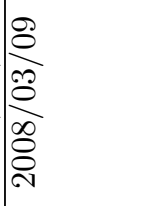 & 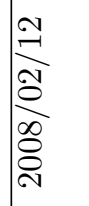 & 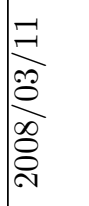 & 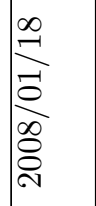 & 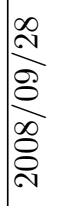 & 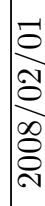 & 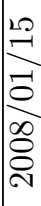 \\
\hline 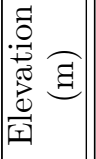 & $\stackrel{ }{12}$ & \begin{tabular}{l}
10 \\
\hdashline
\end{tabular} & 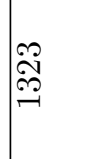 & $\frac{\vec{\infty}}{\infty}$ & 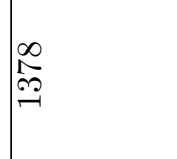 & 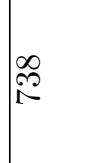 & $\mid \begin{array}{l}\hat{\mathscr{O}} \\
\vec{\delta}\end{array}$ & 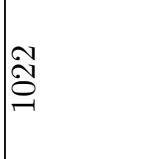 & 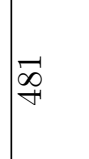 & 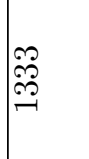 & 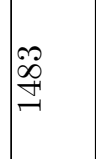 & 18 & $\mathscr{R}$ & $\stackrel{\vec{R}}{\mathbb{R}}$ \\
\hline 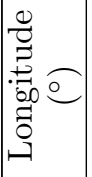 & 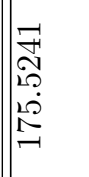 & 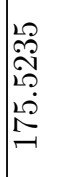 & 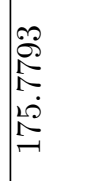 & $\begin{array}{l}1 \\
0 \\
0 \\
0 \\
0 \\
10 \\
10 \\
-1\end{array}$ & 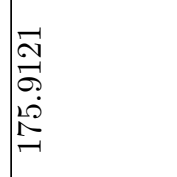 & 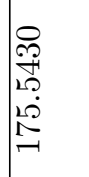 & 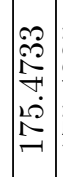 & 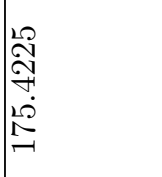 & $\begin{array}{l}\infty \\
\infty \\
0 \\
0 \\
20 \\
10 \\
-1 \\
-1\end{array}$ & 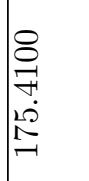 & 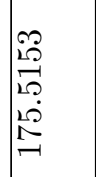 & 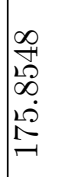 & 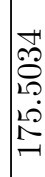 & 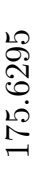 \\
\hline 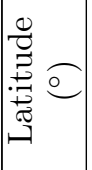 & 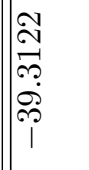 & 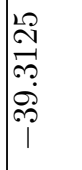 & 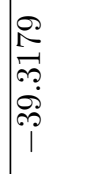 & $\begin{array}{l}\vec{\infty} \\
\stackrel{+}{\not} \\
\therefore \\
\infty \\
1\end{array}$ & 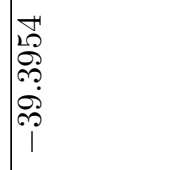 & 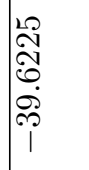 & 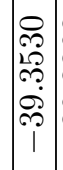 & 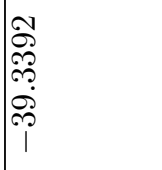 & $\begin{array}{l}\infty \\
\infty \\
\infty \\
2 \\
\infty \\
\infty \\
\infty \\
i \\
1\end{array}$ & $\begin{array}{l}\overrightarrow{\tilde{O}} \\
\tilde{D} \\
\tilde{a} \\
\ddot{p} \\
1\end{array}$ & 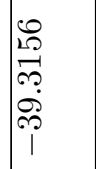 & $\begin{array}{l}\stackrel{9}{+} \\
0 \\
20 \\
0 \\
0 \\
0 \\
1\end{array}$ & 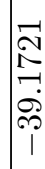 & 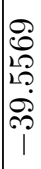 \\
\hline 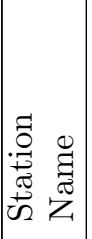 & 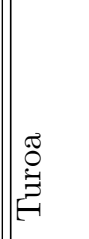 & 宽 & 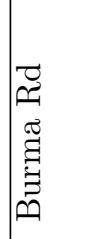 & 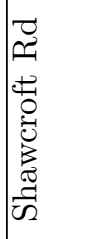 & 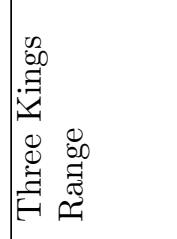 & 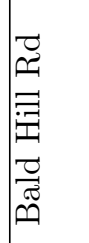 & 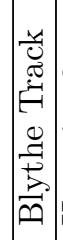 & 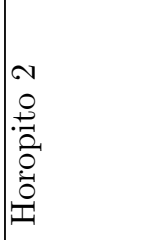 & 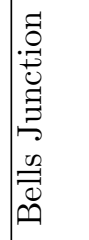 & 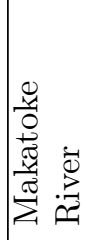 & 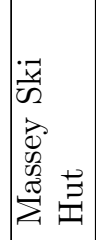 & 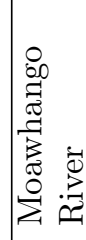 & 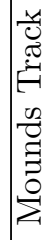 & ָ \\
\hline 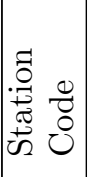 & 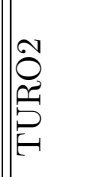 & 疍 & 缹 & 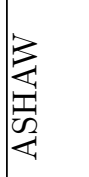 & 焉 & 焉 & $\begin{array}{l}E \\
5 \\
3 \\
0\end{array}$ & 莡 & 国 & 崖 & 憵 & 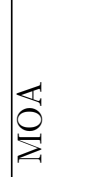 & & i \\
\hline & 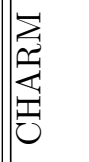 & 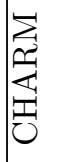 & 蛋 & 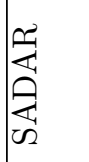 & 空 & 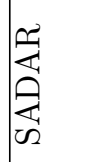 & 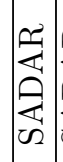 & 究 & 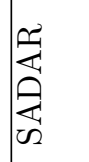 & 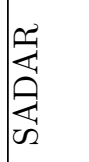 & 疍 & 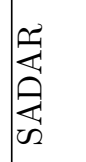 & & \\
\hline
\end{tabular}




\begin{tabular}{|c|c|c|c|c|c|c|c|c|c|c|c|c|c|c|c|c|}
\hline 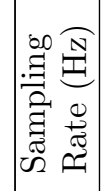 & 8 & $\stackrel{\Xi}{-}$ & $\stackrel{尺}{Ð}$ & $\underset{-}{\circledR}$ & 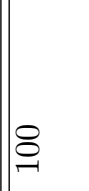 & $\stackrel{8}{\circ}$ & 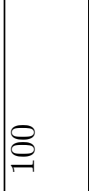 & 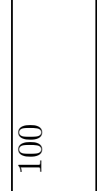 & $\stackrel{8}{-}$ & $\stackrel{8}{\circ}$ & 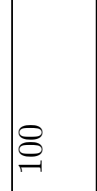 & 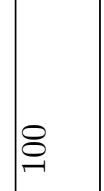 & $\stackrel{8}{\circ}$ & 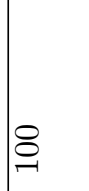 & $\stackrel{8}{-}$ & $\stackrel{8}{2}$ \\
\hline 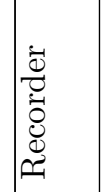 & 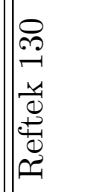 & 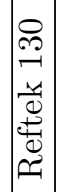 & 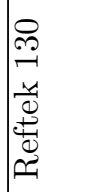 & 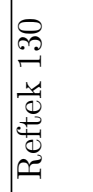 & 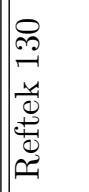 & 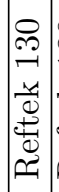 & 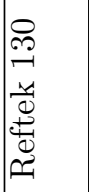 & 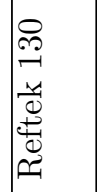 & 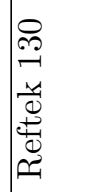 & 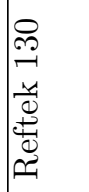 & 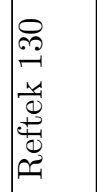 & 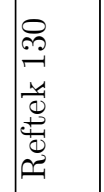 & 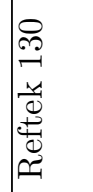 & 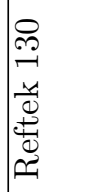 & 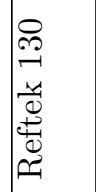 & 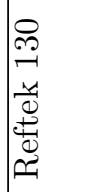 \\
\hline 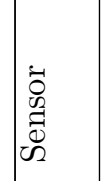 & 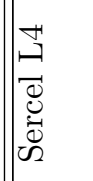 & 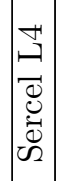 & 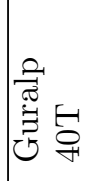 & 苗 & 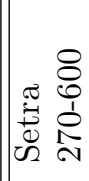 & 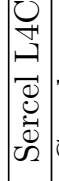 & 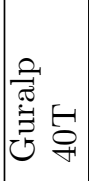 & 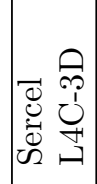 & 悹 & 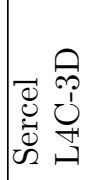 & 窇 & 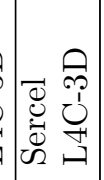 & 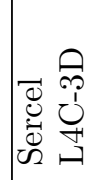 & 悹 & 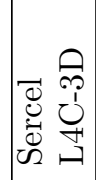 & 胥 \\
\hline$\underbrace{0}$ & 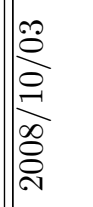 & 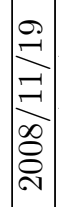 & 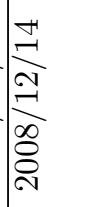 & 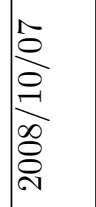 & 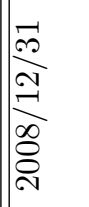 & 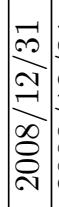 & 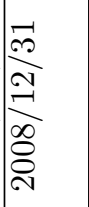 & 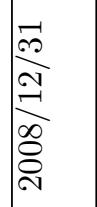 & 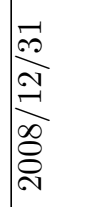 & 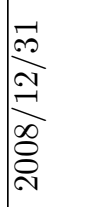 & 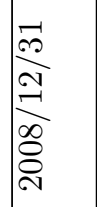 & 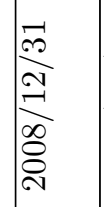 & 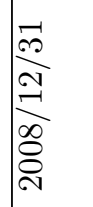 & 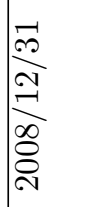 & 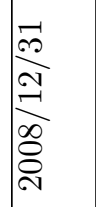 & 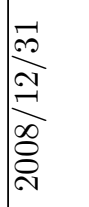 \\
\hline 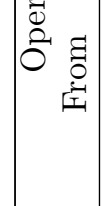 & 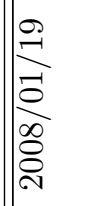 & 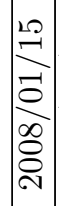 & 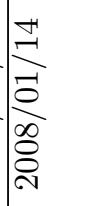 & $\begin{array}{l}0 \\
\stackrel{1}{-} \\
\overrightarrow{0} \\
\frac{\infty}{\infty} \\
\stackrel{0}{2} \\
\sim\end{array}$ & 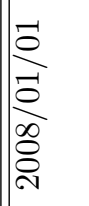 & $\mid \begin{array}{c}\overrightarrow{0} \\
\overrightarrow{0} \\
0 \\
0 \\
0 \\
0 \\
\sim\end{array}$ & $\frac{\overrightarrow{0}}{\overrightarrow{0}}$ & 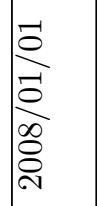 & 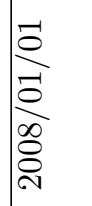 & $\begin{array}{l}\vec{b} \\
\overrightarrow{0} \\
\frac{0}{\infty} \\
0 \\
\stackrel{0}{2}\end{array}$ & 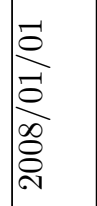 & $\frac{\vec{\rho}}{\vec{\rho}}$ & 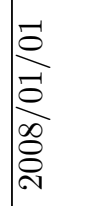 & 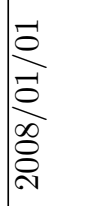 & $\begin{array}{l}\frac{\overrightarrow{0}}{2} \\
\overrightarrow{0} \\
\frac{\infty}{0} \\
\stackrel{0}{\alpha}\end{array}$ & 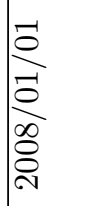 \\
\hline 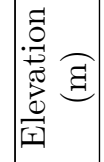 & 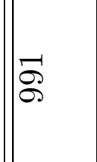 & 20 & $\begin{array}{l}\mathscr{\infty} \\
\infty \\
\infty\end{array}$ & $\stackrel{\infty}{\infty}$ & $\| \stackrel{\leftrightarrow}{\stackrel{m}{二}}$ & $\begin{array}{c}\mathcal{O} \\
\mathscr{d} \\
\mathcal{N}\end{array}$ & $\underset{\sim}{\stackrel{\leftrightarrow}{\sim}}$ & 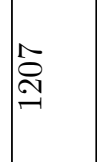 & $\underset{\infty}{\mathbb{\infty}}$ & $\underset{\infty}{\stackrel{f}{\infty}}$ & $\mid \begin{array}{l}1 \\
\stackrel{2}{2} \\
\exists \\
\neg\end{array}$ & $\begin{array}{l}0 \\
0 \\
19 \\
-1\end{array}$ & $\left.\right|_{R} ^{R}$ & ఫ్ & $\underset{f}{\stackrel{0}{Z}}$ & 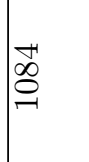 \\
\hline 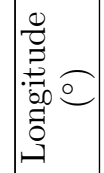 & $\mid \begin{array}{l}0 \\
0 \\
0 \\
2 \\
20 \\
1 \\
1 \\
-1 \\
-1\end{array}$ & $\begin{array}{l}0 \\
0 \\
0 \\
0 \\
20 \\
10 \\
-1\end{array}$ & 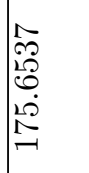 & 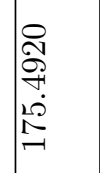 & 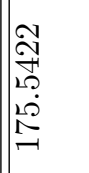 & 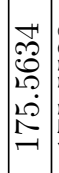 & 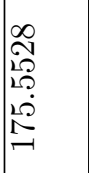 & 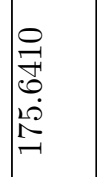 & $\begin{array}{l}\hat{N} \\
\stackrel{1}{1} \\
1 \\
10 \\
10 \\
-1\end{array}$ & 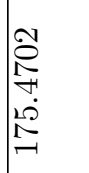 & $\begin{array}{l}0 \\
\stackrel{0}{0} \\
8 \\
0 \\
10 \\
10 \\
-1\end{array}$ & 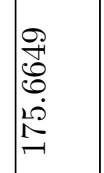 & $\begin{array}{l}01 \\
0 \\
0 \\
0 \\
20 \\
10 \\
10 \\
-1\end{array}$ & $\begin{array}{l}10 \\
0 \\
1 \\
71 \\
10 \\
1 \\
-1 \\
0\end{array}$ & $\begin{array}{l}0 \\
13 \\
0 \\
0 \\
10 \\
1 \\
-1\end{array}$ & 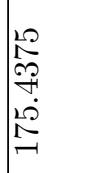 \\
\hline 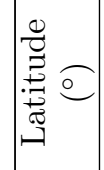 & 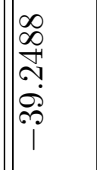 & 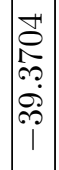 & 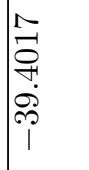 & 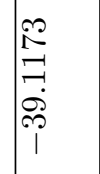 & 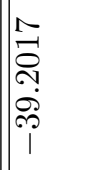 & 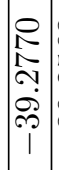 & 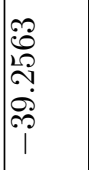 & 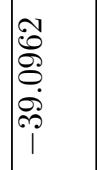 & 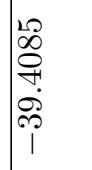 & 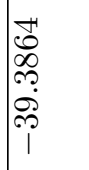 & 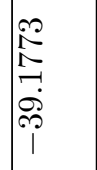 & \begin{tabular}{l}
$\stackrel{9}{9}$ \\
$\overrightarrow{0}$ \\
-1 \\
\hdashline \\
9 \\
1
\end{tabular} & 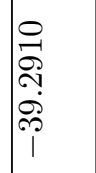 & \begin{tabular}{l}
0 \\
8 \\
0 \\
$\infty$ \\
$\infty$ \\
\hdashline \\
\hdashline \\
1
\end{tabular} & 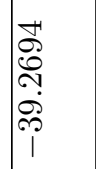 & 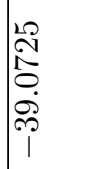 \\
\hline 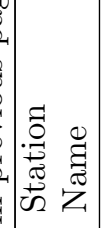 & 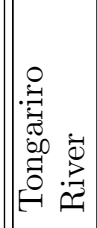 & 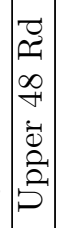 & 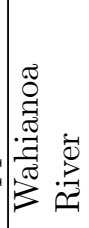 & 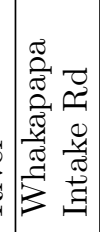 & 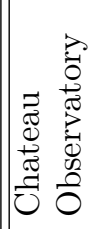 & 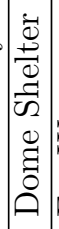 & 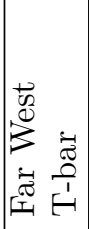 & 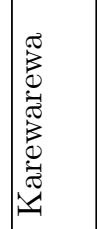 & 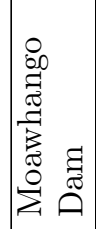 & 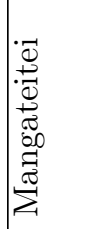 & 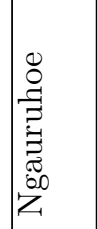 & 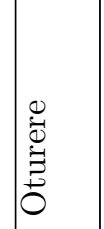 & 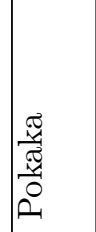 & 总 & 总 & 营 \\
\hline 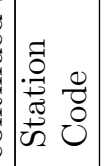 & 萜 & 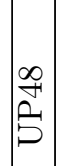 & $\underset{⿱ 乛}{3}$ & 态 & $\mid \begin{array}{l}N \\
0 \\
0\end{array}$ & 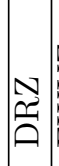 & $\sum_{i=1}^{N}$ & 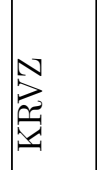 & 点 & $\sum_{i}^{N}$ & 总 & P & $\underset{N}{N}$ & in & $\sum_{3}^{N}$ & $\sum_{i=1}^{N}$ \\
\hline & 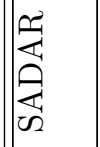 & $\mid \begin{array}{l}\text { 空 } \\
\text { 岕 } \\
\text { n }\end{array}$ & 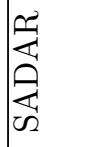 & 窟 & $\mid$\begin{tabular}{|l}
$\mid \overrightarrow{0}$ \\
$\overrightarrow{0}$ \\
0 \\
0 \\
0 \\
0
\end{tabular} & $\mid \begin{array}{l}0 \\
0 \\
\vdots \\
0 \\
0 \\
0 \\
0\end{array}$ & 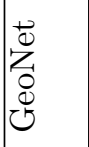 & $\begin{array}{l}0 \\
0 \\
0 \\
0 \\
0 \\
0 \\
0\end{array}$ & $\begin{array}{l}\overrightarrow{0} \\
\ddot{0} \\
0 \\
0 \\
0 \\
ن\end{array}$ & $\begin{array}{l}\overrightarrow{0} \\
\ddot{0} \\
0 \\
0 \\
0 \\
0\end{array}$ & $\begin{array}{l}\overrightarrow{0} \\
\overrightarrow{0} \\
\dot{0} \\
0 \\
0 \\
0\end{array}$ & 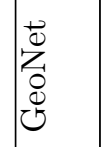 & $\begin{array}{l}\overrightarrow{0} \\
0 \\
0 \\
0 \\
0 \\
0 \\
0\end{array}$ & 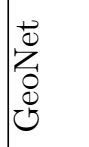 & $\begin{array}{l}\overrightarrow{0} \\
\ddot{0} \\
0 \\
0 \\
0 \\
0\end{array}$ & 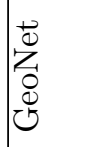 \\
\hline
\end{tabular}




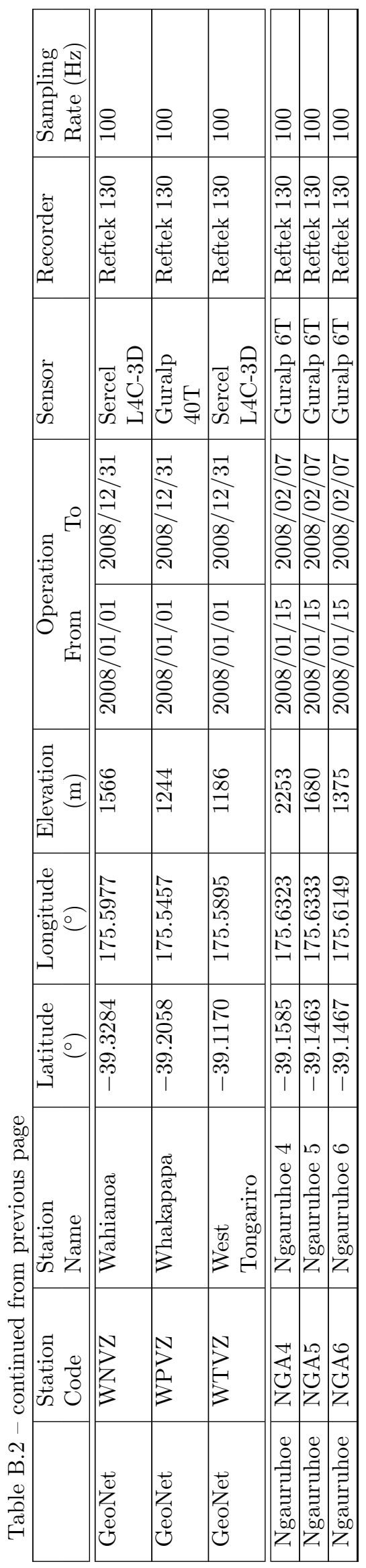





\section{Appendix: Data acquisition}

\section{C.1. Deployment logistics}

Sites for the SADAR seismometers were chosen based on several criteria. Approximate locations were first identified on a map to fill in the gaps of the permanent network and, where possible, reoccupy previous station's locations. Where previous locations were reoccupied, the exact coordinates were used. Where no previous station had been located, sites were chosen based on:

- the ability to secure the sensor onto in situ bedrock for coupling;

- $180^{\circ}$ of clear view of the northern sky for maximum solar panel efficiency;

- the site not being close to any sources of noise (e.g. running water);

- the site not being readily visible from public areas to avoid interference;

- the site not being in an area that has a lot of livestock.

In most cases not all of these criteria could be fulfilled and so a trade-off between points usually resulted in suitable sites. When a suitable site was identified, permission was obtained from the landowners and the seismometers were installed.

Each seismometer was placed on a ceramic plate in a hole between 0.5 and $1.5 \mathrm{~m}$ deep. The holes were intended to reach in situ bedrock if it was available. The ceramic plates were cemented to the rock at all of the sites except those within the Tongariro National Park boundary. The seismometers were orientated to true north and levelled (Figure C.1). A water-tight container was then secured around each seismometer so that rain and groundwater did not affect the instruments. Every seismometer was connected to a Reftek RT130, which was also placed in a waterproof package (Figure C.2). The systems were powered (in all occasions bar one) by a solar panel and two $12 \mathrm{~V}, 65 \mathrm{kWh}$ sealed lead acid batteries, with the voltage controlled by a regulator. In one case, a solar panel was impractical due to the site's proximity 


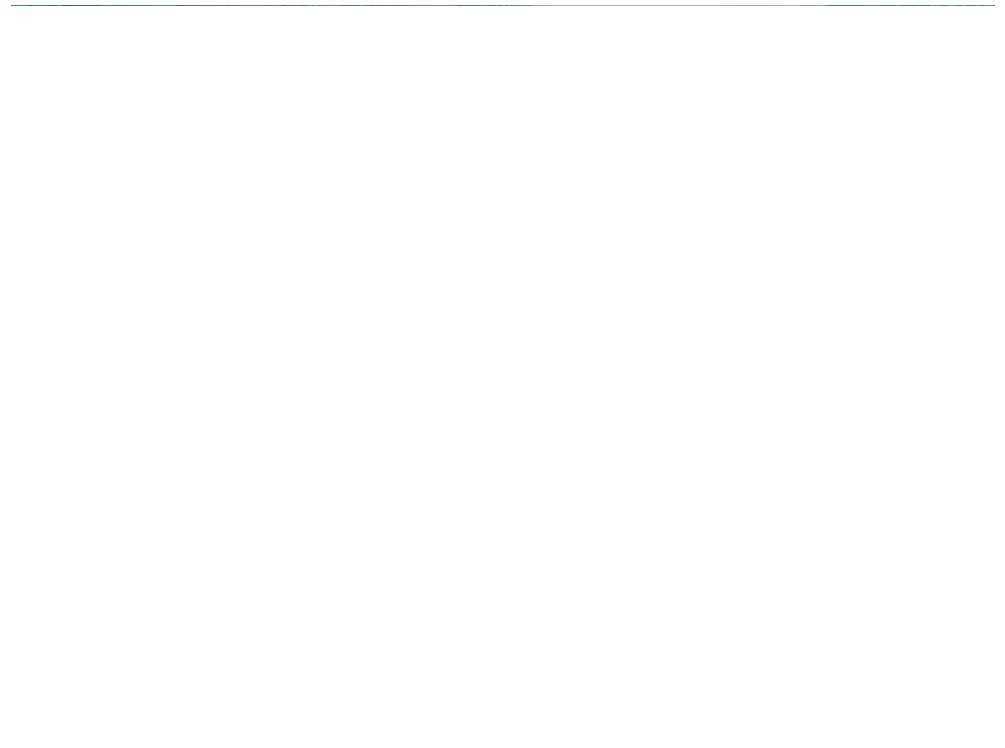

Figure C.1. Guralp (CMG3-ESP) in waterproof casing and cemented onto bedrock, aligned to north and levelled at station MOA.

to a walking track so alkali cells were used. The Reftek timing and accurate site locations were obtained from continuous GPS units. In some cases electric fences were erected to discourage livestock from investigating the station (Figure C.3).

The continuous data were recorded by Reftek RT-130s on SD memory cards at 100 Hz with unitary gain in RT-130 format. The sites were serviced and data downloaded approximately every three months.

\section{C.2. Data processing manual}

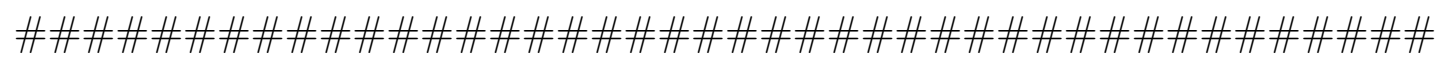
Author: Jessica Johnson, jessica.johnson@vuw.ac.nz

Date: $30 / 01 / 09$

Program: rt2sac_ms

Purose: To convert data from rt130 from the refteks to sac data cut around events listed from GeoNet Quakesearch

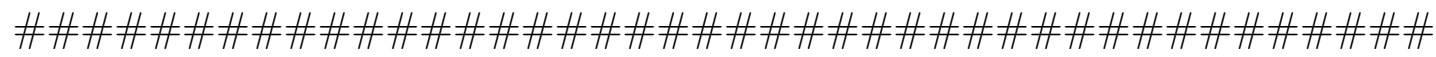

Note: I have chosen to convert the data through Mseed rather than SegY because ms 2 sac provides more headers in the final product than segy2sac (hence the 'ms' 


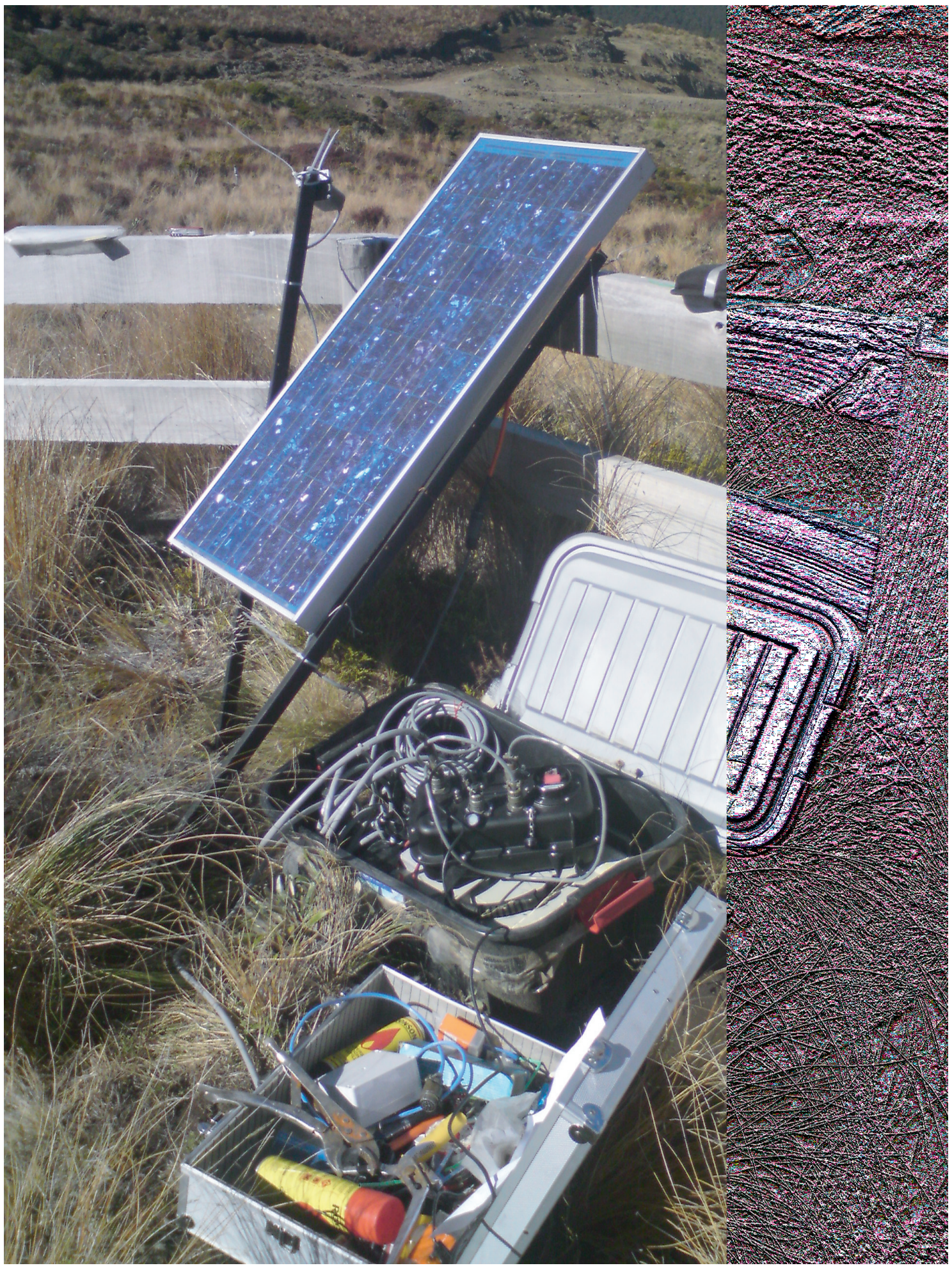

Figure C.2. Reftek seismograph, batteries and cables in plastic casing next to solar panel and GPS unit at station ABUR. 


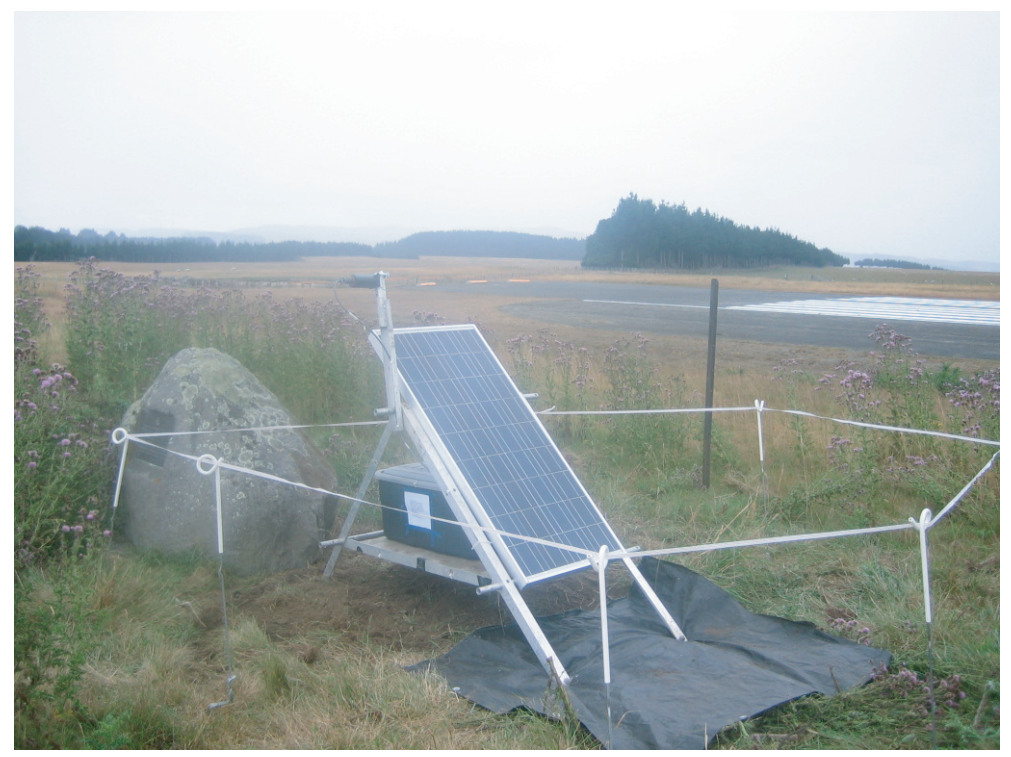

Figure C.3. Complete set up of solar panel, plastic casing and GPS unit surrounded by electric fencing at station ASHAW.

in the title). I have also written a working script that uses SegY, please see that script if you with to use it, it may need some work.

- Preparation:

The RT130 data should be in a directory named after the das number, which is in a directory named after the site e.g. MAKA/915D/'raw data'. You will need to conduct a quakesearch on the geonet website (http://magma.geonet.org . $\mathrm{nz} /$ resources/quakesearch/) with the full CSV output. This information can be converted into the format needed with the script mk_evtfile.sh. Note: this is set up for time over the leap day in 2008. This creates the file 'evtfile' and can be located in the working directory (one level above the 'sitename' directory) or within the 'sitename' directory. You will need to make a 'dasfile' with the details of the das used at each station. This can be one file with all of the information or many files with one das in each. The format is:

$$
\begin{array}{ll}
\text { \#das } & \text { chan } \\
0912 B & 1 C 1,1 C 2,1 C 3 \\
0915 A & 1 C 1,1 C 2,1 C 3 \\
\text { etc } &
\end{array}
$$

This can also be located in the working directory or within the 'sitename' directory. 
- Running:

The program $r t 2 s a c \_m s_{-} j j$.sh must be invoked in the working directory (one level above the 'sitename' directory) with the sitename and das\#

e.g. rt2sac_ms $s_{-} j$.sh MAKA $915 \mathrm{D}$

rt2sac_ms $s_{-} j$.sh keeps track of the steps that it has performed in das\#.log and echos most steps to stout.

It will create the rest of the file structure:

REF_DATA

Mseed_DATA

SAC_DATA

Err_files

Log_files

PCF_files

Stations

It will also create a file in the directory Stations called 'station.info':

$\begin{array}{llllll}\text { \#st_das } & \text { end_dasDS } & \text { s_rate } & \text { format } & \text { chan } & \text { preamp_gain } \\ 9 F 99 & 9 F 99 & 100 & c 0 & 1 & 1 \\ 9 F 99 & 9 F 99 & 100 & c 0 & 2 & 1 \\ 9 F 99 & 9 F 99 & 100 & c 0 & 3 & 1\end{array}$

If the sample rate, format or pre-amp gain is different to those listed, this must be changed in the code.

- rt130cut:

Once this is all set up rt130cut (passcal code) is invoked in REF_DATA:

rt130cut $-\mathrm{r}$ wd/'sitename'/das\# -d das\#

This creates large data files in REF_DATA. More than one ref file could be created if there is a gap in the data. $r t 2 s a s_{-} s_{-} j j . s h$ checks that there is at least one .ref file. If there is not, it gives a warning. 
- ref $2 \mathrm{mseed}$ :

In the Mseed_DATA directory ref2mseed (passcal code) is run:

ref2mseed $-f$../REF_DATA/*.ref -1 wd/'site'/Stations/station.info

This makes miniseed data files in the directory Mseed_DATA in folders named per day. It also makes a $\log$ file, which has the state of health information and a .err file, which has information about any time jumps. If there are no time jumps then .err may not be created. The .log file is moved to directory Log_files and the .err file (if it exists) is moved to the Err_files directory.

- refrate:

The program refrate (passcal code) uses the .log file to make a file das\#.rating in the PCF files directory, which will be used to correct the timing. If there is no errors there will be no .err file and refrate will not run. If this is the case, rt2sac_ms_jj.sh makes the file with the correct headings but it will be empty.

- mk_pos:

mk_pos is a shell script (written by Sonja Greve) that uses MATLAB to find the accurate location of the station from the state of health files (i.e. from the GPS antenna). It makes the file das\#.pos, which is then moved to Stations directory (used later).

- tkeqcut:

tkeqcut is a gui (passcal code), which has replaced eqcut. It therefore cannot be used easily in a command line (qmerge might be used in a later version).

The following should be entered in the windows that pop up:

Data Directories: Mseed_DATA

DAS file: Stations/dasfile

Event file: Events/eq.local

click 'Build Trace db')

When this has counted the data files, enter the following:

Sample Rate of Input Data: 100 
Input Record Length: 3600

Output Record Length: 600

Parent of Output Directory: (default)

PCF File: PCF_files/das\#.rating

Leave both Tolerances as 0.0

Now click "Cut Events'"

This created a directory EqCut with one folder per Earthquake and cut mseed files.

- run_ms2sac.sh:

run ms $2 \mathrm{sac} . \mathrm{sh}$ is a shell script (written by me) that makes cut sac files in the directory SAC_DATA using ms2sac (passcal code). It must be run in the working directory and have the sitename and das\# entered

e.g. run_ms2sac.sh MAKA 915D

Other Notes:

cleanup.sh, when run in the wd followed by the sitename, removes all of the products of rt2sac_ms_jj.sh to start afresh. 



\section{Appendix: Scripts}

\section{D.1. CWB data retrieval}

\#! /bin/bash

$\begin{array}{lll}\text { \# } & \text { Script: } & \text { cwb_getevents.sh } \\ \text { \# Invoke with: } & \text {./cwb_getevents.sh station } \\ \text { \# Purpose: } & \text { To download data from GeoNet } \\ \text { \# Author: } & \text { Jess Johnson } \\ \text { \# Date: } & 17 / 04 / 10 \\ \text { \# } \text { Inputs: } & \text { station ID code } \\ \text { \# } & \text { earthquakes.cusp: list of earthquake cusp IDs } \\ \text { \# Outputs: } & \text { sac files with automatic picks in headers } \\ \text { \# Other programs: } & \text { java } \\ \text { \# } & & \text { GeoNetCWBQuery-2.2.2-bin.jar }\end{array}$

\#run this where you want the data.

stat $=\$ 1$

\#read list of cusp IDs

while read id

do

\# check if this id has already been downloaded (this way you can stop it midway and don't need to repeat any downloads).

if [ ! -f \$id.done ]

then

echo \$id

\# put the data into a folder named for the cusp id.

if [ ! $-d$ \$id ]

then

mkdir \$id

fi

cd \$id

\# download the data (the -o tells it the name of the downloaded file, if you want it 
named differently change this bit.

\# At the moment it will just give STAT.COMP.SAC

\#Also change the path for the jar file to match wherever you keep it.

java -jar /software/CWB/GeoNetCWBQuery-2.2.2-bin.jar -event

geonet:\$id -s "NZ\$stat..H...'

cd . .

\# make a flag to show that this ID has been downloaded in case you need to stop and start the process.

touch \$id.done

fi

\# make this your list of cusp ids.

done $<$ earthquakes.cusp

cd . . 


\section{D.2. Phase picking}

\#!/bin/bash

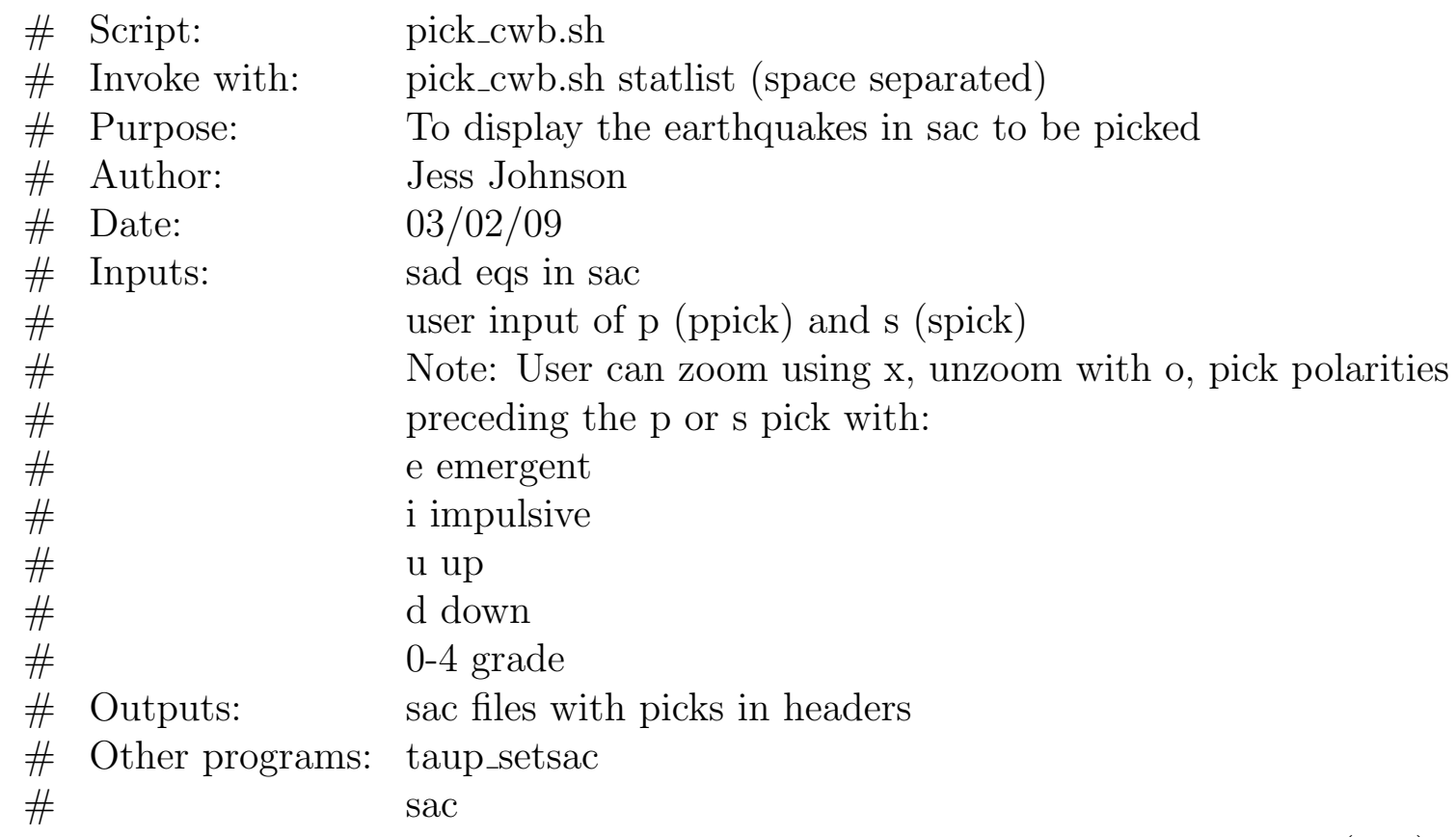

date

for stat in $\$ 1$

do

\# make an empty file to remind you which station you are picking

touch \$stat=doing

if [ $-d$ \$stat ]

then

cd \$stat

\# This loops over every earthquake but it is set up specifically for cusp ids

for eq in ???????

do

if [ ! - $f$ \$eq.done ]

then

if $[-\mathrm{d}$ \$eq $]$

then

cd $\$$ eq

echo \$eq

\# taup_setsac uses a $1 D$ earth model to estimate the $P$ and $S$ arrivals on the \# seismograms and puts them into the sac headers as t8 and $t 9$ respectively for comp in \$stat.?.SAC

do 


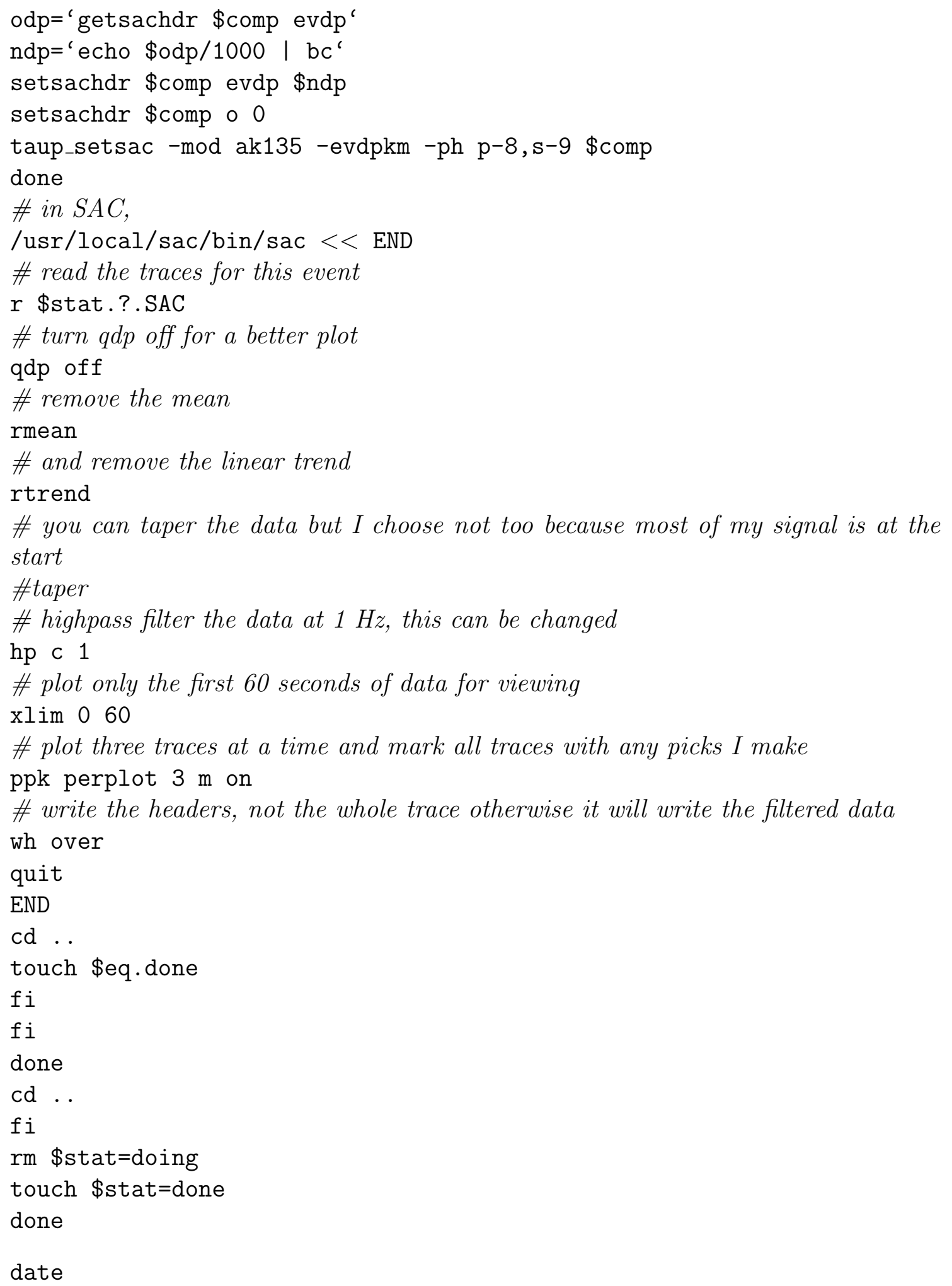


E. Appendix: Parameters used in multiplet identification, double-difference relocation, anisotropy analysis, and Coulomb modelling 


\begin{tabular}{|c|c|c|c|c|c|c|c|c|c|c|}
\hline 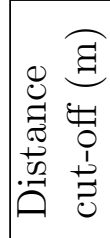 & & 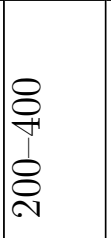 & & 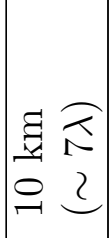 & & 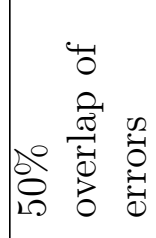 & & $\stackrel{20}{\rightarrow-1}$ & & $\stackrel{\sim}{\curvearrowright}$ \\
\hline 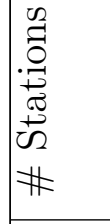 & & $\stackrel{ }{\circ}$ & & 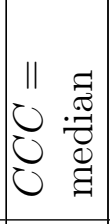 & F & & & & & \\
\hline 己气 & & & & 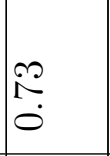 & $\ddot{\theta}$ & & $\ddot{\circ}$ & 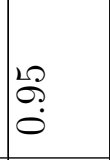 & $\infty$ & $\begin{array}{l}\infty \\
\stackrel{0}{0} \\
0\end{array}$ \\
\hline 离 & & 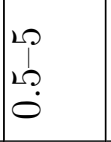 & & & $\begin{array}{l}\mathcal{I} \\
\mathcal{I} \\
\dot{H}\end{array}$ & & & & & \\
\hline 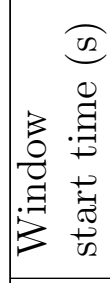 & & & & 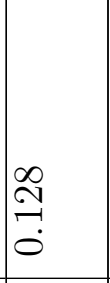 & & & & & 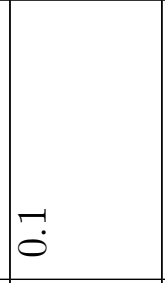 & \\
\hline 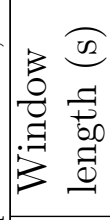 & & & $\stackrel{\sim}{\sim}$ & 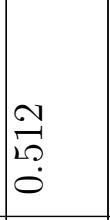 & $\stackrel{\infty}{\stackrel{\infty}{\sim}}$ & & $\begin{array}{l}20 \\
\text { id }\end{array}$ & $\begin{array}{l}\vec{H} \\
\dot{0} \\
-1\end{array}$ & $\stackrel{\vec{i}}{\vec{i}}$ & \\
\hline 总 & \begin{tabular}{|l}
$\exists$ \\
50 \\
.5 \\
.
\end{tabular} & $\begin{array}{l}0 \\
0 \\
0 \\
3 \\
.0\end{array}$ & 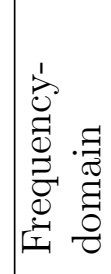 & 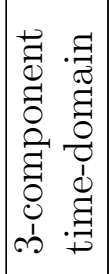 & 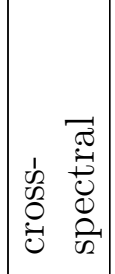 & 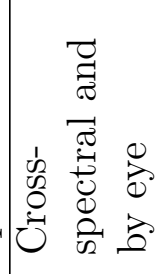 & 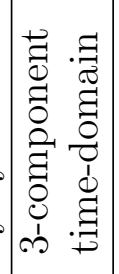 & 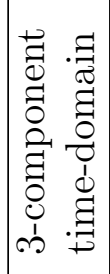 & 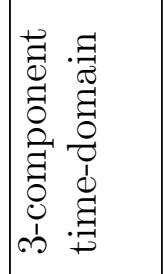 & 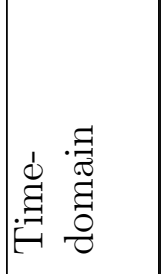 \\
\hline 贯 & 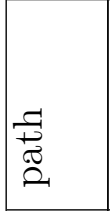 & 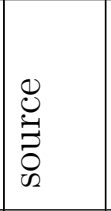 & 宽 & 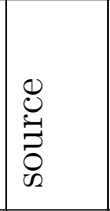 & 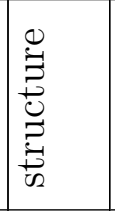 & $\begin{array}{l}\vec{F} \\
\stackrel{\vec{\Xi}}{\tilde{a}}\end{array}$ & 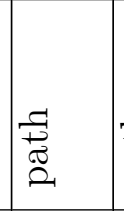 & 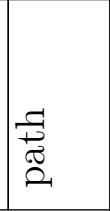 & 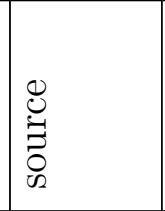 & 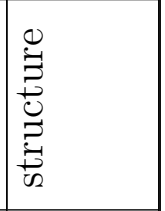 \\
\hline 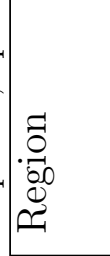 & 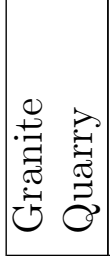 & . & 营 & . & 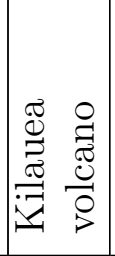 & : & . & . & 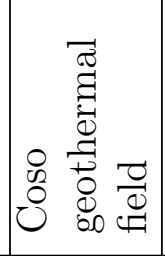 & . \\
\hline$\vec{g}$ & 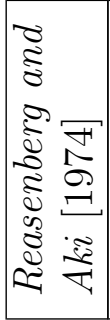 & 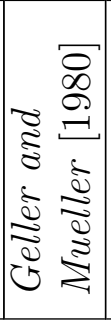 & 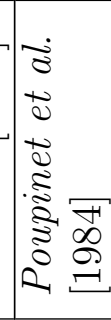 & 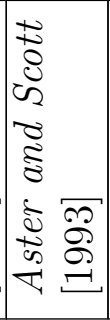 & 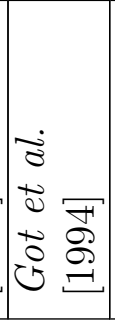 & 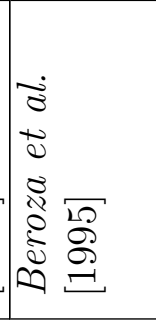 & 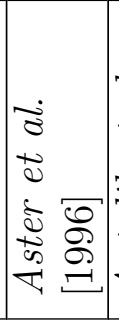 & 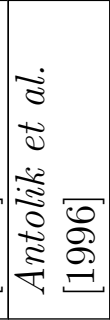 & 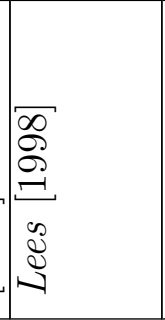 & 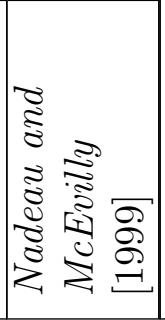 \\
\hline
\end{tabular}




\begin{tabular}{|c|c|c|c|c|c|c|c|c|c|c|c|}
\hline 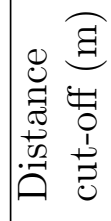 & & & & & $\begin{array}{l}\infty \\
0 \\
\cdots \\
\infty \\
\infty\end{array}$ & & & 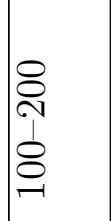 & $\begin{array}{l}\infty \\
0 \\
x \\
0 \\
0\end{array}$ & 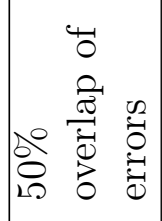 & 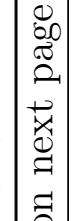 \\
\hline 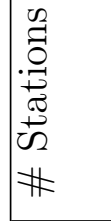 & & & $\odot$ & & & & & 10 & $\infty$ & & 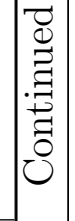 \\
\hline 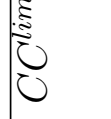 & $\stackrel{\infty}{\infty}$ & $\stackrel{2}{\stackrel{2}{0}}$ & 占 & $\stackrel{?}{0}$ & $\stackrel{\infty}{\infty}$ & $\begin{array}{l}\infty \\
\stackrel{0}{0} \\
0\end{array}$ & & 决 & 占 & & \\
\hline 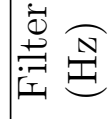 & & $\begin{array}{l}0 \\
\ddots \\
\dashv \\
-1\end{array}$ & $\stackrel{+}{\stackrel{H}{\prime}}$ & & $\infty$ & مَ & & $\infty$ & & & \\
\hline 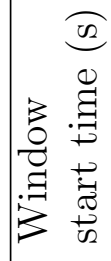 & & 0 & 0 & & & $\neg$ & & 0 & $\stackrel{10}{0}$ & & \\
\hline 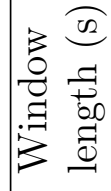 & تִ & $\underset{\sim}{O}$ & $\stackrel{\llcorner 2}{\rightarrow}$ & $\frac{\overrightarrow{\tilde{\sigma}}}{\frac{\pi}{n}}$ & 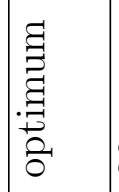 & $\stackrel{\curvearrowright}{\curvearrowright}$ & $\stackrel{\leftrightarrow}{\sim}$ & 10 & $\stackrel{\circ}{\circ}$ & & \\
\hline 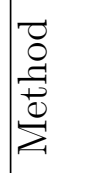 & 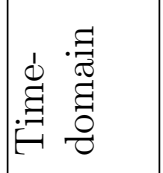 & 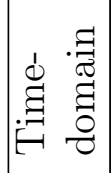 & 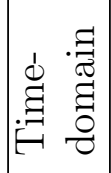 & 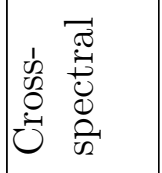 & 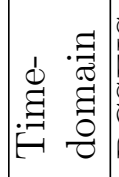 & 记 & 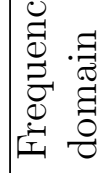 & 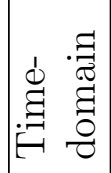 & 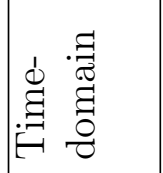 & & \\
\hline 寻 & 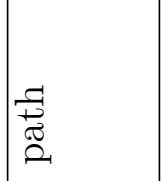 & 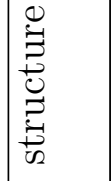 & 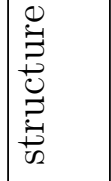 & 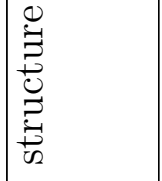 & $\begin{array}{l}0 \\
0 \\
\Xi \\
0 \\
0 \\
\infty\end{array}$ & 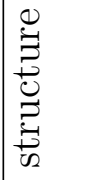 & 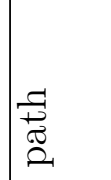 & 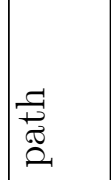 & 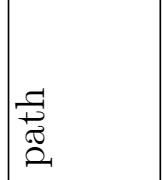 & $\begin{array}{l}0 \\
0 \\
\vdots \\
0 \\
\infty\end{array}$ & \\
\hline 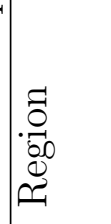 & 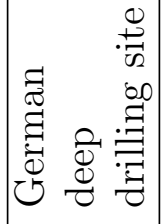 & 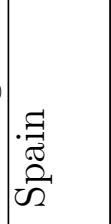 & 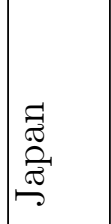 & 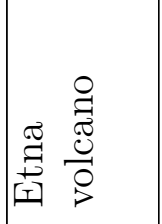 & 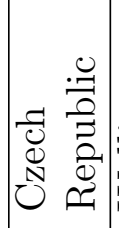 & 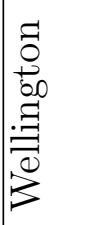 & . & 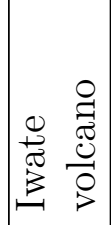 & 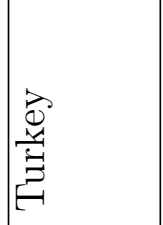 & . & \\
\hline$\frac{3}{0}$ & 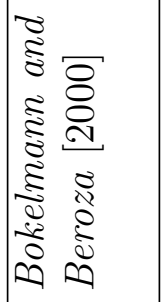 & 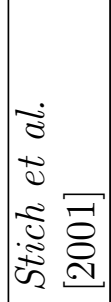 & 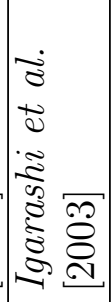 & 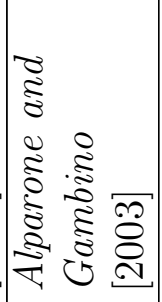 & 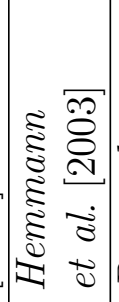 & 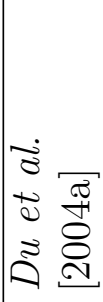 & 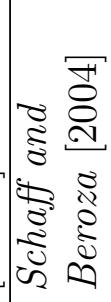 & 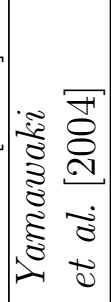 & 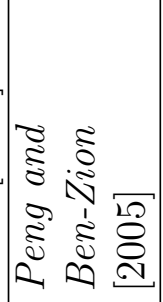 & 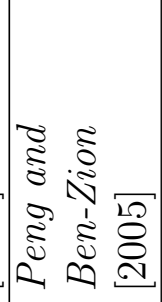 & \\
\hline
\end{tabular}




\begin{tabular}{|c|c|c|c|c|c|c|c|c|c|c|c|}
\hline 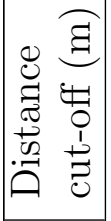 & | & & & & & & & & $\begin{array}{l}0 \\
0 \\
\cdots \\
x \\
0 \\
-1\end{array}$ & $\frac{\not}{x}$ & $=$ \\
\hline 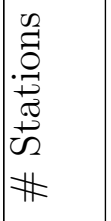 & $\neg$ & -1 & & & & -1 & & & & 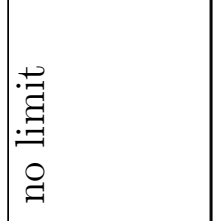 & \\
\hline ర్. & $\stackrel{\theta}{0}$ & $\infty$ & $\stackrel{r}{0}$ & & $\stackrel{0}{0}$ & $\stackrel{N}{0}$ & $\stackrel{\infty}{\infty}$ & $\infty$ & 占? & $\stackrel{20}{\mathscr{2}}$ & \\
\hline 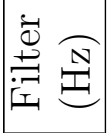 & $\frac{10}{1}$ & $\begin{array}{l}\stackrel{0}{\Xi} \\
\stackrel{\Xi}{\Xi}\end{array}$ & $\begin{array}{l}20 \\
\sim \\
\sim \\
0\end{array}$ & $\stackrel{\infty}{\stackrel{\Lambda}{\Lambda}}$ & $\stackrel{\sim}{־}$ & $\stackrel{20}{T}$ & 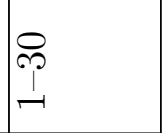 & $\begin{array}{l}20 \\
12 \\
0 \\
0\end{array}$ & $\begin{array}{l}\stackrel{0}{0} \\
\stackrel{\Xi}{*}\end{array}$ & $\begin{array}{l}\stackrel{0}{0} \\
\stackrel{\Xi}{a}\end{array}$ & \\
\hline 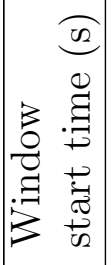 & 0 & & & $\stackrel{20}{0}$ & 0 & $\stackrel{20}{\circ}$ & & & 0 & & \\
\hline 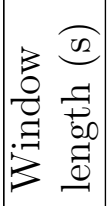 & 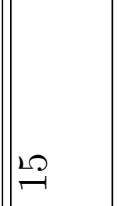 & $\infty$ & $\infty$ & Le & $\underset{0}{0}$ & 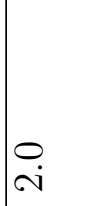 & $\stackrel{\circ}{\stackrel{0}{*}}$ & 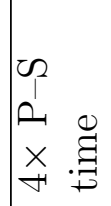 & 20 & 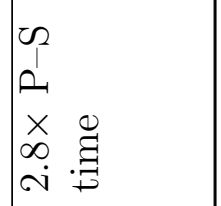 & \\
\hline 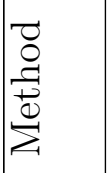 & 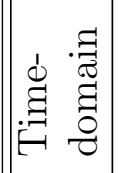 & 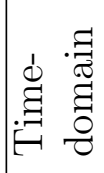 & 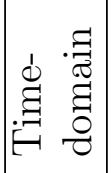 & 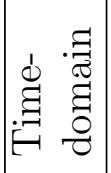 & 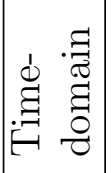 & 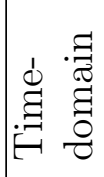 & 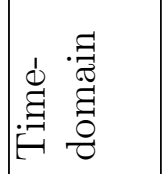 & 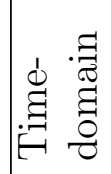 & 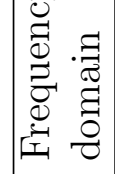 & 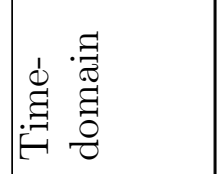 & \\
\hline 灵 & 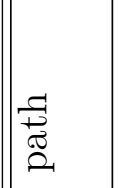 & $\begin{array}{l}\stackrel{0}{0} \\
\stackrel{\Xi}{0} \\
0 \\
\infty\end{array}$ & 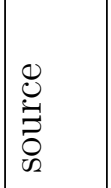 & 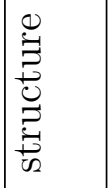 & 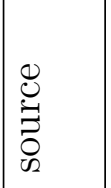 & 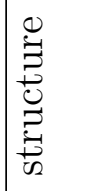 & 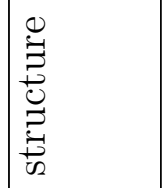 & 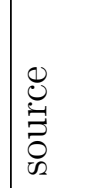 & 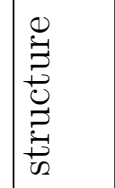 & 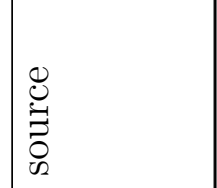 & \\
\hline 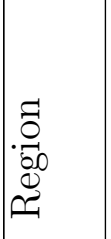 & 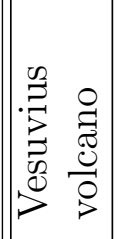 & $\begin{array}{l}\frac{\pi}{3} \\
\frac{\pi}{\sqrt{2}} \\
\frac{\pi}{4} \\
\end{array}$ & 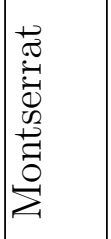 & 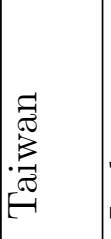 & $\stackrel{\vec{D}}{ \pm}$ & $\begin{array}{l}\vec{\nexists} \\
\widetilde{\widetilde{a}} \\
\tilde{\tilde{n}}\end{array}$ & 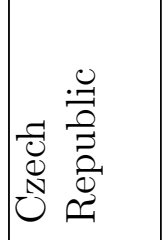 & $\stackrel{\widetilde{\Xi}}{\Xi}$ & . & 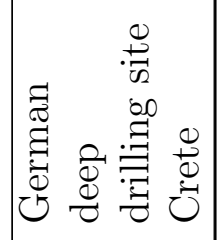 & \\
\hline 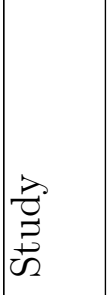 & 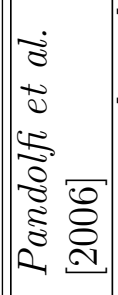 & 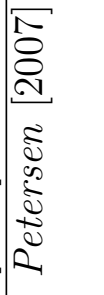 & 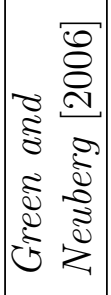 & 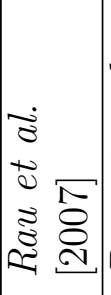 & 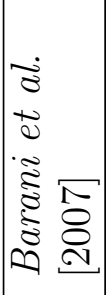 & 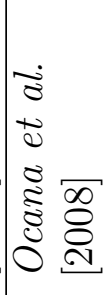 & 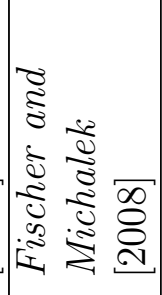 & 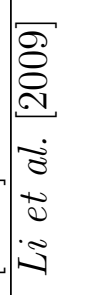 & 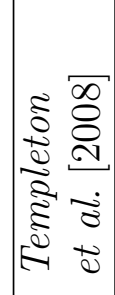 & 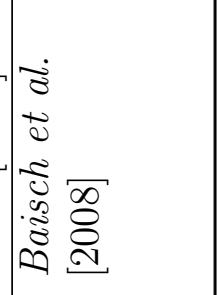 & \\
\hline
\end{tabular}




\begin{tabular}{|c|c|c|c|c|c|c|c|}
\hline 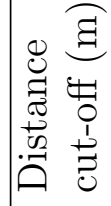 & & 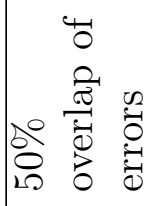 & & & 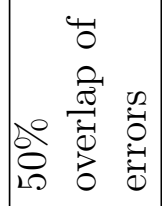 & & \\
\hline 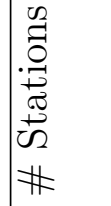 & & $\infty$ & $\infty$ & & 10 & $\neg$ & $\sim$ \\
\hline 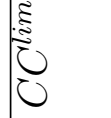 & $\stackrel{\infty}{0}$ & $\ddot{0}$ & $\ddot{0}$ & $\ddot{0}$ & 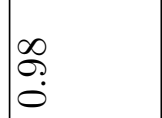 & 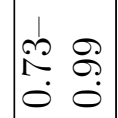 & $\stackrel{\check{c}}{\circ}$ \\
\hline 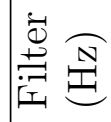 & 8 & 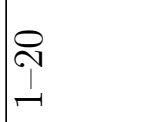 & $\begin{array}{l}\infty \\
-1 \\
10\end{array}$ & $\stackrel{20}{11}$ & & $\begin{array}{l}\infty \\
10 \\
0 \\
0\end{array}$ & \\
\hline 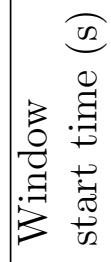 & $\stackrel{\overbrace{}}{\circ}$ & - & $\stackrel{\sim}{O}$ & & & $\stackrel{\sim}{O}$ & $\curvearrowright$ \\
\hline 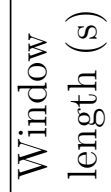 & 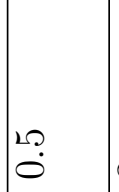 & $\infty$ & $\underset{i}{O}$ & $\stackrel{\sim}{\sim}$ & 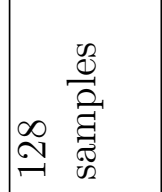 & نج & $a$ \\
\hline 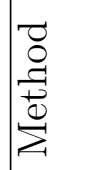 & 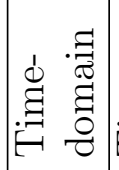 & 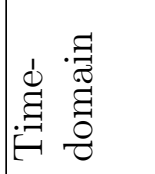 & 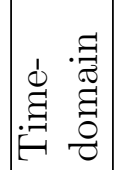 & 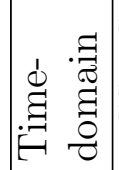 & $\begin{array}{l}0 \Omega \\
\text { II } \\
0 \\
0 \\
0\end{array}$ & 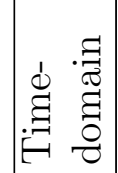 & 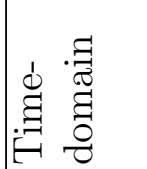 \\
\hline 浔 & 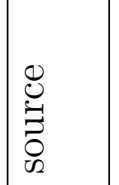 & 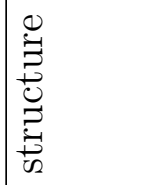 & 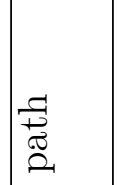 & 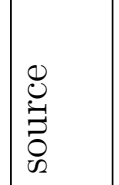 & 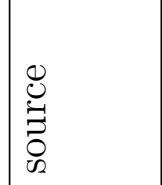 & $\frac{\theta}{\frac{\theta}{ \pm}}$ & 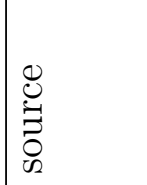 \\
\hline $\begin{array}{l}07 \\
.0 \\
.00 \\
\stackrel{8}{10} \\
.1\end{array}$ & 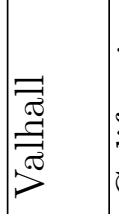 & . & 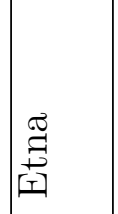 & 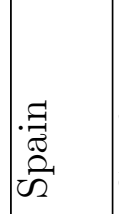 & . & 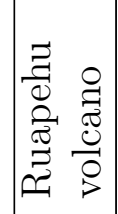 & 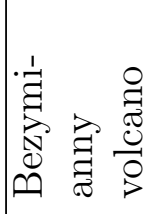 \\
\hline$\frac{3}{\frac{B}{3}}$ & 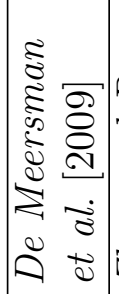 & 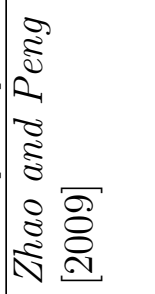 & 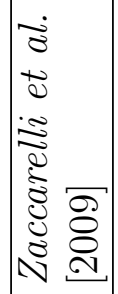 & 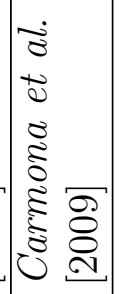 & 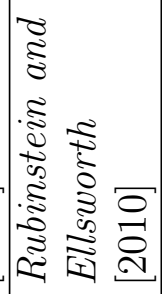 & 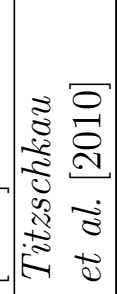 & 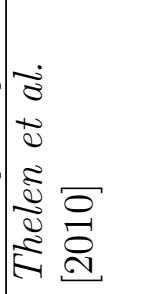 \\
\hline
\end{tabular}




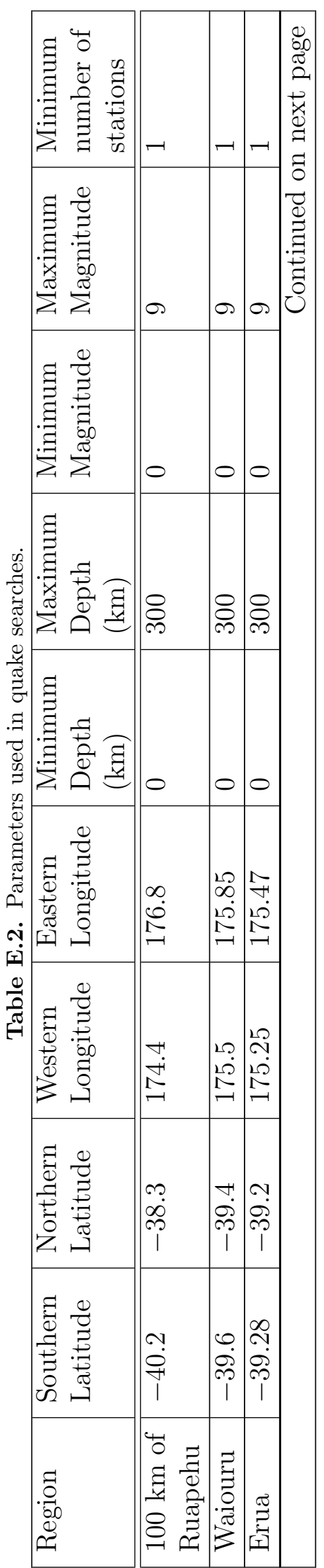


Table E.3. Parameters used during double-difference relocation.

\begin{tabular}{|c|c|c|}
\hline Parameter & Description & Typical Value \\
\hline $\begin{array}{l}\text { MIN- } \\
\text { WGHT }\end{array}$ & Minimum picking weight & (0 \\
\hline MAXDIST & $\begin{array}{l}\text { Maximum distance (in } \mathrm{km} \text { ) between } \\
\text { event pair and station }\end{array}$ & 400 \\
\hline MAXSEP & $\begin{array}{l}\text { Maximum hypocentral separation } \\
\text { between event pairs (in km) }\end{array}$ & 4 \\
\hline MAXNGH & $\begin{array}{l}\text { Maximum number of neighbours per } \\
\text { event }\end{array}$ & 4 \\
\hline MINLNK & $\begin{array}{l}\text { Minimum number of links required to } \\
\text { define a neighbour }\end{array}$ & 5 \\
\hline MINOBS & Minimum number of links per pair saved & 5 \\
\hline MAXOBS & Maximum number of links per pair saved & 20 \\
\hline IDAT & $\begin{array}{l}\text { Data type: } 1=\text { cross-correlation data } \\
\text { only; } 2=\text { absolute (catalogue) data only; } \\
3=\text { cross-correlation and catalogue data }\end{array}$ & 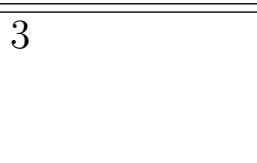 \\
\hline IPHA & $\begin{array}{l}\text { Phase }: 1=\mathrm{P} \text { wave; } 2=\mathrm{S} \text { wave; } 3=\mathrm{P} \& \mathrm{~S} \\
\text { wave }\end{array}$ & 3 \\
\hline DIST & $\begin{array}{l}\text { Maximum distance between cluster } \\
\text { centroid and stations }\end{array}$ & 400 \\
\hline OBSCC & $\begin{array}{l}\text { Minimum number of cross-correlation } \\
\text { links per event to form a continuous } \\
\text { cluster }\end{array}$ & 0 \\
\hline OBSCT & $\begin{array}{l}\text { Minimum number of catalogue links per } \\
\text { event to form a continuous cluster } \\
0=\text { no clustering performed. If } \\
\text { IDAT }=3 \text {, the sum of OBSCC and } \\
\text { OBSCT is taken and used for both }\end{array}$ & 8 \\
\hline ISTART & $\begin{array}{l}\text { Initial locations: } 1 \text { =start from cluster } \\
\text { centroid; } 2=\text { start from catalogue } \\
\text { locations }\end{array}$ & 2 \\
\hline ISOLV & $\begin{array}{l}\text { Least squares solution: } 1=\mathrm{SVD} \\
2=\mathrm{LSQR}\end{array}$ & 1 \\
\hline NSET & $\begin{array}{l}\text { Number of sets of iterations with the } \\
\text { following specifications }\end{array}$ & 2 \\
\hline NITER & $\begin{array}{l}\text { Number of iterations for the set of } \\
\text { weighting parameters that follow }\end{array}$ & 5 \\
\hline WTCCP & Weight for cross-correlation $\mathrm{P}$ wave data & 1 \\
\hline WTCCS & Weight for cross-correlation $\mathrm{S}$ wave data & 0.5 \\
\hline WTCTP & Weight for catalogue $\mathrm{P}$ wave data & 0.1 \\
\hline WTCTS & $\begin{array}{l}\text { Weight for catalogue } S \text { wave data } \\
-9=\text { data not used }\end{array}$ & -9 \\
\hline
\end{tabular}


Table E.3 - continued from previous page

\begin{tabular}{|c|c|c|}
\hline Parameter & Description & Typical Value \\
\hline WRCC & $\begin{array}{l}\text { Cut-off threshold for outliers located on } \\
\text { the tails of the cross-correlation data }\end{array}$ & (6 \\
\hline WRCT & $\begin{array}{l}\text { Cut-off threshold for outliers located on } \\
\text { the tails of the catalogue data } \\
0<1=\text { absolute threshold in seconds; } \\
\geq 1=\text { factor to multiply standard } \\
\text { deviation; }-9=\text { no outliers removed }\end{array}$ & 4 \\
\hline WDCC & $\begin{array}{l}\text { Maximum event separation distance (in } \\
\mathrm{km} \text { ) for cross-correlation data }\end{array}$ & 4 \\
\hline WDCT & $\begin{array}{l}\text { Maximum event separation distance (in } \\
\mathrm{km} \text { ) for catalogue data } \\
-9=\text { not activated }\end{array}$ & 10 \\
\hline DAMP & Damping (only for $\mathbf{I S O L V}=2$ ) & -9 \\
\hline NLAY & Number of layers in velocity model & 6 \\
\hline RATIO & $V_{P} / V_{S}$ ratio, constant for all layers & 1.73 \\
\hline TOP & $\begin{array}{l}\text { Depths to top of layers (in km), space } \\
\text { separated }\end{array}$ & $0.0 \quad 1.0$ \\
\hline VEL & $\begin{array}{l}\text { Layer velocities (in } \mathrm{km} / \mathrm{s} \text { ), space } \\
\text { separated }\end{array}$ & $3.7 \quad 4.6$ \\
\hline CID & Index of cluster to be relocated $(0=$ all $)$ & 0 \\
\hline ID & $\begin{array}{l}\text { ID of events to be relocated ( } 8 \text { per line), } \\
\text { blank for all events }\end{array}$ & \\
\hline
\end{tabular}




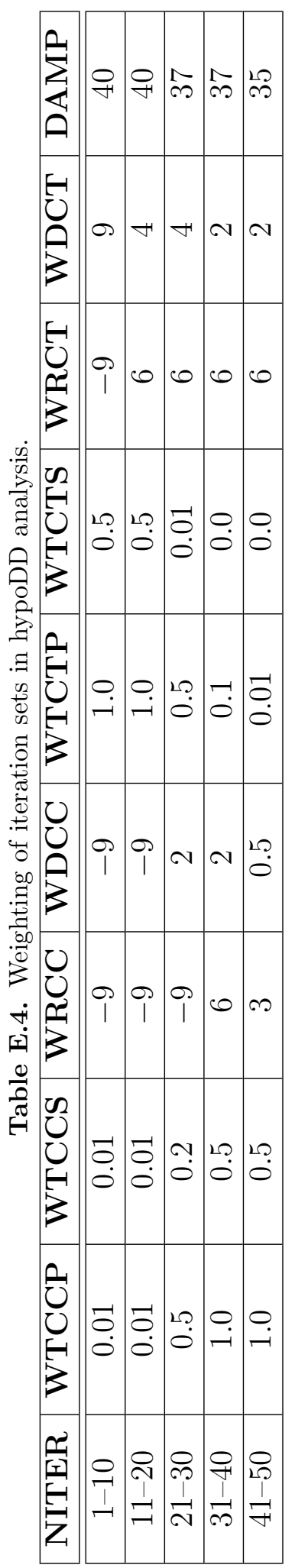


Table E.5. Filters tested during MFAST [after Savage et al., 2010b]

\begin{tabular}{|c|c|c|c|}
\hline Filter Number & Low Frequency $(\mathrm{Hz})$ & High Frequency $(\mathrm{Hz})$ & Bandwidth (Octave) \\
\hline \hline 1 & 0.4 & 4.0 & 5 \\
\hline 2 & 0.5 & 5.0 & 5 \\
\hline 3 & 0.2 & 3.0 & 7.5 \\
\hline 4 & 0.3 & 3.0 & 5 \\
\hline 5 & 0.5 & 4.0 & 2.5 \\
\hline 6 & 0.6 & 3.0 & 3.75 \\
\hline 7 & 0.8 & 6.0 & 1.5 \\
\hline 8 & 1.0 & 3.0 & 2.5 \\
\hline 9 & 1.0 & 5.0 & 4 \\
\hline 10 & 1.0 & 8.0 & 0.75 \\
\hline 11 & 2.0 & 3.0 & 1.5 \\
\hline 12 & 2.0 & 6.0 & 1.3 \\
\hline 13 & 3.0 & 8.0 & 1.25 \\
\hline 14 & 4.0 & 10.0 & \\
\hline
\end{tabular}

Table E.6. Quality criteria for grading during MFAST [after Savage et al., 2010b]

\begin{tabular}{|c|c|}
\hline Grade Name & Criterion \\
\hline$\overline{\mathrm{N}}$ (null) & $\begin{array}{l}\text { If } \phi \text { is between }-20^{\circ} \text { to } 20^{\circ} \text { or } 70^{\circ} \text { to } 110^{\circ} \text { of the } \\
\text { incoming polarisation }\end{array}$ \\
\hline Dcl (Cluster D grade) & $\begin{array}{l}\text { If there is any cluster } \mathrm{k} \text { for which the following holds: } \\
N(k)>N\left(k_{\text {best }}\right) / 2 \\
\operatorname{var}(k)<5 \times \operatorname{var}\left(k_{\text {best }}\right) \\
\delta \mathrm{t}_{\text {diff }}(k)>\delta \mathrm{t}_{\max } / 4 \text { or } \pi / 4<\phi_{\text {diff }}(k)<3 \pi / 4\end{array}$ \\
\hline Ccl (Cluster C grade) & $\begin{array}{l}\text { Not Dcl } \\
N(k)>N\left(k_{\text {best }}\right) / 2 \\
\operatorname{var}(k)<5 \times \operatorname{var}\left(k_{\text {best }}\right) \\
\delta \mathrm{t}_{\text {diff }}(k)>\delta \mathrm{t}_{\max } / 8 \text { or } \pi / 8<\phi_{\text {diff }}(k)<7 \pi / 8\end{array}$ \\
\hline Bcl (Cluster B grade) & $\begin{array}{l}\text { Not Ccl } \\
N(k)>N_{\min } \\
\operatorname{var}(k)<5 \times \operatorname{var}\left(k_{\text {best }}\right) \\
\delta \mathrm{t}_{\text {diff }}(k)>\delta \mathrm{t}_{\max } / 8 \text { or } \pi / 8<\phi_{\text {diff }}(k)<7 \pi / 8\end{array}$ \\
\hline Acl (Cluster A grade) & Not Bcl \\
\hline $\mathrm{AB}_{\mathrm{PAR}}$ & $\begin{array}{l}\text { Not } \mathrm{N} \\
\delta \mathrm{t}<0.8 \times \delta \mathrm{t}_{\max } \\
\mathrm{SNR}>3 \\
d \phi<25, \text { where } d \phi=\text { standard deviation of } \phi\end{array}$ \\
\hline & Continued on next page \\
\hline
\end{tabular}


Table E. 6 - continued from previous page

\begin{tabular}{|l|l|}
\hline Grade Name & Criterion \\
\hline \hline A $_{\text {PAR }}$ & $\delta \mathrm{t}<0.8 \times \delta \mathrm{t}_{\max }$ \\
& $\mathrm{SNR}>4$ \\
& $d \phi<10$ \\
\hline B & Bcl \\
& Not N \\
& $\delta \mathrm{t}<0.8 \times \delta \mathrm{t}_{\max }$ \\
& $\mathrm{SNR}>3$ \\
& $d \phi<25$ \\
\hline A & Acl \\
& $\delta \mathrm{t}<0.8 \times \delta \mathrm{t}_{\text {max }}$ \\
& $\mathrm{SNR}>4$ \\
& $d \phi<10$ \\
\hline AB & A or B \\
\hline Eng8 & Maximum value of contour energy plots is greater than \\
& 8 \\
\hline
\end{tabular}


Table E.7. Parameters used in Coulomb 3.1 modelling.

\begin{tabular}{|c|c|c|c|}
\hline Description & Parameter & Typical value & Units \\
\hline Poisson's ratio & $\nu$ or PR & 0.25 & \\
\hline Young's Modulous & $\mathrm{E}$ & $8 \times 10^{5}$ & bar \\
\hline Calculation Depth & Calc & 2 & $\mathrm{~km}$ \\
\hline Coefficient of Friction & $\mu$ or FRIC & 0.8 & \\
\hline \multicolumn{4}{|l|}{ Regional stress } \\
\hline Axis of maximum principle stress & S1 & & \\
\hline $\begin{array}{l}\text { Axis of intermediate principle } \\
\text { stress }\end{array}$ & S2 & & \\
\hline Axis of minimum principle stress & S3 & & \\
\hline Azimuth & DR & 035 & $\circ$ \\
\hline Plunge & $\overline{\mathrm{DP}}$ & 0 & $\overline{0}$ \\
\hline Surface stress & IN & 0 & bar \\
\hline Vertical gradient & GD & 225.4 & $\mathrm{bar} / \mathrm{km}$ \\
\hline Total number of sources & \# fixed & 1 & \\
\hline $\begin{array}{l}\text { Type of source ( } 200 \text { for a dyke, } \\
500 \text { for a Mogi-point source) }\end{array}$ & Kode & 200 & \\
\hline Right lateral slip & rt.lat or tensile & 0 & $\mathrm{~m}$ \\
\hline Opening or closing & $\begin{array}{l}\text { reverse or } \\
\text { tensile or inflate }\end{array}$ & 1 or $5 \times 10^{7}$ & $\mathrm{~m}$ or $\mathrm{m}^{3}$ \\
\hline Dip of source & dip & 90 & $\circ$ \\
\hline Depth to top of source & Top & 0.5 & $\mathrm{~km}$ \\
\hline Depth to bottom of source & Bot & 6 & $\mathrm{~km}$ \\
\hline $\begin{array}{l}\text { West limit of source with respect } \\
\text { to the centre }\end{array}$ & X-start & -0.1 & $\mathrm{~km}$ \\
\hline $\begin{array}{l}\text { South limit of source with } \\
\text { respect to the centre }\end{array}$ & Y-start & -0.1 & $\mathrm{~km}$ \\
\hline $\begin{array}{l}\text { East limit of source with respect } \\
\text { to the centre }\end{array}$ & X-fin & 2.1 & $\mathrm{~km}$ \\
\hline $\begin{array}{l}\text { North Limit of source with } \\
\text { respect to the centre }\end{array}$ & Y-fin & 4.1 & $\mathrm{~km}$ \\
\hline $\begin{array}{l}\text { increment of grid for calculation } \\
\text { in the } \mathrm{x} \text { direction }\end{array}$ & $\mathrm{x}$-increment & 1 & $\mathrm{~km}$ \\
\hline $\begin{array}{l}\text { increment of grid for calculation } \\
\text { in the } \mathrm{y} \text { direction }\end{array}$ & y-increment & 1 & $\mathrm{~km}$ \\
\hline
\end{tabular}


F. Appendix: Earthquake multiplets 


\begin{tabular}{|c|c|c|c|}
\hline & 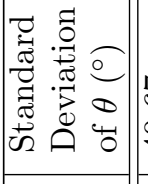 & 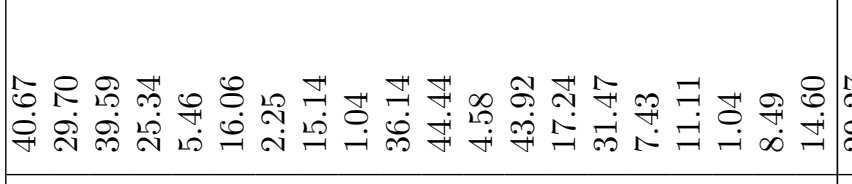 & 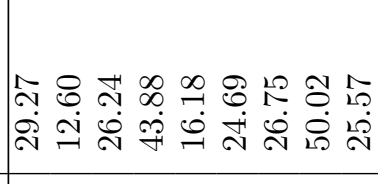 \\
\hline & 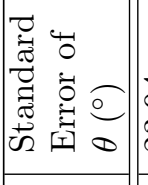 & 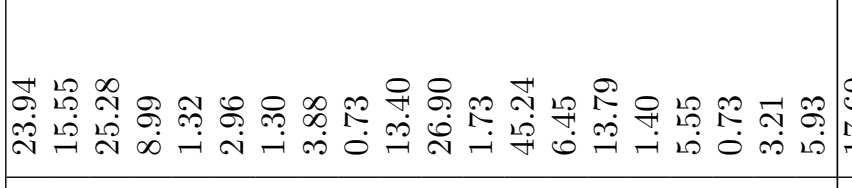 & 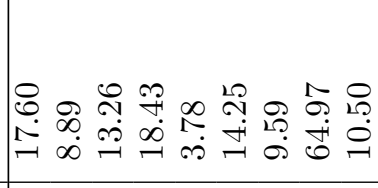 \\
\hline & 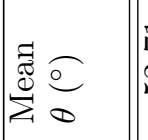 & 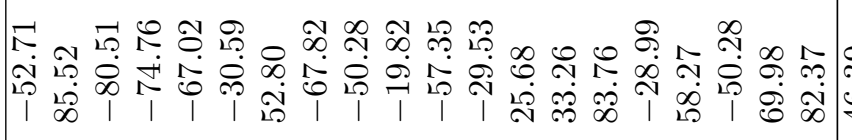 & 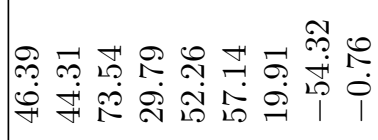 \\
\hline & 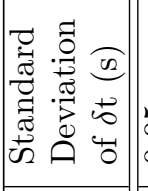 & 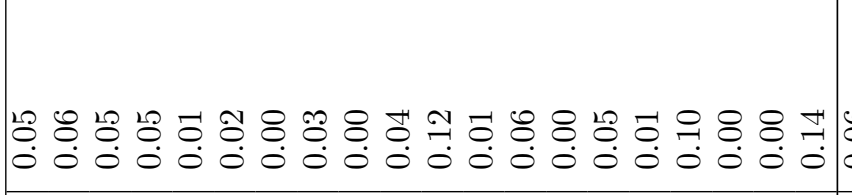 & 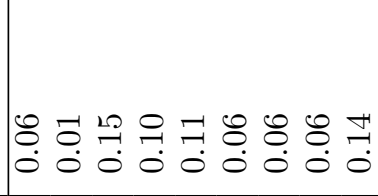 \\
\hline & 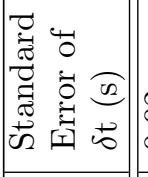 & 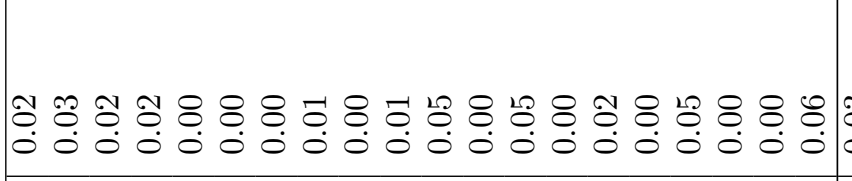 & 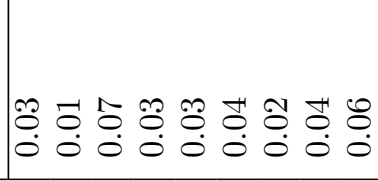 \\
\hline & 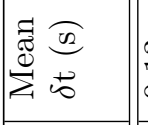 & 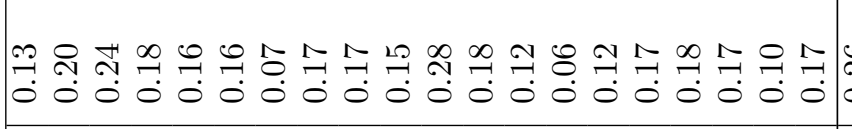 & 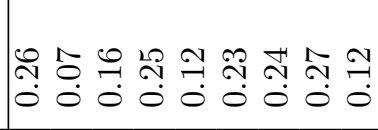 \\
\hline & 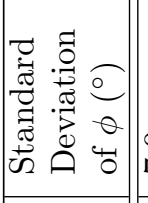 & 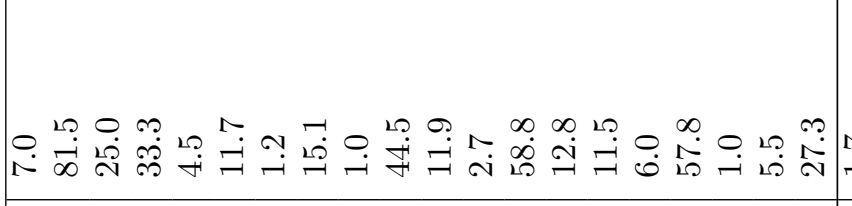 & 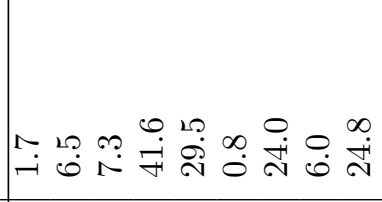 \\
\hline & 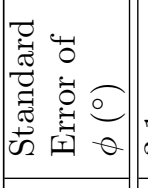 & 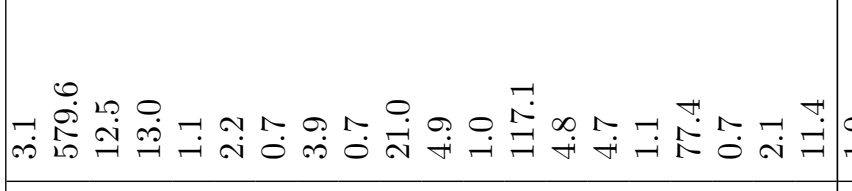 & 茾 \\
\hline & 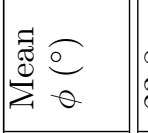 & 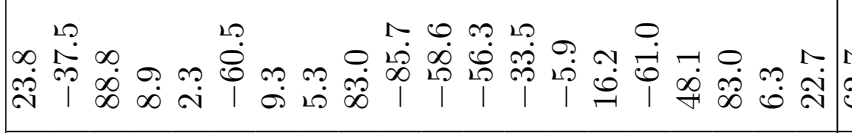 & 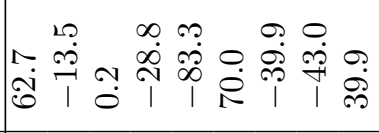 \\
\hline & 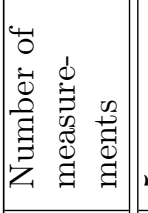 & 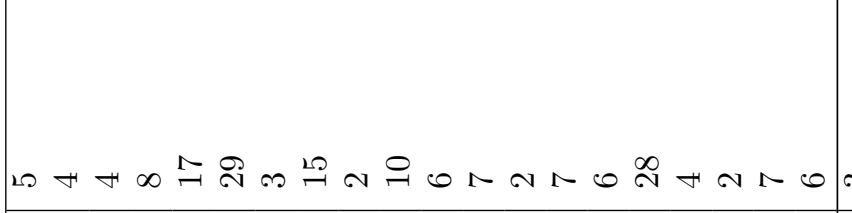 & $\infty \sim \nsucc \stackrel{\infty}{\sim} \infty \infty \sim 0$ \\
\hline & 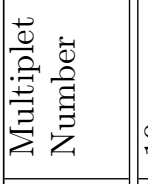 & 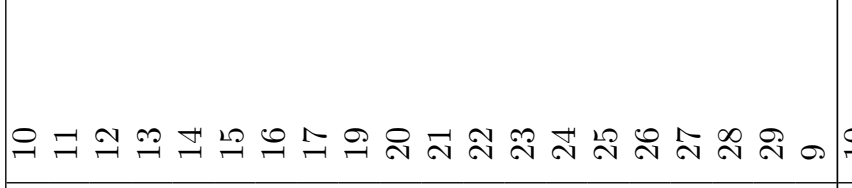 & 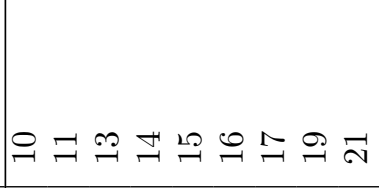 \\
\hline &. & 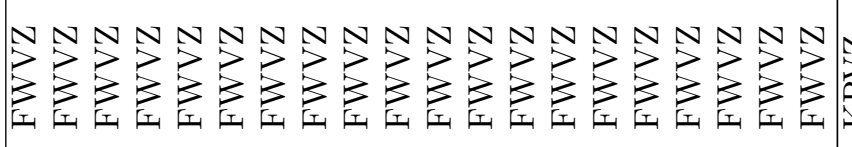 & 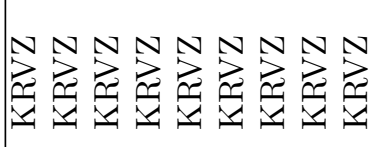 \\
\hline
\end{tabular}




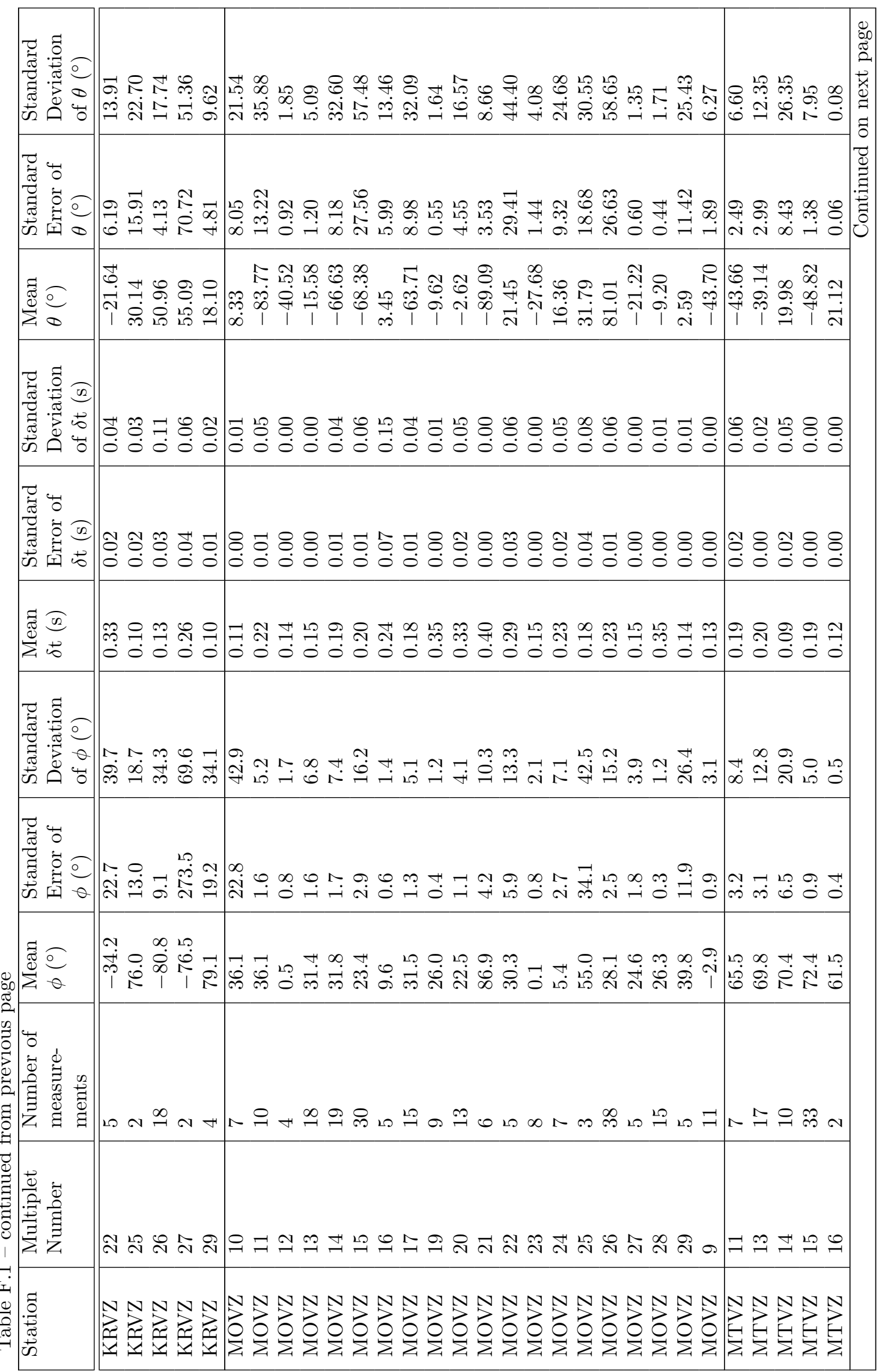




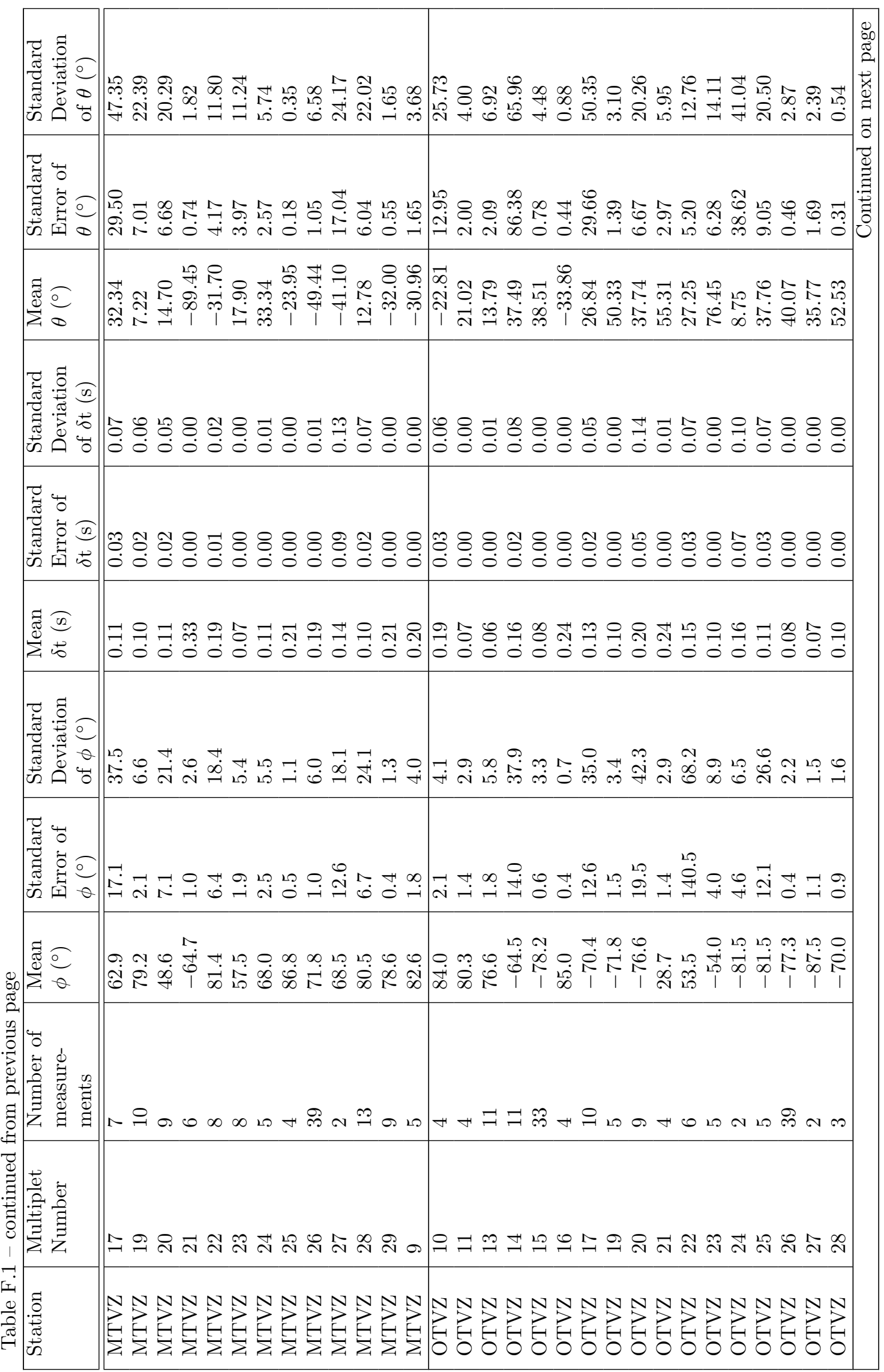




\begin{tabular}{|c|c|c|c|}
\hline 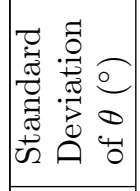 & 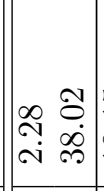 & 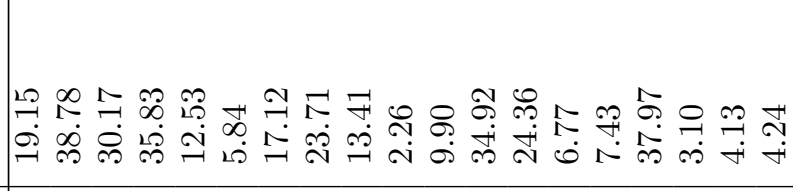 & 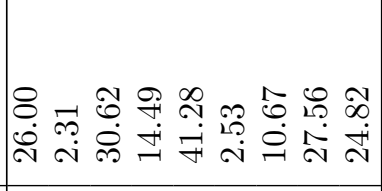 \\
\hline 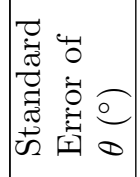 & 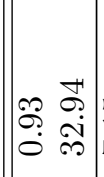 & 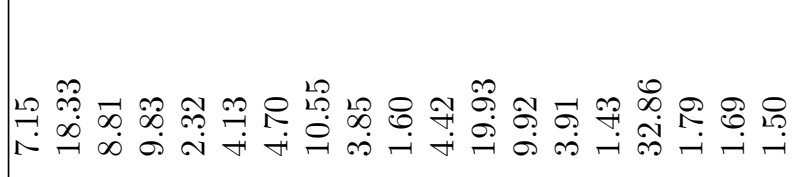 & 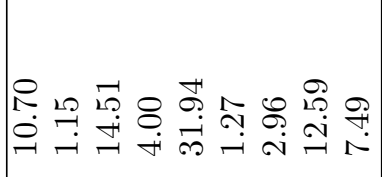 \\
\hline 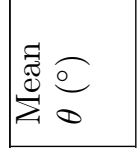 & $\mid$\begin{tabular}{ll|}
$\infty$ & 0 \\
$\infty$ & 0 \\
$\infty$ & 0 \\
$\infty$ & 0 \\
\end{tabular} & 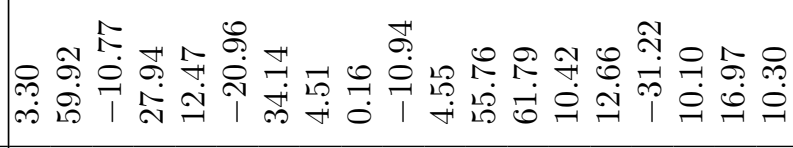 & 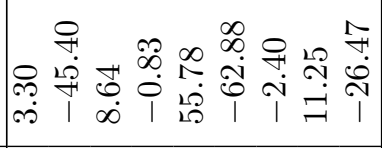 \\
\hline 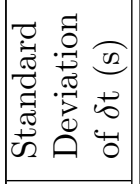 & $\left|\begin{array}{ll|} & \\
0 & \infty \\
\hdashline & \infty \\
0 & 0 \\
0\end{array}\right|$ & 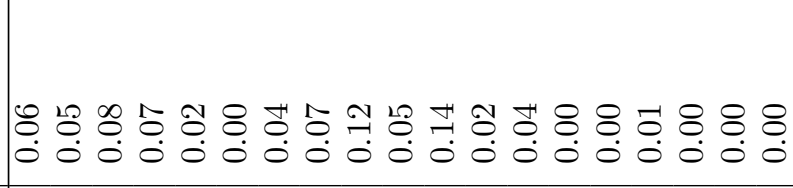 & 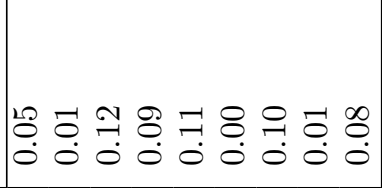 \\
\hline 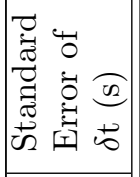 & $\mid$\begin{tabular}{ll|} 
& \\
0 & 8 \\
0 & 8 \\
0 & 0 \\
\end{tabular} & 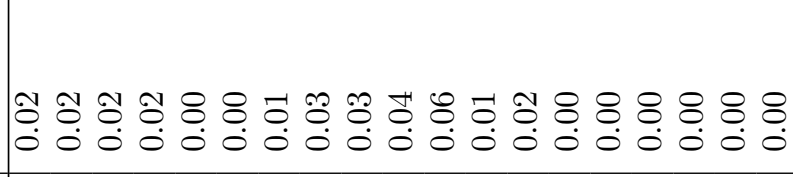 & 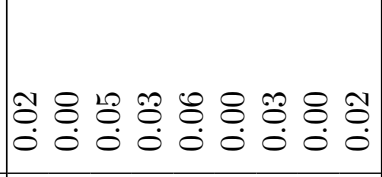 \\
\hline 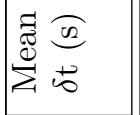 & $\mid \begin{array}{lll}1 & 12 \\
0 & : \\
0 & 0 \\
0\end{array}$ & 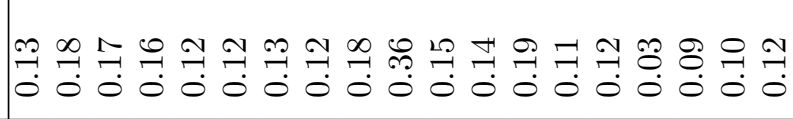 & 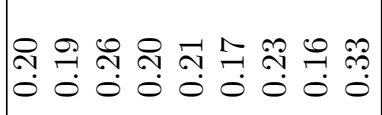 \\
\hline 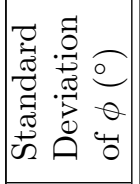 & $\mid$\begin{tabular}{ll|}
$\infty$ & 0 \\
$\infty$ & $\stackrel{\dot{\varphi}}{-}$
\end{tabular} & 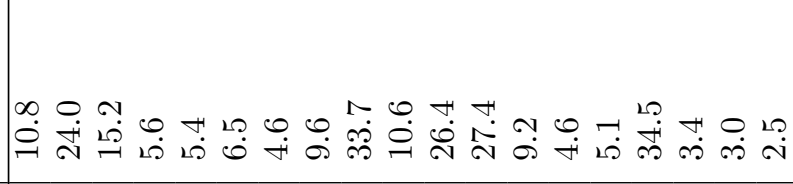 & 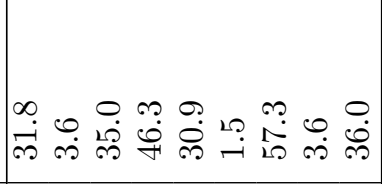 \\
\hline 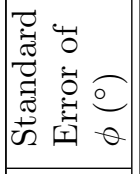 & $\mid$ & Fُ & 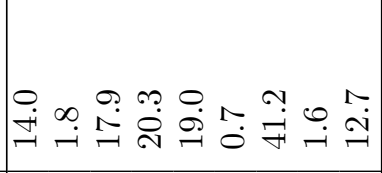 \\
\hline 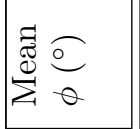 & 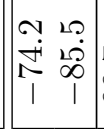 & 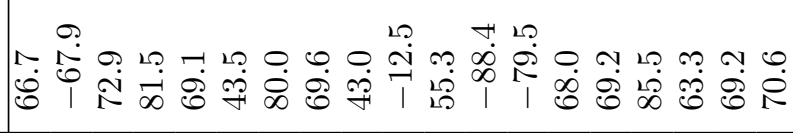 & 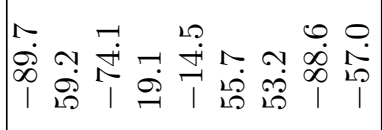 \\
\hline 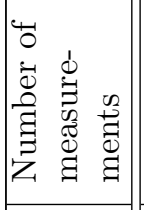 & $\mid$ & 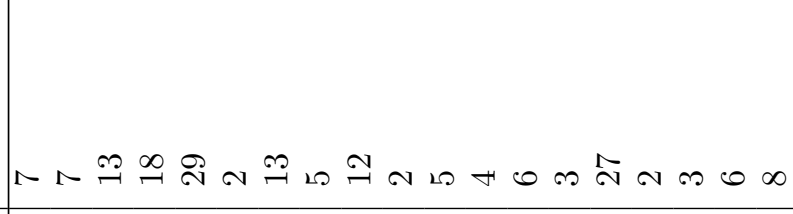 & 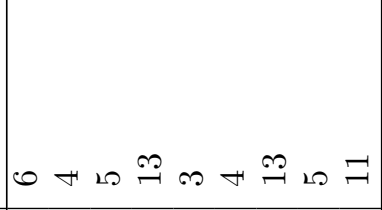 \\
\hline 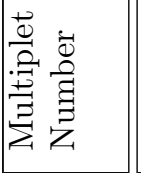 & Pr & 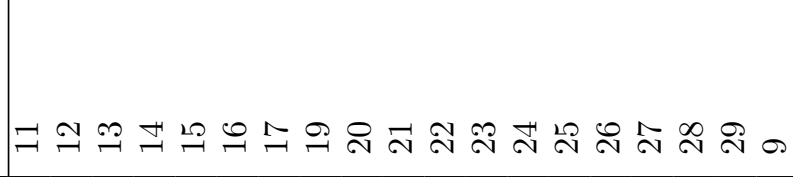 & 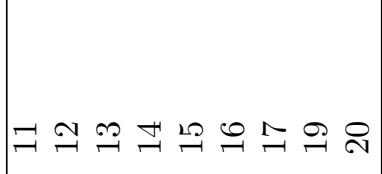 \\
\hline 营 & $\begin{array}{ll}N & N \\
2 & 2 \\
-1 & 0 \\
0 & 0\end{array}$ & 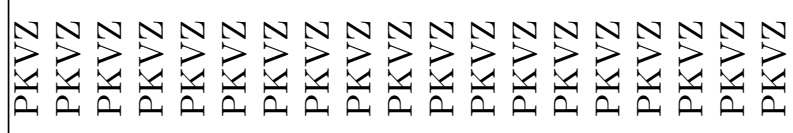 & 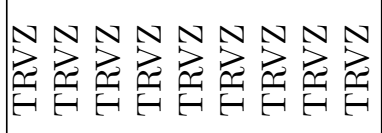 \\
\hline
\end{tabular}




\begin{tabular}{|c|c|c|c|}
\hline 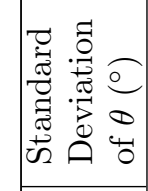 & 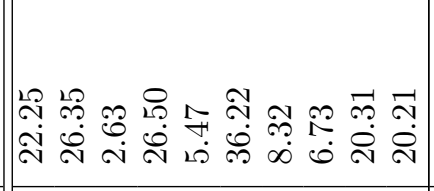 & 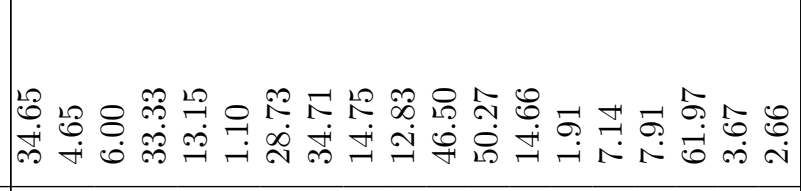 & 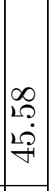 \\
\hline 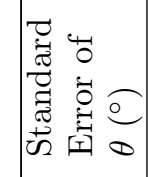 & 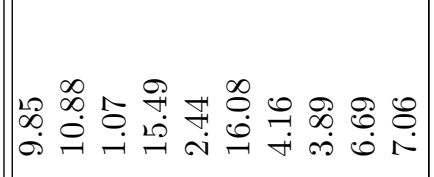 & 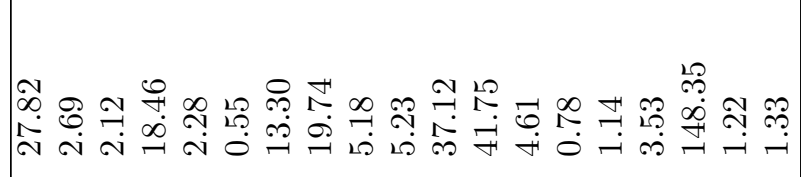 & : \\
\hline 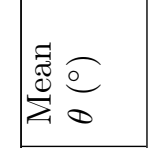 & 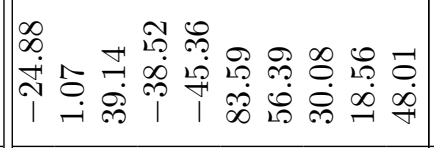 & 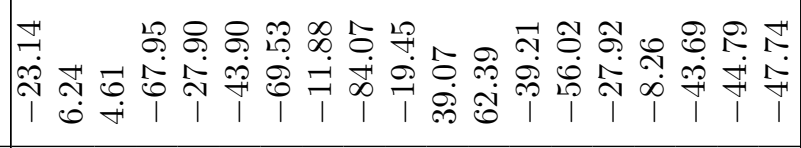 & $\begin{array}{c}2 \\
9 \\
2 \infty \\
20 \\
1 \\
1 \\
\end{array}$ \\
\hline 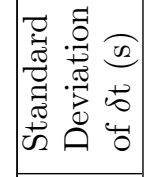 & 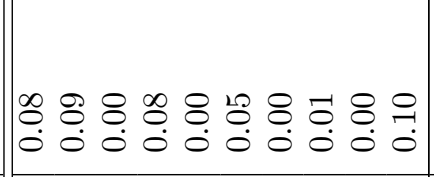 & 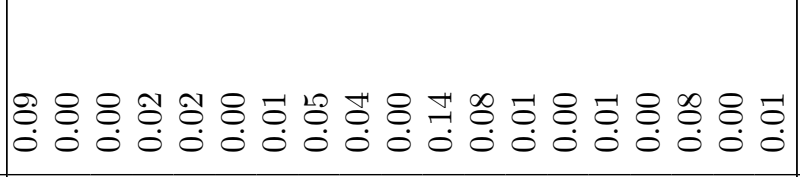 & 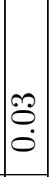 \\
\hline 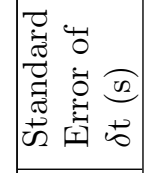 & 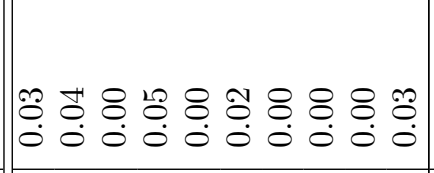 & 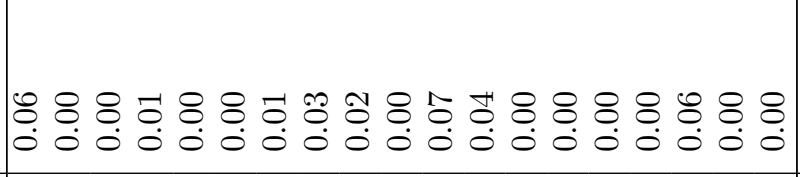 & $\begin{array}{l}0 \\
0 \\
0\end{array}$ \\
\hline 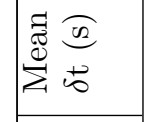 & 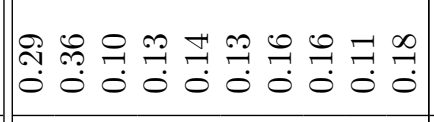 & 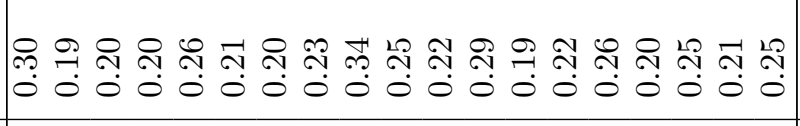 & $\begin{array}{l}0 \\
0 \\
0 \\
\end{array}$ \\
\hline 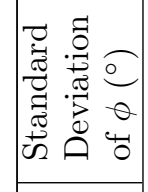 & 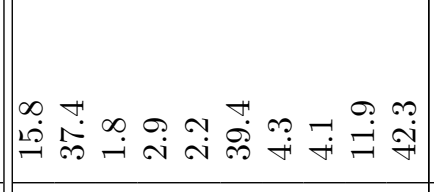 & 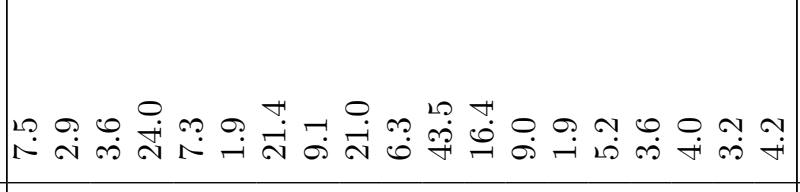 & $\stackrel{L a}{\sim}$ \\
\hline 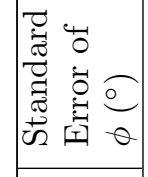 & 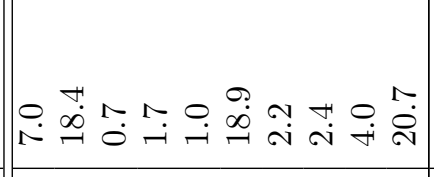 & 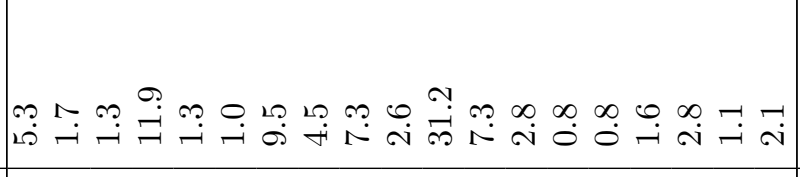 & 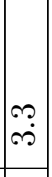 \\
\hline 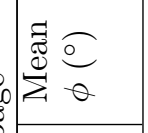 & 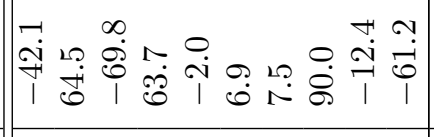 & 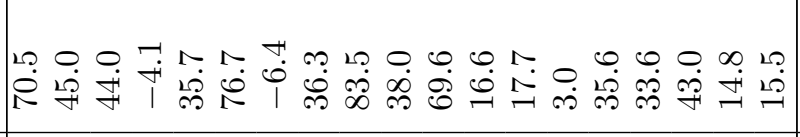 & 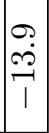 \\
\hline 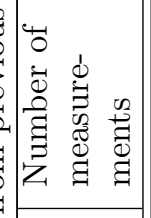 & 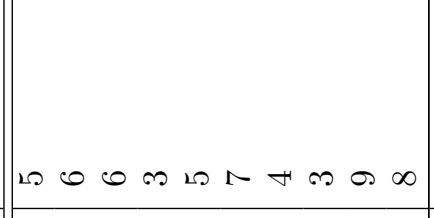 & 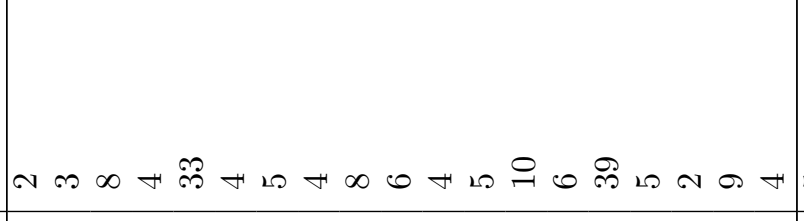 & 20 \\
\hline 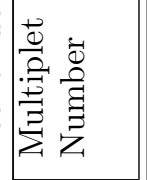 & 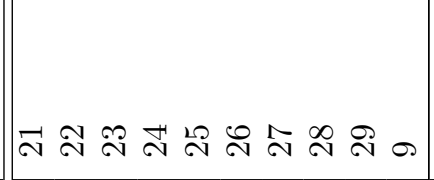 & 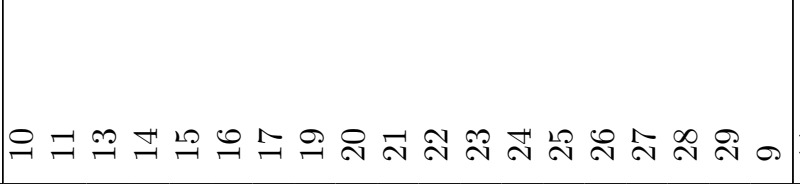 & $\exists$ \\
\hline 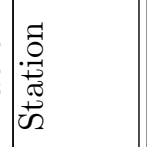 & 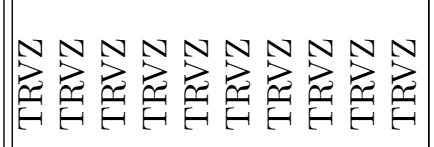 & $\begin{array}{l}N \\
N \\
3\end{array}$ & \\
\hline
\end{tabular}




\begin{tabular}{|c|c|c|}
\hline 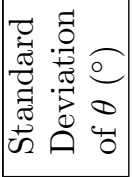 & 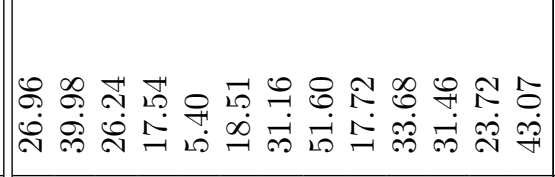 & 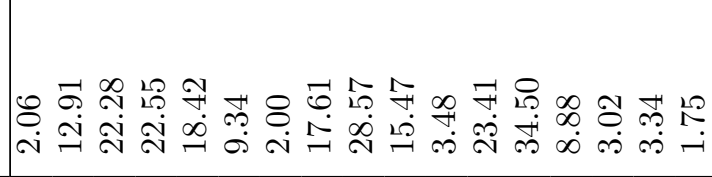 \\
\hline 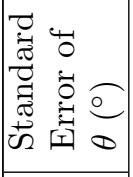 & 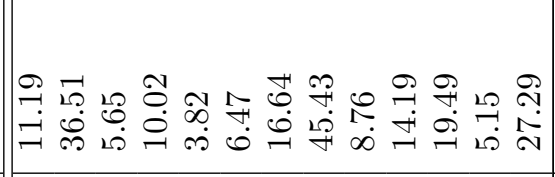 & 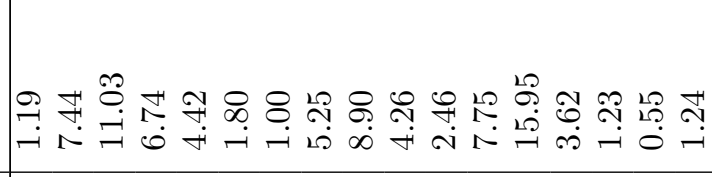 \\
\hline 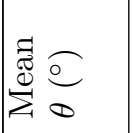 & 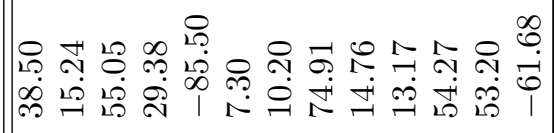 & 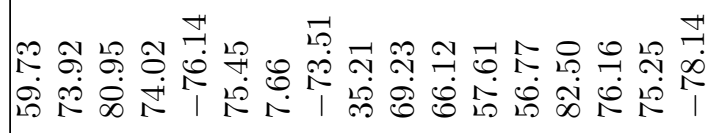 \\
\hline 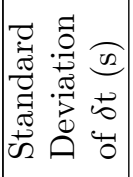 & 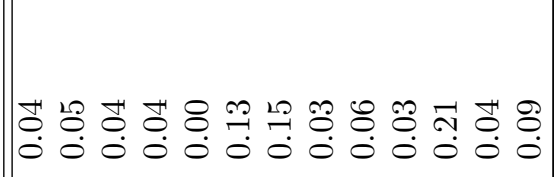 & 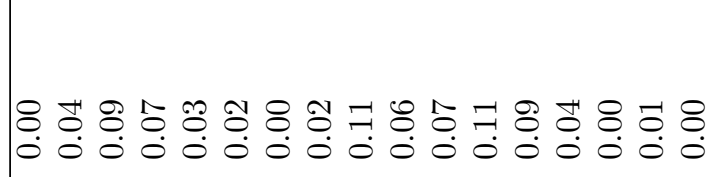 \\
\hline 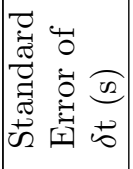 & 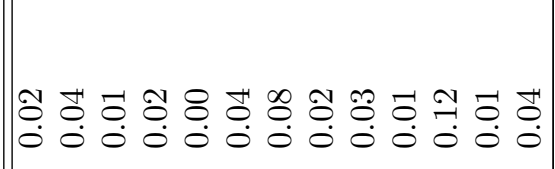 & 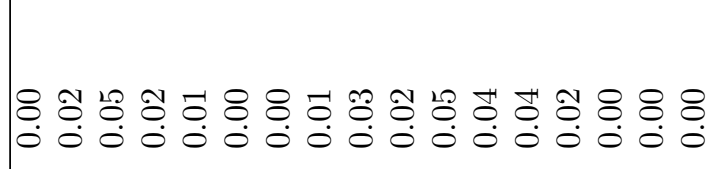 \\
\hline 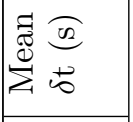 & 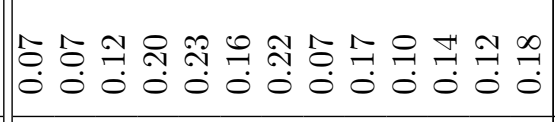 & 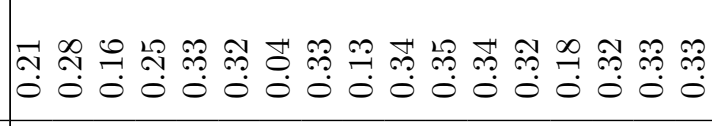 \\
\hline 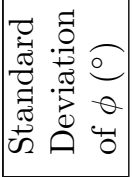 & 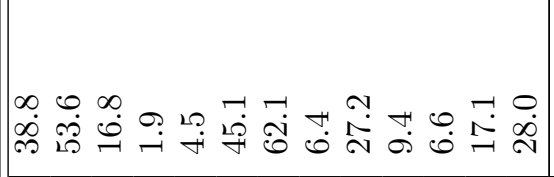 & 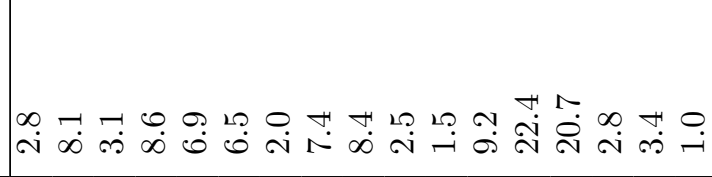 \\
\hline 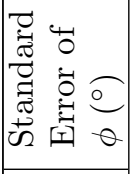 & 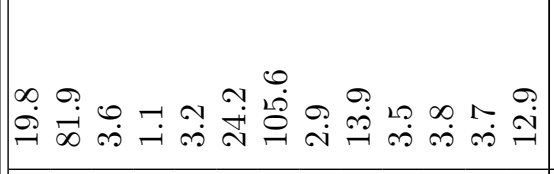 & 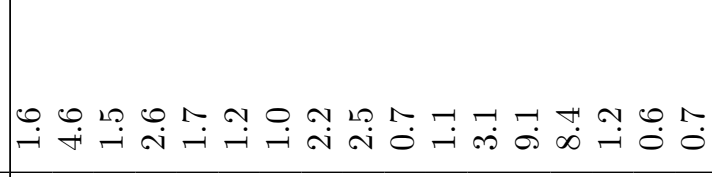 \\
\hline 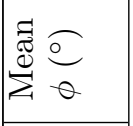 & 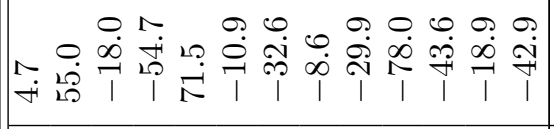 & 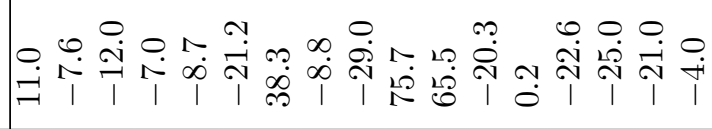 \\
\hline 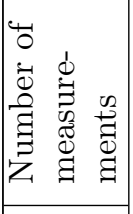 & 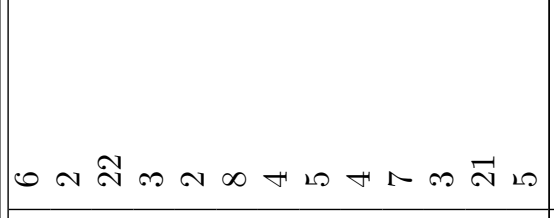 & 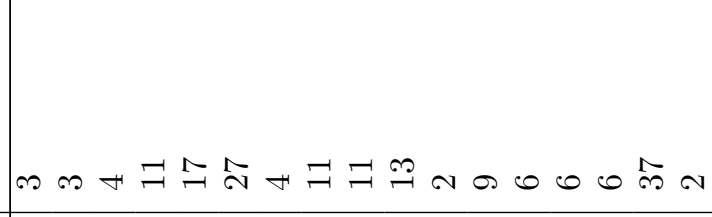 \\
\hline 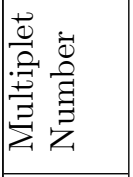 & 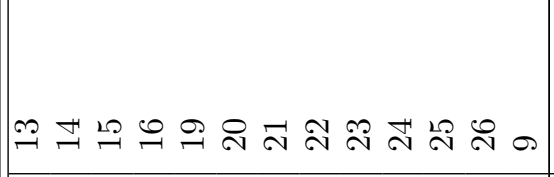 & 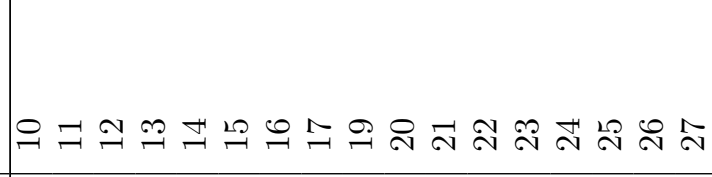 \\
\hline 总 & 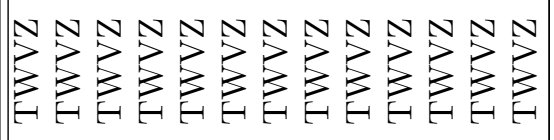 & 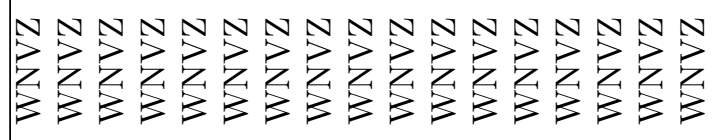 \\
\hline
\end{tabular}




\begin{tabular}{|c|c|c|}
\hline 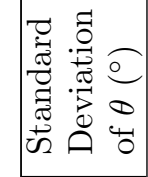 & 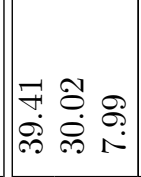 & 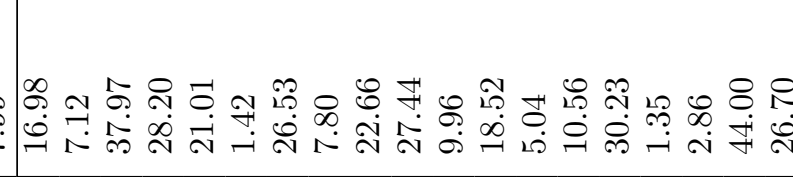 \\
\hline 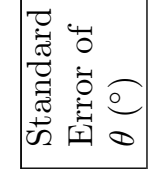 & 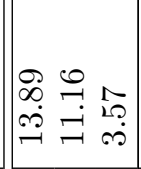 & 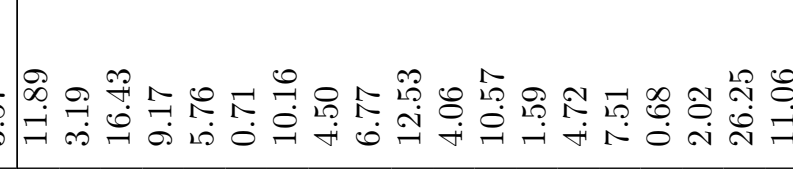 \\
\hline 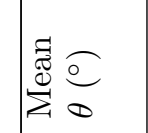 & 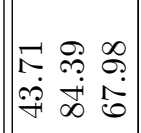 & 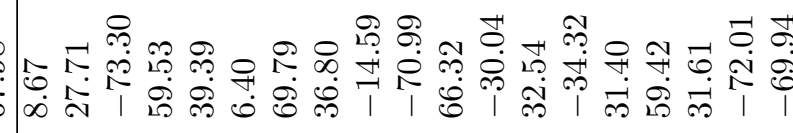 \\
\hline 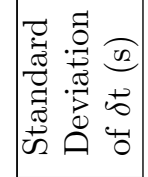 & 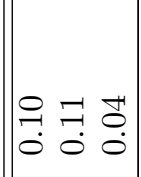 & 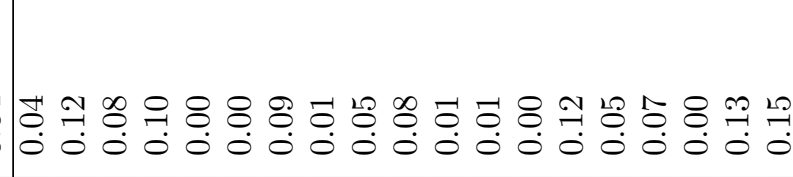 \\
\hline 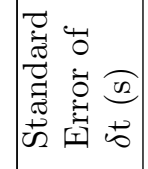 & 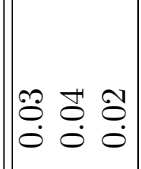 & 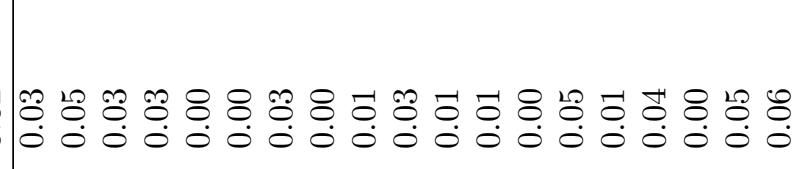 \\
\hline 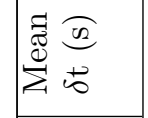 & 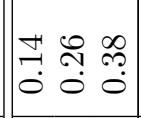 & 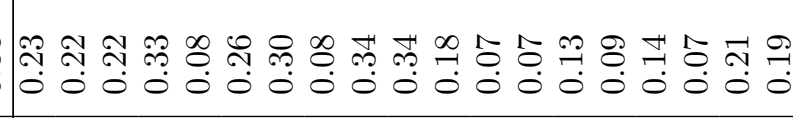 \\
\hline 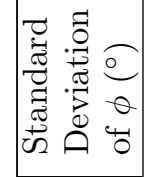 & 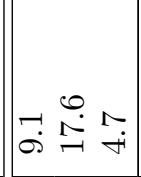 & 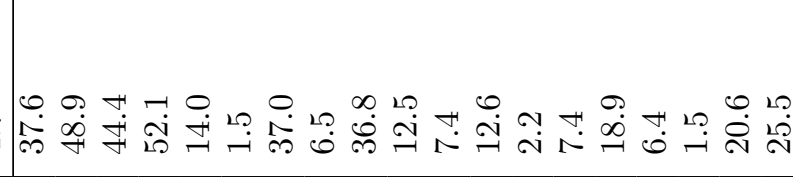 \\
\hline 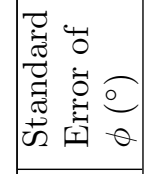 & 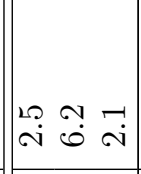 & 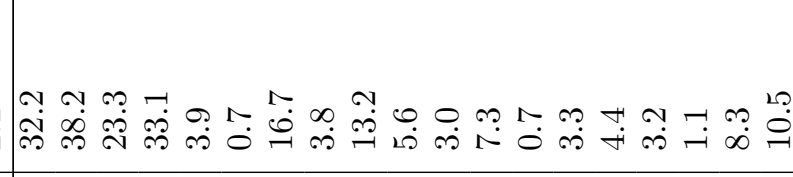 \\
\hline 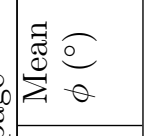 & 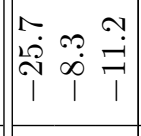 & 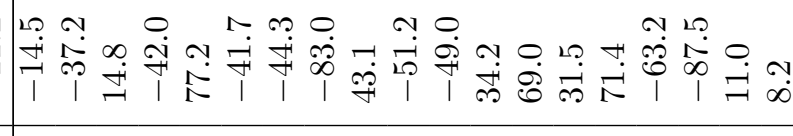 \\
\hline 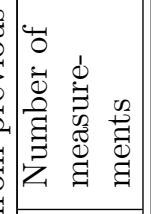 & 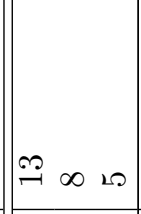 & 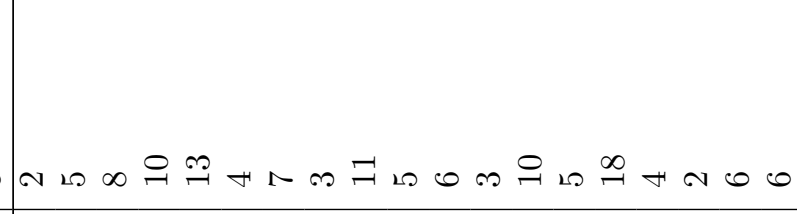 \\
\hline 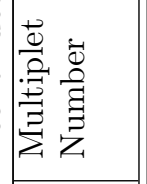 & 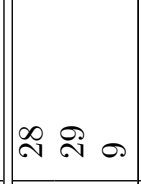 & 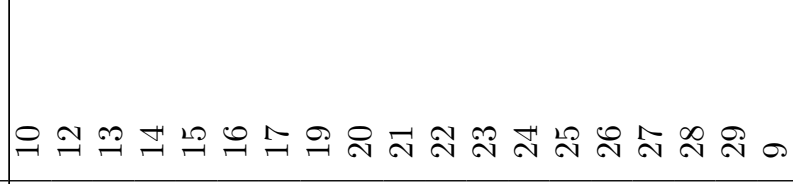 \\
\hline . & 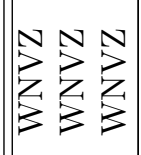 & 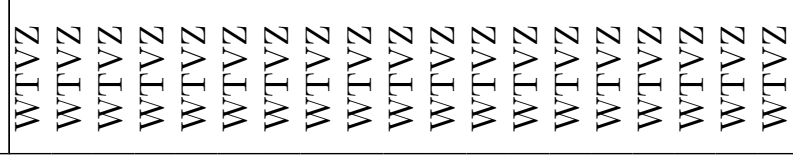 \\
\hline
\end{tabular}




\section{Multiplet 1}
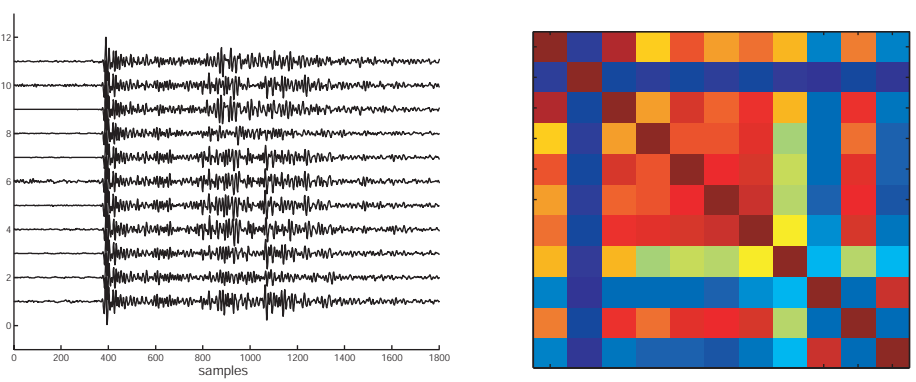

\section{Multiplet 2}
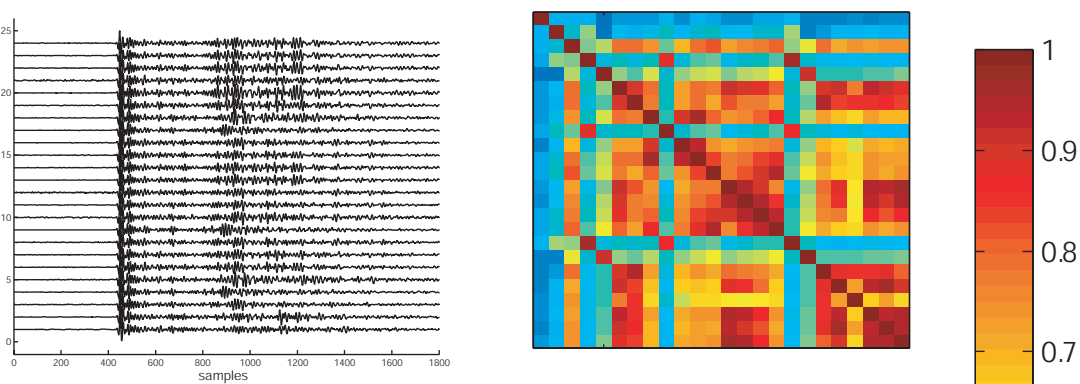

Multiplet 3
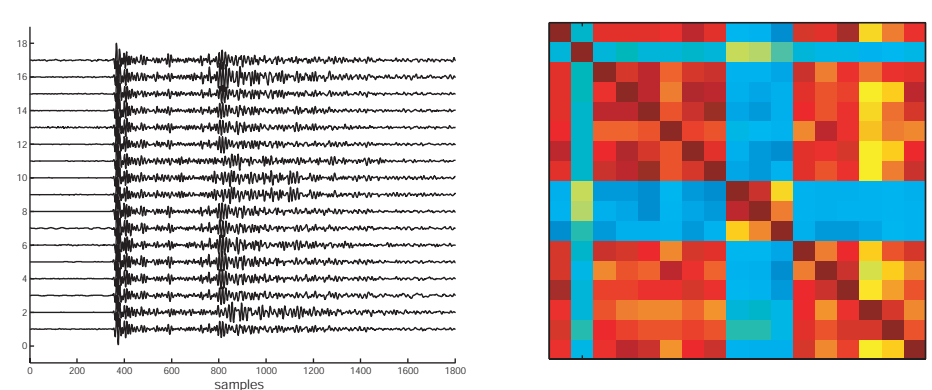

\section{Multiplet 4}
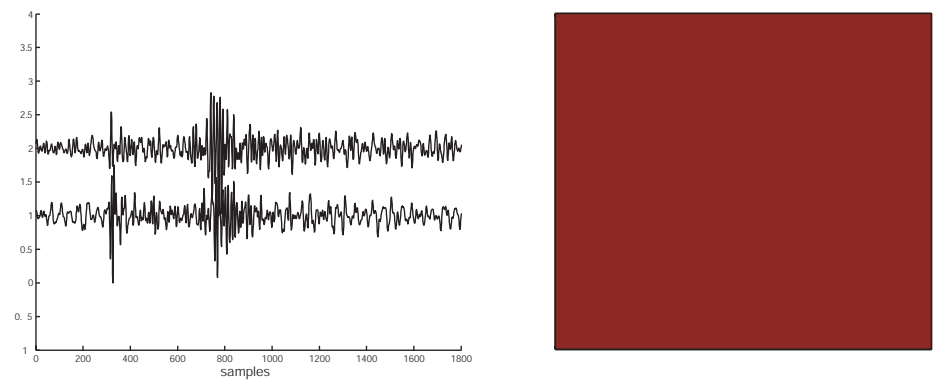

\section{Multiplet 5}
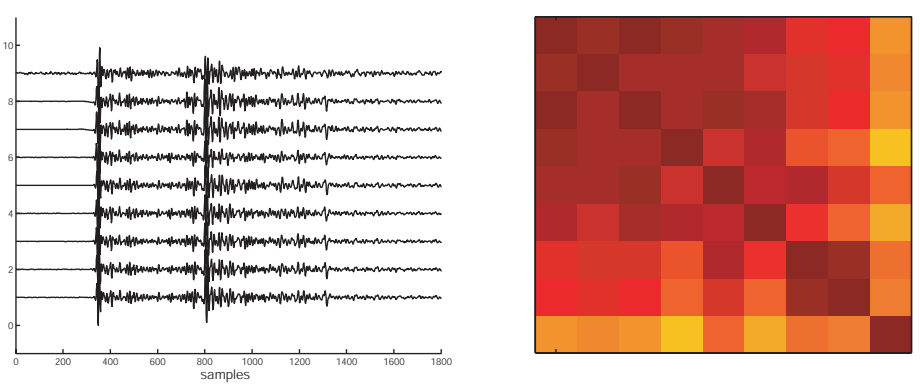

Figure F.1. Waveforms of earthquakes belonging the multiplets, recorded at station TUVZ and filtered 1-10 Hz, and cross-correlation matrices for the multiplets using the whole waveform. Colours represent cross-correlation coefficient. 
Multiplet 6

Multiplet 7

Multiplet 8

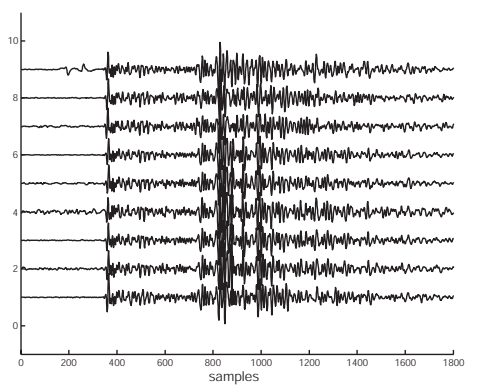

Multiplet 9
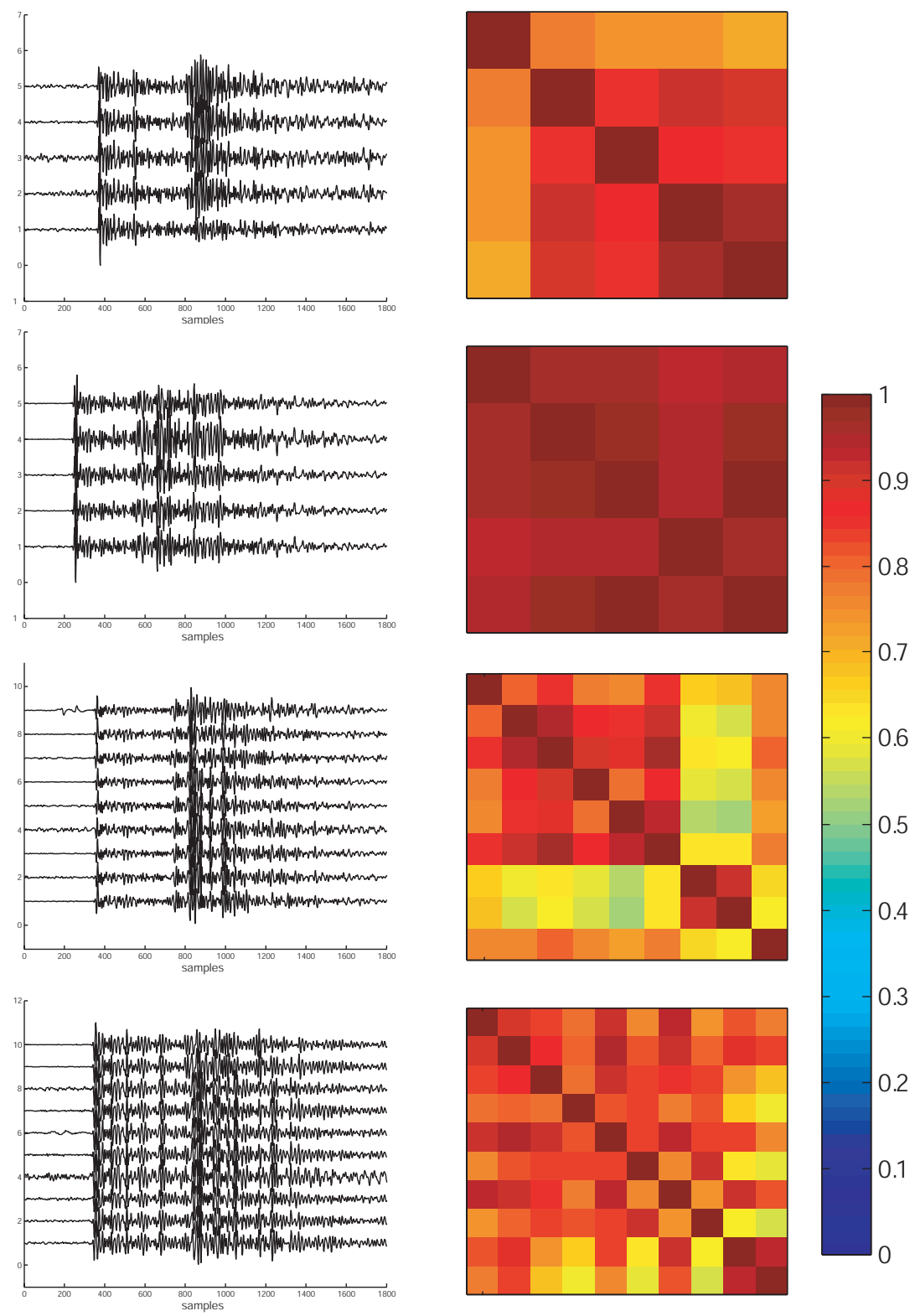

$-0.6$
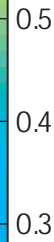

0.2

0.1

\section{Multiplet 10}
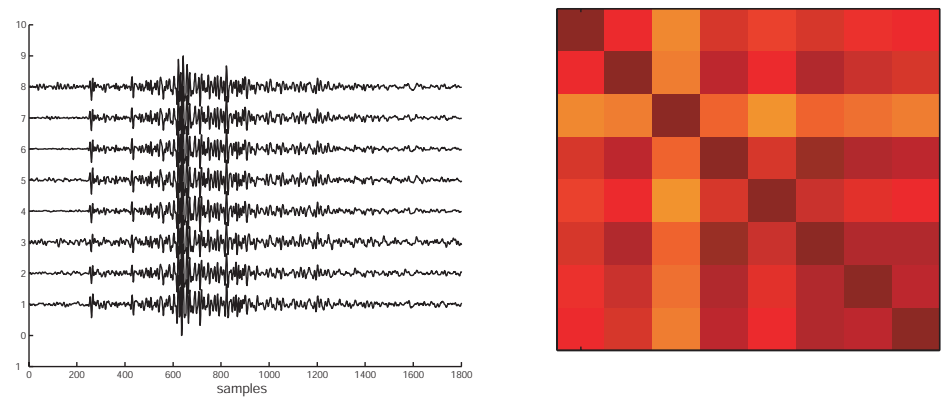

Figure F.2. Waveforms of earthquakes belonging the multiplets, recorded at station TUVZ and filtered 1-10 Hz, and cross-correlation matrices for the multiplets using the whole waveform. Colours represent cross-correlation coefficient. 
Multiplet 11

\section{Multiplet 12}
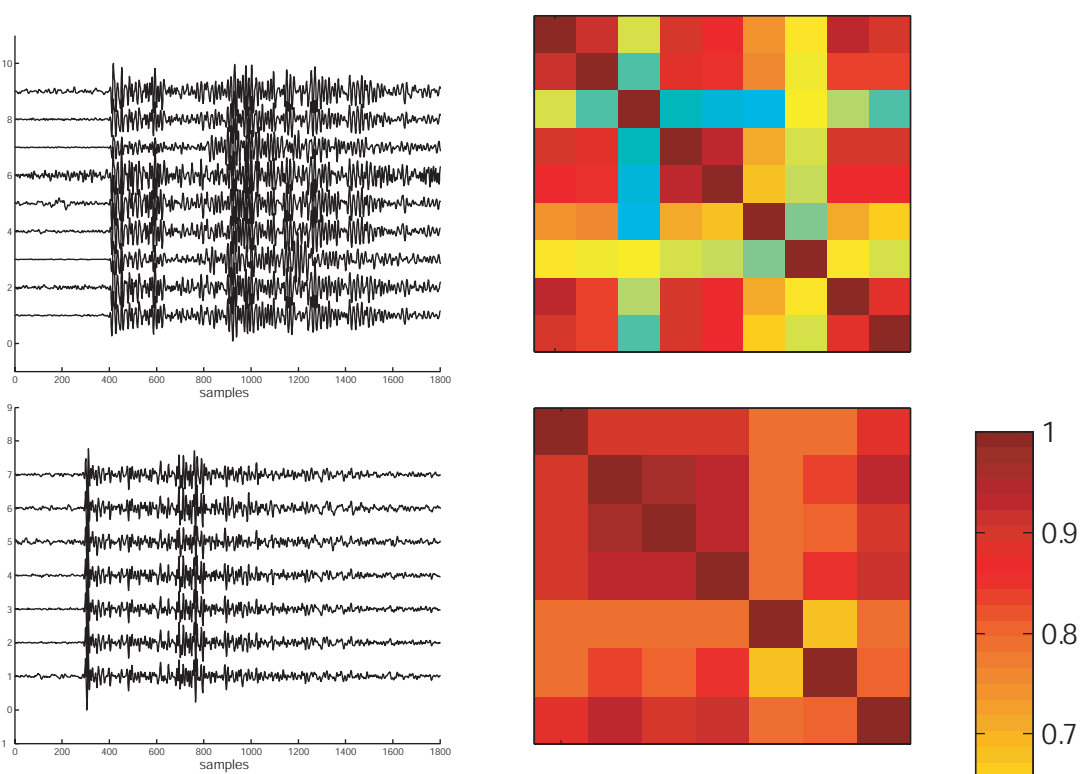

Multiplet 13
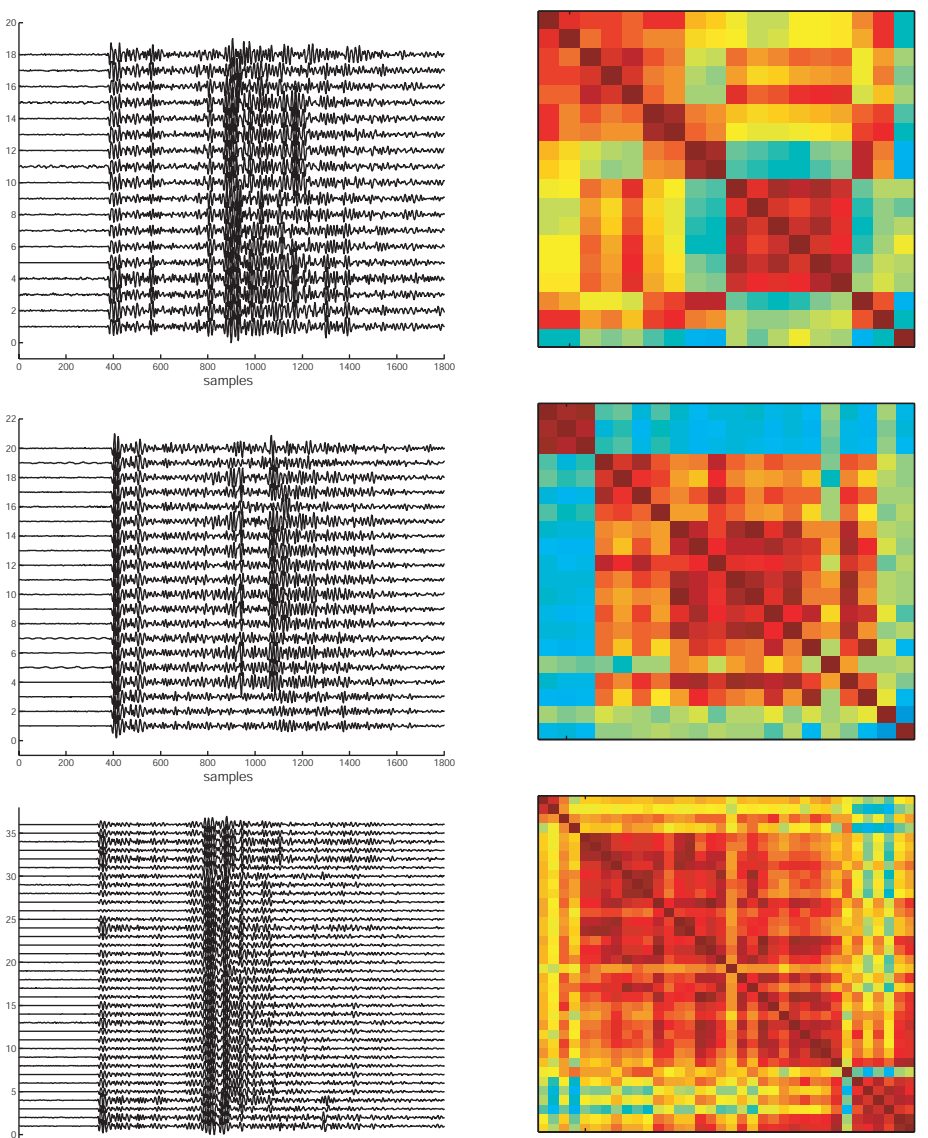

Multiplet 15
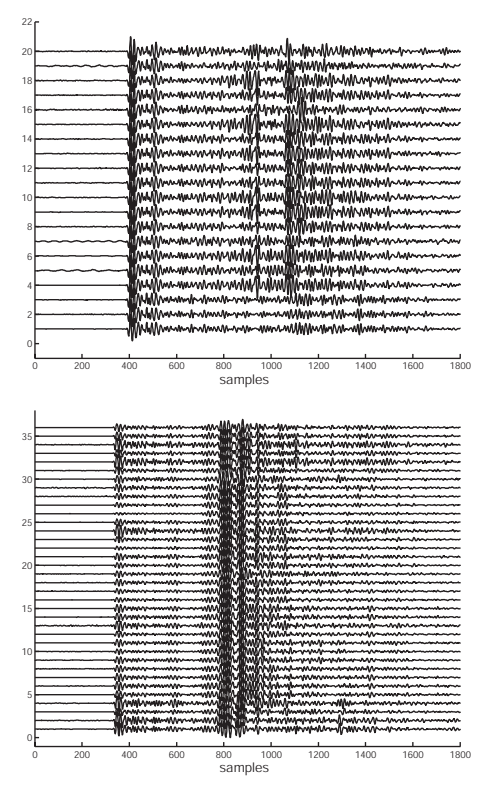

Multiplet 14

Figure F.3. Waveforms of earthquakes belonging the multiplets, recorded at station TUVZ and filtered 1-10 Hz, and cross-correlation matrices for the multiplets using the whole waveform. Colours represent cross-correlation coefficient. 
Multiplet 16
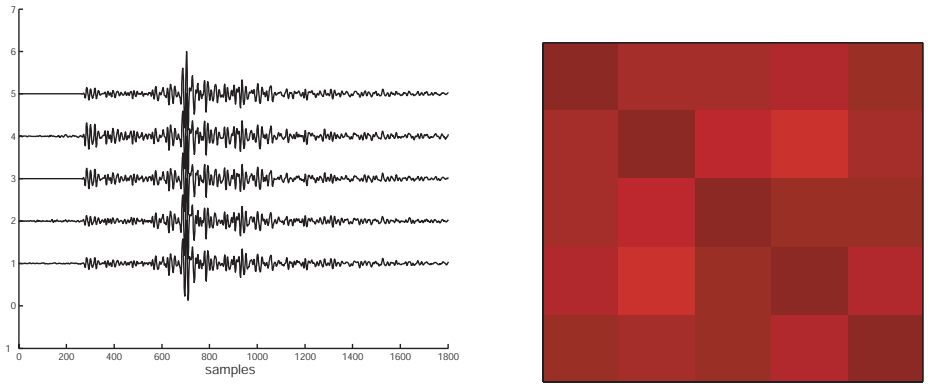

\section{Multiplet 17}

\section{Multiplet 18}
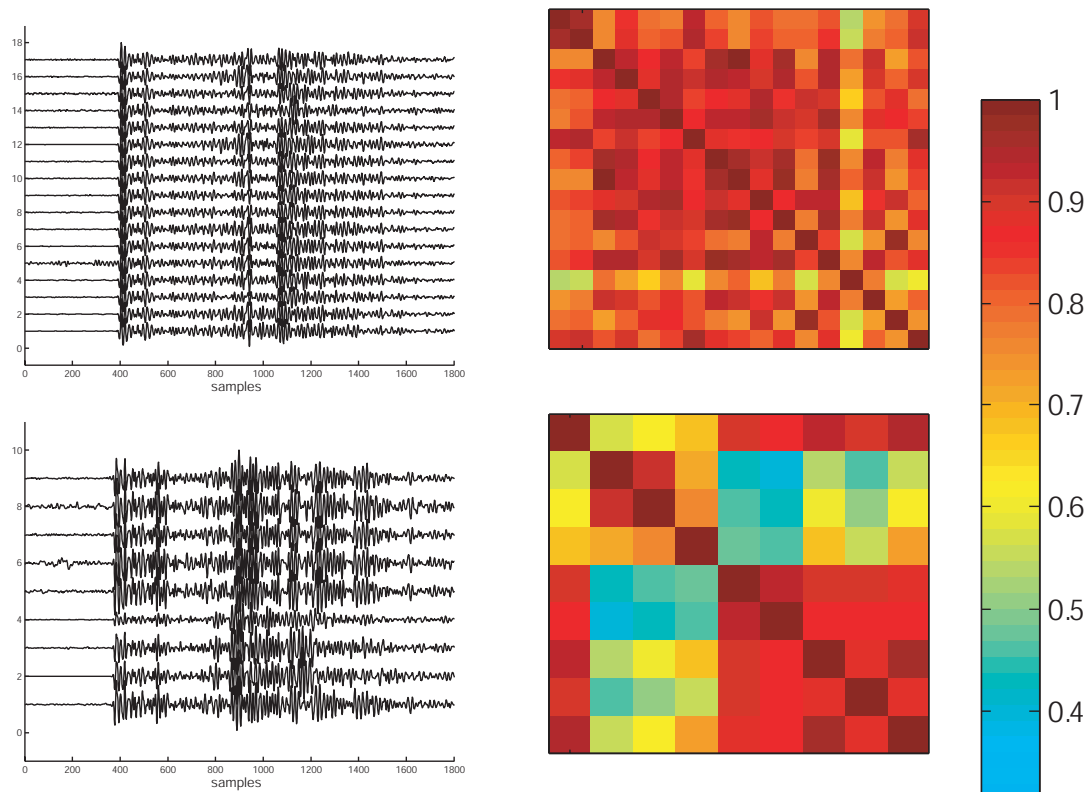

\section{Multiplet 19}
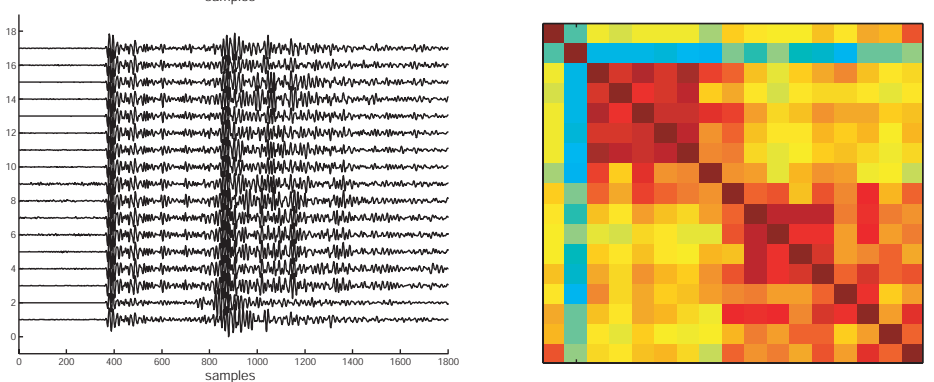

\section{Multiplet 20}
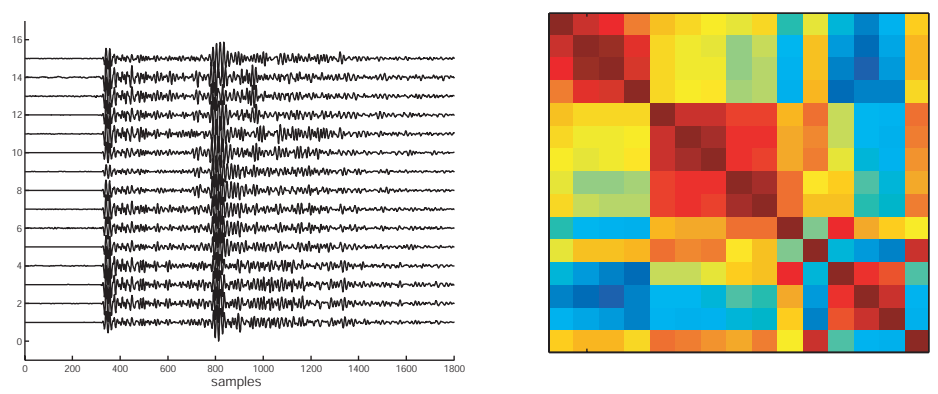

Figure F.4. Waveforms of earthquakes belonging the multiplets, recorded at station TUVZ and filtered 1-10 Hz, and cross-correlation matrices for the multiplets using the whole waveform. Colours represent cross-correlation coefficient. 
Multiplet 21

Multiplet 22

Multiplet 23

Multiplet 24
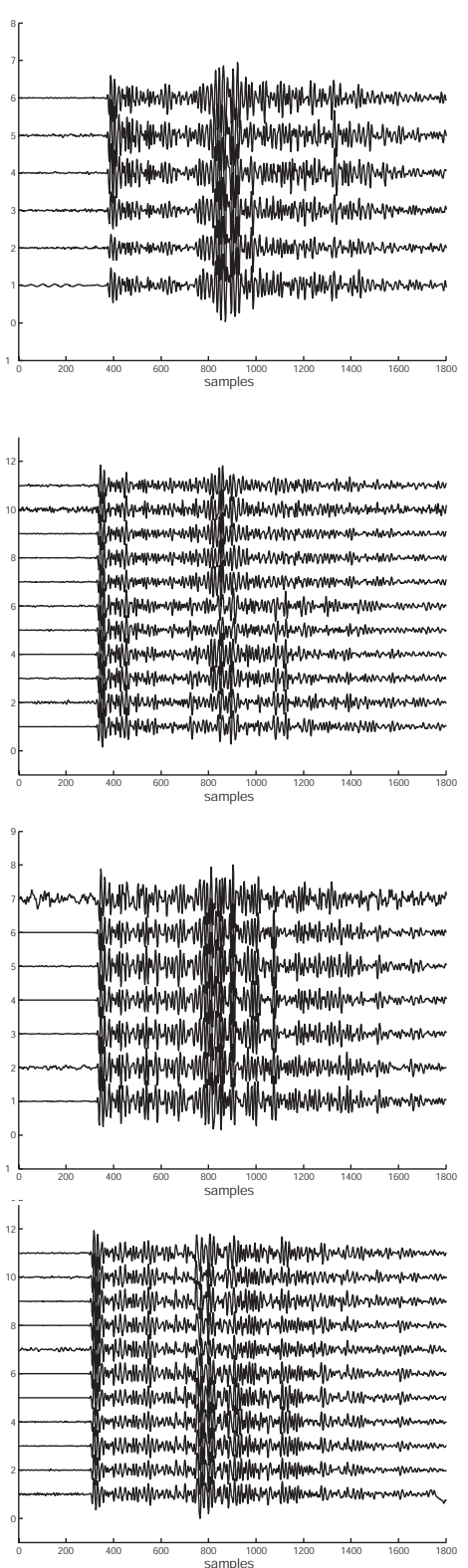

\section{Multiplet 25}

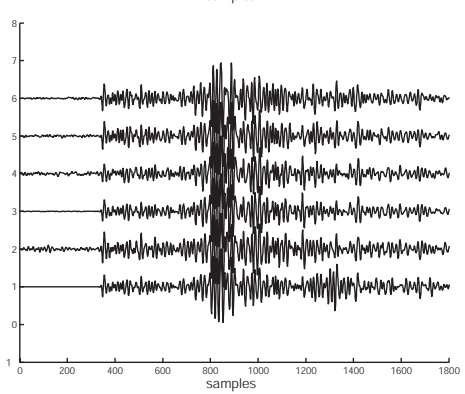

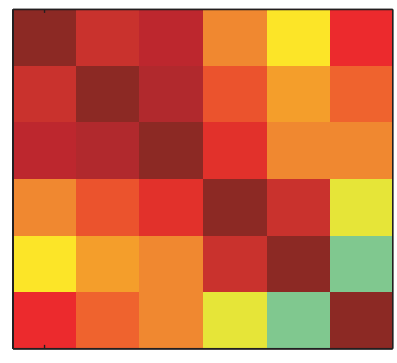
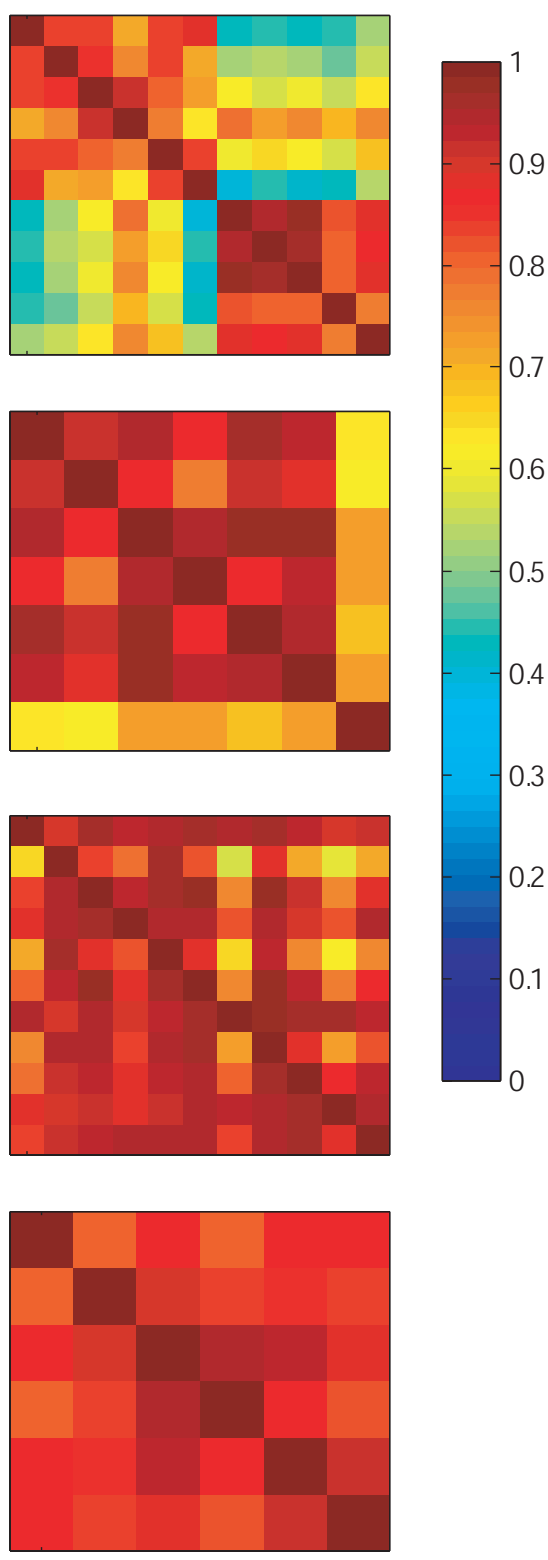

Figure F.5. Waveforms of earthquakes belonging the multiplets, recorded at station TUVZ and filtered 1-10 Hz, and cross-correlation matrices for the multiplets using the whole waveform. Colours represent cross-correlation coefficient. 
Multiplet 26
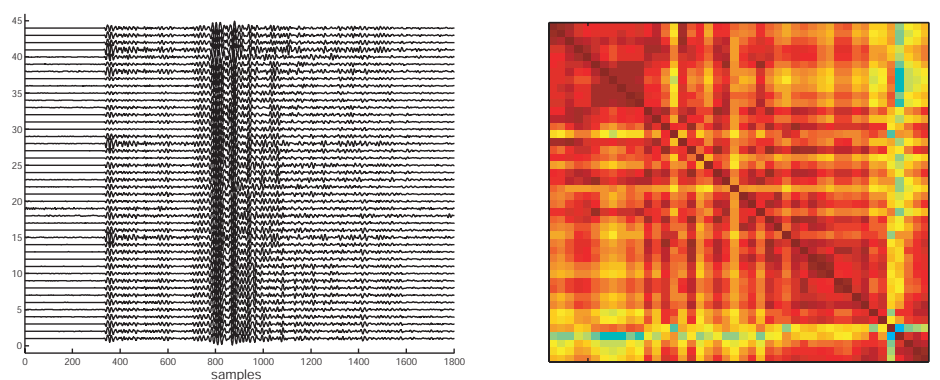

Multiplet 27
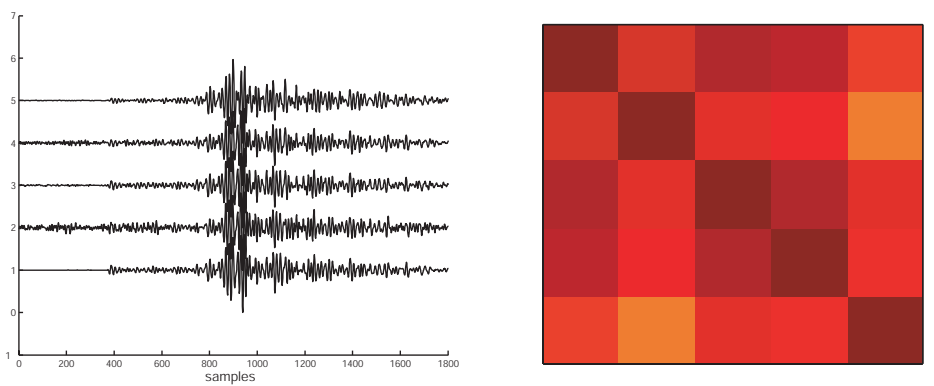

\section{Multiplet 28}

\section{Multiplet 29}
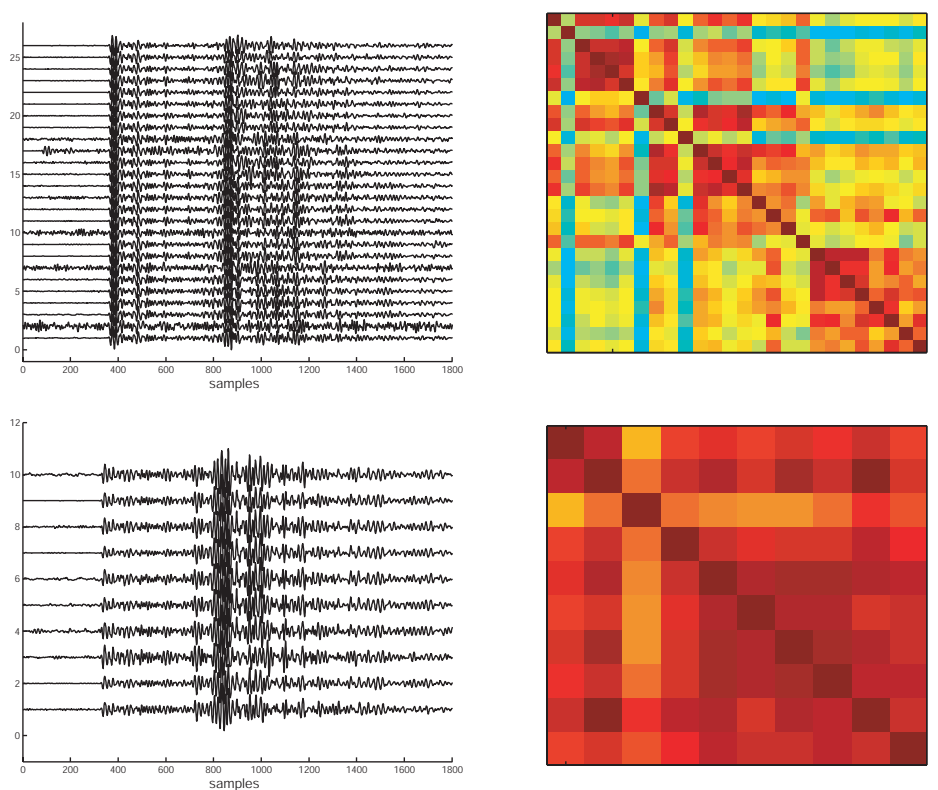

Figure F.6. Waveforms of earthquakes belonging the multiplets, recorded at station TUVZ and filtered 1-10 Hz, and cross-correlation matrices for the multiplets using the whole waveform. Colours represent cross-correlation coefficient. 


\section{G. The Erua earthquake cluster and seismic anisotropy in the Ruapehu region, New Zealand}

Keats, B. S., J. H. Johnson \& M. K. Savage (2011) The Erua earthquake cluster and seismic anisotropy in the Ruapehu region, New Zealand. Geophys. Res. Lett., vol. 38, L16315, doi:10.1029/2011GL049014¹.

\section{Abstract}

We use seismicity generated from the Erua earthquake cluster (a consistently active area of seismicity about $20 \mathrm{~km}$ to the west of Mount Ruapehu) over the last 12 years to study seismic anisotropy in the Ruapehu region. In particular, we search for changes associated with two minor phreatic eruptions on the 4th of October 2006 and the 25th of September 2007. The seismicity rate, magnitude of completeness, focal mechanisms and b-value of the cluster are also examined to investigate whether the characteristics of the seismicity changed over the duration of the study. The earthquakes were relocated using hypoDD, and were found to show some shallow structure with a westward dip in the depth of shallow seismicity. Shear wave splitting results revealed a decrease in delay time in the 2006-2007 period at all stations and a significant variation in the fast shear wave orientation at one station in the same time period. The b-value also increased significantly from $1.0 \pm 0.2$ in 2004 to a peak of $1.8 \pm 0.2$ in 2007 , but no other parameters were found to vary significantly over this time period. We attribute these changes to an increase in pore-fluid pressure in the Erua region due to fluid movement and suggest that this fluid movement may be associated with the eruptions in 2006 and 2007.

\footnotetext{
${ }^{1}$ Based on Brook Keats' Honors Thesis, the manuscript from which I prepared for publication and was corresponding author
} 


\section{G.1. Introduction}

Understanding the temporal evolution of the stresses underlying tectonic processes remains one of the fundamental goals of geophysics. The large changes in stress accompanying magma movement around volcanoes may cause changes in seismic properties, and understanding the relationship between changes in different processes is one of the ways by which we can ultimately understand volcanic activity and mitigate hazards [Roman et al., 2006]. Here we examine Mount Ruapehu, an andesitic stratovolcano in the centre of the North Island of New Zealand at the southern end of the Taupo Volcanic Zone (TVZ). Large magmatic eruptions have occurred several times over the last century, the largest of which were a series of phreatomagmatic-magmatic eruptions in 1995-1996 [Johnston et al., 2000]. Minor phreatic and phreatomagmatic eruptions are also relatively common, the most recent of which occurred on the 4th of October 2006 and on the 25th of September 2007 [Jolly et al., 2010; Mordret et al., 2010].

A shear wave in an anisotropic medium will be split into a fast and slow component, with the fast orientation $\phi$ and delay time $\delta t$. Seismic anisotropy in the Earth's crust can be caused by alignment of minerals, layering of materials, fractures or stress-aligned microcracks [Crampin, 1994]. An applied stress field can cause microcracks to preferentially open parallel to the maximum compressive stress, causing the medium to become seismically anisotropic. This mechanism is the only one that allows seismic anisotropy to vary on observable time scales [Crampin and Zatsepin, 1997].

Previous studies by Miller and Savage [2001] and Gerst and Savage [2004] have found that the 1995-1996 eruptions of Ruapehu were accompanied by a change in seismic anisotropy. Liu et al. [2004] demonstrated that spatial variations in seismic sources can be misinterpreted as temporal changes in anisotropy as different ray paths sample different regions of the anisotropic medium. We use seismicity from the Erua earthquake cluster, a consistently active area of seismicity about $20 \mathrm{~km}$ to the west of Ruapehu, to measure seismic anisotropy over the last 12 years. Restricting the location of the seismic sources (earthquakes) to a cluster minimises these spatial variations to ensure observed changes are legitimately temporal. We compare the anisotropy with relocated hypocentres and b-values. 


\section{G.2. Data}

Earthquakes from the Erua earthquake cluster were recorded on the GeoNet permanent seismic network in the Ruapehu region [www.geonet.org.nz]. We have defined the Erua earthquake cluster as a rectangle bounded by the latitudes $39.200^{\circ} \mathrm{S}$ and $39.283^{\circ} \mathrm{S}$, and the longitudes $175.250^{\circ} \mathrm{E}$ and $175.467^{\circ} \mathrm{E}$ (Figure G.1). A total of 283 earthquakes with local magnitude $\left(M_{L}\right)$ greater than 2 were recorded in this area on GeoNet's national seismograph network between March 1998 and June 2010. Four stations (FWVZ, MOVZ, TWVZ and WNVZ) were selected for analysis based on their location, spatial distribution and period of operation. There were 242 crustal earthquakes at depths shallower than $40 \mathrm{~km}$, and 41 deep earthquakes (70-250 km) in the Wadati-Benioff zone, created by the subduction of the Pacific plate under the Australian plate beneath the North Island of New Zealand. Due to a low velocity surface layer, incidence angles of all measurements were less than the critical angle at which S-P conversions can interfere with the waveform [Nuttli, 1961].

\section{G.3. Method}

The earthquakes in the Erua cluster were relocated using hypoDD [Waldhauser, 2001], a double difference earthquake relocation algorithm. The algorithm was applied to catalogue phase data and differential times obtained with the Bispectrum Cross-correlation package for SEISmic events [BCSEIS, Du et al., 2004a]. The weightings in hypoDD were set so that catalogue picks were weighted heavily for the initial iterations and were significantly down-weighted later, while the cross correlation times were weighted weakly at the beginning and heavily at the end. This technique constrained the relative positions without sacrificing highly accurate cross correlation data [Waldhauser, 2001]. The velocity model used for the relocation algorithm is from Hurst and McGinty [1999] (see Table G.1). To improve azimuthal coverage of the stations for the relocations, three stations to the west of the cluster (HIZ, VRZ and WAZ) were included in the analysis (inset of Figure G.1).

We used the method of shear wave splitting (SWS) analysis to obtain measurements of seismic anisotropy. An automated program developed by Savage et al. [2010b], and based on the algorithms of Silver and Chan [1991] and Teanby et al. [2004a], was used to perform all SWS measurements in this study. The program grades each measurement and marks any null measurements in which no splitting result 


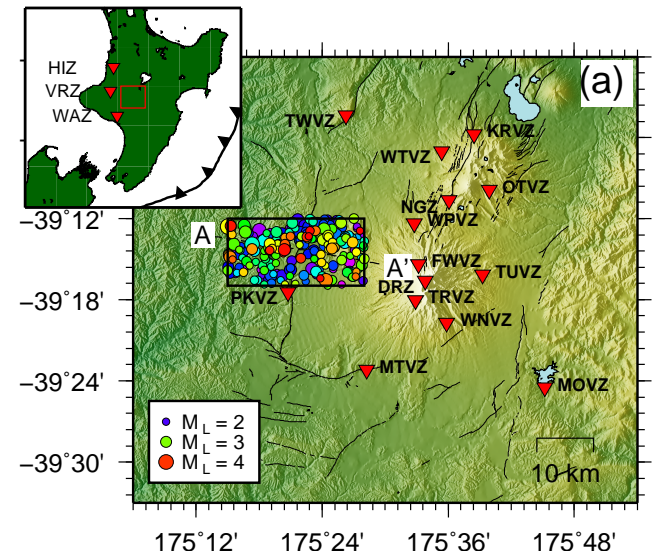

(b)
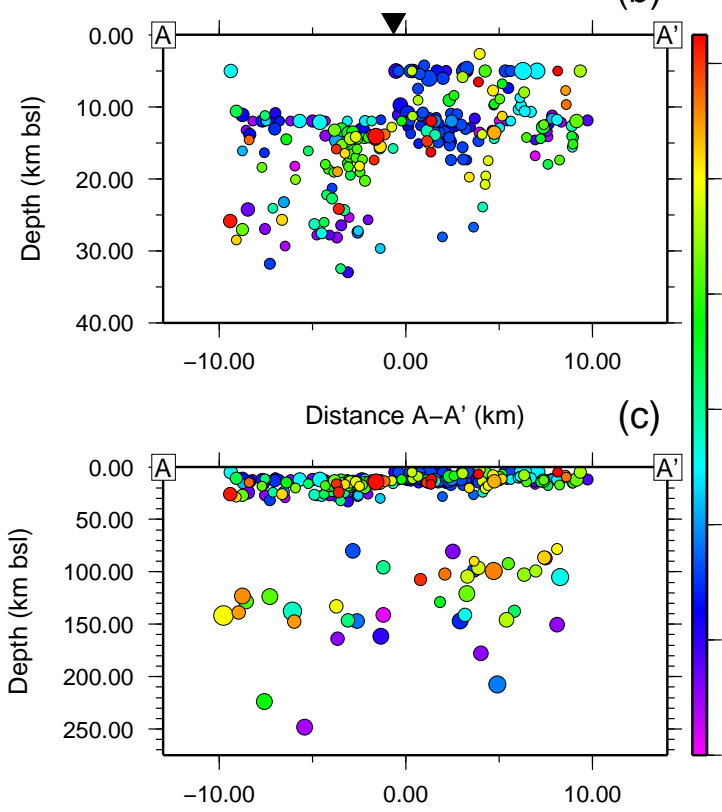

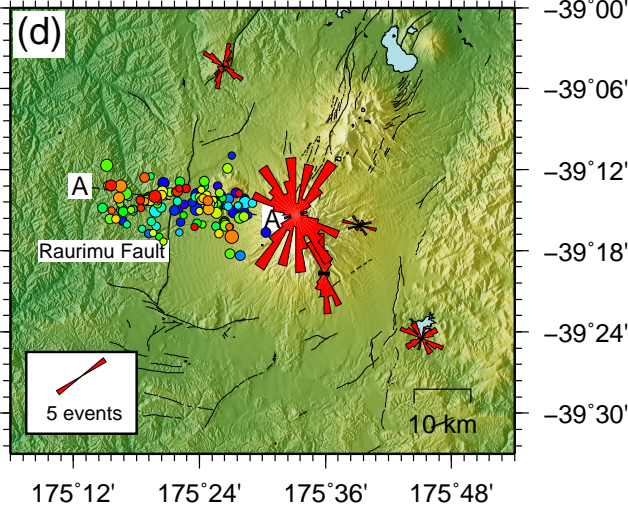

(e)
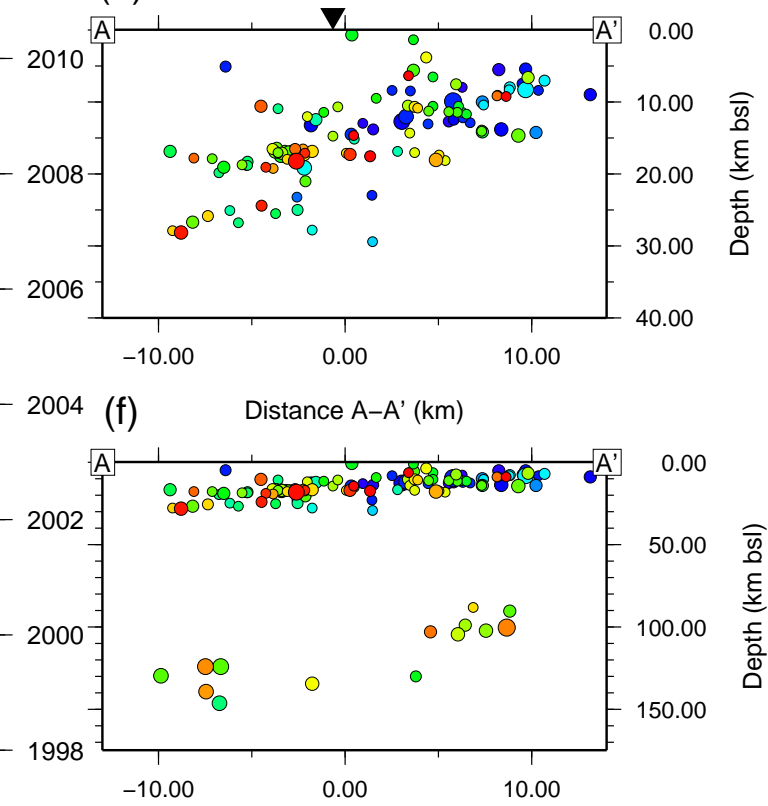

Figure G.1. (a) Map of the Ruapehu region showing seismic stations in GeoNet's permanent network (red inverted triangles). Catalogue earthquake locations in the Erua earthquake cluster are colour coded by origin time and scaled by magnitude, and active faults from GNS Science active fault database are displayed in black. Inset shows study region in New Zealand and three additional stations. (b) Cross section along A-A' showing catalogue locations and depths of earthquakes in the Erua earthquake cluster shallower than $40 \mathrm{~km}$. Black inverted triangle marks the location of the surface expression of the Raurimu fault. (c) Cross section along A-A' showing catalogue locations and depths of all earthquakes in the Erua earthquake cluster. (d) Map of the Ruapehu region showing shear wave splitting $\phi$ results from shallow $(<40 \mathrm{~km})$ earthquakes in the Erua cluster plotted as red rose diagrams (circular histograms) at the station that they were recorded and scaled by the number of measurements. Relocated earthquakes are colour coded by origin time and scaled by magnitude. (e) Cross section along $\mathrm{A}-\mathrm{A}^{\prime}$ showing earthquake relocations of earthquakes shallower than $40 \mathrm{~km}$. Black inverted triangle marks the location of the surface expression of the Raurimu fault. (f) Cross section along $\mathrm{A}-\mathrm{A}^{\prime}$ showing relocations. 
Table G.1. Velocity model used during earthquake relocation [after Hurst and McGinty, 1999]. $v_{P} / v_{S}$ is 1.73 .

\begin{tabular}{ll}
\hline $\begin{array}{l}\text { Depth to bottom of layer } \\
(\mathrm{km} \text { b.s.l. })\end{array}$ & $\begin{array}{l}\mathrm{P} \text { wave velocity } \\
(\mathrm{km} / \mathrm{s})\end{array}$ \\
\hline 1.5 & 3.2 \\
5.5 & 5.5 \\
14.5 & 5.95 \\
32.5 & 6.5 \\
halfspace & 8.1 \\
\hline
\end{tabular}

is obtained. Only non-null results with a measurement grade of A or B and delay time smaller than $0.5 \mathrm{~s}$ were included. Measurements that differed substantially across filters were removed, and at most one measurement is presented for each earthquake-station pair. Refer to Savage et al. [2010b] for details on these quality control steps.

The seismicity rate, magnitude of completeness $\left(M_{c}\right)$, and b-value of the Erua earthquake cluster were examined with time using ZMAP [Wiemer, 2001]. The magnitude of completeness is calculated for the whole catalogue using the Maximum curvature method. The uncertainty on $M_{c}$ was calculated using 100 bootstrap calculations. A catalogue of events with $M_{L} \geq 2$ was used to calculate a moving b-value with time. The b-value was calculated using the maximum likelihood method and plotted against time using a window of 40 events and a five event overlap (Figure G.2). The uncertainty on the b-value was also calculated by bootstrapping.

\section{G.4. Results}

\section{Earthquake Relocation}

Using hypoDD, $87 \%$ of the earthquakes in the catalogue were relocatable (Figure G.1). Average hypocenter uncertainties were $44.6 \mathrm{~m}, 47.0 \mathrm{~m}$ and $109.9 \mathrm{~m}$ in the $\mathrm{x}, \mathrm{y}$ and $\mathrm{z}$ directions respectively calculated with the singular value decomposition (SVD) method.

Relocated earthquakes in the subducted slab showed earthquake depths around 100$150 \mathrm{~km}$ and depth increasing to the west along the direction of the dip of the slab.

Earthquake depths within the shallow cluster gradually increased to the west. All earthquakes were shallower than $20 \mathrm{~km}$ on the eastern side of the Raurimu fault but 
Table G.2. A summary of the statistical analysis results for each time period at station FWVZ, showing the average value and $90 \%$ confidence interval for the fast orientation $\phi$ and delay time $\delta t$. The $\bar{R}$ value is a statistical measure of dispersion for circular datasets. If this value is above a critical value $\left(R_{\text {crit }}\right)$, which is a function of the number of measurements $(n)$, then a preferential orientation is present for data following a von Mises distribution [Davis, 1986].

\begin{tabular}{ccccc}
\hline Parameter & Period 1 & Period 2 & Period 3 & Period 4 \\
\hline Time & $1999-001-2000-200$ & $2001-001-2004-320$ & $2005-150-2006-300$ & $2007-150-2011-001$ \\
$n$ & 15 & 22 & 16 & 14 \\
$\phi\left(^{\circ}\right)$ & $3.0 \pm 12.1$ & $-36.8 \pm 18.6$ & $46.3 \pm 10.2$ & $-50.5 \pm 23.3$ \\
$\delta t(s)$ & $0.109 \pm 0.024$ & $0.122 \pm 0.020$ & $0.083 \pm 0.015$ & $0.137 \pm 0.037$ \\
$\bar{R}$ & 0.625 & 0.372 & 0.692 & 0.372 \\
$R_{\text {crit }}$ & 0.391 & 0.323 & 0.379 & 0.405 \\
\hline
\end{tabular}

on the western side some were deeper than $30 \mathrm{~km}$ and only one was shallower than $10 \mathrm{~km}$.

\section{Shear Wave Splitting}

SWS results were calculated using deep and shallow sources at individual stations. The orientation of $\phi$ for deep events did not vary significantly with time at any of the stations analysed [Keats, 2010].

The results for shallow earthquakes are displayed as red rose diagrams in Figure G.1 (d). These results were more numerous and varied between stations. At FWVZ, the station operating for the longest period of time, there were considerably more SWS results than for other stations and an interesting variation in $\phi$ and $\delta t$ with time was observed (Figure G.2). Around $2005 \phi$ changed significantly and $\delta t$ also decreased. Both parameters appeared to have reverted back to their original values by mid-2007. These changes are of interest because they precede the two minor eruptions at Ruapehu in late 2006 and late 2007.

There are some gaps in the plot, which are due to the lack of good shear wave splitting measurements at these times, which in turn are caused by a combination of fewer earthquakes, noisier waveforms, and more null measurements (Figure G.3). These factors could be partially caused by processes leading up to the eruption, but we do not have enough other evidence to be definitive.

The results at FWVZ were divided into four time periods based on the changes observed (Figure G.2). These periods were statistically analysed for significance (Table G.2). The analysis showed a preferential orientation in $\phi$ in the first, second 


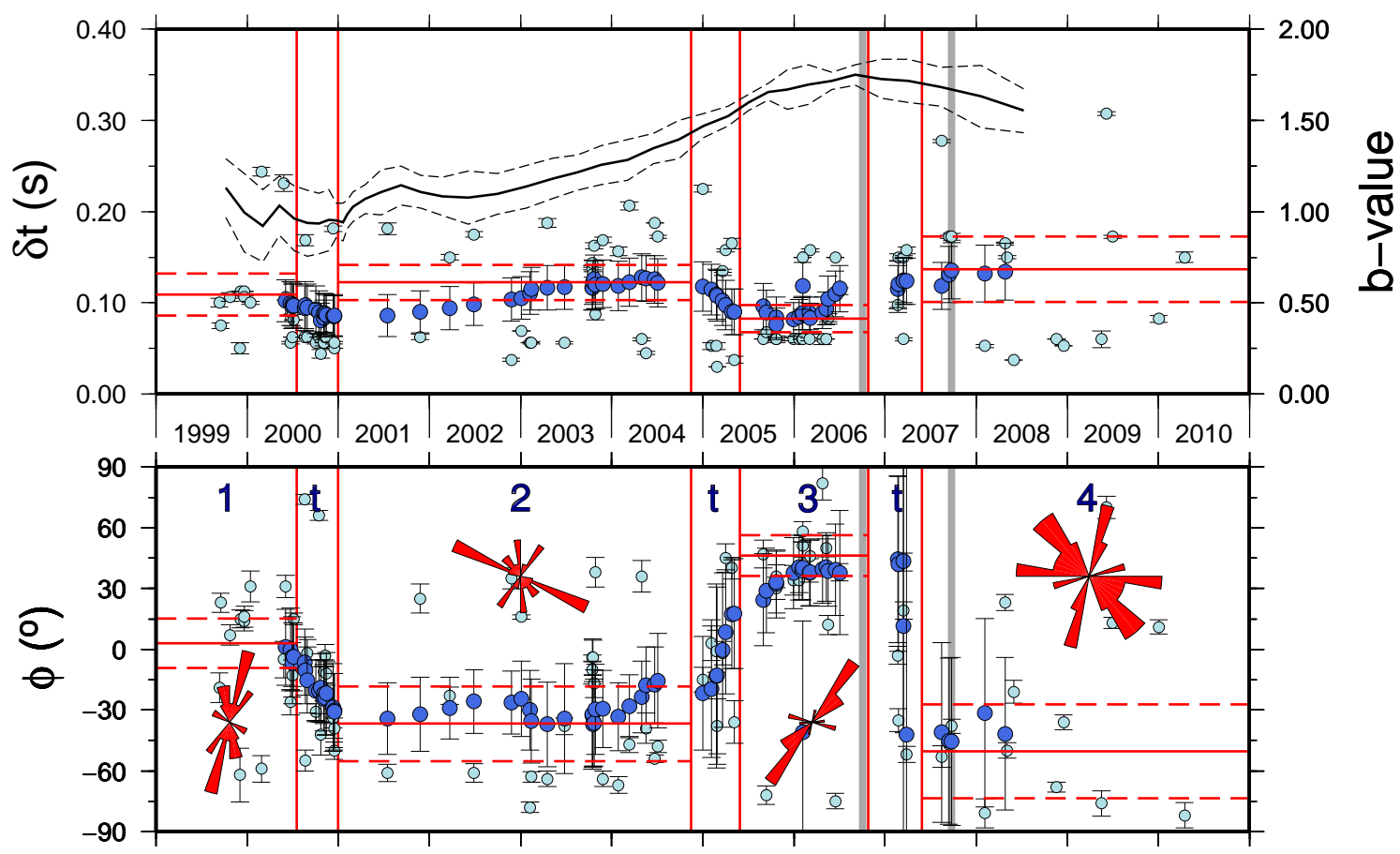

Figure G.2. Moving average plot of fast polarisation $(\phi)$ and delay time $(\delta t)$ using earthquakes within the Erua swarm at station FWVZ. Individual measurements for $\phi$ and $\delta t$ are displayed in light blue and 10 point moving averages are displayed in dark blue. The error bars indicate $95 \%$ confidence intervals. The four time periods, marked by the numbers $1-4$, and three transition zones, marked by a $\mathbf{t}$, are indicated with vertical red lines and the mean for each period are shown by the red horizontal bars with $90 \%$ confidence interval (dashed red lines). The times of the two phreatomagmatic eruptions that occurred are also marked with grey bars. Rose diagrams of $\phi$ are displayed in their respective time periods. The b-value for the Erua swarm catalogue is also plotted against time in black at the top using a window of 40 events and an 8 event overlap. Dashed black lines indicate $95 \%$ confidence interval. 


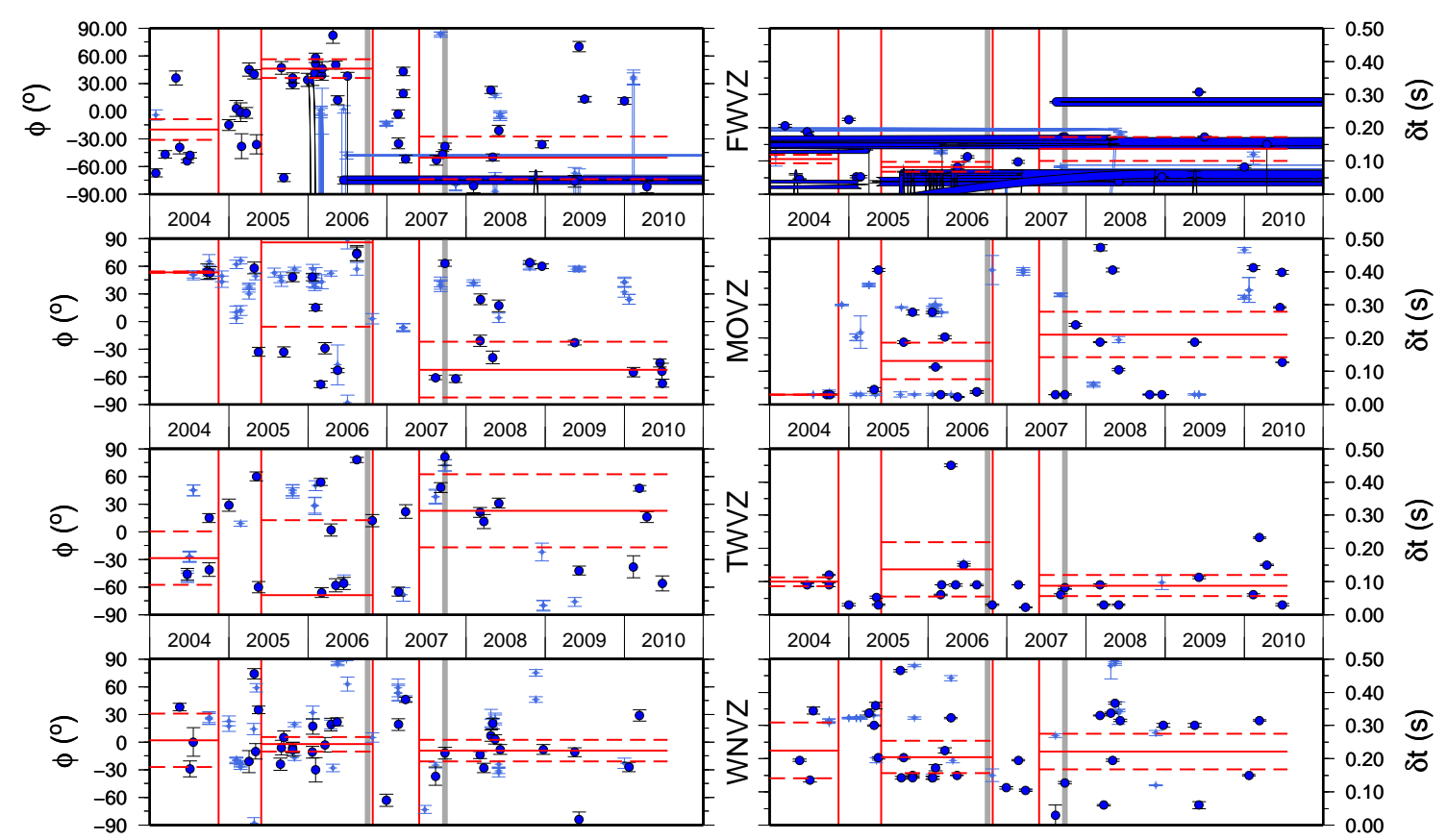

Figure G.3. Moving average results at stations FWVZ, MOVZ, TWVZ and WNVZ from 2004 to 2011. Individual measurements for $\phi$ and $\delta t$ are displayed in dark blue and null results are in pale blue. The error bars indicate $95 \%$ confidence intervals. Grey vertical bars indicate the times of the two phreatomagmatic eruptions. Red vertical bars indicate the same time periods as those in Figure G.2 and Table G.2. Red horizontal bars indicate the mean values with $90 \%$ confidence interval (dashed lines) for each time period. The reason that few nulls appear on the plot of $\delta t$ at TWVZ is that they have values that are larger than $0.5 \mathrm{~s}$ and so are not plotted.

and third time periods following a von Mises distribution [Davis, 1986]. The fourth time period had no preferred orientation. The mean value of $\phi$ changed significantly at the $90 \%$ confidence level from $-36.8 \pm 18.6^{\circ}$ to $46.3 \pm 10.2^{\circ}$ between periods two and three before becoming more scattered in period four. This change in $\phi$ was accompanied by a decrease in $\delta t$ from $0.122 \pm 0.020 \mathrm{~s}$ to $0.083 \pm 0.015 \mathrm{~s}$ between the second and third time period and an increase back to $0.137 \pm 0.037 \mathrm{~s}$ in the fourth time period. The moving averages in Figure G.2 display an apparent increase in $\delta t$ at FWVZ before the 2006 eruption. However, this is an artefact of the moving average window as it includes data from the other time periods and so smoothes the transitions, as can be seen from the data with no moving average plotted in Figure G.3.

No significant variations in $\phi$ or $\delta t$ were observed at stations MOVZ, TWVZ and WNVZ using the same time periods (Figure G.3). 


\section{$M_{c}$ and $b$-value}

The magnitude of completeness was found to be $M_{c}=1.6 \pm 0.05$, so the dataset of earthquakes with $M_{L} \geq 2$ that was used can be considered to be complete.

For crustal earthquakes b-values typically have values of $\sim 1$ for tectonic earthquakes, though they tend to be higher in volcanic regions [Wiemer and Wyss, 2002]. Figure G.2 shows that the b-value of the swarm changes systematically with time. B-values began to increase significantly in 2004 from $\sim 1$ up to a peak of $\sim 1.8$ at the end of 2006 before beginning to decrease again.

\section{G.5. Discussion and conclusions}

The Erua earthquake cluster lies around the Raurimu fault, a north-south oriented normal fault that is down-thrown to the east [Villamor and Berryman, 2006]. Seismicity in the cluster does not however, seem to be generated from this fault, with earthquake locations distributed evenly around it. The step in shallow seismicity (Figure G.1) showed shallower earthquakes on the down-thrown eastern side, indicating that the step is not due to a seismogenic structure displaced by the fault. The Raurimu fault has been interpreted to be a shallow structure $(\sim 100 \mathrm{~m})$ [Horspool, 2003]. It is therefore not surprising that there is no seismic expression of the Raurimu fault at depth. The shallow seismicity on the east of the Raurimu fault is typical of the TVZ. The transition in the depth of the earthquakes is likely due to the change in geothermal gradient from within the TVZ to the cooler, thicker crust outside the TVZ. The deeper seismicity to the west may be part of a system known as the Taranaki-Ruapehu line, a line of earthquakes thought to be due to high strain rates associated with the rapid change in material properties across a step in crustal thickness [Salmon et al., 2011].

At station FWVZ a significant rotation of $\phi$ and decrease in $\delta t$ was observed preceding the 2006 and 2007 eruptions. Examination of the earthquakes with time showed that locations within the cluster were sufficiently random with no migration occurring (Figure G.1). Small seismogenic zones within the Erua cluster also returned changing $\phi$ over time, confirming that the observed changes at FWVZ were not due to changes in the source location (Figure G.4). Sherburn et al. [2009] created a catalogue of earthquake focal mechanisms across New Zealand between 2004 and 2009, 31 of which were in the region of the Erua earthquake cluster. The focal 


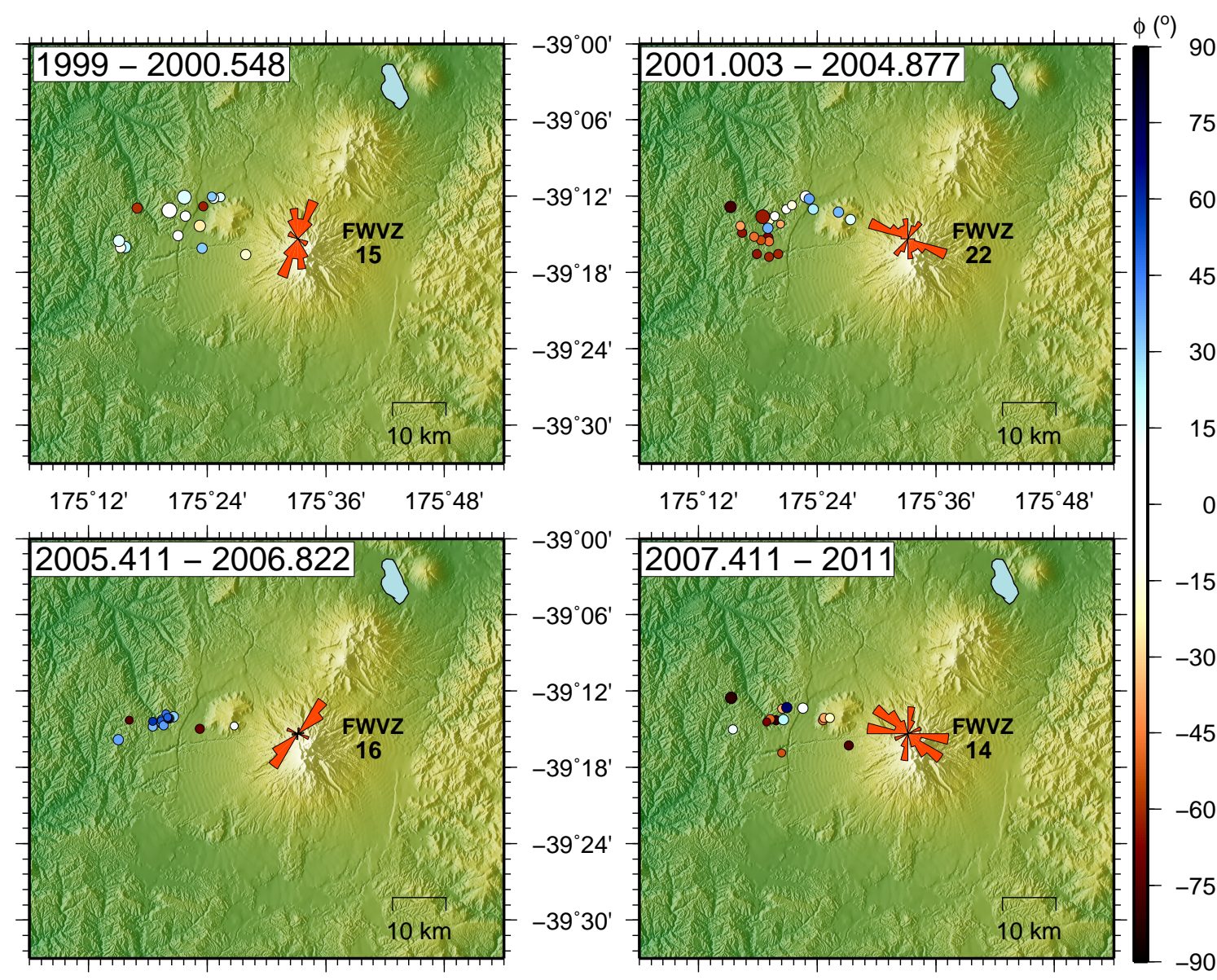

Figure G.4. Maps of earthquake location, scaled by magnitude and coloured by fast direction of anisotropy $(\phi)$ recorded at station FWVZ. Panels show data for each of the time periods from in Figure G.2 and Table G.2. Red rose diagrams summarise the $\phi$ measurements for each time period.

mechanisms show predominant normal faulting with no obvious change in source mechanism with time.

Variations in the seismicity rate and b-values of nearby earthquake swarms were observed at Mount Ruapehu accompanying the 1995-1996 eruptions [Hurst and McGinty, 1999; Hayes et al., 2004]. Changes in seismicity at proximal swarms have also been observed at other volcanoes around the world such as Augustine Volcano in Alaska [Jacobs and McNutt, 2010], and Unzen and Kuju volcanoes in Japan [Shimizu et al., 1992; Sudo et al., 1998]. Using the Erua cluster, we do not observe an increase in the seismicity rate in the period around the 2006 and 2007 eruptions, yet the increase in b-value beginning in 2004 indicates that the nature of the seismicity changed before and during the eruptive period with an increase in the number of low magnitude earthquakes relative to the number of high magnitude earthquakes. An increase in b-value is expected to accompany an increase in pore-fluid pressure or an increase in thermal gradient [Jacobs and McNutt, 2010]. An increase in pore-fluid 
pressure would also decrease the crack aspect-ratio [Zatsepin and Crampin, 1997], therefore making the rock more isotropic and hence account for the smaller $\delta t$ s from the SWS results. This effect could also account for the variation in $\phi$ observed at FWVZ: In periods two and four, $\phi$ had an orientation similar to the local stress field found by Sherburn et al. [2009]. We therefore infer that the anisotropy was caused by stress-aligned microcracks at these times. During period three, $\phi$ changed to a significantly different orientation. The orientation of $\phi$ in period three is sub-parallel to the Raurimu fault, indicating that the stress-induced orientation of $\phi$ could have been replaced by structurally controlled anisotropy. This would not be observed at stations farther from the cluster because there was strong anisotropy local to those stations (Chapter 4). Results using deep (>70 km) earthquakes would also fail to display a change in SWS parameters because the changes occur in the crust near the hypocenters of the shallow $(<40 \mathrm{~km})$ earthquakes, which is unsampled when using the deeper earthquakes (Figure G.1). We do not see seismicity on the Raurimu fault induced by the increase in pore-fluid pressure because the fault is much shallower than the hypocenters [Horspool, 2003].

A similar mechanism was proposed by Crampin et al. [2002] to explain " $90^{\circ}$-flips" in $\phi$. They suggest that as the pore-fluid pressure approaches the maximum horizontal stress and the crack aspect-ratio decreases, the delay times will approach zero. At this point the anisotropy becomes negative and the fast direction will flip $90^{\circ}$ to $\mathrm{S}_{H \max }$. However, according to this model the anisotropy will continue to be increasingly negative with increasing pore-fluid pressure up to about $2 \%$ anisotropy. The reason that the mechanism presented in this paper differs is because we observe a transition period of several months between the two dominant fast orientations and we observe the changes in delay time in the transition periods, and stable delay times when $\phi$ is stable. If this were a "flip" mechanism, we should observe decreasing delay time with a stable fast direction until a threshold delay time and then a sudden change to a direction which is orthogonal and an accompanying increase in delay time. In general these two mechanisms are very similar in causation but have different outcomes.

Other temporal changes in SWS parameters at volcanoes have suggested that the local stress changes due to magma emplacement. Miller and Savage [2001], Gerst and Savage [2004] and Roman et al. [2011] observed a rotation of $\phi$ attributed to a rotation of maximum horizontal stress. Bianco et al. [2006] and Volti and Crampin [2003] both observed increasing delay times prior to volcanic eruption, suggesting increasing differential stress, rather than increasing pore-fluid pressure. We do not 
find evidence that either of these models are appropriate for the data presented here.

Mordret et al. [2010] reported decreases in isotropic Rayleigh wave speed, using noise cross-correlations, between some station pairs in two-week periods at the time of the 2006 and 2007 eruptions, suggesting that cracks opened or filled with fluids around that period. The paths with the most significant Rayleigh wave speed variations did not coincide with the paths that contain the biggest decreases in delay time that we see, although it is likely that our observations have the same mechanism.

We propose that the variations in b-value, $\phi$ and $\delta t$ observed in this study were due to fluid movement associated with volcanic activity in 2006 and 2007, similar to that in 1995-1996 [Hayes et al., 2004]. In this model we propose that it is the regional fluid movement that affected both the Erua earthquake swarm and the magmatic system at Mt. Ruapehu, rather than volcanic activity affecting the fluid movement in the region. This fluid movement led to an increase in pore-fluid pressure in the Erua region. The temporal changes in seismic anisotropy observed indicate that monitoring seismic anisotropy as part of an eruption forecasting system holds potential. The nature of seismic swarms near active volcanoes seems to be linked to volcanic activity and should be taken into consideration in the monitoring process. 